Supporting Information

\title{
Modular Total Synthesis of ISO-ARChazolids AND ARCHAZOLOGS
}

Stephan Scheeff, Solenne Rivière, Johal Ruiz, Simon Dedenbach and Dirk Menche*

Kekulé-Institut für Organische Chemie und Biochemie, Universität Bonn Gerhard-Domagk-Str. 1, D-53121 Bonn, Germany

*Corresponding author: dirk.menche@uni-bonn.de 


\section{Contents}

1 Additional Information to chemical synthesis and compound characterization data S3

1.1 Analytical Setup . . . . . . . . . . . . . . . . . . . . . . . . . . S3

1.2 Time resolved NMR studies for the isomerization of archazolid B to F . . . . . . . . . . . . . . S4

1.3 Additional analytical data . . . . . . . . . . . . . . . . . . . . . . . . . . S9

1.3.1 NOE data for compound $95 \ldots \ldots \ldots$. . . . . . . . . . . . . . . . . . . . . . . . S9

1.3.2 Determination of purity of compound 25a . . . . . . . . . . . . . . . . . . . . S10

1.3.3 NOE data for compound 33a . . . . . . . . . . . . . . . . . . . . . . . S11

1.3.4 Mosher ester analysis for compound 53e . . . . . . . . . . . . . . . . . . . . . . . . . S11

1.3 .5 NOE data for compound 63 . . . . . . . . . . . . . . . . . . . . . . . . . . . . . . . . . S12

1.3.6 Mosher ester analysis for compound 65a . . . . . . . . . . . . . . . . . . . . . . S13

1.3.7 NOE data for compound 64 . . . . . . . . . . . . . . . . . . . . . . . . . . . . . . S14

1.3.8 NOE data for compound 75 . . . . . . . . . . . . . . . . . . . . . . . . . . . . . S15

1.4 Determination of relative configuration of iso archazolid $\mathrm{A} 1$ and $\mathrm{A} 2 \ldots \ldots$. . . . . . . . . . . . . S15

1.4 .1 Predicted Parameters . . . . . . . . . . . . . . . . . . . . . . . . . . . . . . . . . . . . . . .

1.4 .2 Experimental Parameters . . . . . . . . . . . . . . . . . . . . . . . . S17

1.4 .3 Copies of Calculated Clusters . . . . . . . . . . . . . . . . . . . . . . . . . S20

2 Copies of NMR spectras $\quad$ S37 


\section{Additional Information to chemical synthesis and compound characterization data}

\subsection{Analytical Setup}

HPLC setup Semi-preperative and analytical HPLC analyses were perfomed on Knauer Wissenschaftliche Geräte $\mathrm{GmbH}$ systems. The solvents for HPLC were purchased in HPLC grade. The spectra were recorded by UV-detection at $230 \mathrm{~nm}$. Ratios between the starting material and the product were obtained by comparison integral of the area of each peak.

Table S1: HPLC configuration for analytical HPLC.

\begin{tabular}{l|l|l}
\hline & System A & System B \\
\hline series & PLATINblue & Azura \\
pumps & binary, HPG P1 system, $10 \mathrm{~mL}$ & binary, HPG P 6.1L, $10 \mathrm{~mL}$ \\
pressure & 750 bar & 700 bar \\
autosampler & AS1 with $10 \mu \mathrm{L}$ injection loop & 3950 with $100 \mu \mathrm{L}$ injection loop \\
mixing chamber & static, SmartMix $350 \mu \mathrm{L}$ & integrated static mixing chamber, $100 \mu \mathrm{L}$ \\
column heater & T1 & CT 2.1 \\
detection type & PDA UV/VIS detection PDA1, $\mathrm{D}_{2} / \mathrm{Hg}$ halo- & $\begin{array}{l}\text { PDA UV/VIS detection DAD } 6.1 \mathrm{~L}, \mathrm{D}_{2} / \mathrm{Hg} \\
\text { halogen lamps, 190-1020 nm }\end{array}$ \\
degasser & gen lamps, 190-1000 nm & analytical 2-channel-online-degasser \\
\hline
\end{tabular}

Table S2: HPLC configuration for analytical HPLC.

\begin{tabular}{l|l}
\hline & System C \\
\hline series & PLATINblue \\
pumps & binary, HPG P1 system, $5 \mathrm{~mL}$ \\
pressure & 1000 bar \\
autosampler & AS1 with $10 \mu \mathrm{L}$ injection loop \\
mixing chamber & static, SmartMix $100 \mu \mathrm{L}$ \\
column heater & T1 \\
detection type & $\begin{array}{l}\text { PDA UV } / \mathrm{VIS} \text { detection PDA1, } \mathrm{D}_{2} / \mathrm{Hg} \text { halo- } \\
\text { gen lamps, 190-1000 } \mathrm{nm} \\
\text { degasser }\end{array}$ \\
\hline
\end{tabular}


Table S3: HPLC configuration for preparative HPLC.

\begin{tabular}{l|l|l}
\hline & System D & System E \\
\hline series & Azura & Smartline \\
pumps & binary, HPG P 6.1L, 50 mL & binary, S-1800, 100 mL \\
pressure & 200-300 bar & 400 bar \\
autosampler & assistant ASM 2.1L with a feed pump P 2.1S & assistant 6000 with a feed pump S-100 \\
mixing chamber & integrated static mixing chamber, 200 $\mu \mathrm{L}$ & static, SmartMix 350 $\mu \mathrm{L}$ \\
detection type & MWL 2.1L, 190-700 $\mathrm{nm}$ & UV-detector S-2550, 190-600 nm \\
degasser & preparative 2-channel-online-degasser & preparative 2-channel-online-degasser \\
\hline
\end{tabular}

Mass Analysis Mass spectra (MS) and High-resolution-mass spectra (HRMS) were recorded on the documented systems in Table S4 at the University Bonn under supervision of Dr. Marianne Engeser.

Table S4: Used MS systems for MS and HRMS.

\begin{tabular}{|c|c|c|}
\hline name & manufacturer & measurements \\
\hline MAT $95 \mathrm{XL}$ & Thermo Finnigan (Bremen) & El \\
\hline MAT 90 & Thermo Finnigan (Bremen) & EI, LIFDI, CI, FAB \\
\hline MALDI autoflex II TOF/TOF & Bruker Daltonik (Bremen) & MALDI \\
\hline micrOTOF-Q & Bruker Daltonik (Bremen) & $\begin{array}{l}\text { ESI, APCI, nano-ESI, } \\
\text { MS/MS, LC-MS, DC- } \\
\text { MS }\end{array}$ \\
\hline Orbitrap XL & Thermo Fisher Scientific (Bremen) & $\begin{array}{l}\text { ESI, APCI, APPI, } \\
\text { nano-ESI }\end{array}$ \\
\hline Apex IV FT-ICR & Bruker Daltonik (Bremen) & $\begin{array}{ll}\text { ESI, } & \text { nano-ESI, } \\
\text { MALDI, EI, Cl }\end{array}$ \\
\hline
\end{tabular}

\subsection{Time resolved NMR studies for the isomerization of archazolid B to F}

\section{Procedure}

To a solution of archazolid B (2) $(1.5 \mathrm{mg}, 2.07 \mu \mathrm{mol}, 1.00 \mathrm{eq})$ in deuterated THF $(0.50 \mathrm{~mL})$ at room temperature in a NMR tube DBU $(0.5 \mathrm{mg}, 3.28 \mu \mathrm{mol}, 1.50 \mathrm{eq})$ was added. The screening of the reaction was obtained by ${ }^{1} \mathrm{H}-\mathrm{NMR}$ $(500 \mathrm{MHz})$ measurements at $298 \mathrm{~K}$.

Nine different integrals of the NMR spectra are used to determine the ratio between archazolid B (2) and F (3). Beside of the both archazolids and DBU no side-product or intermediate could be identified. For all collected NMR-spectra see page S38. 
Table S5: Selection of signals and signal range for kinetic examination of isomerization.

\begin{tabular}{l|c|c|l}
\hline signal & signal range [ppm] & archazolid & position \\
\hline 1 & $7.160-7.120$ & B & C4'-H $^{\prime}$ \\
2 & $7.100-7.055$ & F & C4'-H \\
3 & $5.175-5.100$ & B & C9-H \\
4 & $4.520-4.450$ & B & C15-H \\
5 & $4.450-4.395$ & F & C15-H \\
6 & $4.080-4.015$ & F & C7-H \\
7 & $4.015-3.935$ & B & C7-H \\
8 & $0.740-0.695$ & F & C8-CH3 \\
9 & $0.695-0.650$ & B & C8-CH3 \\
\hline
\end{tabular}

The integrals were determined with MestReNova software after automatic baseline correction. The percentages of the archazolids were obtained by adding the corresponding integrals and division through the sum of all integrals:

$$
\begin{gathered}
x_{B}=\frac{1}{5}\left(\int_{7.120}^{7.160} f(x) d x+\int_{5.100}^{5.175} f(x) d x+\int_{4.450}^{4.520} f(x) d x+\int_{3.935}^{4.015} f(x) d x+\frac{1}{3} \int_{0.650}^{0.695} f(x) d x\right) \\
x_{F}=\frac{1}{4}\left(\int_{7.055}^{7.100} f(x) d x+\int_{4.395}^{4.450} f(x) d x+\int_{4.015}^{4.080} f(x) d x+\frac{1}{3} \int_{0.695}^{0.740} f(x) d x\right) \\
{[\operatorname{archB}]=\frac{x_{B}}{x_{B}+x_{F}}} \\
{[\operatorname{arch} F]=\frac{x_{F}}{x_{B}+x_{F}}}
\end{gathered}
$$

The standard deviation was calculated by using following equation:

$$
s=\sqrt{\frac{n}{n-1} \sum_{i=1}^{n} x_{1}-\bar{x}}
$$

Table S6: Integrals of signals in the isomerization of archazolid B (2) to archazolid F (3).

\begin{tabular}{l|c|c|c|c|c|c|c|c|c}
\hline & \multicolumn{10}{c}{ signal } \\
time [min] & 1 & 2 & 3 & 4 & 5 & 6 & 7 & 8 & 9 \\
\hline 46.4 & 9.29 & 0.82 & 10.03 & 9.83 & 0.71 & 0.88 & 9.84 & 4.33 & 31.72 \\
69.4 & 9.20 & 1.46 & 9.86 & 9.74 & 1.31 & 1.58 & 9.71 & 6.45 & 31.58 \\
93.4 & 9.23 & 2.23 & 9.94 & 9.79 & 2.03 & 2.39 & 9.71 & 8.76 & 31.75 \\
116.4 & 9.20 & 3.04 & 9.92 & 9.80 & 2.79 & 3.29 & 9.75 & 11.17 & 31.75 \\
139.4 & 9.17 & 3.78 & 9.87 & 9.71 & 3.51 & 4.09 & 9.60 & 13.71 & 31.80 \\
162.4 & 9.08 & 4.57 & 9.75 & 9.72 & 4.29 & 5.02 & 9.59 & 16.39 & 31.77 \\
186.4 & 9.03 & 5.47 & 9.69 & 9.67 & 5.13 & 6.02 & 9.54 & 19.21 & 31.74 \\
209.4 & 8.94 & 6.34 & 9.49 & 9.58 & 5.98 & 7.05 & 9.51 & 22.04 & 31.55 \\
232.4 & 8.89 & 7.32 & 9.45 & 9.56 & 6.89 & 8.11 & 9.40 & 25.22 & 31.63 \\
255.4 & 8.84 & 8.36 & 9.39 & 9.50 & 7.86 & 9.21 & 9.28 & 28.59 & 31.72 \\
\hline
\end{tabular}


Table S6: Integrals of signals in the isomerization of archazolid B (2) to archazolid F (3).

\begin{tabular}{l|c|c|c|c|c|c|c|c|c}
\hline \multirow{2}{*}{ time [min] } & \multicolumn{10}{|c}{ signal } \\
\hline 277.4 & 8.07 & 8.69 & 8.55 & 8.78 & 8.23 & 9.67 & 8.65 & 29.43 & 29.01 \\
\hline 300.4 & 7.23 & 8.83 & 7.71 & 7.81 & 8.32 & 9.68 & 7.57 & 29.81 & 26.40 \\
323.4 & 6.46 & 8.81 & 6.78 & 6.77 & 8.30 & 9.71 & 6.81 & 29.58 & 23.75 \\
\hline 347.4 & 5.87 & 8.95 & 6.24 & 6.39 & 8.48 & 9.87 & 6.17 & 30.07 & 21.91 \\
\hline 370.4 & 5.33 & 9.00 & 5.67 & 5.79 & 8.53 & 9.91 & 5.57 & 30.09 & 20.00 \\
\hline 393.4 & 4.83 & 8.97 & 5.08 & 5.32 & 8.51 & 9.95 & 5.12 & 29.82 & 18.20 \\
416.4 & 4.35 & 8.85 & 4.39 & 4.79 & 8.34 & 9.82 & 4.65 & 29.33 & 16.47 \\
\hline 440.4 & 4.04 & 9.06 & 4.22 & 4.44 & 8.59 & 9.97 & 4.26 & 29.96 & 15.57 \\
\hline 463.4 & 3.69 & 9.01 & 3.75 & 4.13 & 8.53 & 9.98 & 3.89 & 29.75 & 14.30 \\
\hline 486.4 & 3.40 & 9.10 & 3.53 & 3.79 & 8.62 & 10.04 & 3.57 & 30.01 & 13.42 \\
509.4 & 3.12 & 9.05 & 3.15 & 3.56 & 8.62 & 10.11 & 3.39 & 29.79 & 12.30 \\
\hline 531.4 & 2.91 & 9.03 & 2.87 & 3.30 & 8.61 & 10.11 & 3.16 & 29.63 & 11.43 \\
\hline 554.4 & 2.65 & 9.06 & 2.62 & 3.07 & 8.62 & 10.14 & 2.93 & 29.71 & 10.71 \\
\hline 577.4 & 2.48 & 9.02 & 2.37 & 2.88 & 8.59 & 10.10 & 2.74 & 29.51 & 9.99 \\
\hline 602.4 & 2.30 & 9.17 & 2.32 & 2.66 & 8.73 & 10.19 & 2.45 & 30.10 & 9.68 \\
\hline 624.4 & 2.12 & 9.14 & 2.10 & 2.48 & 8.72 & 10.19 & 2.33 & 29.87 & 8.90 \\
647.4 & 2.00 & 9.07 & 1.83 & 2.33 & 8.63 & 10.11 & 2.15 & 29.64 & 8.42 \\
\hline 670.4 & 1.85 & 9.11 & 1.72 & 2.17 & 8.67 & 10.14 & 2.01 & 29.75 & 8.00 \\
\hline 694.4 & 1.72 & 9.08 & 1.54 & 2.06 & 8.62 & 10.13 & 1.89 & 29.59 & 7.53 \\
\hline 717.4 & 1.63 & 9.13 & 1.46 & 1.97 & 8.71 & 10.24 & 1.85 & 29.79 & 7.14 \\
\hline
\end{tabular}

Table S7: Percentage distribution of 2 and 3.

\begin{tabular}{l|c|c|c|c}
\hline time [min] & archazolid B [\%] & \pm & archazolid F [\%] & \pm \\
\hline 46.40 & 91.1 & 0.5 & 8.9 & 0.3 \\
69.40 & 85.8 & 0.5 & 14.2 & 0.4 \\
\hline 93.40 & 80.5 & 0.5 & 19.5 & 0.4 \\
116.40 & 75.4 & 0.5 & 24.6 & 0.4 \\
\hline 139.40 & 71.1 & 0.5 & 28.9 & 0.5 \\
162.40 & 66.8 & 0.5 & 33.2 & 0.5 \\
\hline 186.40 & 62.8 & 0.6 & 37.2 & 0.6 \\
\hline 209.40 & 59.0 & 0.6 & 41.0 & 0.6 \\
\hline 232.40 & 55.5 & 0.6 & 44.5 & 0.7 \\
\hline 255.40 & 52.1 & 0.6 & 47.9 & 0.8 \\
\hline 277.40 & 49.0 & 0.6 & 51.0 & 0.8 \\
300.40 & 46.0 & 0.6 & 54.0 & 0.7 \\
\hline 323.40 & 43.1 & 0.6 & 56.9 & 0.7 \\
347.40 & 40.7 & 0.6 & 59.3 & 0.7 \\
\hline 370.40 & 38.3 & 0.5 & 61.7 & 0.7 \\
393.40 & 36.1 & 0.5 & 63.9 & 0.7 \\
416.40 & 34.0 & 0.5 & 66.0 & 0.7 \\
440.40 & 32.0 & 0.4 & 68.0 & 0.7 \\
\hline 463.40 & 30.2 & 0.4 & 69.8 & 0.7 \\
\hline & & & & \\
\hline
\end{tabular}


Table S7: Percentage distribution of 2 and 3.

\begin{tabular}{l|c|c|c|c}
\hline time [min] & archazolid B [\%] & \pm & archazolid F [\%] & \pm \\
\hline 486.40 & 28.4 & 0.4 & 71.6 & 0.7 \\
509.40 & 26.9 & 0.4 & 73.1 & 0.7 \\
531.40 & 25.4 & 0.4 & 74.6 & 0.7 \\
554.40 & 23.9 & 0.4 & 76.1 & 0.7 \\
577.40 & 22.7 & 0.4 & 77.3 & 0.7 \\
602.40 & 21.4 & 0.4 & 78.6 & 0.7 \\
624.40 & 20.2 & 0.4 & 79.8 & 0.7 \\
647.40 & 19.1 & 0.4 & 80.9 & 0.7 \\
670.40 & 18.0 & 0.4 & 82.0 & 0.7 \\
694.40 & 17.1 & 0.4 & 82.9 & 0.7 \\
717.40 & 16.4 & 0.4 & 83.6 & 0.7 \\
\hline
\end{tabular}

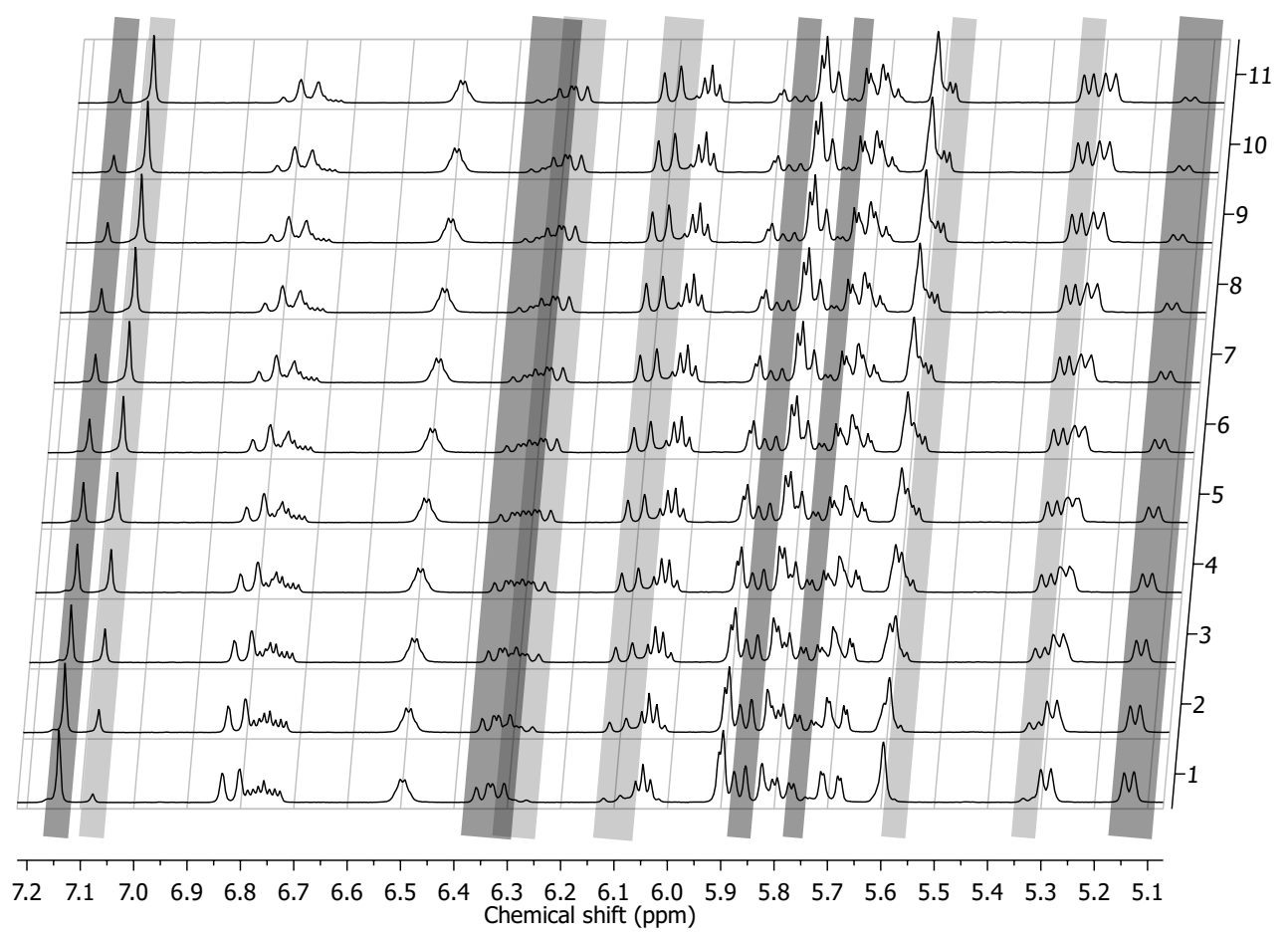

Figure S1. Time-dependence NMR studies: archazolid B (2) is marked black, archazolid F (3) gray. Unmarked signals refer to both of the archazolids. 
Nucleus: $1 \mathrm{H}$

Frequency: $500.13 \mathrm{MHz}$

Solvent: THF

Temperature: $297.9 \mathrm{~K}$

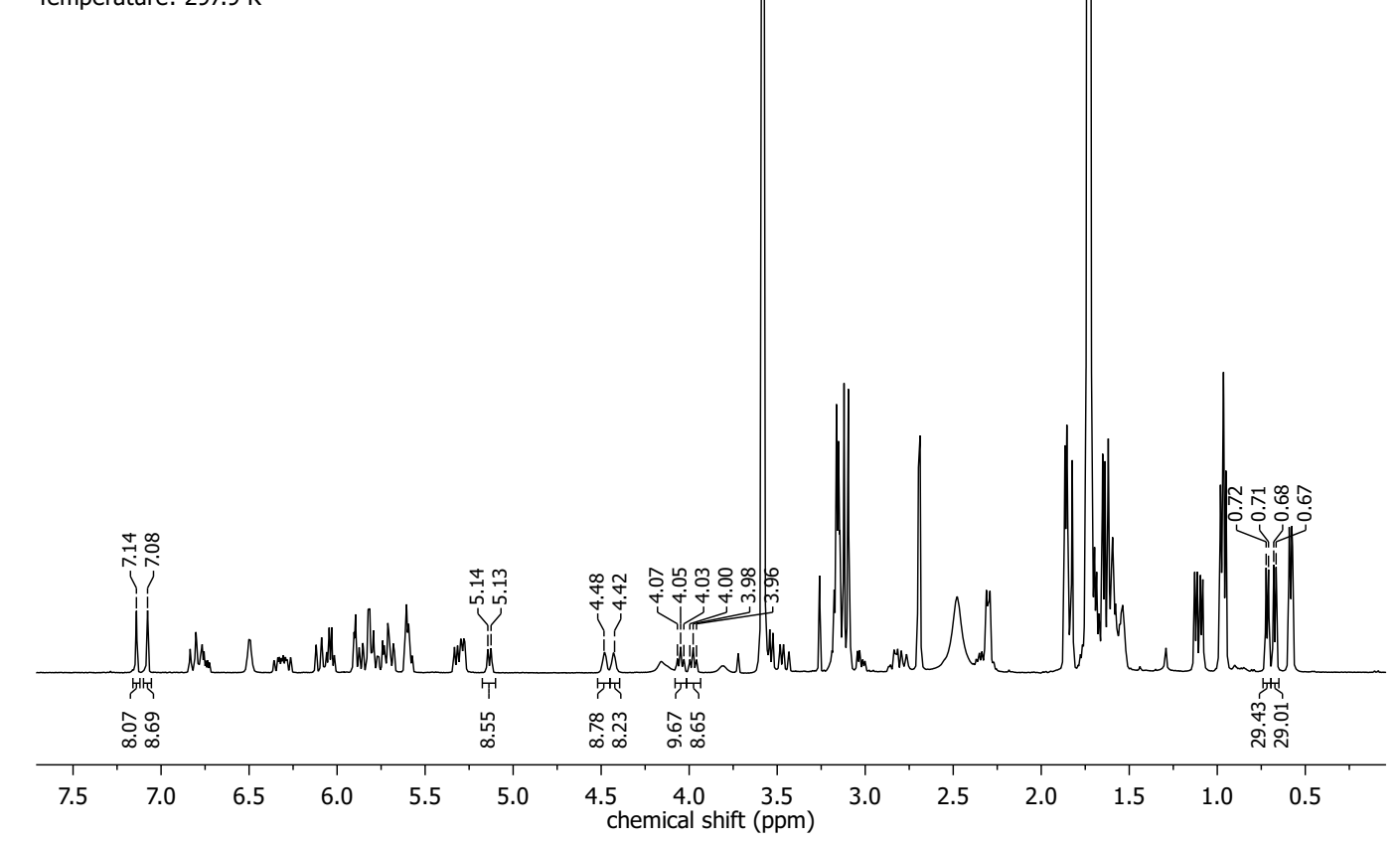

Figure S2. NMR-Spectra at $t=277.4 \mathrm{~min}$. Integrals used for the calculation are marked. 


\subsection{Additional analytical data}

\subsubsection{NOE data for compound 95}

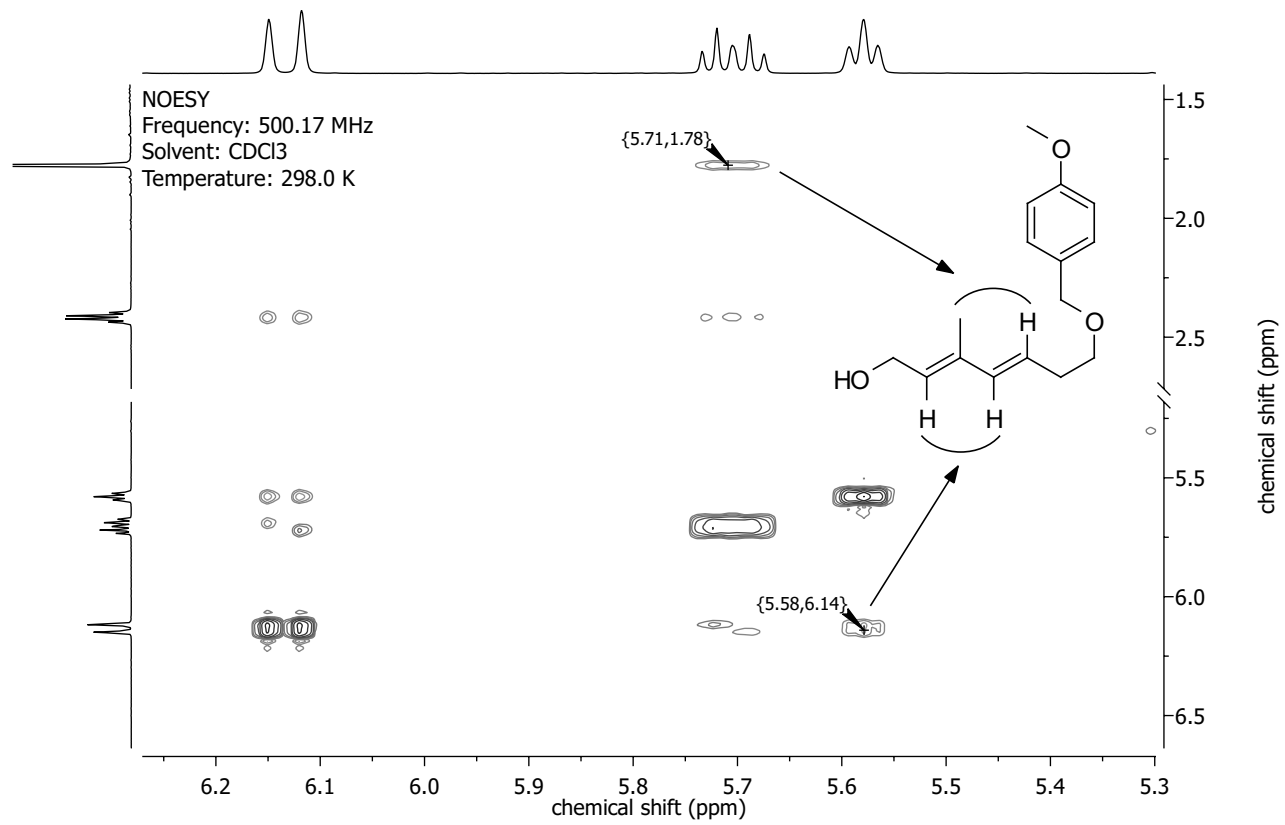

Figure S3. NOESY analysis proves the conformation of $\mathbf{9 5 .}$ 


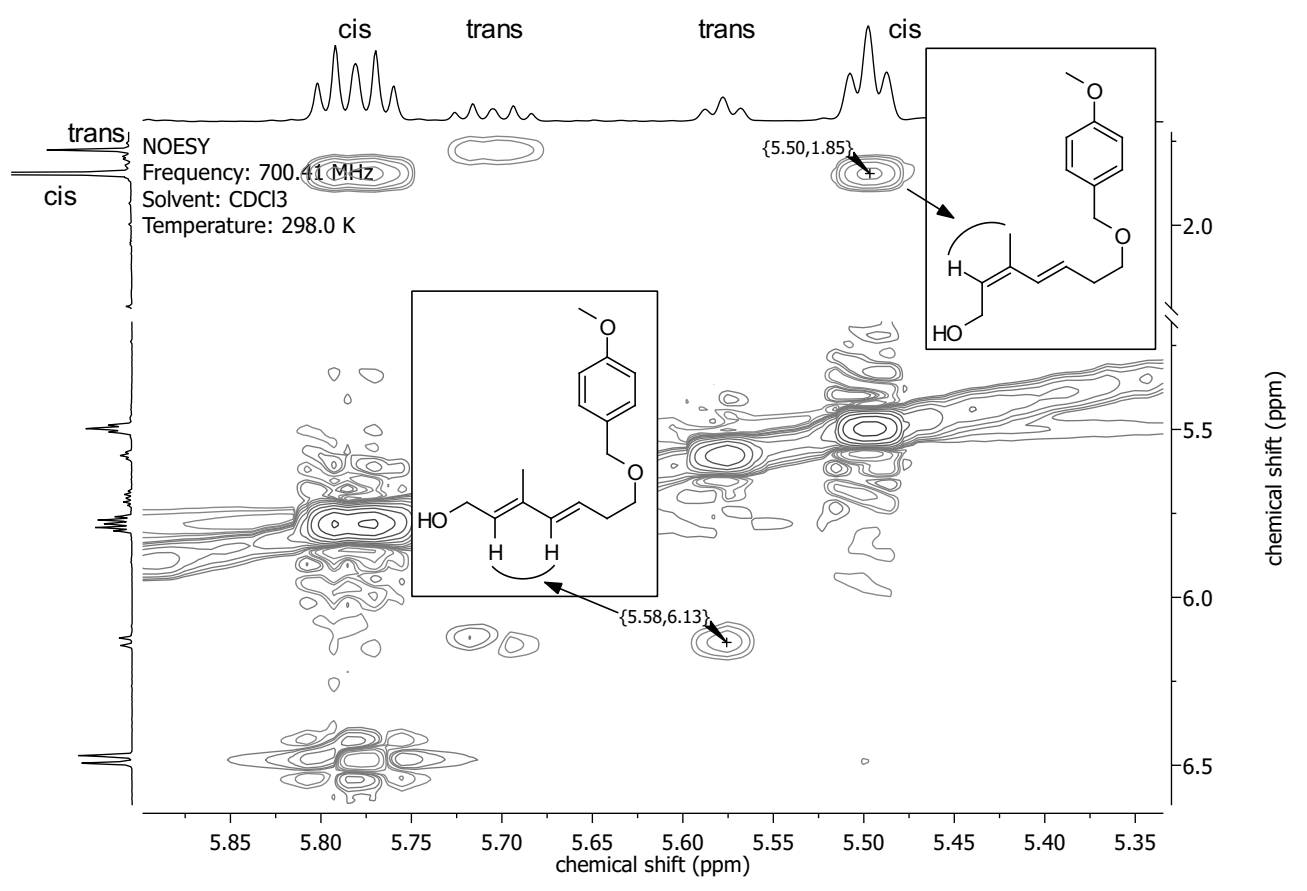

Figure S4. Direct comparison of a mixture of $(E, E) 95$ and $(Z, E) 95$ (main) in NOESY spectrum. Indicated signals show proof of correct assignment.

\subsubsection{Determination of purity of compound 25a}

Nucleus: $1 \mathrm{H}$

Frequency: $500.17 \mathrm{MHz}$

Solvent: $\mathrm{CD} 2 \mathrm{Cl} 2$

Temperature: $298.0 \mathrm{~K}$

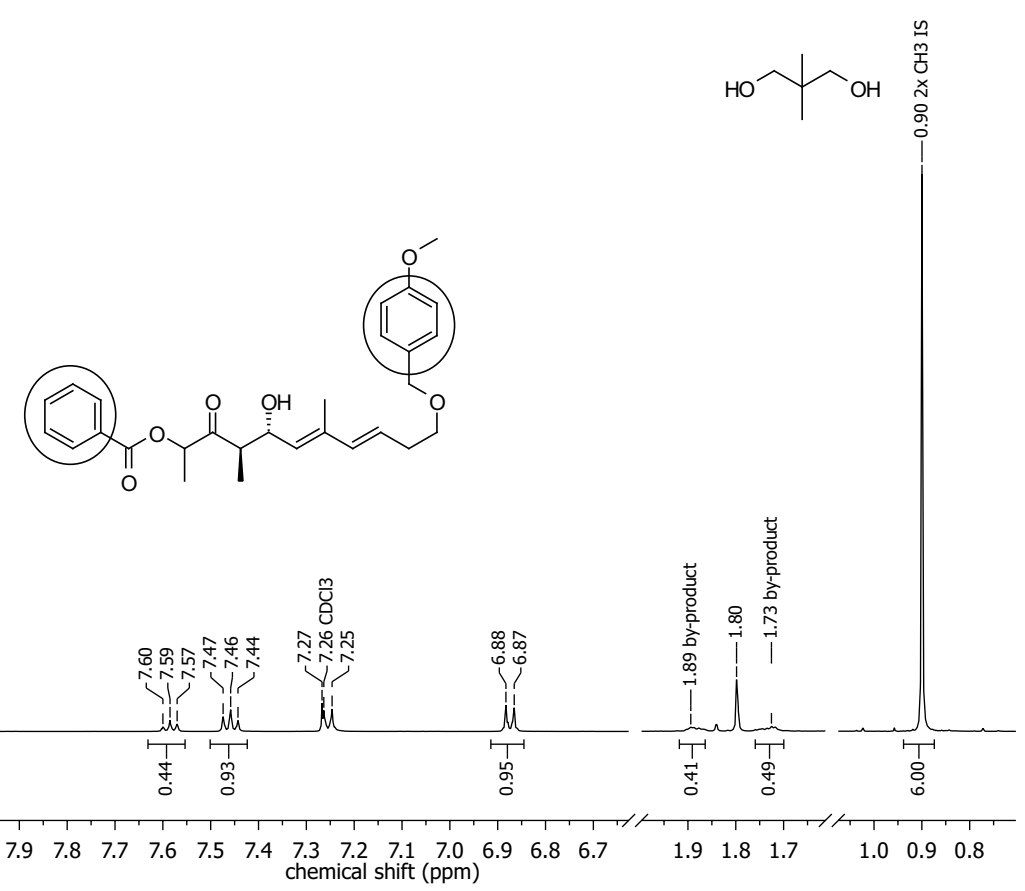

Figure S5. Determination of the purity of compound 25a with internal standard (2,2-dimethyl-1,3-propanediol). 
The purity was determined by the following equations:

$$
\begin{gathered}
\text { Purity }=\frac{m_{\text {product }}}{m_{\text {mixture }}}=\frac{n_{I S} \times M_{\text {product }}}{m_{\text {mixture }}} \times \frac{\text { integral }_{\text {product }} \times \text { protons }_{I S}}{\text { integral }_{I S} \times \text { protons }_{\text {product }}} \\
\text { Purity }=\frac{81.61 \mu \mathrm{mol} \times 466.57 \mathrm{~g} / \mathrm{mol}}{22.3 \mathrm{mg}} \times \frac{(0.90+0.44+0.93+0.95) \times 6}{6.00 \times 7}
\end{gathered}
$$

$$
\text { Purity }=79 w t . \%
$$

\subsubsection{NOE data for compound 33a}

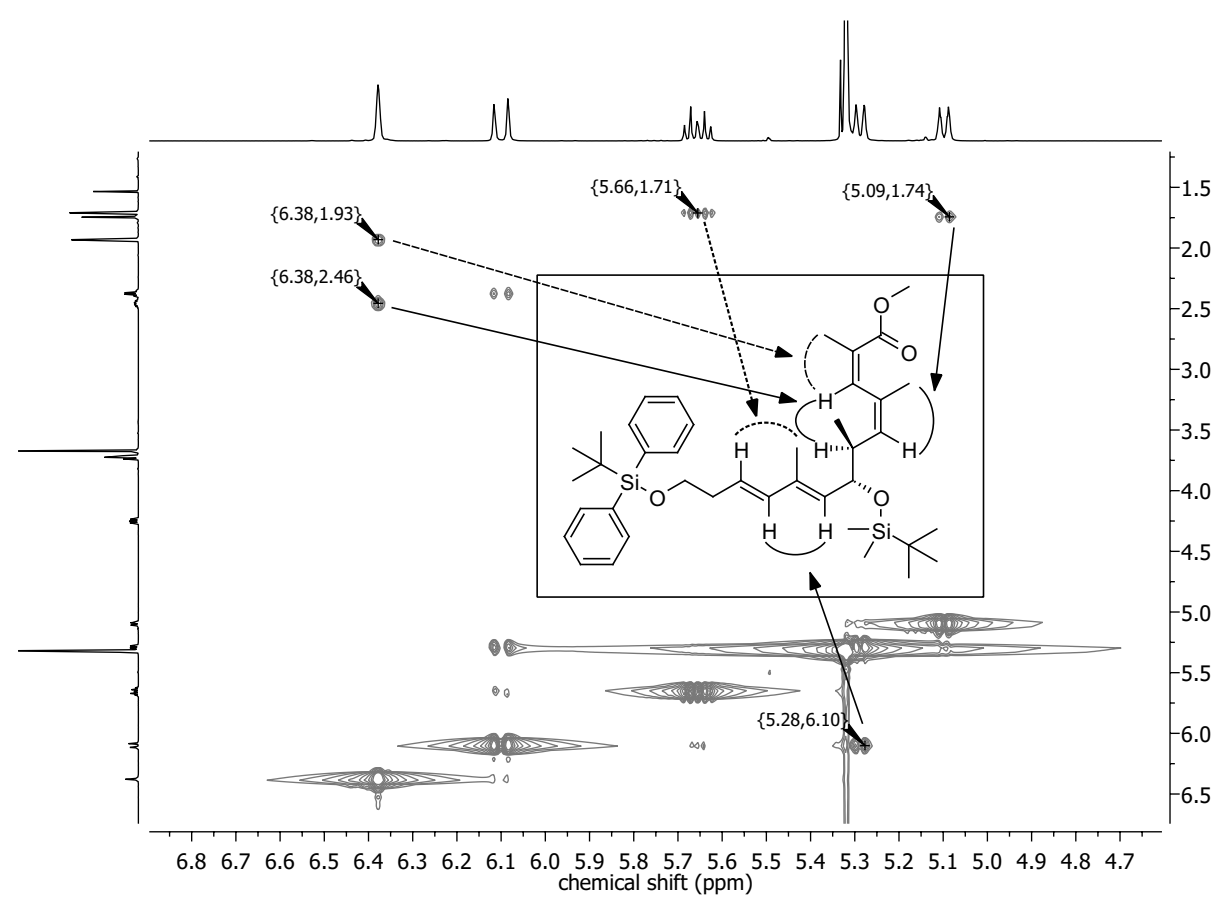

Figure S6. NOESY analysis for determining the conformation of $(Z, Z)$ unsaturated ester 33a.

\subsubsection{Mosher ester analysis for compound 53e}

Table S8: NMR data for Mosher's analysis of alcohol 53e.

\begin{tabular}{l|c|c|c}
\hline position & $\delta_{H}[\mathrm{ppm}](S)$ & $\delta_{H}[\mathrm{ppm}](R)$ & $\Delta \delta^{S-R}$ \\
\hline 21 & 5.63 & 5.77 & -0.14 \\
22 & 2.97 & 3.00 & -0.03 \\
23 & 5.97 & 6.02 & -0.05 \\
$4^{\prime}$ & 7.08 & 6.89 & +0.19 \\
$5^{\prime}$ & 5.04 & 5.02 & +0.02 \\
$6^{\prime}$ & 1.76 & 1.73 & +0.03 \\
\hline
\end{tabular}


The determination of the absolute configuration was accomplished by the accepted model for the analysis of the Mosher ester. Therefore the collected NMR-Data for the two Mosher ester derivatives were compared as shown in Table S8. Determination of the difference between each signal led to the determination of the absolute configuration as shown in Scheme S1. A detailed theoretical background can be found in literature. ${ }^{a}$

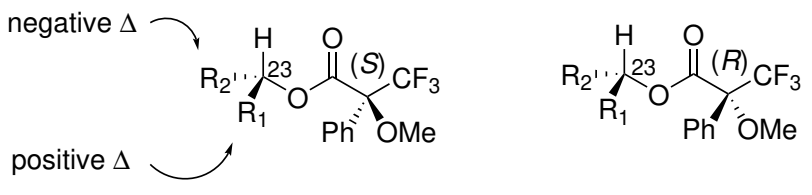

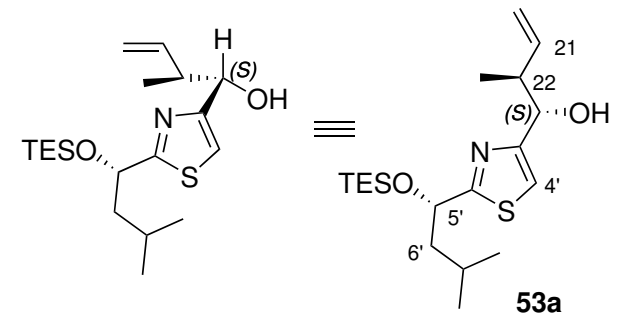

Scheme S1. Proposed conformation in the Mosher's analysis of alcohol 53e.

\subsubsection{NOE data for compound 63}

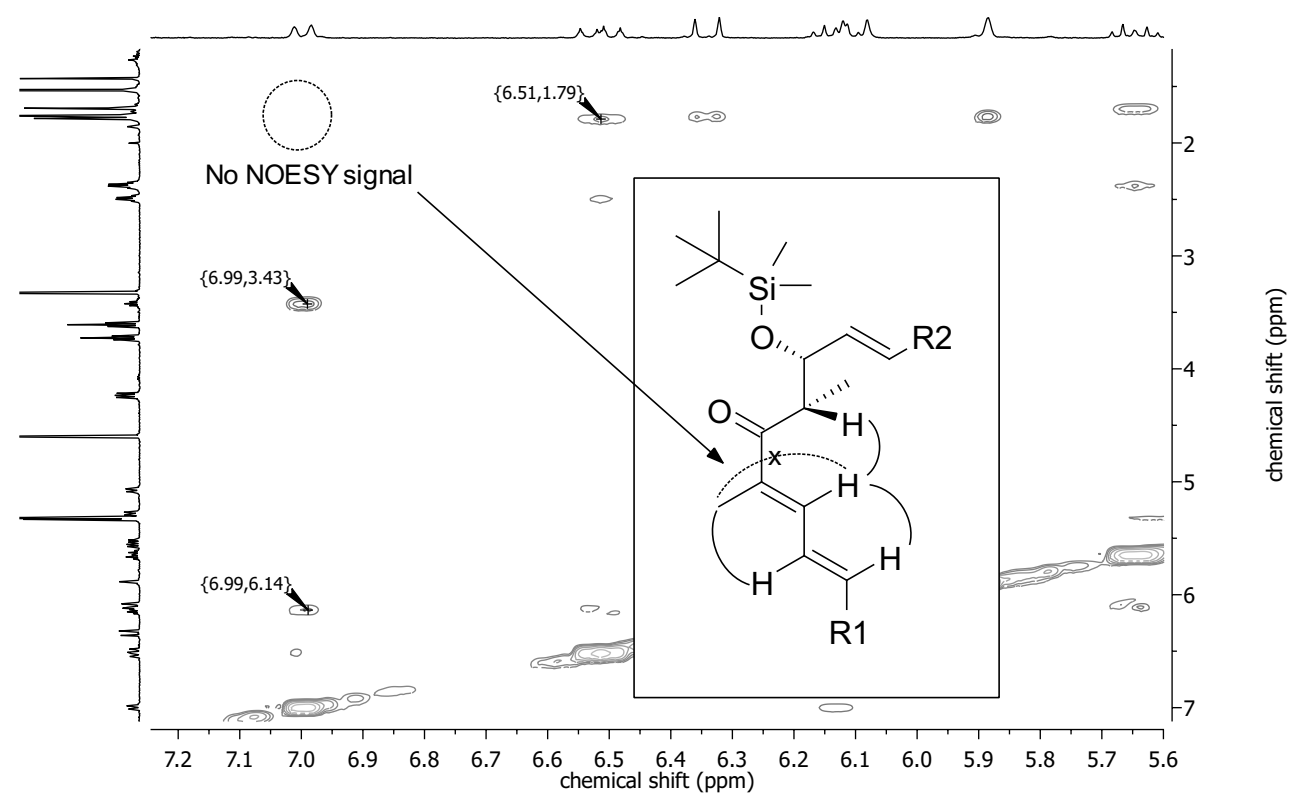

Figure S7. NOESY spektrum of compound $\mathbf{6 3}$ for determination of (18E)-conformation. No NOESY signal could be detected between proton on C19 and Me18.

\footnotetext{
a J. A. Dale, H. S. Mosher, J. Am. Chem. Soc. 1973, 95, 512-519; T. R. Hoye, C. S. Jeffrey, F. Shao, Nat. Protoc. 2007, 2, $2451-2458$.
} 


\subsubsection{Mosher ester analysis for compound $65 a$}

The determination of the absolute configuration was accomplished by the accepted model for the analysis of the Mosher ester. Therefore the collected NMR-Data for the two Mosher ester derivatives were compared as shown in Table S9. Determination of the difference between each signal led to the determination of the absolute configuration as shown in Scheme S2. A detailed theoretical background can be found in literature. ${ }^{b}$

Table S9: NMR data for Mosher's analysis of alcohol 65a.

\begin{tabular}{|c|c|c|c|}
\hline position & $\delta_{H}[\mathrm{ppm}](S)$ & $\delta_{H}[\mathrm{ppm}](R)$ & $\Delta \delta^{S-R}$ \\
\hline 1 & 3.73 & 3.72 & +0.01 \\
\hline 2 & 2.37 & 2.37 & 0.00 \\
\hline 3 & 5.64 & 5.64 & 0.00 \\
\hline 4 & 6.10 & 6.09 & +0.01 \\
\hline Me5 & 1.67 & 1.67 & 0.00 \\
\hline 6 & 5.26 & 5.27 & -0.01 \\
\hline 7 & 4.20 & 4.21 & -0.01 \\
\hline 8 & 2.32 & 2.37 & -0.05 \\
\hline Me8 & 0.77 & 0.81 & -0.04 \\
\hline 9 & 5.01 & 5.05 & -0.04 \\
\hline Me10 & 1.77 & 1.78 & -0.01 \\
\hline 11 & 5.83 & 5.86 & -0.03 \\
\hline Me12 & 1.82 & 1.84 & -0.02 \\
\hline 13 & 6.22 & 6.38 & -0.16 \\
\hline 14 & 5.68 & 5.72 & -0.04 \\
\hline 15 & 4.15 & 4.34 & -0.19 \\
\hline 16 & 1.81 & 1.87 & -0.06 \\
\hline Me16 & 0.74 & 0.75 & -0.01 \\
\hline 17 & 5.29 & 5.29 & 0.00 \\
\hline Me18 & 1.62 & 1.42 & +0.20 \\
\hline 19 & 6.13 & 6.08 & +0.05 \\
\hline 20 & 6.32 & 6.27 & +0.05 \\
\hline 21 & 5.79 & 5.78 & +0.01 \\
\hline 22 & 2.40 & 2.40 & 0.00 \\
\hline 23 & 3.57 & 3.58 & -0.01 \\
\hline
\end{tabular}

\footnotetext{
bJ. A. Dale, H. S. Mosher, J. Am. Chem. Soc. 1973, 95, 512-519; T. R. Hoye, C. S. Jeffrey, F. Shao, Nat. Protoc. 2007, 2, $2451-2458$.
} 

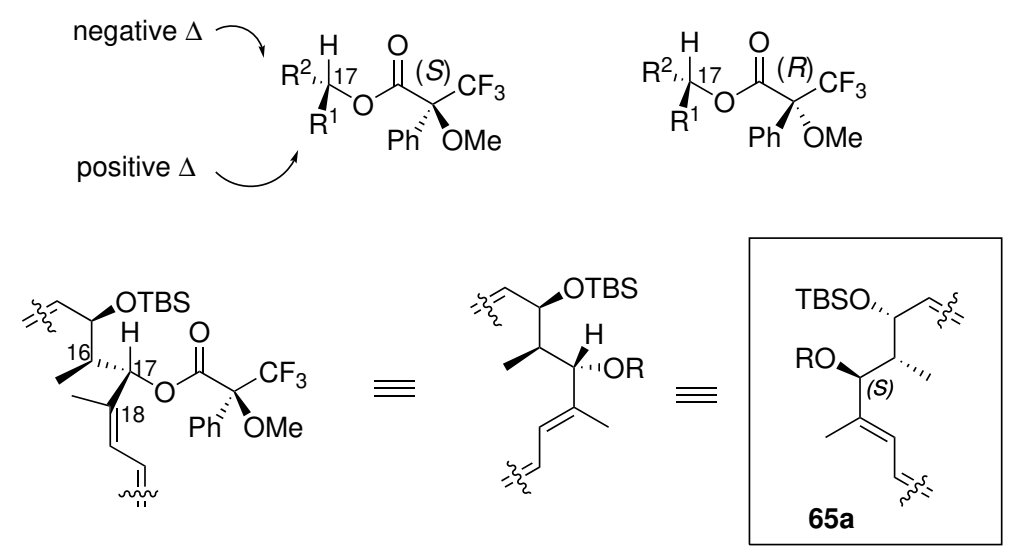

Scheme S2. Proposed conformation in the Mosher's analysis of alcohol 65a.

\subsubsection{NOE data for compound 64}

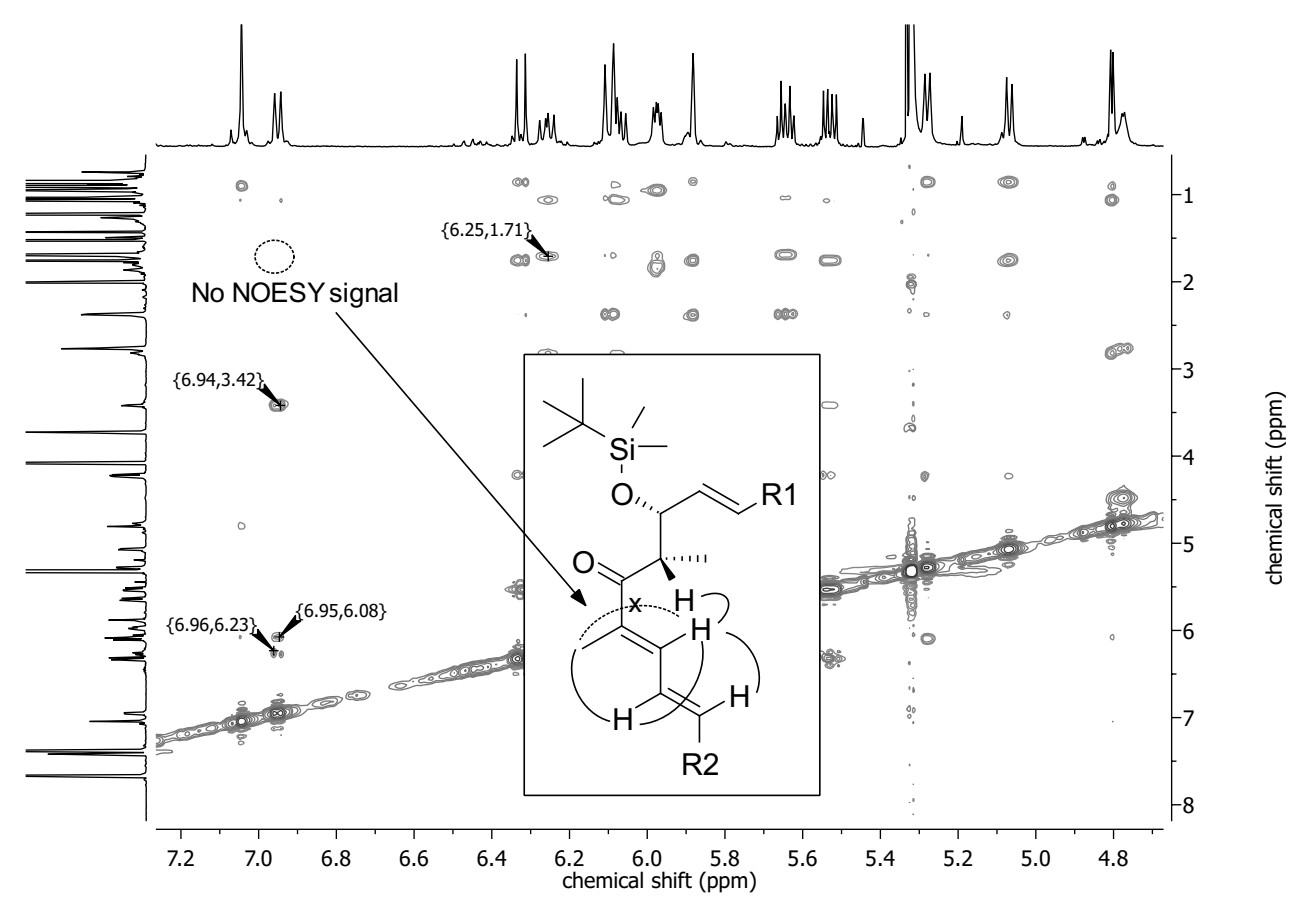

Figure S8. NOESY spektrum of compound $\mathbf{6 4}$ for determination of (18E)-conformation. No NOESY signal could be detected between proton on C19 and Me18. 


\subsubsection{NOE data for compound 75}

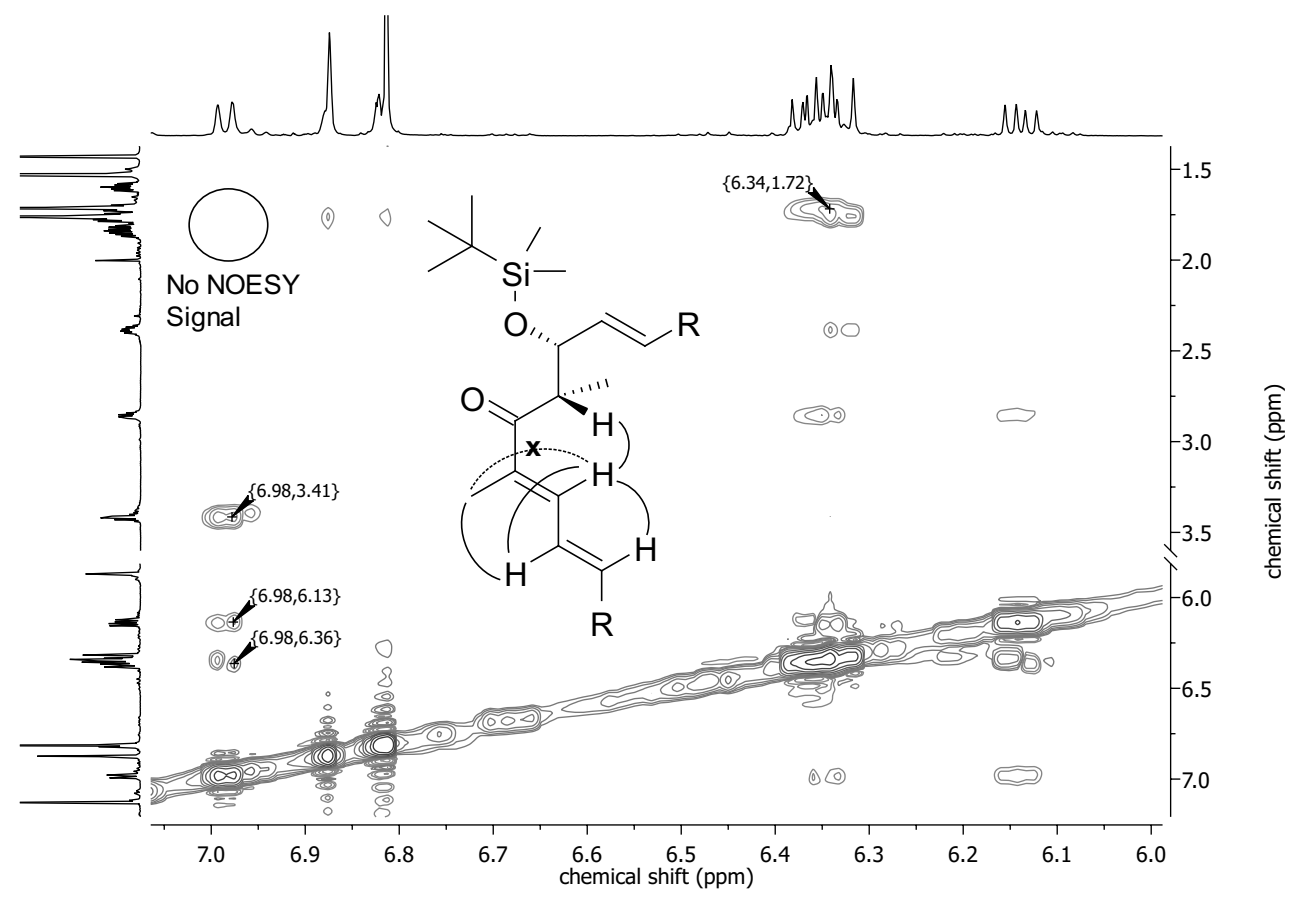

Figure S9. NOESY spektrum of compound 75 for determination of (18E)-conformation. No NOESY signal could be detected between proton on C19 and Me18.

\subsection{Determination of relative configuration of iso archazolid A1 and A2}

The both epimers iso-Archazolid $\mathrm{A}_{1}$ and $\mathrm{A}_{2}$ were calculated using Maestro MacroModel (version 10.5, Schrödinger, LLC, New York, 2016) and minimized with 2500 iterations with the Polak-Ribiere conjugate gradient algorithm (PRCG), OPLS2008 force field and chloroform solvent model. The conformational search calculations were performed via Merck Molecular Force Field (mmffs) and chloroform solvent model. The maximum number of steps was set to 1000 with automatic setup parameters.

The calculated conformers were clustered by visual similarity. For the analysis and the comparison with the experimental data only clusters were analysed which were consistent with the characteristic data in the region C2-C4 obtained by NOESY NMR (see Scheme S3). 


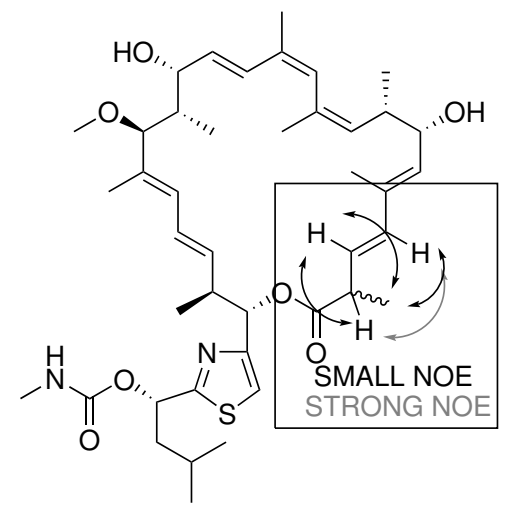

Scheme S3. Structural motif in the region C2-C4 in both epimers.

\subsubsection{Predicted Parameters}

Table S10: Predicted data for the $(S)$-Isomere.

\begin{tabular}{l||c|c|c|c|c||c}
\hline & cluster 1 & cluster 2 & cluster 3 & cluster 4 & cluster 5 & range \\
\hline Me2-2 $[\AA]$ & 2.8 & 2.7 & 2.7 & 2.7 & 2.7 & $2.7-2.8$ \\
Me2-3 $[\AA]$ & 3.2 & 3.1 & 3.2 & 3.2 & 3.2 & $3.1-3.2$ \\
Me2-4 $[\AA]]$ & 4.3 & 4.3 & 4.3 & 4.3 & 4.3 & 4.3 \\
$2-3[\AA]$ & 3.1 & 3.1 & 3.1 & 3.1 & 3.1 & 3.1 \\
$2-4[\AA]$ & 2.4 & 2.4 & 2.4 & 2.3 & 2.3 & $2.3-2.4$ \\
$13-15[\AA]$ & 2.4 & 2.4 & 2.4 & 2.4 & 2.4 & 2.4 \\
$14-15[\AA]$ & 3.1 & 3.1 & 3.1 & 3.1 & 3.1 & 3.1 \\
$20-M e 22[\AA]$ & 4.2 & 4.2 & 4.2 & 4.3 & 4.3 & $4.2-4.3$ \\
\hline$J_{3}(2-3)[\mathrm{Hz}]$ & 11.9 & 11.9 & 11.9 & 11.9 & 11.9 & 11.9 \\
$J_{3}(14-15)[\mathrm{Hz}]$ & 9.9 & 10 & 9.9 & 9.6 & 9.8 & $9.6-10.0$ \\
\hline representing & $24 \%$ & $19 \%$ & $12 \%$ & $6 \%$ & $2 \%$ & $63 \%$ \\
\hline
\end{tabular}

Table S11: Predicted data for the $(R)$-Isomere.

\begin{tabular}{l||c|c|c|c||c}
\hline & cluster 1 & cluster 2 & cluster 3 & cluster 4 & range \\
\hline Me2-2 $[\AA]$ & 2.7 & 2.7 & 2.7 & 2.7 & 2.7 \\
Me2-3 $[\AA]$ & 3.4 & 3 & 3.1 & 3.2 & $3.0-3.4$ \\
Me2-4 $[\AA]$ & 4.6 & 4.6 & 4.4 & 4.4 & $4.4-4.6$ \\
$2-3[\AA]$ & 3 & 3 & 3.1 & 3.1 & $3.0-3.1$ \\
$2-4[\AA]$ & 2.4 & 2.4 & 2.3 & 2.3 & $2.3-2.4$ \\
$13-15[\AA]$ & 3.7 & 3.7 & 3.6 & 3.6 & $3.6-3.7$ \\
$14-15[\AA]$ & 2.3 & 2.3 & 2.4 & 2.4 & $2.3-2.4$ \\
$20-\mathrm{Me} 22[\AA]$ & 2.8 & 2.8 & 4.2 & 4.1 & $2.8-4.2$ \\
\hline$J_{3}(2-3)[\mathrm{Hz}]$ & 10.1 & 9.4 & 11.3 & 11.5 & $9.4-11.5$ \\
$J_{3}(14-15)[\mathrm{Hz}]$ & 5.4 & 5 & 4.8 & 4.7 & $4.7-5.4$ \\
\hline representing & $34 \%$ & $21 \%$ & $19 \%$ & $13 \%$ & $87 \%$ \\
\hline
\end{tabular}




\subsubsection{Experimental Parameters}

Table S12: Experimental NMR-data for iso-archazolid $A_{1}$ (5) and iso-archazolid $A_{2}$ (6). Normed on NOESY interaction $\mathrm{Me} 2 \rightarrow 2$.

\begin{tabular}{l||c|c|c||c|c|c}
\hline \multicolumn{1}{l||}{} & \multicolumn{3}{c}{ iso-archazolid $\mathrm{A}_{1}$} & \multicolumn{3}{c}{ iso-archazolid $\mathrm{A}_{2}$} \\
& $x_{1}$ & $x_{2}$ & $\left\|\frac{1}{2}\left(x_{1}+x_{2}\right)\right\|$ & $x_{3}$ & $x_{4}$ & $\left\|\frac{1}{2}\left(x_{3}+x_{4}\right)\right\|$ \\
\hline $\mathrm{Me} 2 \rightarrow 2$ & 10.0 & 10.6 & 1.00 & 10.0 & 10.9 & 1.00 \\
$\mathrm{Me} 2 \rightarrow 3$ & 4.04 & 4.15 & 0.40 & 4.04 & 3.79 & 0.37 \\
$\mathrm{Me} 2 \rightarrow 4$ & 2.73 & 2.19 & 0.24 & 3.49 & 3.42 & 0.33 \\
$\mathrm{Me} 2 \rightarrow 23$ & - & - & - & - & 0.30 & 0.03 \\
$2 \rightarrow 3$ & 3.54 & 3.44 & 0.34 & 2.78 & 3.27 & 0.29 \\
$2 \rightarrow 4$ & 6.77 & 6.06 & 0.62 & 8.15 & 7.79 & 0.76 \\
$13 \rightarrow 15$ & 9.34 & 9.24 & 0.90 & 3.93 & 3.62 & 0.36 \\
$14 \rightarrow 15$ & 3.25 & 2.72 & 0.29 & 5.79 & 5.98 & 0.56 \\
$20 \rightarrow \mathrm{Me} 22$ & 3.23 & 3.09 & 0.31 & 8.21 & 7.93 & 0.77 \\
\hline$J_{3}(2-3)[\mathrm{Hz}]$ & - & - & 8.8 & - & - & 8.3 \\
$J_{3}(14-15)[\mathrm{Hz}]$ & - & - & 7.8 & - & - & 4.2 \\
\hline
\end{tabular}

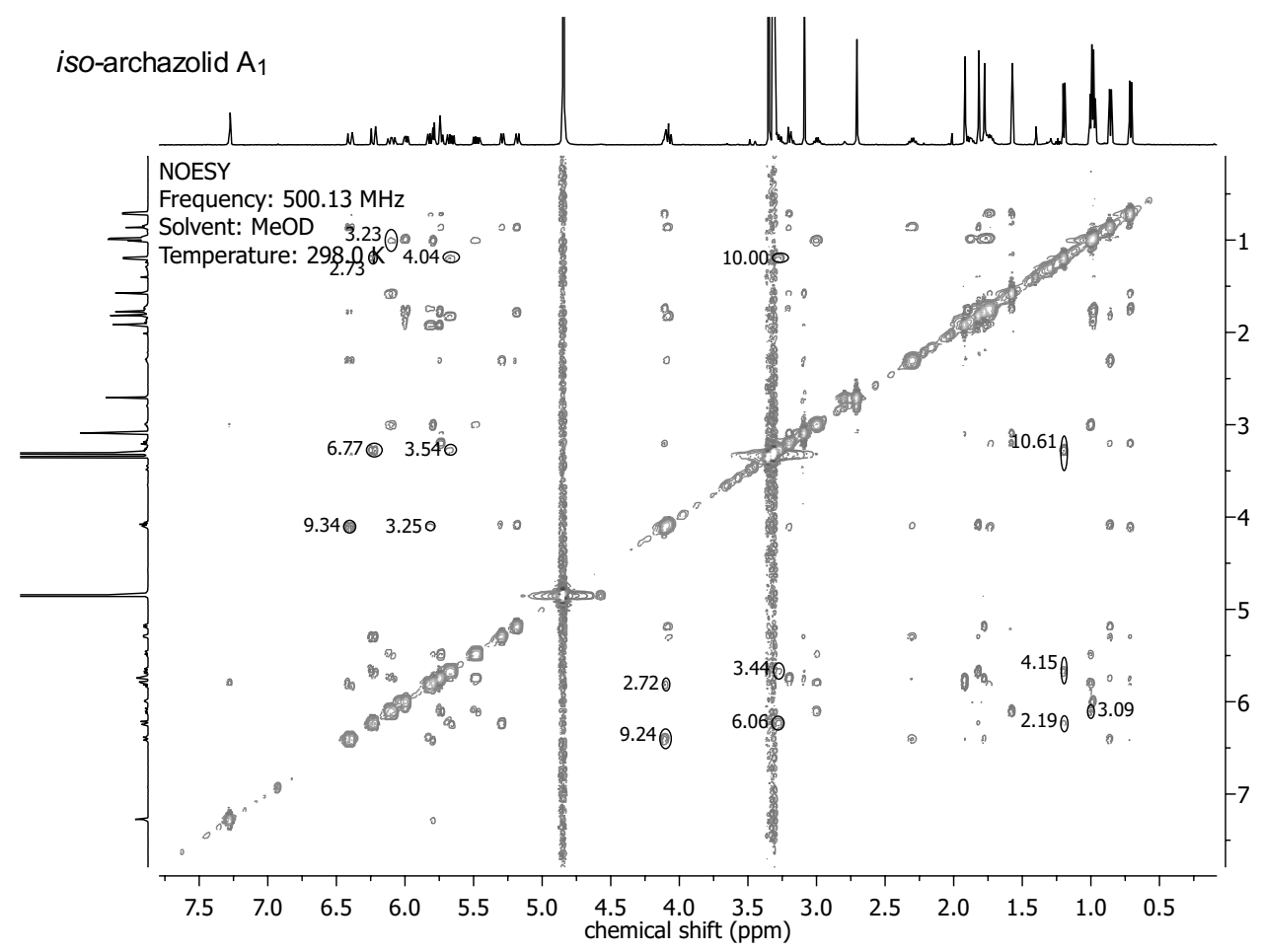

Figure S10. NMR-NOESY Spectra for iso-archazolid $A_{1}$. 


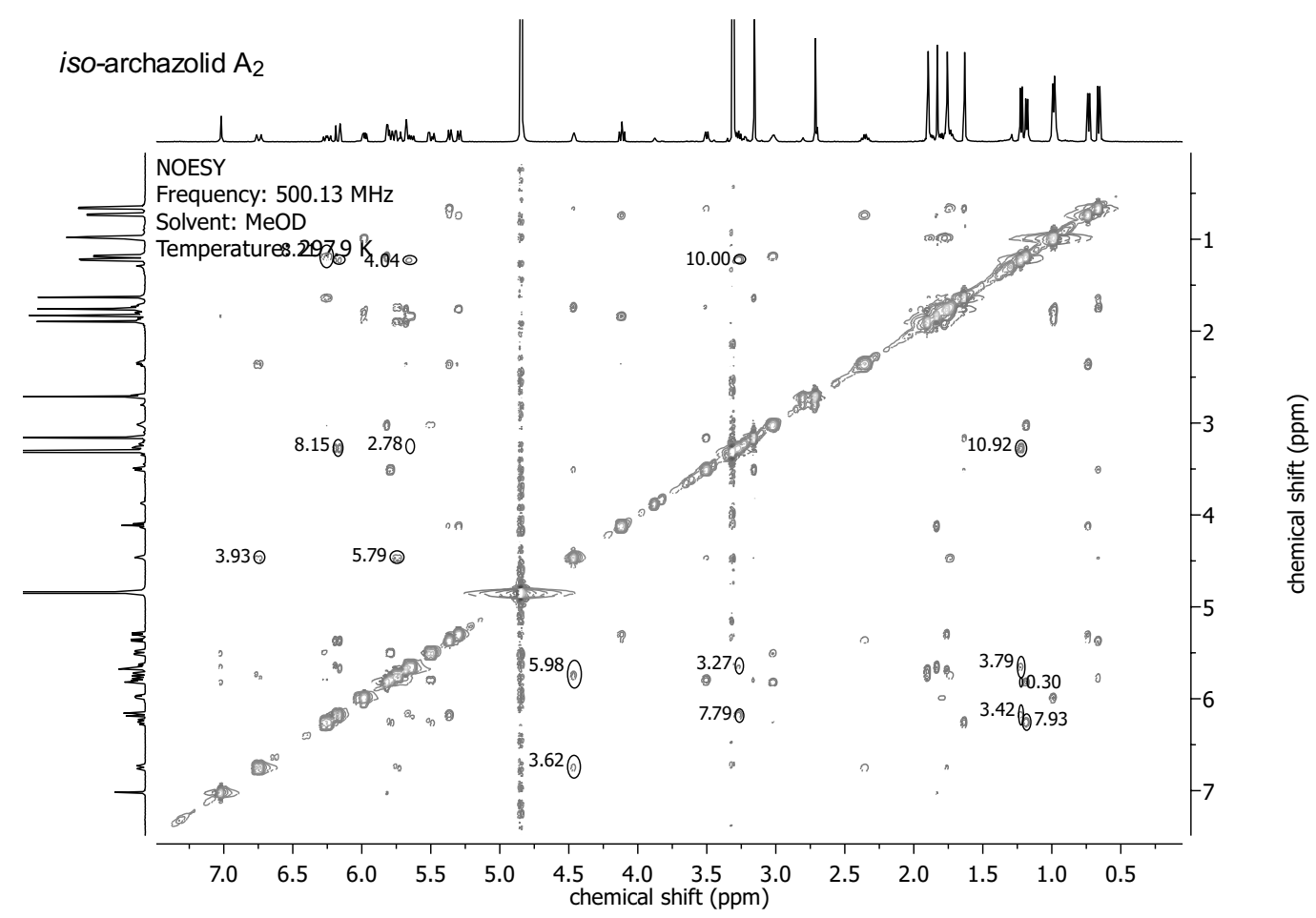

Figure S11. NMR-NOESY Spectra for iso-archazolid $A_{2}$. 


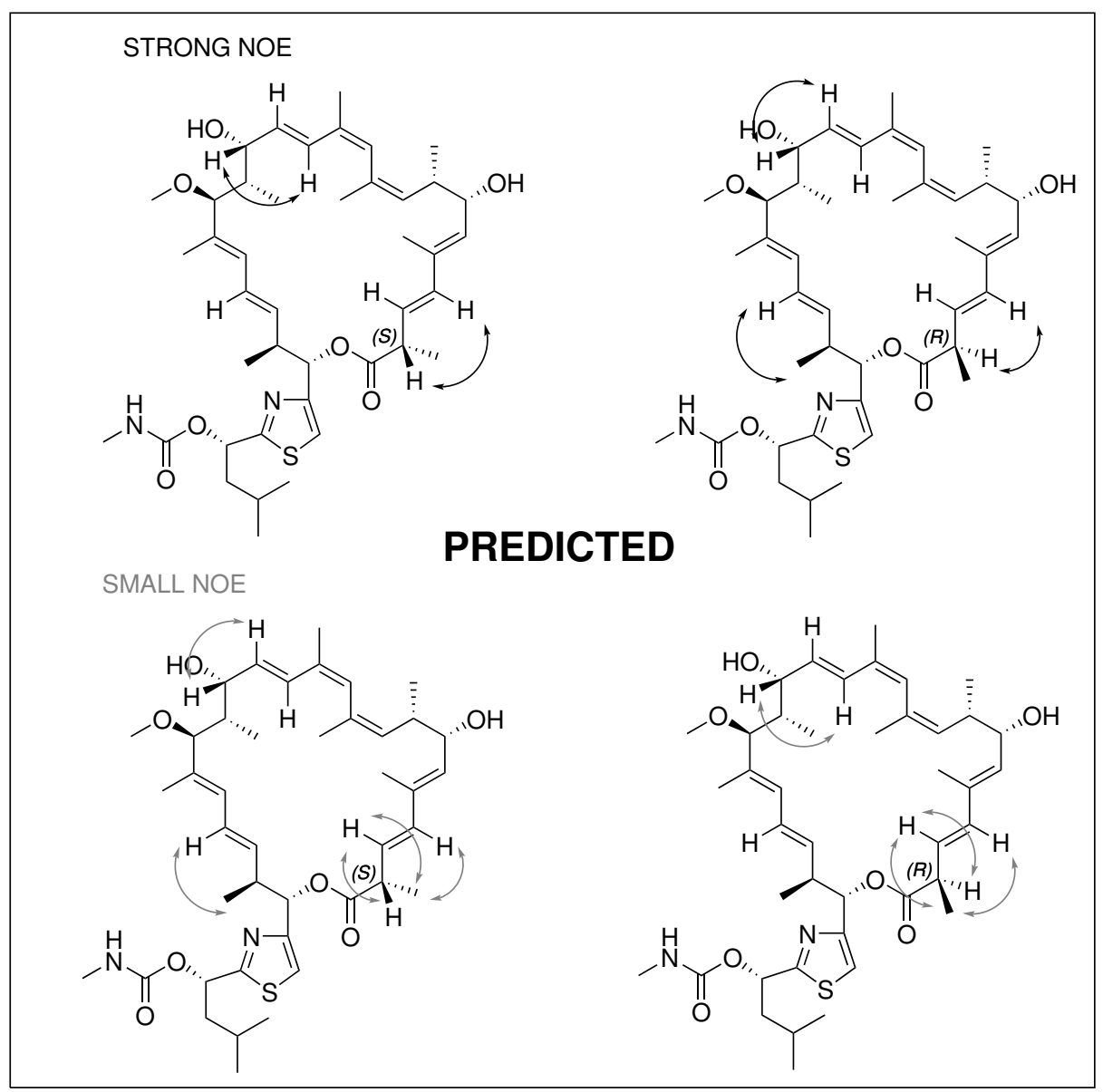

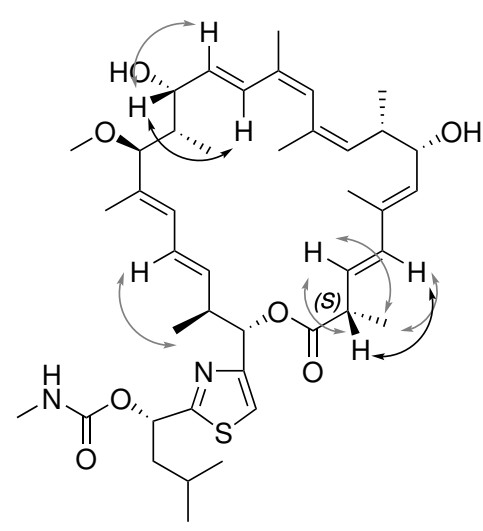

Iso-Archazolid $\mathrm{A}_{1}$

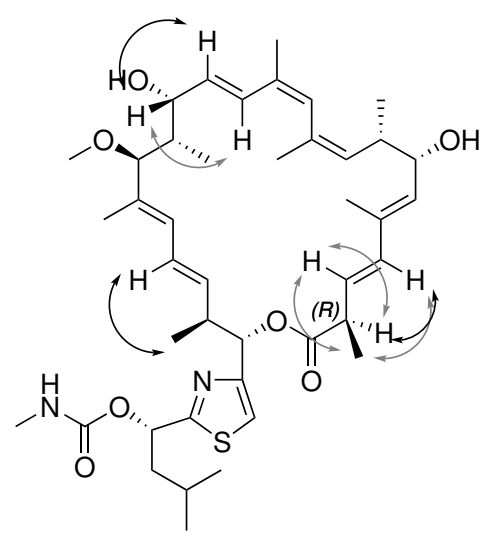

Iso-Archazolid $\mathrm{A}_{2}$

Scheme S4. Graphical comparison of predicted and experimental properties. 


\subsubsection{Copies of Calculated Clusters}
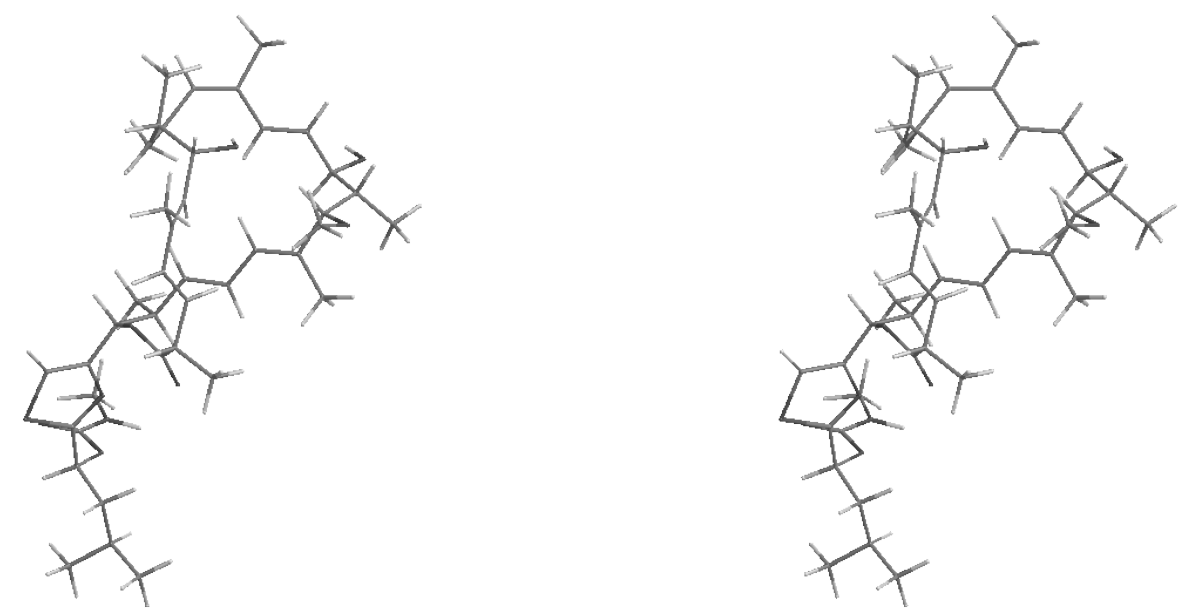

Scheme S5. Conformation of Cluster 1 of $(S)$-iso-archazolid A.

Table S13: Coordinates of Cluster 1 of $(S)$-iso-archazolid A.

\begin{tabular}{l|c|c|c||c|c|c|c}
\hline atom & $\mathrm{X}$ & $\mathrm{Y}$ & $\mathrm{Z}$ & atom & $\mathrm{X}$ & $\mathrm{Y}$ & $\mathrm{Z}$ \\
\hline $\mathrm{C}(1)$ & -7.586 & -7.486 & 0.508 & $\mathrm{C}(58)$ & -4.462 & -9.250 & 2.551 \\
$\mathrm{H}(2)$ & -9.609 & -9.157 & -0.271 & $\mathrm{C}(59)$ & -7.557 & -8.996 & 0.436 \\
$\mathrm{H}(3)$ & -8.975 & -10.641 & 0.455 & $\mathrm{H}(60)$ & -7.124 & -9.335 & -0.513 \\
$\mathrm{C}(4)$ & -7.026 & -6.685 & -0.414 & $\mathrm{C}(61)$ & -8.978 & -9.548 & 0.534 \\
$\mathrm{H}(5)$ & -8.062 & -7.055 & 1.387 & $\mathrm{H}(62)$ & -9.446 & -9.289 & 1.490 \\
$\mathrm{H}(6)$ & -6.559 & -7.124 & -1.296 & $\mathrm{C}(63)$ & -6.668 & -9.558 & 1.553 \\
$\mathrm{C}(7)$ & -4.042 & -3.155 & 3.777 & $\mathrm{O}(64)$ & -7.004 & -10.438 & 2.335 \\
$\mathrm{C}(8)$ & -3.243 & -4.079 & 4.663 & $\mathrm{O}(65)$ & -5.447 & -8.956 & 1.523 \\
$\mathrm{H}(9)$ & -2.337 & -3.576 & 5.020 & $\mathrm{C}(66)$ & -3.878 & -10.637 & 2.374 \\
$\mathrm{H}(10)$ & -3.822 & -4.402 & 5.533 & $\mathrm{C}(67)$ & -3.075 & -11.036 & 1.321 \\
$\mathrm{H}(11)$ & -2.924 & -4.983 & 4.131 & $\mathrm{~S}(68)$ & -2.627 & -12.678 & 1.496 \\
$\mathrm{C}(12)$ & -4.436 & -1.904 & 4.396 & $\mathrm{C}(69)$ & -3.526 & -12.759 & 2.961 \\
$\mathrm{C}(13)$ & -5.491 & -1.672 & 5.206 & $\mathrm{~N}(70)$ & -4.133 & -11.639 & 3.303 \\
\hline $\mathrm{H}(14)$ & -3.787 & -1.069 & 4.126 & $\mathrm{H}(71)$ & -2.738 & -10.450 & 0.475 \\
$\mathrm{C}(15)$ & -5.749 & -0.260 & 5.677 & $\mathrm{C}(72)$ & -3.571 & -14.049 & 3.751 \\
$\mathrm{H}(16)$ & -6.696 & 0.116 & 5.276 & $\mathrm{H}(73)$ & -2.684 & -14.662 & 3.549 \\
$\mathrm{H}(17)$ & -5.783 & -0.214 & 6.772 & $\mathrm{C}(74)$ & -4.841 & -14.845 & 3.401 \\
\hline $\mathrm{H}(18)$ & -4.965 & 0.435 & 5.354 & $\mathrm{H}(75)$ & -4.837 & -15.089 & 2.330 \\
$\mathrm{C}(19)$ & -6.379 & -2.723 & 5.696 & $\mathrm{H}(76)$ & -5.718 & -14.204 & 3.571 \\
\hline $\mathrm{C}(20)$ & -7.576 & -2.537 & 6.279 & $\mathrm{C}(77)$ & -5.034 & -16.135 & 4.225 \\
$\mathrm{H}(21)$ & -7.986 & -1.535 & 6.387 & $\mathrm{H}(78)$ & -5.074 & -15.878 & 5.291 \\
\hline $\mathrm{H}(22)$ & -6.019 & -3.744 & 5.593 & $\mathrm{C}(79)$ & -3.896 & -17.135 & 4.017 \\
$\mathrm{C}(23)$ & -8.429 & -3.635 & 6.858 & $\mathrm{H}(80)$ & -2.948 & -16.746 & 4.401 \\
\hline $\mathrm{H}(24)$ & -7.870 & -4.573 & 6.944 & $\mathrm{H}(81)$ & -4.099 & -18.070 & 4.550 \\
$\mathrm{C}(25)$ & -9.744 & -3.864 & 6.072 & $\mathrm{H}(82)$ & -3.764 & -17.372 & 2.956 \\
$\mathrm{H}(26)$ & -10.208 & -2.882 & 5.889 & $\mathrm{C}(83)$ & -6.368 & -16.788 & 3.857 \\
$\mathrm{O}(27)$ & -8.732 & -3.219 & 8.196 & $\mathrm{H}(84)$ & -6.390 & -17.081 & 2.801 \\
\hline & & & & & continued on the next page
\end{tabular}


Table S13: Coordinates of Cluster 1 of $(S)$-iso-archazolid A.

\begin{tabular}{l|c|c|c||c|c|c|c}
\hline atom & $\mathrm{X}$ & $\mathrm{Y}$ & $\mathrm{Z}$ & $\mathrm{atom}$ & $\mathrm{X}$ & $\mathrm{Y}$ & $\mathrm{Z}$ \\
\hline $\mathrm{H}(28)$ & -7.868 & -3.121 & 8.648 & $\mathrm{H}(85)$ & -6.542 & -17.685 & 4.460 \\
$\mathrm{C}(29)$ & -9.489 & -4.505 & 4.675 & $\mathrm{H}(86)$ & -7.201 & -16.100 & 4.036 \\
$\mathrm{H}(30)$ & -8.788 & -3.845 & 4.151 & $\mathrm{O}(87)$ & -3.629 & -13.707 & 5.154 \\
$\mathrm{C}(31)$ & -10.779 & -4.625 & 6.917 & $\mathrm{C}(88)$ & -2.452 & -13.381 & 5.732 \\
$\mathrm{H}(32)$ & -10.361 & -5.545 & 7.337 & $\mathrm{O}(89)$ & -1.363 & -13.390 & 5.176 \\
$\mathrm{H}(33)$ & -11.119 & -4.010 & 7.757 & $\mathrm{~N}(90)$ & -2.670 & -13.045 & 7.030 \\
$\mathrm{H}(34)$ & -11.668 & -4.878 & 6.332 & $\mathrm{C}(91)$ & -1.634 & -12.620 & 7.936 \\
$\mathrm{C}(35)$ & -8.931 & -5.931 & 4.698 & $\mathrm{H}(92)$ & -0.655 & -12.978 & 7.607 \\
$\mathrm{O}(36)$ & -10.726 & -4.482 & 3.932 & $\mathrm{H}(93)$ & -1.633 & -11.528 & 7.968 \\
$\mathrm{C}(37)$ & -10.515 & -4.448 & 2.521 & $\mathrm{H}(94)$ & -1.860 & -13.014 & 8.929 \\
\hline $\mathrm{H}(38)$ & -11.493 & -4.485 & 2.033 & $\mathrm{H}(95)$ & -3.633 & -13.058 & 7.349 \\
$\mathrm{H}(39)$ & -10.023 & -3.515 & 2.234 & $\mathrm{H}(96)$ & -7.140 & -3.457 & -1.598 \\
$\mathrm{H}(40)$ & -9.929 & -5.308 & 2.186 & $\mathrm{C}(97)$ & -6.975 & -5.230 & -0.332 \\
$\mathrm{C}(41)$ & -7.635 & -6.146 & 4.388 & $\mathrm{C}(98)$ & -7.257 & -4.542 & -1.641 \\
\hline $\mathrm{C}(42)$ & -9.882 & -7.057 & 5.023 & $\mathrm{H}(99)$ & -8.284 & -4.746 & -1.962 \\
$\mathrm{H}(43)$ & -10.928 & -6.763 & 4.896 & $\mathrm{H}(100)$ & -6.576 & -4.903 & -2.420 \\
\hline $\mathrm{H}(44)$ & -9.731 & -7.913 & 4.357 & $\mathrm{C}(101)$ & -6.627 & -4.616 & 0.818 \\
$\mathrm{H}(45)$ & -9.745 & -7.387 & 6.057 & $\mathrm{H}(102)$ & -6.413 & -5.239 & 1.685 \\
\hline $\mathrm{H}(46)$ & -7.014 & -5.293 & 4.118 & $\mathrm{C}(103)$ & -6.463 & -3.144 & 1.084 \\
$\mathrm{C}(47)$ & -6.957 & -7.423 & 4.385 & $\mathrm{H}(104)$ & -6.835 & -2.560 & 0.238 \\
\hline $\mathrm{C}(48)$ & -5.671 & -7.564 & 4.024 & $\mathrm{C}(105)$ & -4.990 & -2.714 & 1.357 \\
$\mathrm{H}(49)$ & -5.089 & -6.696 & 3.718 & $\mathrm{H}(106)$ & -4.411 & -2.988 & 0.462 \\
\hline $\mathrm{H}(50)$ & -7.519 & -8.304 & 4.685 & $\mathrm{C}(107)$ & -4.316 & -3.481 & 2.497 \\
$\mathrm{C}(51)$ & -4.959 & -8.907 & 3.988 & $\mathrm{H}(108)$ & -3.967 & -4.462 & 2.163 \\
$\mathrm{H}(52)$ & -5.655 & -9.685 & 4.327 & $\mathrm{O}(109)$ & -7.330 & -2.814 & 2.173 \\
$\mathrm{C}(53)$ & -3.800 & -8.863 & 4.994 & $\mathrm{H}(110)$ & -6.775 & -2.812 & 2.976 \\
\hline $\mathrm{H}(54)$ & -4.162 & -8.602 & 5.995 & $\mathrm{C}(111)$ & -4.891 & -1.185 & 1.435 \\
$\mathrm{H}(55)$ & -3.297 & -9.831 & 5.074 & $\mathrm{H}(112)$ & -5.550 & -0.761 & 2.199 \\
\hline $\mathrm{H}(56)$ & -3.049 & -8.118 & 4.706 & $\mathrm{H}(113)$ & -3.864 & -0.862 & 1.634 \\
$\mathrm{H}(57)$ & -3.642 & -8.551 & 2.329 & $\mathrm{H}(114)$ & -5.188 & -0.741 & 0.478 \\
\hline & & & & & & & \\
\hline
\end{tabular}



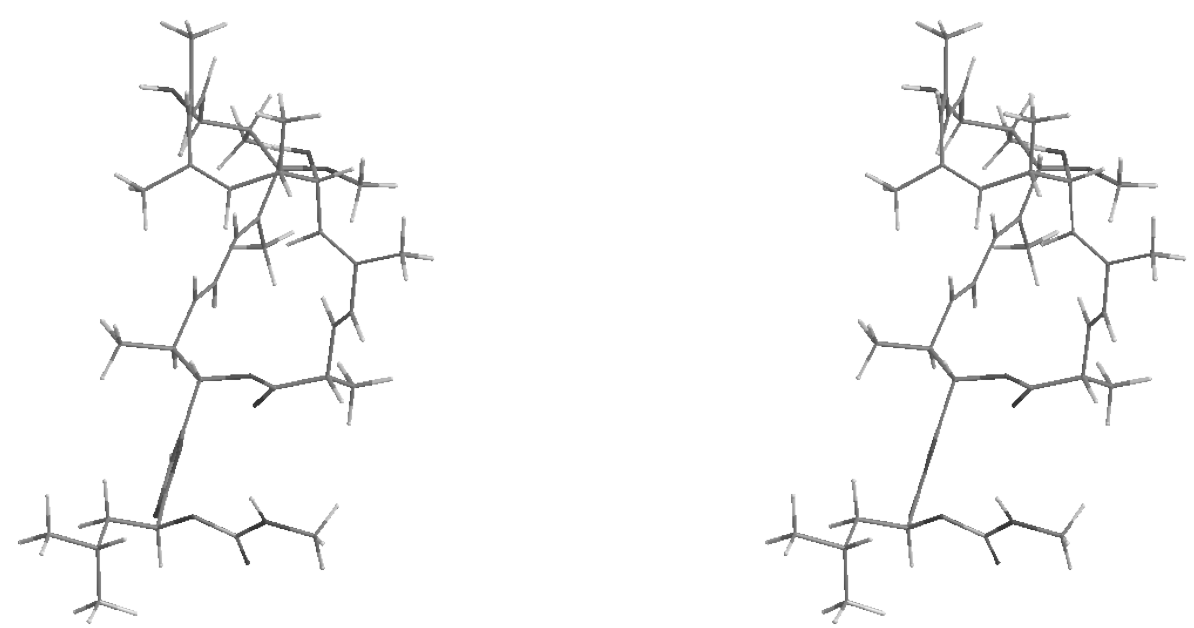

Scheme S6. Conformation of Cluster 2 of (S)-iso-archazolid A.

Table S14: Coordinates of Cluster 2 of $(S)$-iso-archazolid A.

\begin{tabular}{l|c|c|c||c|c|c|c}
\hline atom & $\mathrm{X}$ & $\mathrm{Y}$ & $\mathrm{Z}$ & $\mathrm{atom}$ & $\mathrm{X}$ & $\mathrm{Y}$ & $\mathrm{Z}$ \\
\hline $\mathrm{C}(1)$ & -8.250 & -8.211 & 1.708 & $\mathrm{C}(58)$ & -5.417 & -9.674 & 4.335 \\
$\mathrm{H}(2)$ & -10.535 & -9.704 & 1.458 & $\mathrm{C}(59)$ & -8.471 & -9.641 & 2.147 \\
$\mathrm{H}(3)$ & -10.137 & -10.933 & 2.669 & $\mathrm{H}(60)$ & -8.117 & -10.346 & 1.384 \\
$\mathrm{C}(4)$ & -7.583 & -7.869 & 0.593 & $\mathrm{C}(61)$ & -9.960 & -9.893 & 2.372 \\
$\mathrm{H}(5)$ & -8.632 & -7.438 & 2.373 & $\mathrm{H}(62)$ & -10.363 & -9.253 & 3.165 \\
$\mathrm{H}(6)$ & -7.212 & -8.649 & -0.073 & $\mathrm{C}(63)$ & -7.665 & -9.924 & 3.420 \\
$\mathrm{C}(7)$ & -3.956 & -3.676 & 3.450 & $\mathrm{O}(64)$ & -8.129 & -10.394 & 4.452 \\
$\mathrm{C}(8)$ & -3.298 & -4.367 & 4.619 & $\mathrm{O}(65)$ & -6.357 & -9.602 & 3.229 \\
$\mathrm{H}(9)$ & -3.146 & -5.436 & 4.430 & $\mathrm{C}(66)$ & -5.049 & -11.108 & 4.660 \\
$\mathrm{H}(10)$ & -2.313 & -3.929 & 4.816 & $\mathrm{C}(67)$ & -5.667 & -11.961 & 5.556 \\
$\mathrm{H}(11)$ & -3.901 & -4.281 & 5.528 & $\mathrm{~S}(68)$ & -4.898 & -13.489 & 5.564 \\
$\mathrm{C}(12)$ & -4.121 & -2.242 & 3.601 & $\mathrm{C}(69)$ & -3.793 & -12.932 & 4.368 \\
$\mathrm{C}(13)$ & -5.102 & -1.582 & 4.253 & $\mathrm{~N}(70)$ & -3.974 & -11.682 & 3.983 \\
\hline $\mathrm{H}(14)$ & -3.350 & -1.665 & 3.089 & $\mathrm{H}(71)$ & -6.521 & -11.767 & 6.192 \\
$\mathrm{C}(15)$ & -5.110 & -0.072 & 4.215 & $\mathrm{C}(72)$ & -2.719 & -13.862 & 3.845 \\
\hline $\mathrm{H}(16)$ & -5.991 & 0.298 & 3.680 & $\mathrm{H}(73)$ & -3.013 & -14.910 & 3.975 \\
$\mathrm{H}(17)$ & -5.110 & 0.343 & 5.229 & $\mathrm{C}(74)$ & -1.390 & -13.605 & 4.577 \\
$\mathrm{H}(18)$ & -4.230 & 0.335 & 3.704 & $\mathrm{H}(75)$ & -1.522 & -13.789 & 5.652 \\
$\mathrm{C}(19)$ & -6.140 & -2.247 & 5.038 & $\mathrm{H}(76)$ & -1.128 & -12.543 & 4.473 \\
$\mathrm{C}(20)$ & -7.275 & -1.682 & 5.485 & $\mathrm{C}(77)$ & -0.200 & -14.436 & 4.052 \\
$\mathrm{H}(21)$ & -7.511 & -0.649 & 5.236 & $\mathrm{H}(78)$ & -0.048 & -14.213 & 2.989 \\
\hline $\mathrm{H}(22)$ & -5.955 & -3.287 & 5.295 & $\mathrm{C}(79)$ & -0.430 & -15.940 & 4.197 \\
$\mathrm{C}(23)$ & -8.283 & -2.366 & 6.370 & $\mathrm{H}(80)$ & -1.254 & -16.280 & 3.563 \\
\hline $\mathrm{H}(24)$ & -7.886 & -3.300 & 6.782 & $\mathrm{H}(81)$ & 0.462 & -16.500 & 3.894 \\
$\mathrm{C}(25)$ & -9.637 & -2.624 & 5.663 & $\mathrm{H}(82)$ & -0.661 & -16.209 & 5.233 \\
$\mathrm{H}(26)$ & -9.937 & -1.698 & 5.149 & $\mathrm{C}(83)$ & 1.077 & -14.035 & 4.796 \\
$\mathrm{O}(27)$ & -8.479 & -1.482 & 7.481 & $\mathrm{H}(84)$ & 0.995 & -14.243 & 5.868 \\
$\mathrm{H}(28)$ & -7.600 & -1.381 & 7.901 & $\mathrm{H}(85)$ & 1.941 & -14.586 & 4.409 \\
$\mathrm{C}(29)$ & -9.527 & -3.729 & 4.569 & $\mathrm{H}(86)$ & 1.281 & -12.967 & 4.671 \\
\hline & & & & $c 0 n t i n u e d$ & on the next page
\end{tabular}


Table S14: Coordinates of Cluster 2 of $(S)$-iso-archazolid A.

\begin{tabular}{c|c|c|c||c|c|c|c}
\hline atom & $\mathrm{X}$ & $\mathrm{Y}$ & $\mathrm{Z}$ & atom & $\mathrm{X}$ & $\mathrm{Y}$ & $\mathrm{Z}$ \\
\hline $\mathrm{H}(30)$ & -8.739 & -3.407 & 3.878 & $\mathrm{O}(87)$ & -2.523 & -13.569 & 2.444 \\
$\mathrm{C}(31)$ & -10.762 & -2.878 & 6.680 & $\mathrm{C}(88)$ & -3.405 & -14.138 & 1.593 \\
\hline $\mathrm{H}(32)$ & -10.492 & -3.661 & 7.395 & $\mathrm{O}(89)$ & -4.325 & -14.877 & 1.915 \\
$\mathrm{H}(33)$ & -10.974 & -1.971 & 7.256 & $\mathrm{~N}(90)$ & -3.104 & -13.765 & 0.322 \\
$\mathrm{H}(34)$ & -11.696 & -3.161 & 6.185 & $\mathrm{C}(91)$ & -3.848 & -14.179 & -0.839 \\
$\mathrm{C}(35)$ & -9.216 & -5.136 & 5.085 & $\mathrm{H}(92)$ & -3.140 & -14.410 & -1.639 \\
$\mathrm{O}(36)$ & -10.761 & -3.750 & 3.820 & $\mathrm{H}(93)$ & -4.464 & -15.056 & -0.623 \\
$\mathrm{C}(37)$ & -10.583 & -4.225 & 2.486 & $\mathrm{H}(94)$ & -4.488 & -13.349 & -1.150 \\
\hline $\mathrm{H}(38)$ & -11.565 & -4.262 & 2.007 & $\mathrm{H}(95)$ & -2.322 & -13.128 & 0.207 \\
$\mathrm{H}(39)$ & -9.951 & -3.535 & 1.921 & $\mathrm{H}(96)$ & -7.182 & -5.257 & -1.609 \\
\hline $\mathrm{H}(40)$ & -10.155 & -5.231 & 2.476 & $\mathrm{C}(97)$ & -7.292 & -6.500 & 0.184 \\
$\mathrm{C}(41)$ & -7.980 & -5.653 & 4.913 & $\mathrm{C}(98)$ & -7.483 & -6.256 & -1.289 \\
$\mathrm{C}(42)$ & -10.335 & -5.915 & 5.731 & $\mathrm{H}(99)$ & -8.538 & -6.381 & -1.558 \\
$\mathrm{H}(43)$ & -10.230 & -5.904 & 6.821 & $\mathrm{H}(100)$ & -6.892 & -6.967 & -1.877 \\
\hline $\mathrm{H}(44)$ & -11.319 & -5.509 & 5.481 & $\mathrm{C}(101)$ & -6.823 & -5.600 & 1.073 \\
$\mathrm{H}(45)$ & -10.347 & -6.956 & 5.391 & $\mathrm{H}(102)$ & -6.695 & -5.921 & 2.106 \\
\hline $\mathrm{H}(46)$ & -7.231 & -5.054 & 4.397 & $\mathrm{C}(103)$ & -6.412 & -4.172 & 0.836 \\
$\mathrm{C}(47)$ & -7.528 & -6.951 & 5.360 & $\mathrm{H}(104)$ & -6.705 & -3.854 & -0.168 \\
\hline $\mathrm{C}(48)$ & -6.292 & -7.415 & 5.117 & $\mathrm{C}(105)$ & -4.881 & -3.923 & 0.995 \\
$\mathrm{H}(49)$ & -5.576 & -6.809 & 4.564 & $\mathrm{H}(106)$ & -4.379 & -4.572 & 0.260 \\
\hline $\mathrm{H}(50)$ & -8.226 & -7.575 & 5.913 & $\mathrm{C}(107)$ & -4.313 & -4.362 & 2.345 \\
$\mathrm{C}(51)$ & -5.822 & -8.793 & 5.555 & $\mathrm{H}(108)$ & -4.141 & -5.442 & 2.371 \\
\hline $\mathrm{H}(52)$ & -6.635 & -9.281 & 6.106 & $\mathrm{O}(109)$ & -7.184 & -3.359 & 1.723 \\
$\mathrm{C}(53)$ & -4.655 & -8.622 & 6.537 & $\mathrm{H}(110)$ & -6.615 & -3.178 & 2.496 \\
$\mathrm{H}(54)$ & -4.944 & -7.985 & 7.381 & $\mathrm{C}(111)$ & -4.531 & -2.494 & 0.559 \\
$\mathrm{H}(55)$ & -4.337 & -9.585 & 6.950 & $\mathrm{H}(112)$ & -5.091 & -1.737 & 1.116 \\
\hline $\mathrm{H}(56)$ & -3.788 & -8.161 & 6.052 & $\mathrm{H}(113)$ & -3.460 & -2.296 & 0.669 \\
$\mathrm{H}(57)$ & -4.506 & -9.221 & 3.919 & $\mathrm{H}(114)$ & -4.776 & -2.354 & -0.501 \\
\hline
\end{tabular}
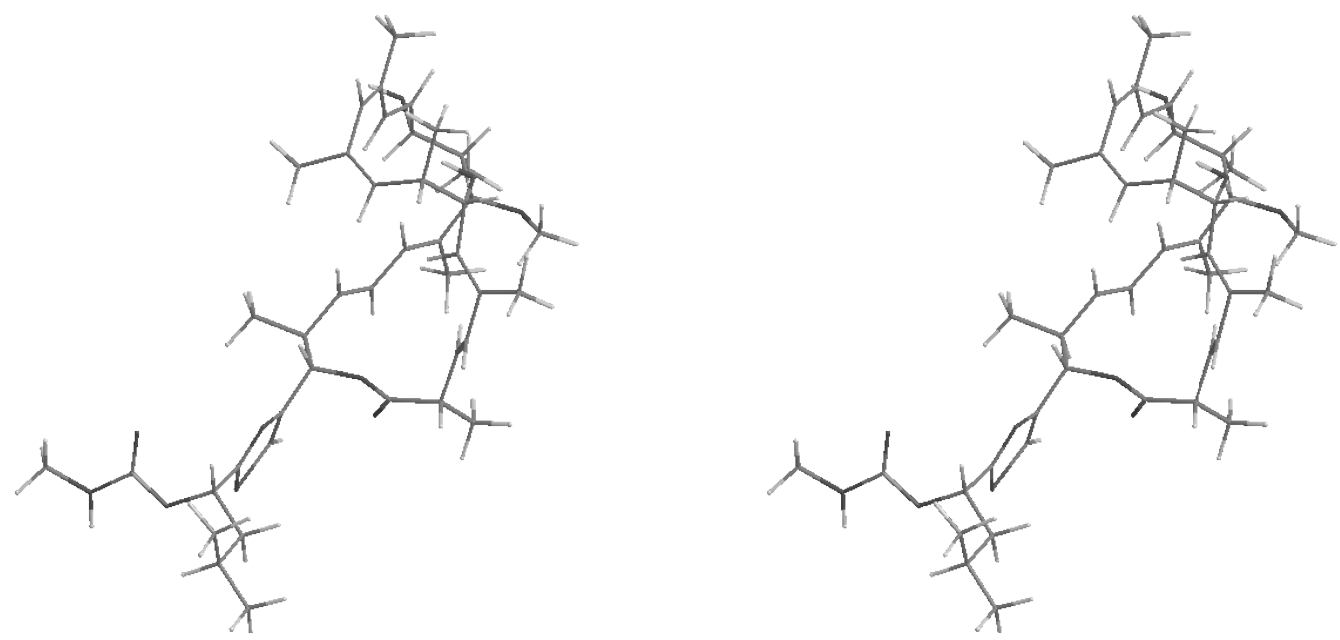

Scheme S7. Conformation of Cluster 3 of (S)-iso-archazolid A. 
Table S15: Coordinates of Cluster 3 of (S)-iso-archazolid A.

\begin{tabular}{|c|c|c|c|c|c|c|c|}
\hline atom & $x$ & $Y$ & Z & atom & $x$ & $Y$ & z \\
\hline$C(1)$ & 8.228 & -7.858 & 1.305 & (58) & -5.567 & -9.635 & J.9 \\
\hline & 0.532 & -9.253 & 809 & 59) & -8.504 & -9.315 & .596 \\
\hline$H(3)$ & 0.224 & -10.602 & 1.914 & (60) & -8.129 & -9.956 & .788 \\
\hline$C(4)$ & -7.497 & -7.432 & 0.262 & $C(61)$ & -10.008 & -9.545 & .726 \\
\hline$H(5)$ & -8.626 & -7.140 & 2.021 & (62) & -10.434 & -8.971 & 2.557 \\
\hline$H(6)$ & -7.110 & -8.157 & -0.455 & 63) & -7.769 & -9.739 & .873 \\
\hline$C(7)$ & -3.926 & -3.615 & 3.658 & (64) & -8.293 & -10.298 & 3.829 \\
\hline$C(8)$ & -3.340 & -4.431 & 4.783 & $\mathrm{O}(65)$ & -6.448 & -9.429 & 2.779 \\
\hline$H(9)$ & -2.354 & -4.043 & 5.062 & $C(66)$ & -5.251 & -11.104 & 4.116 \\
\hline $\mathrm{H}(10)$ & -3.980 & -4.411 & 5.670 & (67) & -5.917 & -12.016 & 4.914 \\
\hline $\mathrm{H}(11)$ & -3.208 & -5.482 & .503 & (68) & -5.195 & -13.562 & 4.793 \\
\hline$C(12)$ & -4.066 & -2.198 & 3.937 & $C(69)$ & -4.039 & -12.932 & 3.681 \\
\hline$C(13)$ & -5.062 & -1.576 & 4.603 & (70) & -4.178 & -11.648 & 3.411 \\
\hline $\mathrm{H}(14)$ & -3.259 & -1.597 & 3.517 & (71) & -6.780 & -11 & 5.545 \\
\hline$C(15)$ & -5.035 & -0.069 & 709 & (72) & -2.969 & -13.813 & .064 \\
\hline$H(16)$ & -5.881 & 0.372 & 4.171 & (73) & -2.204 & -13.166 & 2.617 \\
\hline $\mathrm{H}(17)$ & -5.073 & 248 & 5.757 & (74) & -3.558 & 14.716 & 1.966 \\
\hline $\mathrm{H}(18)$ & -4.122 & 359 & .279 & (75) & -4.035 & -14.092 & 1.198 \\
\hline$C(19)$ & -6.151 & 2.283 & 274 & (76) & -4.352 & 39 & 2.399 \\
\hline$C(20)$ & -7.292 & -1.732 & 5.721 & (77) & -2.534 & -15 & 1.305 \\
\hline $\mathrm{H}(21)$ & -7.493 & -0.675 & 5.562 & 8) & -2.092 & 06 & 2.076 \\
\hline $\mathrm{H}(22)$ & -6.002 & -3.348 & 5.439 & (79) & -1.403 & -14.907 & 0.607 \\
\hline$C(23)$ & -8.357 & -2.468 & 493 & (80) & -0.786 & 660 & .326 \\
\hline $\mathrm{H}(24)$ & -8.000 & -3.446 & .833 & $\mathrm{H}(\varepsilon$ & -0.741 & 00 & 0.077 \\
\hline$C(25)$ & -9.682 & -2.624 & 5.706 & 2) & -1.796 & 91 & -0.123 \\
\hline $\mathrm{H}(26)$ & -9.935 & -1.647 & 5.266 & (8) & -3.246 & -16.570 & 0.297 \\
\hline $\mathrm{O}(27)$ & -8.584 & -1.685 & 7.672 & r) & -3.703 & -15.986 & -0.509 \\
\hline $\mathrm{H}(28)$ & -7.724 & -1.648 & 8.139 & (85) & -2.542 & -17.278 & -0.153 \\
\hline$C(29)$ & -9.546 & -3.627 & 4.521 & (86) & -4.035 & 17.151 & 0.785 \\
\hline $\mathrm{H}(30)$ & -8.719 & -3.266 & 3.898 & (8) & -2.394 & -14.645 & 4.092 \\
\hline$C(31)$ & 0.859 & -2.938 & 6.644 & $C(8)$ & -1.365 & 12 & 4.788 \\
\hline $\mathrm{H}(32)$ & -10.641 & -3.790 & 7.295 & (89) & -0.894 & -12.997 & 4.620 \\
\hline $\mathrm{H}(33$ & -11.076 & -2.081 & 7.291 & ) & -0.937 & & 5.708 \\
\hline $\mathrm{H}(34$ & -11.775 & -3.149 & 6.083 & $C(91)$ & 0.140 & -14.763 & 6.631 \\
\hline$C(35$ & -9.293 & $-5.0 \varepsilon$ & 4.919 & 14 & 67 & & 6.879 \\
\hline $\mathrm{O}(36)$ & -10.743 & -3.546 & 3.719 & (93) & 0.875 & -14.084 & 6.191 \\
\hline$C(37)$ & -10.514 & -3.904 & 2.356 & (94) & -0.277 & -14.314 & 7.535 \\
\hline $\mathrm{H}(3$ & -11.473 & -3.868 & 1.832 & (95) & -1.440 & -15.894 & 5.758 \\
\hline $\mathrm{H}(39)$ & -9.837 & -3.187 & 1.885 & $H(96)$ & -6.912 & -4.645 & -1.667 \\
\hline $\mathrm{H}(40)$ & -10.114 & -4.918 & 2.274 & $C(97)$ & -7.155 & -6.039 & -0.003 \\
\hline$C(41)$ & -8.063 & -5.617 & 4.755 & $C(98)$ & -7.255 & -5.659 & -1.457 \\
\hline$C(4$ & -10 & -5 . & & & -8.295 & -5 & -1.795 \\
\hline $\mathrm{H}(43$ & -11.421 & -5.429 & 5.189 & 111 & -6.647 & -6.332 & -2.073 \\
\hline $\mathrm{H}(44)$ & -10.483 & -6.888 & 4.997 & $C(101)$ & -6.720 & -5.235 & 0.988 \\
\hline
\end{tabular}

continued on the next page 
Table S15: Coordinates of Cluster 3 of $(S)$-iso-archazolid A.

\begin{tabular}{c|c|c|c||c|c|c|c}
\hline atom & $\mathrm{X}$ & $\mathrm{Y}$ & $\mathrm{Z}$ & atom & $\mathrm{X}$ & $\mathrm{Y}$ & $\mathrm{Z}$ \\
\hline $\mathrm{H}(45)$ & -10.403 & -5.984 & 6.527 & $\mathrm{H}(102)$ & -6.657 & -5.650 & 1.993 \\
$\mathrm{H}(46)$ & -7.275 & -4.995 & 4.332 & $\mathrm{C}(103)$ & -6.270 & -3.802 & 0.904 \\
$\mathrm{C}(47)$ & -7.664 & -6.963 & 5.098 & $\mathrm{H}(104)$ & -6.511 & -3.384 & -0.077 \\
$\mathrm{C}(48)$ & -6.429 & -7.438 & 4.870 & $\mathrm{C}(105)$ & -4.743 & -3.606 & 1.152 \\
$\mathrm{H}(49)$ & -5.673 & -6.802 & 4.411 & $\mathrm{H}(106)$ & -4.222 & -4.196 & 0.382 \\
$\mathrm{H}(50)$ & -8.404 & -7.616 & 5.556 & $\mathrm{C}(107)$ & -4.247 & -4.185 & 2.478 \\
$\mathrm{C}(51)$ & -6.014 & -8.863 & 5.196 & $\mathrm{H}(108)$ & -4.100 & -5.266 & 2.409 \\
$\mathrm{H}(52)$ & -6.866 & -9.378 & 5.656 & $\mathrm{O}(109)$ & -7.064 & -3.056 & 1.830 \\
$\mathrm{C}(53)$ & -4.896 & -8.819 & 6.246 & $\mathrm{H}(110)$ & -6.526 & -2.958 & 2.639 \\
$\mathrm{H}(54)$ & -5.214 & -8.258 & 7.132 & $\mathrm{C}(111)$ & -4.344 & -2.152 & 0.870 \\
$\mathrm{H}(55)$ & -4.622 & -9.825 & 6.580 & $\mathrm{H}(112)$ & -4.912 & -1.437 & 1.472 \\
$\mathrm{H}(56)$ & -3.995 & -8.337 & 5.851 & $\mathrm{H}(113)$ & -3.275 & -1.992 & 1.045 \\
$\mathrm{H}(57)$ & -4.625 & -9.170 & 3.593 & $\mathrm{H}(114)$ & -4.538 & -1.907 & -0.181 \\
\hline
\end{tabular}
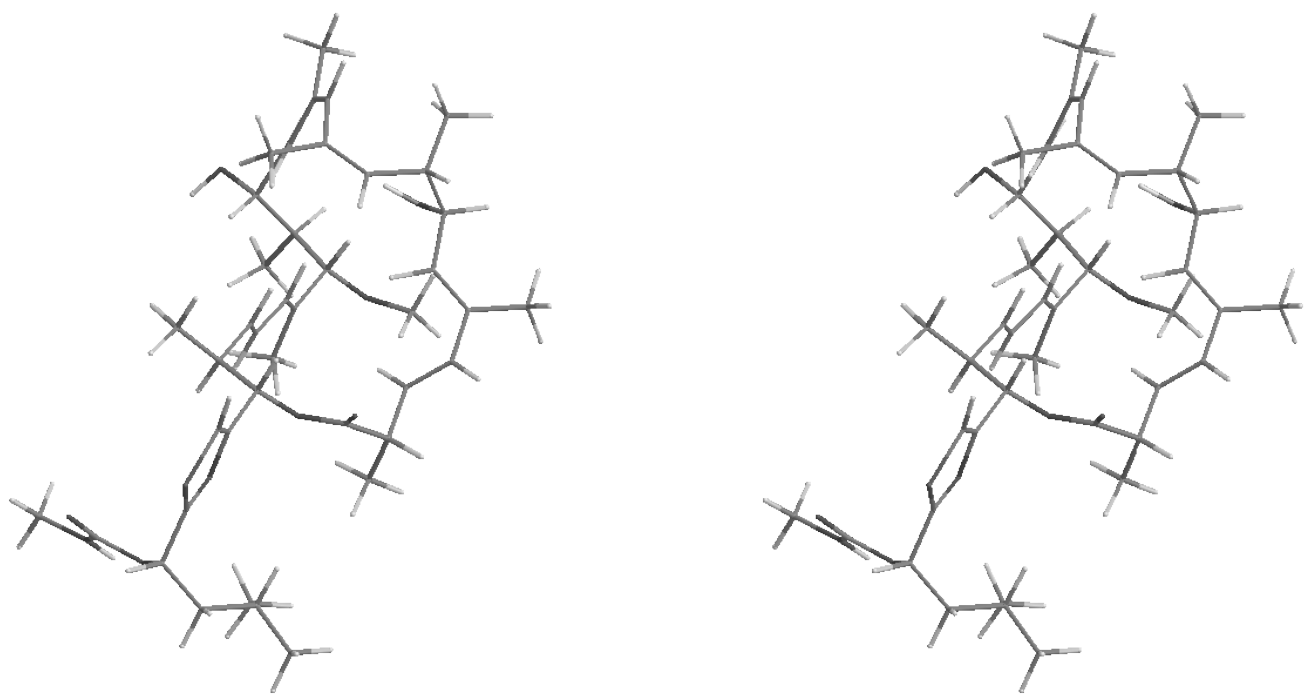

Scheme S8. Conformation of Cluster 4 of $(S)$-iso-archazolid A.

Table S16: Coordinates of Cluster 4 of (S)-iso-archazolid A.

\begin{tabular}{l|c|c|c||c|c|c|c}
\hline atom & $\mathrm{X}$ & $\mathrm{Y}$ & $\mathrm{Z}$ & atom & $\mathrm{X}$ & $\mathrm{Y}$ & $\mathrm{Z}$ \\
\hline $\mathrm{C}(1)$ & -7.312 & -7.960 & 0.751 & $\mathrm{C}(58)$ & -3.970 & -9.317 & 2.900 \\
$\mathrm{H}(2)$ & -8.834 & -10.318 & 1.043 & $\mathrm{C}(59)$ & -6.908 & -9.410 & 0.622 \\
$\mathrm{H}(3)$ & -7.445 & -11.363 & 1.397 & $\mathrm{H}(60)$ & -6.964 & -9.735 & -0.425 \\
$\mathrm{C}(4)$ & -7.405 & -7.117 & -0.290 & $\mathrm{C}(61)$ & -7.811 & -10.332 & 1.436 \\
$\mathrm{H}(5)$ & -7.480 & -7.596 & 1.764 & $\mathrm{H}(62)$ & -7.856 & -10.038 & 2.491 \\
$\mathrm{H}(6)$ & -7.237 & -7.494 & -1.300 & $\mathrm{C}(63)$ & -5.441 & -9.507 & 1.032 \\
$\mathrm{C}(7)$ & -4.355 & -2.730 & 3.085 & $\mathrm{O}(64)$ & -4.505 & -9.475 & 0.238 \\
$\mathrm{C}(8)$ & -3.260 & -3.408 & 3.871 & $\mathrm{O}(65)$ & -5.308 & -9.523 & 2.383 \\
$\mathrm{H}(9)$ & -2.409 & -2.730 & 4.001 & $\mathrm{C}(66)$ & -3.224 & -10.629 & 2.946 \\
\hline
\end{tabular}


Table S16: Coordinates of Cluster 4 of (S)-iso-archazolid A.

\begin{tabular}{|c|c|c|c|c|c|c|c|}
\hline om & $x$ & Y & Z & atom & $x$ & $Y$ & Z \\
\hline $\mathrm{H}(10)$ & -3.610 & 716 & .861 & $C(67)$ & -1.958 & -10.871 & 2.446 \\
\hline $\mathrm{H}(11)$ & -2.887 & 4.305 & .365 & $5(68)$ & -1.521 & -12.507 & 2.696 \\
\hline$C(12)$ & -4.849 & 1.496 & 665 & $C(69)$ & -3.046 & -12.795 & 3.442 \\
\hline (13) & -5.777 & 1.345 & 634 & (70) & -3.835 & -11.739 & 3.519 \\
\hline (14) & -4.409 & .604 & 216 & (71) & -1.286 & -10.180 & 1.954 \\
\hline C(15) & -6.192 & 0.053 & 5.032 & (72) & -3.392 & -14.177 & 3.947 \\
\hline$H(16)$ & -7.243 & 0.230 & 4.780 & $\mathrm{H}(73)$ & -2.489 & -14.698 & 4.294 \\
\hline (17) & -6.052 & 210 & 107 & (74) & -4.035 & -15.057 & 2.857 \\
\hline (18) & -5.605 & 0.822 & .517 & (75) & -4.328 & -16.009 & 3.319 \\
\hline$C(19)$ & -6.373 & -2.463 & 5.362 & (76) & -3.261 & -15.295 & 2.115 \\
\hline$C(20)$ & -7.467 & -2.401 & 6.141 & $(7 i)$ & -5.260 & -14.489 & 2.100 \\
\hline $\mathrm{H}(21)$ & -8.017 & -1.468 & 6.242 & (78) & -4.977 & -13.553 & 1.604 \\
\hline $\mathrm{H}(22)$ & -5.864 & -3.421 & 276 & (79) & -6.462 & -14.208 & 3.001 \\
\hline$C(23)$ & -8.007 & -3.541 & 6.963 & $\mathrm{H}(80)$ & -6.271 & -13.367 & 3.673 \\
\hline $\mathrm{H}(24)$ & -7.263 & -4.341 & 7.050 & 5) & -7.342 & -13.941 & 2.405 \\
\hline$C(25)$ & -9.354 & -4.088 & 6.426 & (82) & -6.720 & -15.084 & 3.606 \\
\hline $\mathrm{H}(26)$ & -9.996 & -3.229 & 6.177 & 10 & -5.673 & -15.473 & 0.999 \\
\hline $\mathrm{O}(27)$ & -8.172 & -2.984 & 8.274 & (84) & -5.987 & -16.434 & 1.421 \\
\hline $\mathrm{H}(28)$ & -8.148 & -3.739 & 8.896 & 5) & -6.507 & -15.073 & 0.411 \\
\hline$C(29)$ & -9.172 & -4.902 & 5.107 & r & -4.842 & -15.659 & 0.311 \\
\hline$H(30)$ & -8.677 & -4.234 & t.393 & (8 & -4.309 & -14.055 & 5.055 \\
\hline$C(31)$ & -10.138 & -4.844 & 7.511 & (88) & -3.765 & -13.712 & 6.244 \\
\hline $\mathrm{H}(32)$ & -9.526 & -5.611 & 7.994 & 0 (००) & -2.573 & -13 & 6.455 \\
\hline $\mathrm{H}(33)$ & -10.473 & -4.1 & 293 & & -4.753 & -13.598 & 7.169 \\
\hline (34) & -11.037 & -5.317 & 7.104 & 9 & -4.539 & -13.235 & 8.546 \\
\hline$C(35)$ & -8.368 & -6.197 & 5.241 & 2) & -5.023 & -12.272 & 8.725 \\
\hline $\mathrm{O}(36)$ & -10.480 & -5.200 & 4.574 & sic & -5.005 & -13 & 9.178 \\
\hline$C(37$ & -10.469 & -5.3 & 159 & & 3.474 & -13.161 & 8.780 \\
\hline $\mathrm{H}(38)$ & -11.47 & -5.666 & 2.847 & (9 & -5.700 & -13.765 & 6.844 \\
\hline $\mathrm{H}(39)$ & -10.213 & -4.438 & 2.663 & (96) & -9.474 & -5.814 & -1.385 \\
\hline $\mathrm{H}(4 \mathrm{C}$ & -9.776 & -6.169 & 2.861 & $\rho(0$ & -7.689 & -5.690 & -0.195 \\
\hline$C(41$ & -7.116 & -6.26 & 4.135 & $C(98)$ & -8.583 & -5.181 & -1.295 \\
\hline$C(42)$ & -9.044 & $-7.37 \varepsilon$ & 5.895 & (00) & -8.055 & -5.195 & -2.255 \\
\hline $\mathrm{H}(43)$ & -8.843 & -8.308 & 5.352 & ) & -8.945 & -4.165 & -1.124 \\
\hline $\mathrm{H}(44)$ & -8.700 & -7.493 & 6.927 & $C(101)$ & -7.114 & -4.927 & 0.758 \\
\hline $\mathrm{H}(45)$ & -10.133 & -7.274 & .908 & (102) & -6.481 & -5.417 & 1.497 \\
\hline$H(46)$ & -6.702 & -5.398 & 4.230 & & -7.182 & -3.432 & 0.923 \\
\hline$C(47)$ & -6.236 & -7.413 & 4.820 & ) & -7.797 & -2.990 & 0.135 \\
\hline$C(48)$ & -5.017 & -7.448 & 4.259 & $C(105)$ & -5.788 & -2.728 & 0.873 \\
\hline (a) & & -6 & 07 & & 37 & -2.993 & -0.096 \\
\hline & & & & & -4.791 & -3.239 & 1.914 \\
\hline$C(51)$ & -4.124 & -8.680 & 4.312 & $\mathrm{H}(108)$ & -4.333 & -4.181 & 1.599 \\
\hline $\mathrm{H}(52)$ & -4.593 & -9.419 & 4.978 & $\mathrm{O}(109)$ & -7.873 & -3.168 & 2.144 \\
\hline$C(53)$ & -2.775 & -8.292 & 4.925 & $\mathrm{H}(110)$ & -7.189 & -2.976 & 2.814 \\
\hline
\end{tabular}

continued on the next page 
Table S16: Coordinates of Cluster 4 of $(S)$-iso-archazolid A.

\begin{tabular}{c|c|c|c||c|c|c|c}
\hline atom & $X$ & $Y$ & $Z$ & atom & $X$ & $Y$ & $Z$ \\
\hline$H(54)$ & -2.913 & -7.802 & 5.896 & $\mathrm{C}(111)$ & -5.971 & -1.205 & 0.818 \\
$\mathrm{H}(55)$ & -2.146 & -9.172 & 5.093 & $\mathrm{H}(112)$ & -6.553 & -0.824 & 1.661 \\
$\mathrm{H}(56)$ & -2.224 & -7.602 & 4.276 & $\mathrm{H}(113)$ & -5.005 & -0.689 & 0.790 \\
$\mathrm{H}(57)$ & -3.437 & -8.604 & 2.256 & $\mathrm{H}(114)$ & -6.511 & -0.925 & -0.094 \\
\hline
\end{tabular}
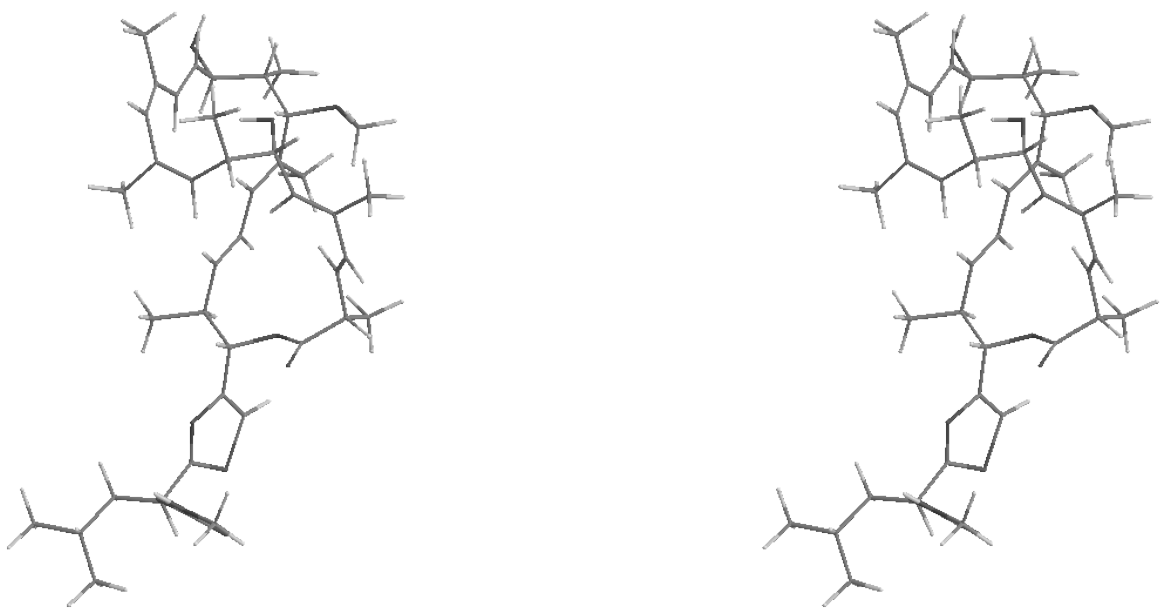

Scheme S9. Conformation of Cluster 5 of $(S)$-iso-archazolid A.

Table S17: Coordinates of Cluster 5 of $(S)$-iso-archazolid A.

\begin{tabular}{l|c|c|c||c|c|c|c}
\hline atom & $\mathrm{X}$ & $\mathrm{Y}$ & $\mathrm{Z}$ & atom & $\mathrm{X}$ & $\mathrm{Y}$ & $\mathrm{Z}$ \\
\hline $\mathrm{C}(1)$ & -7.963 & -7.754 & 0.950 & $\mathrm{C}(58)$ & -5.858 & -9.979 & 3.838 \\
$\mathrm{H}(2)$ & -10.289 & -9.340 & 1.026 & $\mathrm{C}(59)$ & -8.120 & -9.256 & 0.978 \\
$\mathrm{H}(3)$ & -9.493 & -10.765 & 1.718 & $\mathrm{H}(60)$ & -8.109 & -9.668 & -0.039 \\
$\mathrm{C}(4)$ & -7.538 & -7.066 & -0.122 & $\mathrm{C}(61)$ & -9.436 & -9.675 & 1.627 \\
$\mathrm{H}(5)$ & -8.168 & -7.234 & 1.885 & $\mathrm{H}(62)$ & -9.554 & -9.253 & 2.632 \\
$\mathrm{H}(6)$ & -7.339 & -7.596 & -1.054 & $\mathrm{C}(63)$ & -6.893 & -9.821 & 1.692 \\
$\mathrm{C}(7)$ & -3.926 & -3.693 & 3.792 & $\mathrm{O}(64)$ & -5.859 & -10.144 & 1.116 \\
$\mathrm{C}(8)$ & -3.386 & -4.616 & 4.856 & $\mathrm{O}(65)$ & -7.057 & -9.815 & 3.040 \\
$\mathrm{H}(9)$ & -2.408 & -4.265 & 5.205 & $\mathrm{C}(66)$ & -5.614 & -11.440 & 4.135 \\
\hline $\mathrm{H}(10)$ & -4.059 & -4.670 & 5.718 & $\mathrm{C}(67)$ & -6.568 & -12.384 & 4.473 \\
$\mathrm{H}(11)$ & -3.251 & -5.637 & 4.485 & $\mathrm{~S}(68)$ & -5.836 & -13.911 & 4.716 \\
$\mathrm{C}(12)$ & -4.063 & -2.307 & 4.195 & $\mathrm{C}(69)$ & -4.298 & -13.224 & 4.359 \\
$\mathrm{C}(13)$ & -5.082 & -1.733 & 4.868 & $\mathrm{~N}(70)$ & -4.316 & -11.935 & 4.078 \\
$\mathrm{H}(14)$ & -3.231 & -1.682 & 3.866 & $\mathrm{H}(71)$ & -7.636 & -12.248 & 4.583 \\
$\mathrm{C}(15)$ & -5.044 & -0.242 & 5.109 & $\mathrm{C}(72)$ & -3.059 & -14.094 & 4.391 \\
$\mathrm{H}(16)$ & -5.853 & 0.258 & 4.567 & $\mathrm{H}(73)$ & -3.313 & -15.143 & 4.198 \\
$\mathrm{H}(17)$ & -5.138 & -0.018 & 6.178 & $\mathrm{C}(74)$ & -2.361 & -13.974 & 5.758 \\
\hline $\mathrm{H}(18)$ & -4.104 & 0.208 & 4.770 & $\mathrm{H}(75)$ & -3.041 & -14.320 & 6.547 \\
$\mathrm{C}(19)$ & -6.205 & -2.482 & 5.427 & $\mathrm{H}(76)$ & -2.161 & -12.913 & 5.962 \\
$\mathrm{C}(20)$ & -7.370 & -1.957 & 5.845 & $\mathrm{C}(77)$ & -1.020 & -14.731 & 5.862 \\
\hline & & & & & continued on the next page
\end{tabular}


Table S17: Coordinates of Cluster 5 of (S)-iso-archazolid A.

\begin{tabular}{|c|c|c|c|c|c|c|c|}
\hline atom & $x$ & Y & Z & atom & X & $Y$ & Z \\
\hline (21) & .556 & .85 & 5.733 & $-178)$ & 27 & -14 & 5.109 \\
\hline (22) & .064 & .556 & 532 & 9) & 176 & -16.233 & 5.628 \\
\hline$C(23)$ & -8.474 & -2.735 & 514 & (80) & 1.489 & -16.446 & 4.601 \\
\hline $\mathrm{H}(24)$ & -8.117 & -3.720 & 6.830 & H(81) & 0.224 & -16 & 5.788 \\
\hline$C(25)$ & -9.746 & -2.881 & 5.639 & (82) & -1.916 & -16.667 & 6.309 \\
\hline 26) & -9.971 & -1.899 & 196 & 3) & 95 & -14 & 7.238 \\
\hline $\mathrm{O}(27)$ & -8.769 & -2.024 & 7.724 & (84) & -1.033 & -14.873 & 8.040 \\
\hline $\mathrm{H}(28)$ & -9.134 & -1.155 & 7.461 & (85) & 581 & -14 & 7.316 \\
\hline$C(29)$ & -9.531 & -3.866 & 4.448 & (86) & -0.242 & -13.415 & 7.412 \\
\hline 30 & -8.675 & -3.485 & 3.879 & & & -13 & 3.379 \\
\hline$C(31)$ & -10.983 & -3.209 & 6.493 & (88) & 2.399 & -14.018 & 2.115 \\
\hline $\mathrm{H}(32)$ & -10.804 & -4.061 & 7.154 & (89) & -3.297 & -14 & 1.782 \\
\hline$H(33)$ & -11.249 & -2.356 & 128 & (90) & .486 & -13 & 1.278 \\
\hline (34 & -11.857 & -3.421 & 5.871 & & .457 & -13. & -0.147 \\
\hline$C(35)$ & -9.280 & -5.325 & 4.838 & 92) & 2.175 & -14.434 & -0.454 \\
\hline$O(36)$ & -10.684 & -3.788 & 3.584 & (93) & 1.697 & -12.722 & -0.636 \\
\hline$C(37$ & -10.387 & -4.148 & 2.236 & (94) & 47 & -13 & -0.429 \\
\hline $\mathrm{H}(38$ & -11.318 & -4.113 & 1.665 & (95 & -0.802 & -12.840 & 1.699 \\
\hline $\mathrm{H}(39)$ & -9.688 & -3.432 & 1.797 & (96) & 8.696 & -5.264 & -1.722 \\
\hline $\mathrm{H}(40)$ & -9.984 & -5.163 & 2.175 & $C(97)$ & -7.276 & -5 & -0.151 \\
\hline$C(41$ & .046 & -5.856 & 4.694 & (9 & 77 & -4 & 1.443 \\
\hline$C(42)$ & -10.459 & -6.135 & 5.321 & (9 & -7.000 & -5.2 & -2.251 \\
\hline $\mathrm{H}(43)$ & -10.454 & -7.145 & 4.896 & 0) & 7.678 & -3.879 & -1.386 \\
\hline $\mathrm{H}(44$ & -10.447 & -6.216 & 6.412 & 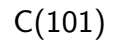 & 558 & -5 & 0.878 \\
\hline (45) & -11.413 & -5.69 & 5.021 & (1c & 18 & -5 & 1.761 \\
\hline (46) & -7.249 & -5.23 & 4.287 & C & -6.187 & -3 & 0.964 \\
\hline$C(47)$ & -7.659 & -7.205 & 5.044 & 4) & 376 & -3 & 0.021 \\
\hline$C(48$ & -6.438 & -7.706 & 4.802 & (100) & 4.663 & -3.442 & 1.274 \\
\hline & -5.67 & $-7.0 \mathrm{~s}$ & 4.317 & (10 & 28 & -3 & 0.467 \\
\hline 50 & -8.402 & -7.841 & 5.520 & & -4 & -4 & 2.554 \\
\hline$C(5$ & -6.055 & -9.141 & 5.135 & & 4.068 & -5.218 & 2.389 \\
\hline $\mathrm{H}(52$ & -6.872 & -9.590 & 5.718 & (109) & -6.997 & -2.928 & 1.938 \\
\hline & -4 & -9 . & 6. & & 90 & -2 & 2.772 \\
\hline & $-4 . c$ & -8.4 & 6.897 & & -4.236 & & 1.141 \\
\hline$H(55)$ & -4.568 & -10.134 & 6.387 & & -4.815 & -1.309 & 1.788 \\
\hline $\mathrm{H}(5$ & -3.931 & -8.756 & 5.474 & 3) & -3.171 & -1.847 & 1.364 \\
\hline $\mathrm{H}$ & -4.994 & -9.571 & 3.293 & $\mathrm{H}(114)$ & -4.391 & -1.629 & 0.111 \\
\hline
\end{tabular}



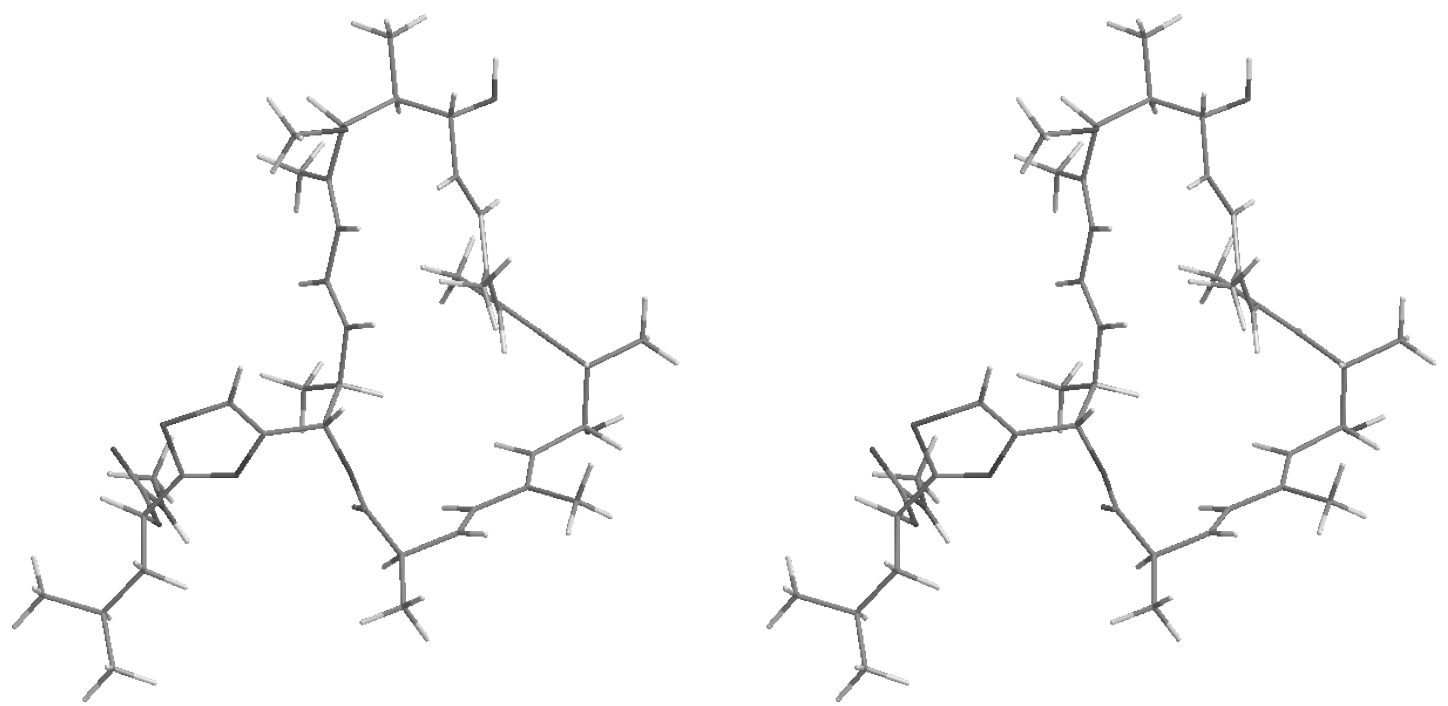

Scheme S10. Conformation of Cluster 1 of $(R)$-iso-archazolid A.

Table S18: Coordinates of Cluster 1 of $(R)$-iso-archazolid A.

\begin{tabular}{l|c|c|c||c|c|c|c}
\hline atom & $\mathrm{X}$ & $\mathrm{Y}$ & $\mathrm{Z}$ & atom & $\mathrm{X}$ & $\mathrm{Y}$ & $\mathrm{Z}$ \\
\hline $\mathrm{C}(1)$ & 1.901 & -0.364 & 5.213 & $\mathrm{H}(58)$ & 5.436 & -1.044 & 2.202 \\
$\mathrm{C}(2)$ & 0.589 & -0.319 & 4.912 & $\mathrm{C}(59)$ & 2.605 & -1.295 & 2.218 \\
$\mathrm{H}(3)$ & 2.567 & 0.375 & 4.791 & $\mathrm{H}(60)$ & 2.889 & -0.338 & 2.630 \\
$\mathrm{H}(4)$ & -0.049 & -1.068 & 5.358 & $\mathrm{C}(61)$ & 2.800 & -3.821 & 2.369 \\
$\mathrm{C}(5)$ & -0.018 & 0.706 & 4.069 & $\mathrm{H}(62)$ & 2.016 & -3.896 & 1.619 \\
$\mathrm{C}(6)$ & -1.254 & 0.551 & 3.543 & $\mathrm{H}(63)$ & 3.708 & -4.256 & 1.949 \\
$\mathrm{H}(7)$ & -1.640 & 1.375 & 2.959 & $\mathrm{H}(64)$ & 2.490 & -4.447 & 3.205 \\
$\mathrm{C}(8)$ & 0.700 & 2.031 & 3.862 & $\mathrm{C}(65)$ & 1.740 & -1.271 & 1.054 \\
$\mathrm{H}(9)$ & 0.067 & 2.771 & 3.371 & $\mathrm{C}(66)$ & 1.127 & -0.143 & 0.653 \\
$\mathrm{H}(10)$ & 1.010 & 2.448 & 4.821 & $\mathrm{H}(67)$ & 1.573 & -2.177 & 0.495 \\
$\mathrm{H}(11)$ & 1.590 & 1.896 & 3.247 & $\mathrm{H}(68)$ & 1.291 & 0.762 & 1.220 \\
\hline $\mathrm{C}(12)$ & -2.081 & -0.651 & 3.561 & $\mathrm{C}(69)$ & 0.147 & 0.004 & -0.502 \\
$\mathrm{C}(13)$ & -3.419 & -0.562 & 3.714 & $\mathrm{H}(70)$ & -0.781 & 0.246 & 0.020 \\
\hline $\mathrm{H}(14)$ & -4.001 & -1.471 & 3.649 & $\mathrm{C}(71)$ & -0.118 & -1.303 & -1.282 \\
$\mathrm{C}(15)$ & -1.485 & -2.010 & 3.234 & $\mathrm{H}(72)$ & -0.508 & -2.083 & -0.628 \\
$\mathrm{H}(16)$ & -2.082 & -2.520 & 2.476 & $\mathrm{H}(73)$ & -0.860 & -1.141 & -2.064 \\
$\mathrm{H}(17)$ & -0.472 & -1.918 & 2.843 & $\mathrm{H}(74)$ & 0.785 & -1.683 & -1.759 \\
$\mathrm{H}(18)$ & -1.454 & -2.638 & 4.124 & $\mathrm{C}(75)$ & 0.503 & 1.233 & -1.380 \\
$\mathrm{C}(19)$ & -4.243 & 0.691 & 4.003 & $\mathrm{H}(76)$ & 0.994 & 1.975 & -0.747 \\
\hline $\mathrm{H}(20)$ & -3.576 & 1.549 & 4.084 & $\mathrm{O}(77)$ & -0.749 & 1.766 & -1.789 \\
$\mathrm{C}(21)$ & -4.934 & 0.568 & 5.372 & $\mathrm{C}(78)$ & 1.386 & 0.917 & -2.568 \\
\hline $\mathrm{H}(22)$ & -4.198 & 0.440 & 6.167 & $\mathrm{~N}(79)$ & 0.839 & 0.586 & -3.817 \\
$\mathrm{H}(23)$ & -5.511 & 1.463 & 5.606 & $\mathrm{C}(80)$ & 1.767 & 0.304 & -4.740 \\
\hline $\mathrm{H}(24)$ & -5.610 & -0.287 & 5.414 & $\mathrm{~S}(81)$ & 3.408 & 0.467 & -4.131 \\
$\mathrm{C}(25)$ & -5.248 & 1.021 & 2.875 & $\mathrm{C}(82)$ & 2.758 & 0.889 & -2.570 \\
$\mathrm{H}(26)$ & -5.807 & 1.911 & 3.165 & $\mathrm{H}(83)$ & 3.423 & 1.093 & -1.741 \\
$\mathrm{C}(27)$ & -4.561 & 1.264 & 1.538 & $\mathrm{C}(84)$ & 1.460 & -0.080 & -6.160 \\
\hline & & & & & continued on the next page
\end{tabular}


Table S18: Coordinates of Cluster 1 of $(R)$-iso-archazolid A.

\begin{tabular}{l|c|c|c||c|c|c|c}
\hline atom & $\mathrm{X}$ & $\mathrm{Y}$ & $\mathrm{Z}$ & atom & $\mathrm{X}$ & $\mathrm{Y}$ & $\mathrm{Z}$ \\
\hline $\mathrm{O}(28)$ & -6.188 & -0.030 & 2.737 & $\mathrm{H}(85)$ & 2.327 & -0.604 & -6.562 \\
$\mathrm{H}(29)$ & -6.653 & -0.106 & 3.564 & $\mathrm{C}(86)$ & 1.164 & 1.150 & -7.040 \\
\hline $\mathrm{C}(30)$ & -4.288 & 2.456 & 0.965 & $\mathrm{H}(87)$ & 0.305 & 1.674 & -6.618 \\
$\mathrm{H}(31)$ & -4.251 & 0.362 & 1.029 & $\mathrm{H}(88)$ & 2.001 & 1.846 & -6.969 \\
$\mathrm{C}(32)$ & -4.693 & 3.761 & 1.640 & $\mathrm{O}(89)$ & 0.319 & -0.938 & -6.139 \\
\hline $\mathrm{H}(33)$ & -4.106 & 3.917 & 2.546 & $\mathrm{C}(90)$ & 0.894 & 0.832 & -8.528 \\
\hline $\mathrm{H}(34)$ & -5.748 & 3.744 & 1.911 & $\mathrm{H}(91)$ & 0.102 & 0.083 & -8.579 \\
$\mathrm{H}(35)$ & -4.561 & 4.631 & 1.000 & $\mathrm{C}(92)$ & 0.382 & 2.078 & -9.266 \\
\hline $\mathrm{C}(36)$ & -3.633 & 2.441 & -0.338 & $\mathrm{H}(93)$ & 1.120 & 2.881 & -9.255 \\
\hline $\mathrm{H}(37)$ & -3.637 & 1.511 & -0.891 & $\mathrm{H}(94)$ & 0.150 & 1.854 & -10.308 \\
\hline $\mathrm{H}(38)$ & -2.969 & 4.443 & -0.417 & $\mathrm{H}(95)$ & -0.531 & 2.462 & -8.807 \\
$\mathrm{C}(39)$ & -0.884 & 3.065 & -2.077 & $\mathrm{C}(96)$ & 2.130 & 0.259 & -9.243 \\
\hline $\mathrm{O}(40)$ & 0.028 & 3.894 & -2.134 & $\mathrm{H}(97)$ & 2.451 & -0.686 & -8.805 \\
$\mathrm{C}(41)$ & 2.590 & -1.366 & 6.126 & $\mathrm{H}(98)$ & 1.918 & 0.064 & -10.296 \\
\hline $\mathrm{H}(42)$ & 3.381 & -0.805 & 6.627 & $\mathrm{H}(99)$ & 2.973 & 0.950 & -9.200 \\
$\mathrm{C}(43)$ & 3.224 & -2.596 & 5.409 & $\mathrm{C}(100)$ & 0.465 & -2.243 & -5.901 \\
\hline $\mathrm{H}(44)$ & 2.410 & -3.283 & 5.173 & $\mathrm{O}(101)$ & 1.534 & -2.820 & -5.697 \\
$\mathrm{O}(45)$ & 1.693 & -1.817 & 7.130 & $\mathrm{~N}(102)$ & -0.779 & -2.834 & -5.928 \\
\hline $\mathrm{H}(46)$ & 2.209 & -2.237 & 7.811 & $\mathrm{H}(103)$ & -1.566 & -2.222 & -6.107 \\
$\mathrm{C}(47)$ & 4.153 & -3.349 & 6.384 & $\mathrm{C}(104)$ & -0.989 & -4.258 & -5.703 \\
\hline $\mathrm{H}(48)$ & 5.008 & -2.734 & 6.665 & $\mathrm{H}(105)$ & -2.040 & -4.512 & -5.838 \\
$\mathrm{H}(49)$ & 3.637 & -3.641 & 7.298 & $\mathrm{H}(106)$ & -0.402 & -4.854 & -6.402 \\
\hline $\mathrm{H}(50)$ & 4.539 & -4.265 & 5.935 & $\mathrm{H}(107)$ & -0.700 & -4.535 & -4.688 \\
$\mathrm{C}(51)$ & 3.983 & -2.318 & 4.074 & $\mathrm{C}(108)$ & -3.035 & 3.494 & -0.926 \\
\hline $\mathrm{H}(52)$ & 4.711 & -3.120 & 3.939 & $\mathrm{C}(109)$ & -2.355 & 3.444 & -2.285 \\
$\mathrm{C}(53)$ & 3.077 & -2.397 & 2.839 & $\mathrm{H}(110)$ & -2.809 & 2.636 & -2.860 \\
\hline $\mathrm{O}(54)$ & 4.725 & -1.102 & 4.170 & $\mathrm{C}(111)$ & -2.540 & 4.757 & -3.064 \\
$\mathrm{C}(55)$ & 5.790 & -0.992 & 3.232 & $\mathrm{H}(112)$ & -2.043 & 4.711 & -4.034 \\
\hline $\mathrm{H}(56)$ & 6.293 & -0.034 & 3.362 & $\mathrm{H}(113)$ & -3.596 & 4.956 & -3.247 \\
$\mathrm{H}(57)$ & 6.531 & -1.779 & 3.383 & $\mathrm{H}(114)$ & -2.129 & 5.610 & -2.521 \\
\hline
\end{tabular}



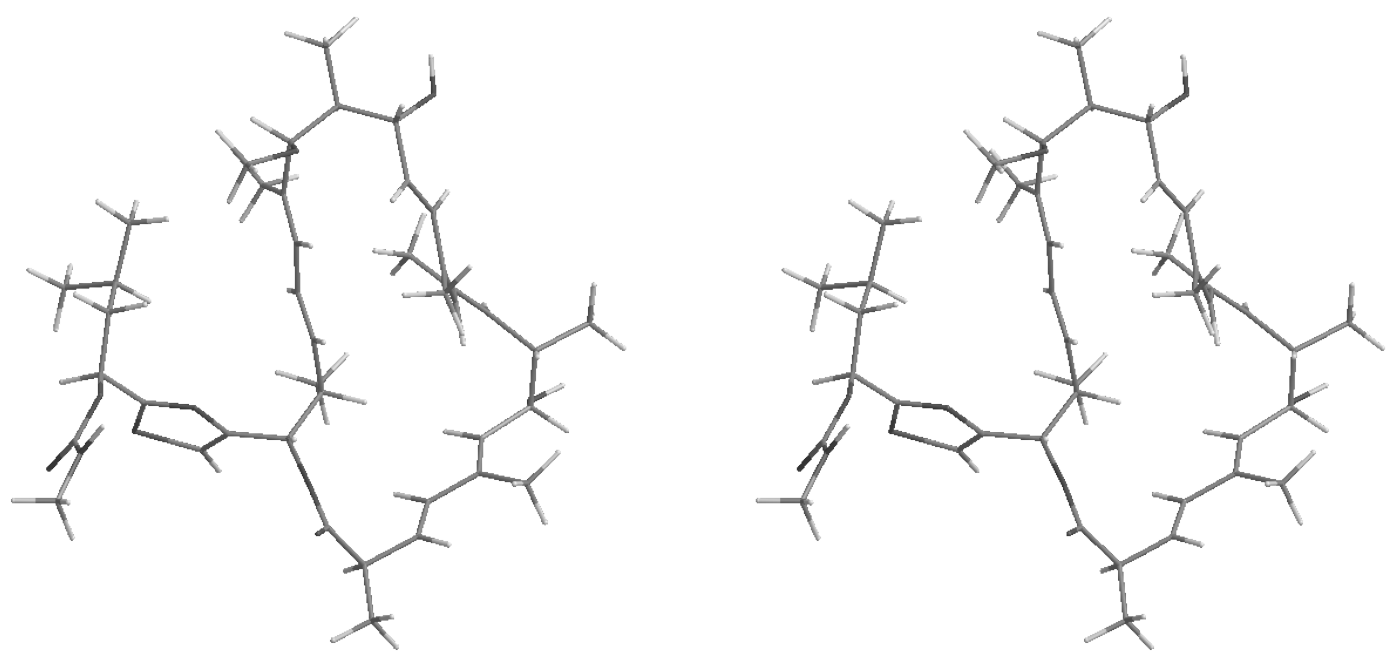

Scheme S11. Conformation of Cluster 2 of $(R)$-iso-archazolid A.

Table S19: Coordinates of Cluster 2 of $(R)$-iso-archazolid A.

\begin{tabular}{l|c|c|c||c|c|c|c}
\hline atom & $\mathrm{X}$ & $\mathrm{Y}$ & $\mathrm{Z}$ & atom & $\mathrm{X}$ & $\mathrm{Y}$ & $\mathrm{Z}$ \\
\hline $\mathrm{C}(1)$ & -4.760 & 0.987 & 5.994 & $\mathrm{H}(58)$ & -2.233 & 0.045 & 2.087 \\
$\mathrm{C}(2)$ & -6.096 & 1.116 & 6.111 & $\mathrm{C}(59)$ & -4.911 & 0.154 & 2.938 \\
$\mathrm{H}(3)$ & -4.225 & 1.650 & 5.330 & $\mathrm{H}(60)$ & -4.314 & 1.045 & 3.068 \\
$\mathrm{H}(4)$ & -6.609 & 0.446 & 6.785 & $\mathrm{C}(61)$ & -5.222 & -2.311 & 3.394 \\
$\mathrm{C}(5)$ & -6.868 & 2.138 & 5.410 & $\mathrm{H}(62)$ & -5.813 & -2.383 & 2.482 \\
$\mathrm{C}(6)$ & -8.207 & 2.045 & 5.246 & $\mathrm{H}(63)$ & -4.529 & -3.152 & 3.378 \\
$\mathrm{H}(7)$ & -8.680 & 2.869 & 4.730 & $\mathrm{H}(64)$ & -5.889 & -2.438 & 4.247 \\
$\mathrm{C}(8)$ & -6.168 & 3.403 & 4.934 & $\mathrm{C}(65)$ & -6.197 & 0.313 & 2.289 \\
$\mathrm{H}(9)$ & -6.874 & 4.180 & 4.639 & $\mathrm{C}(66)$ & -6.627 & 1.492 & 1.808 \\
$\mathrm{H}(10)$ & -5.541 & 3.812 & 5.726 & $\mathrm{H}(67)$ & -6.871 & -0.527 & 2.265 \\
$\mathrm{H}(11)$ & -5.534 & 3.187 & 4.075 & $\mathrm{H}(68)$ & -5.968 & 2.348 & 1.852 \\
$\mathrm{C}(12)$ & -9.077 & 0.913 & 5.552 & $\mathrm{C}(69)$ & -8.015 & 1.780 & 1.253 \\
$\mathrm{C}(13)$ & -10.355 & 1.114 & 5.940 & $\mathrm{H}(70)$ & -8.379 & 2.591 & 1.886 \\
\hline $\mathrm{H}(14)$ & -10.983 & 0.246 & 6.089 & $\mathrm{C}(71)$ & -9.017 & 0.615 & 1.433 \\
$\mathrm{C}(15)$ & -8.633 & -0.514 & 5.278 & $\mathrm{H}(72)$ & -9.113 & 0.340 & 2.483 \\
\hline $\mathrm{H}(16)$ & -9.424 & -1.079 & 4.781 & $\mathrm{H}(73)$ & -10.012 & 0.891 & 1.087 \\
$\mathrm{H}(17)$ & -7.759 & -0.541 & 4.627 & $\mathrm{H}(74)$ & -8.715 & -0.275 & 0.881 \\
\hline $\mathrm{H}(18)$ & -8.386 & -1.024 & 6.209 & $\mathrm{C}(75)$ & -7.956 & 2.341 & -0.188 \\
$\mathrm{C}(19)$ & -11.046 & 2.442 & 6.242 & $\mathrm{H}(76)$ & -7.212 & 3.139 & -0.195 \\
$\mathrm{H}(20)$ & -10.352 & 3.262 & 6.062 & $\mathrm{O}(77)$ & -9.244 & 2.879 & -0.440 \\
$\mathrm{C}(21)$ & -11.403 & 2.521 & 7.736 & $\mathrm{C}(78)$ & -7.575 & 1.312 & -1.223 \\
$\mathrm{H}(22)$ & -10.508 & 2.432 & 8.353 & $\mathrm{~N}(79)$ & -6.231 & 1.141 & -1.570 \\
$\mathrm{H}(23)$ & -11.871 & 3.475 & 7.980 & $\mathrm{C}(80)$ & -6.033 & 0.123 & -2.416 \\
\hline $\mathrm{H}(24)$ & -12.086 & 1.726 & 8.036 & $\mathrm{~S}(81)$ & -7.527 & -0.679 & -2.884 \\
$\mathrm{C}(25)$ & -12.281 & 2.698 & 5.344 & $\mathrm{C}(82)$ & -8.407 & 0.434 & -1.873 \\
\hline $\mathrm{H}(26)$ & -12.753 & 3.633 & 5.650 & $\mathrm{H}(83)$ & -9.482 & 0.356 & -1.779 \\
$\mathrm{C}(27)$ & -11.939 & 2.795 & 3.864 & $\mathrm{C}(84)$ & -4.678 & -0.317 & -2.896 \\
$\mathrm{O}(28)$ & -13.247 & 1.681 & 5.541 & $\mathrm{H}(85)$ & -4.818 & -1.031 & -3.709 \\
\hline & & & & & & & \\
\hline
\end{tabular}


Table S19: Coordinates of Cluster 2 of $(R)$-iso-archazolid A.

\begin{tabular}{l|c|c|c||c|c|c|c}
\hline atom & $\mathrm{X}$ & $\mathrm{Y}$ & $\mathrm{Z}$ & atom & $\mathrm{X}$ & $\mathrm{Y}$ & $\mathrm{Z}$ \\
\hline $\mathrm{H}(29)$ & -13.508 & 1.709 & 6.456 & $\mathrm{C}(86)$ & -3.824 & -0.986 & -1.796 \\
$\mathrm{C}(30)$ & -11.638 & 3.912 & 3.165 & $\mathrm{H}(87)$ & -2.866 & -1.284 & -2.223 \\
$\mathrm{H}(31)$ & -11.976 & 1.851 & 3.337 & $\mathrm{H}(88)$ & -3.592 & -0.226 & -1.048 \\
$\mathrm{C}(32)$ & -11.527 & 5.277 & 3.834 & $\mathrm{O}(89)$ & -3.991 & 0.838 & -3.375 \\
$\mathrm{H}(33)$ & -11.420 & 5.215 & 4.916 & $\mathrm{C}(90)$ & -4.472 & -2.191 & -1.076 \\
$\mathrm{H}(34)$ & -10.647 & 5.811 & 3.478 & $\mathrm{H}(91)$ & -5.424 & -1.869 & -0.651 \\
$\mathrm{H}(35)$ & -12.408 & 5.879 & 3.611 & $\mathrm{C}(92)$ & -4.750 & -3.366 & -2.028 \\
\hline $\mathrm{C}(36)$ & -11.435 & 3.776 & 1.728 & $\mathrm{H}(93)$ & -3.834 & -3.731 & -2.494 \\
$\mathrm{H}(37)$ & -11.569 & 2.797 & 1.289 & $\mathrm{H}(94)$ & -5.203 & -4.202 & -1.493 \\
\hline $\mathrm{H}(38)$ & -10.926 & 5.772 & 1.274 & $\mathrm{H}(95)$ & -5.441 & -3.088 & -2.824 \\
$\mathrm{C}(39)$ & -9.408 & 4.151 & -0.814 & $\mathrm{C}(96)$ & -3.594 & -2.652 & 0.099 \\
\hline $\mathrm{O}(40)$ & -8.526 & 4.908 & -1.226 & $\mathrm{H}(97)$ & -3.432 & -1.841 & 0.810 \\
$\mathrm{C}(41)$ & -3.867 & -0.018 & 6.703 & $\mathrm{H}(98)$ & -4.063 & -3.471 & 0.645 \\
$\mathrm{H}(42)$ & -2.957 & 0.533 & 6.947 & $\mathrm{H}(99)$ & -2.616 & -2.995 & -0.241 \\
$\mathrm{C}(43)$ & -3.480 & -1.277 & 5.867 & $\mathrm{C}(100)$ & -4.221 & 1.293 & -4.609 \\
\hline $\mathrm{H}(44)$ & -4.318 & -1.973 & 5.927 & $\mathrm{O}(101)$ & -4.993 & 0.791 & -5.426 \\
$\mathrm{O}(45)$ & -4.451 & -0.430 & 7.931 & $\mathrm{~N}(102)$ & -3.446 & 2.415 & -4.812 \\
\hline $\mathrm{H}(46)$ & -3.773 & -0.865 & 8.440 & $\mathrm{H}(103)$ & -2.860 & 2.704 & -4.038 \\
$\mathrm{C}(47)$ & -2.285 & -1.998 & 6.524 & $\mathrm{C}(104)$ & -3.448 & 3.168 & -6.058 \\
\hline $\mathrm{H}(48)$ & -1.391 & -1.374 & 6.504 & $\mathrm{H}(105)$ & -2.779 & 4.025 & -5.984 \\
$\mathrm{H}(49)$ & -2.488 & -2.256 & 7.563 & $\mathrm{H}(106)$ & -3.112 & 2.545 & -6.888 \\
\hline $\mathrm{H}(50)$ & -2.050 & -2.928 & 6.007 & $\mathrm{H}(107)$ & -4.449 & 3.537 & -6.289 \\
$\mathrm{C}(51)$ & -3.191 & -1.058 & 4.348 & $\mathrm{C}(108)$ & -11.089 & 4.775 & 0.893 \\
$\mathrm{H}(52)$ & -2.655 & -1.939 & 3.990 & $\mathrm{C}(109)$ & -10.857 & 4.607 & -0.602 \\
$\mathrm{C}(53)$ & -4.466 & -0.992 & 3.497 & $\mathrm{H}(110)$ & -11.498 & 3.797 & -0.953 \\
\hline $\mathrm{O}(54)$ & -2.324 & 0.064 & 4.180 & $\mathrm{C}(111)$ & -11.210 & 5.885 & -1.381 \\
$\mathrm{C}(55)$ & -1.585 & 0.054 & 2.964 & $\mathrm{H}(112)$ & -11.029 & 5.756 & -2.449 \\
\hline $\mathrm{H}(56)$ & -0.966 & 0.950 & 2.907 & $\mathrm{H}(113)$ & -12.262 & 6.141 & -1.255 \\
$\mathrm{H}(57)$ & -0.921 & -0.810 & 2.912 & $\mathrm{H}(114)$ & -10.617 & 6.737 & -1.045 \\
\hline & & & & & & & \\
\hline
\end{tabular}



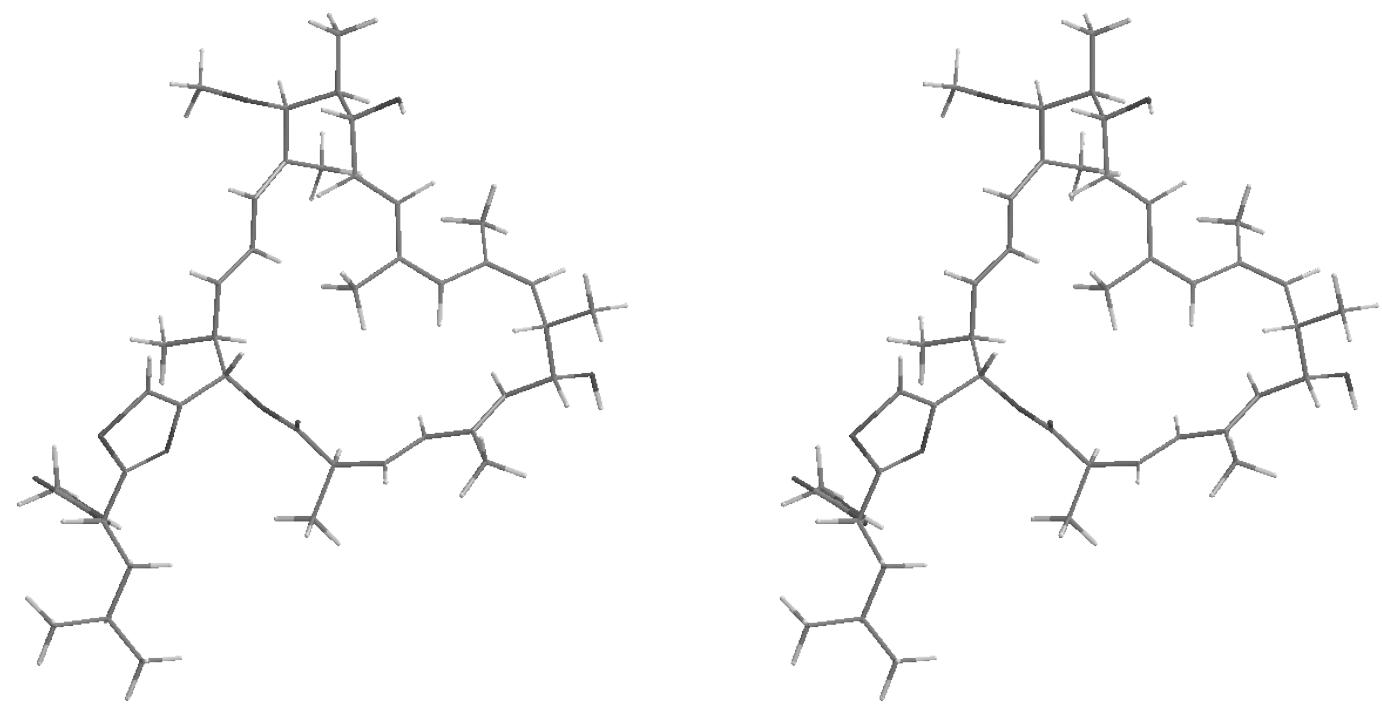

Scheme S12. Conformation of Cluster 3 of $(R)$-iso-archazolid A.

Table S20: Coordinates of Cluster 3 of $(R)$-iso-archazolid A.

\begin{tabular}{l|c|c|c||c|c|c|c}
\hline atom & $\mathrm{X}$ & $\mathrm{Y}$ & $\mathrm{Z}$ & atom & $\mathrm{X}$ & $\mathrm{Y}$ & $\mathrm{Z}$ \\
\hline $\mathrm{C}(1)$ & -5.318 & 0.904 & 6.577 & $\mathrm{H}(58)$ & -1.853 & -2.433 & 4.550 \\
$\mathrm{C}(2)$ & -6.620 & 1.231 & 6.692 & $\mathrm{C}(59)$ & -4.343 & -1.154 & 4.044 \\
$\mathrm{H}(3)$ & -4.822 & 1.031 & 5.626 & $\mathrm{H}(60)$ & -3.425 & -0.587 & 3.999 \\
\hline $\mathrm{H}(4)$ & -7.077 & 1.105 & 7.662 & $\mathrm{C}(61)$ & -5.876 & -2.649 & 5.399 \\
$\mathrm{C}(5)$ & -7.428 & 1.796 & 5.618 & $\mathrm{H}(62)$ & -6.341 & -2.967 & 4.468 \\
$\mathrm{C}(6)$ & -8.775 & 1.881 & 5.716 & $\mathrm{H}(63)$ & -5.642 & -3.557 & 5.954 \\
$\mathrm{H}(7)$ & -9.278 & 2.360 & 4.890 & $\mathrm{H}(64)$ & -6.612 & -2.084 & 5.971 \\
\hline $\mathrm{C}(8)$ & -6.747 & 2.399 & 4.399 & $\mathrm{C}(65)$ & -5.194 & -1.104 & 2.869 \\
$\mathrm{H}(9)$ & -7.436 & 2.980 & 3.785 & $\mathrm{C}(66)$ & -4.818 & -0.501 & 1.728 \\
\hline $\mathrm{H}(10)$ & -5.937 & 3.065 & 4.702 & $\mathrm{H}(67)$ & -6.179 & -1.545 & 2.902 \\
$\mathrm{H}(11)$ & -6.327 & 1.612 & 3.772 & $\mathrm{H}(68)$ & -3.842 & -0.038 & 1.664 \\
\hline $\mathrm{C}(12)$ & -9.644 & 1.278 & 6.725 & $\mathrm{C}(69)$ & -5.674 & -0.416 & 0.477 \\
$\mathrm{C}(13)$ & -10.796 & 1.878 & 7.094 & $\mathrm{H}(70)$ & -6.610 & -0.919 & 0.727 \\
\hline $\mathrm{H}(14)$ & -11.455 & 1.347 & 7.768 & $\mathrm{C}(71)$ & -5.033 & -1.234 & -0.662 \\
$\mathrm{C}(15)$ & -9.392 & -0.137 & 7.222 & $\mathrm{H}(72)$ & -4.892 & -2.273 & -0.363 \\
\hline $\mathrm{H}(16)$ & -10.317 & -0.715 & 7.233 & $\mathrm{H}(73)$ & -5.666 & -1.236 & -1.550 \\
$\mathrm{H}(17)$ & -8.688 & -0.665 & 6.580 & $\mathrm{H}(74)$ & -4.057 & -0.840 & -0.946 \\
\hline $\mathrm{H}(18)$ & -8.992 & -0.121 & 8.237 & $\mathrm{C}(75)$ & -6.007 & 1.069 & 0.144 \\
$\mathrm{C}(19)$ & -11.305 & 3.261 & 6.695 & $\mathrm{H}(76)$ & -5.721 & 1.671 & 1.009 \\
\hline $\mathrm{H}(20)$ & -10.479 & 3.819 & 6.251 & $\mathrm{O}(77)$ & -7.421 & 1.151 & -0.002 \\
$\mathrm{C}(21)$ & -11.725 & 4.057 & 7.942 & $\mathrm{C}(78)$ & -5.317 & 1.620 & -1.086 \\
\hline $\mathrm{H}(22)$ & -10.900 & 4.140 & 8.650 & $\mathrm{~N}(79)$ & -5.904 & 1.496 & -2.354 \\
$\mathrm{H}(23)$ & -12.034 & 5.068 & 7.678 & $\mathrm{C}(80)$ & -5.154 & 2.011 & -3.335 \\
\hline $\mathrm{H}(24)$ & -12.559 & 3.580 & 8.458 & $\mathrm{~S}(81)$ & -3.660 & 2.729 & -2.746 \\
$\mathrm{C}(25)$ & -12.435 & 3.204 & 5.638 & $\mathrm{C}(82)$ & -4.102 & 2.256 & -1.127 \\
\hline $\mathrm{H}(26)$ & -12.799 & 4.217 & 5.469 & $\mathrm{H}(83)$ & -3.443 & 2.477 & -0.297 \\
$\mathrm{C}(27)$ & -11.918 & 2.611 & 4.338 & $\mathrm{C}(84)$ & -5.550 & 2.020 & -4.785 \\
\hline & & & & & continued on the next page \\
& & & & & & \\
& & & & & &
\end{tabular}


Table S20: Coordinates of Cluster 3 of $(R)$-iso-archazolid A.

\begin{tabular}{l|c|c|c||c|c|c|c}
\hline atom & $\mathrm{X}$ & $\mathrm{Y}$ & $\mathrm{Z}$ & atom & $\mathrm{X}$ & $\mathrm{Y}$ & $\mathrm{Z}$ \\
\hline $\mathrm{O}(28)$ & -13.526 & 2.437 & 6.117 & $\mathrm{H}(85)$ & -4.644 & 2.126 & -5.381 \\
\hline $\mathrm{H}(29)$ & -14.264 & 2.589 & 5.533 & $\mathrm{C}(86)$ & -6.509 & 3.181 & -5.112 \\
\hline $\mathrm{C}(30)$ & -11.582 & 3.276 & 3.213 & $\mathrm{H}(87)$ & -7.406 & 3.066 & -4.502 \\
$\mathrm{H}(31)$ & -11.719 & 1.548 & 4.387 & $\mathrm{H}(88)$ & -6.051 & 4.119 & -4.793 \\
\hline $\mathrm{C}(32)$ & -11.806 & 4.778 & 3.084 & $\mathrm{O}(89)$ & -6.196 & 0.778 & -5.065 \\
\hline $\mathrm{H}(33)$ & -11.751 & 5.124 & 2.053 & $\mathrm{C}(90)$ & -6.908 & 3.292 & -6.600 \\
\hline $\mathrm{H}(34)$ & -12.799 & 5.057 & 3.436 & $\mathrm{H}(91)$ & -7.308 & 2.328 & -6.919 \\
\hline $\mathrm{H}(35)$ & -11.065 & 5.325 & 3.668 & $\mathrm{C}(92)$ & -8.029 & 4.328 & -6.785 \\
\hline $\mathrm{C}(36)$ & -10.947 & 2.503 & 2.154 & $\mathrm{H}(93)$ & -7.705 & 5.325 & -6.485 \\
\hline $\mathrm{H}(37)$ & -10.937 & 1.426 & 2.255 & $\mathrm{H}(94)$ & -8.349 & 4.382 & -7.826 \\
\hline $\mathrm{H}(38)$ & -10.259 & 4.082 & 0.925 & $\mathrm{H}(95)$ & -8.906 & 4.072 & -6.189 \\
\hline $\mathrm{C}(39)$ & -8.068 & 2.279 & 0.316 & $\mathrm{C}(96)$ & -5.713 & 3.632 & -7.507 \\
\hline $\mathrm{O}(40)$ & -7.545 & 3.313 & 0.740 & $\mathrm{H}(97)$ & -4.950 & 2.854 & -7.480 \\
\hline $\mathrm{C}(41)$ & -4.426 & 0.380 & 7.692 & $\mathrm{H}(98)$ & -6.026 & 3.731 & -8.547 \\
\hline $\mathrm{H}(42)$ & -3.444 & 0.839 & 7.560 & $\mathrm{H}(99)$ & -5.243 & 4.571 & -7.214 \\
\hline $\mathrm{C}(43)$ & -4.267 & -1.170 & 7.698 & $\mathrm{C}(100)$ & -5.472 & -0.305 & -5.357 \\
\hline $\mathrm{H}(44)$ & -5.258 & -1.600 & 7.847 & $\mathrm{O}(101)$ & -4.243 & -0.345 & -5.438 \\
\hline $\mathrm{O}(45)$ & -4.926 & 0.813 & 8.951 & $\mathrm{~N}(102)$ & -6.331 & -1.364 & -5.554 \\
\hline $\mathrm{H}(46)$ & -4.871 & 1.764 & 8.971 & $\mathrm{H}(103)$ & -7.319 & -1.169 & -5.448 \\
\hline $\mathrm{C}(47)$ & -3.415 & -1.635 & 8.901 & $\mathrm{C}(104)$ & -5.875 & -2.708 & -5.882 \\
\hline $\mathrm{H}(48)$ & -2.411 & -1.213 & 8.859 & $\mathrm{H}(105)$ & -5.215 & -3.095 & -5.104 \\
\hline $\mathrm{H}(49)$ & -3.859 & -1.341 & 9.852 & $\mathrm{H}(106)$ & -6.726 & -3.384 & -5.973 \\
\hline $\mathrm{H}(50)$ & -3.318 & -2.720 & 8.924 & $\mathrm{H}(107)$ & -5.332 & -2.714 & -6.828 \\
\hline $\mathrm{C}(51)$ & -3.672 & -1.794 & 6.400 & $\mathrm{C}(108)$ & -10.327 & 3.015 & 1.075 \\
\hline $\mathrm{H}(52)$ & -3.482 & -2.847 & 6.621 & $\mathrm{C}(109)$ & -9.577 & 2.173 & 0.060 \\
\hline $\mathrm{C}(53)$ & -4.617 & -1.815 & 5.191 & $\mathrm{H}(110)$ & -9.857 & 1.130 & 0.216 \\
\hline $\mathrm{O}(54)$ & -2.416 & -1.172 & 6.124 & $\mathrm{C}(111)$ & -9.926 & 2.558 & -1.386 \\
\hline $\mathrm{C}(55)$ & -1.472 & -2.027 & 5.488 & $\mathrm{H}(112)$ & -9.382 & 1.938 & -2.100 \\
\hline $\mathrm{H}(56)$ & -0.564 & -1.467 & 5.264 & $\mathrm{H}(113)$ & -10.990 & 2.425 & -1.579 \\
$\mathrm{H}(57)$ & -1.197 & -2.859 & 6.137 & $\mathrm{H}(114)$ & -9.675 & 3.599 & -1.593 \\
\hline & & & & & & & \\
\hline
\end{tabular}
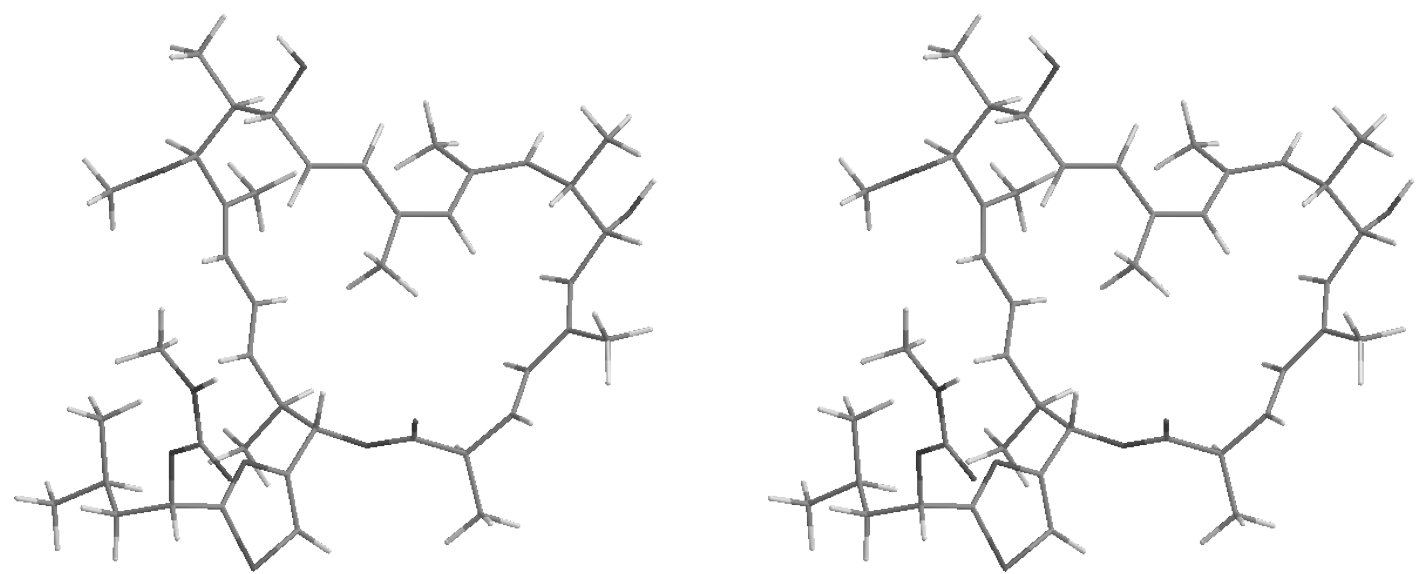

Scheme S13. Conformation of Cluster 4 of $(R)$-iso-archazolid A. 
Table S21: Coordinates of Cluster 4 of $(R)$-iso-archazolid A.

\begin{tabular}{|c|c|c|c|c|c|c|c|}
\hline atom & $x$ & Y & Z & atom & $x$ & Y & Z \\
\hline$C(1)$ & -5.187 & 1.070 & 5.113 & $H(58)$ & -2.598 & -2.493 & 2.376 \\
\hline$C(2)$ & -6.391 & 1.433 & 5.597 & $C(59)$ & -5.140 & -1.263 & 2.660 \\
\hline $\mathrm{H}(3)$ & -5.015 & 1.098 & 4.047 & $H(60)$ & -4.306 & -0.729 & 2.227 \\
\hline $\mathrm{H}(4)$ & -6.520 & 1.410 & 6.669 & $C(61)$ & -6.073 & -2.567 & 4.617 \\
\hline$C(5)$ & -7.496 & 1.919 & 4.778 & $H(62)$ & -6.827 & -2.973 & 3.945 \\
\hline$C(6)$ & -8.752 & 2.021 & 5.270 & $\mathrm{H}(63)$ & -5.648 & -3.415 & 5.155 \\
\hline $\mathrm{H}(7)$ & -9.490 & 2.439 & 4.602 & $\mathrm{H}(64)$ & -6.573 & -1.924 & 5.340 \\
\hline$C(8)$ & -7.224 & 2.427 & 3.370 & $C(65)$ & -6.369 & -1.277 & 1.891 \\
\hline $\mathrm{H}(9)$ & -8.062 & 2.999 & 2.968 & $C(66)$ & -6.430 & -0.840 & 0.622 \\
\hline $\mathrm{H}(10)$ & -6.348 & 3.076 & 3.357 & $\mathrm{H}(67)$ & -7.289 & -1.595 & 2.360 \\
\hline $\mathrm{H}(11)$ & -7.040 & 1.590 & 2.696 & $H(68)$ & -5.531 & -0.494 & 0.130 \\
\hline$C(12)$ & -9.266 & 1.497 & 6.534 & $C(69)$ & -7.701 & -0.759 & -0.203 \\
\hline$C(13)$ & -10.260 & 2.130 & 7.193 & $\mathrm{H}(70)$ & -8.507 & -1 . & 0.476 \\
\hline $\mathrm{H}(14)$ & -10.673 & 1.651 & 8.070 & $C(71)$ & -7 . & -1 & 44 \\
\hline$C(15)$ & -8.852 & 0.120 & 7.029 & $\mathrm{H}(72)$ & -7.602 & -2.806 & -0.949 \\
\hline $\mathrm{H}(16)$ & -9.718 & -0.448 & 7.371 & & -8.599 & -1.742 & -1.932 \\
\hline $\mathrm{H}(17)$ & -8.377 & -0.459 & 6.237 & & -6.841 & & -2.019 \\
\hline $\mathrm{H}(18)$ & -8.153 & 0.206 & 7.861 & & -7.957 & 0.710 & -0.640 \\
\hline$C(19)$ & -10.880 & 3.490 & 6.877 & $\mathrm{H}(76)$ & -7.456 & 1.351 & 0.088 \\
\hline $\mathrm{H}(20)$ & -10.233 & 4.009 & 6.167 & $\mathrm{O}(77)$ & -9.358 & 0.938 & -0.543 \\
\hline$C(21)$ & -10.907 & 4.368 & 8.139 & $C(78)$ & -7.445 & 1.046 & -2.021 \\
\hline $\mathrm{H}(22)$ & -9.902 & 4.506 & 8.540 & $N(79)$ & -6.077 & 1.259 & -2.214 \\
\hline $\mathrm{H}(23)$ & -11.308 & 5.359 & 7.922 & $C(80)$ & -5.752 & 1.501 & -3.491 \\
\hline $\mathrm{H}(24)$ & -11.518 & 3.928 & 8.928 & $\mathrm{~S}(81)$ & -7.152 & 1.512 & -4.556 \\
\hline$C(25)$ & -12.278 & 3.380 & 6.222 & $C(82)$ & -8.168 & 1.158 & -3.184 \\
\hline $\mathrm{H}(26)$ & -12.695 & 4.383 & 6.129 & $\mathrm{H}(83)$ & -9.235 & 1.037 & -3.313 \\
\hline$C(27)$ & -12.186 & 2.733 & 4.849 & $C(84)$ & -4.343 & 1.672 & -3.995 \\
\hline $\mathrm{O}(28)$ & -13.155 & 2.638 & 7.049 & $\mathrm{H}(85)$ & -4.361 & 2.394 & -4.813 \\
\hline $\mathrm{H}(29)$ & -13.223 & 3.099 & 7.879 & $C(86)$ & -3.706 & 0.368 & -4.533 \\
\hline$C(30)$ & -12.217 & 3.356 & 3.652 & $\mathrm{H}(87)$ & -4.179 & 0.173 & -5.496 \\
\hline $\mathrm{H}(31)$ & -11.991 & 1.670 & 4.875 & $\mathrm{H}(88)$ & -2.655 & 0.546 & -4.759 \\
\hline$C(32)$ & -12.463 & 4.857 & 3.549 & $\mathrm{O}(89)$ & -3.543 & 2.181 & -2.929 \\
\hline $\mathrm{H}(33)$ & -13.315 & 5.155 & 4.160 & $C(90)$ & -3.861 & -0.918 & -3.678 \\
\hline $\mathrm{H}(34)$ & -12.699 & 5.179 & 2.536 & $\mathrm{H}(91)$ & -4.920 & -1.071 & -3.467 \\
\hline $\mathrm{H}(35)$ & -11.586 & 5.410 & 3.886 & $C(92)$ & -3.392 & -2.147 & -4.472 \\
\hline$C(36)$ & -11.967 & 2.539 & 2.472 & $\mathrm{H}(93)$ & -2.333 & -2.080 & -4.723 \\
\hline $\mathrm{H}(37)$ & -11.961 & 1.465 & 2.597 & $\mathrm{H}(94)$ & -3.540 & -3.066 & -3.903 \\
\hline $\mathrm{H}(38)$ & -11.651 & 4.069 & 1.045 & $\mathrm{H}(95)$ & -3.947 & -2.254 & -5.405 \\
\hline$C(39)$ & -9.818 & 2.125 & -0.126 & $C(96)$ & -3.119 & -0.849 & -2.332 \\
\hline $\mathrm{O}(40)$ & -9.133 & 3.130 & 0.082 & $\mathrm{H}(97)$ & -3.535 & -0.080 & -1.681 \\
\hline$C(41)$ & -3.982 & 0.635 & 5.936 & $\mathrm{H}(98)$ & -3.197 & -1.792 & -1.790 \\
\hline $\mathrm{H}(42)$ & -3.105 & 1.073 & 5.457 & $\mathrm{H}(99)$ & -2.059 & -0.633 & -2.469 \\
\hline$C(43)$ & -3.792 & -0.908 & 6.036 & $C(100)$ & -3.494 & 3.496 & -2.673 \\
\hline $\mathrm{H}(44)$ & -4.659 & -1.311 & 6.560 & $\mathrm{O}(101)$ & -4.081 & 4.365 & -3.316 \\
\hline
\end{tabular}


Table S21: Coordinates of Cluster 4 of $(R)$-iso-archazolid A.

\begin{tabular}{c|c|c|c||c|c|c|c}
\hline atom & $\mathrm{X}$ & $\mathrm{Y}$ & $\mathrm{Z}$ & atom & $\mathrm{X}$ & $\mathrm{Y}$ & $\mathrm{Z}$ \\
\hline $\mathrm{O}(45)$ & -4.064 & 1.187 & 7.243 & $\mathrm{~N}(102)$ & -2.680 & 3.741 & -1.580 \\
$\mathrm{H}(46)$ & -3.219 & 1.056 & 7.662 & $\mathrm{H}(103)$ & -2.581 & 4.713 & -1.315 \\
$\mathrm{C}(47)$ & -2.567 & -1.255 & 6.912 & $\mathrm{C}(104)$ & -1.992 & 2.714 & -0.800 \\
$\mathrm{H}(48)$ & -1.650 & -0.838 & 6.493 & $\mathrm{H}(105)$ & -1.410 & 3.175 & -0.002 \\
$\mathrm{H}(49)$ & -2.670 & -0.883 & 7.931 & $\mathrm{H}(106)$ & -2.707 & 2.027 & -0.343 \\
$\mathrm{H}(50)$ & -2.430 & -2.334 & 6.991 & $\mathrm{H}(107)$ & -1.310 & 2.137 & -1.425 \\
$\mathrm{C}(51)$ & -3.668 & -1.667 & 4.680 & $\mathrm{C}(108)$ & -11.704 & 3.008 & 1.238 \\
$\mathrm{H}(52)$ & -3.385 & -2.695 & 4.921 & $\mathrm{C}(109)$ & -11.340 & 2.122 & 0.061 \\
$\mathrm{C}(53)$ & -4.977 & -1.797 & 3.891 & $\mathrm{H}(110)$ & -11.632 & 1.101 & 0.310 \\
$\mathrm{O}(54)$ & -2.607 & -1.083 & 3.923 & $\mathrm{C}(111)$ & -12.068 & 2.536 & -1.226 \\
$\mathrm{C}(55)$ & -1.923 & -2.000 & 3.076 & $\mathrm{H}(112)$ & -11.807 & 1.875 & -2.055 \\
$\mathrm{H}(56)$ & -1.169 & -1.470 & 2.495 & $\mathrm{H}(113)$ & -13.149 & 2.488 & -1.096 \\
$\mathrm{H}(57)$ & -1.413 & -2.767 & 3.661 & $\mathrm{H}(114)$ & -11.811 & 3.554 & -1.520 \\
\hline
\end{tabular}


2 Copies of NMR spectras 
NMR-Spectra for kinetic analysis of isomerization

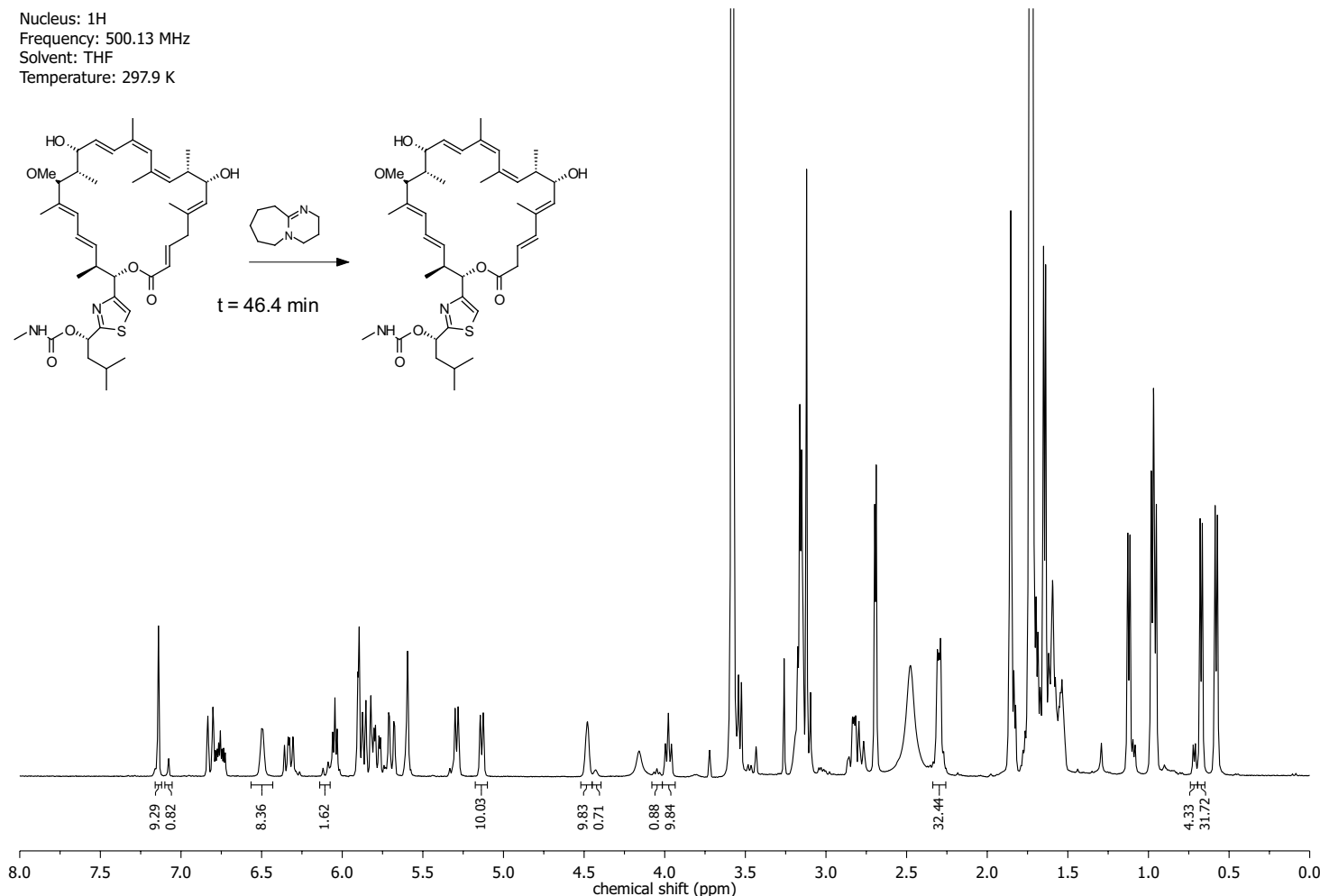

Nucleus: $1 \mathrm{H}$

Frequency: $500.13 \mathrm{MHz}$

Temperature: $297.9 \mathrm{~K}$
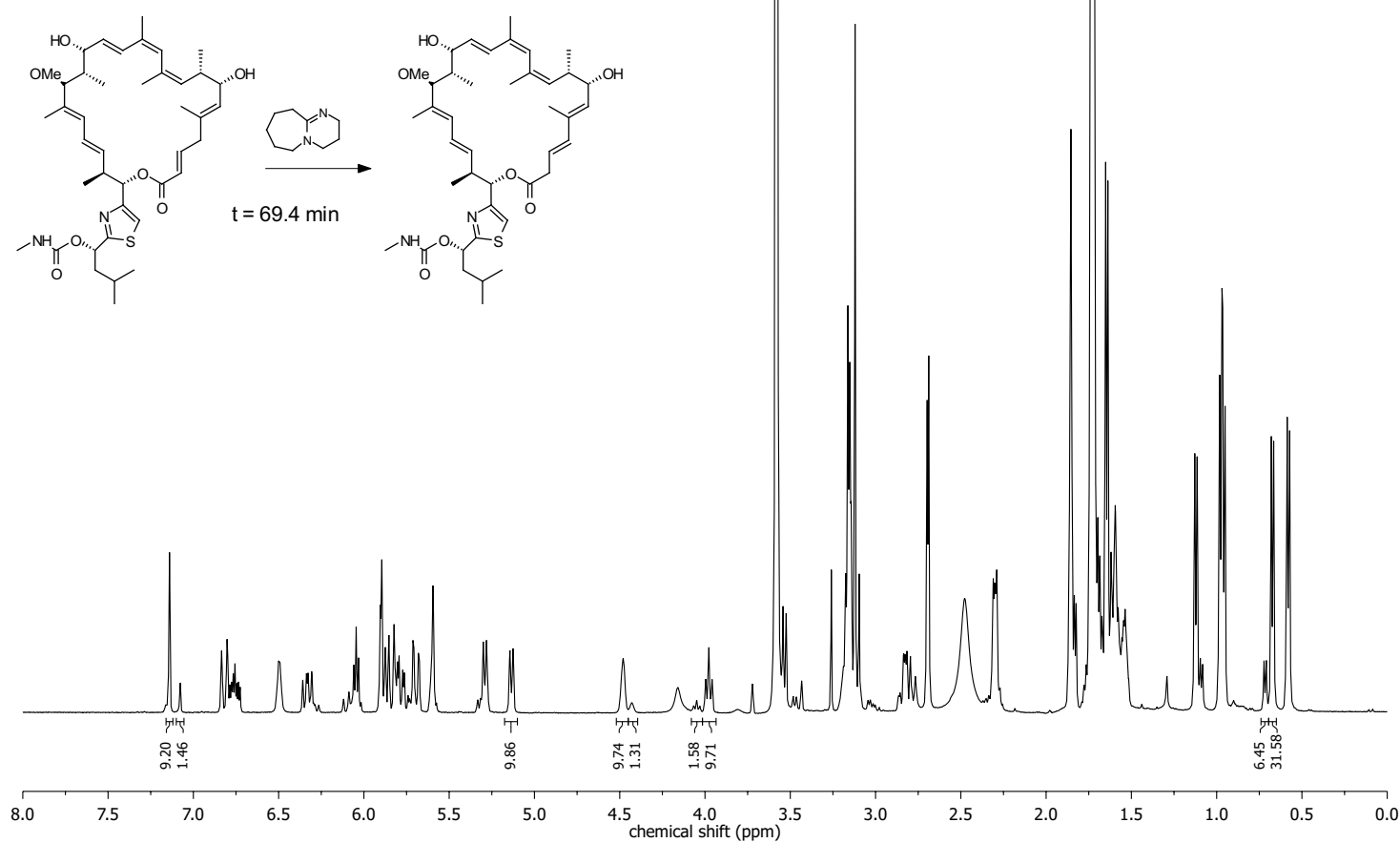


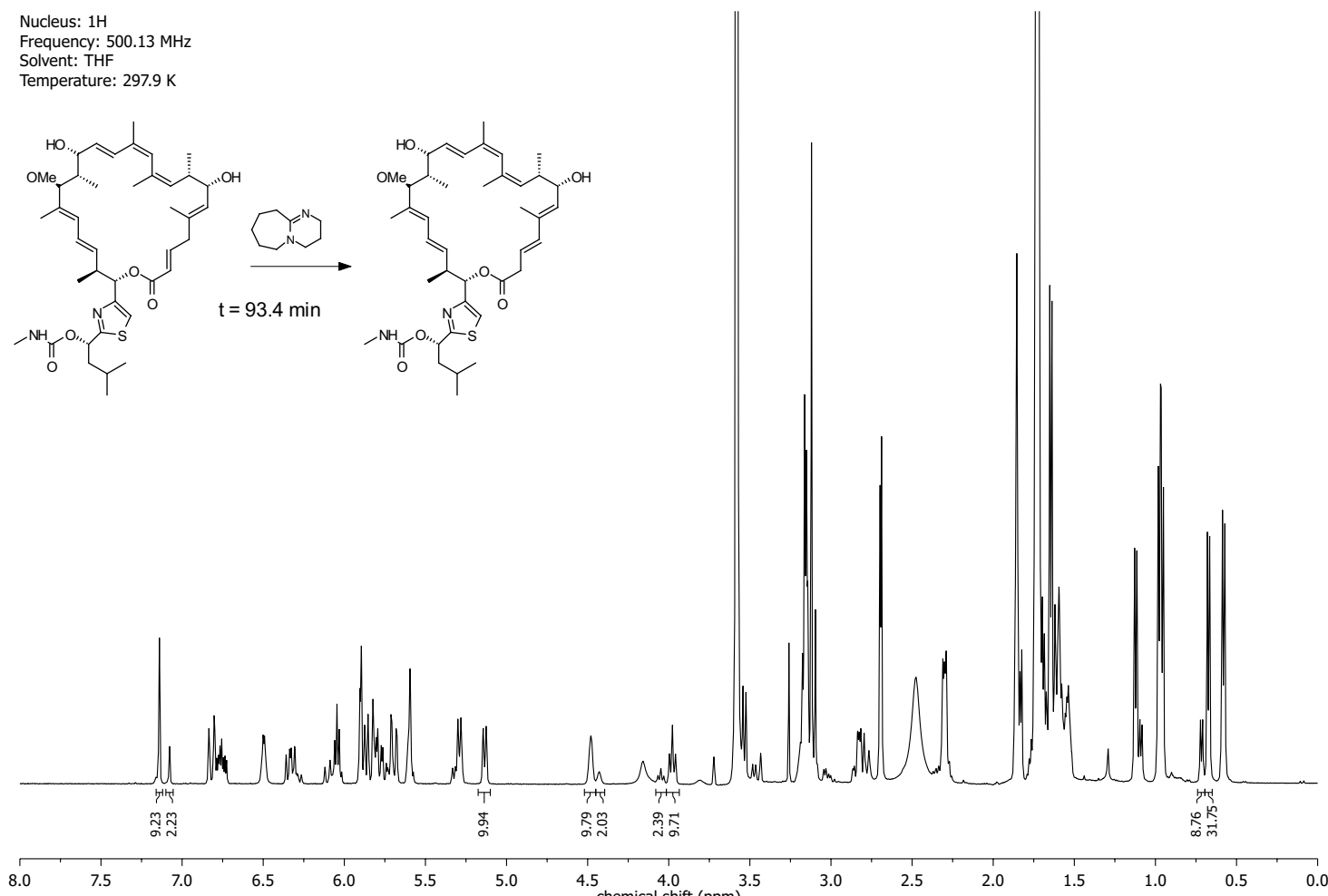

Nucleus: $1 \mathrm{H}$

Frequency: $500.13 \mathrm{MHz}$

Temperature: $297.9 \mathrm{~K}$
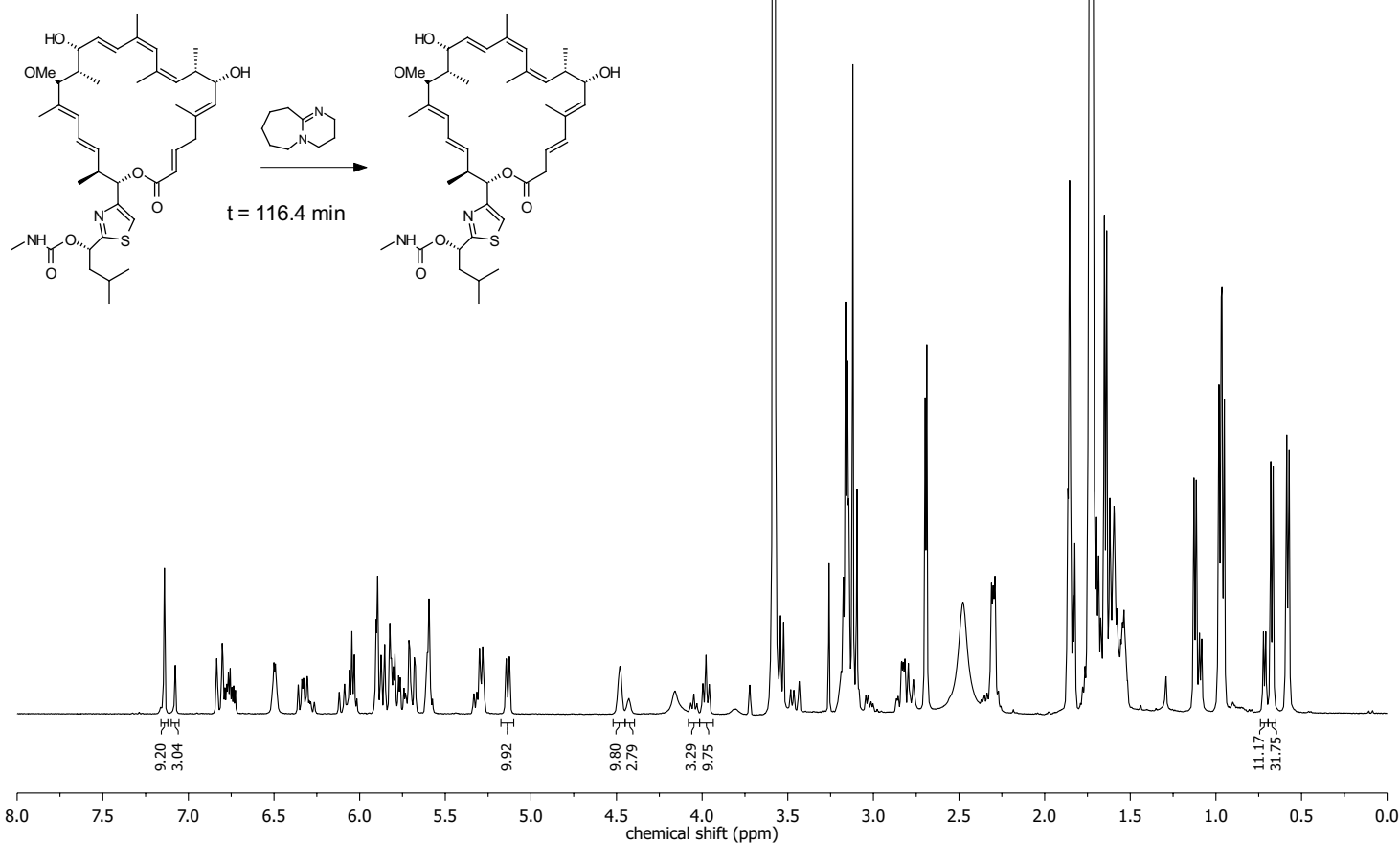


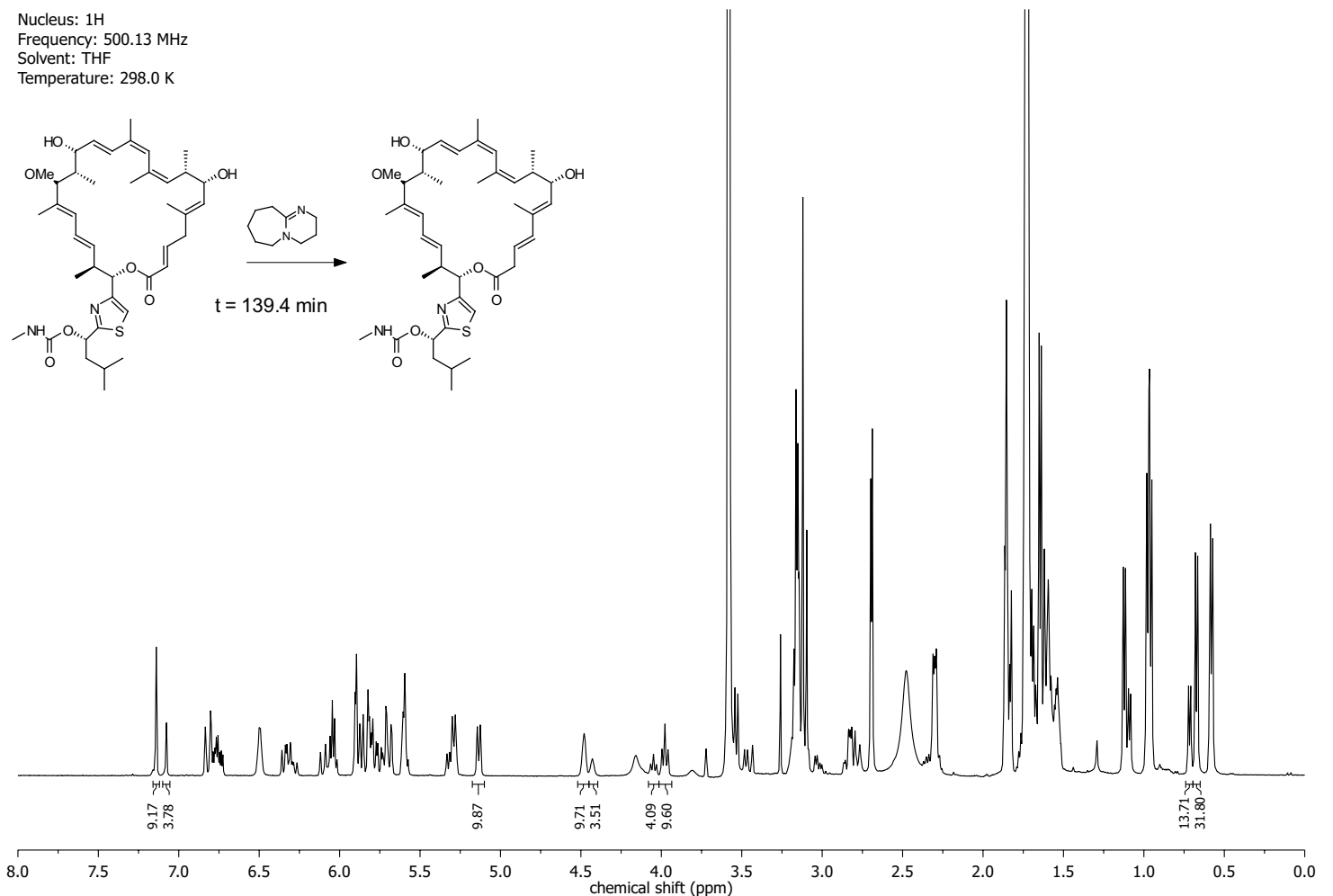

Nucleus: $1 \mathrm{H}$

requency: $500.13 \mathrm{MHz}$

Solvent: THF $297.9 \mathrm{~K}$
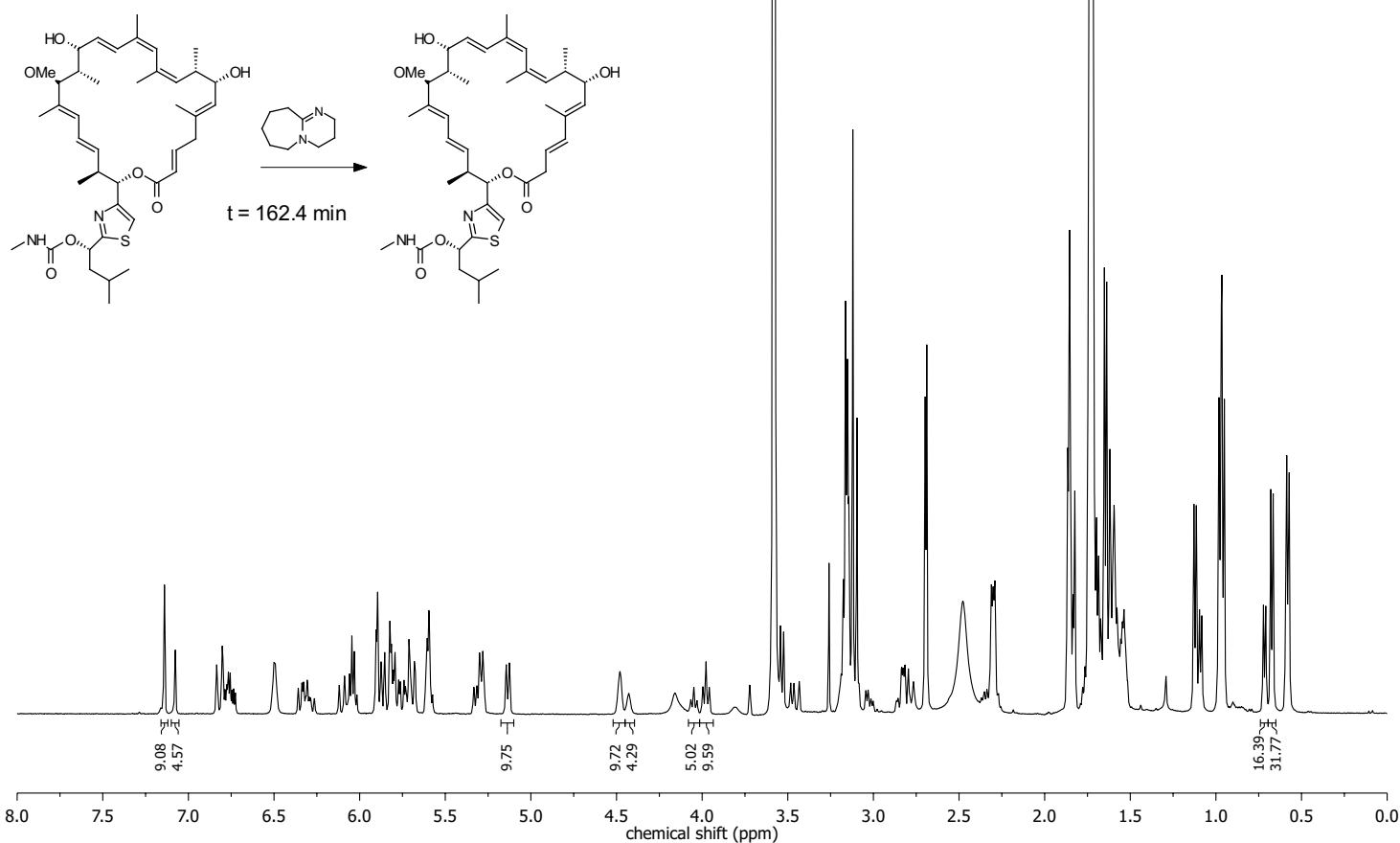


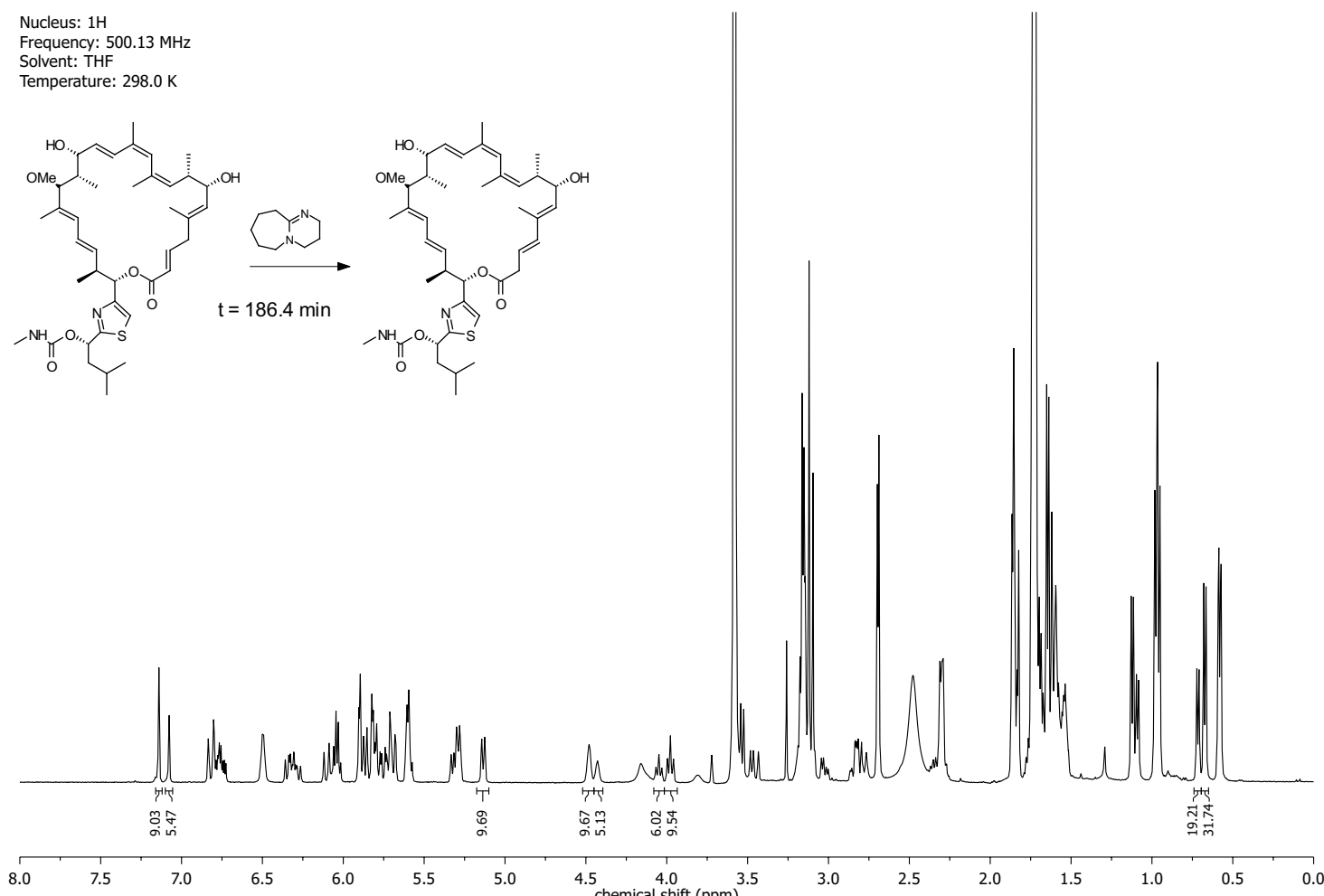

Nucleus: $1 \mathrm{H}$

requency: $500.13 \mathrm{MHz}$

Solvent: THF $297.9 \mathrm{~K}$
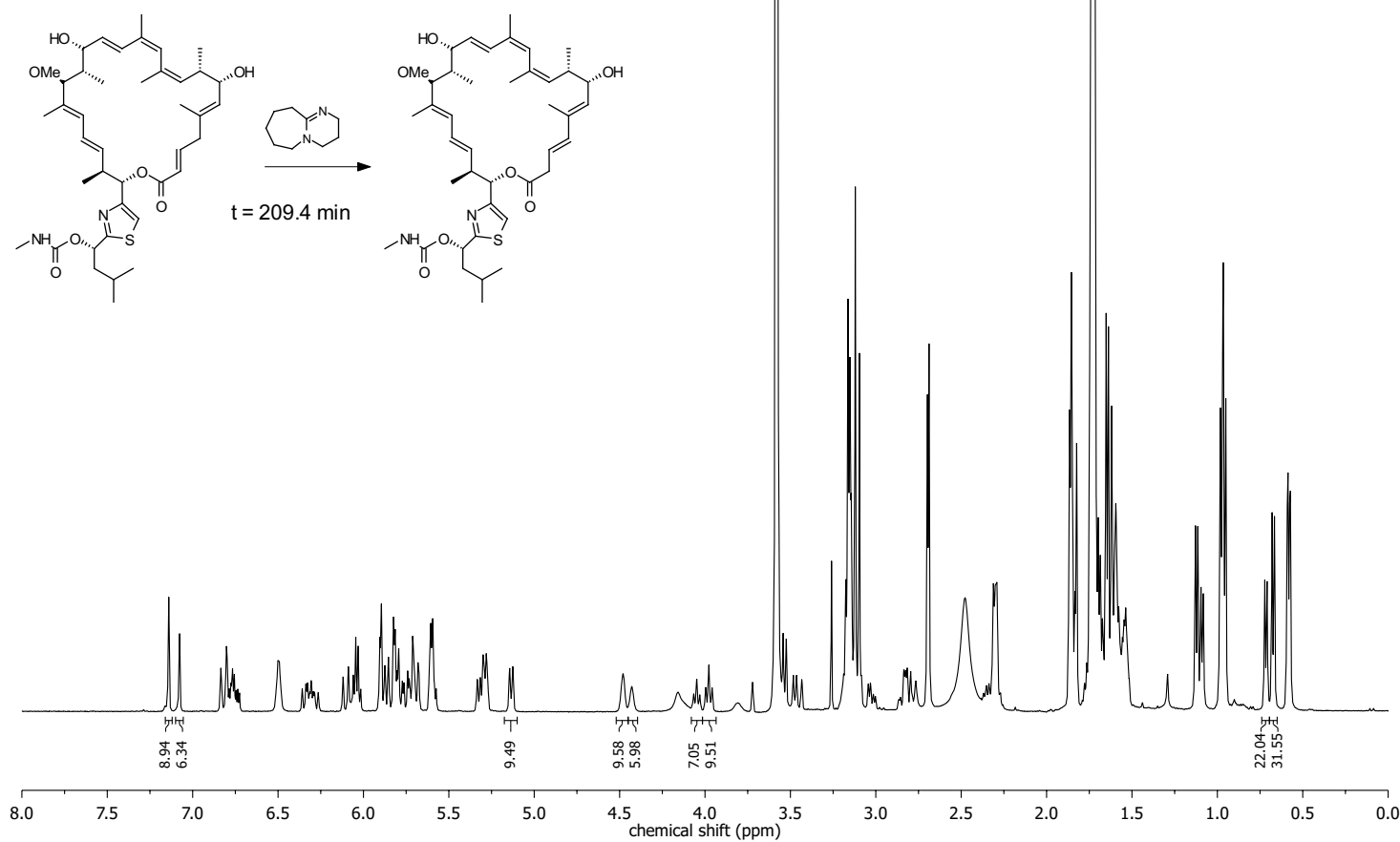


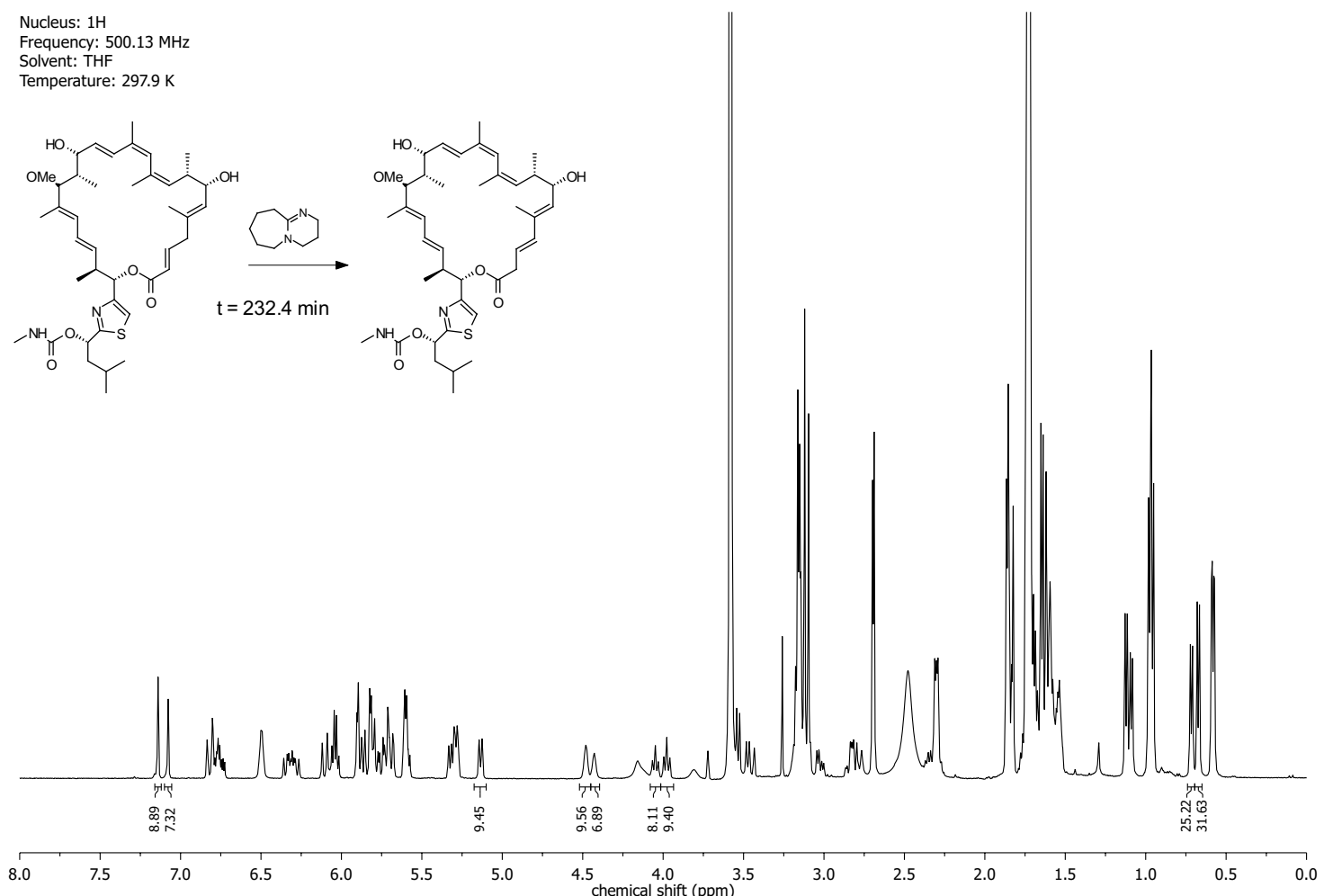

Nucleus: $1 \mathrm{H}$

requency: $500.13 \mathrm{MHz}$

Solvent: THF $297.9 \mathrm{~K}$
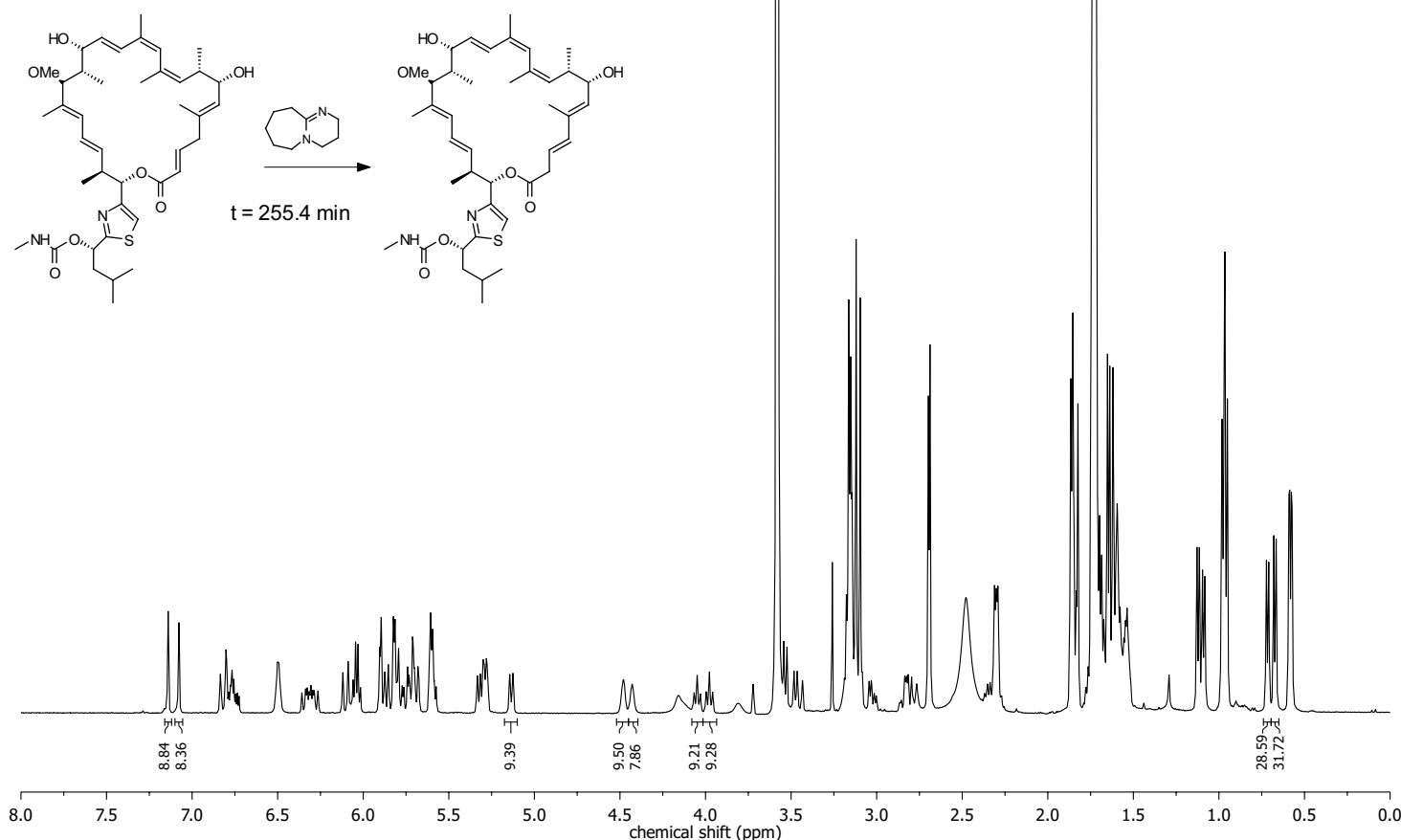
Nucleus: $1 \mathrm{H}$

Frequency: $500.13 \mathrm{MHz}$

Temperature: $297.9 \mathrm{~K}$

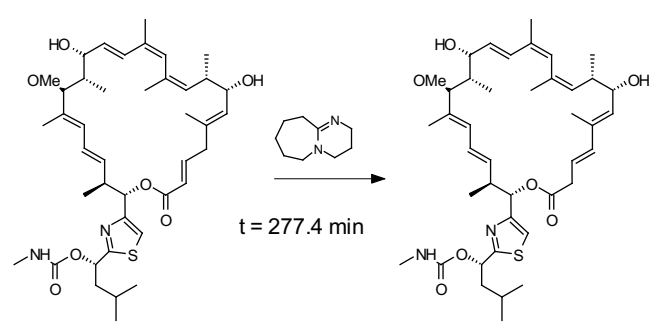

Frequency: $500.13 \mathrm{MH}$

Solvent: THF $298.0 \mathrm{~K}$
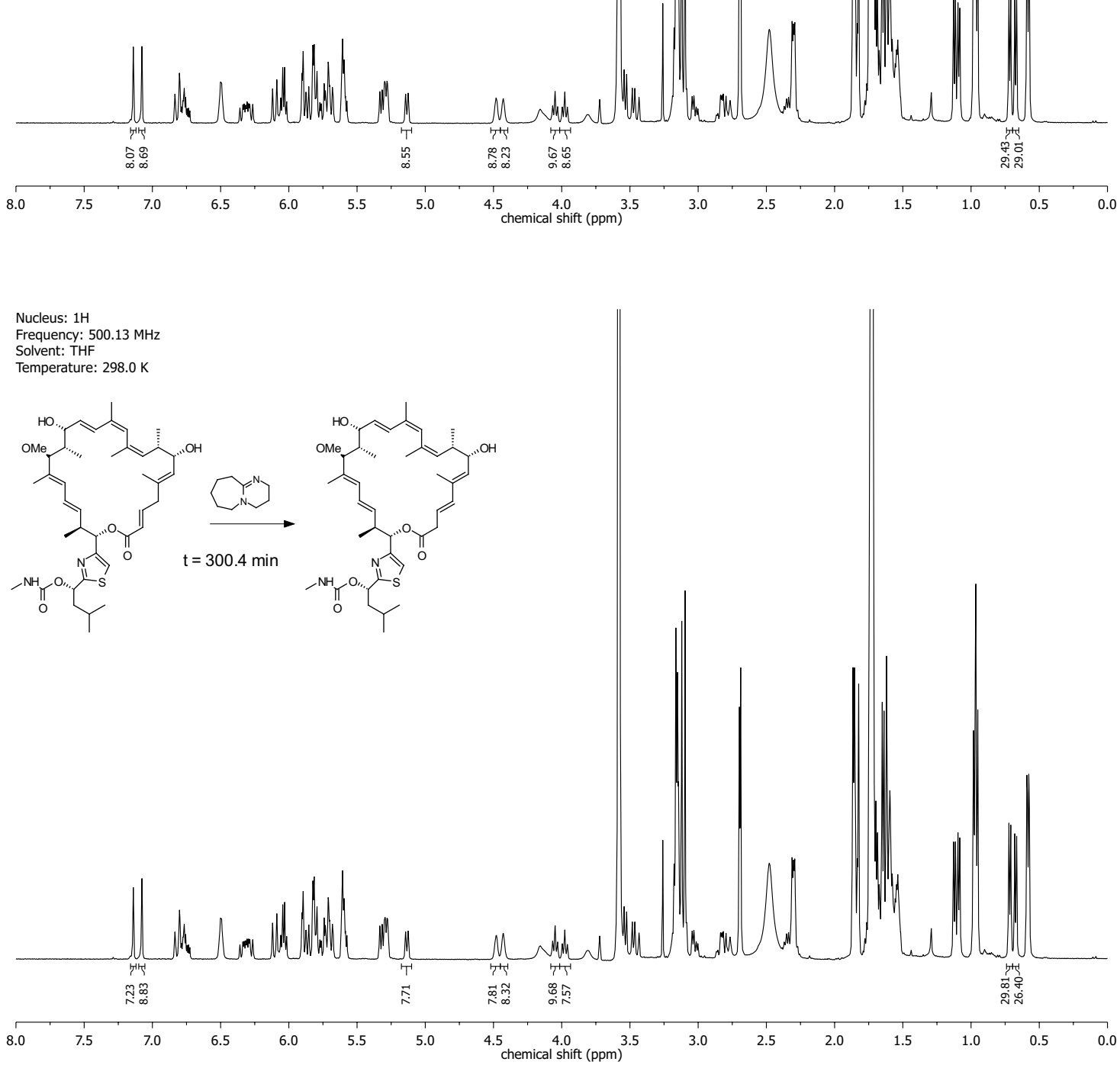
Nucleus: $1 \mathrm{H}$

Frequency: $500.13 \mathrm{MHz}$

Temperature: $297.9 \mathrm{~K}$
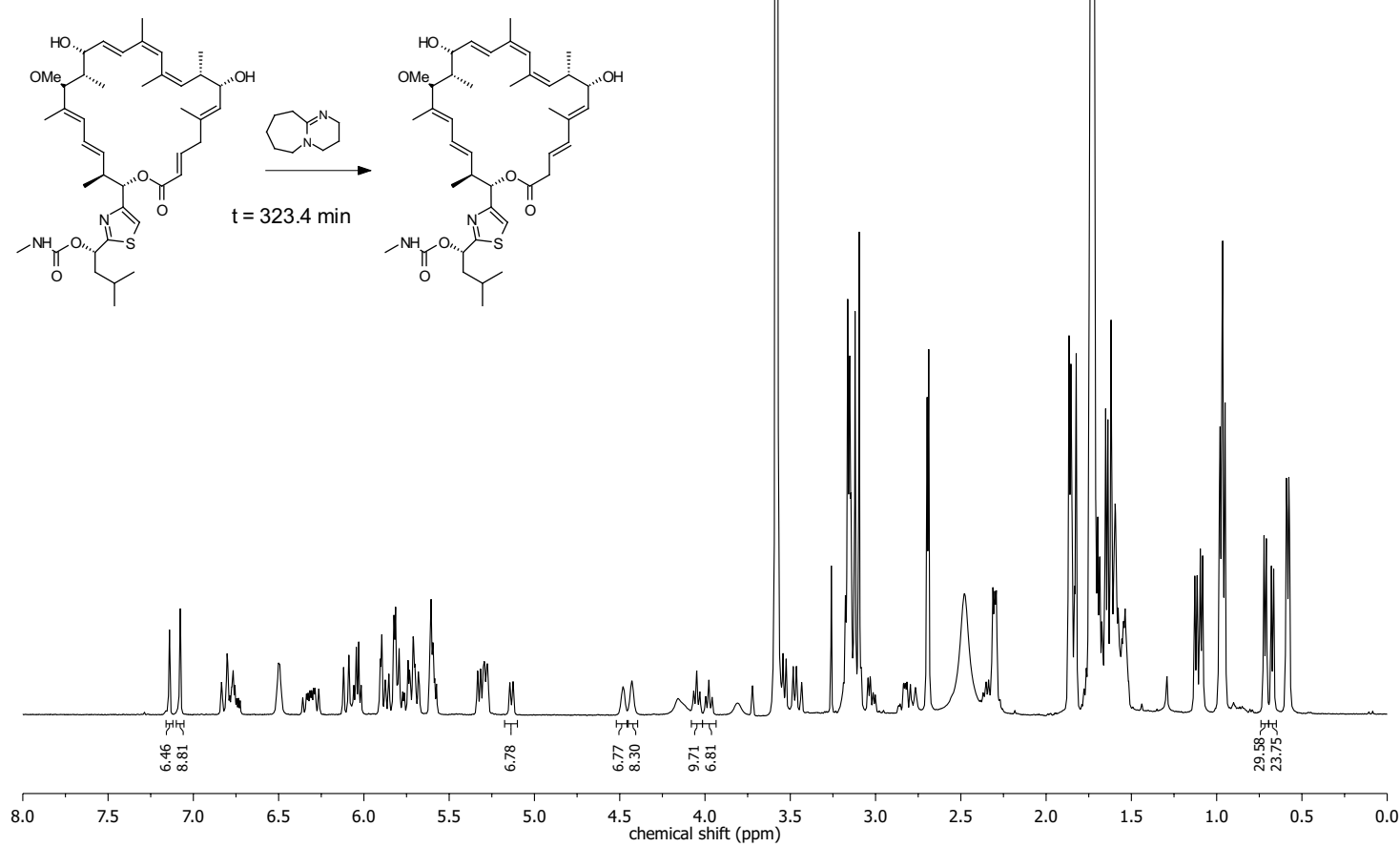

Nucleus: $1 \mathrm{H}$

requency: $500.13 \mathrm{MHz}$

Solvent: THF $297.9 \mathrm{~K}$
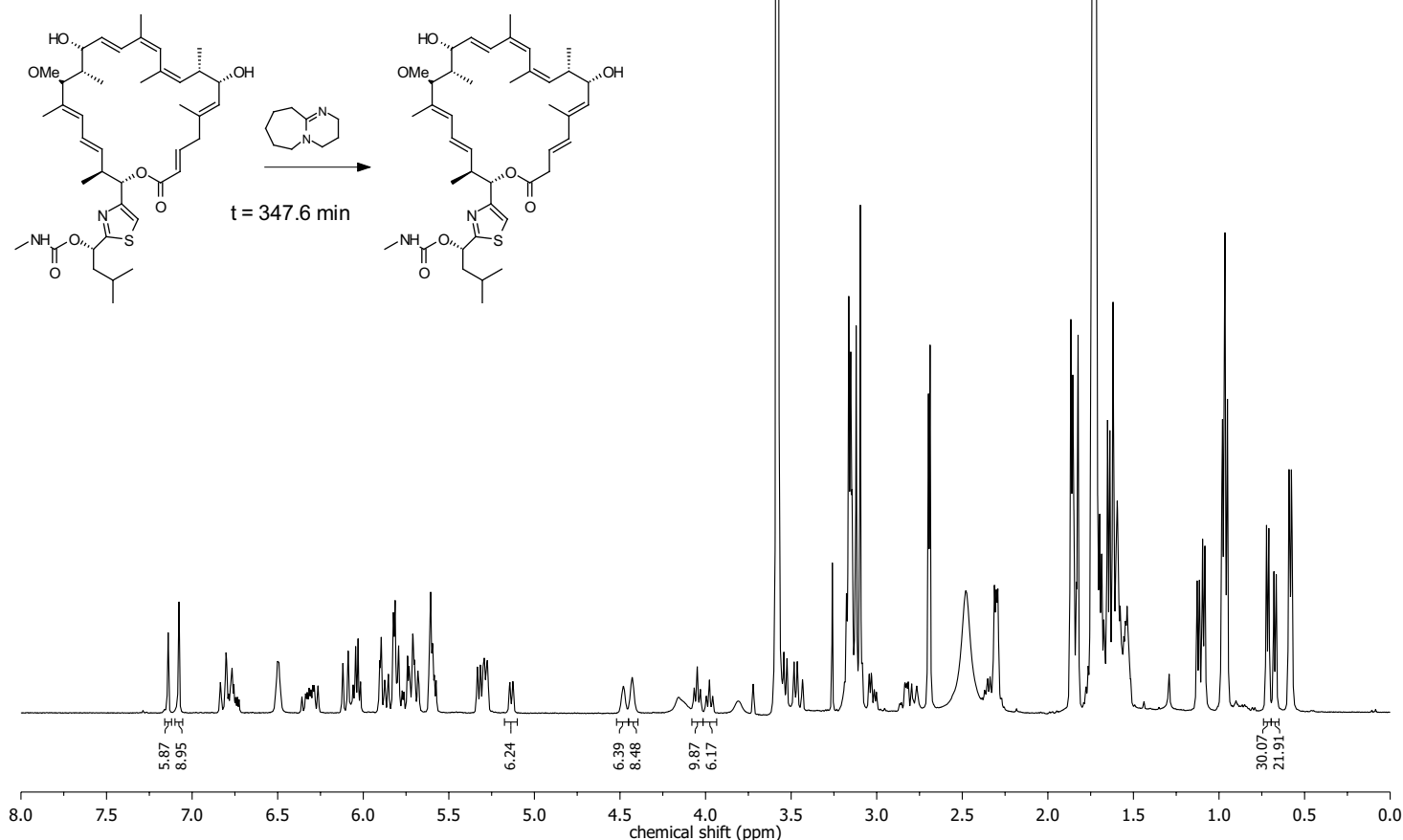
Nucleus: $1 \mathrm{H}$

Frequency: $500.13 \mathrm{MHz}$

Temperature: $297.9 \mathrm{~K}$
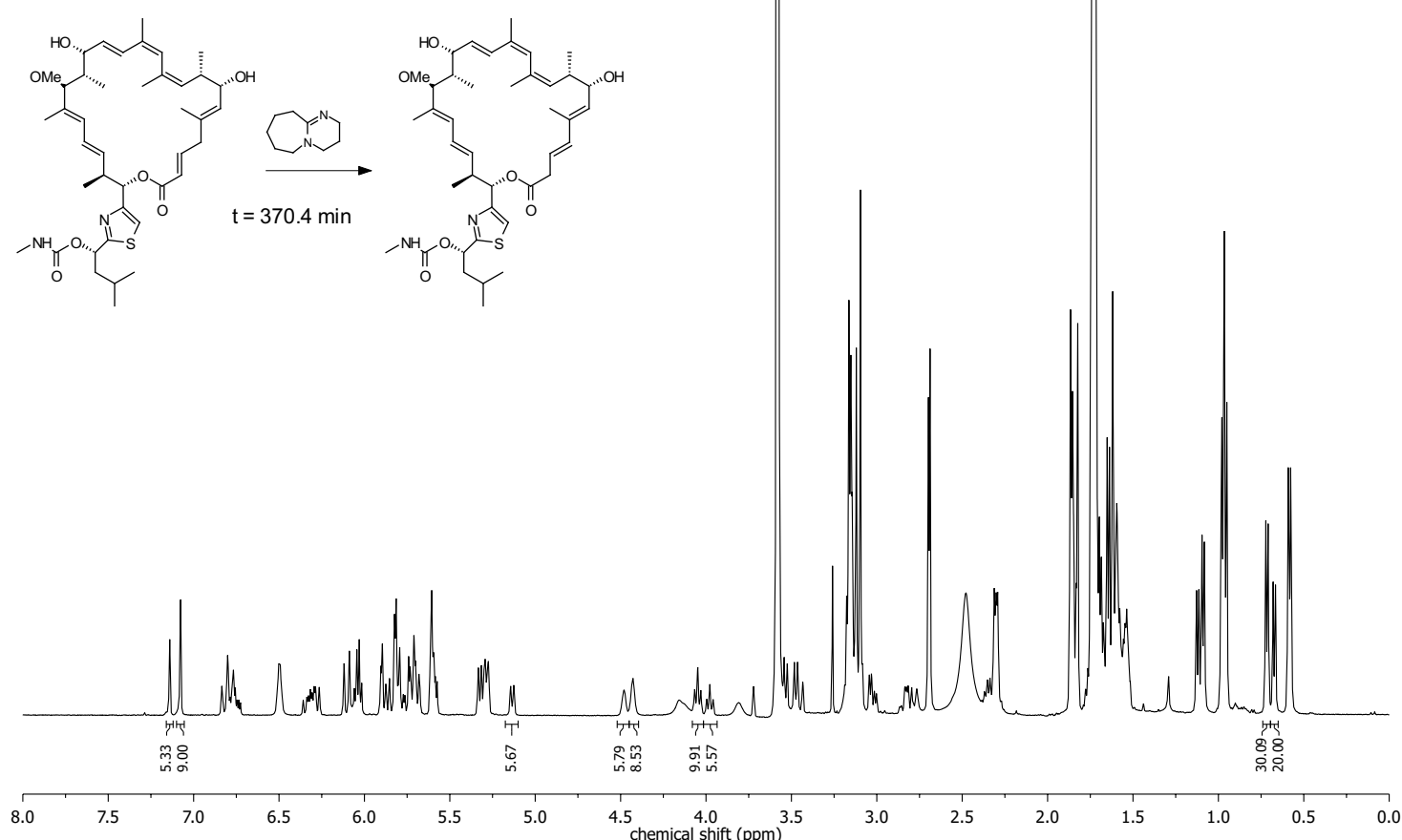

Nucleus: $1 \mathrm{H}$

requency: $500.13 \mathrm{MHz}$

Temperature: $298.0 \mathrm{~K}$
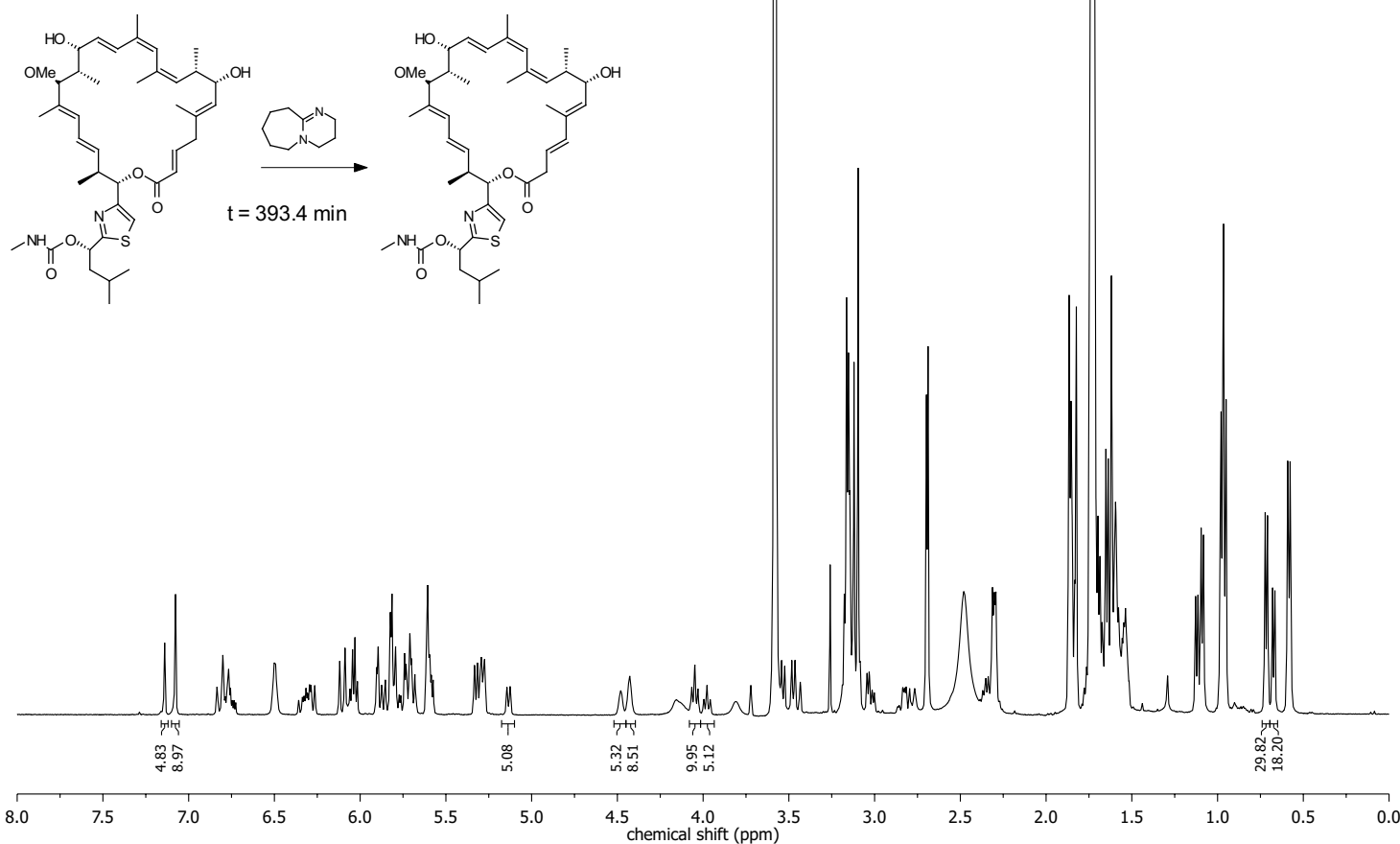
Nucleus: $1 \mathrm{H}$

Frequency: $500.13 \mathrm{MHz}$

Temperature: $297.9 \mathrm{~K}$
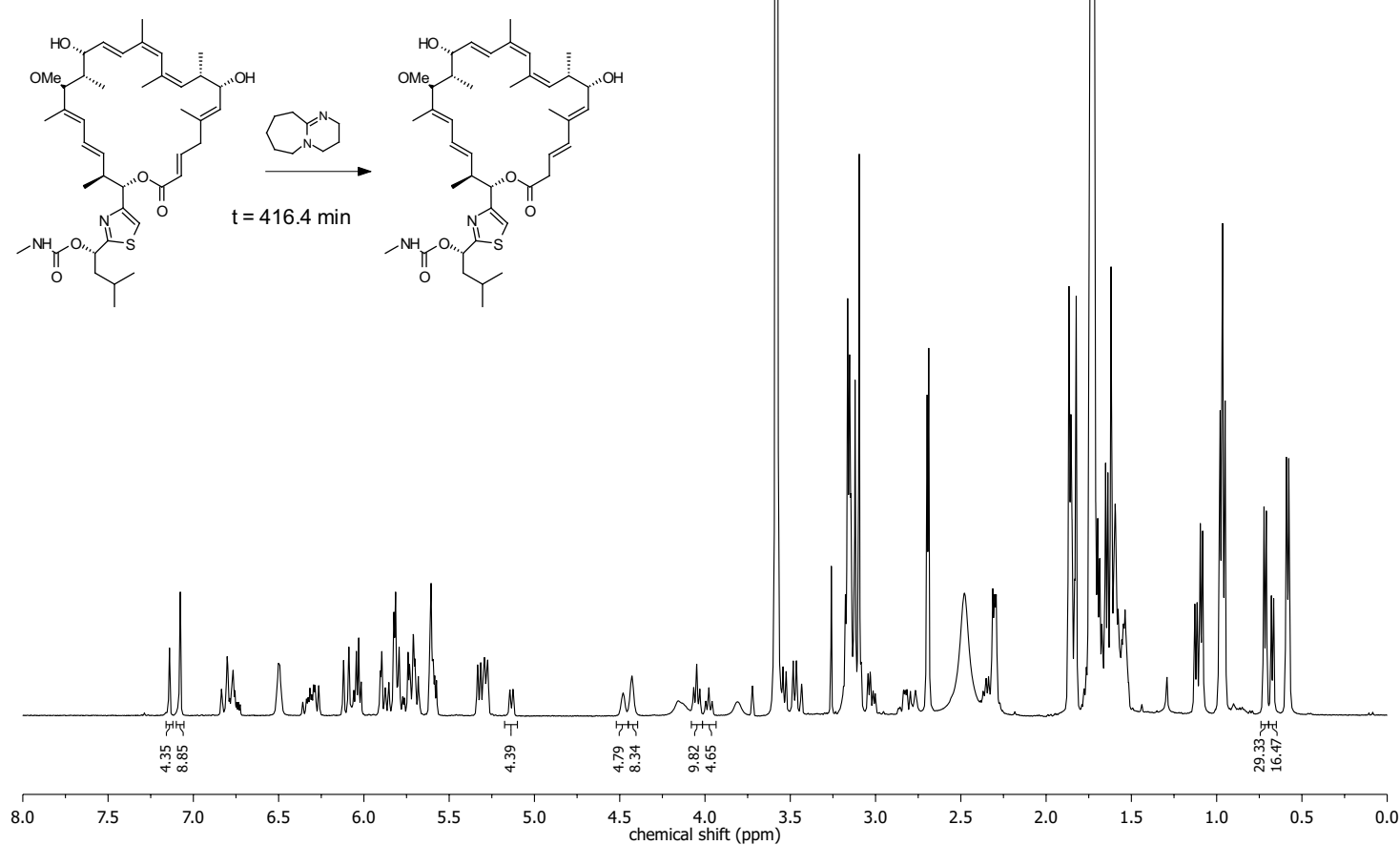

Nucleus: $1 \mathrm{H}$

requency: $500.13 \mathrm{MHz}$

Temperature: $297.9 \mathrm{~K}$
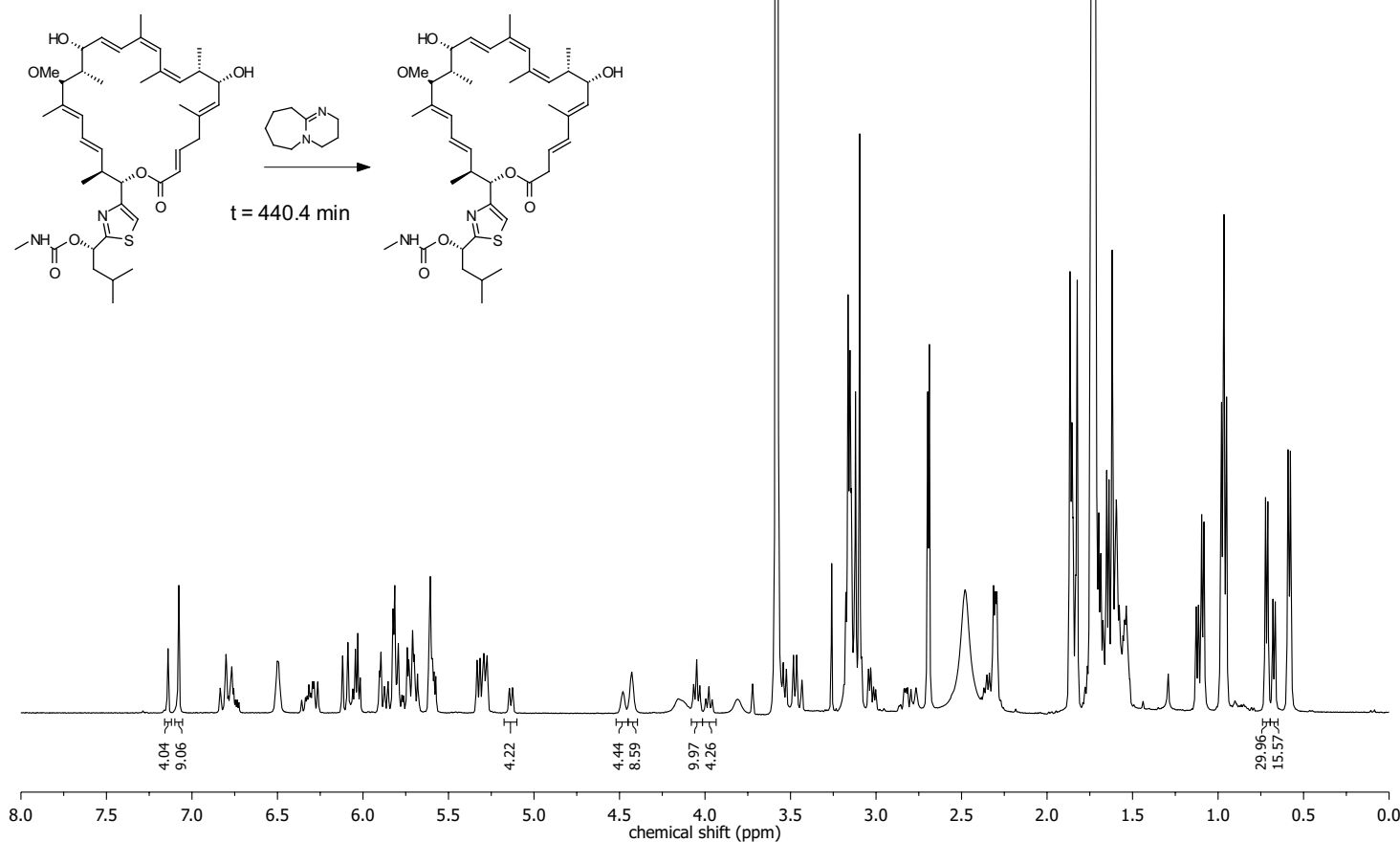
Nucleus: $1 \mathrm{H}$

Frequency: $500.13 \mathrm{MHz}$

Temperature: $298.0 \mathrm{~K}$
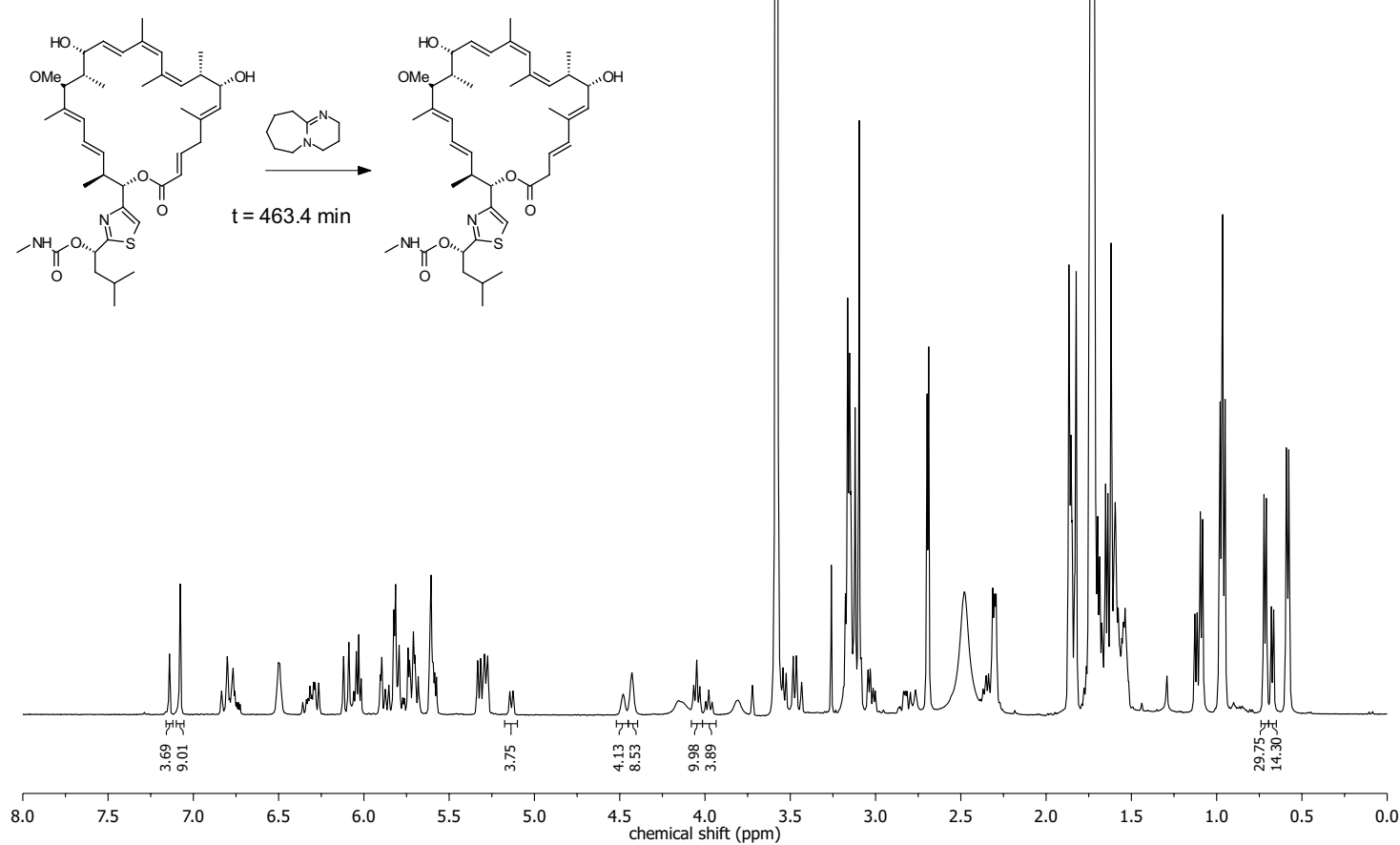

Nucleus: $1 \mathrm{H}$

requency: $500.13 \mathrm{MHz}$

Temperature: $297.9 \mathrm{~K}$
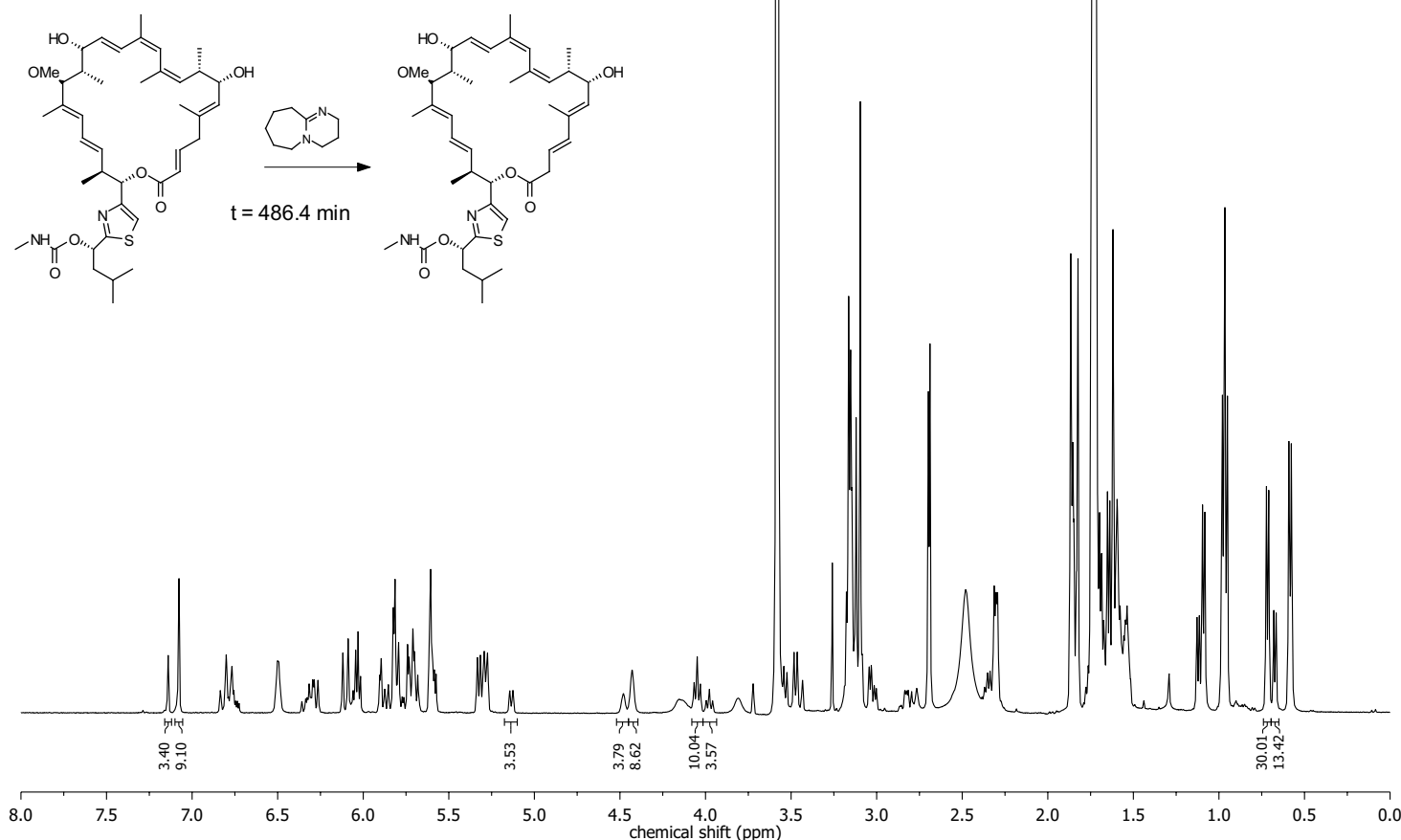
Nucleus: $1 \mathrm{H}$

Frequency: $500.13 \mathrm{MHz}$

Temperature: $297.9 \mathrm{~K}$
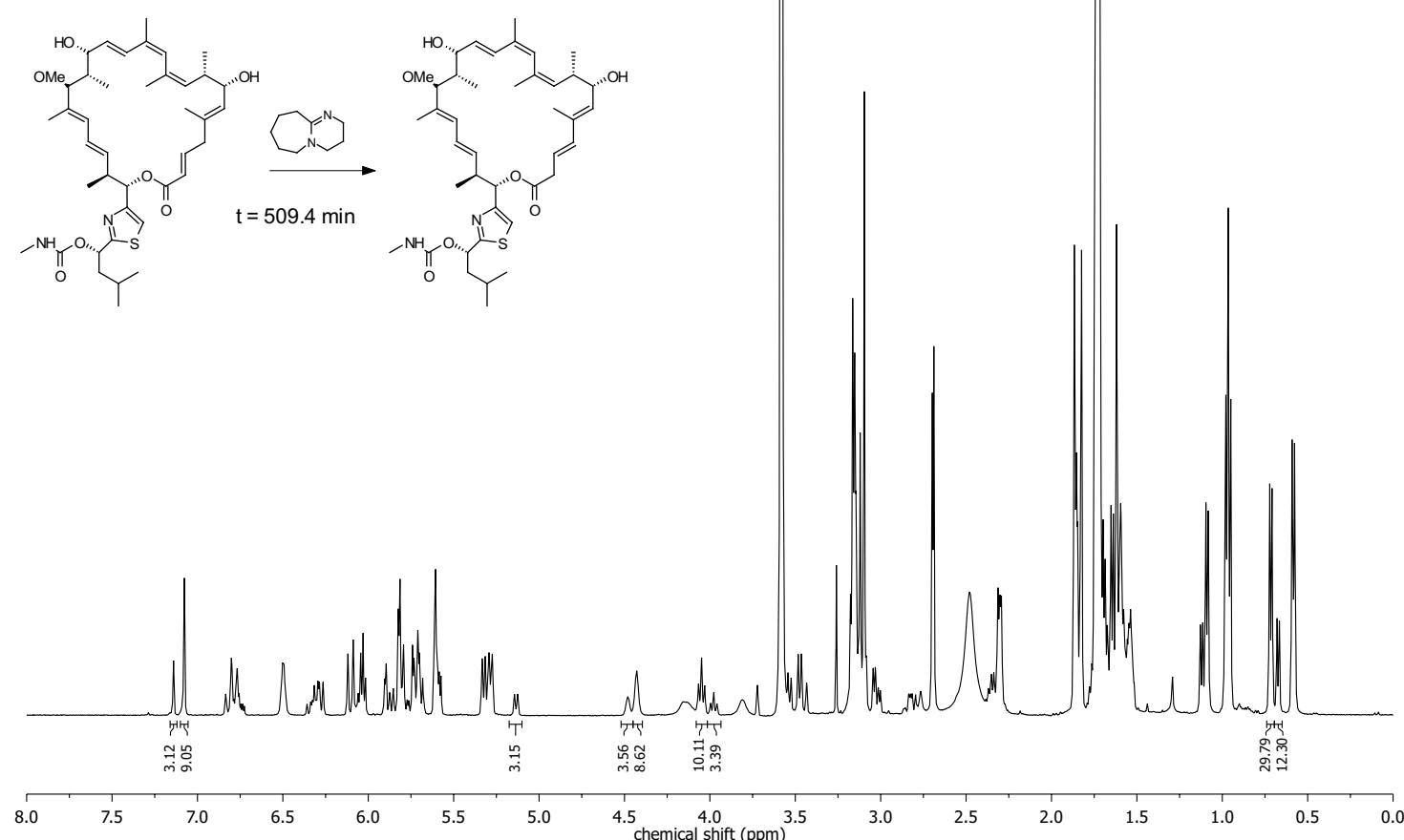

Nucleus: $1 \mathrm{H}$

requency: $500.13 \mathrm{MHz}$

Temperature: $297.9 \mathrm{~K}$
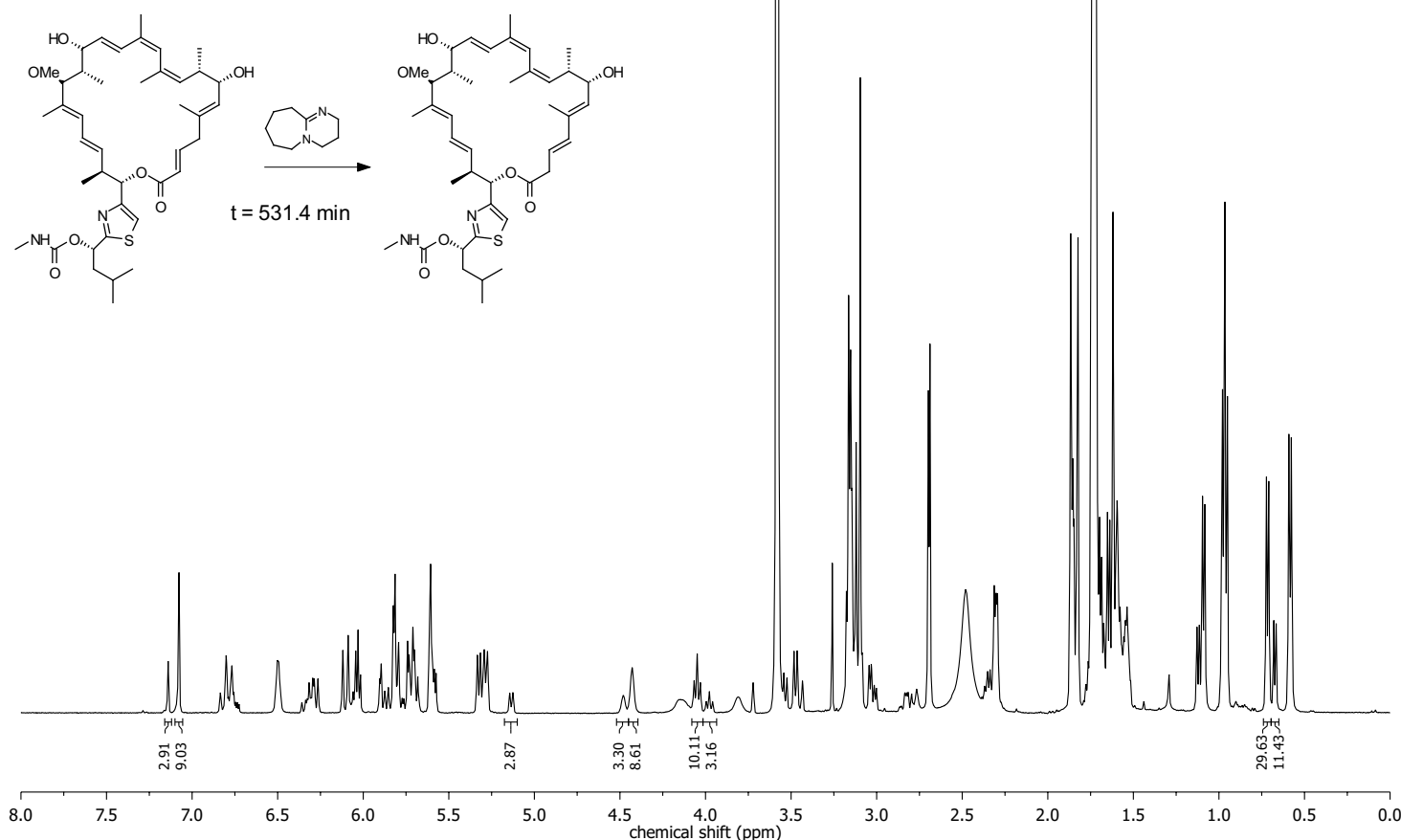
Nucleus: $1 \mathrm{H}$

Frequency: $500.13 \mathrm{MHz}$

Temperature: $297.9 \mathrm{~K}$
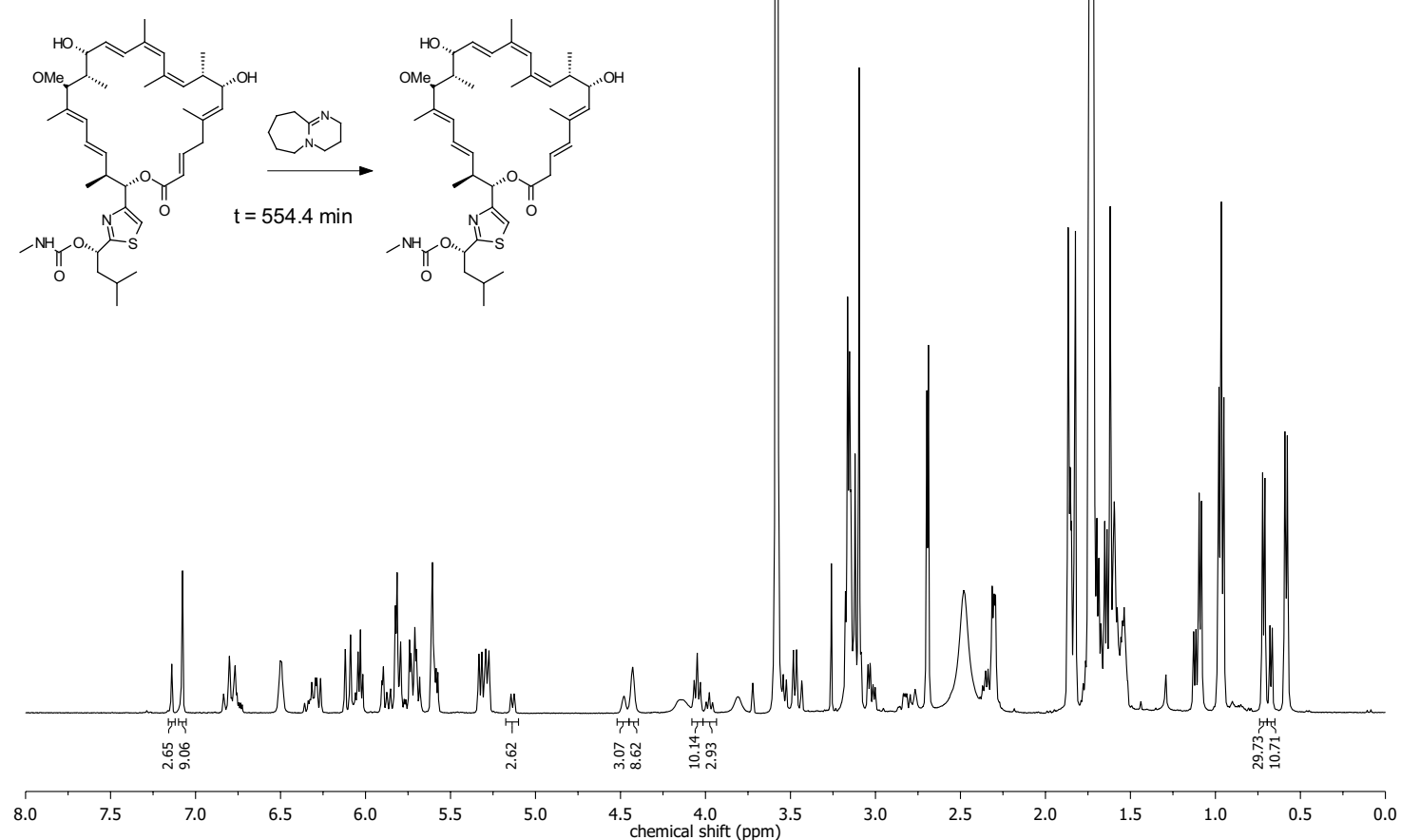

Nucleus: $1 \mathrm{H}$

requency: $500.13 \mathrm{MHz}$

Solvent: THF $297.9 \mathrm{~K}$
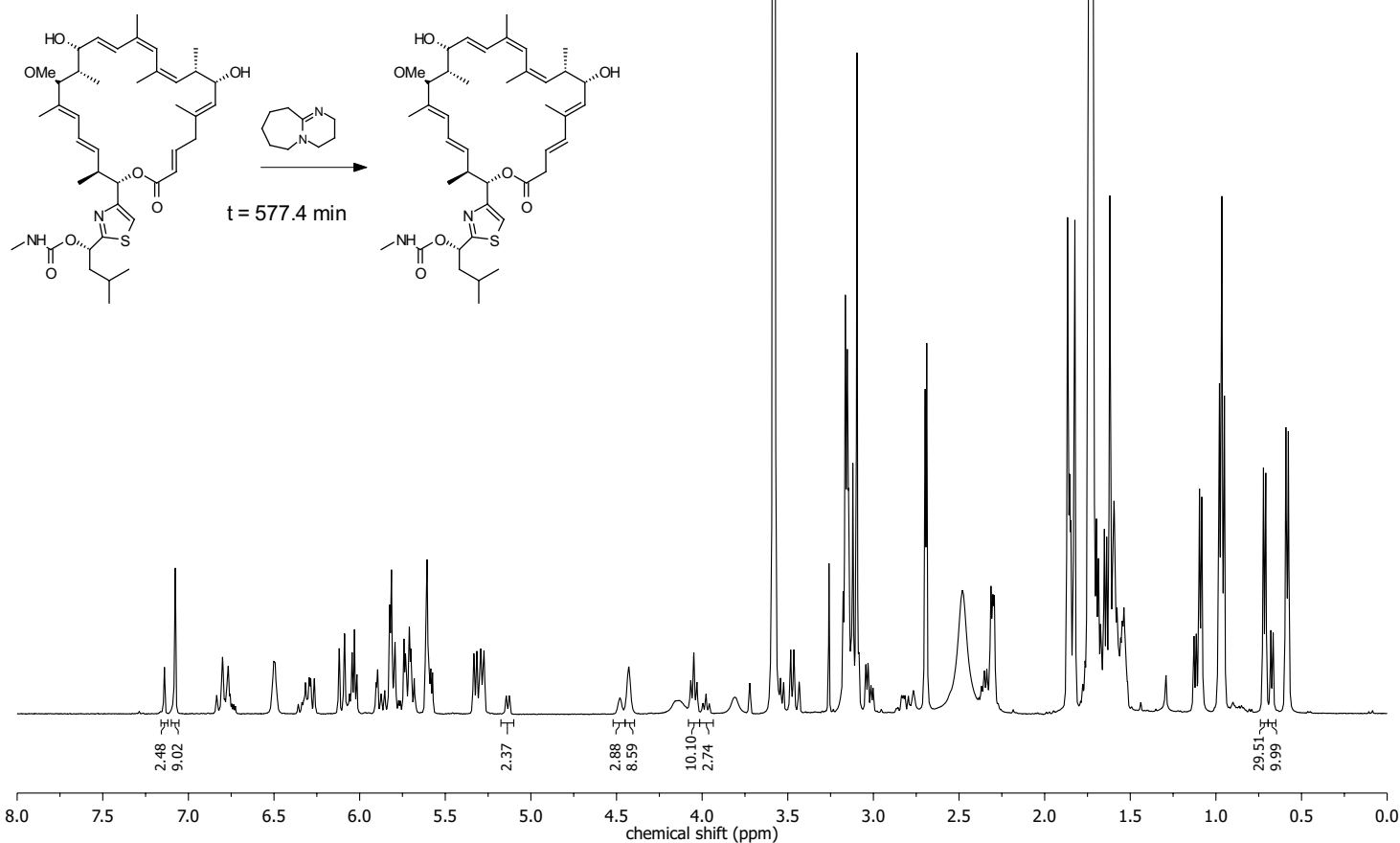
Nucleus: $1 \mathrm{H}$

Frequency: $500.13 \mathrm{MHz}$

Temperature: $298.0 \mathrm{~K}$
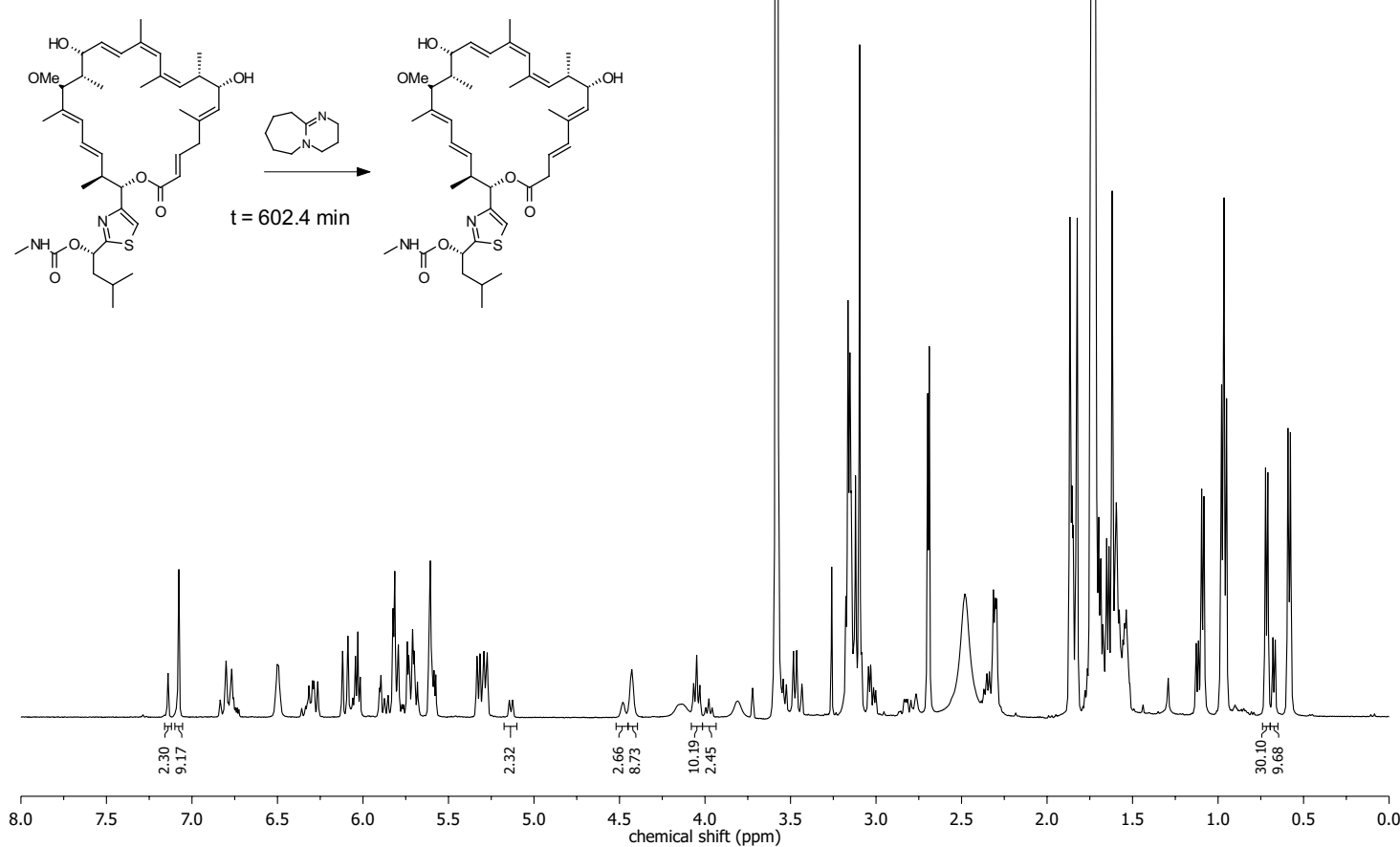

Nucleus: $1 \mathrm{H}$

requency: $500.13 \mathrm{MHz}$

Temperature: $298.0 \mathrm{~K}$
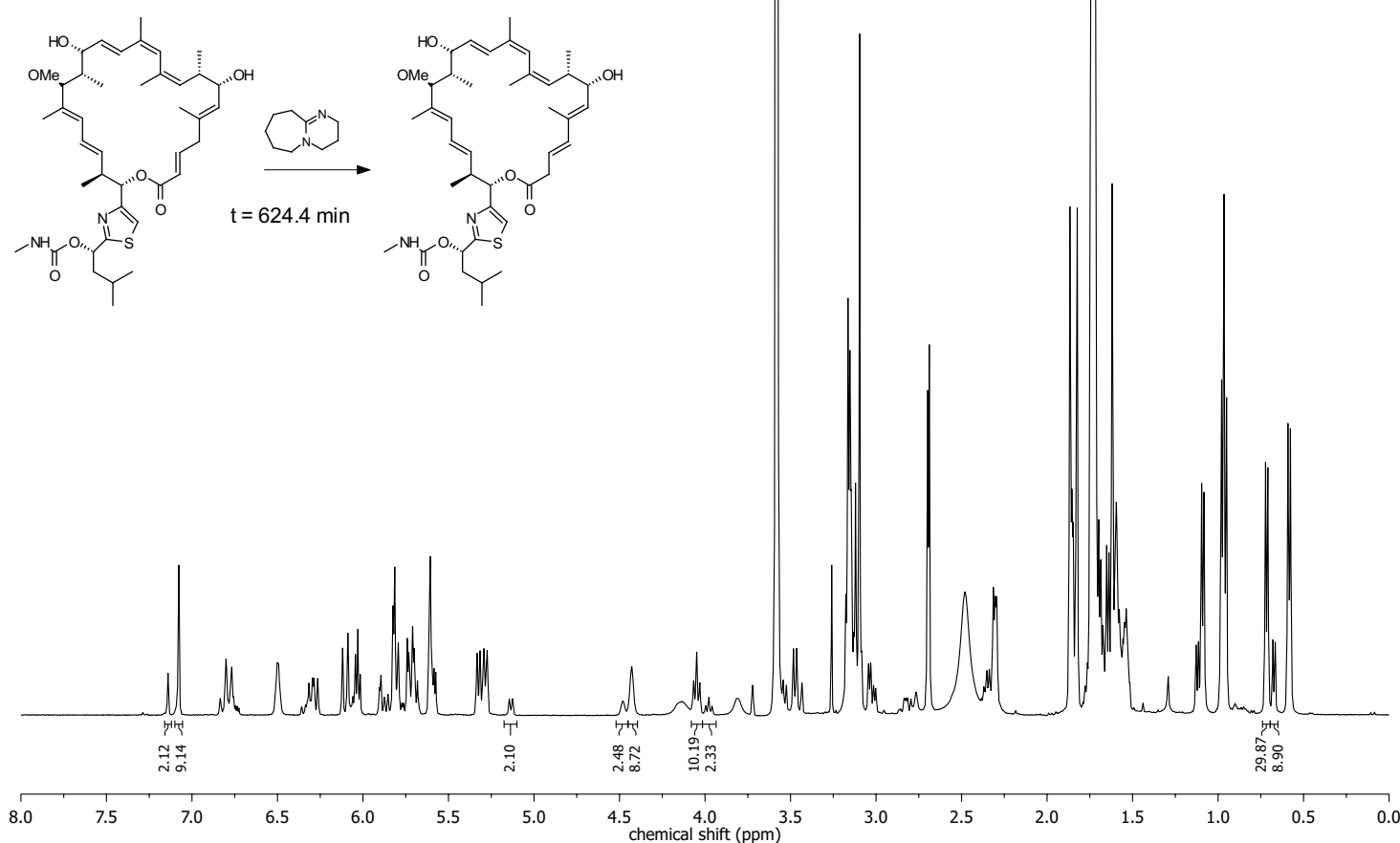
Nucleus: $1 \mathrm{H}$

Frequency: $500.13 \mathrm{MHz}$

Temperature: $297.9 \mathrm{~K}$
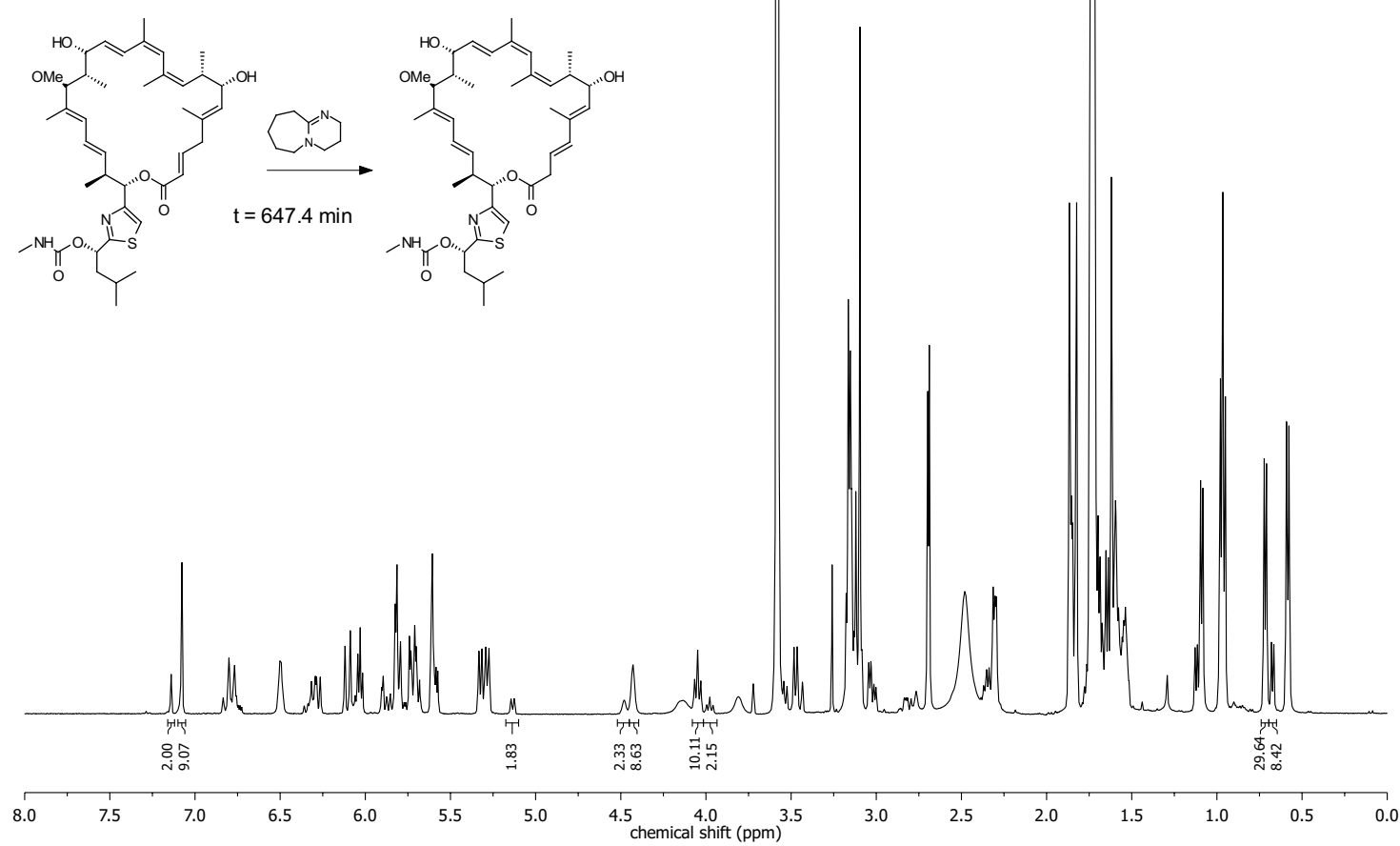

Nucleus: $1 \mathrm{H}$

requency: $500.13 \mathrm{MHz}$

Temperature: $297.9 \mathrm{~K}$
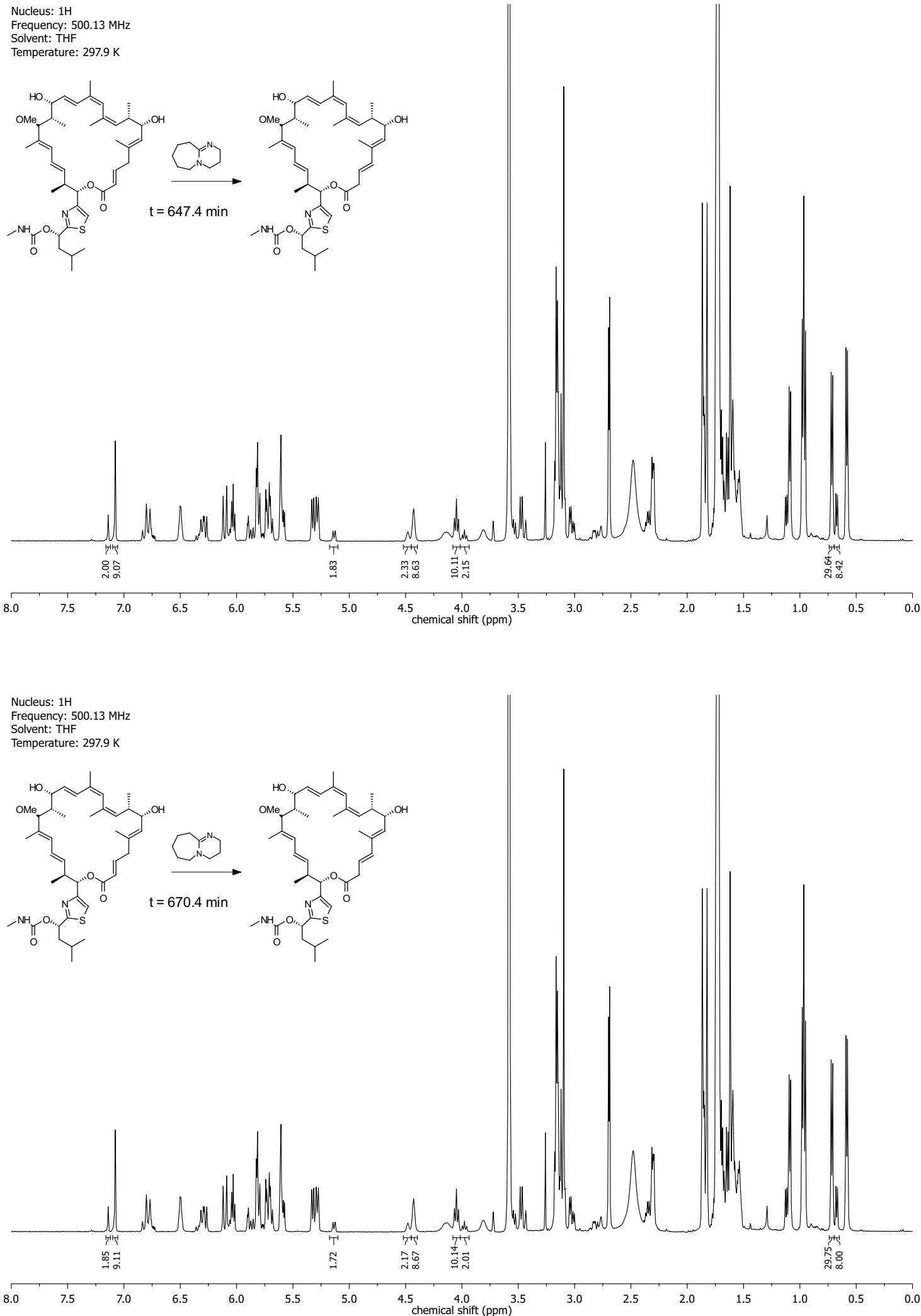
Nucleus: $1 \mathrm{H}$

Frequency: $500.13 \mathrm{MHz}$

Temperature: $298.0 \mathrm{~K}$
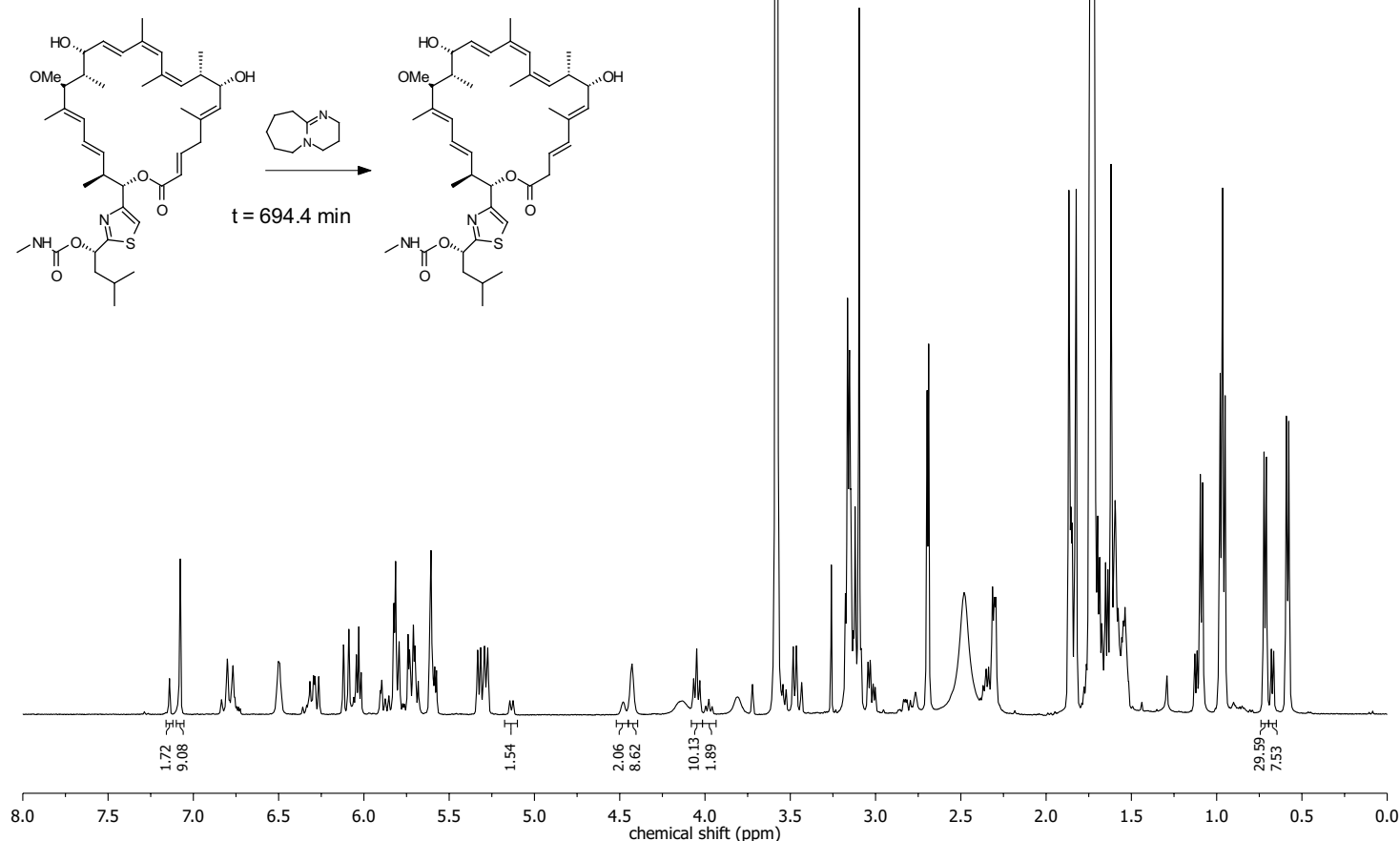

Nucleus: $1 \mathrm{H}$

requency: $500.13 \mathrm{MHz}$

Temperature: $297.9 \mathrm{~K}$
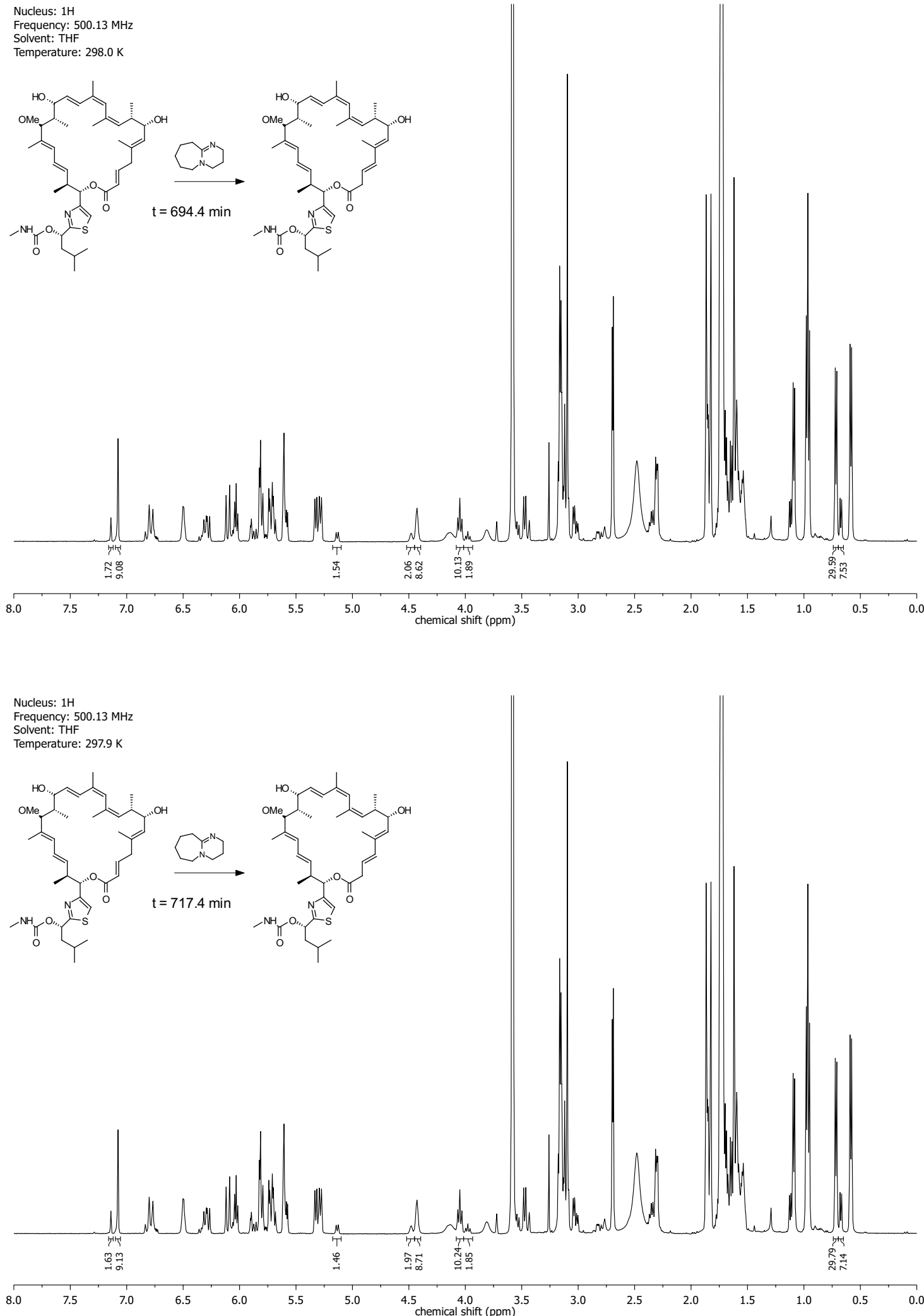
NMR-Spectra for Compound 94

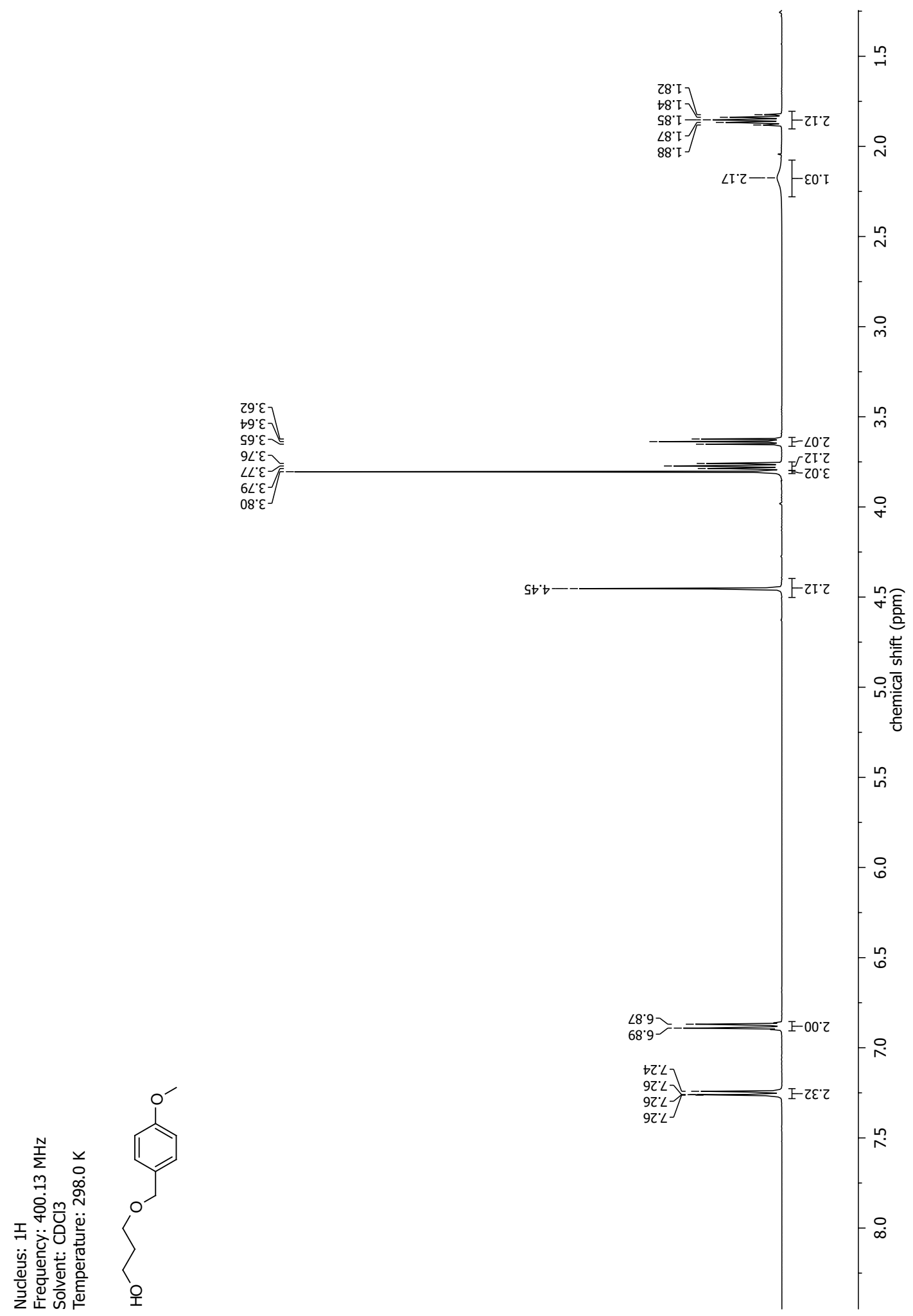


ว'ฉ६-

t'ss-

2'29-

$\varepsilon \cdot 69-$

$8 \cdot \varepsilon L$
$8.9 L$
$7: 4]$

s\&u

$0 .+\mathrm{LI}-$

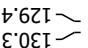

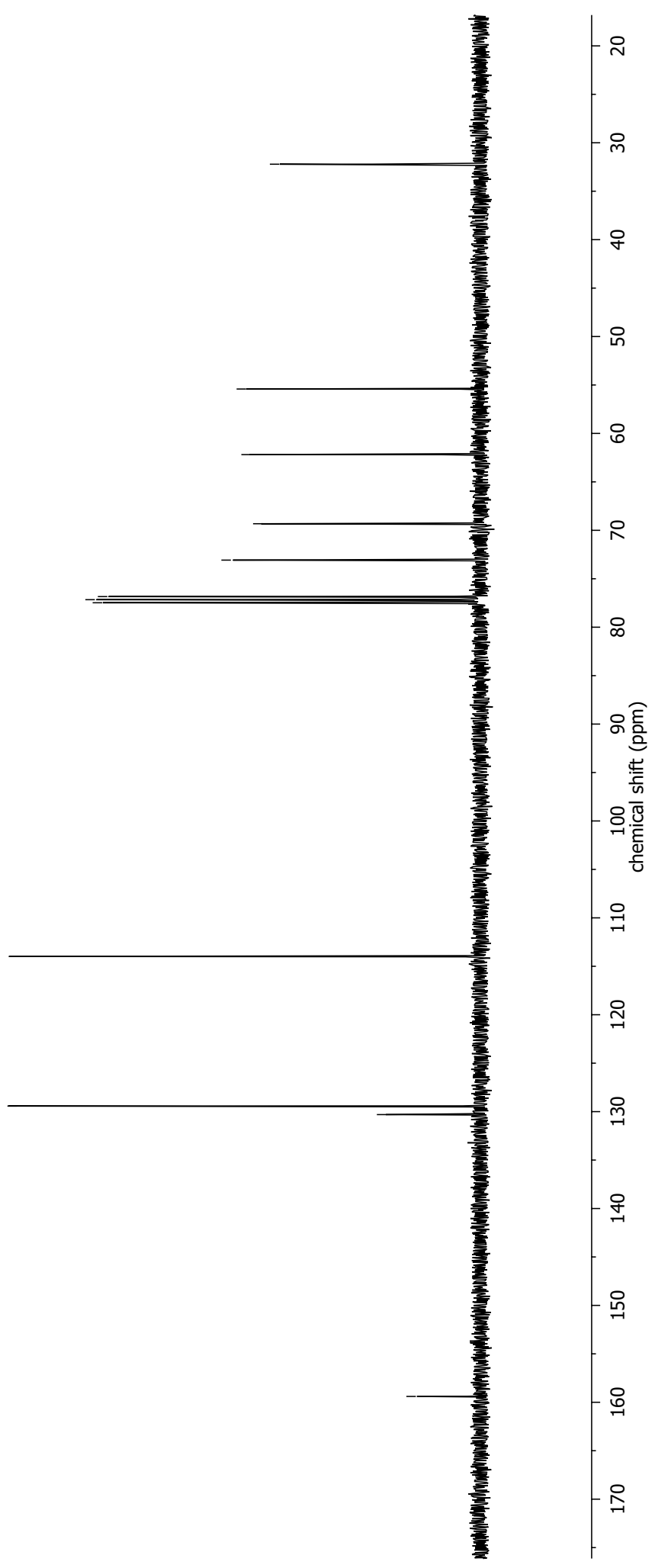


NMR-Spectra for Compound 13

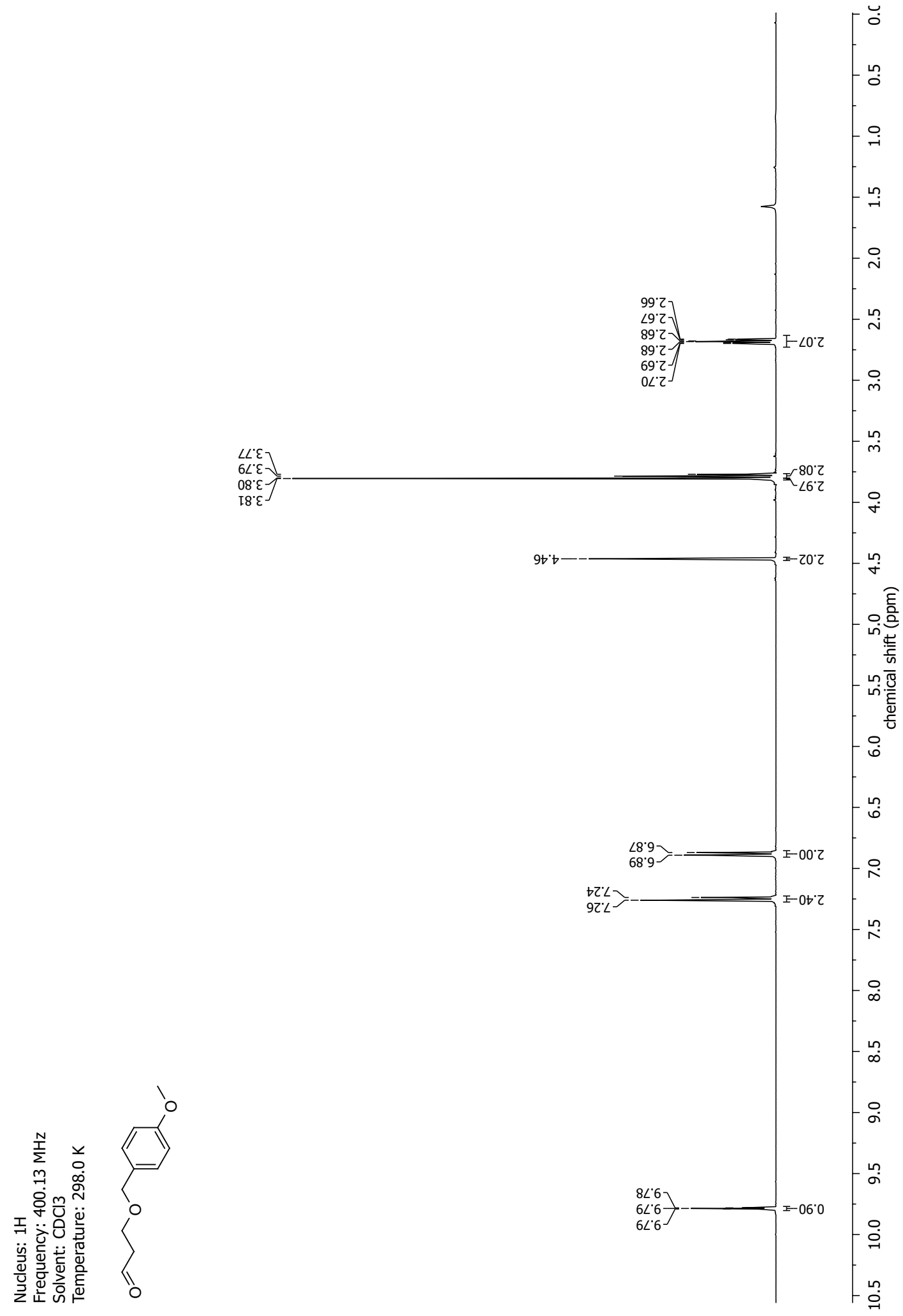




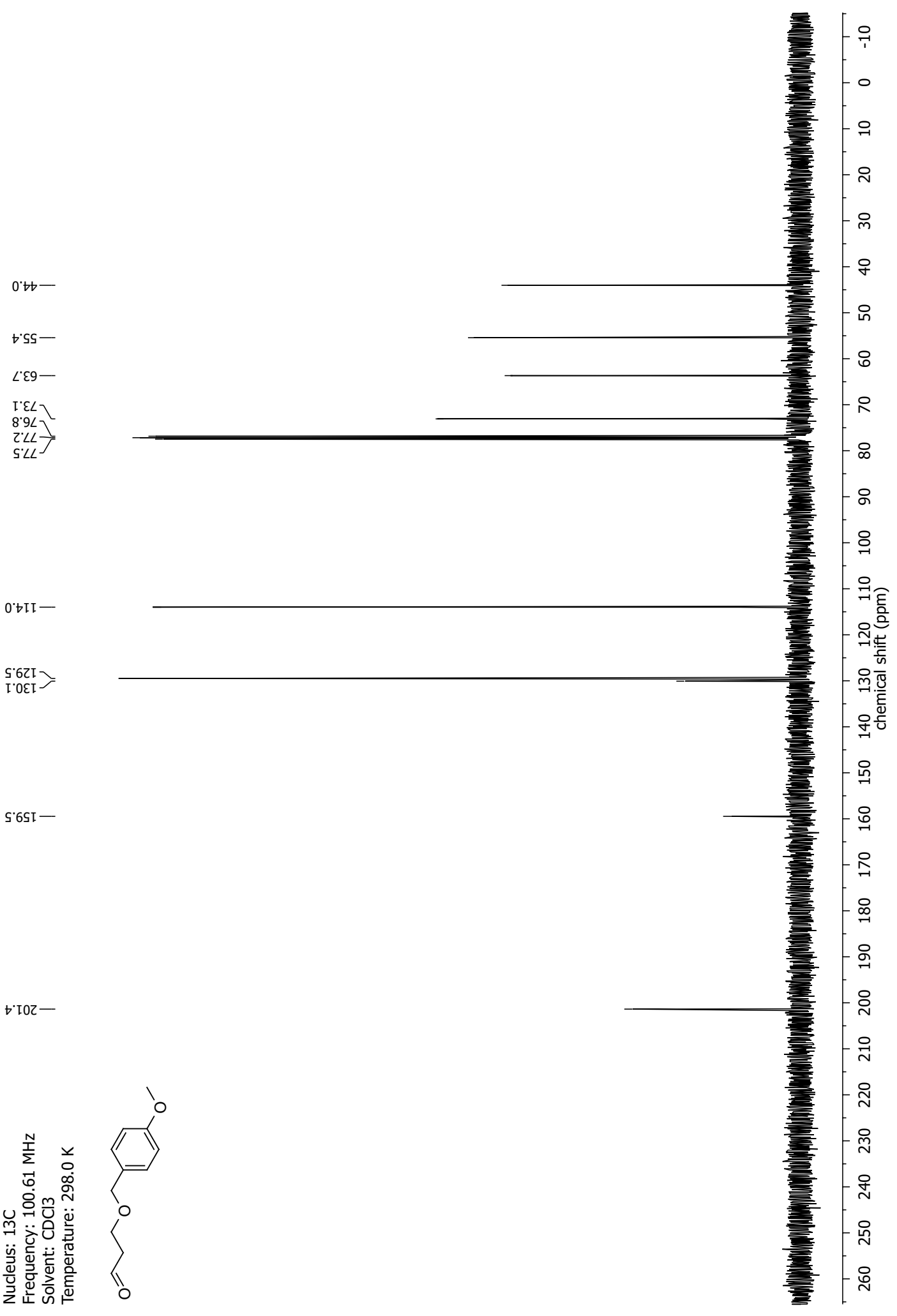


NMR-Spectra for Compound 15

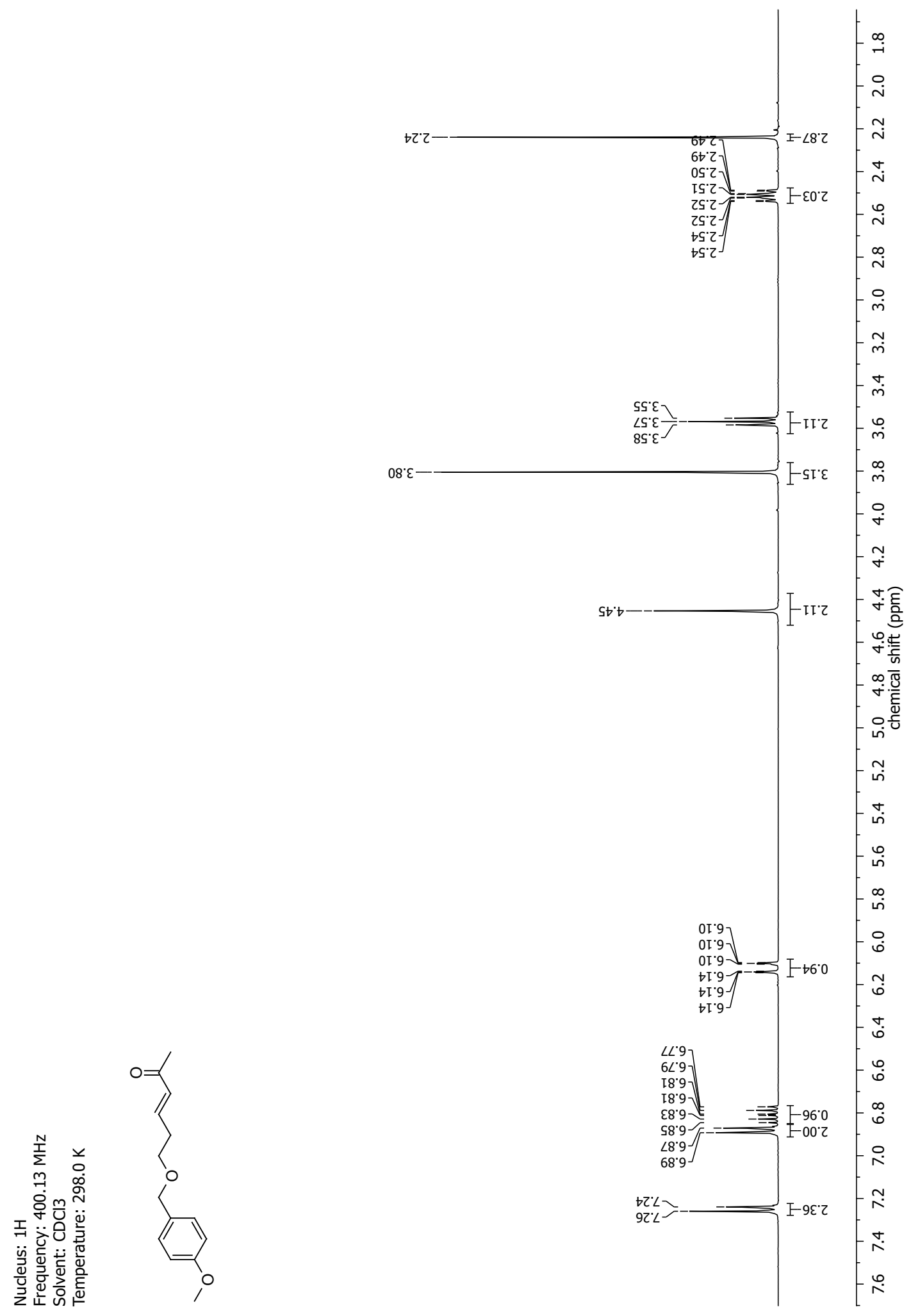




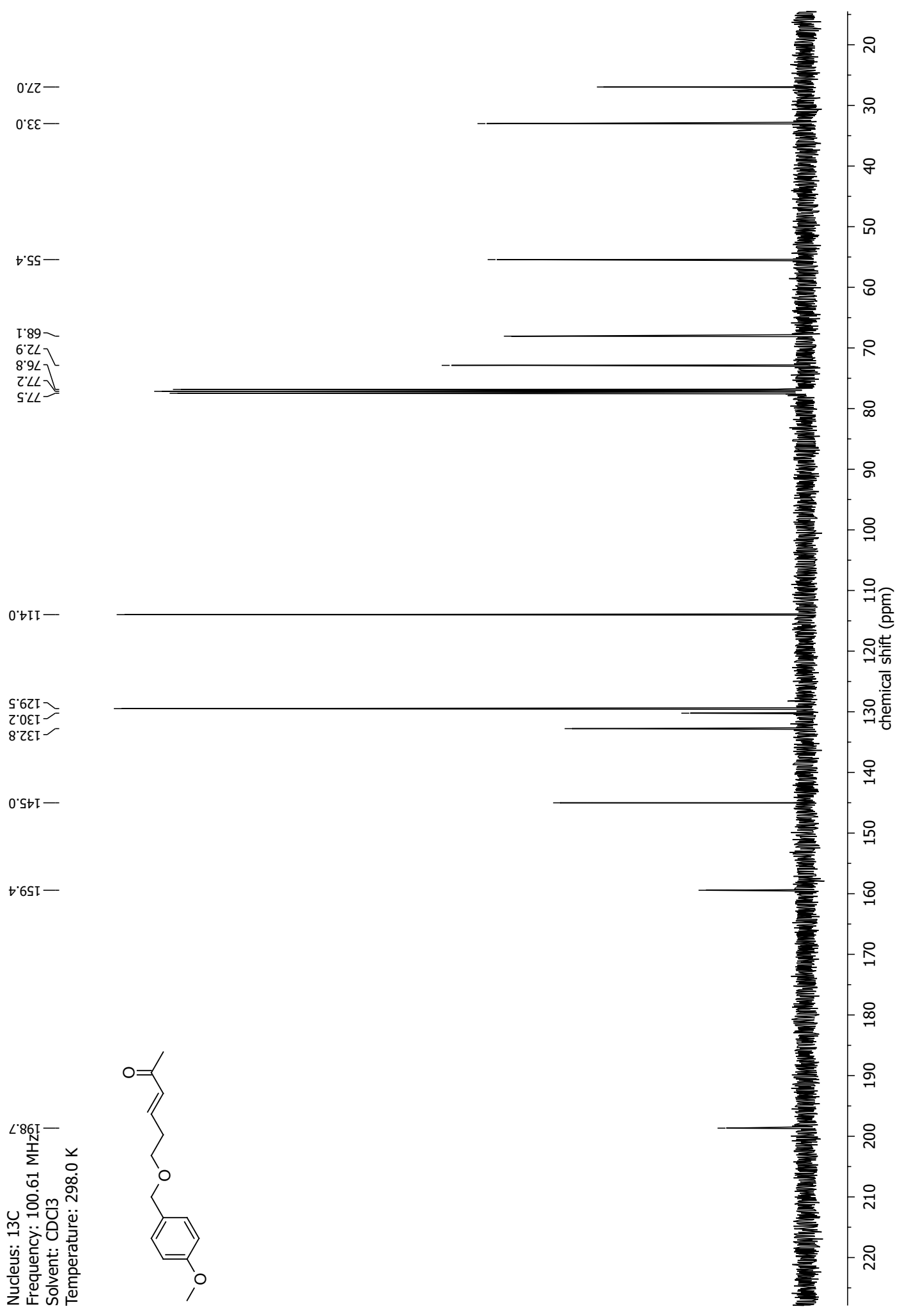


NMR-Spectra for Compound 21

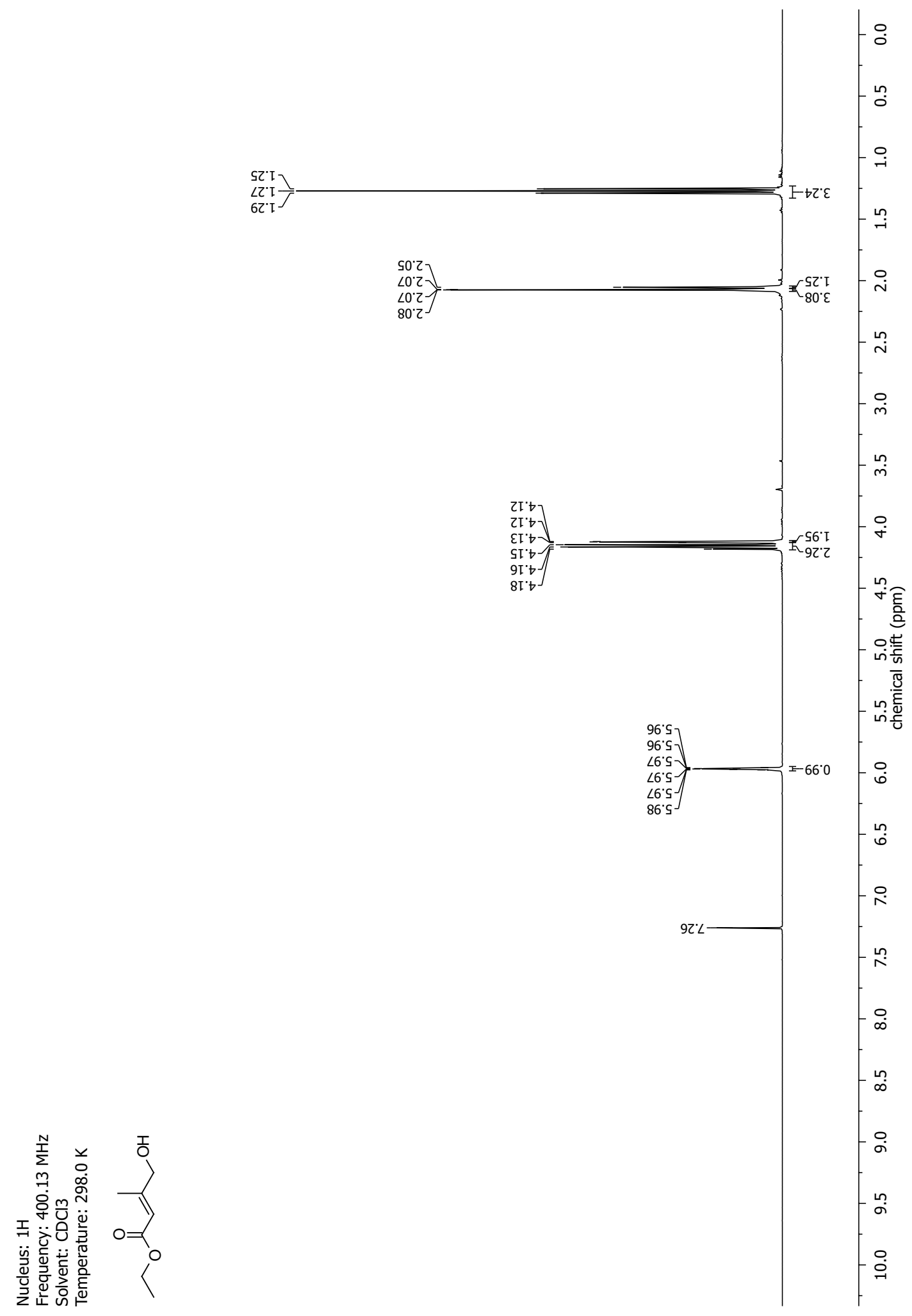


$\underset{\text { t'tiI }}{\text { L'SI }}$

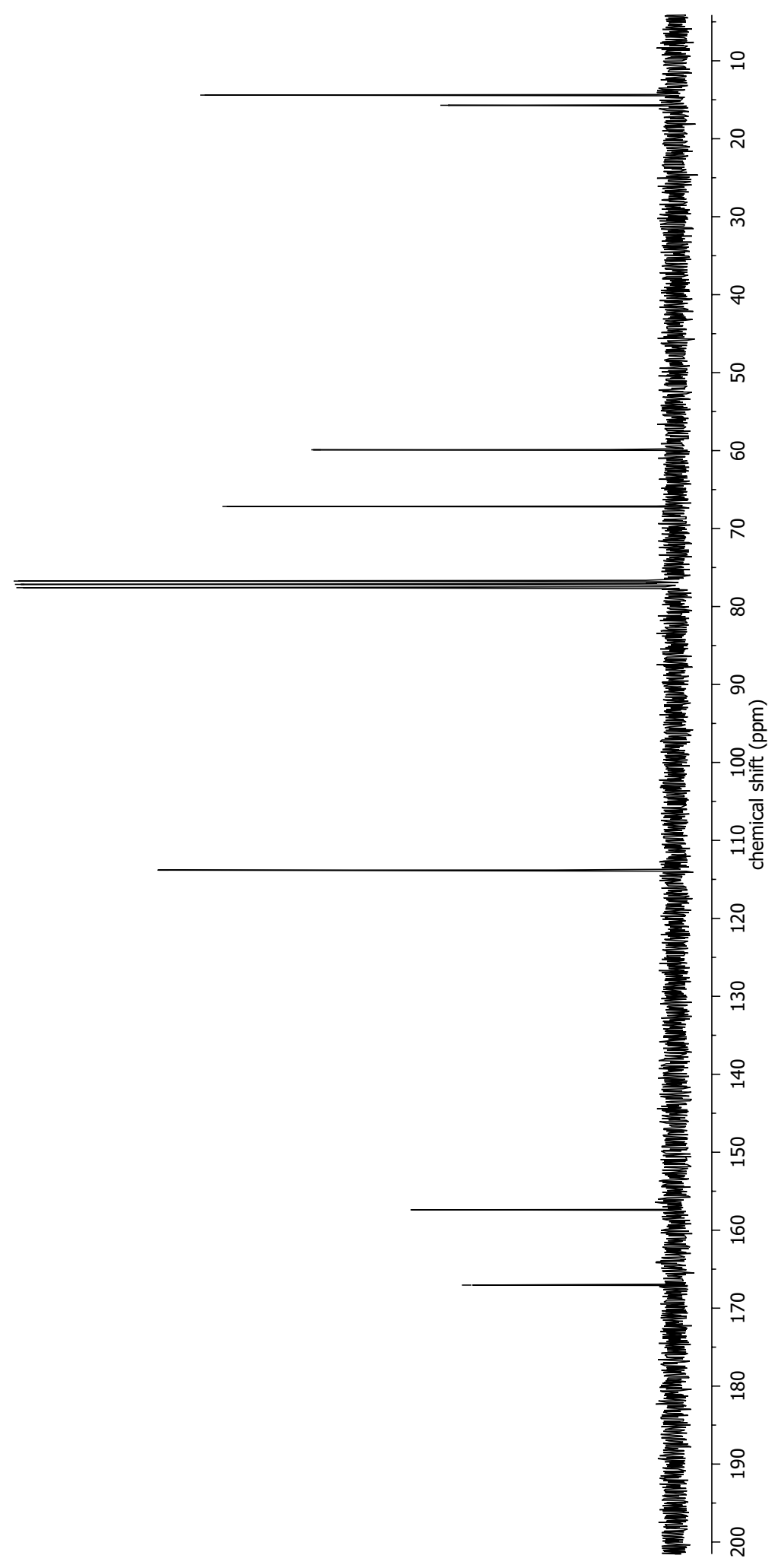


NMR-Spectra for Compound 22

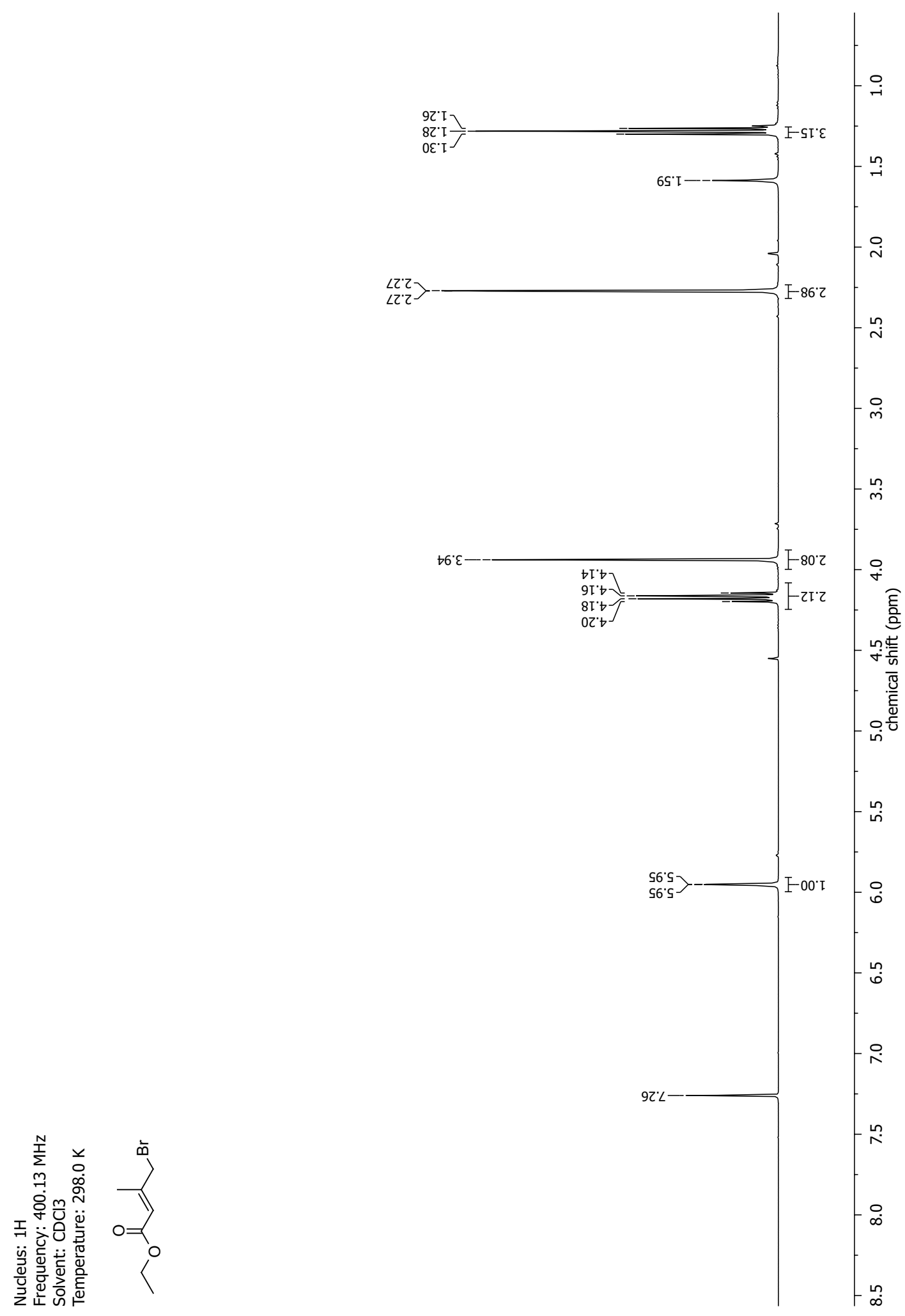




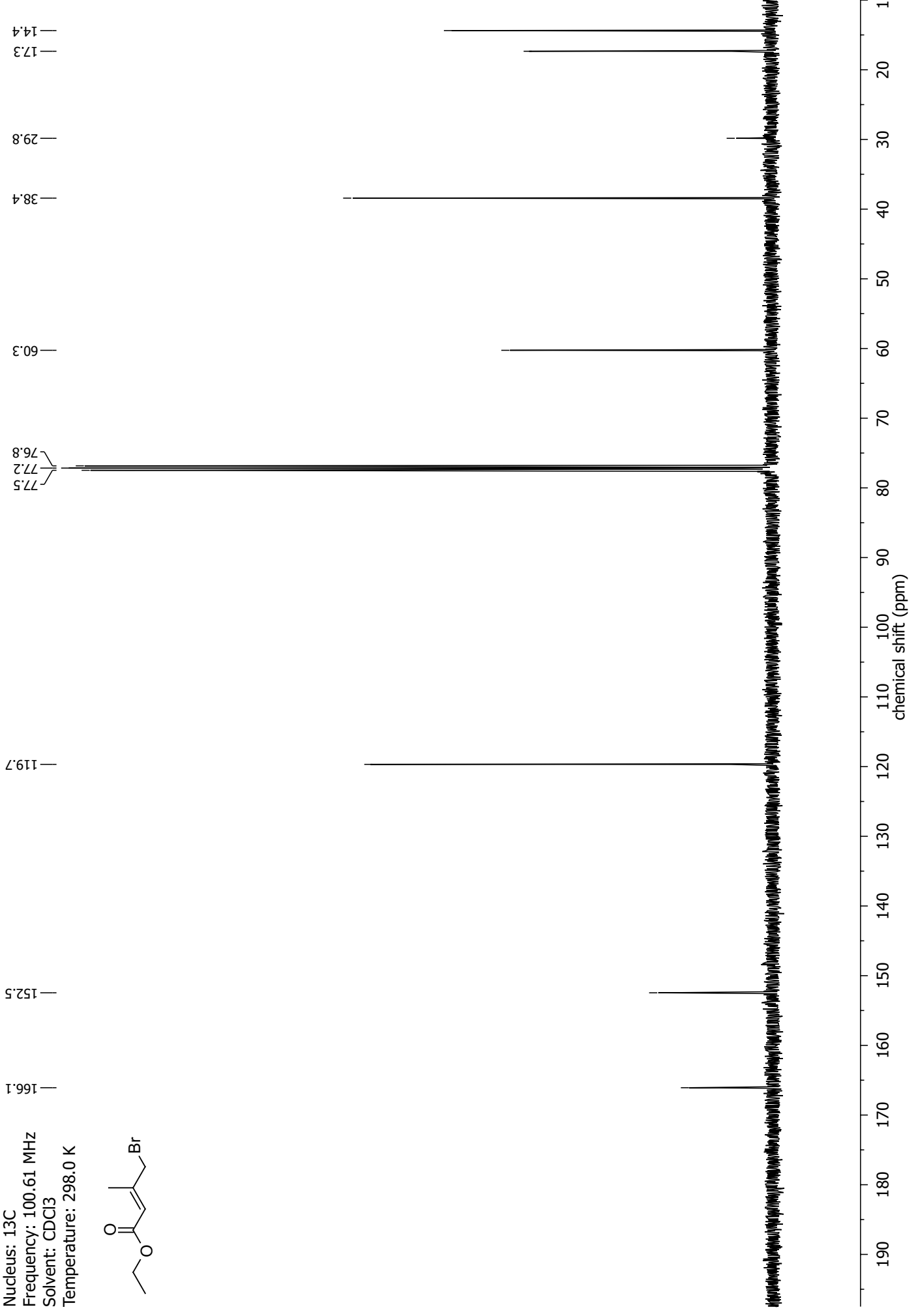


NMR-Spectrum for Compound 19a

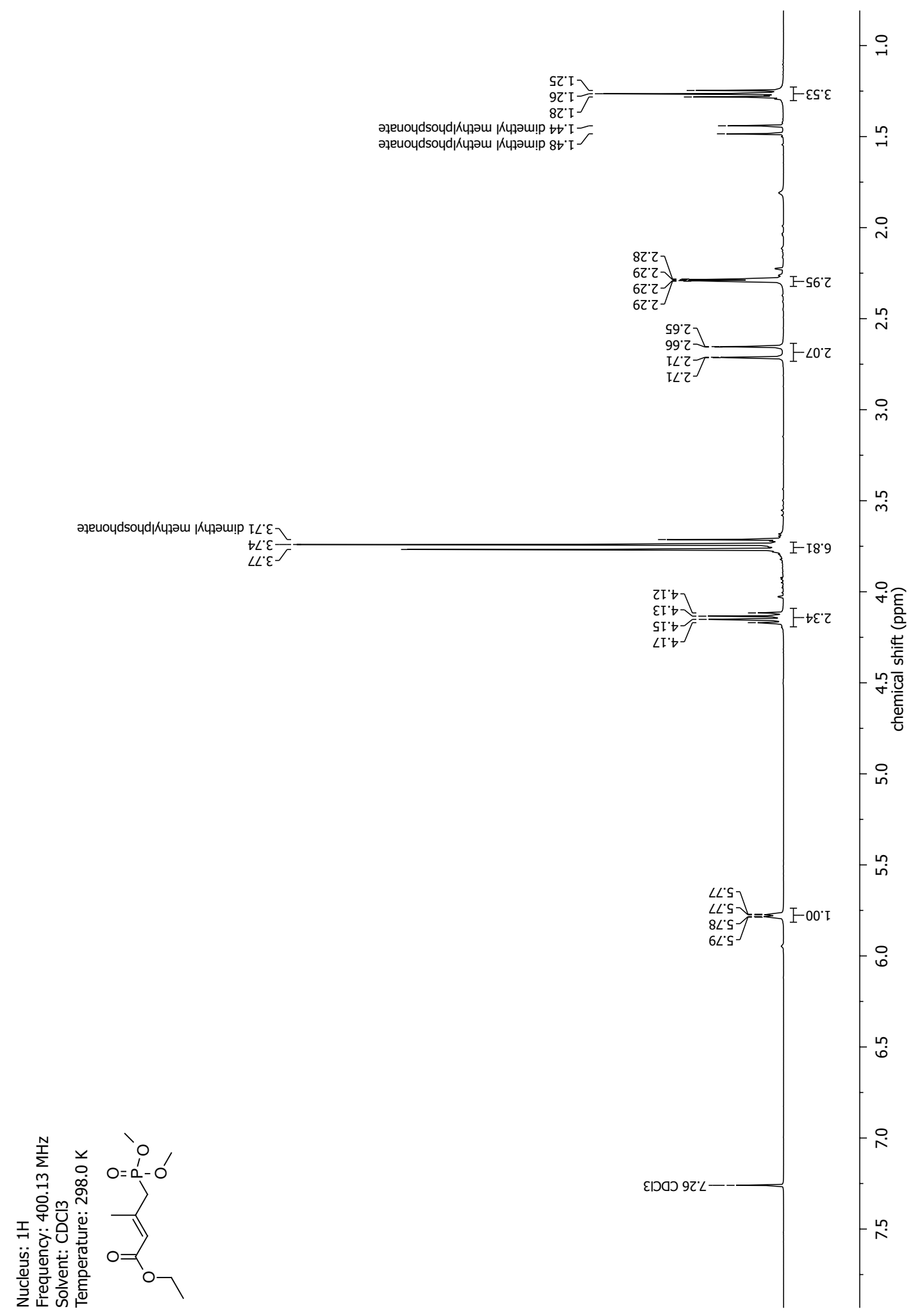


NMR-Spectra for Compound 19b

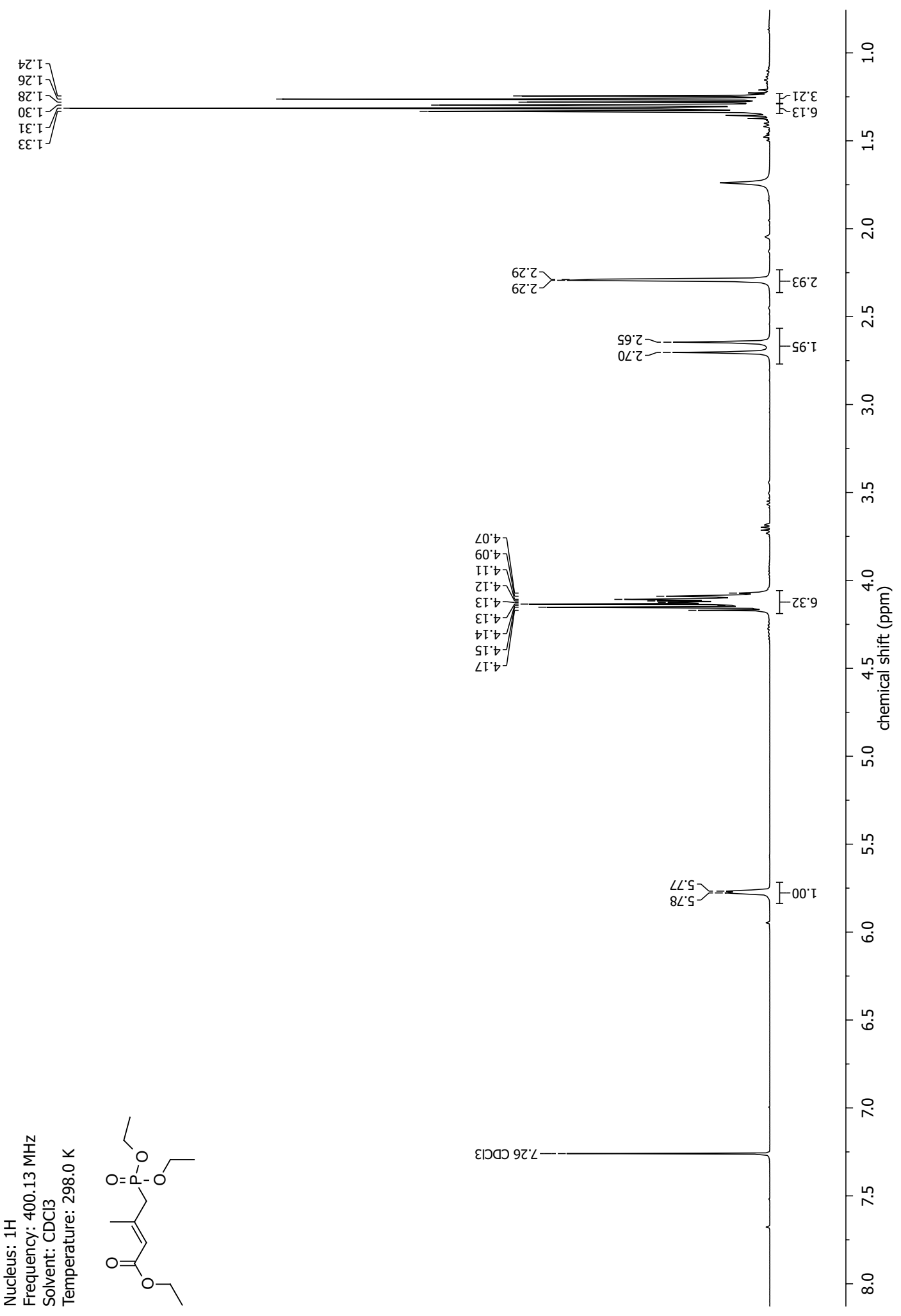




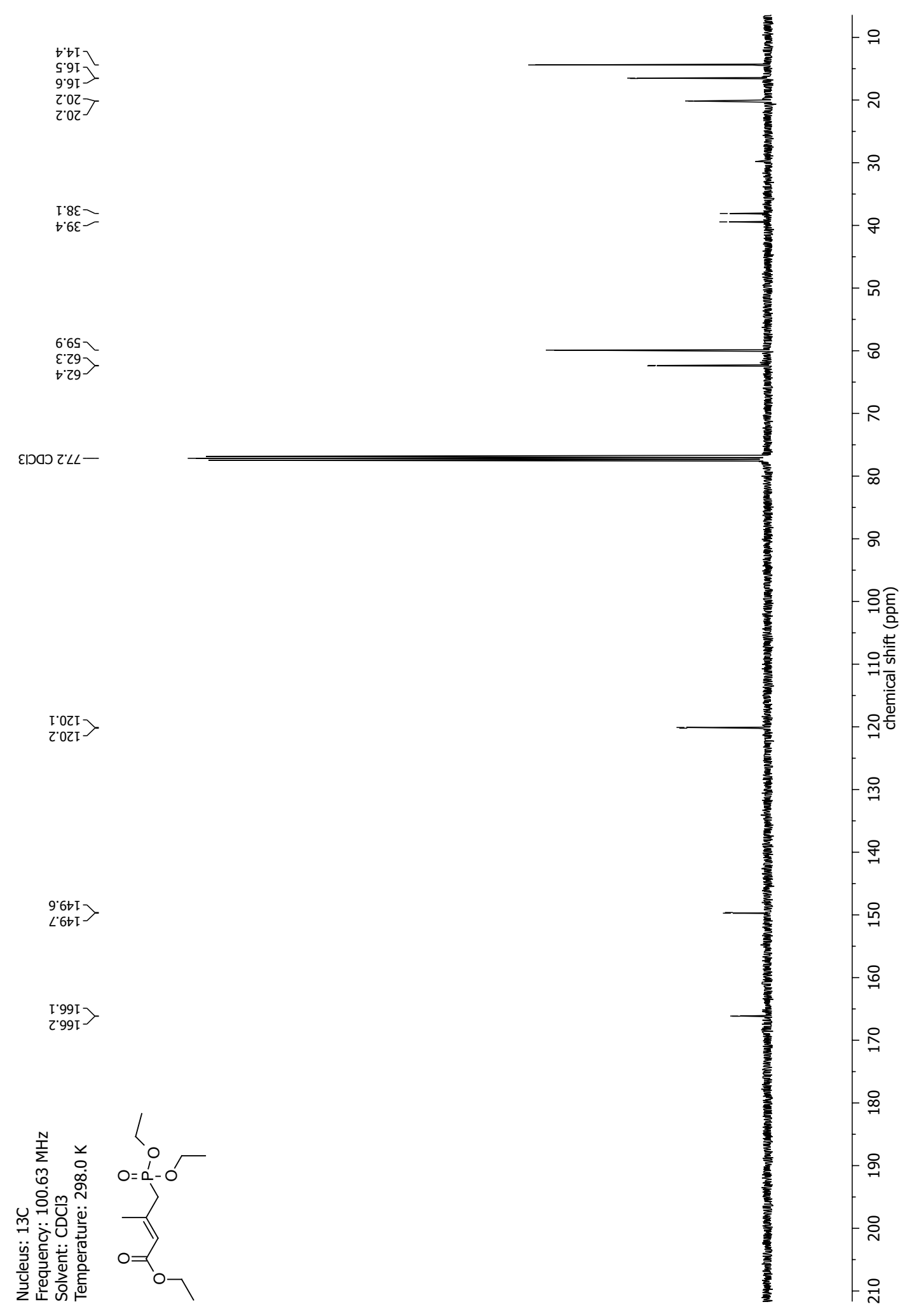


NMR-Spectra for Compound 19c

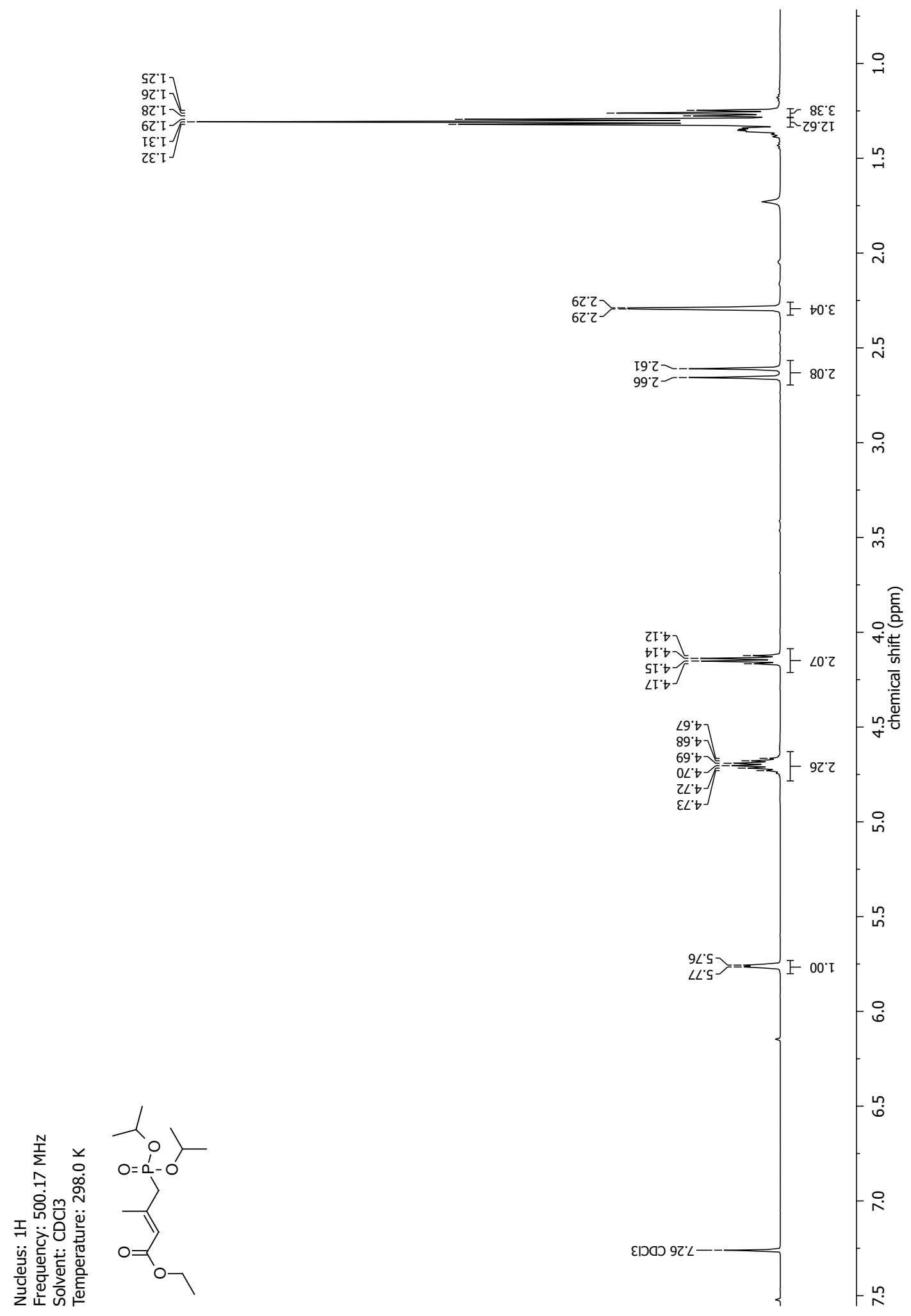




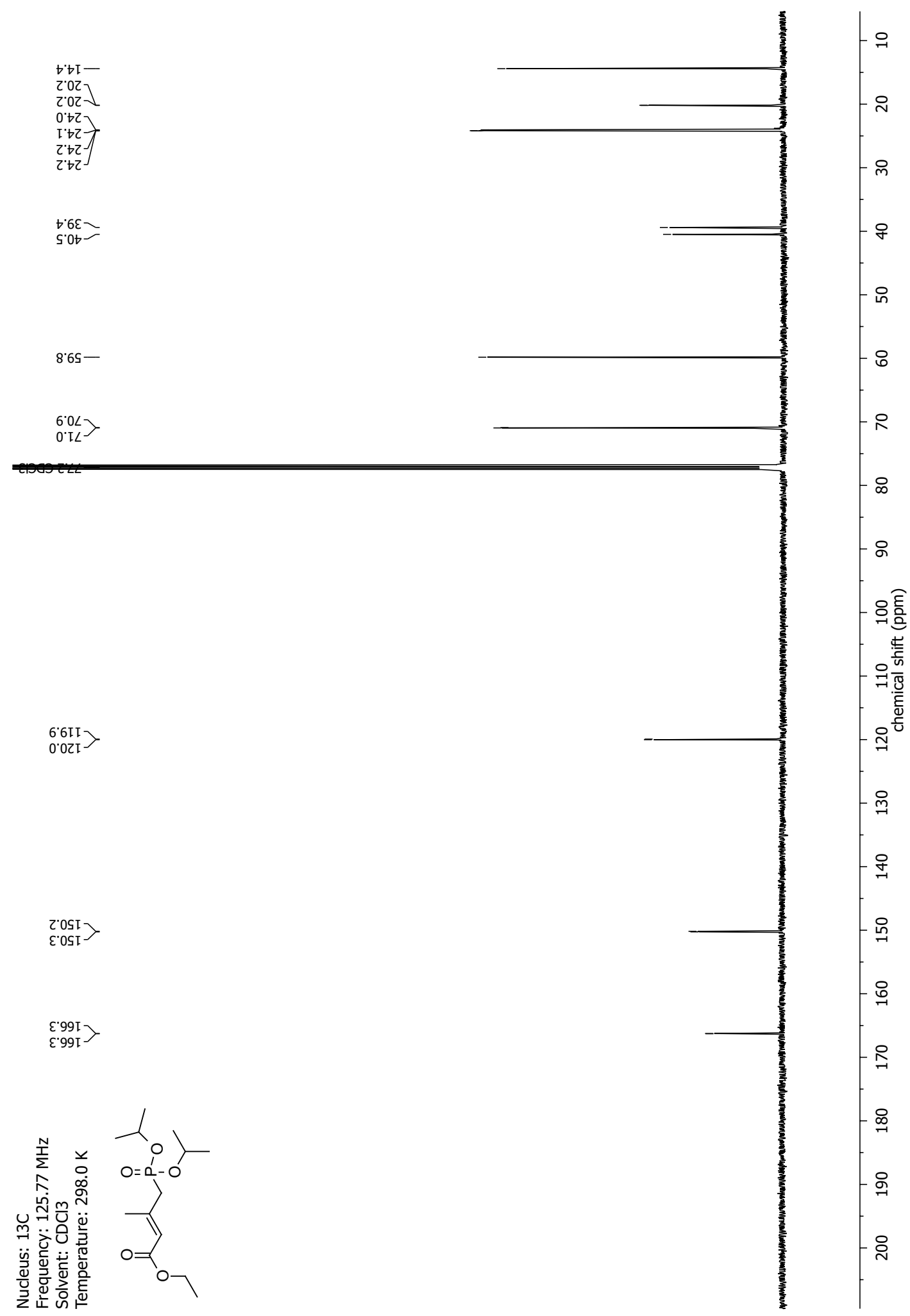


NMR-Spectra for Compound 17

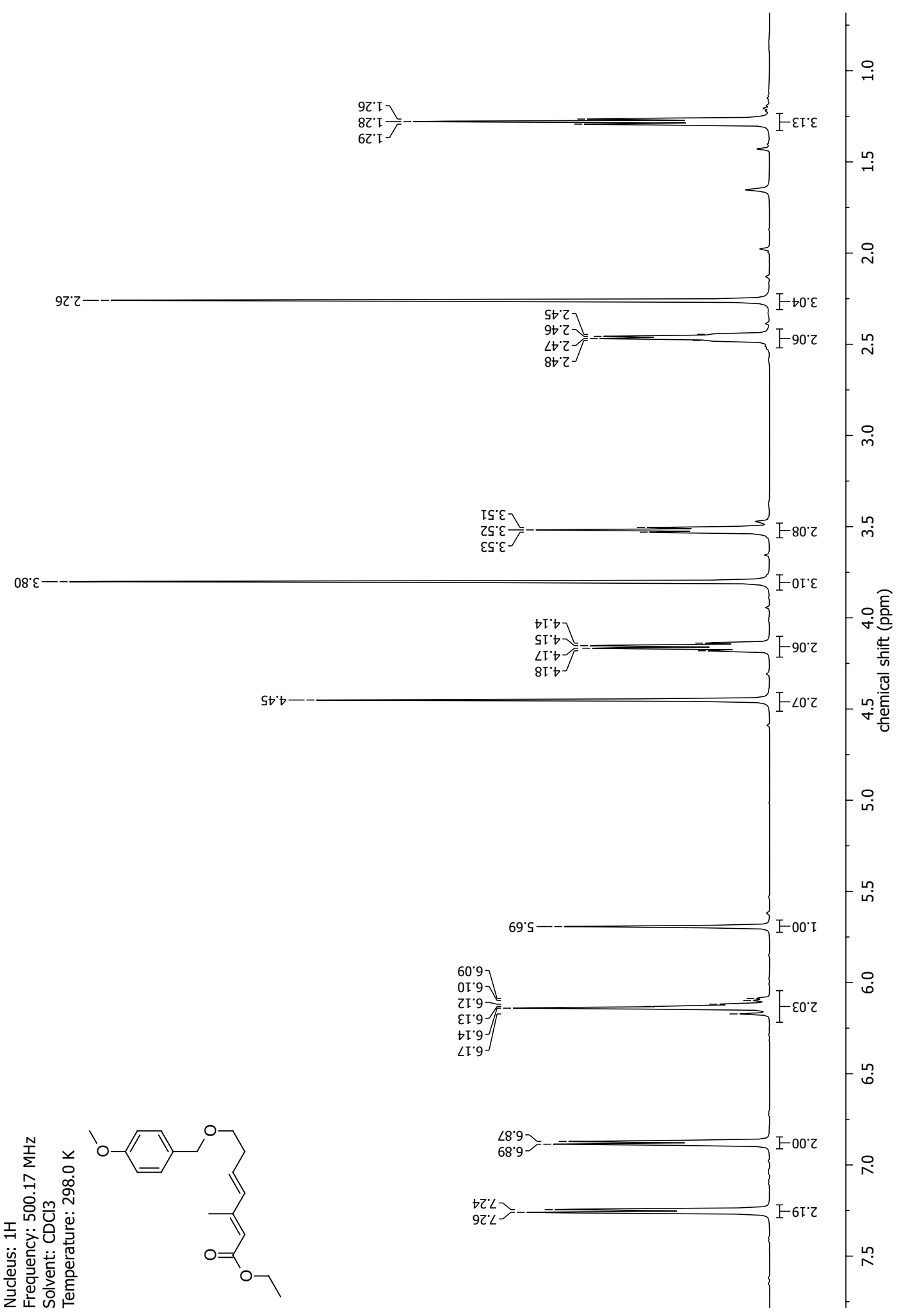



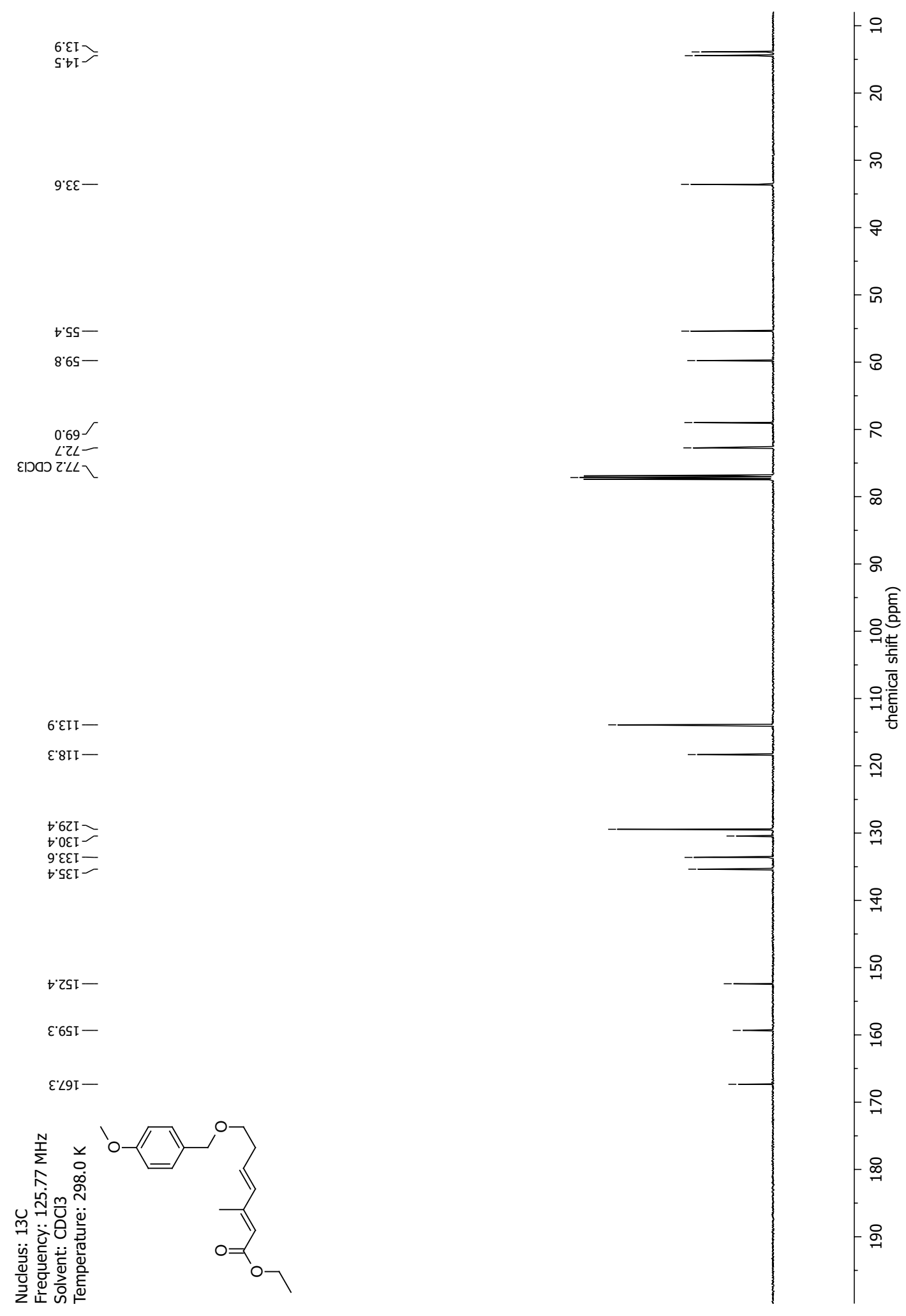
NMR-Spectra for Compound 95

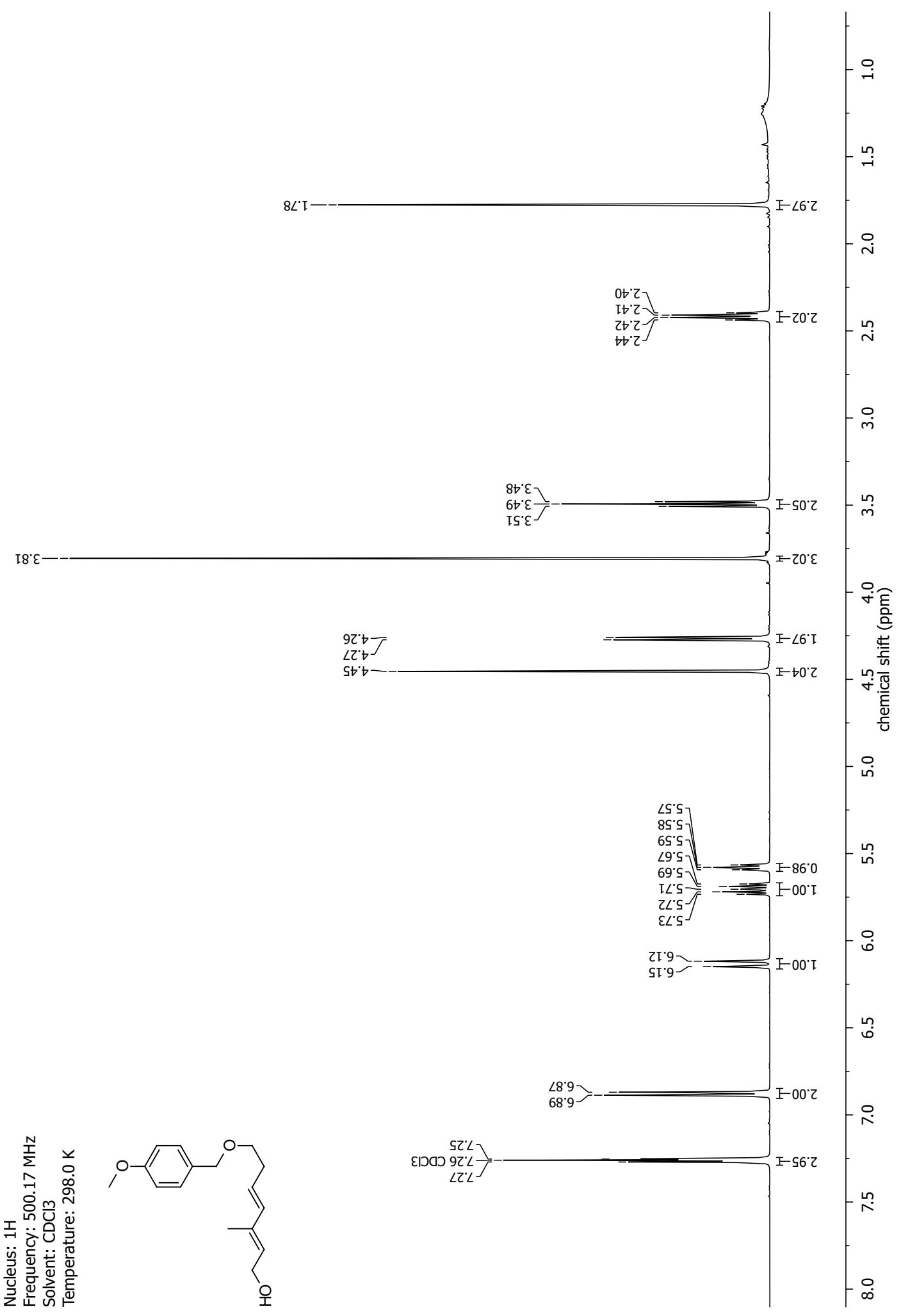




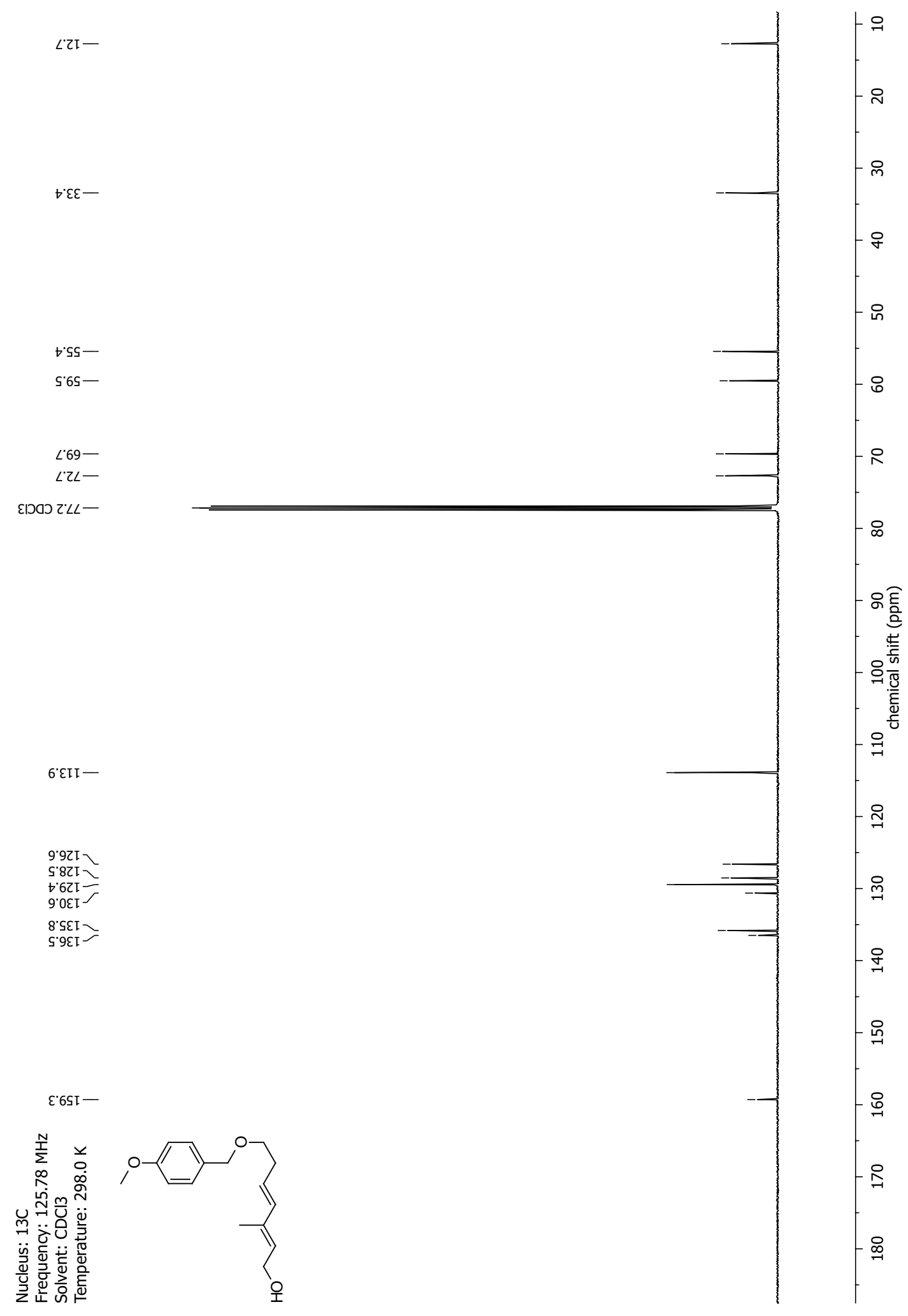




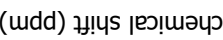

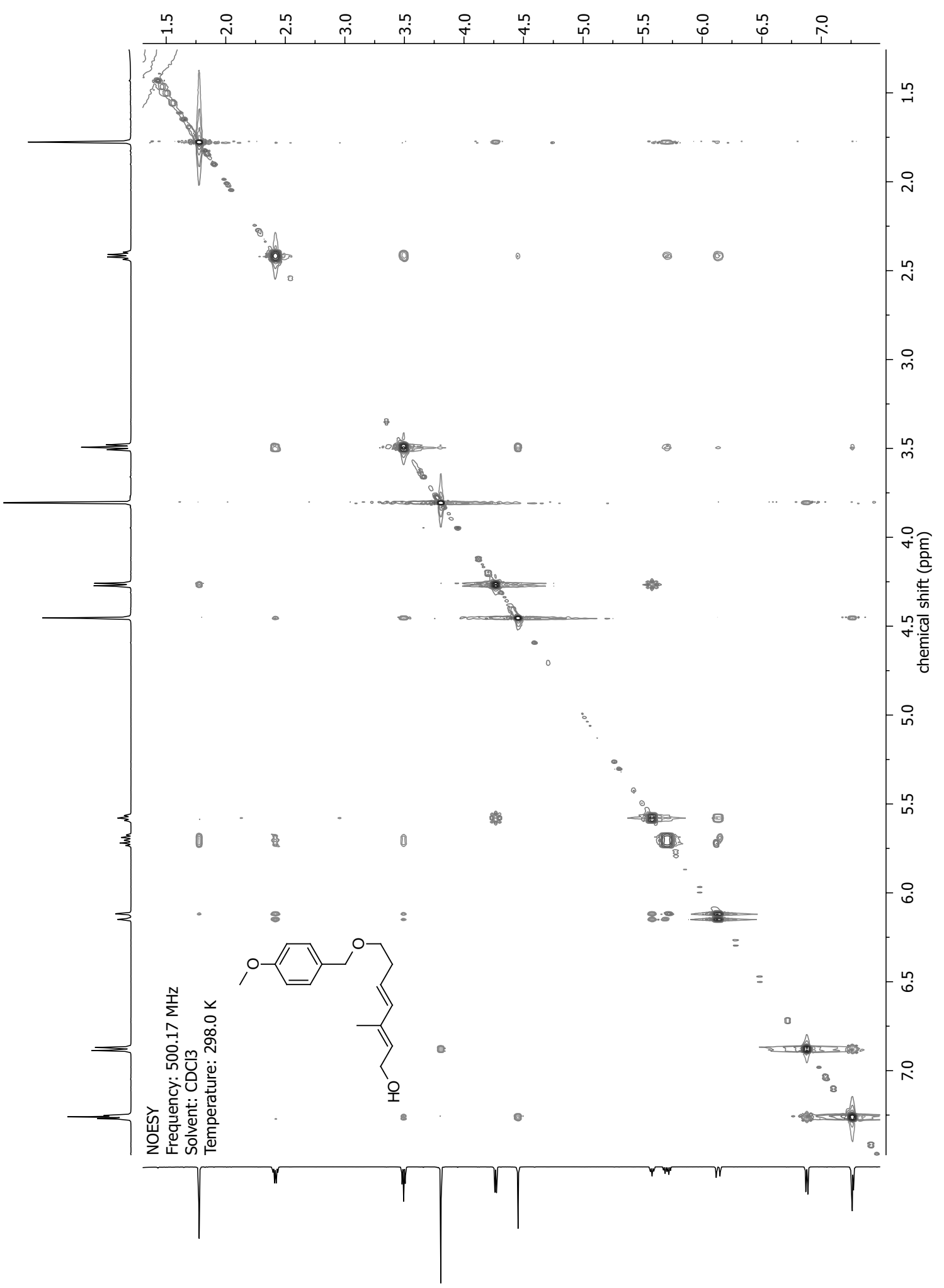


NMR-Spectra for Compound 23

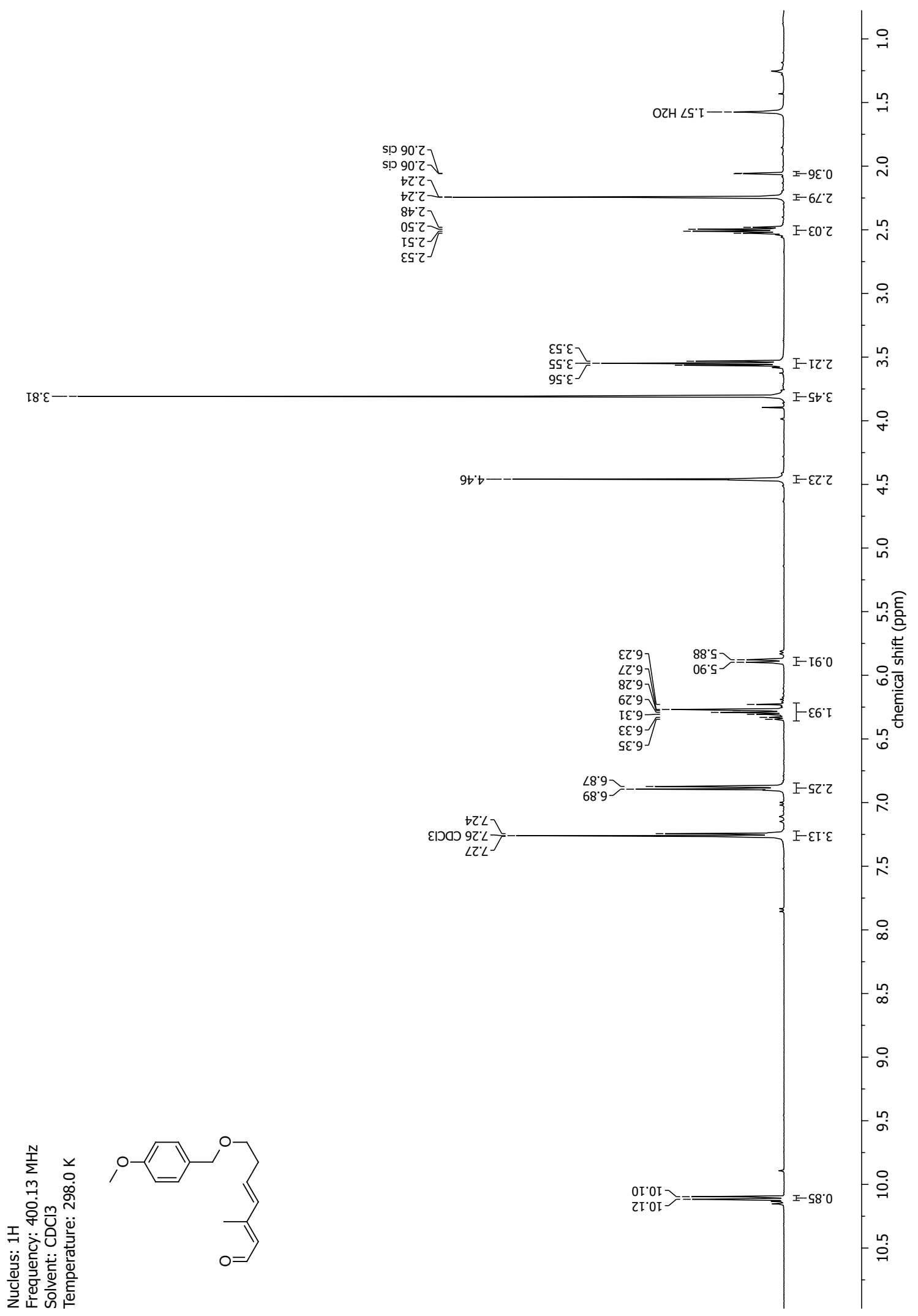




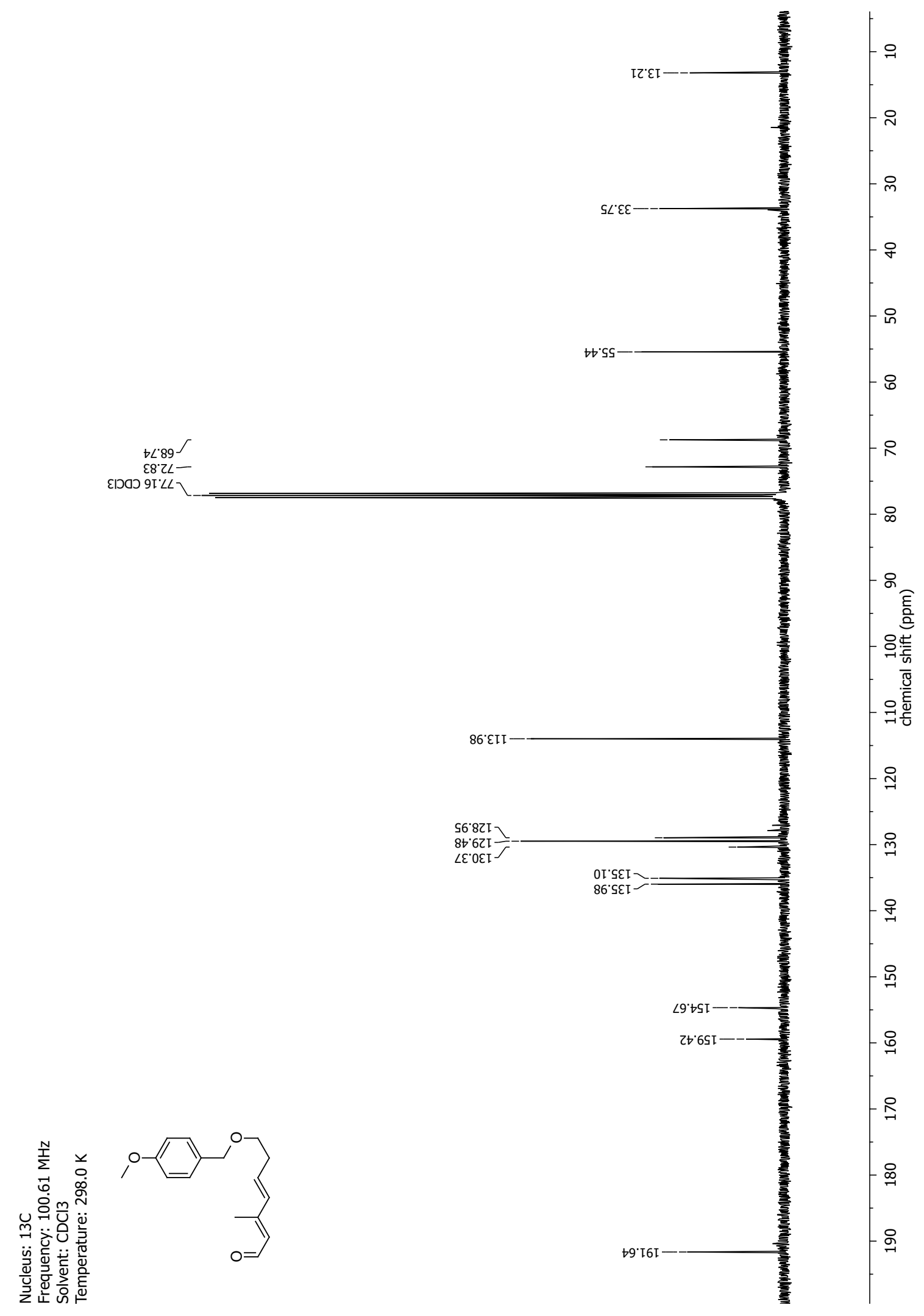


NMR-Spectra for Compound 25a

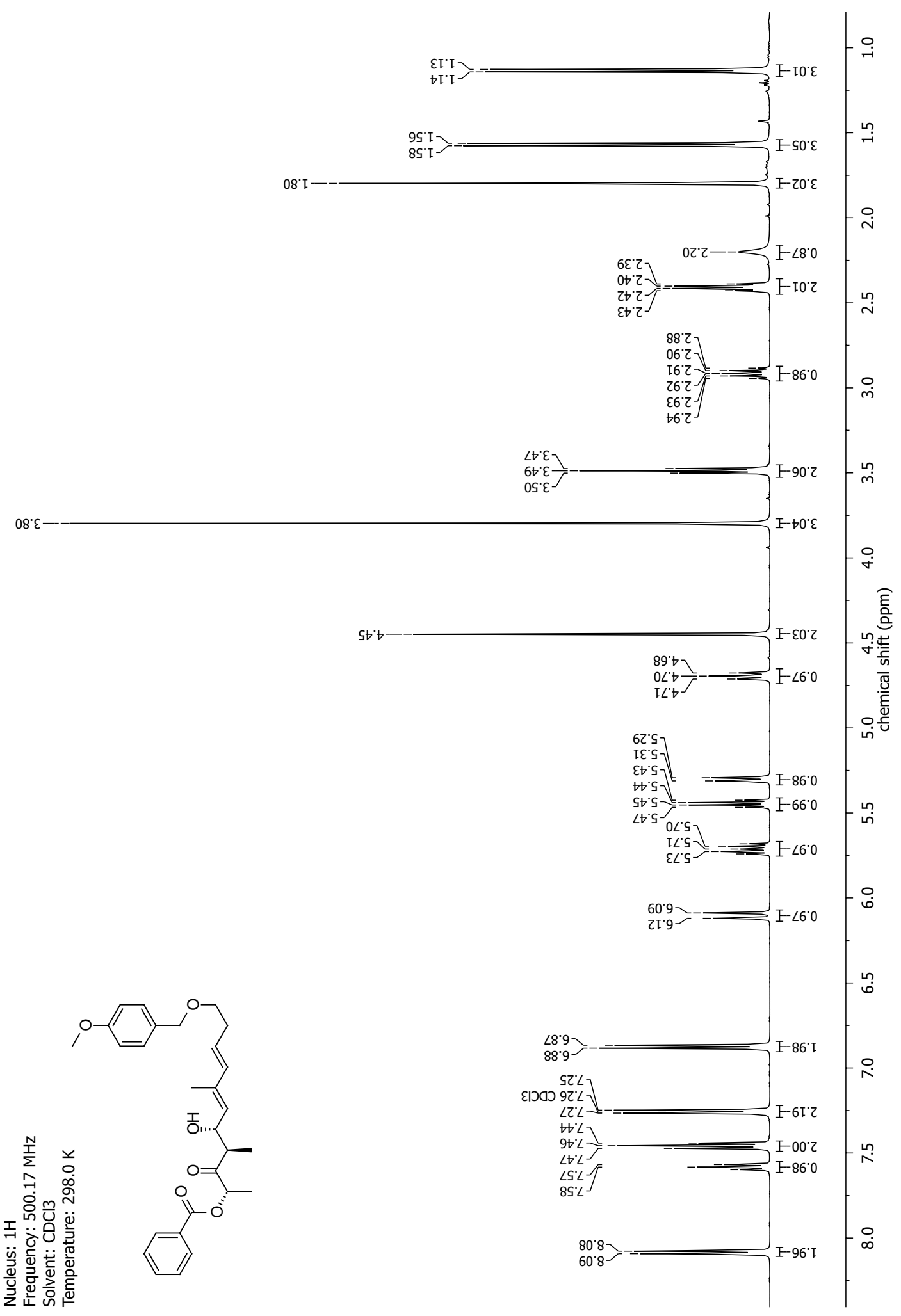




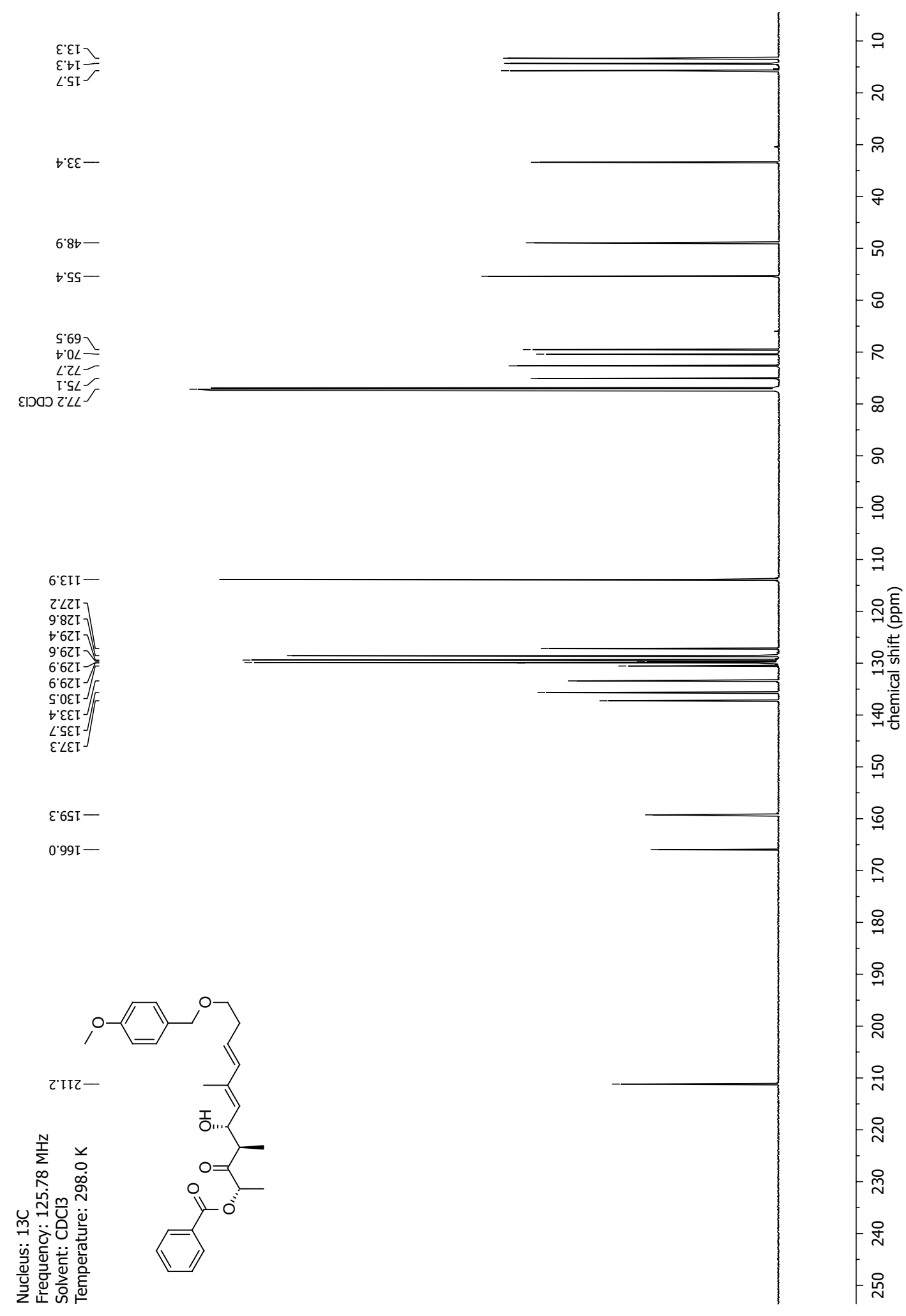


NMR-Spectra for Compound 28a

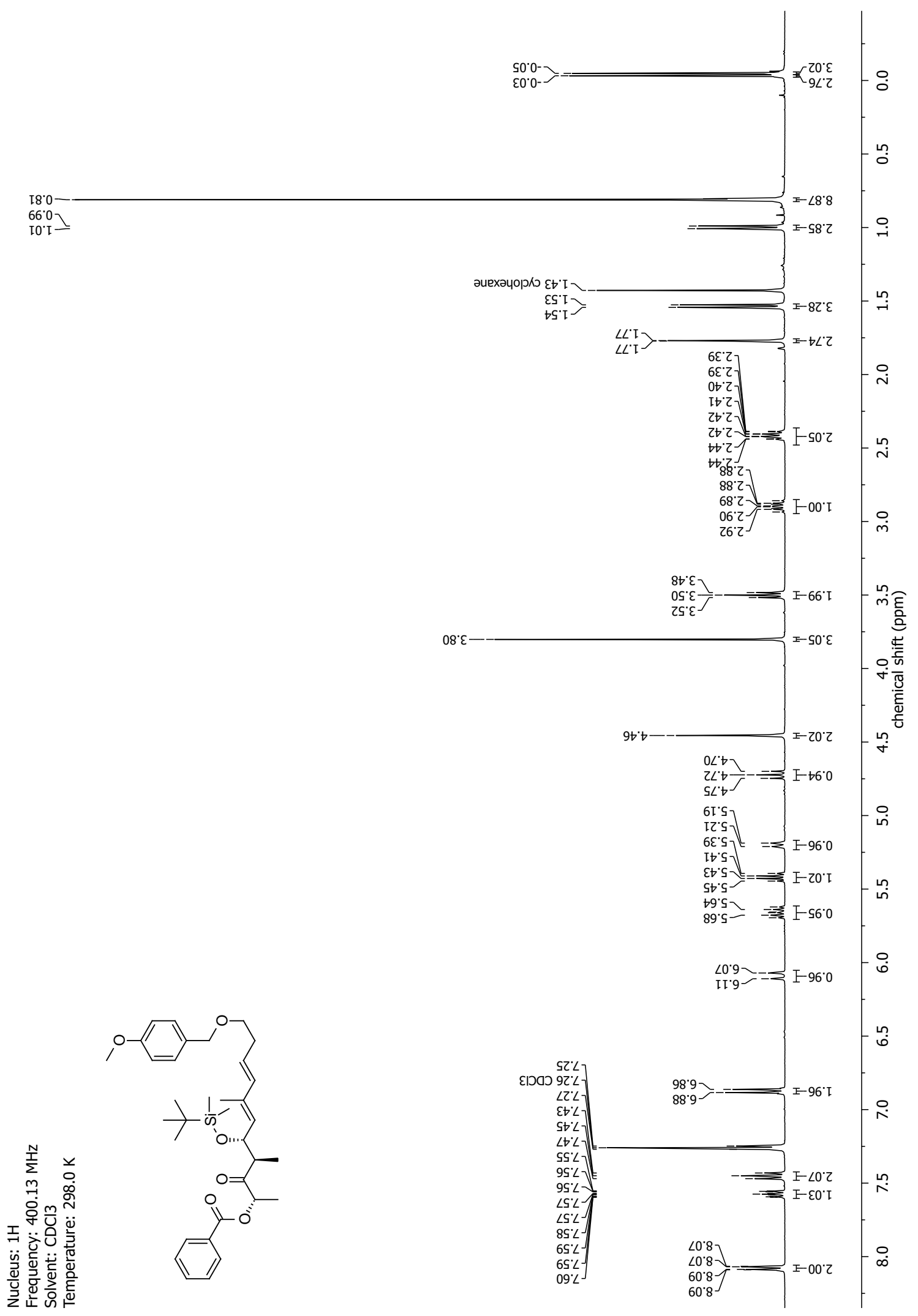




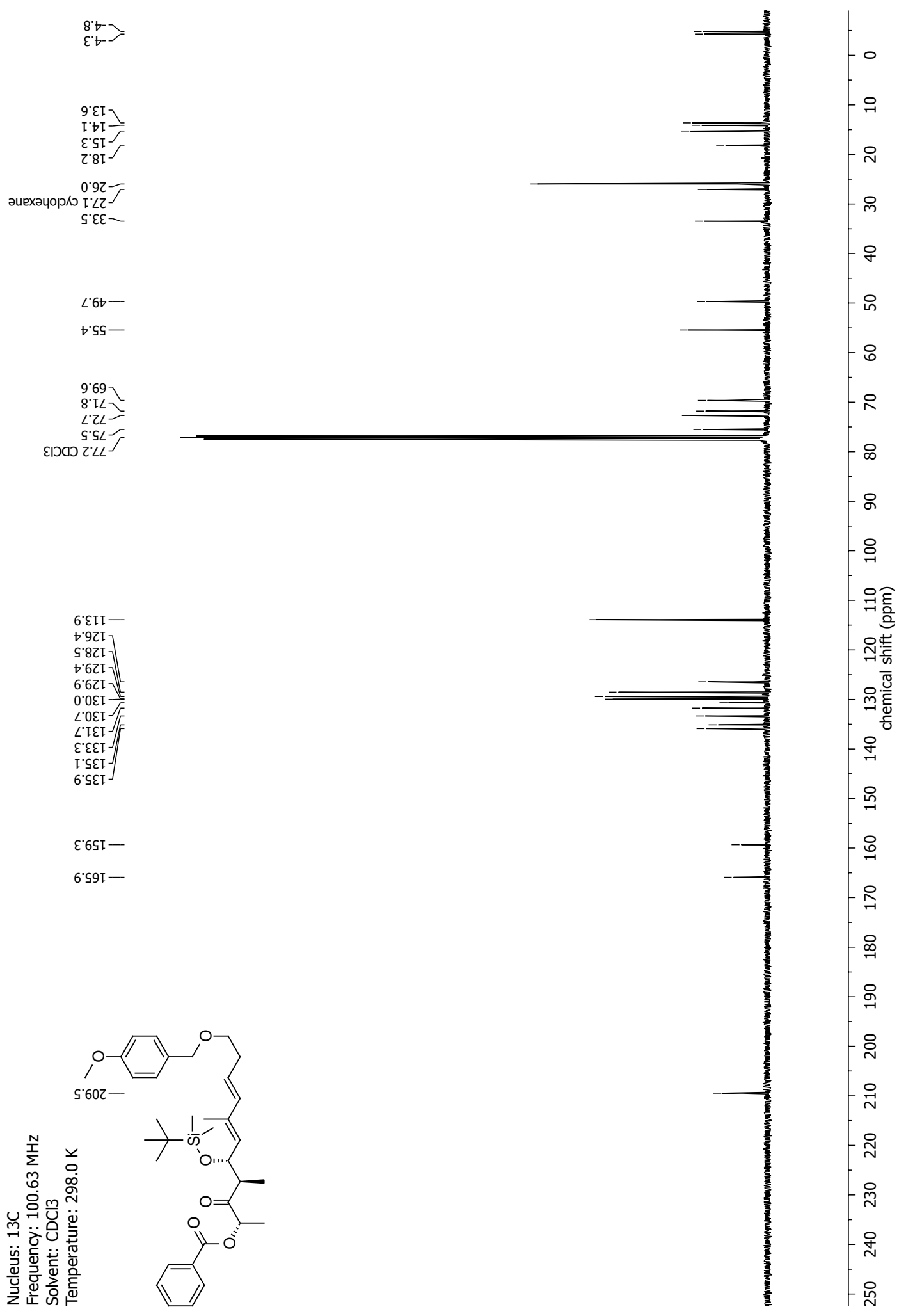


NMR-Spectra for Compound 96

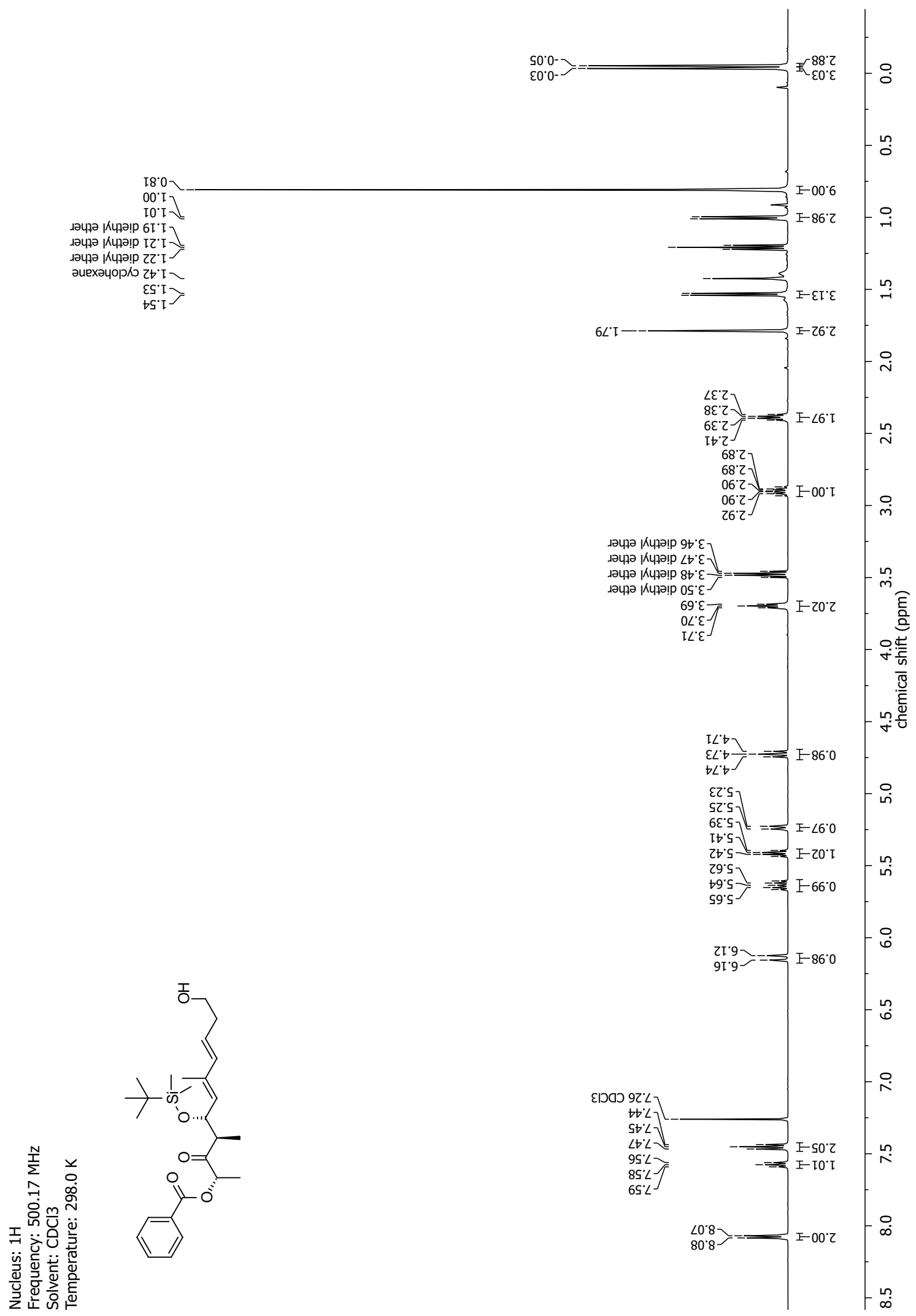




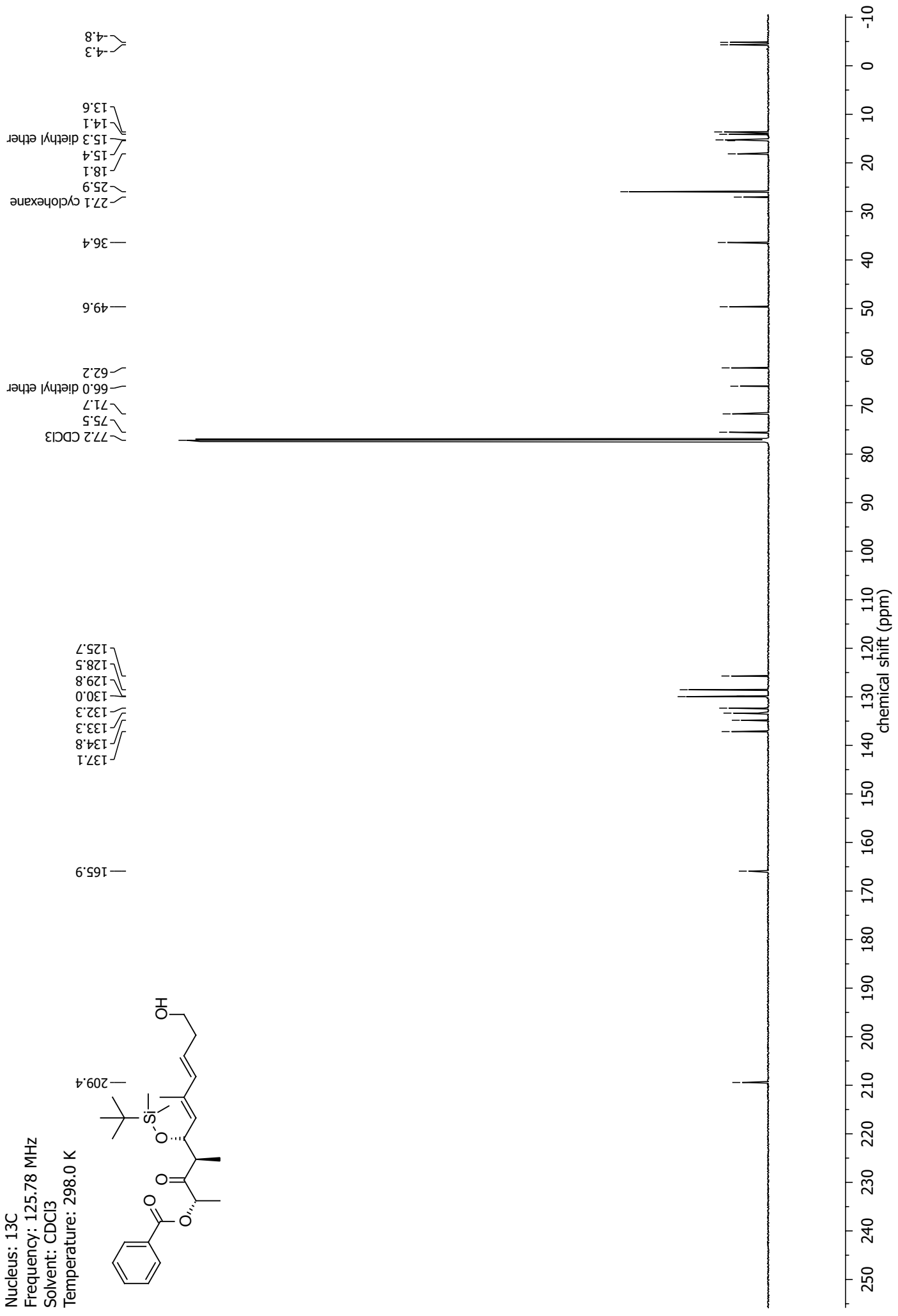


NMR-Spectra for Compound 28c

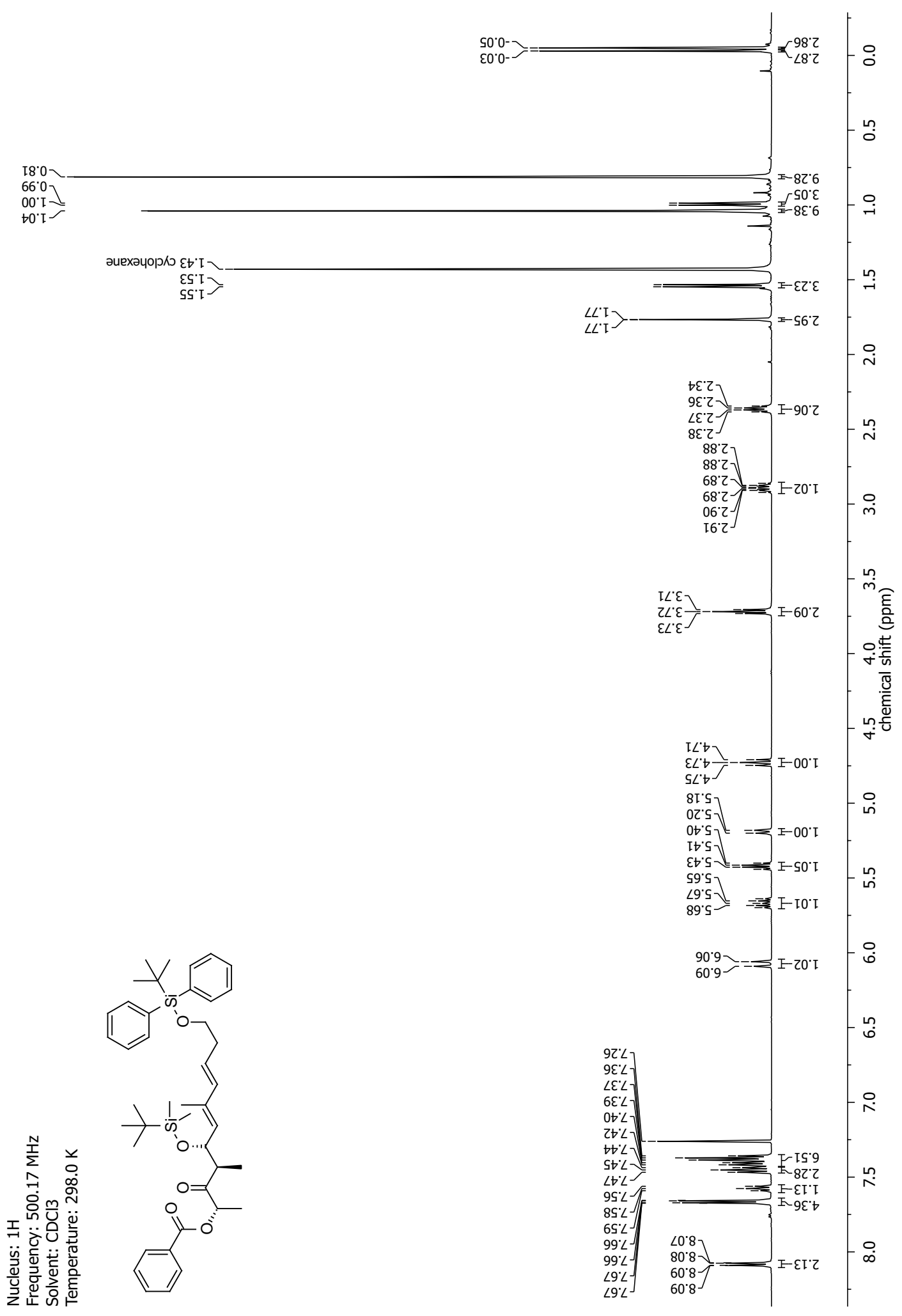




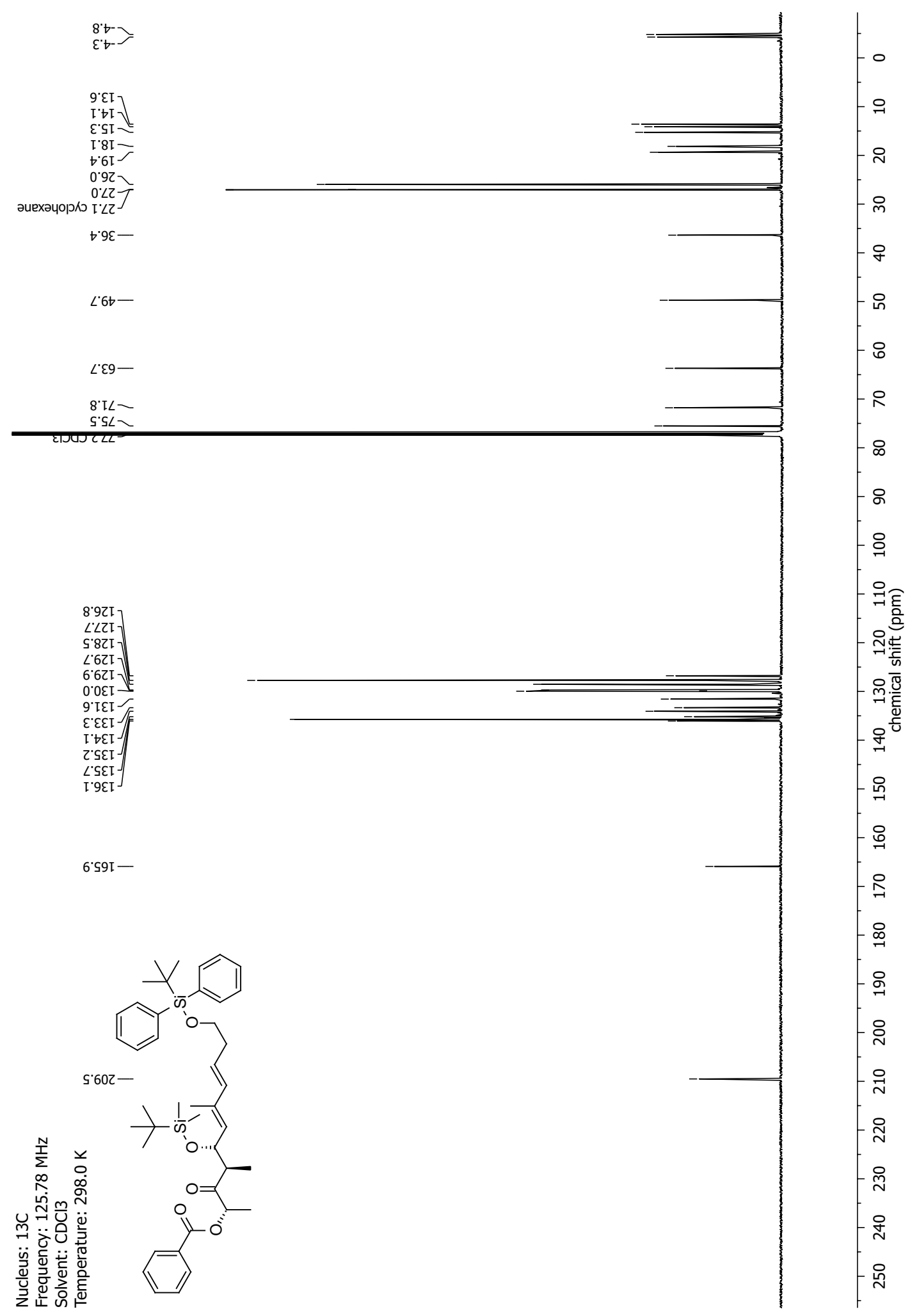


NMR-Spectra for Compound 97

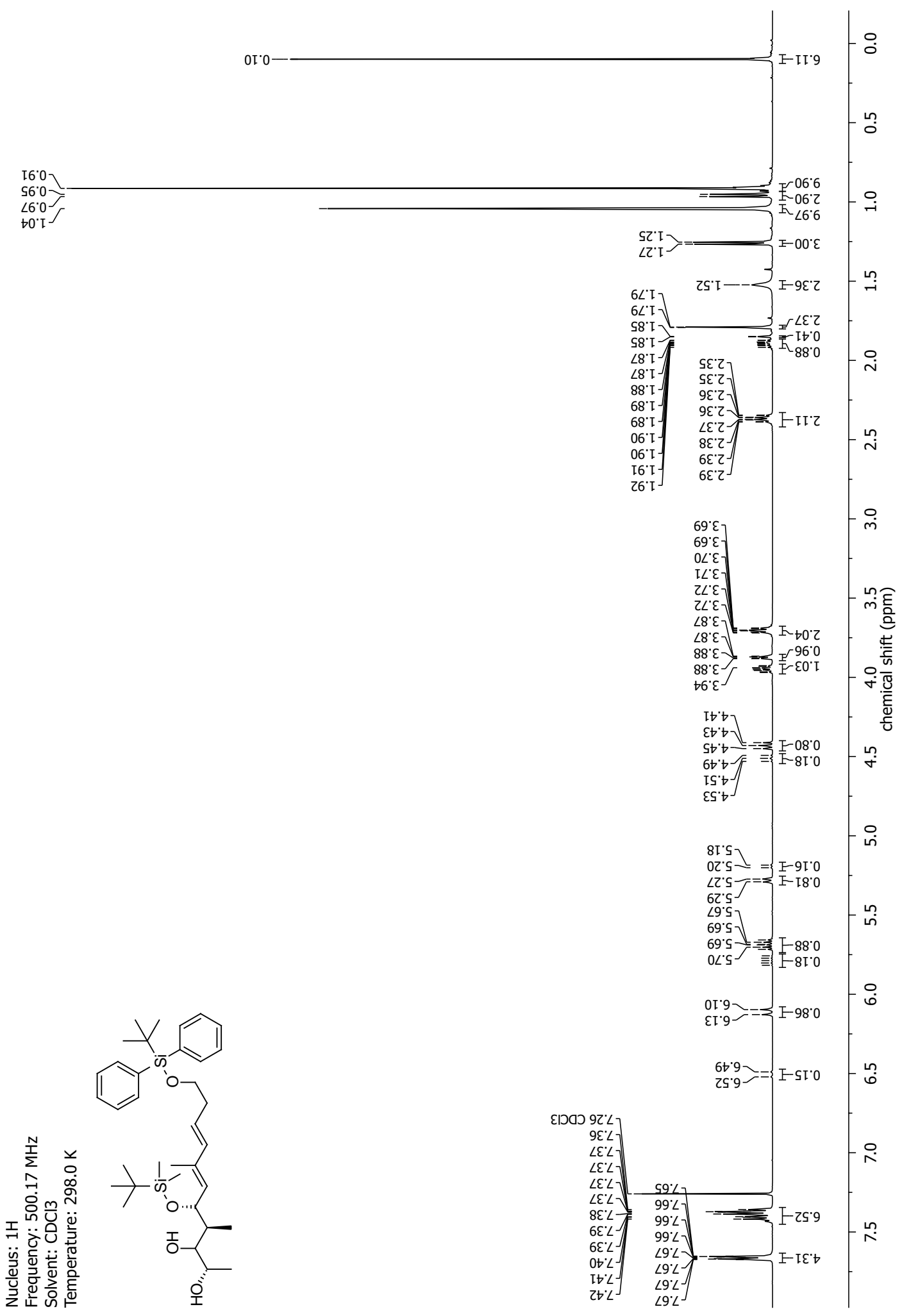




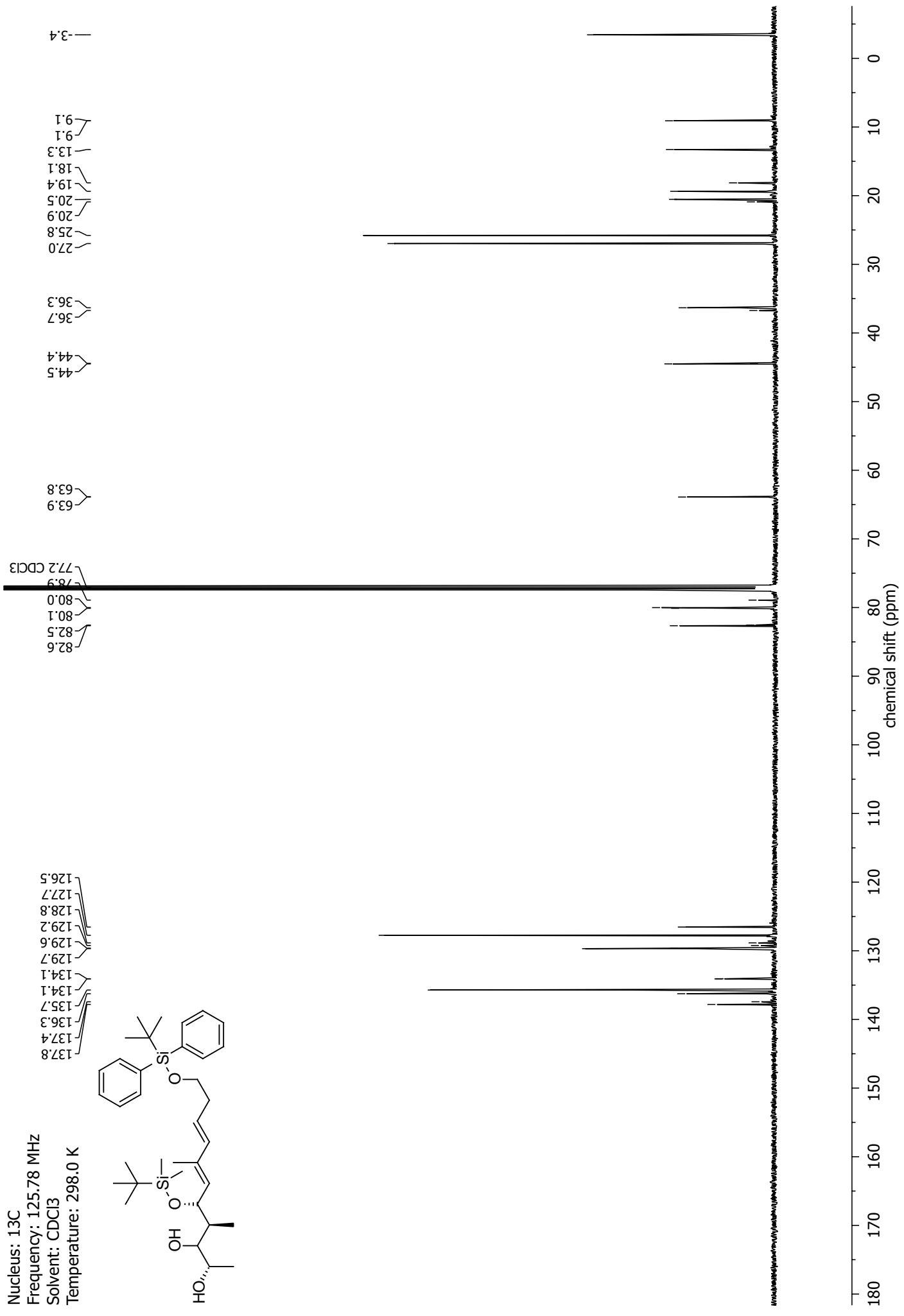


NMR-Spectra for Compound 29a

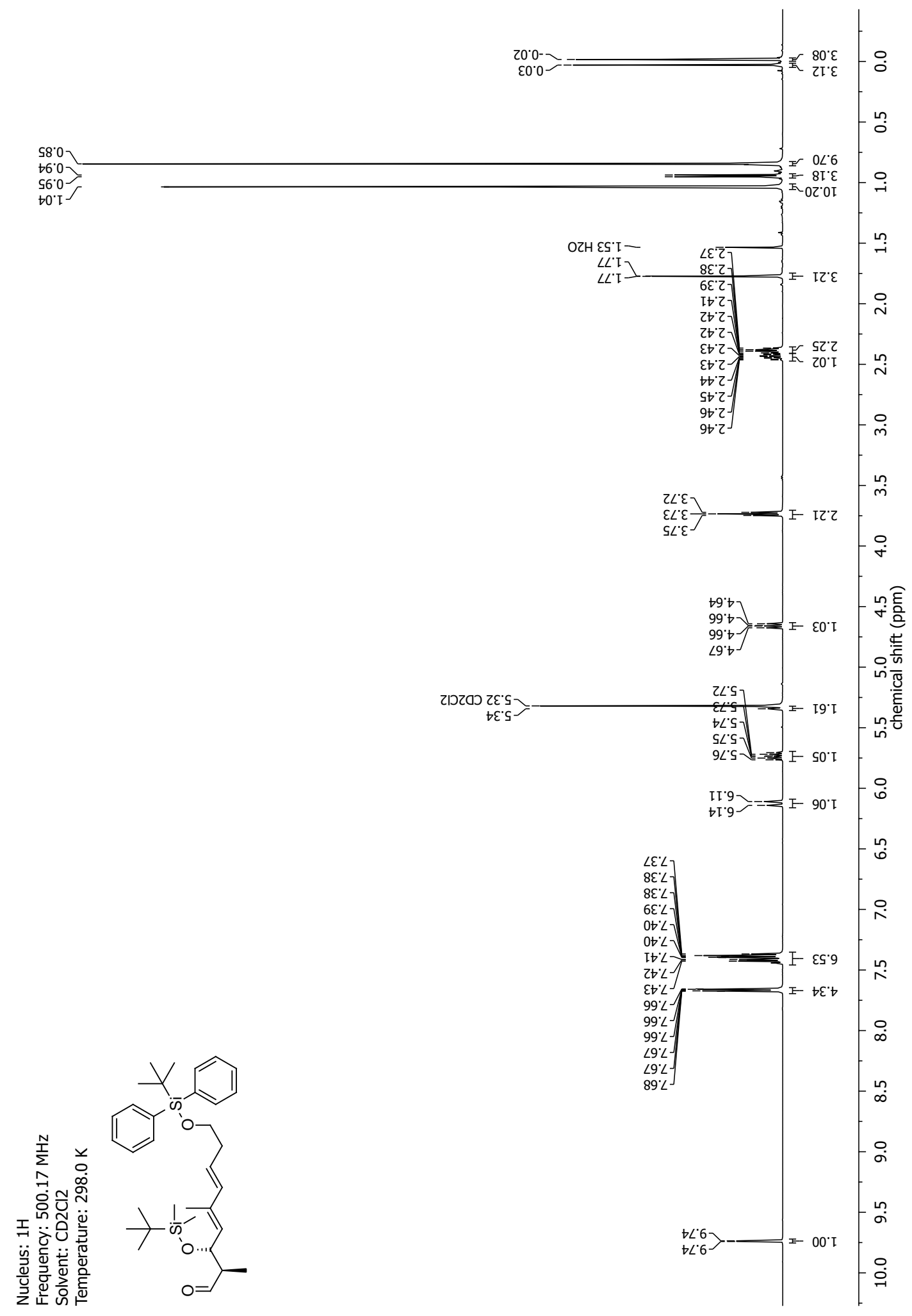




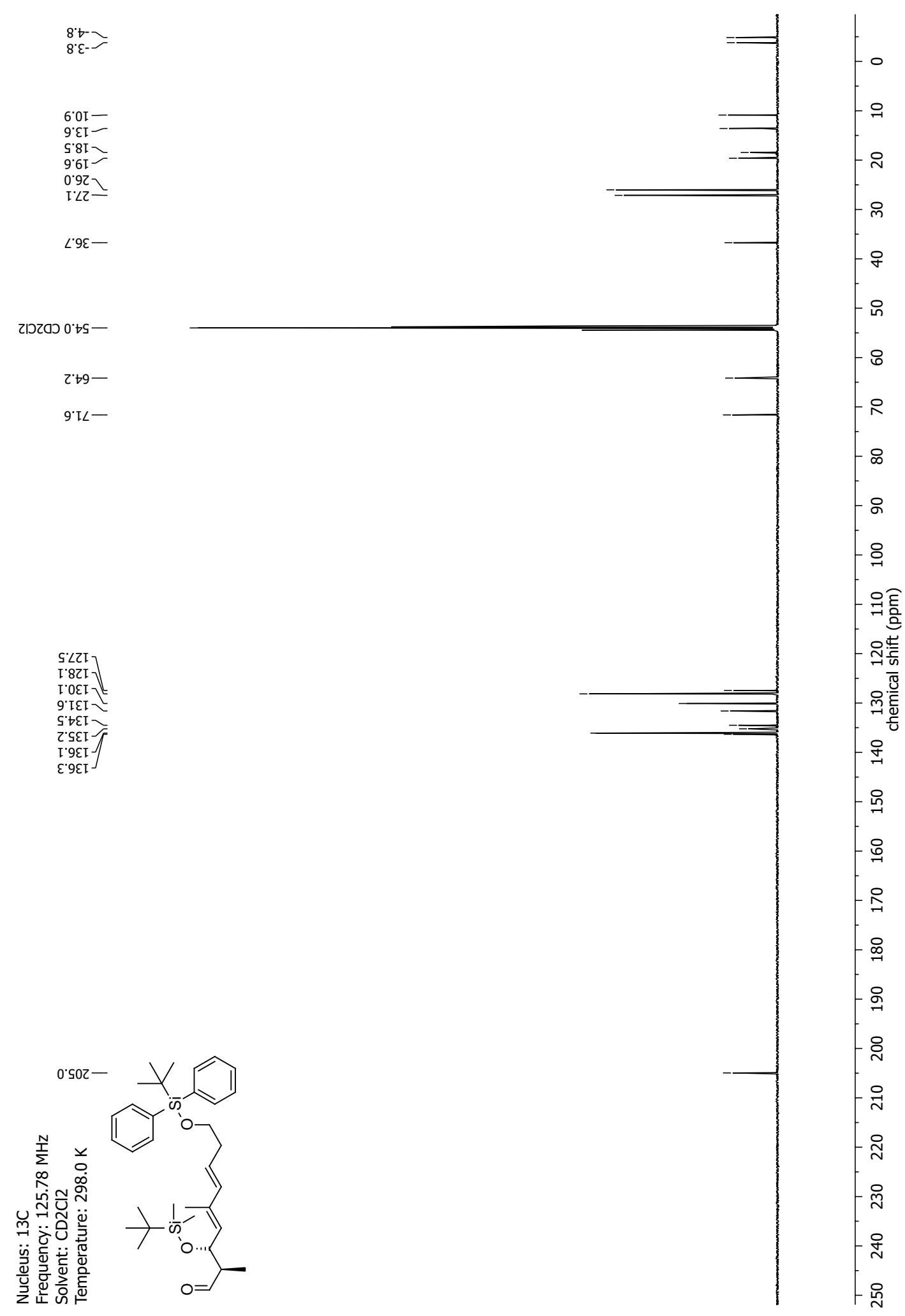


NMR-Spectra for Compound 31a

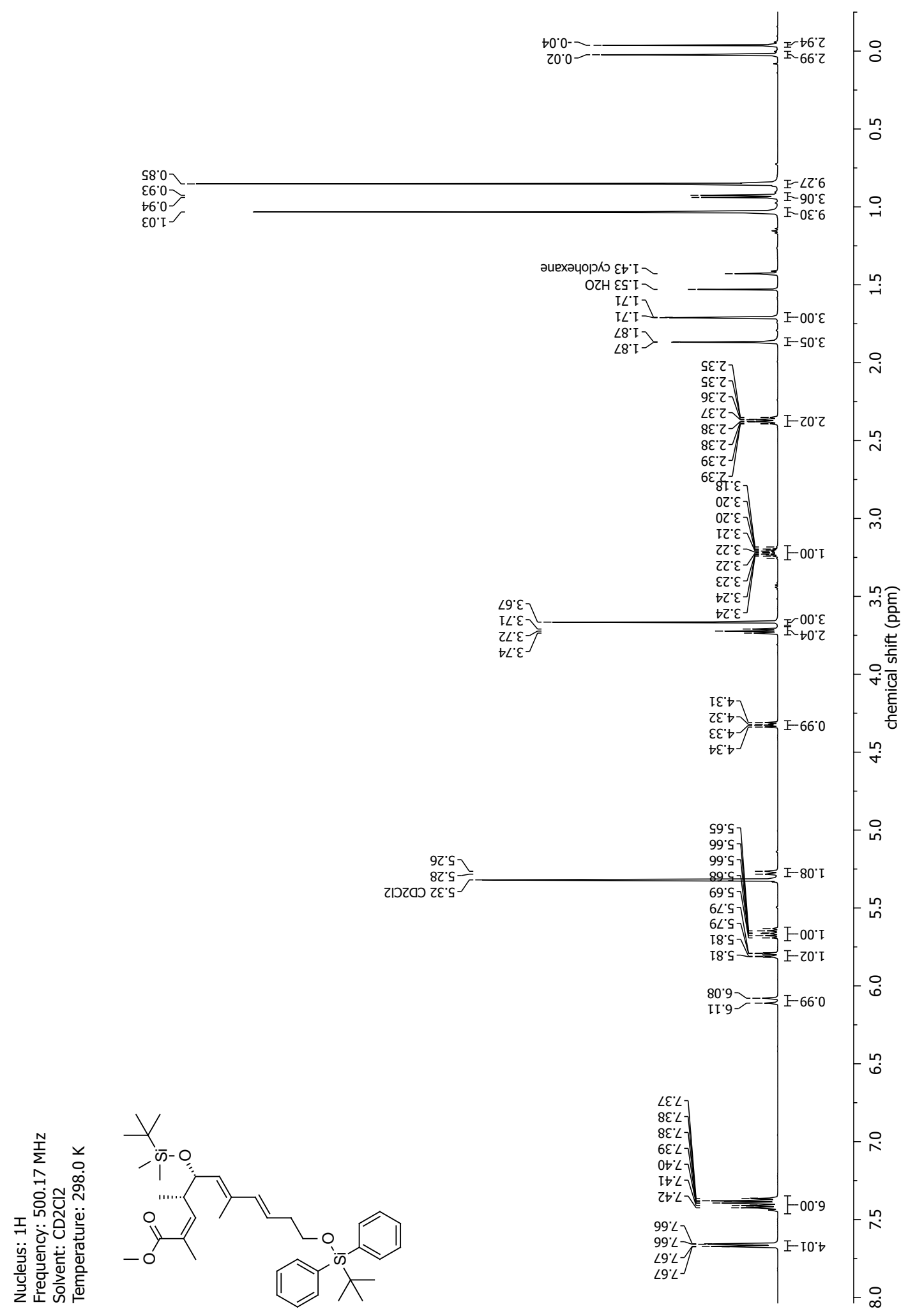




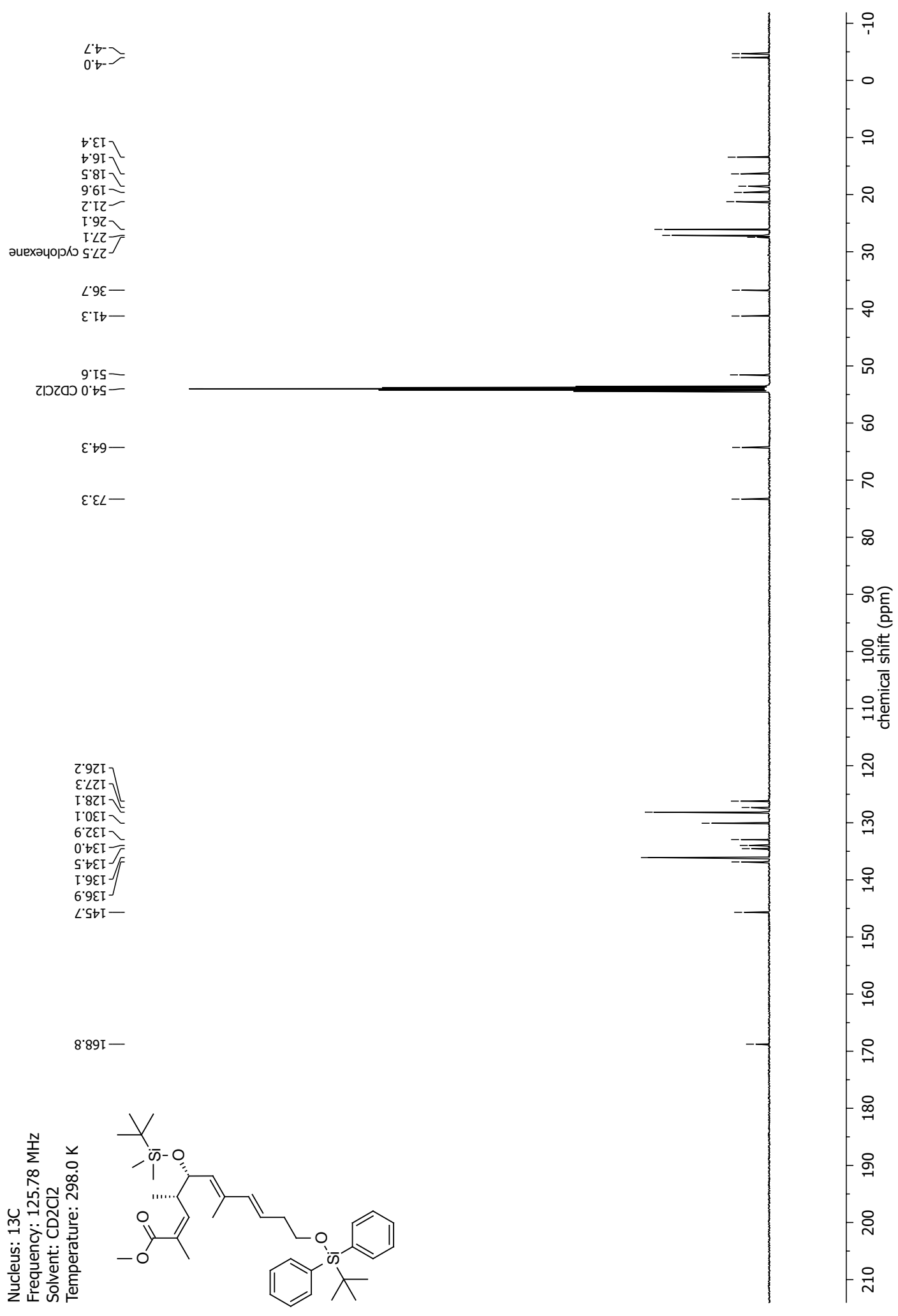


NMR-Spectra for Compound 32a

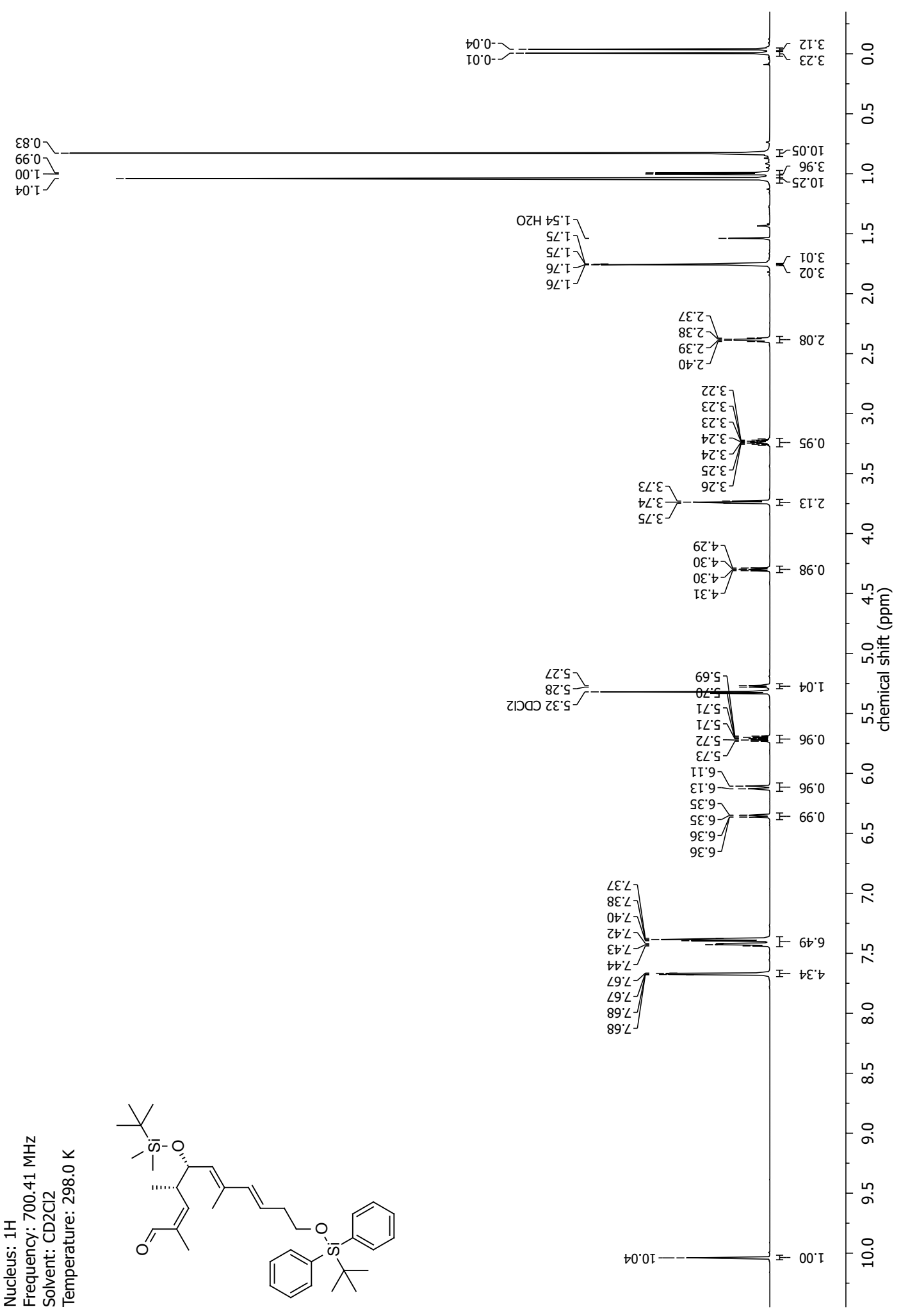




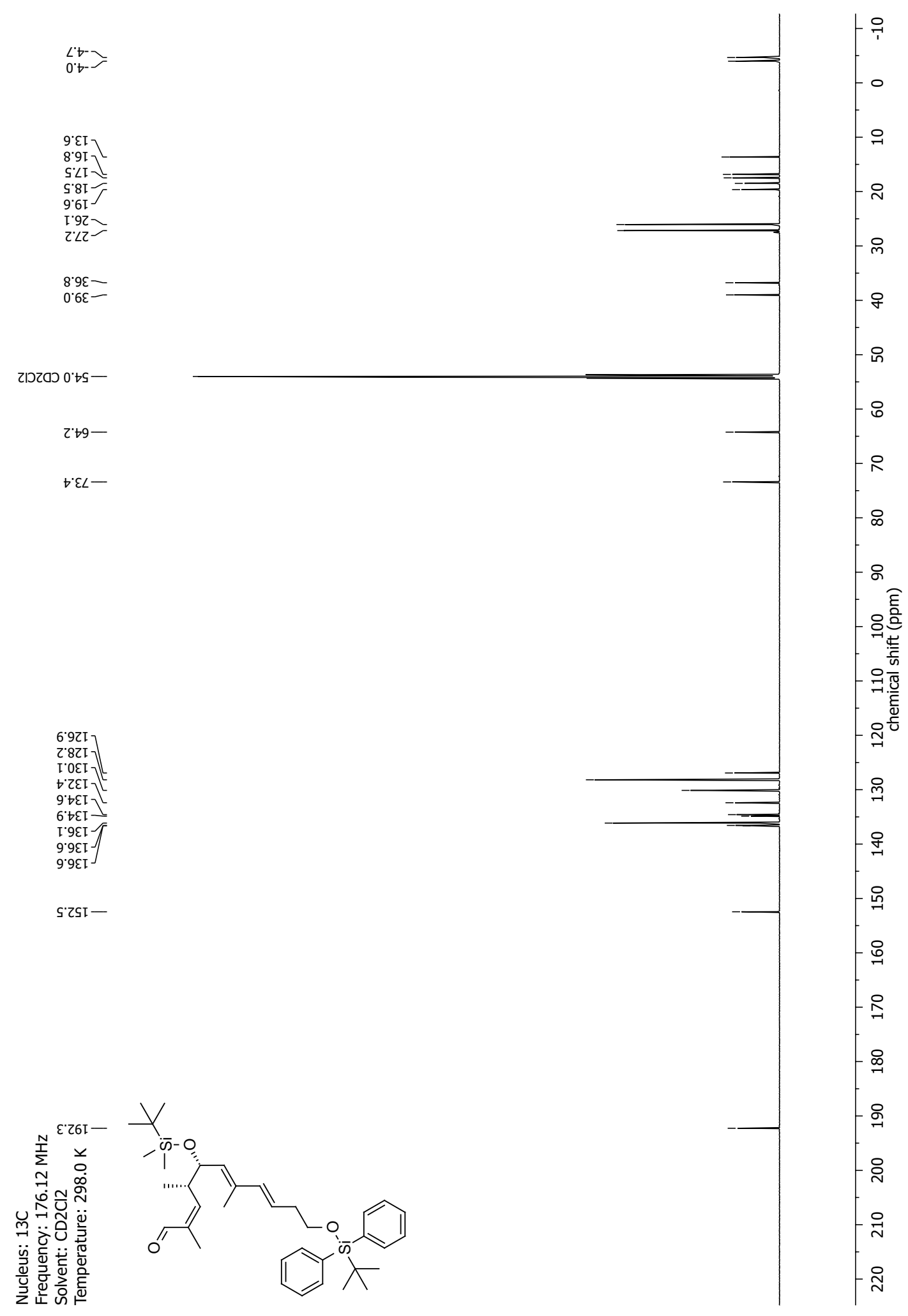


NMR-Spectra for Compound 33a

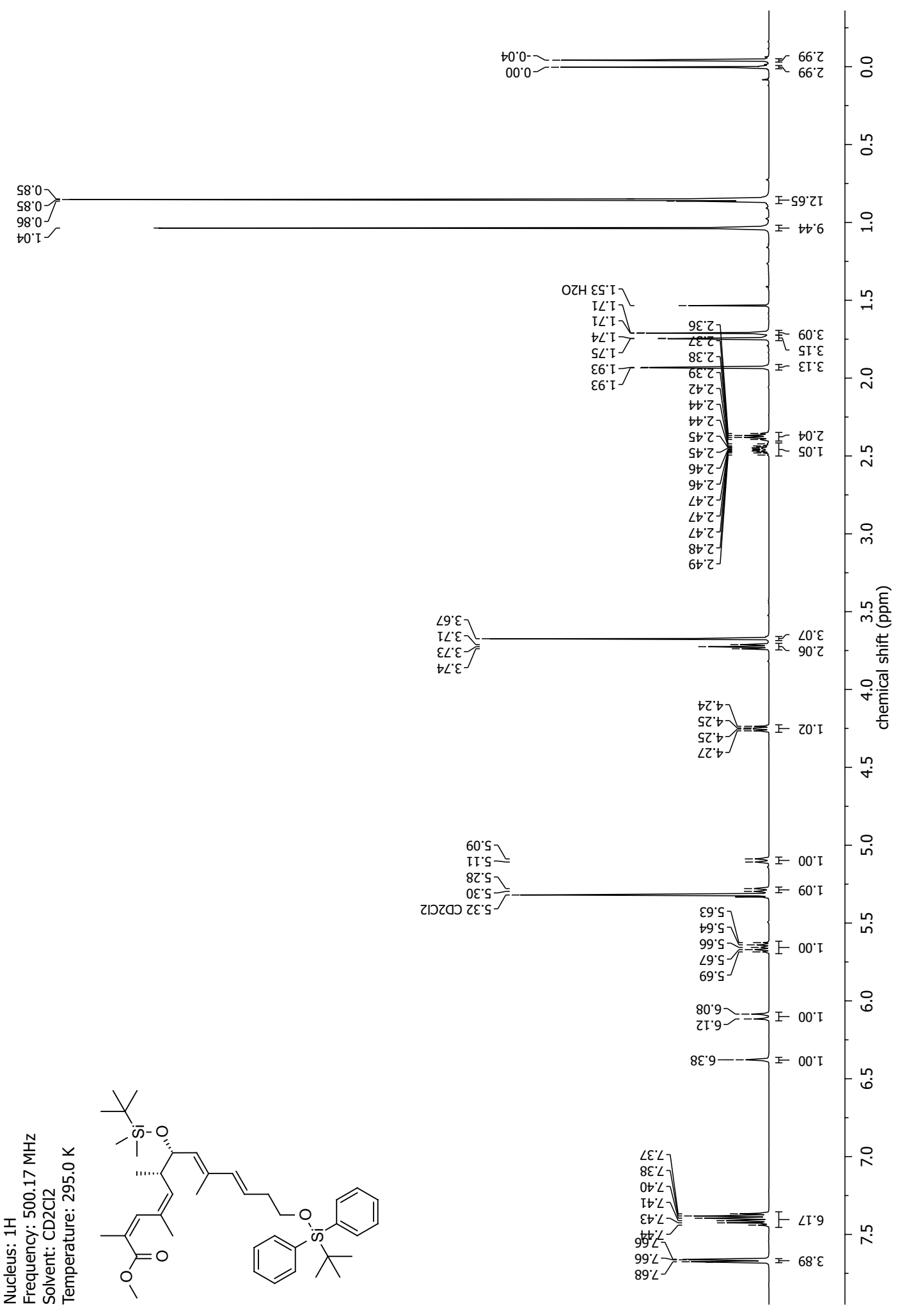




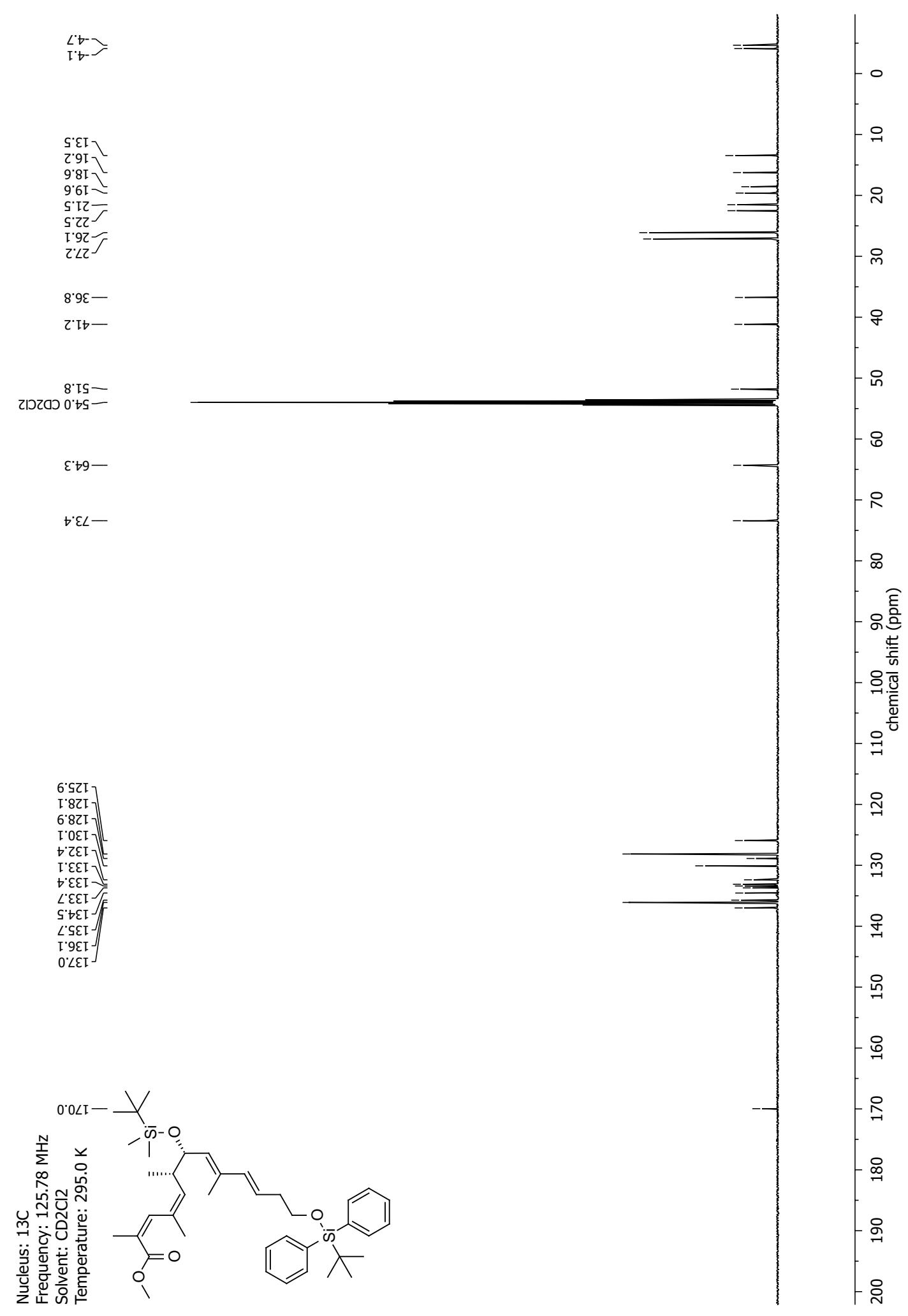


(udd) भ!บऽ ןอว!щәцว

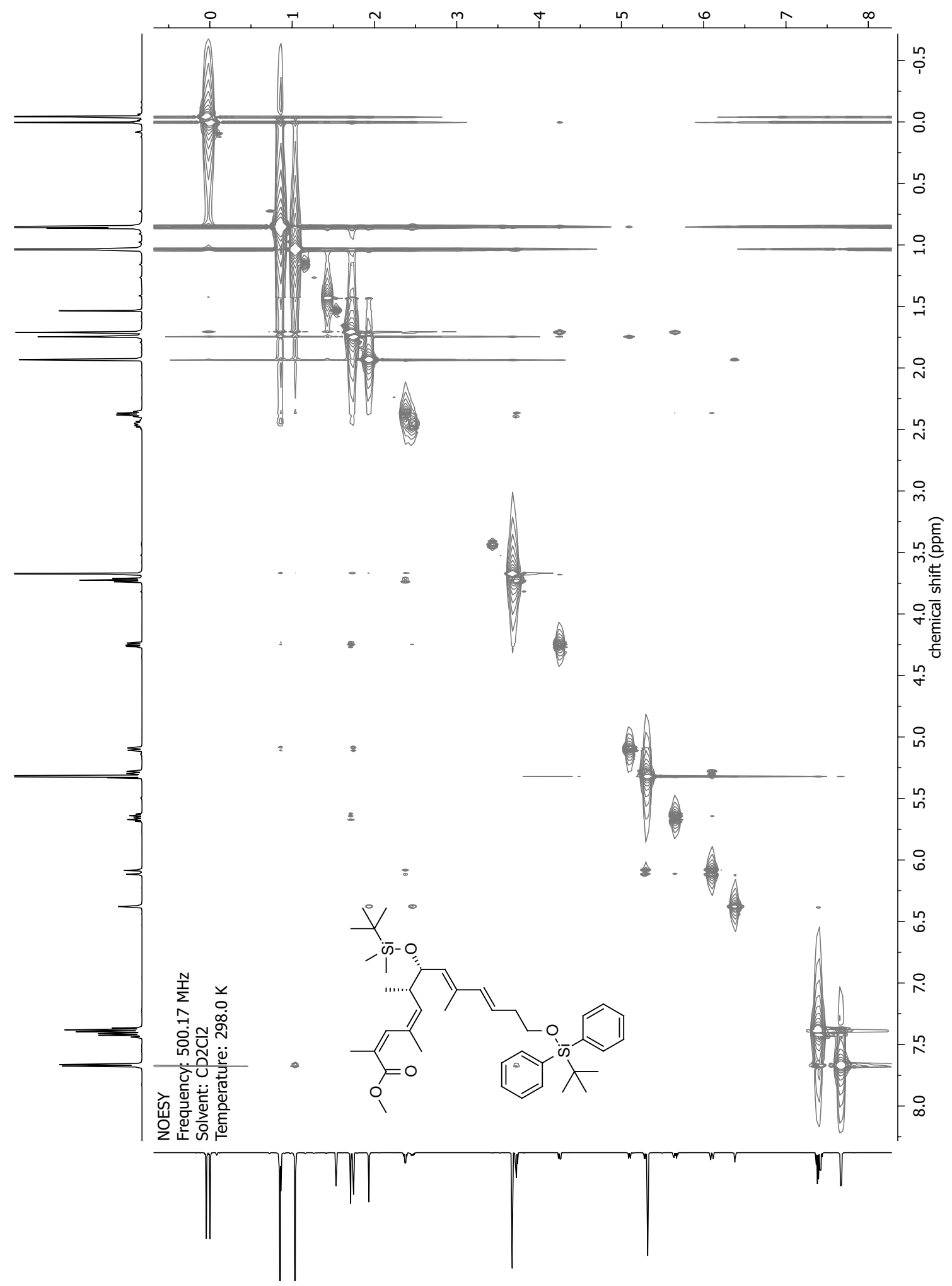


NMR-Spectra for Compound 98

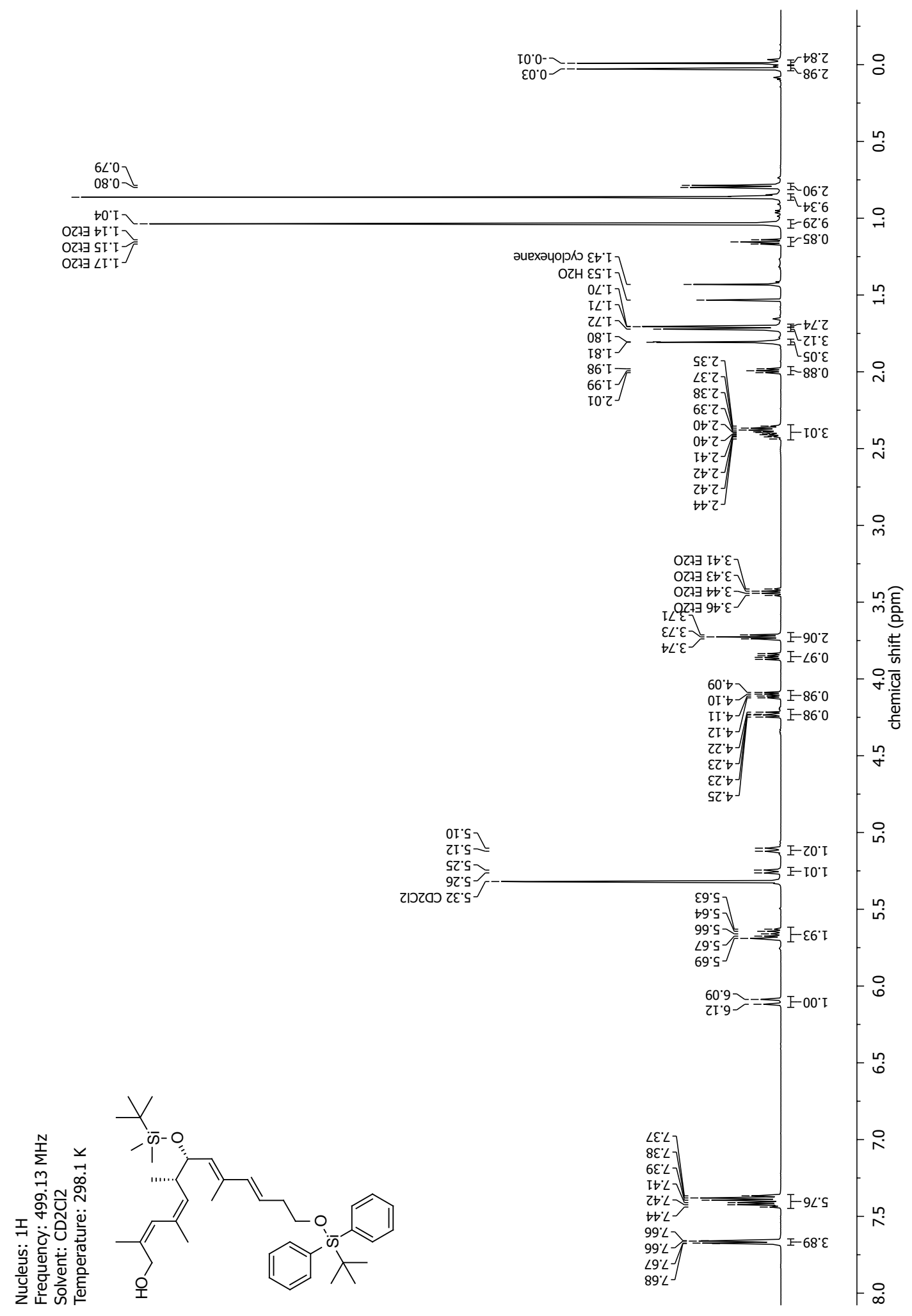




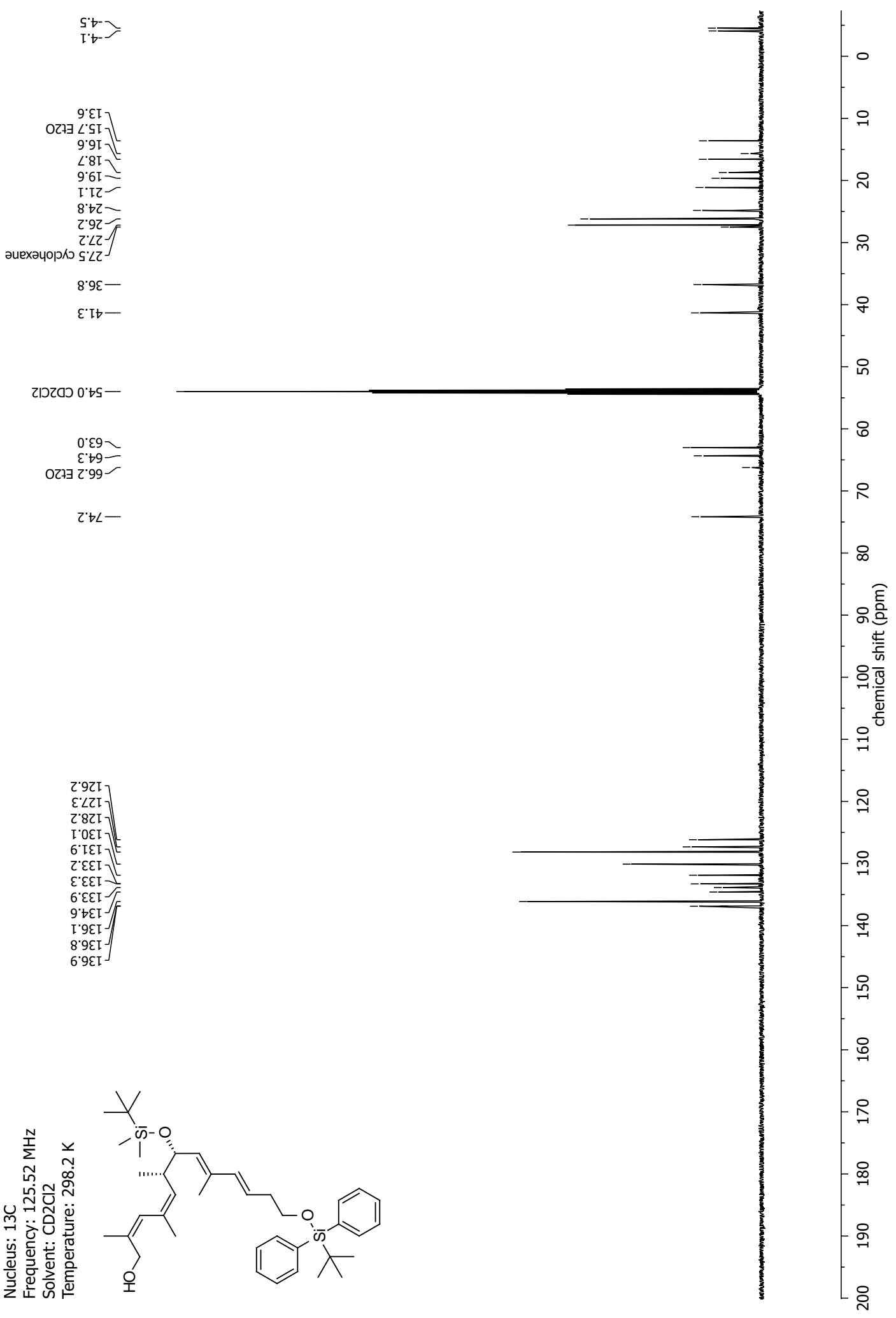


NMR-Spectra for Compound 34a

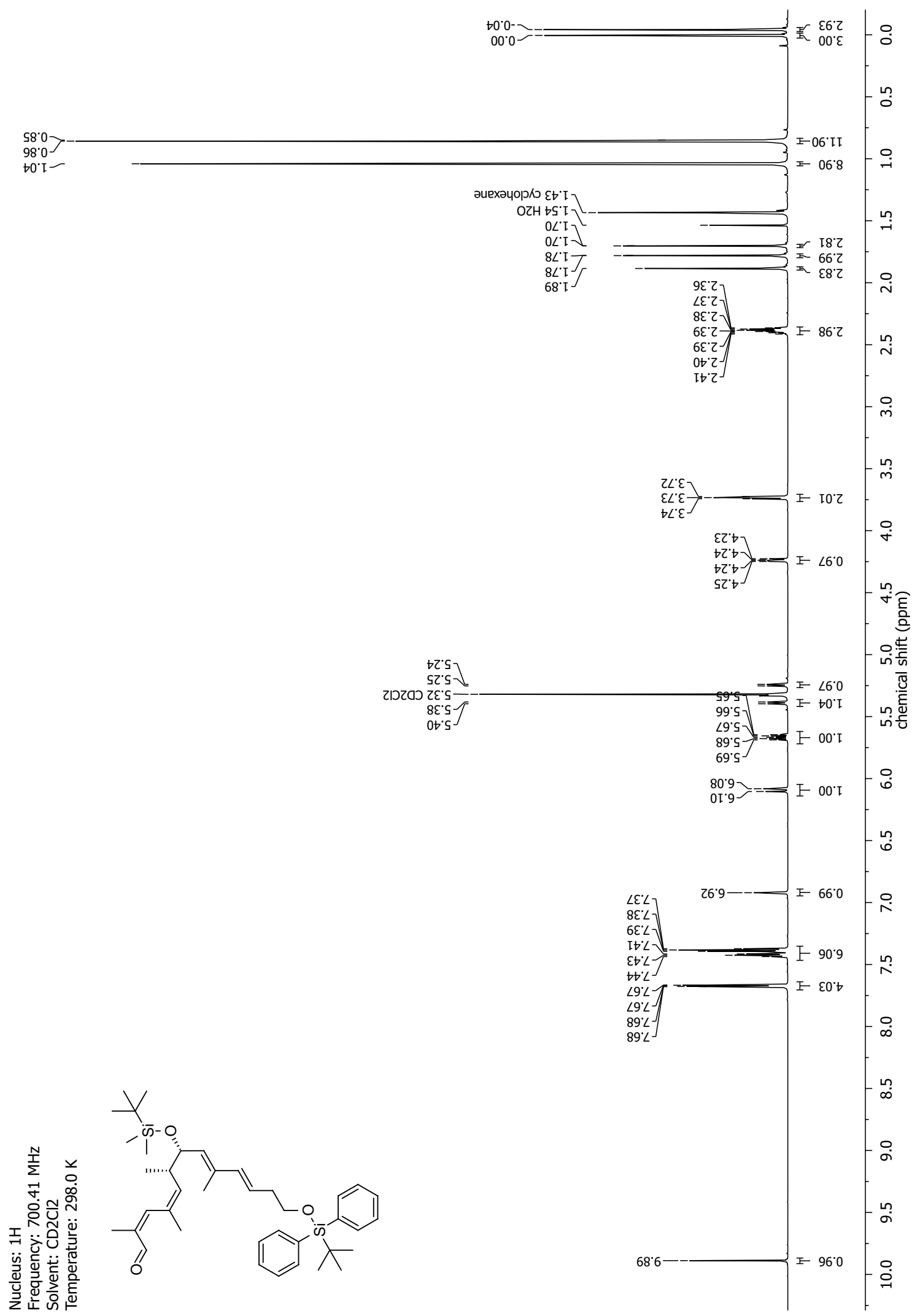




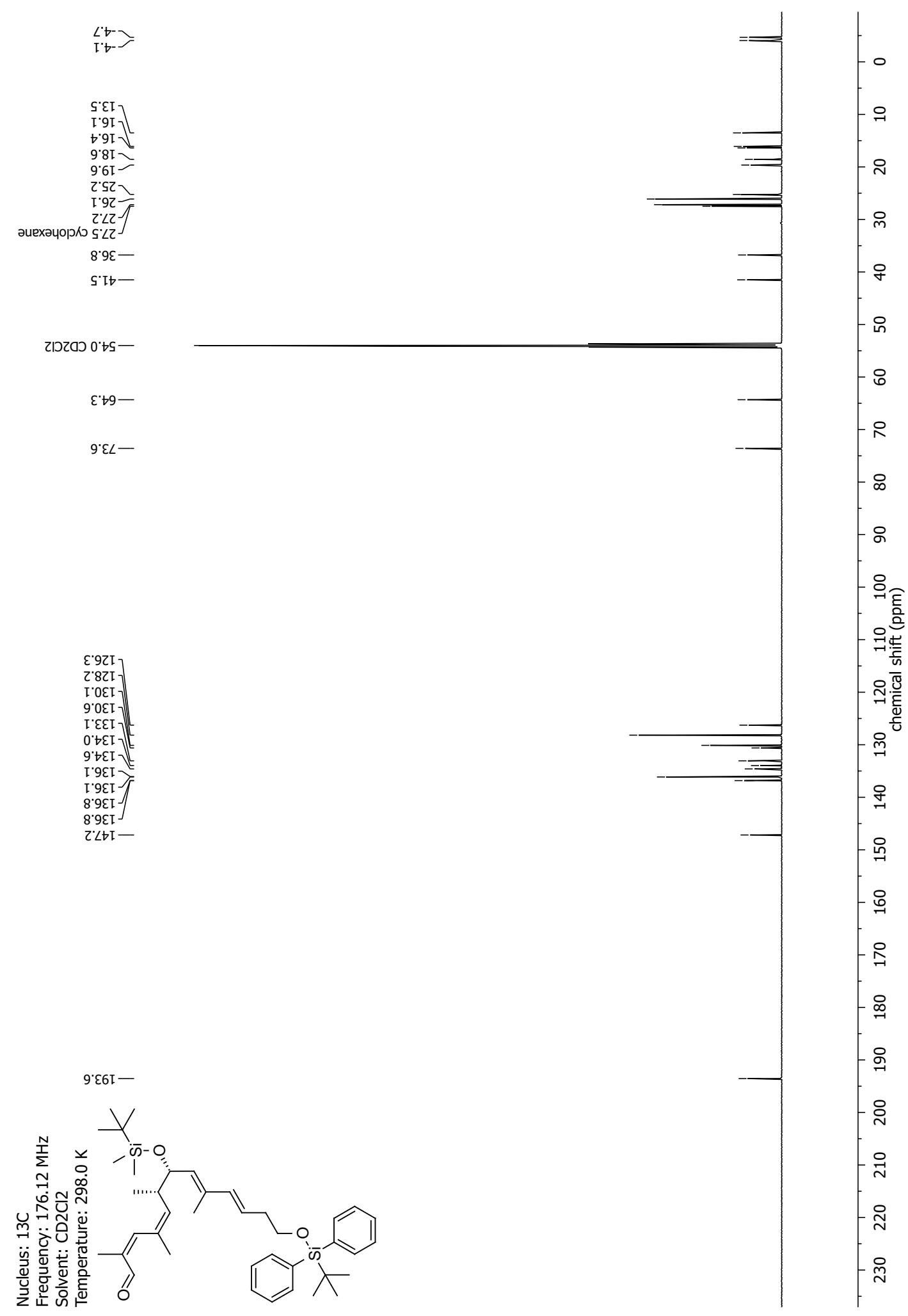


NMR-Spectra for Compound 36a

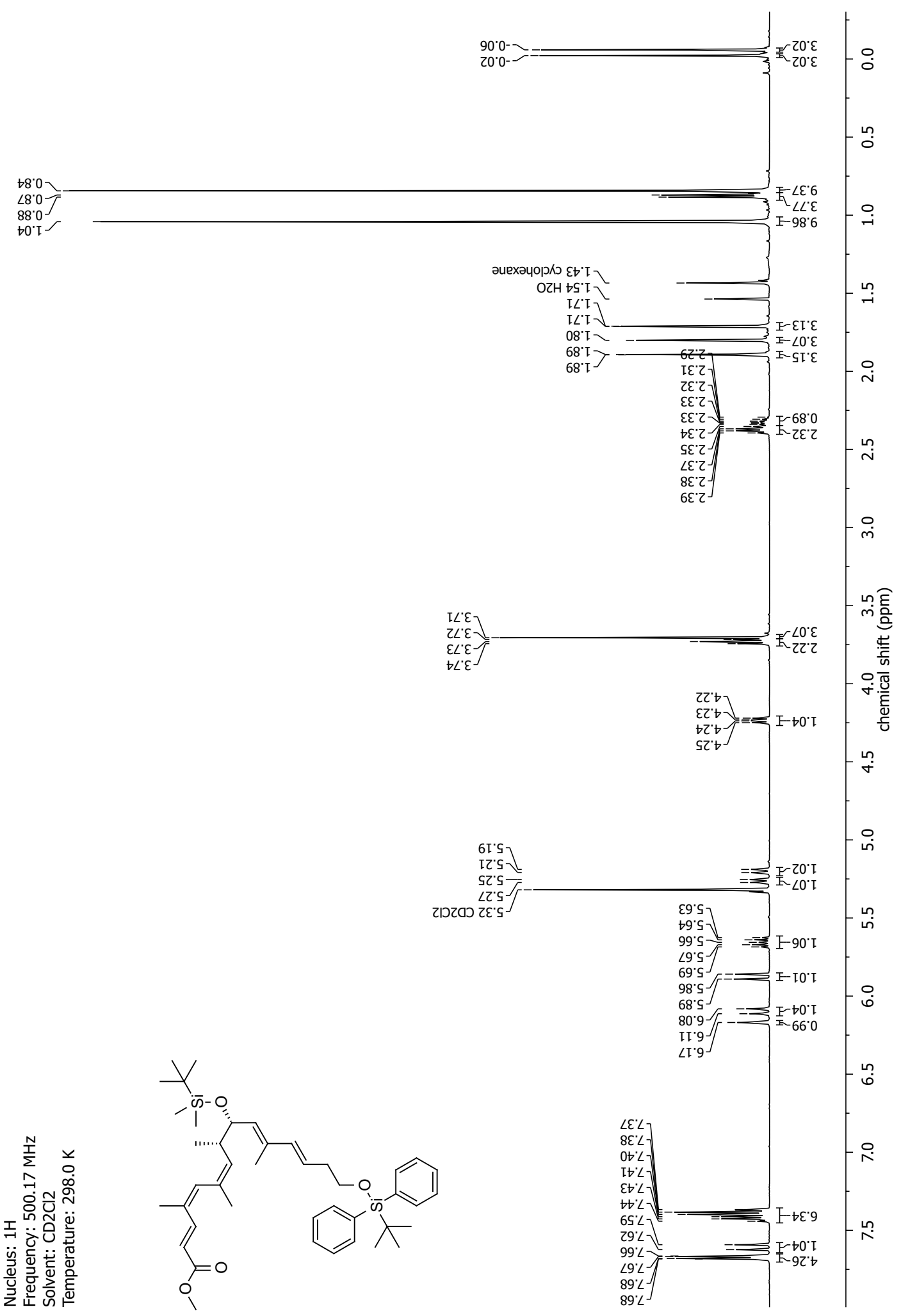




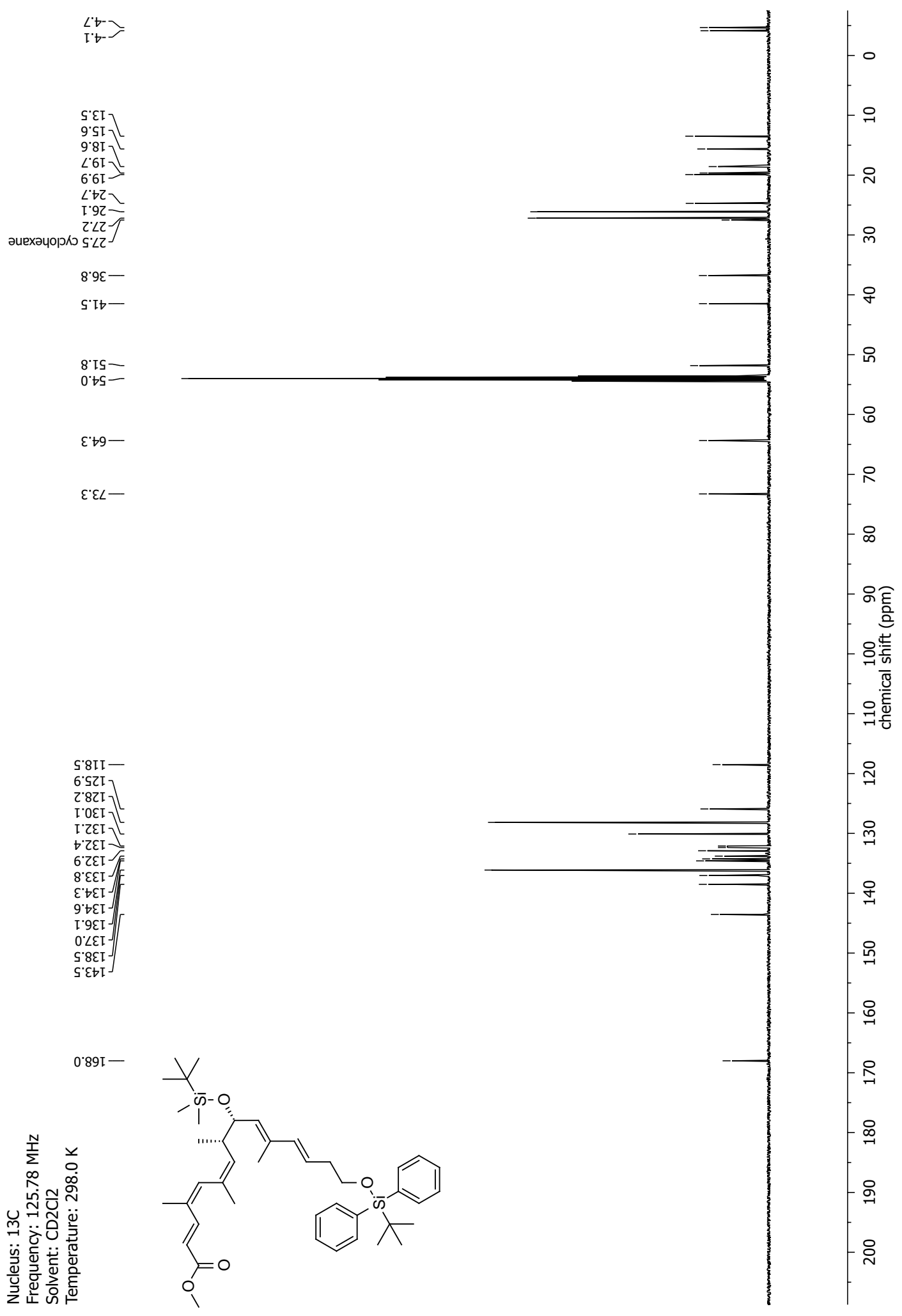


NMR-Spectra for Compound 9a

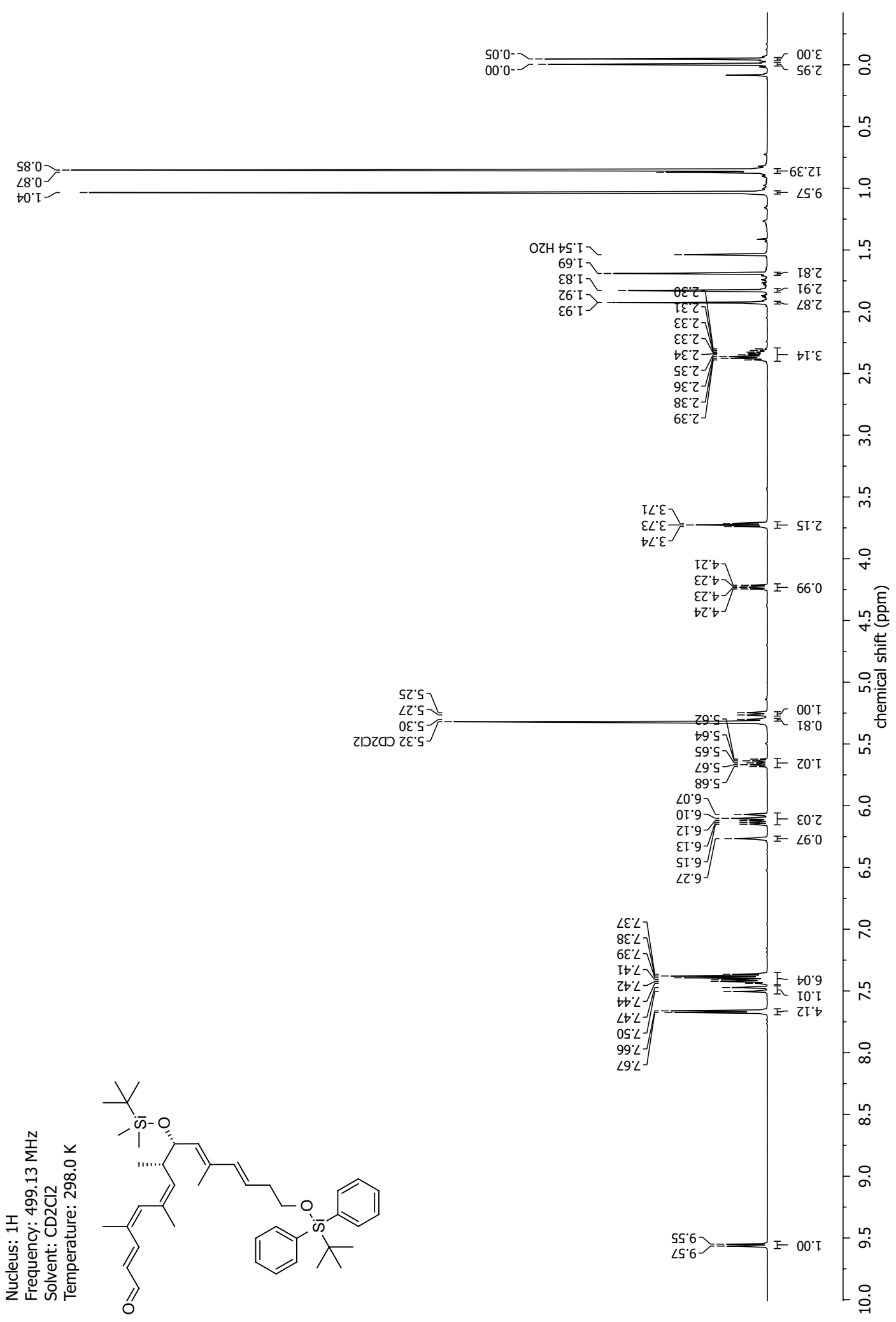




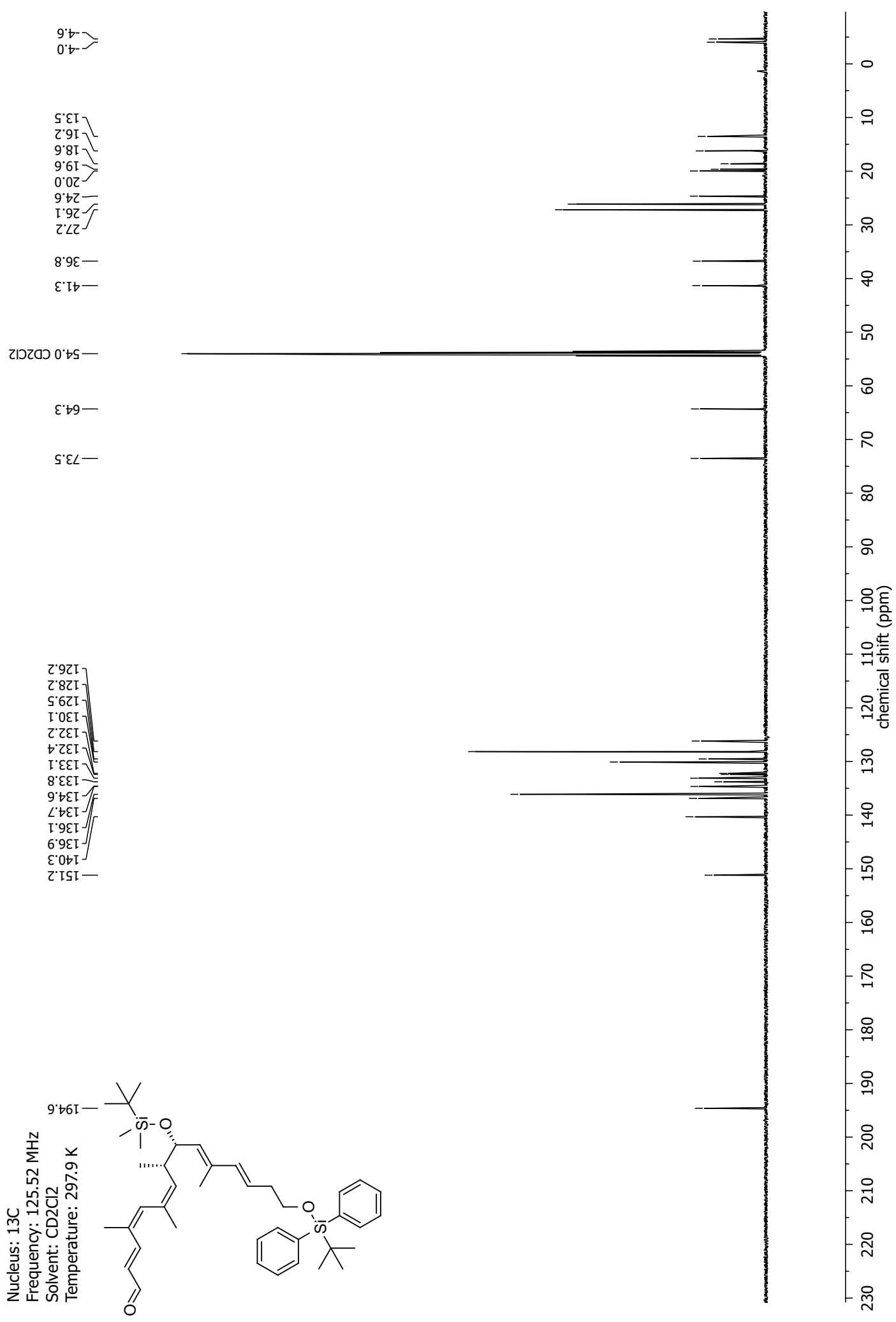


NMR-Spectra for Compound 38

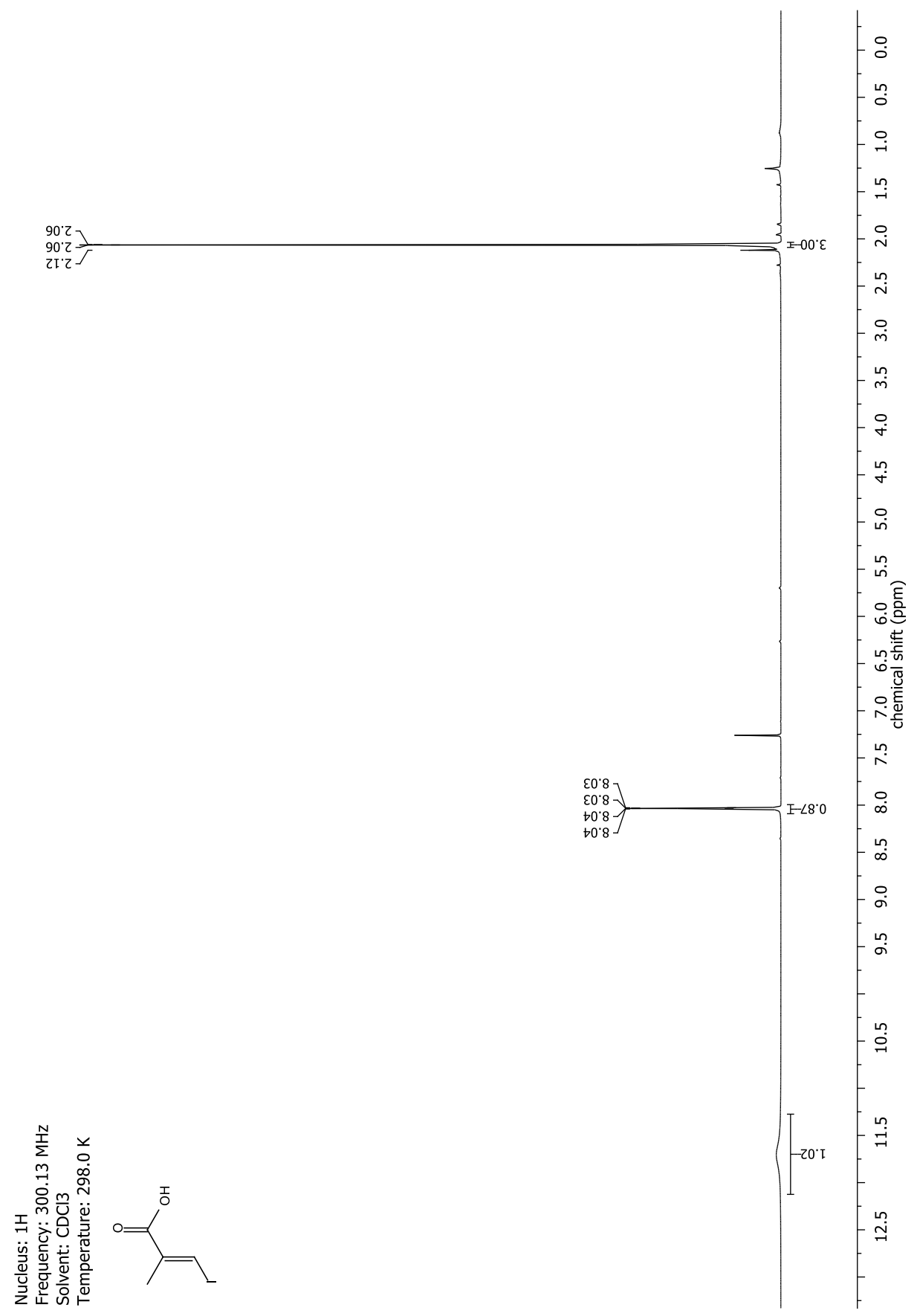




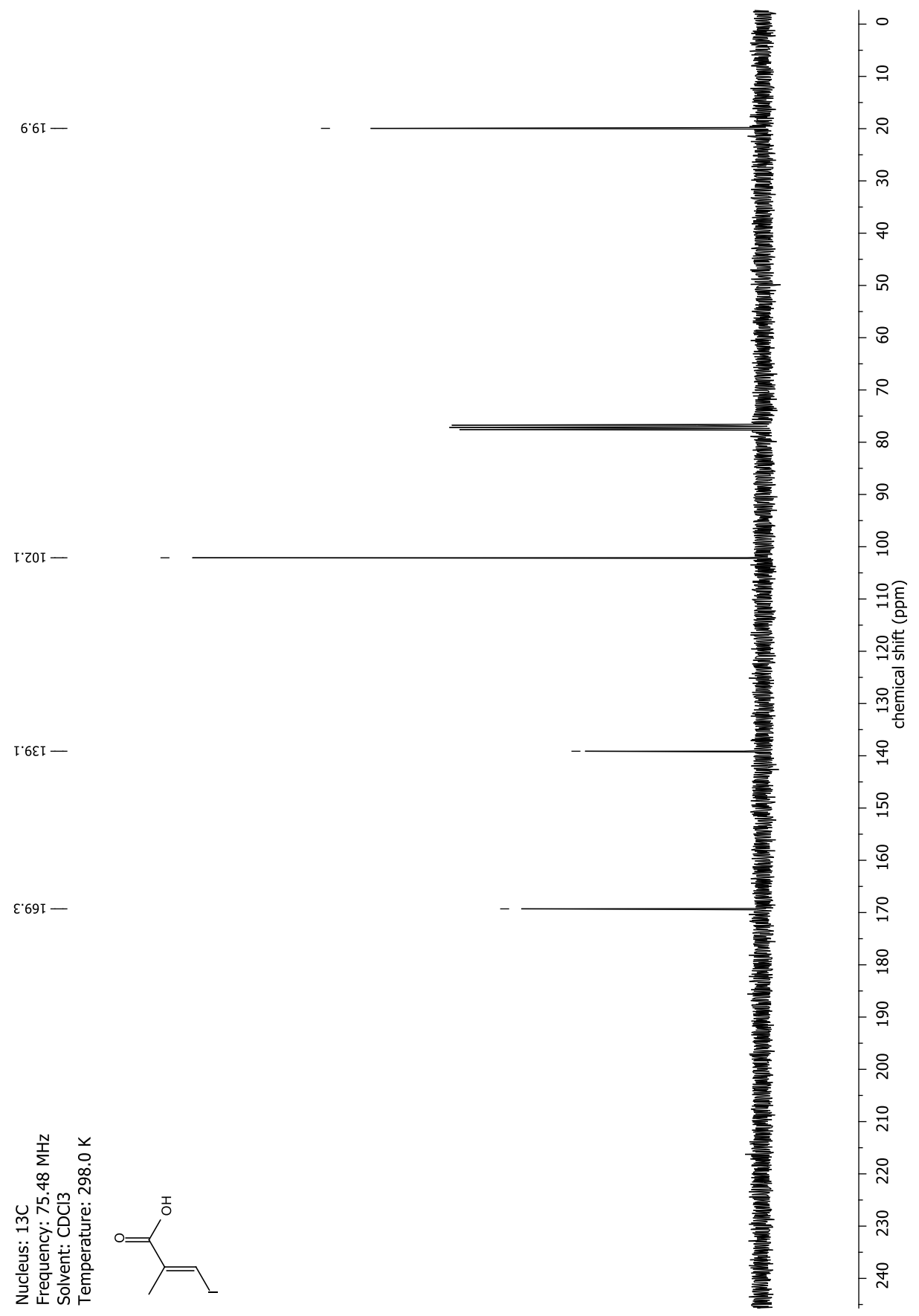


NMR-Spectra for Compound 99

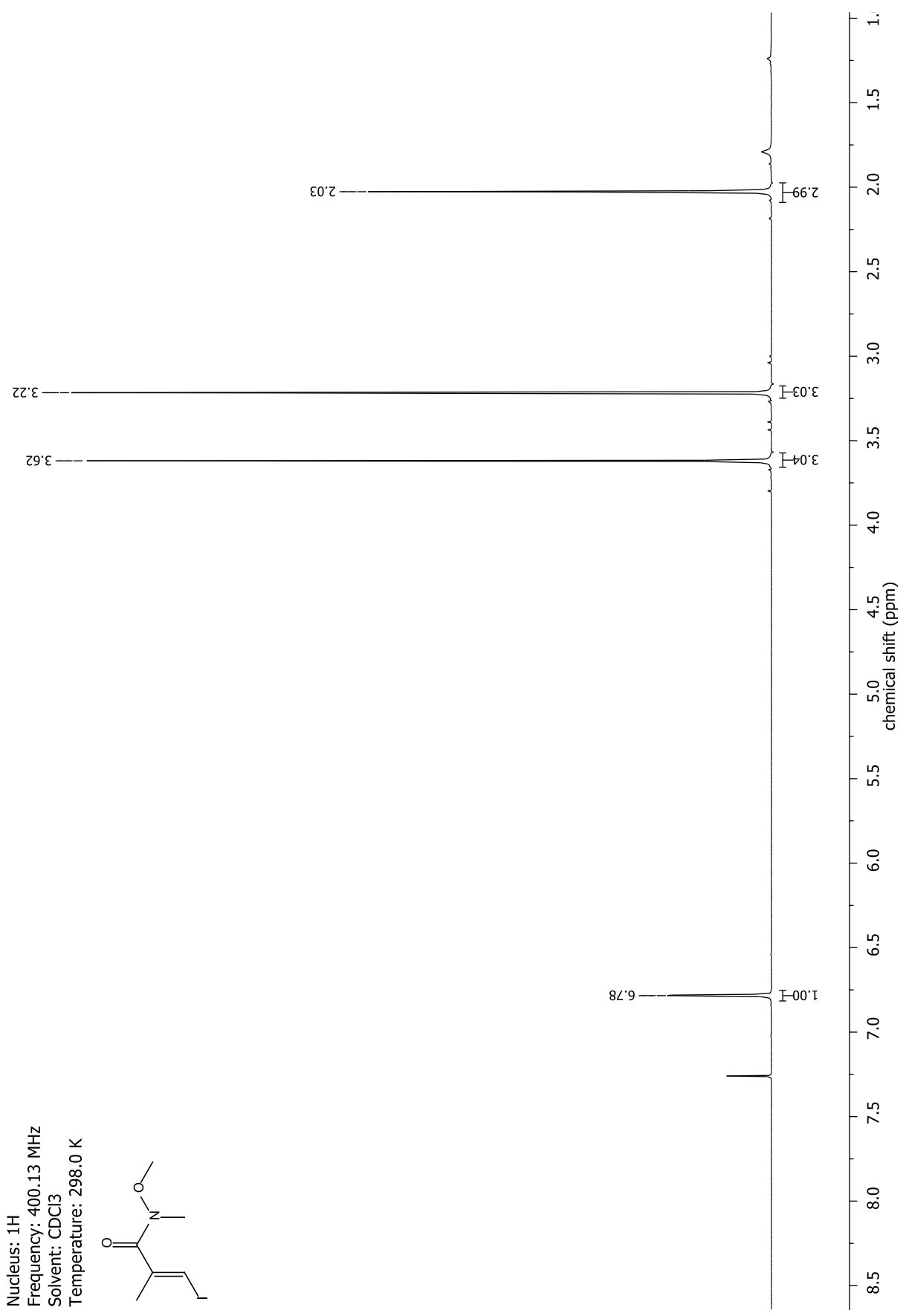


$\varepsilon: 2 Z$

$\varepsilon$ ¿ะ -

เ.'9-

$0_{0}^{\circ} 9 L>$

$0<L L$
$\varepsilon<L$

L'98 -

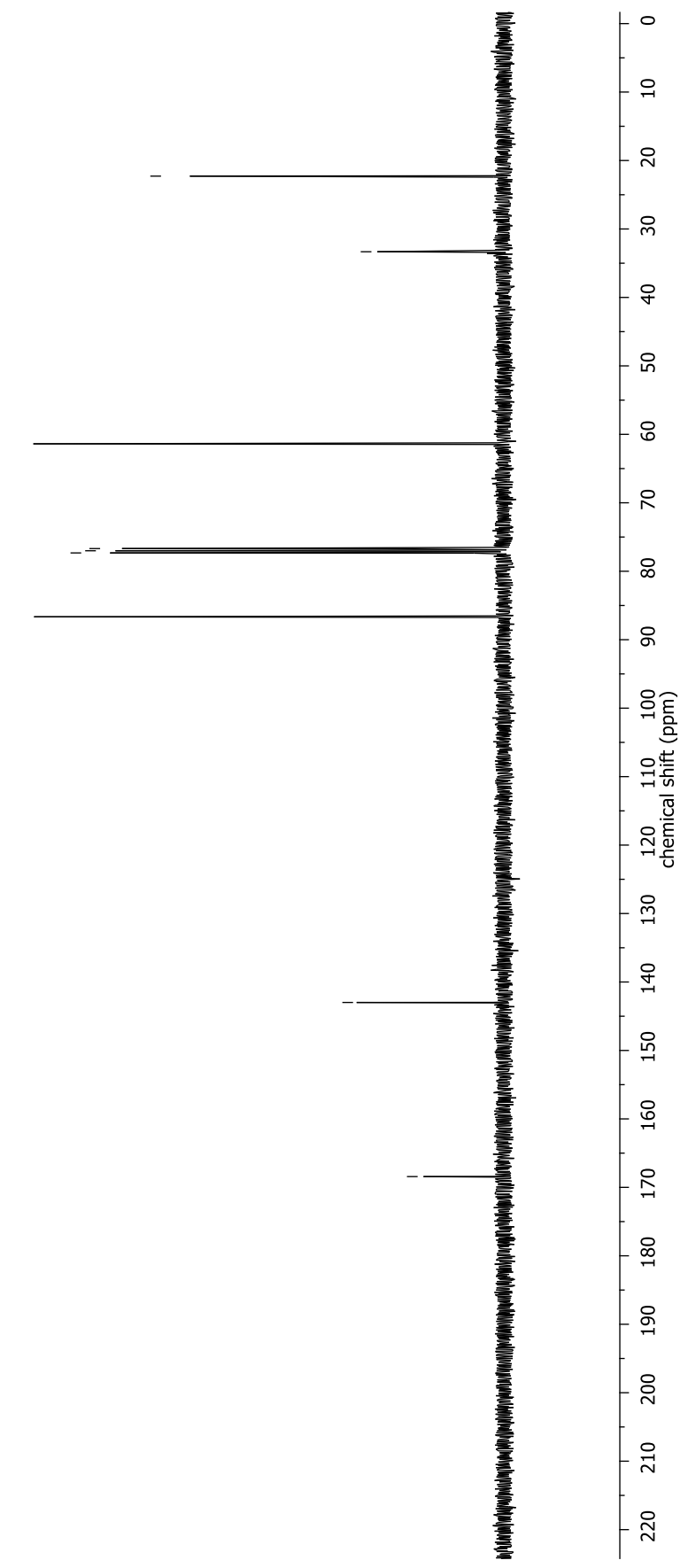


NMR-Spectra for Compound 10

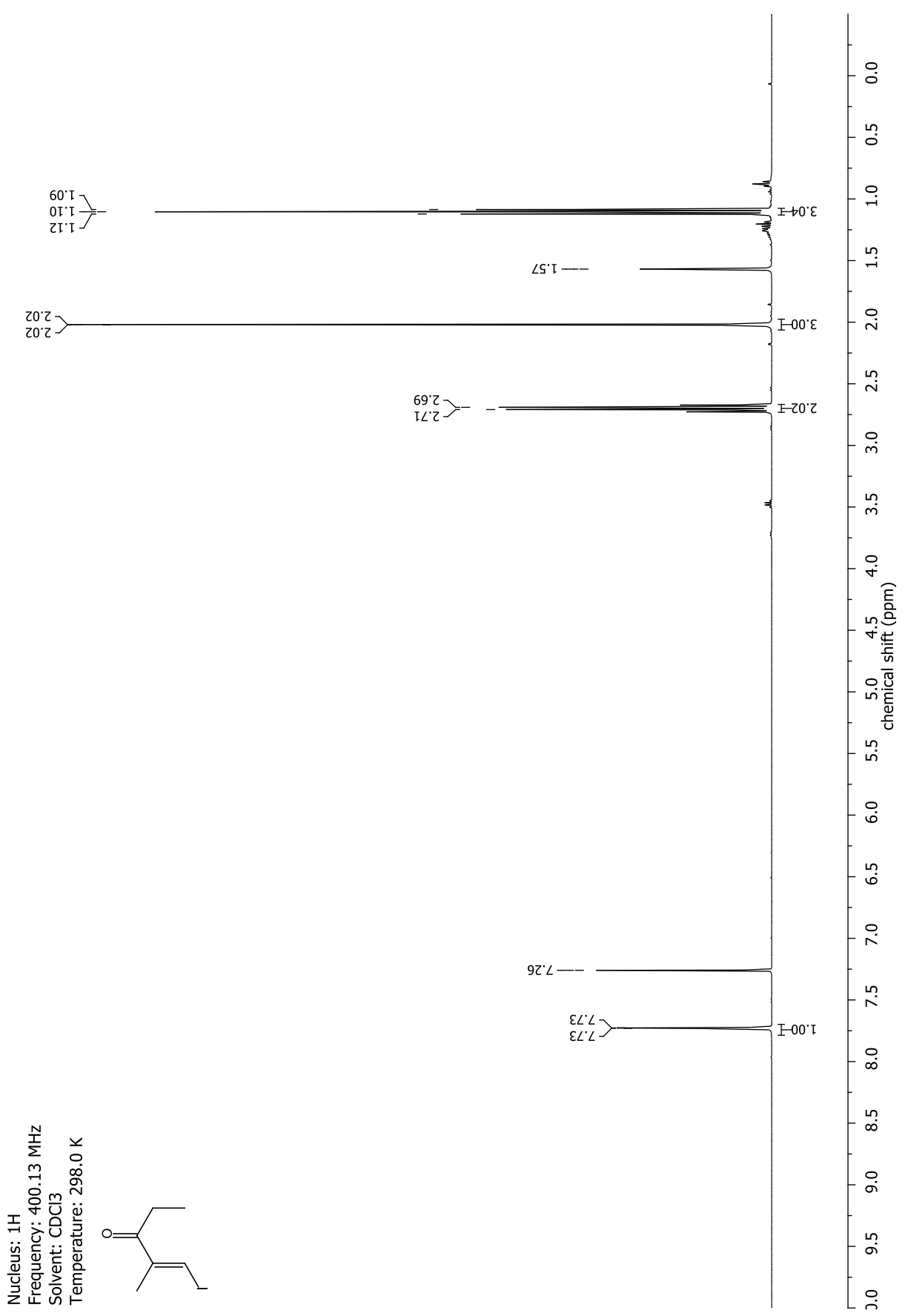




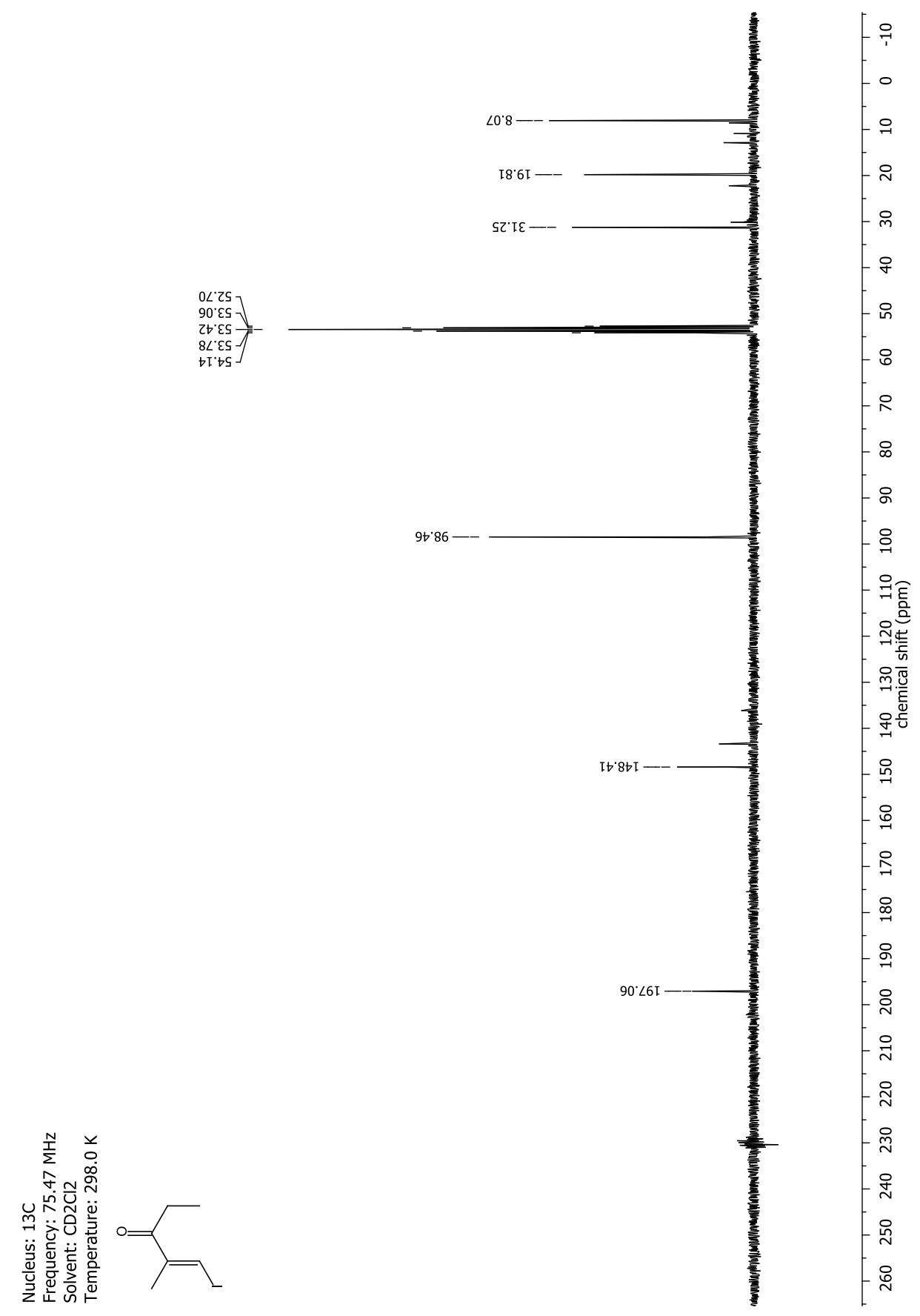


NMR-Spectra for Compound 41

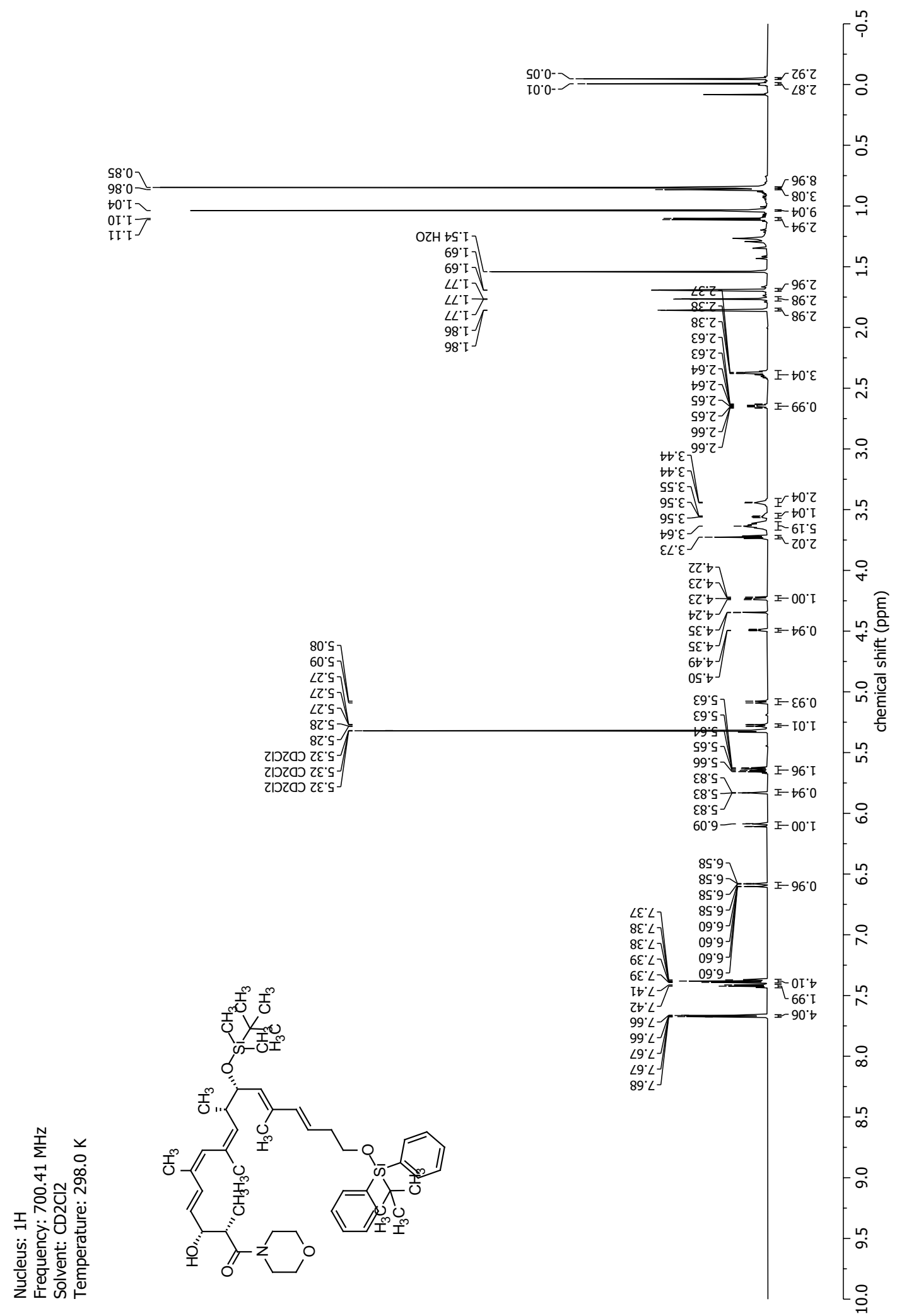




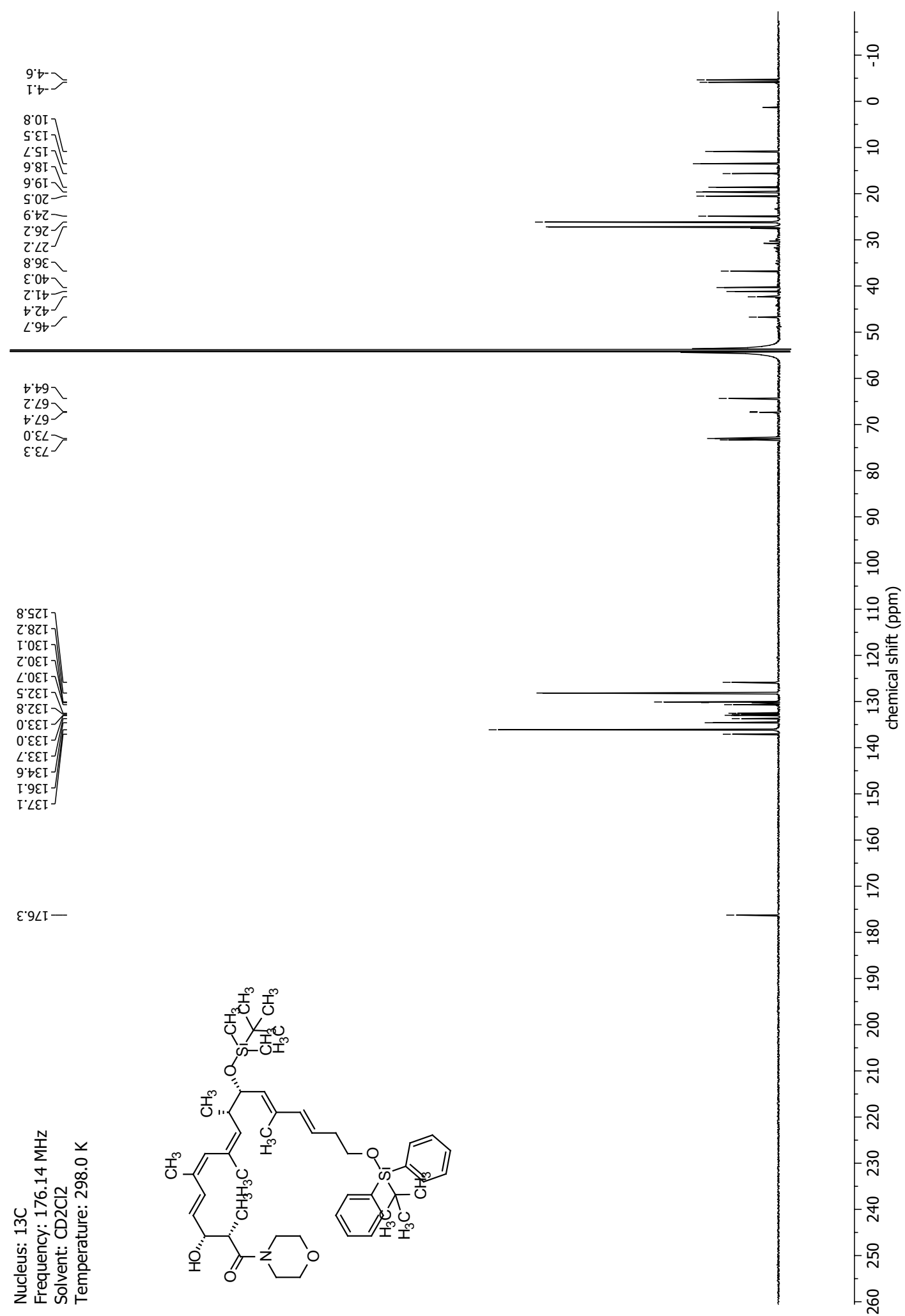


NMR-Spectra for Compound 44

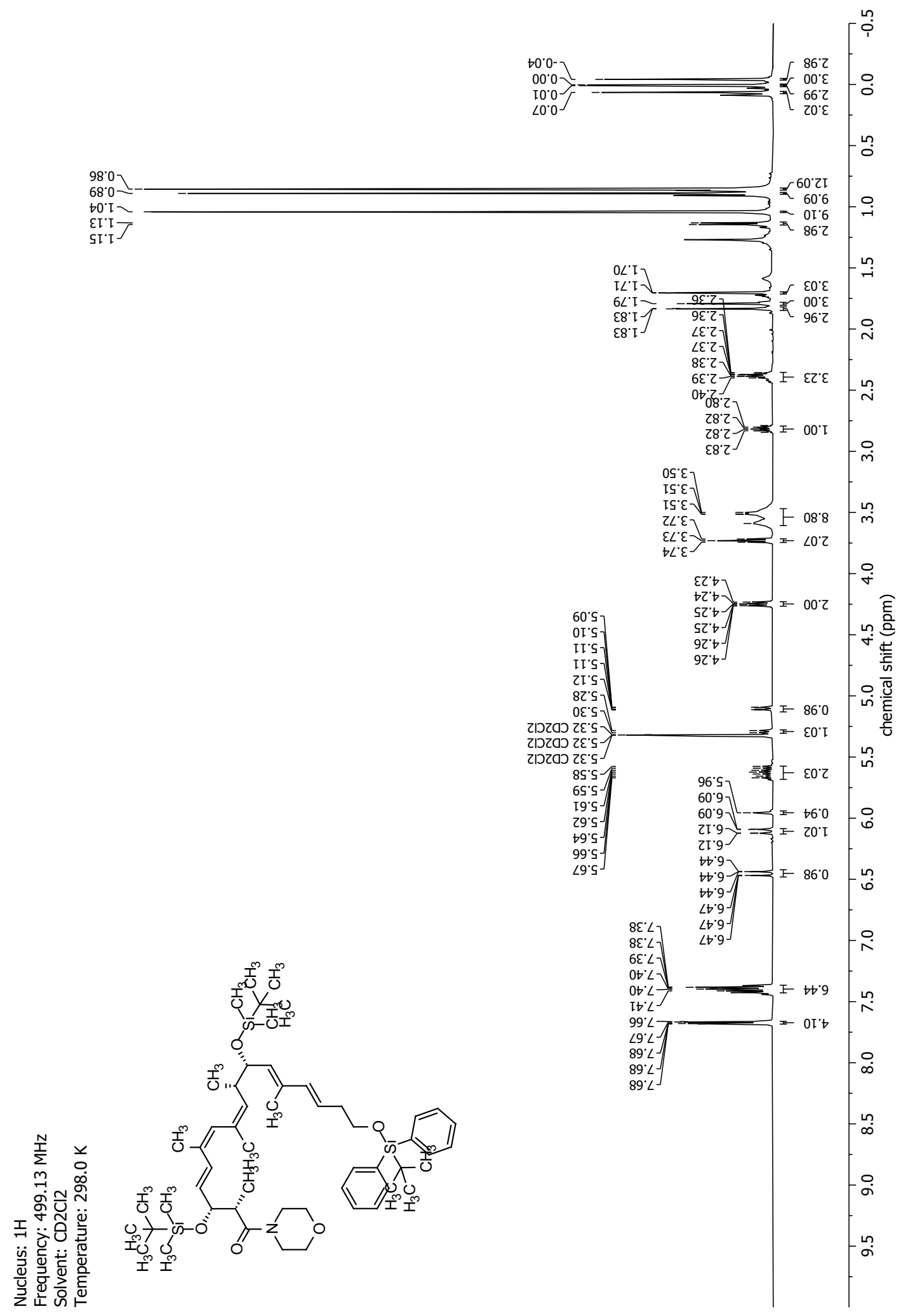




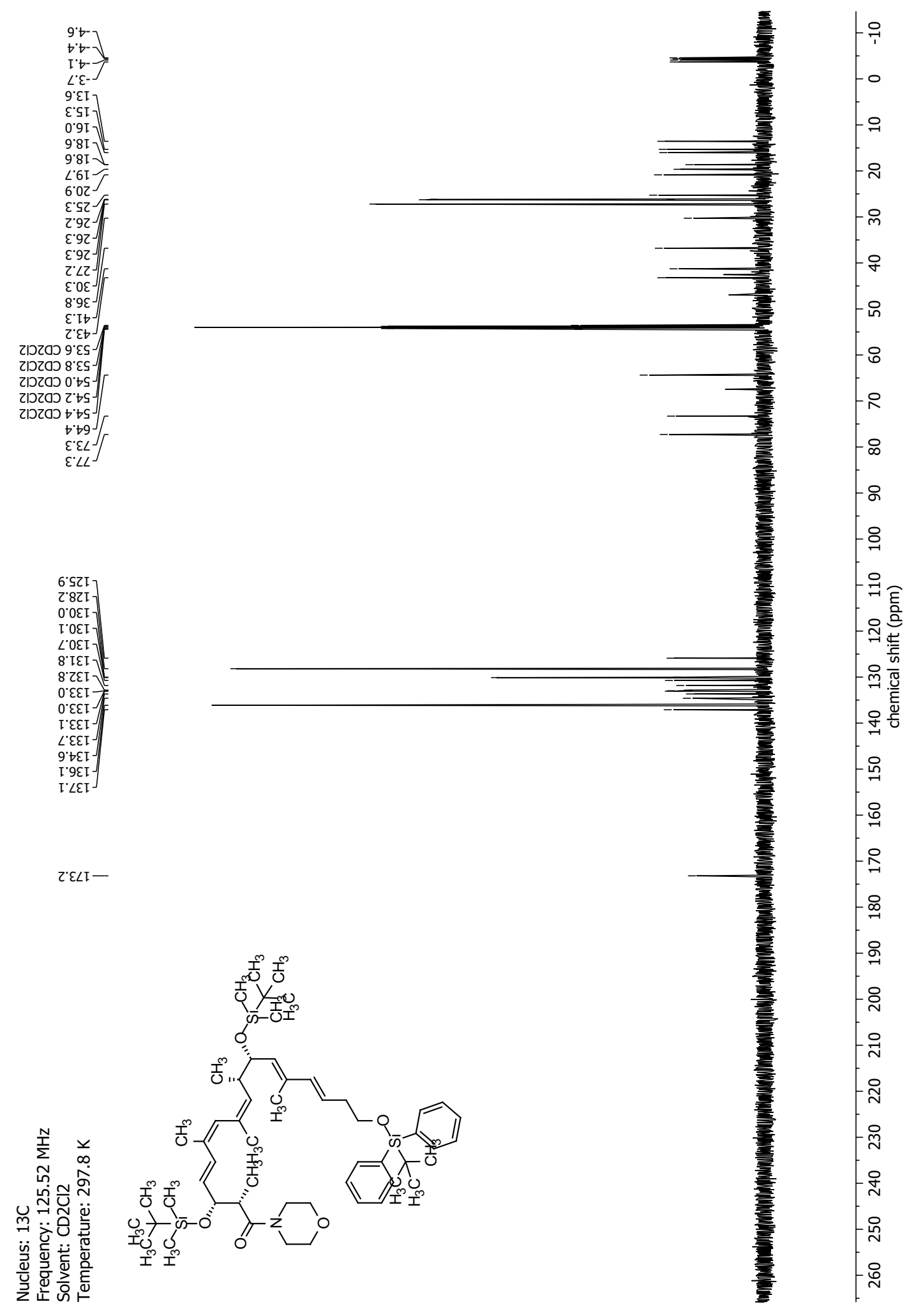


NMR-Spectra for Compound 43

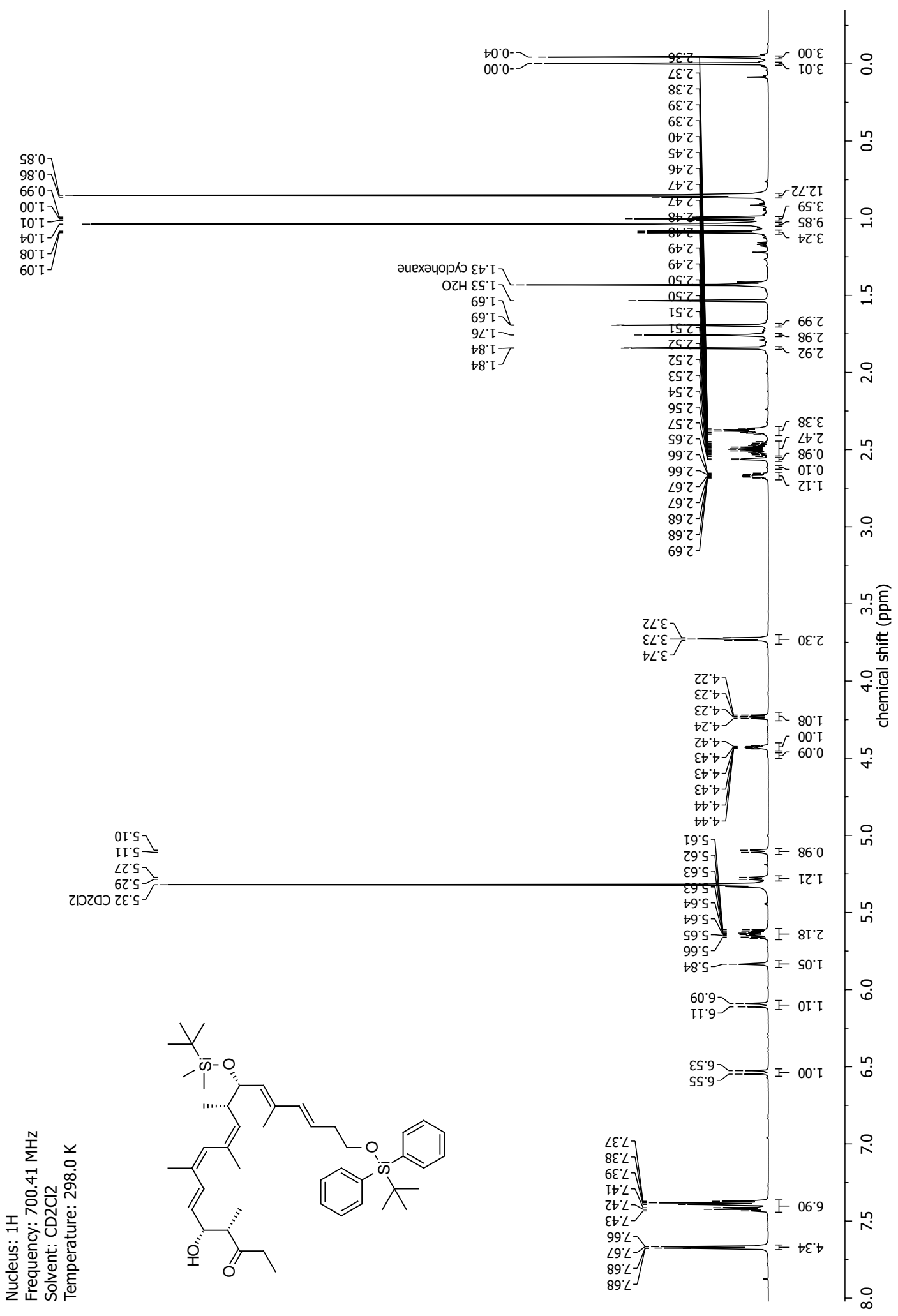




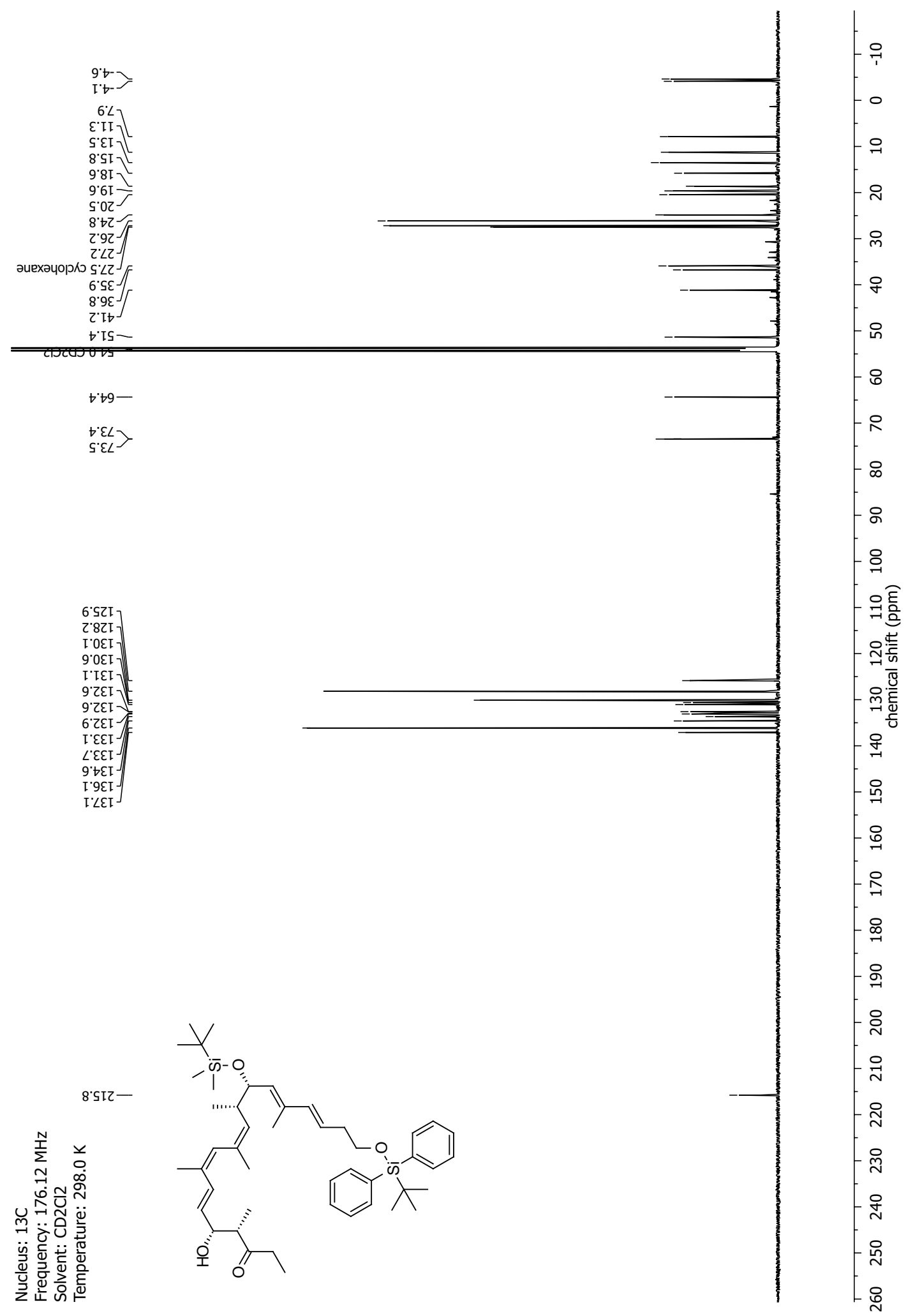


NMR-Spectra for Compound 45

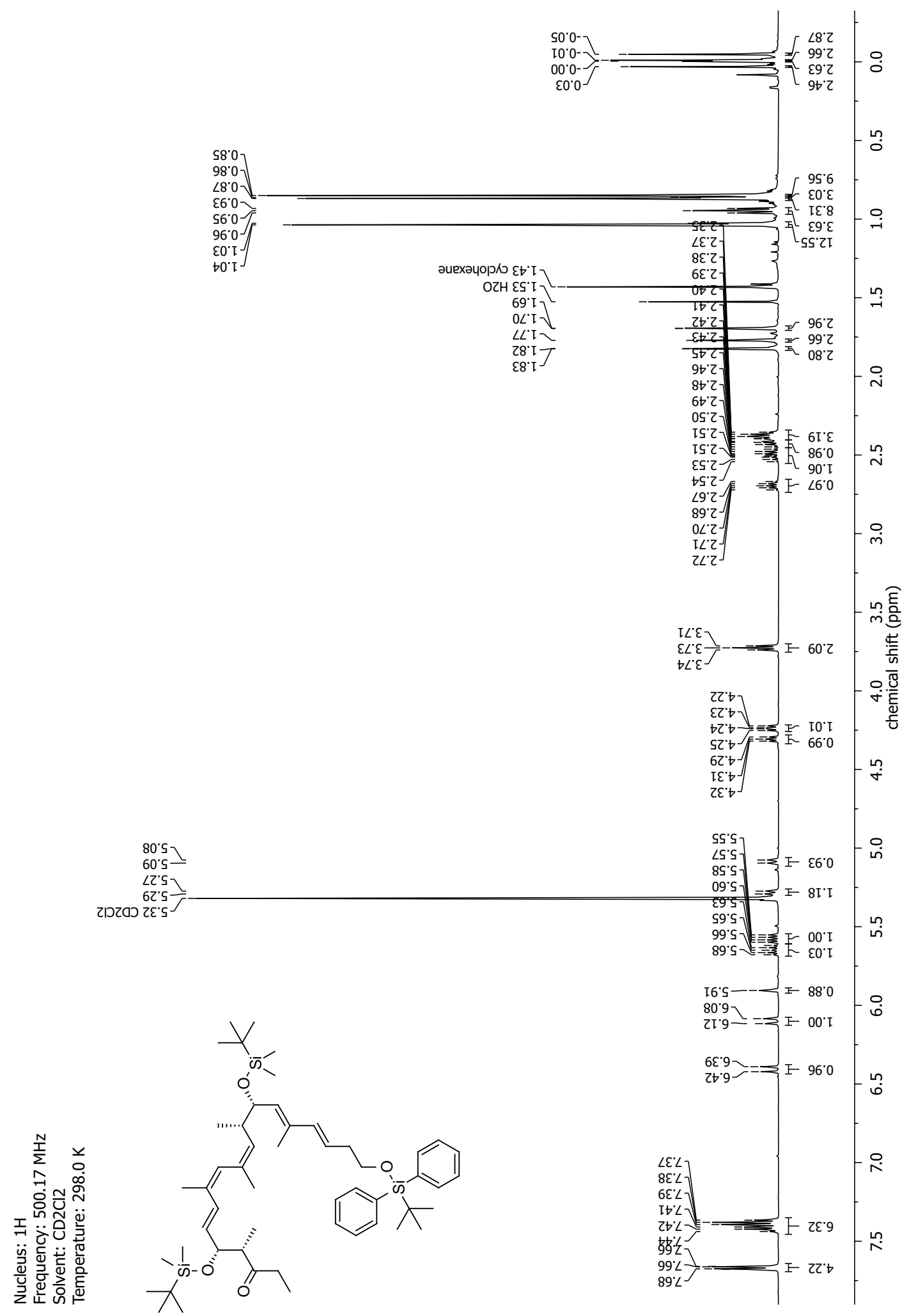




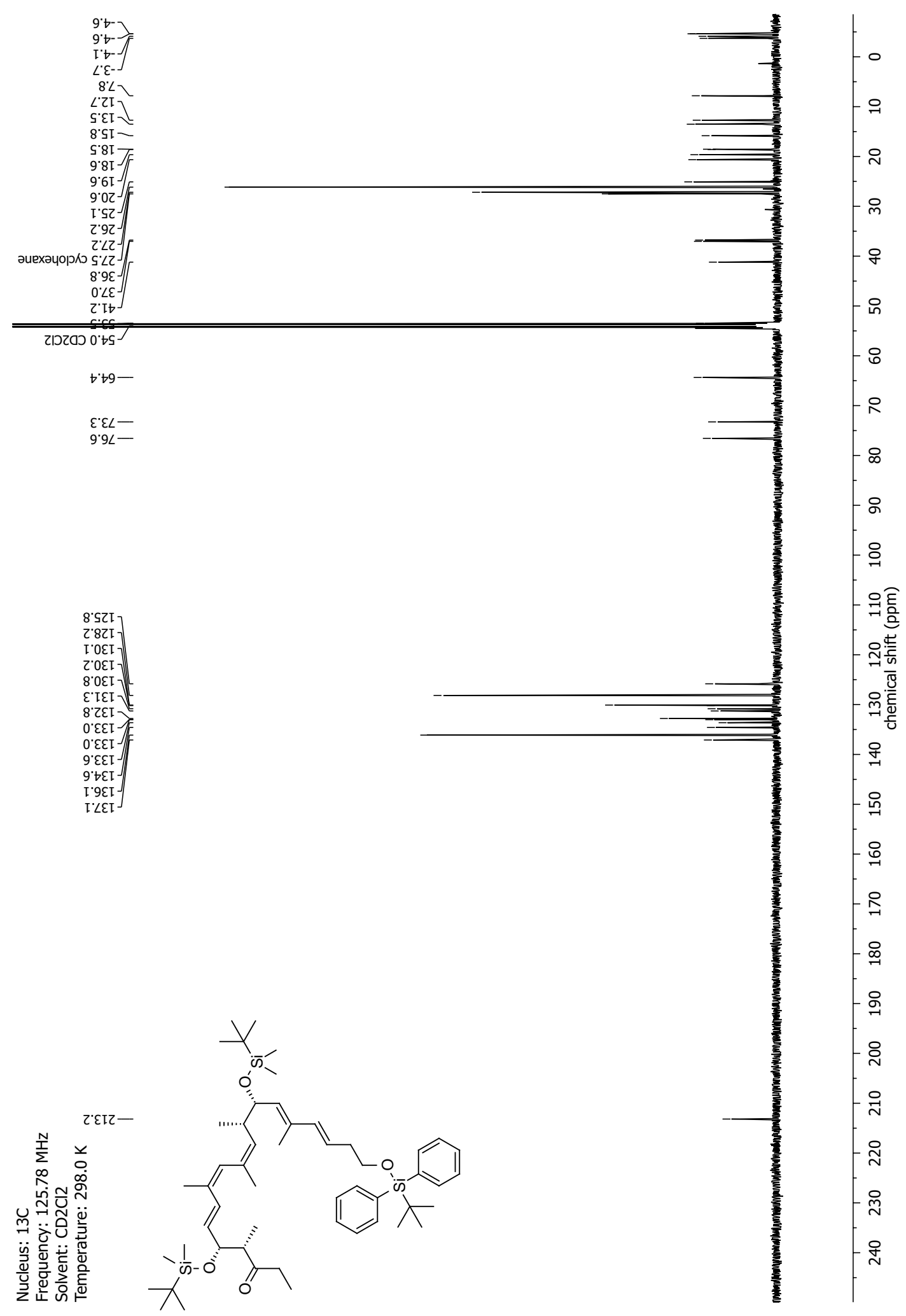


NMR-Spectra for Compound 50

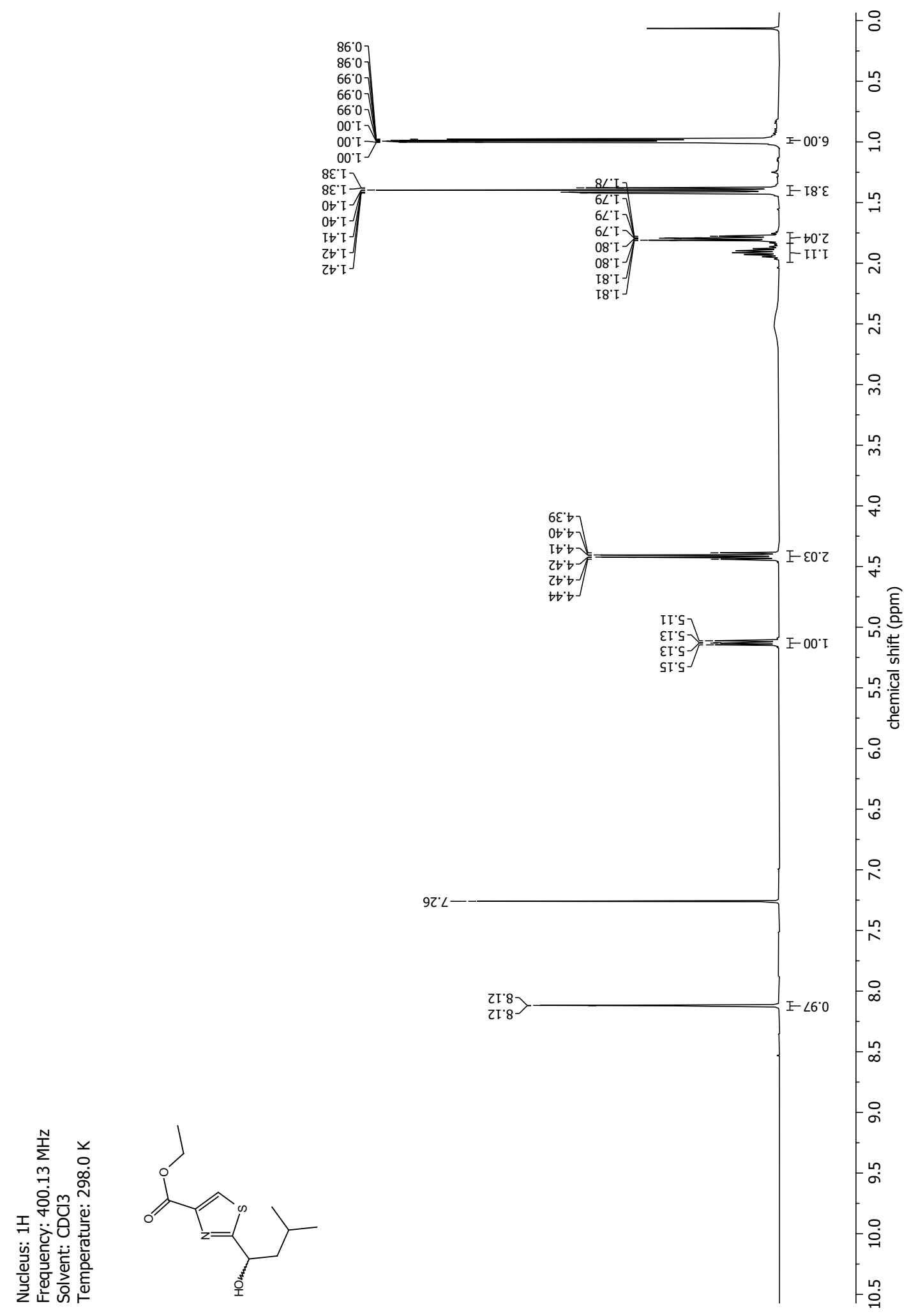




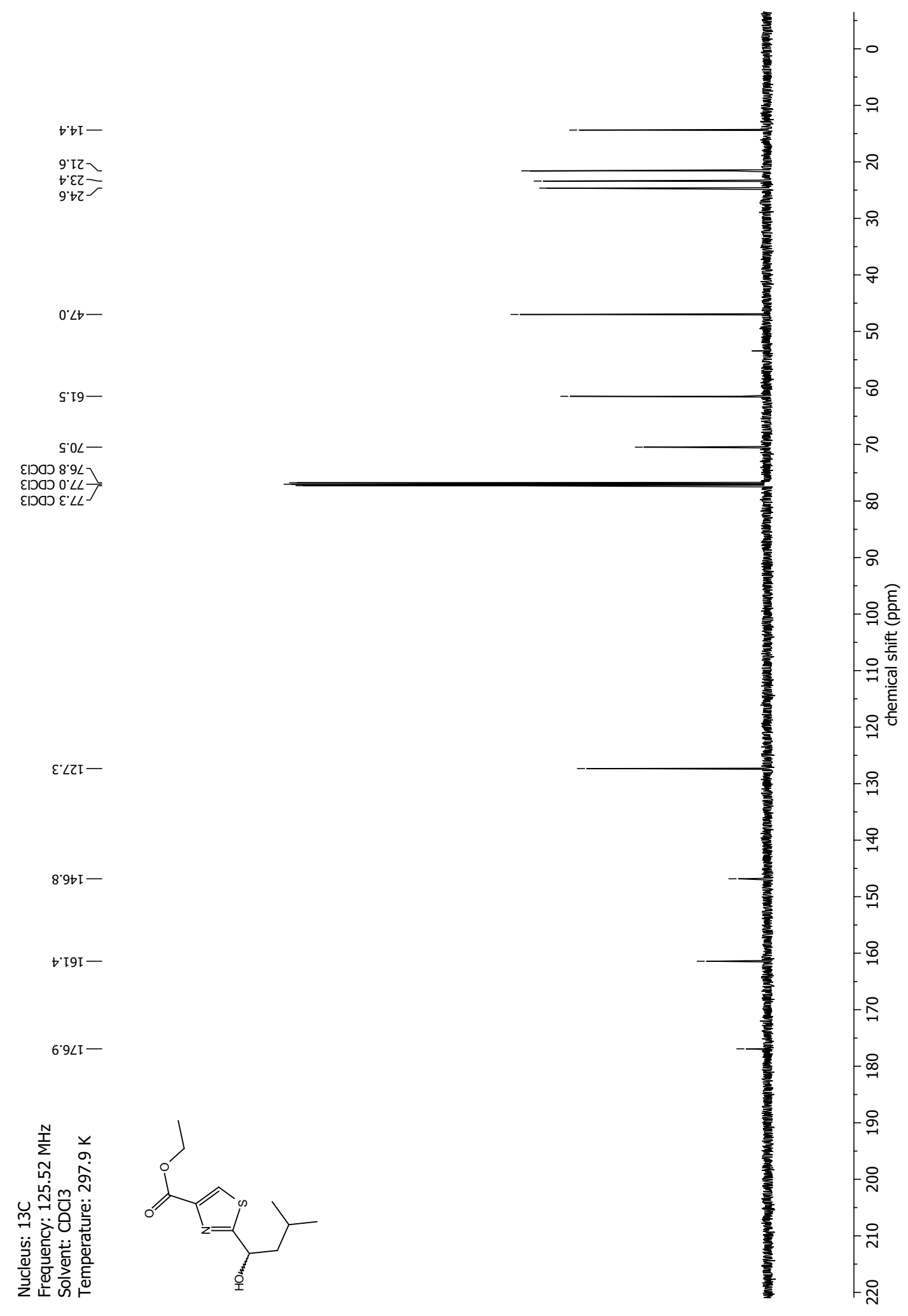


NMR-Spectra for Compound 100

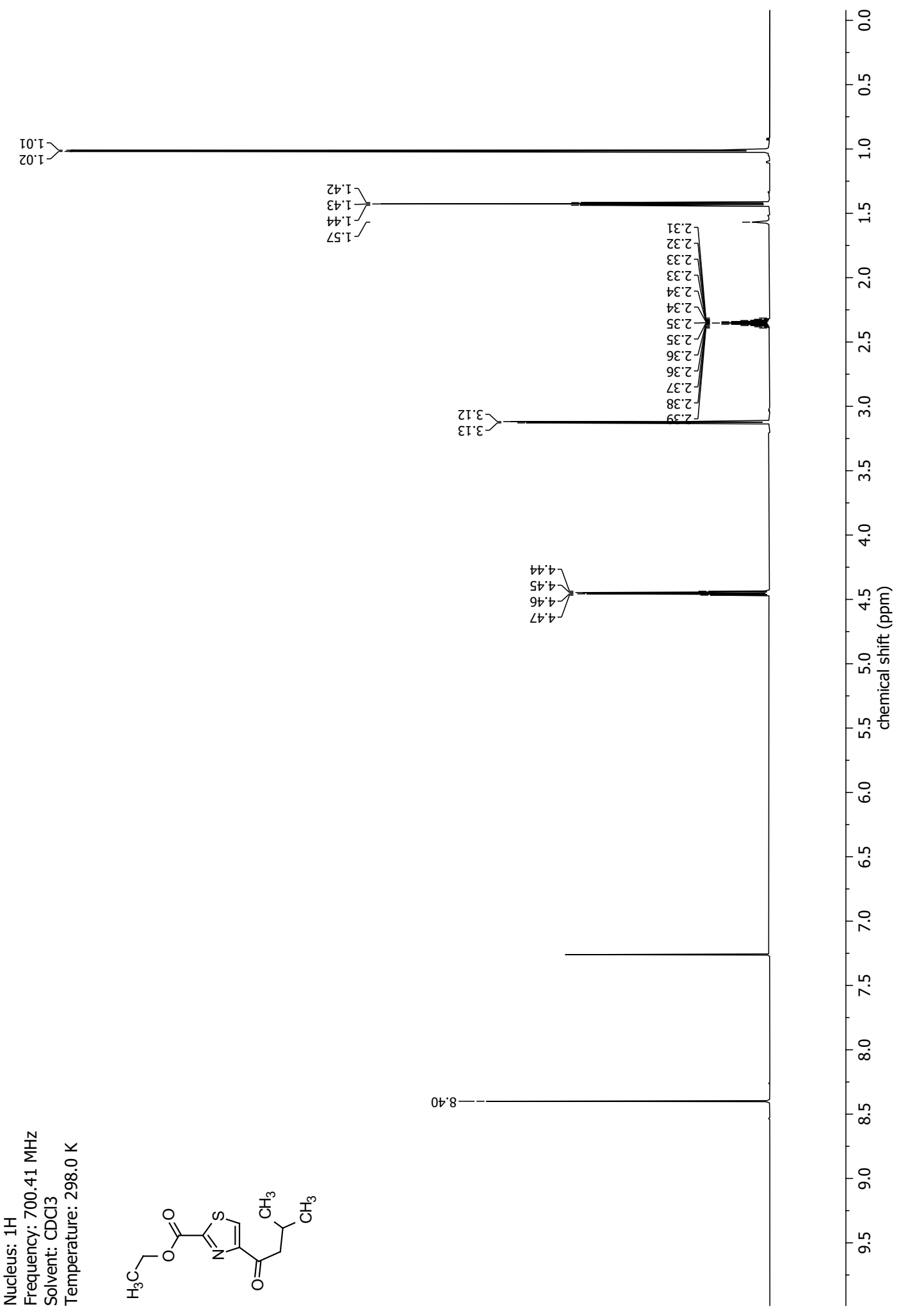




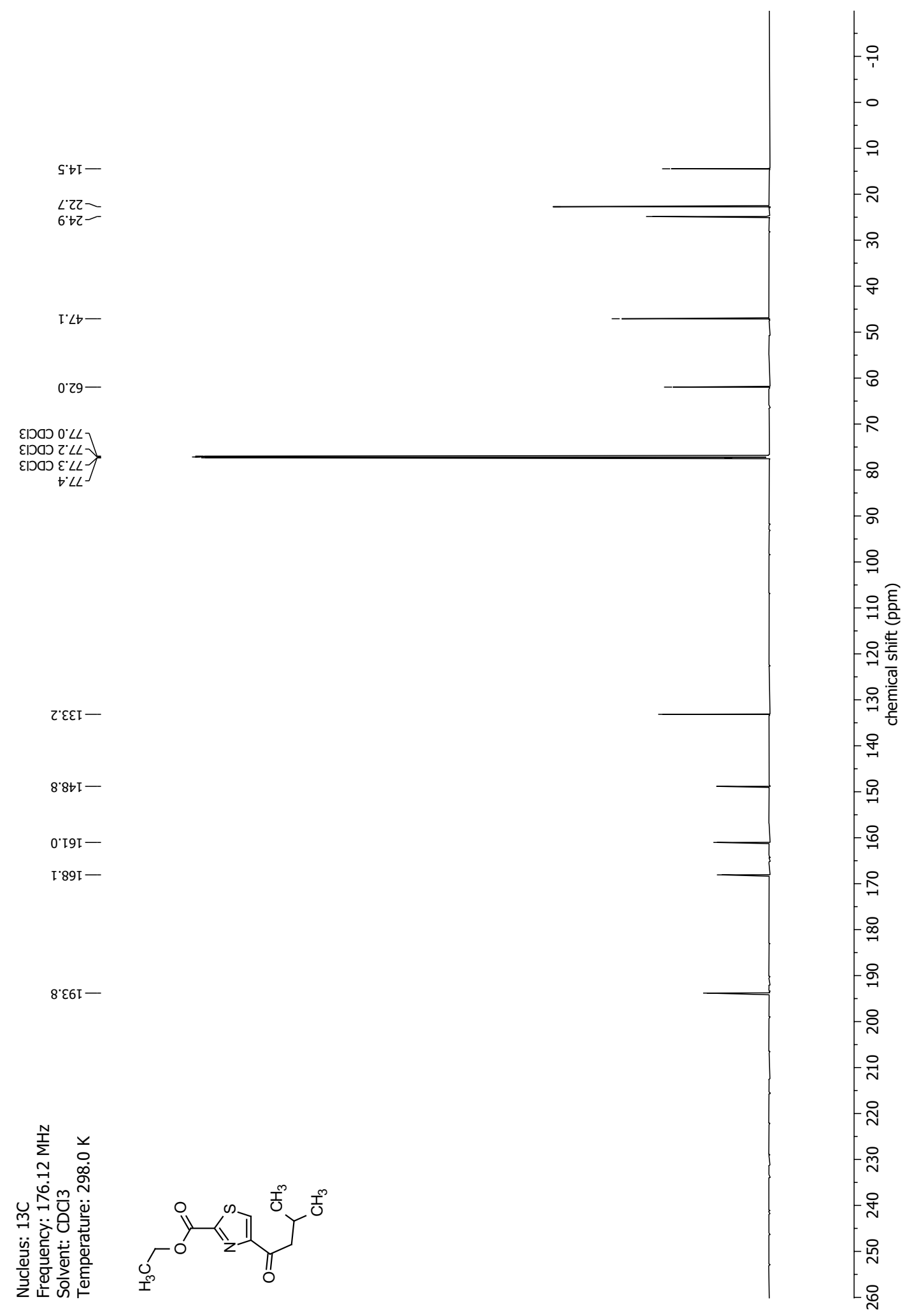


NMR-Spectra for Compound 47

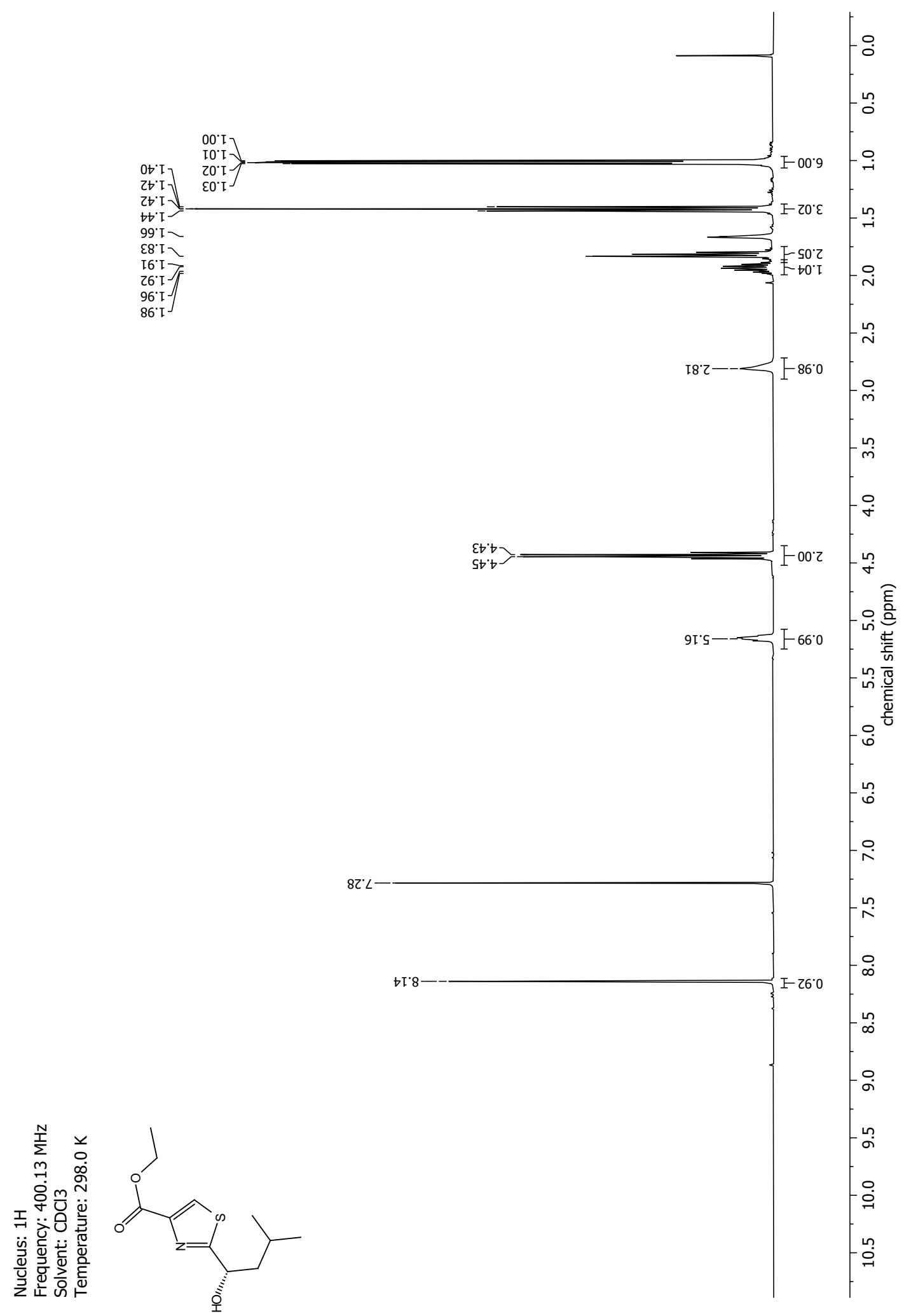




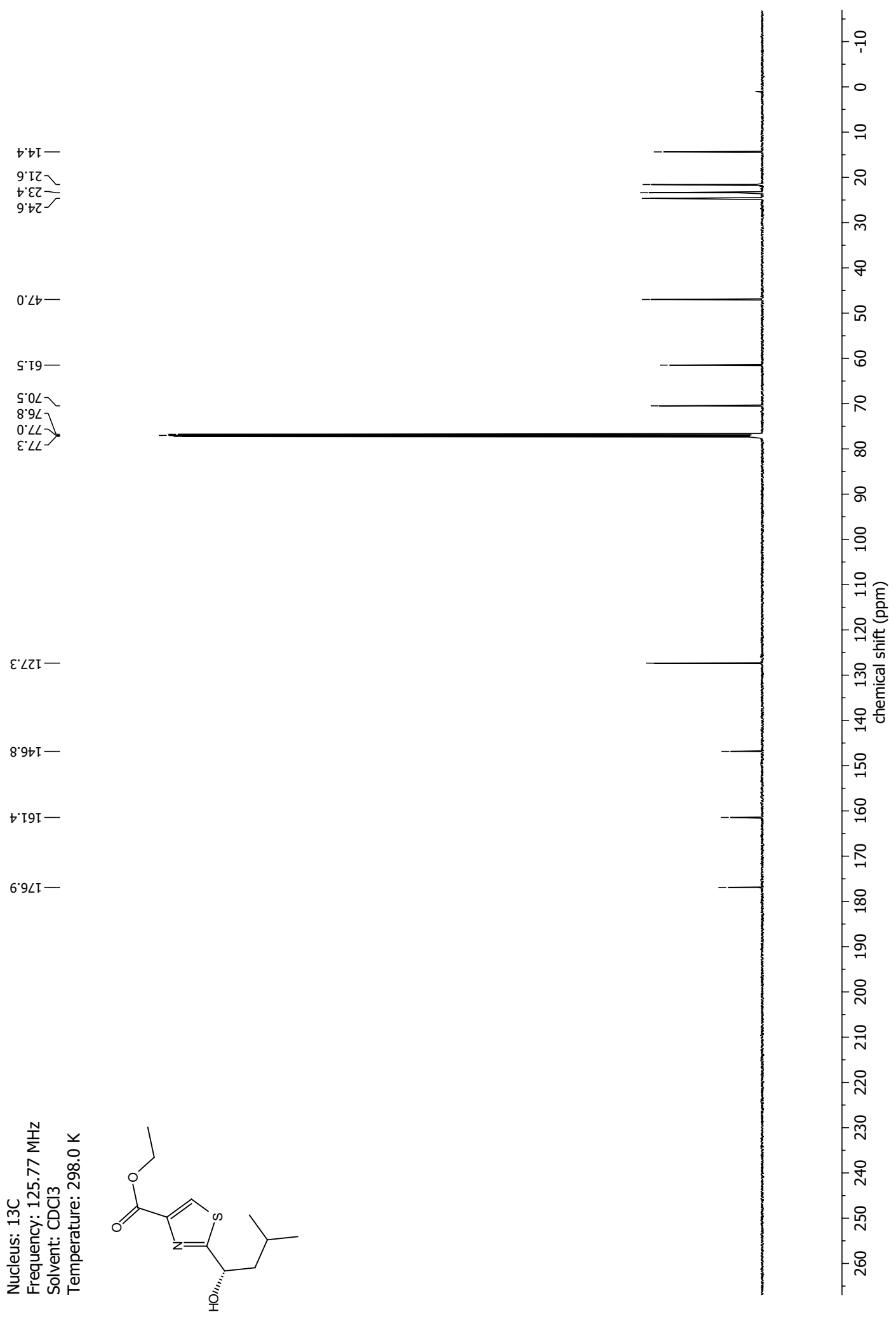


NMR-Spectra for Compound 51a

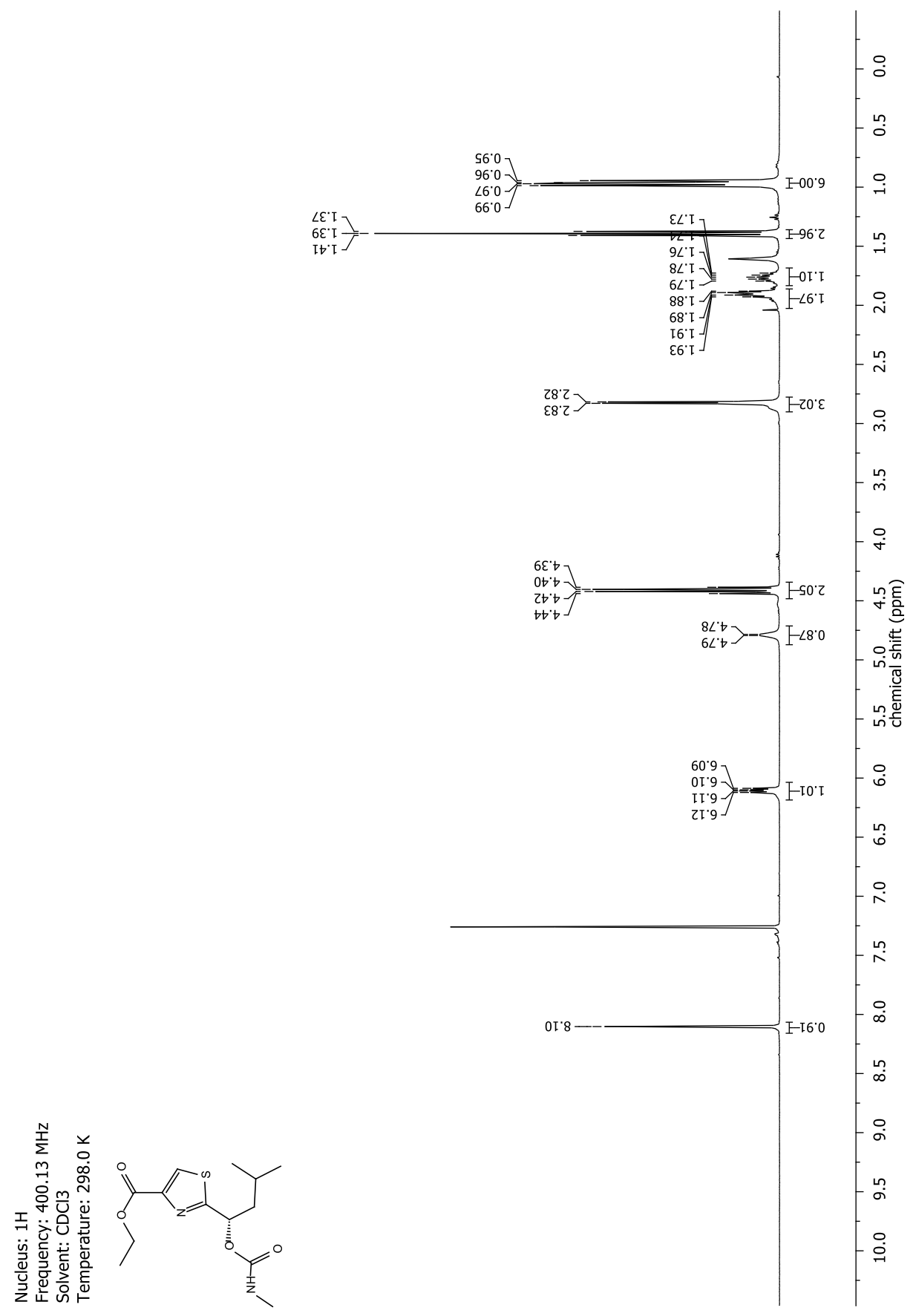




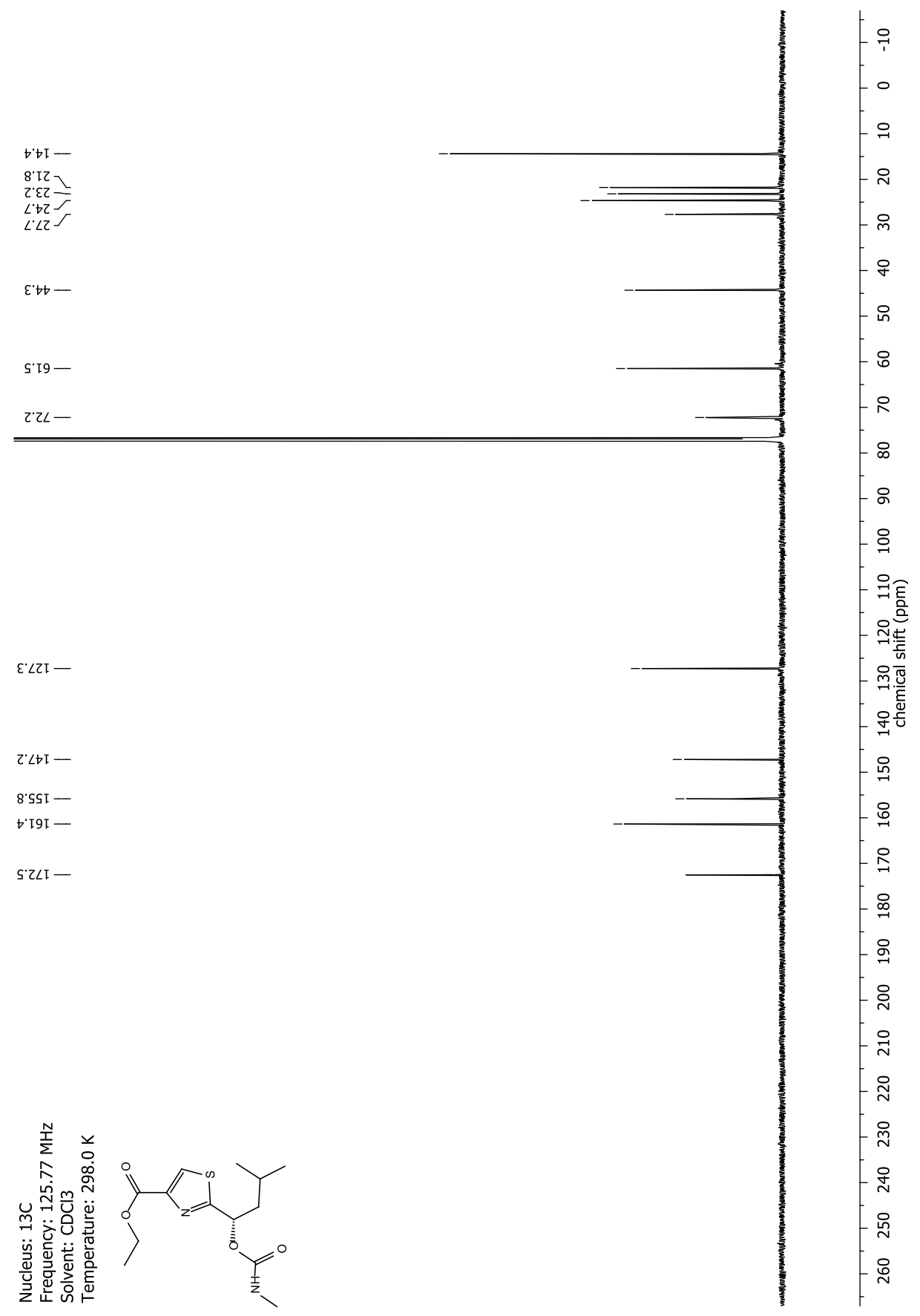


NMR-Spectra for Compound 52a

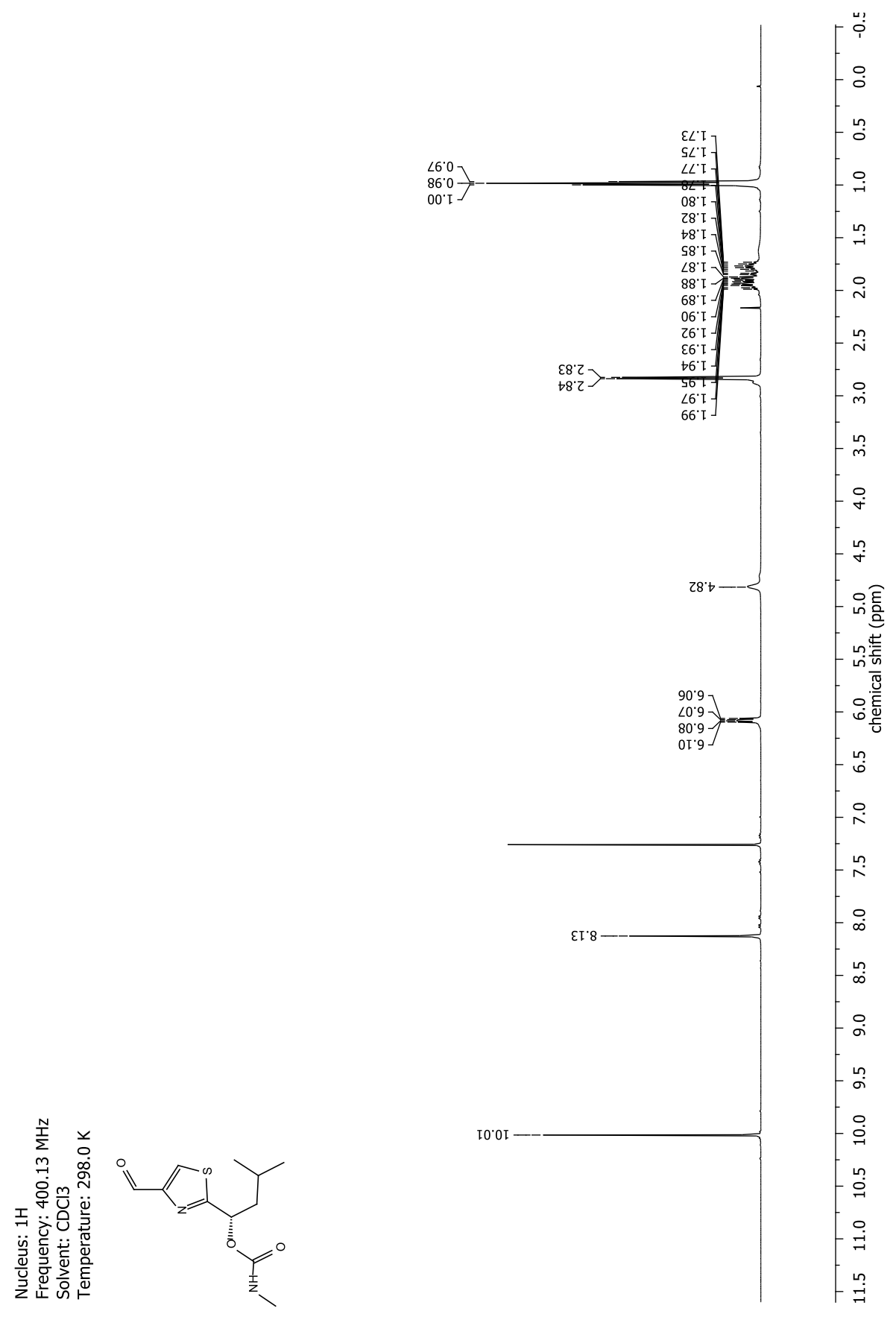




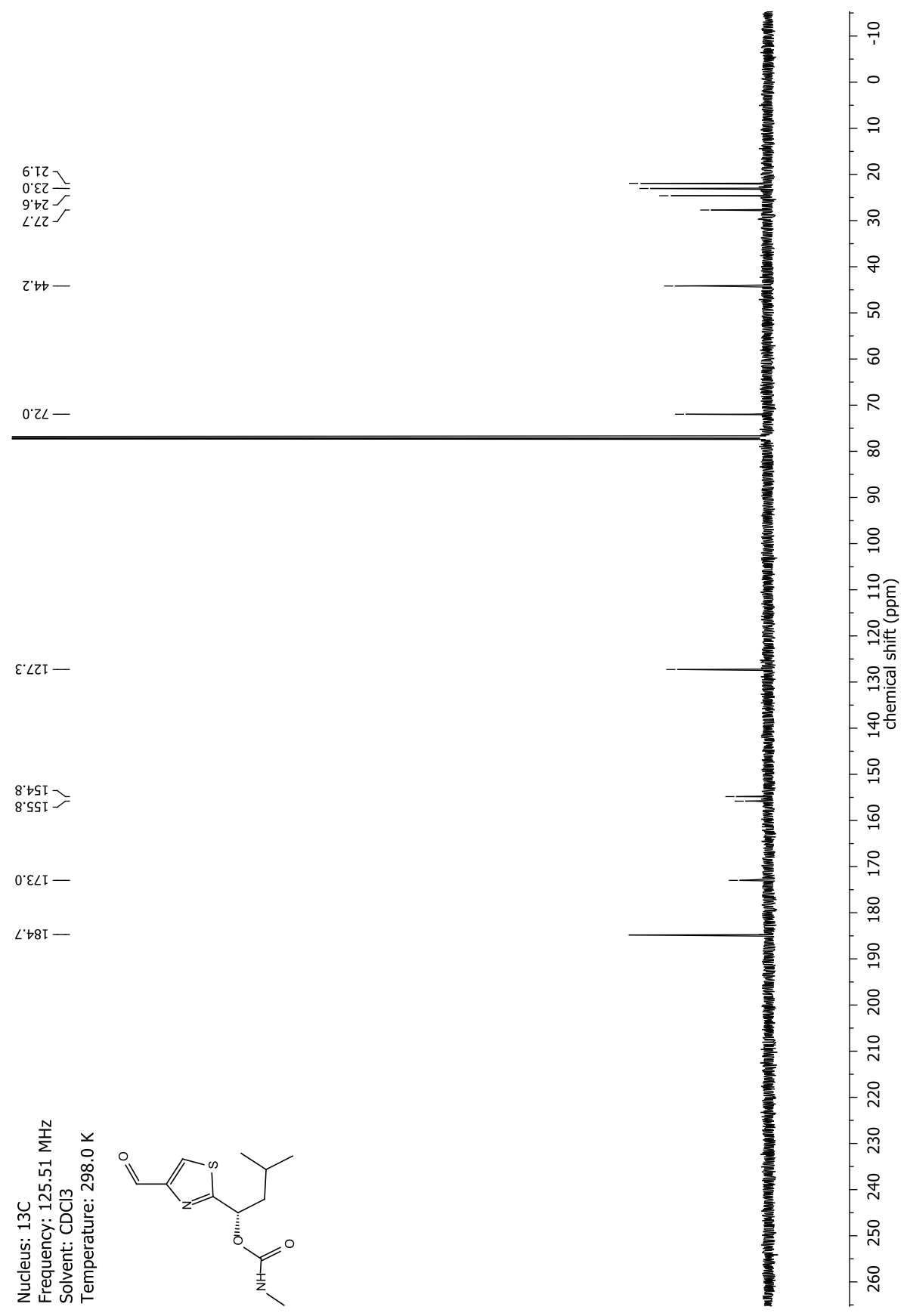


NMR-Spectra for Compound 53a

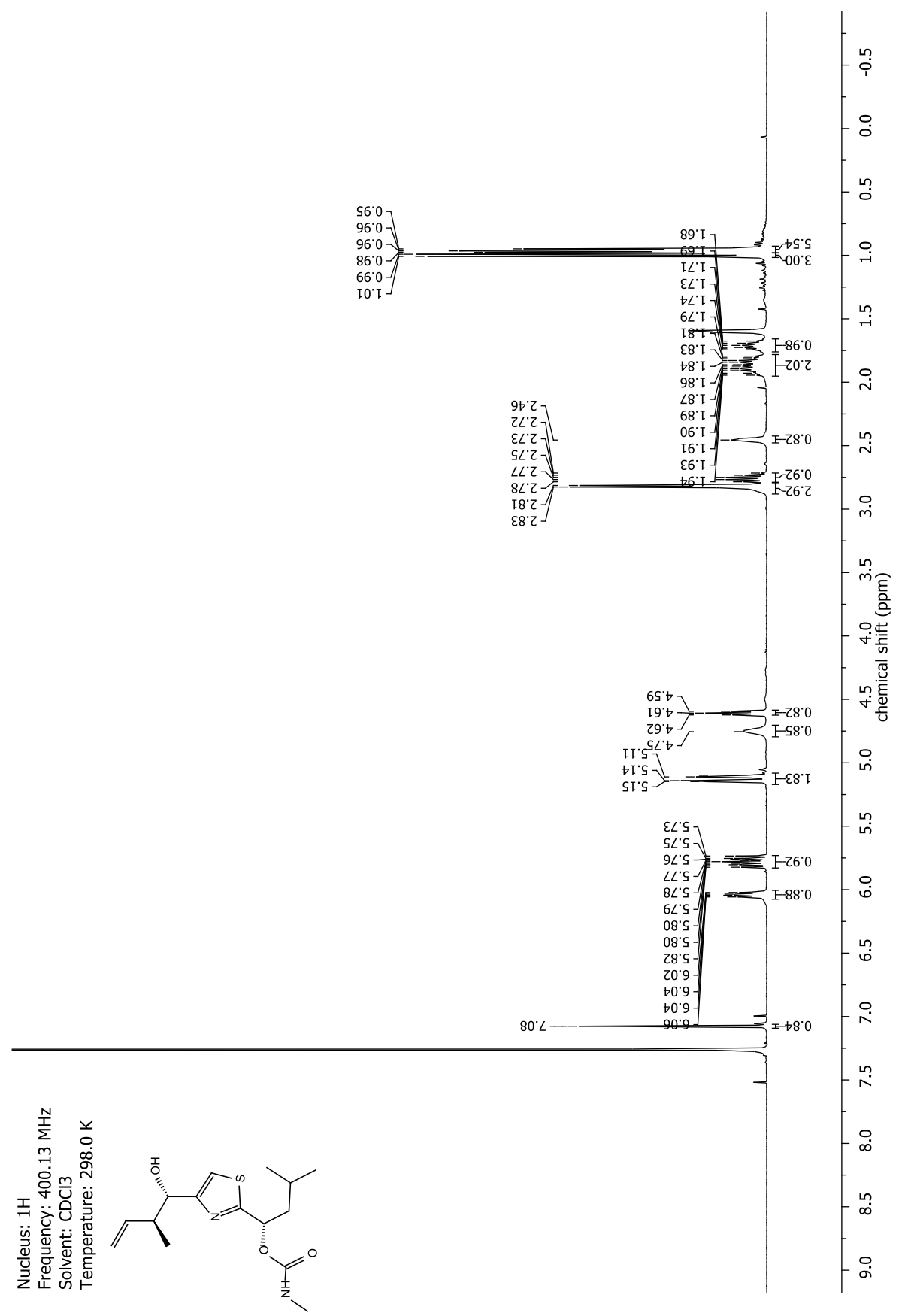




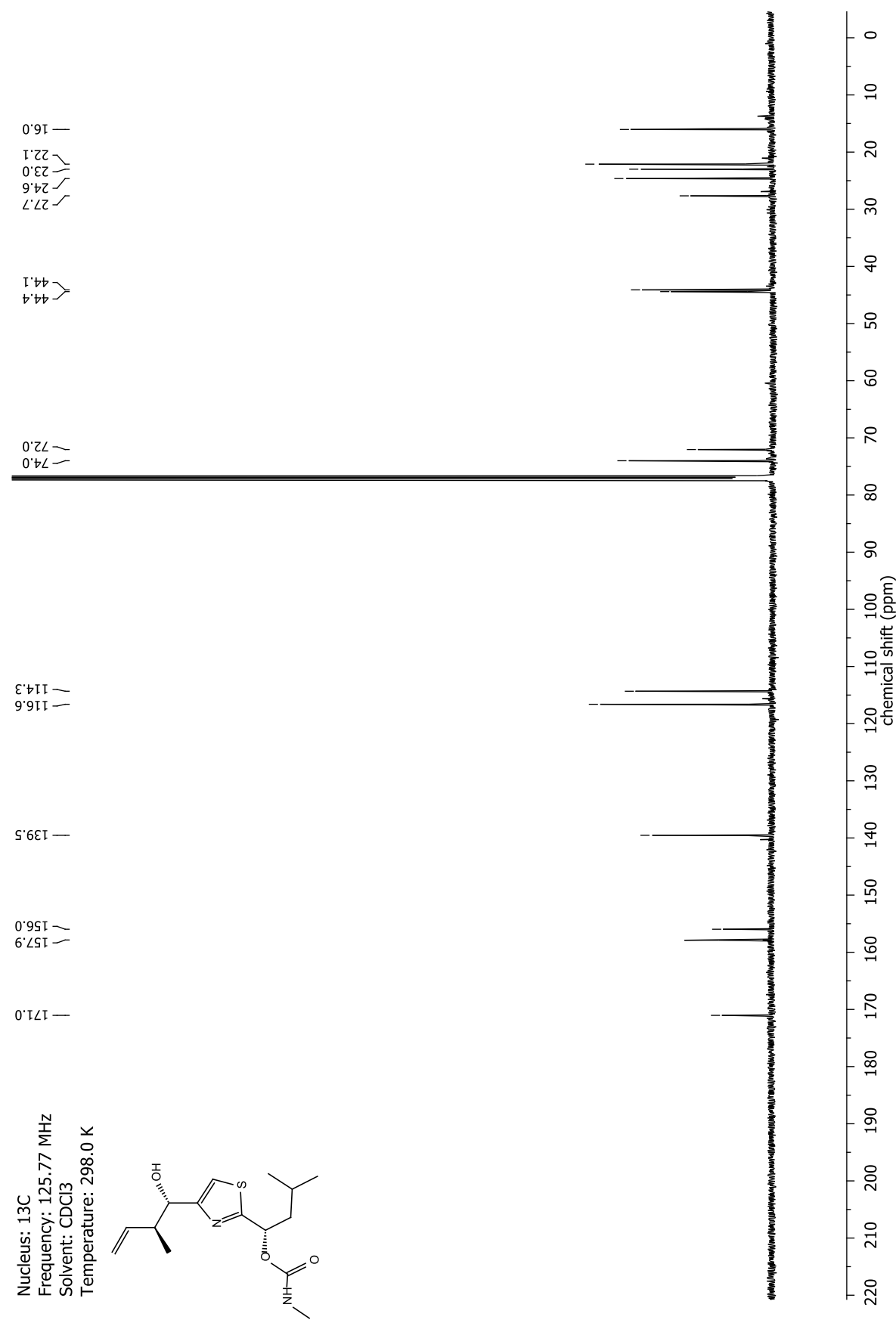


NMR-Spectra for Compound 54a

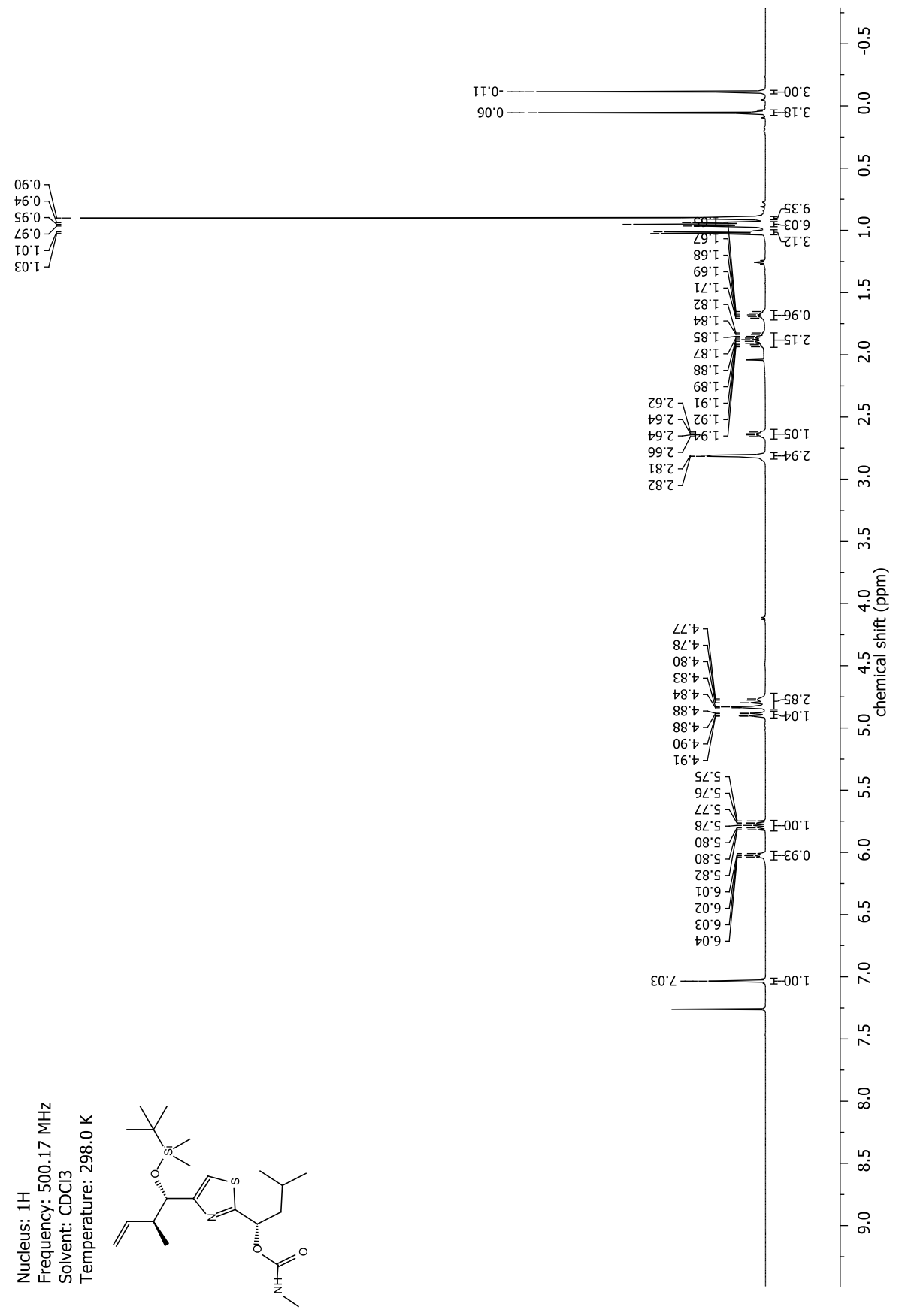




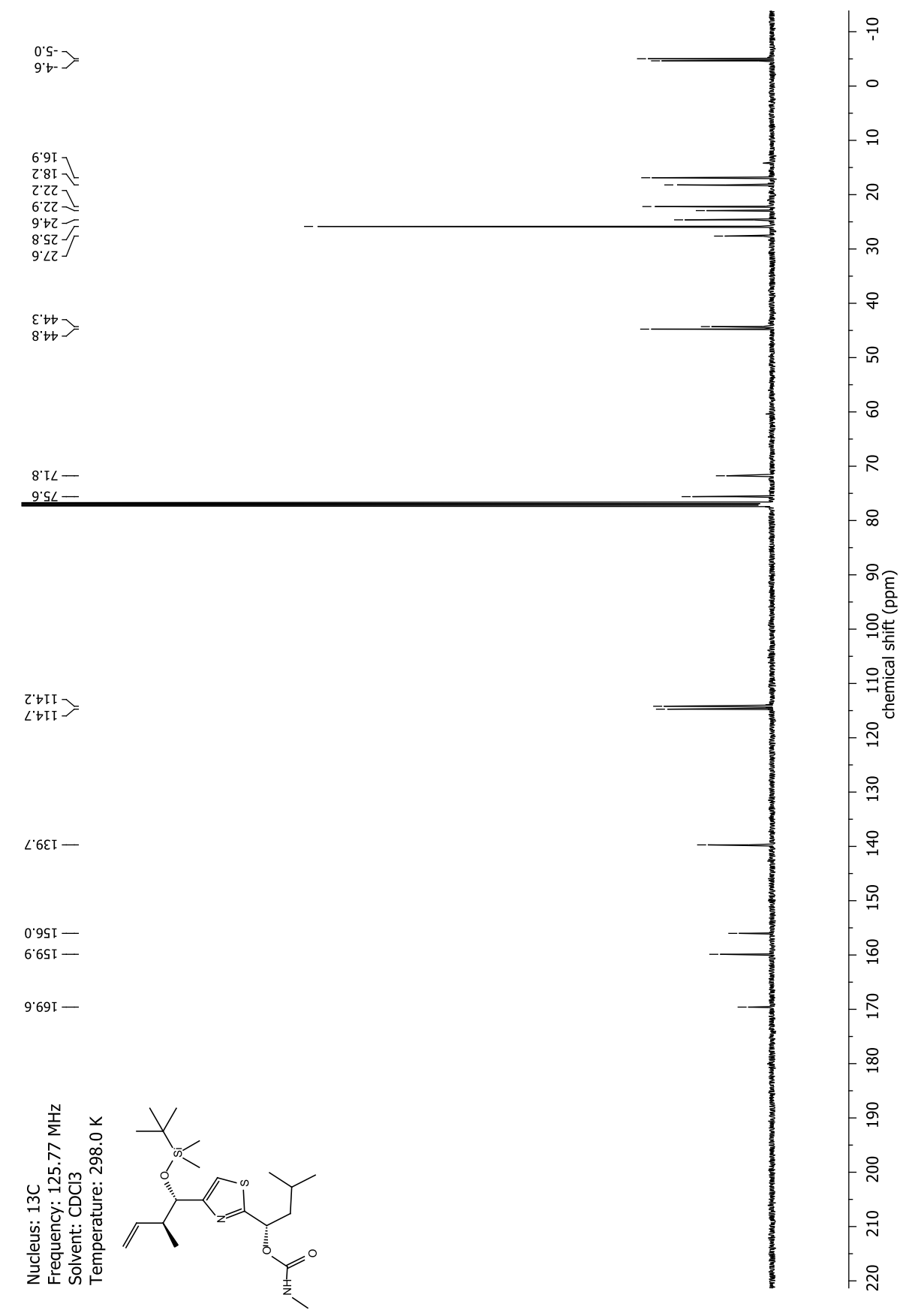


NMR-Spectra for Compound 46a

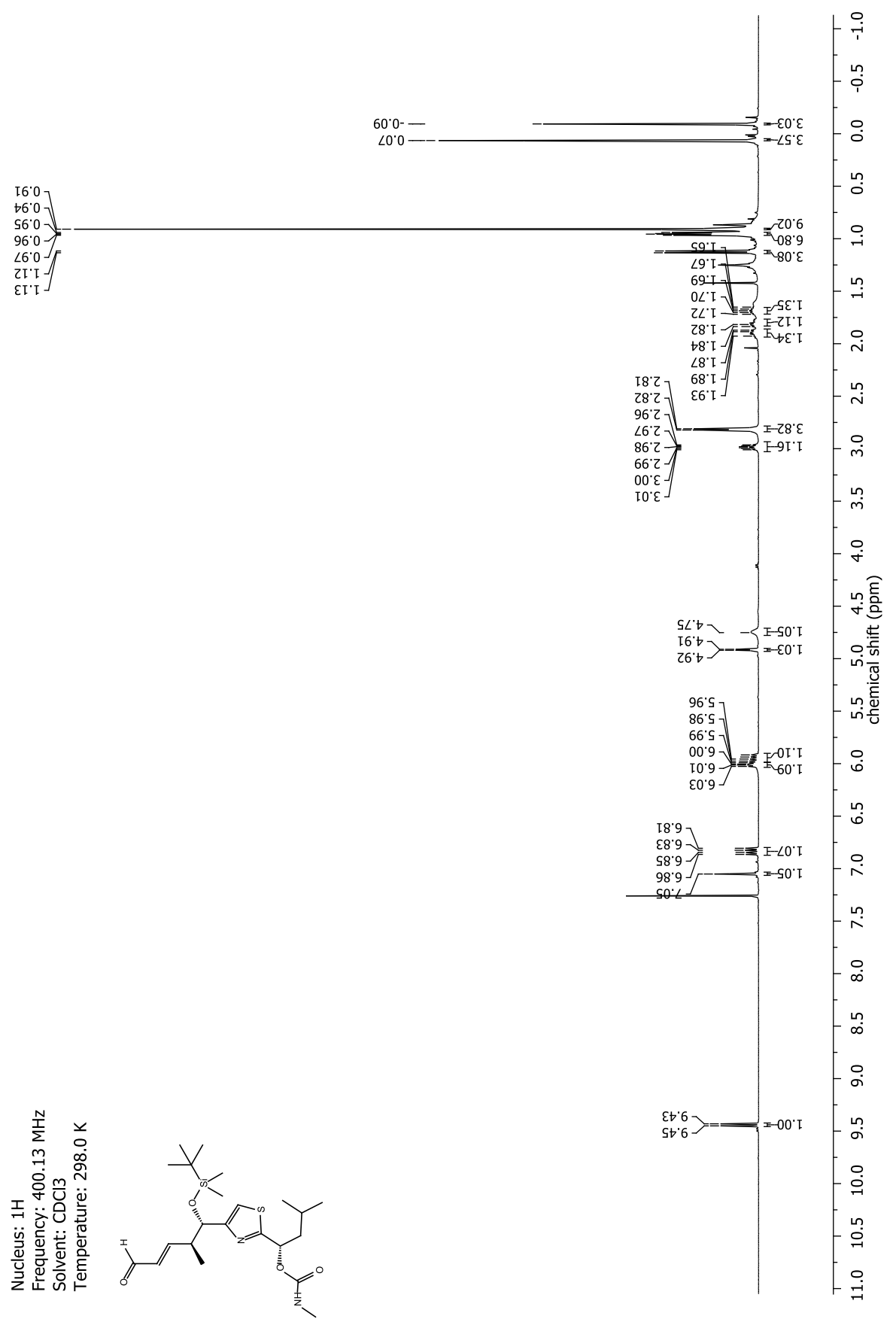




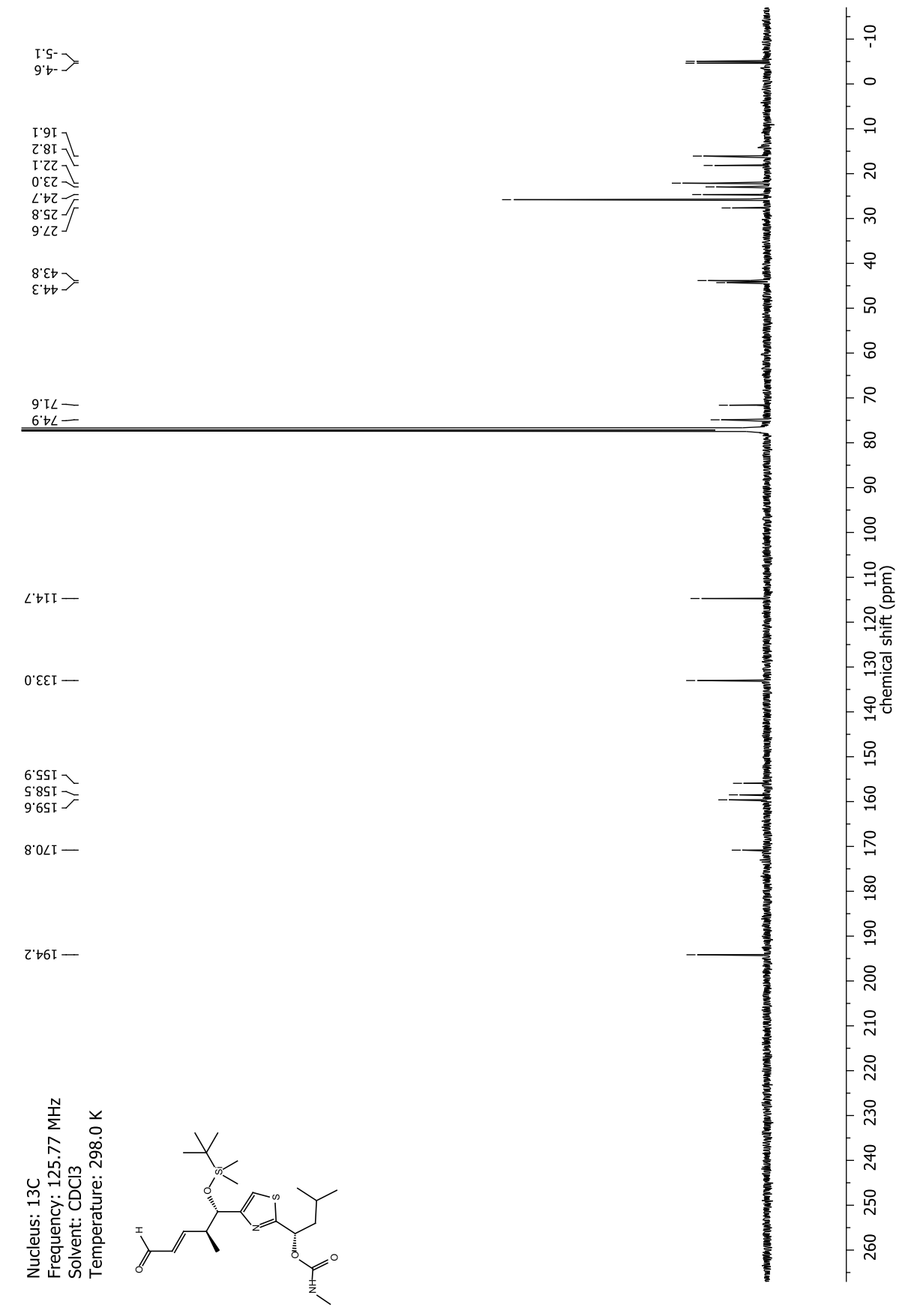


NMR-Spectra for Compound 51c

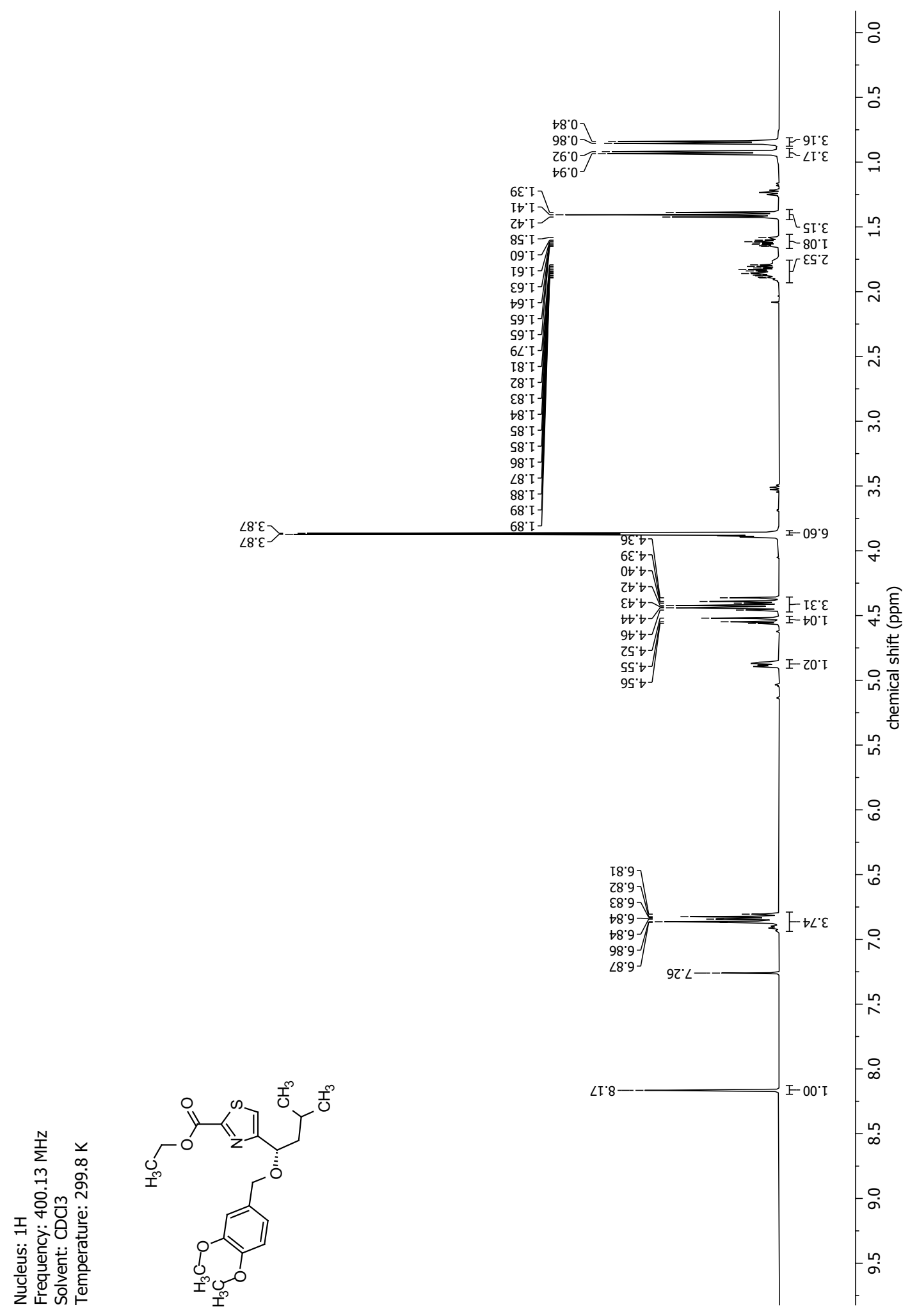




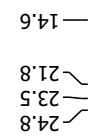

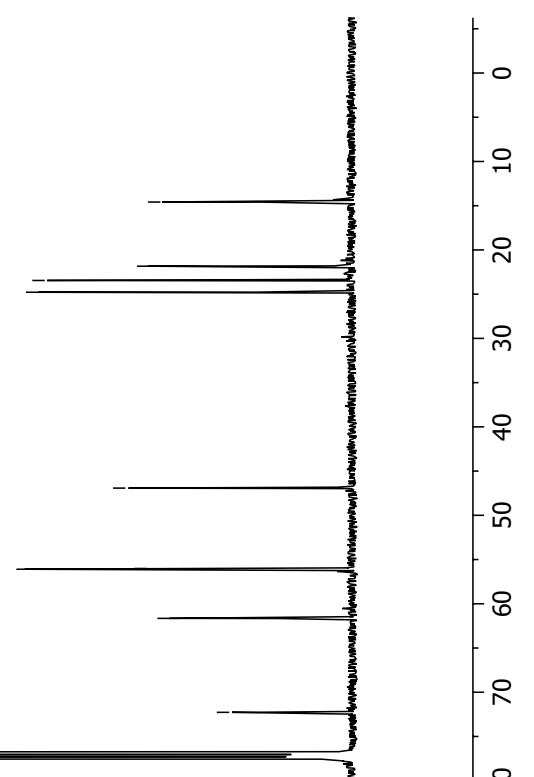

${ }^{0.95}$ T.95

L'I9-

$\varepsilon 2 L>$
$69 L$
6

$t: L{ }^{\prime}$

I'LII
9 'III

0.tZI-

$0.821-$

I.0८T二

$0.4 \mathrm{LT}>$

$\tau^{0} 66 t 5$

S'T9I-

S'9டI-
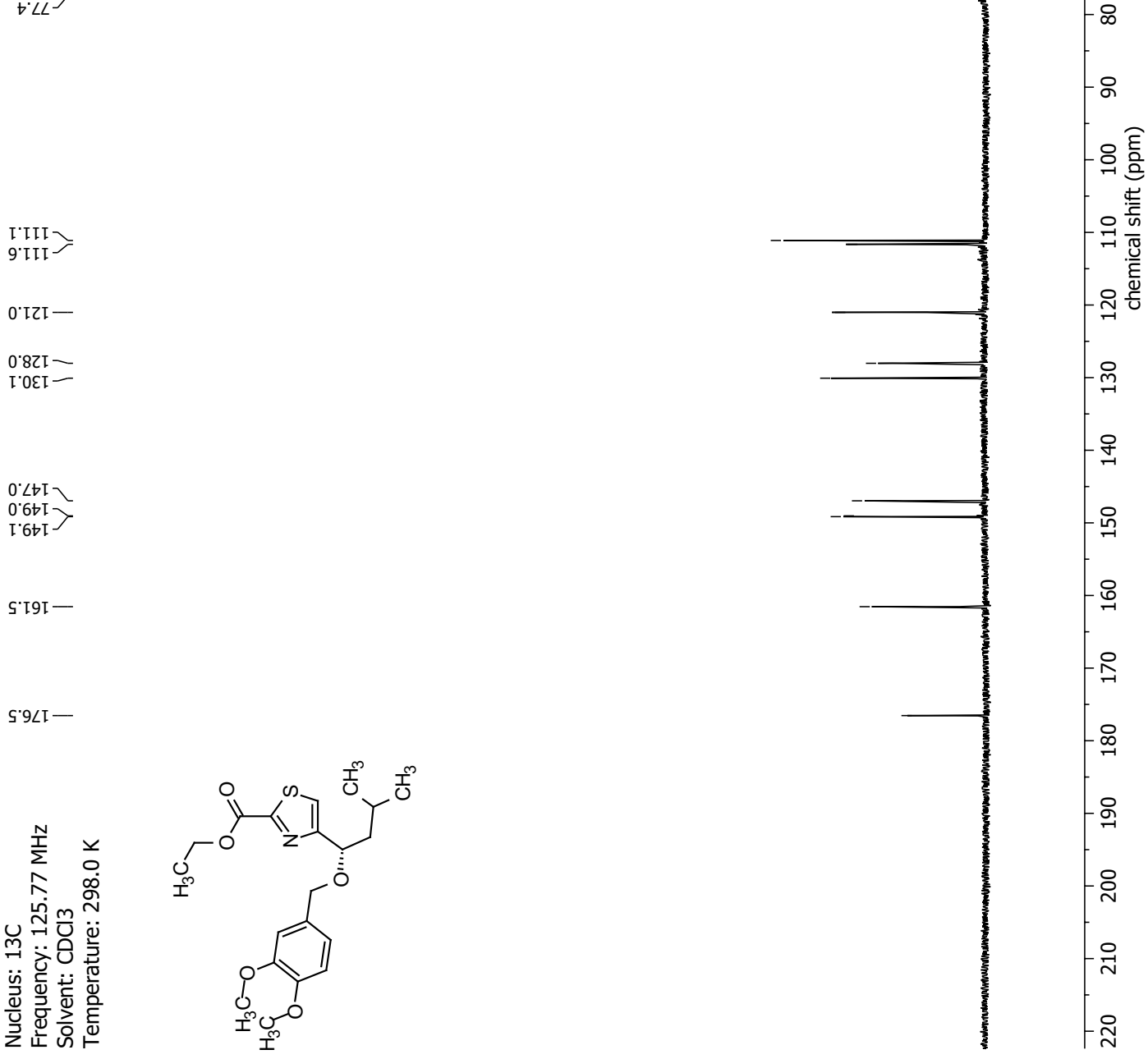
NMR-Spectra for Compound 101

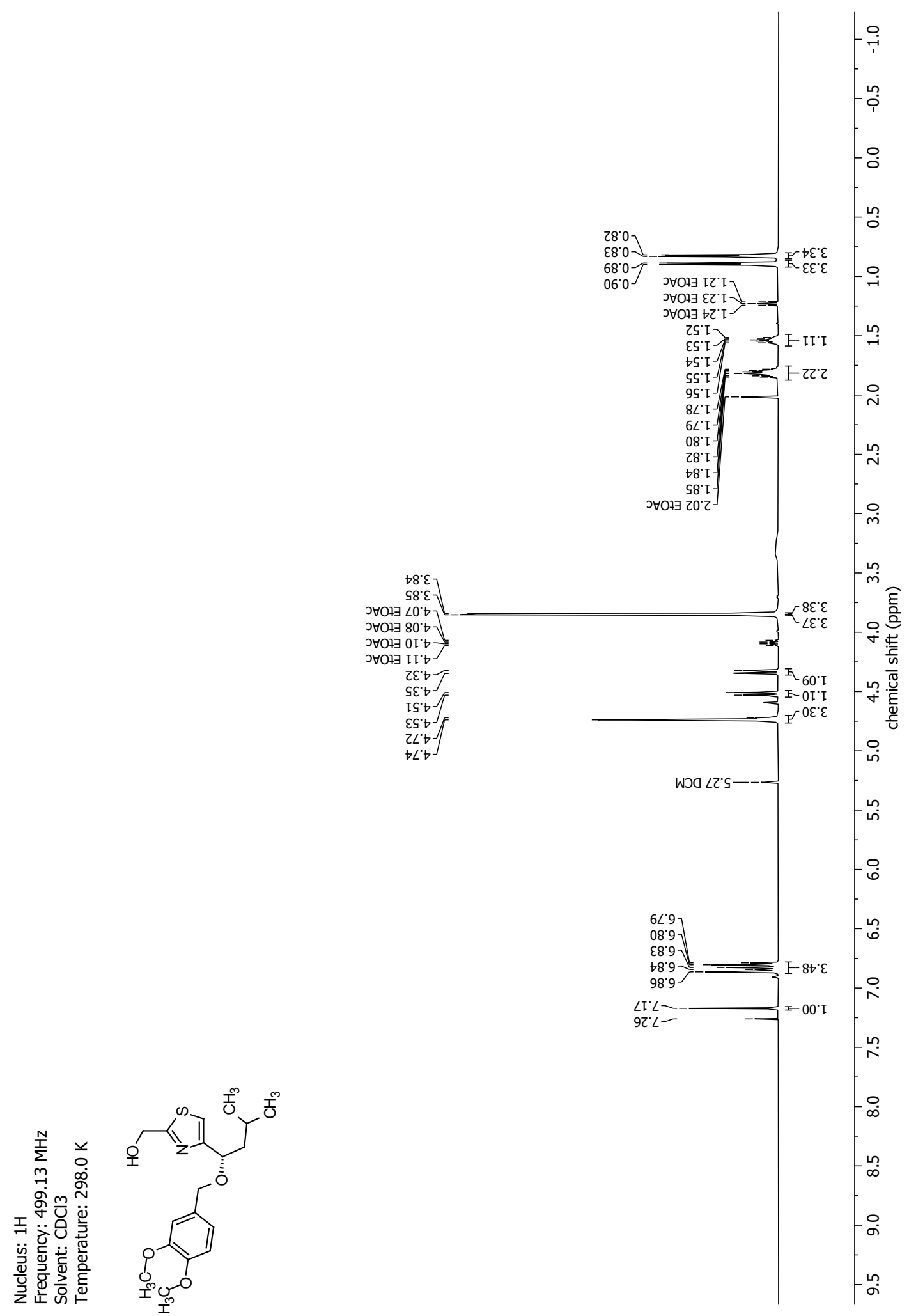




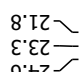

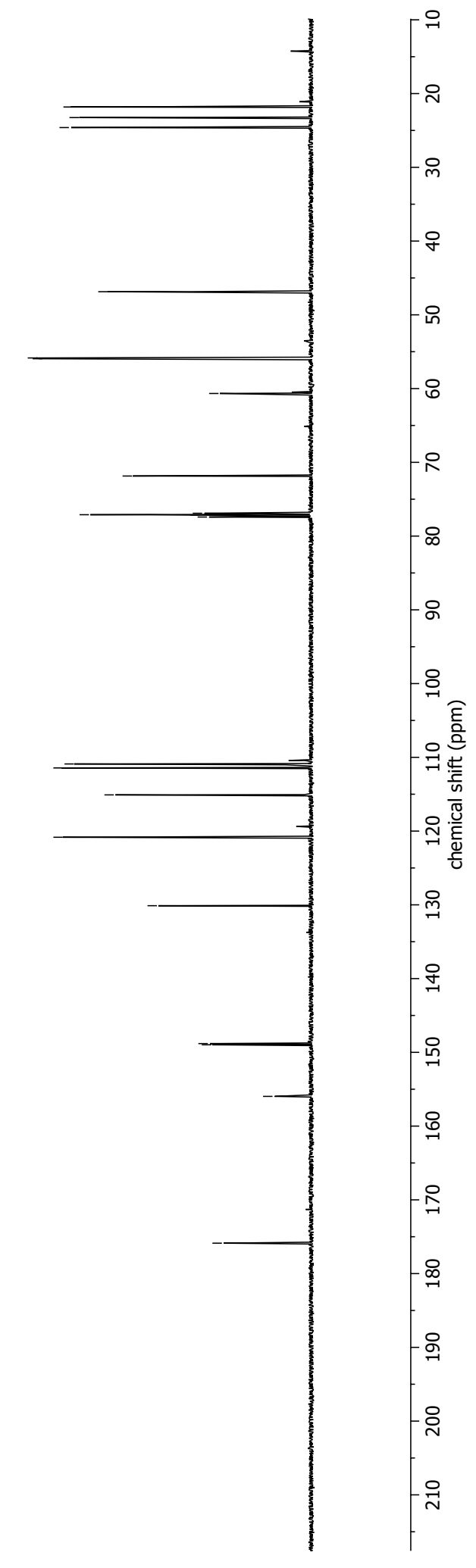

6.0II

t'III
I'SI

8.0ZI -

I’0عI-

$\begin{aligned} & 8.8 b I \\ & 0.6 b I\end{aligned}>$

$0.9 S I-$

6.SLI-

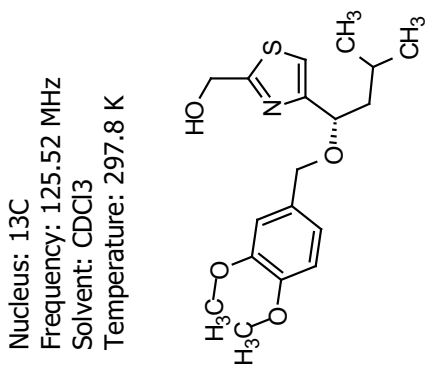


NMR-Spectra for Compound 52c

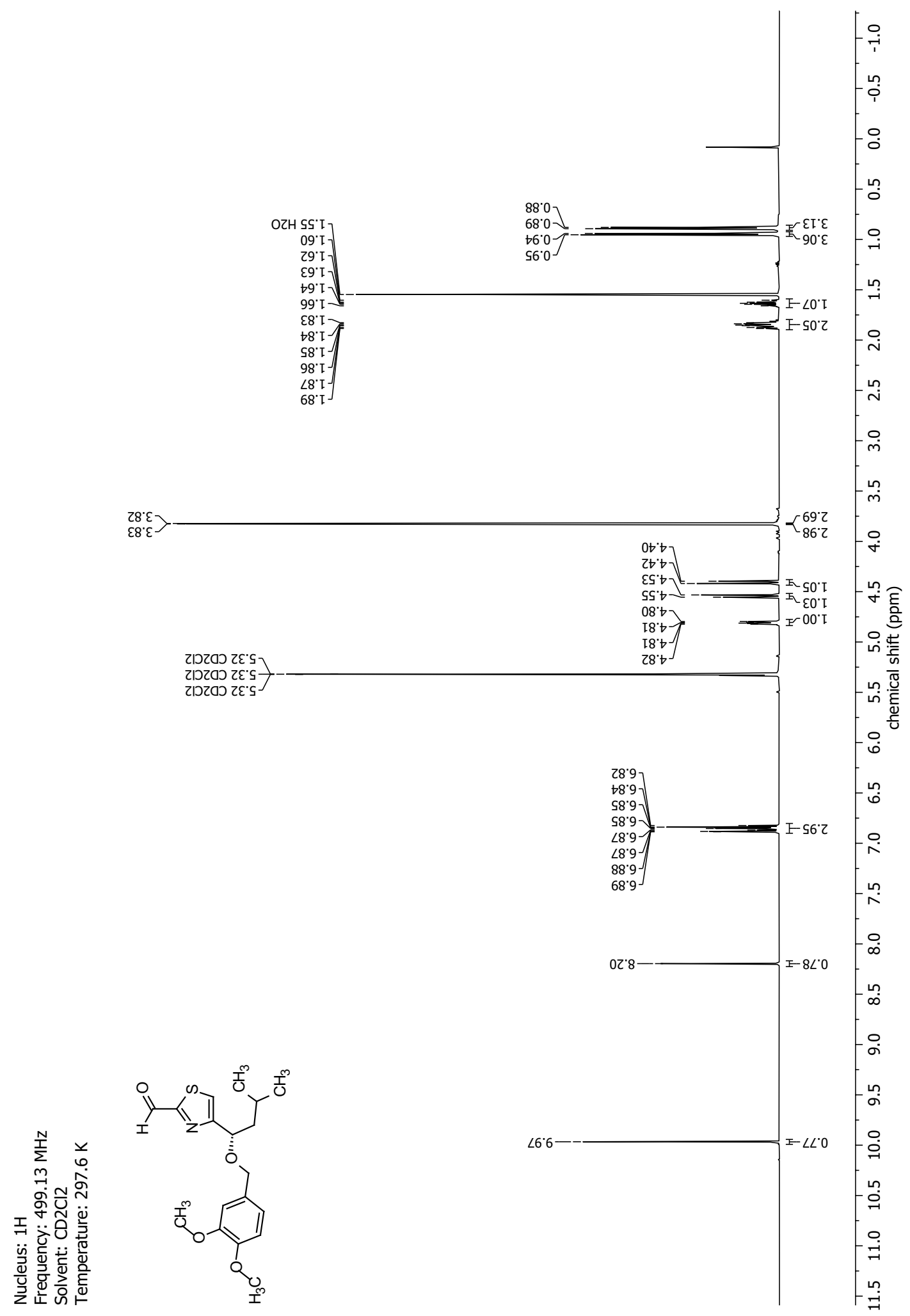




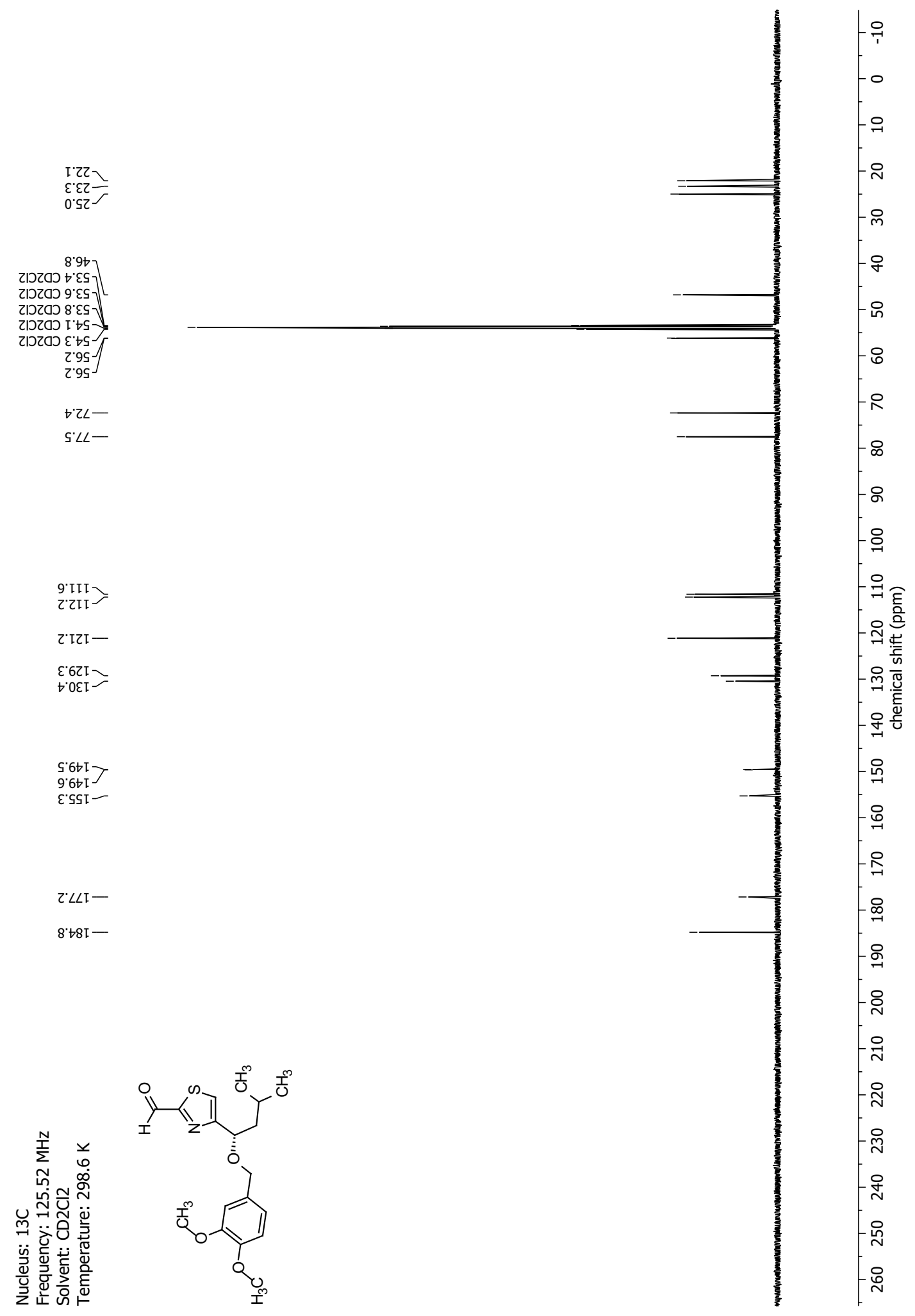


NMR-Spectra for Compound 53c

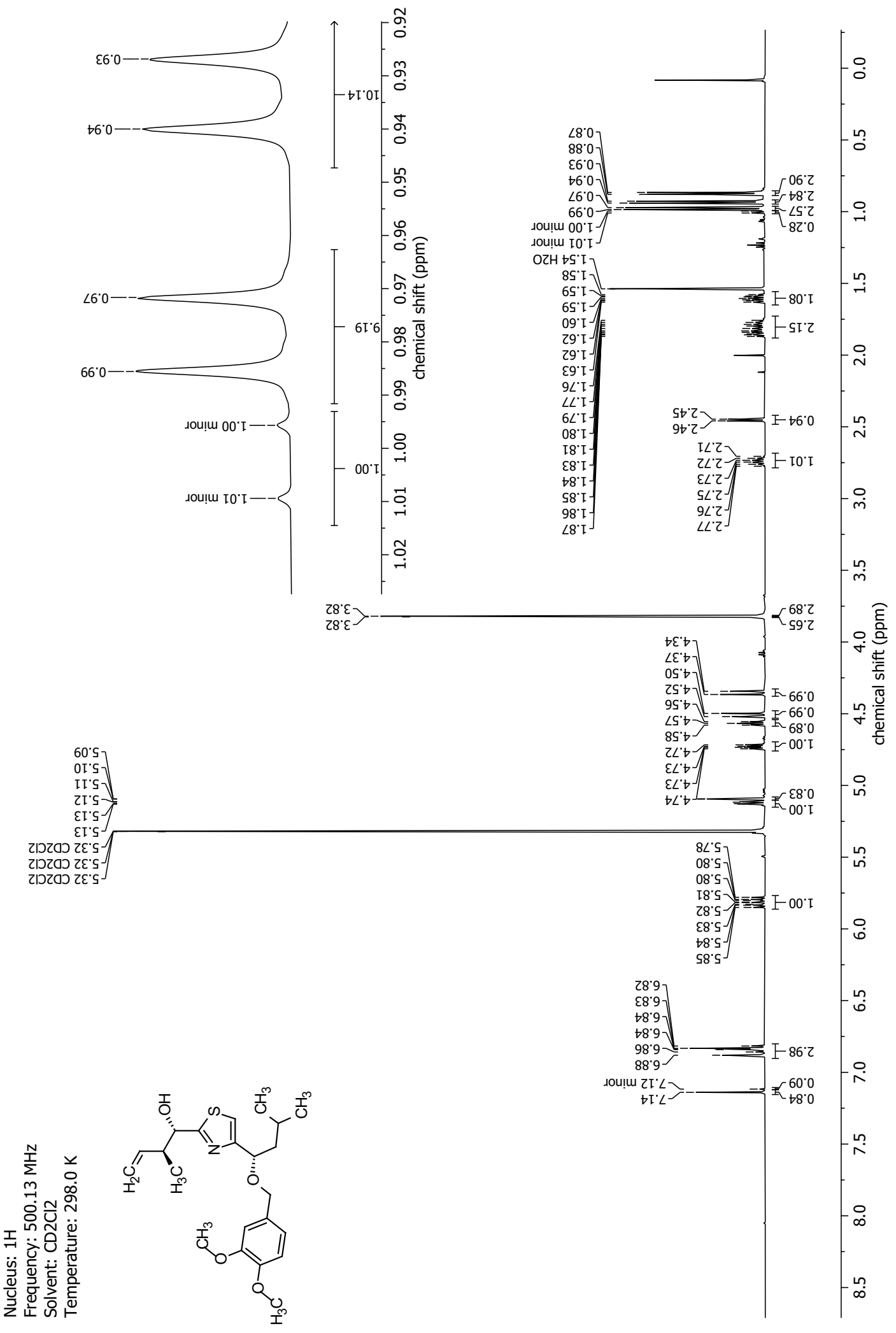




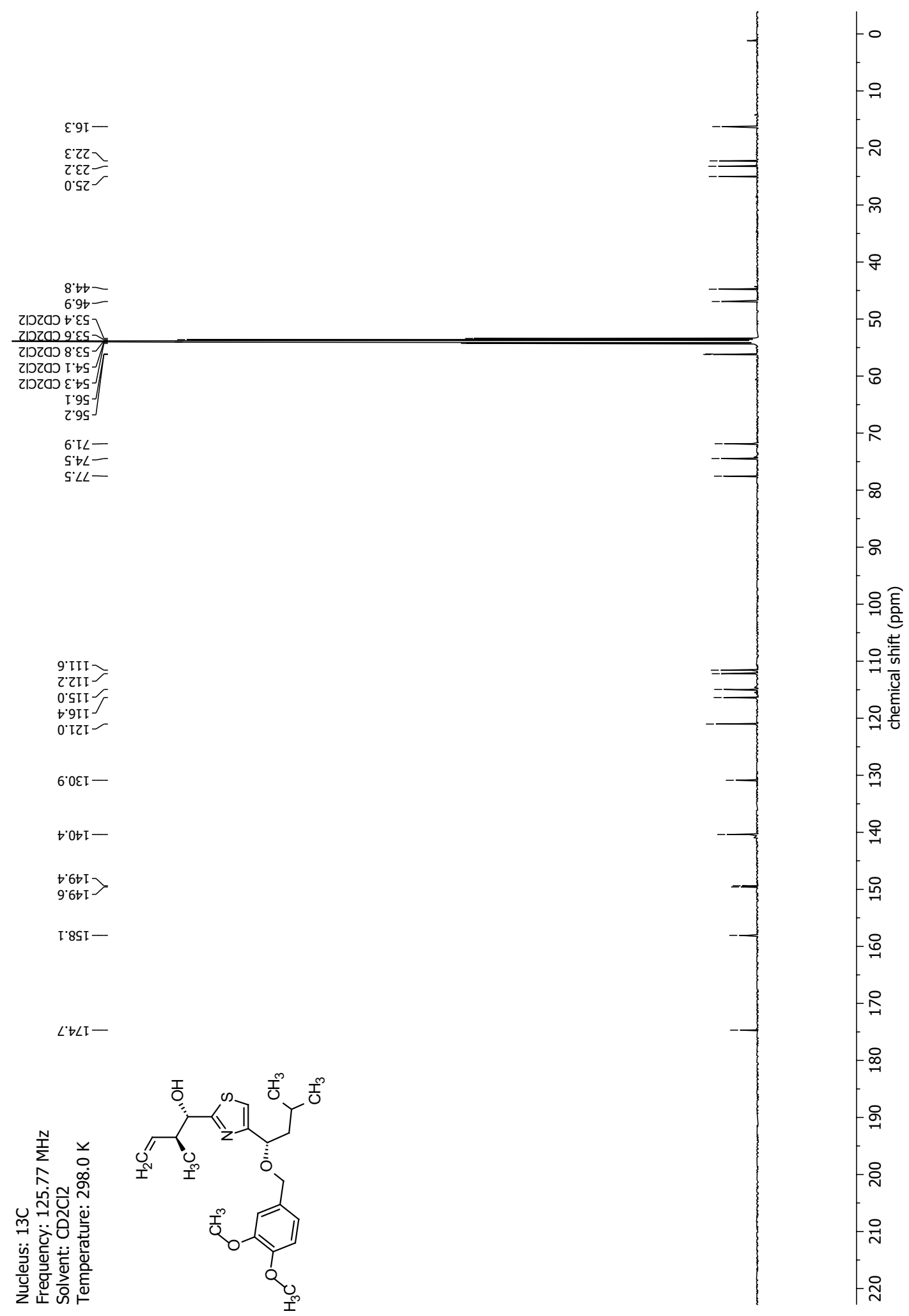


NMR-Spectra for Compound 54c

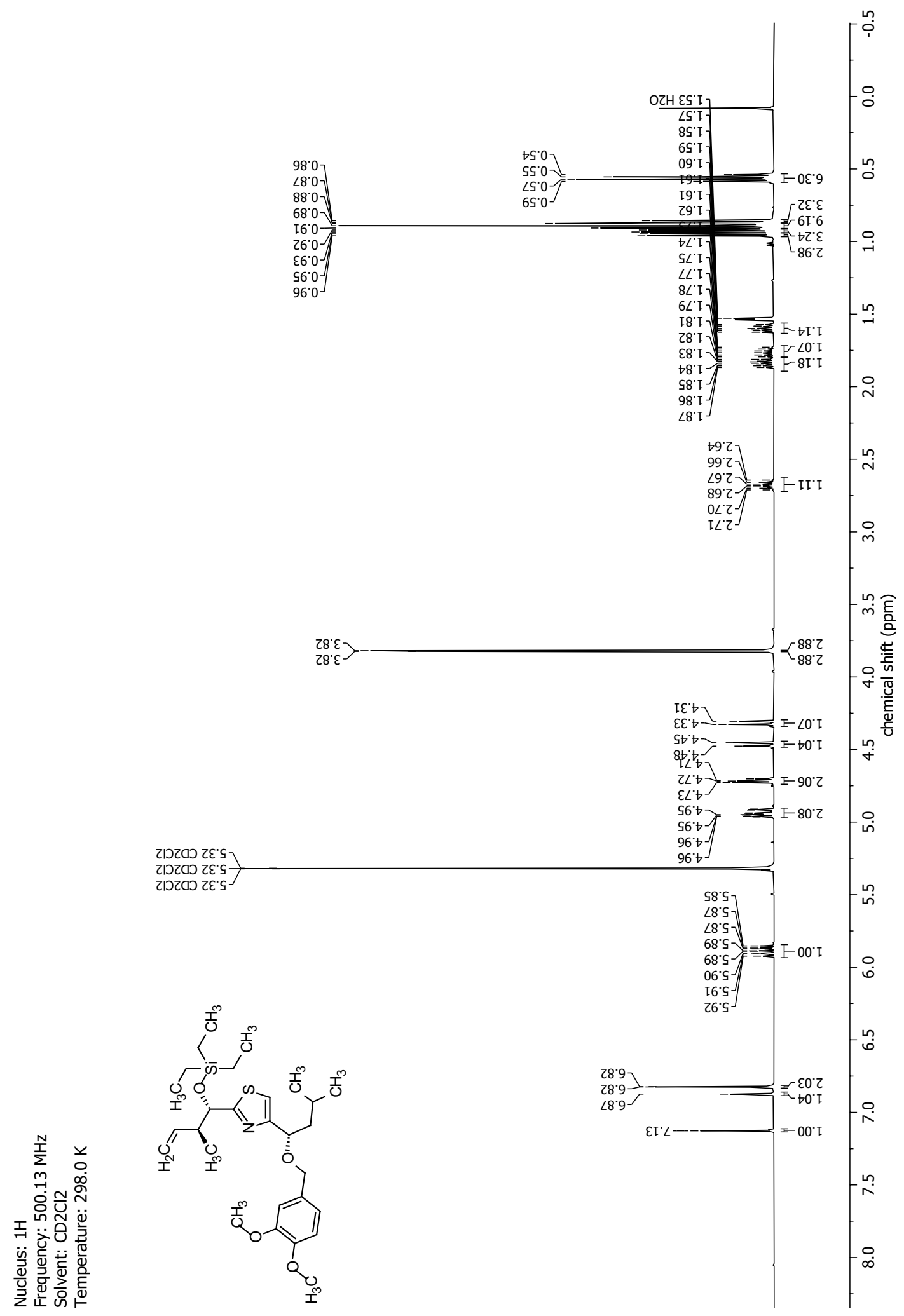




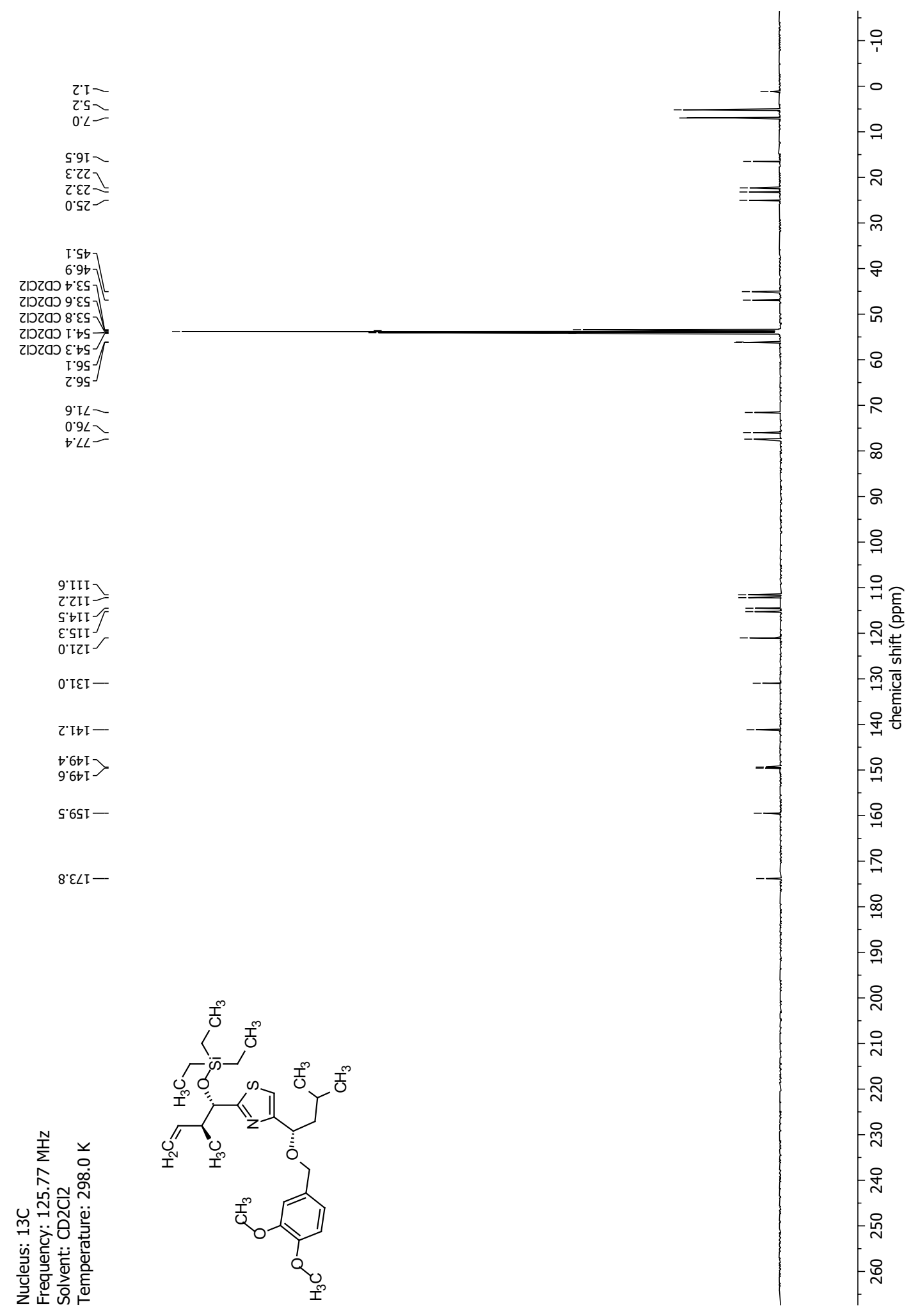


NMR-Spectra for Compound 46c

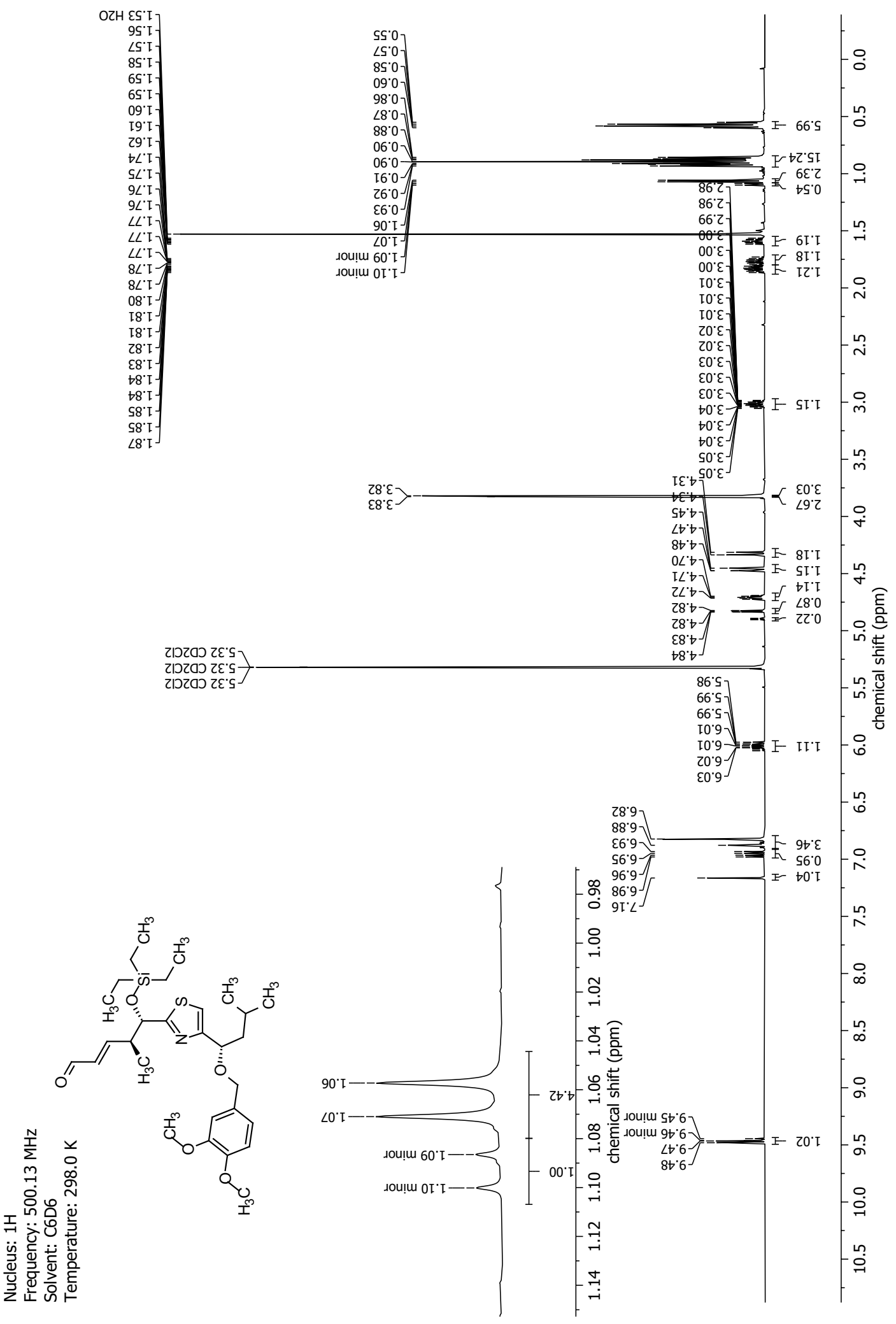




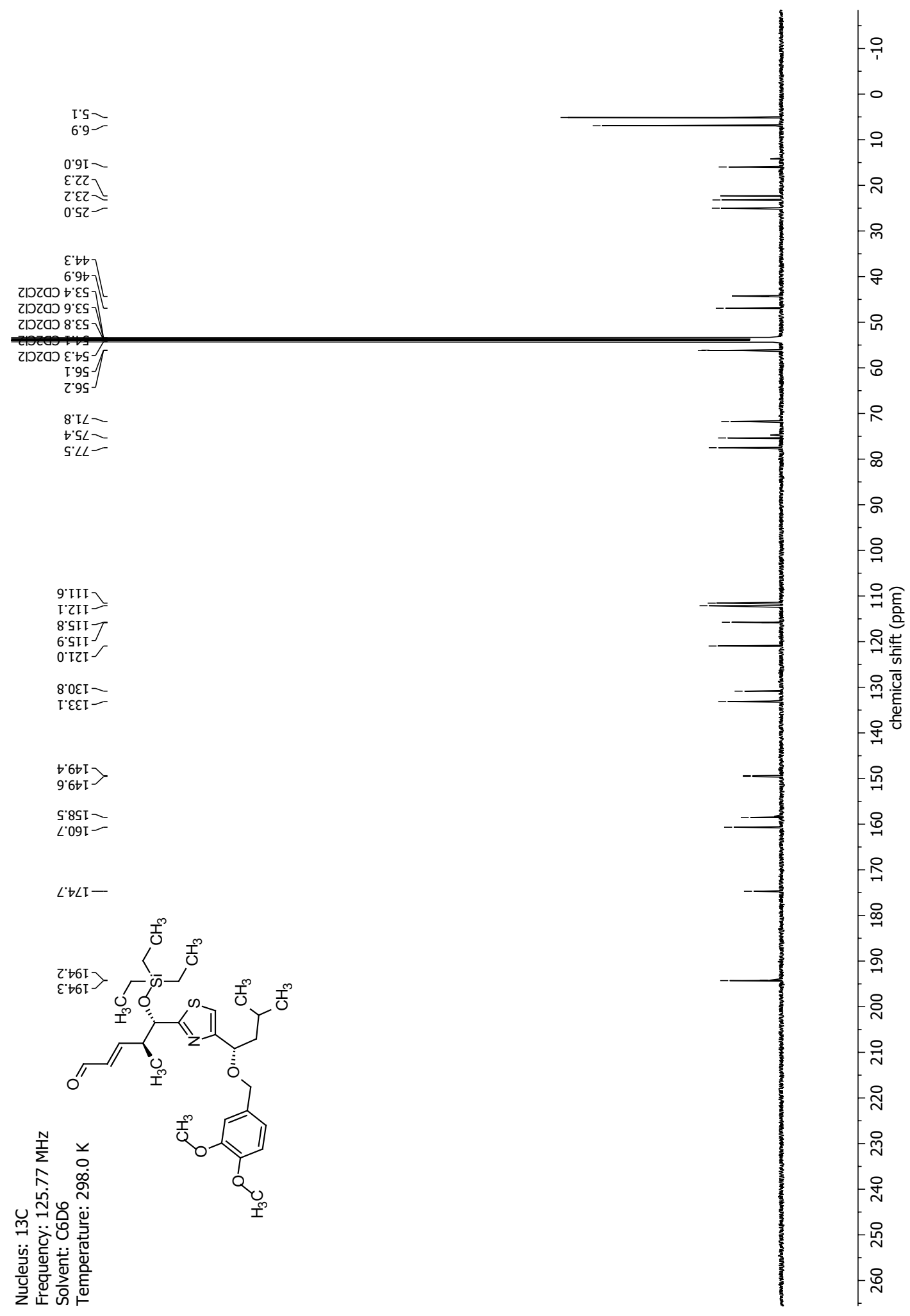


NMR-Spectra for Compound 102

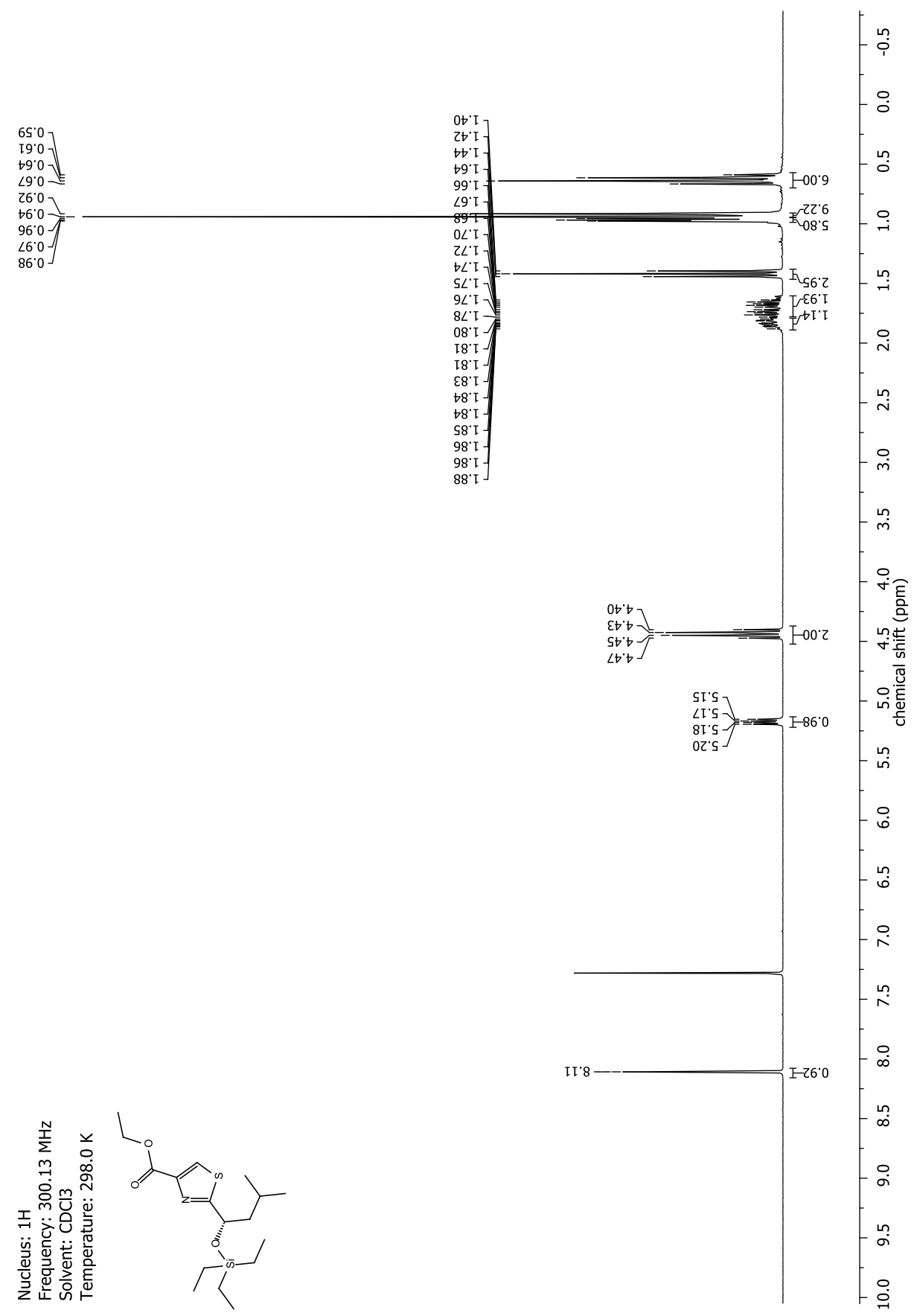




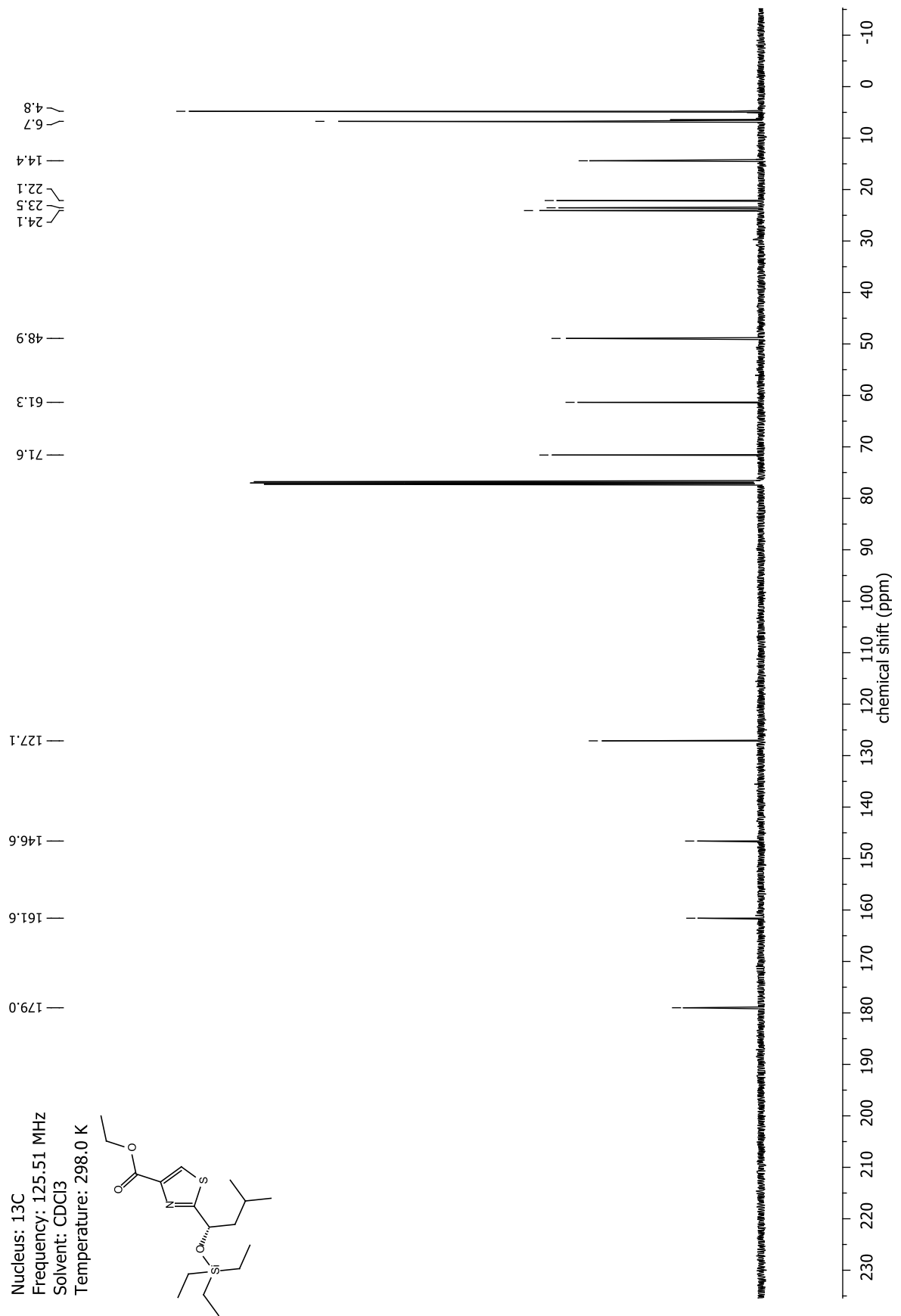


NMR-Spectra for Compound 52e

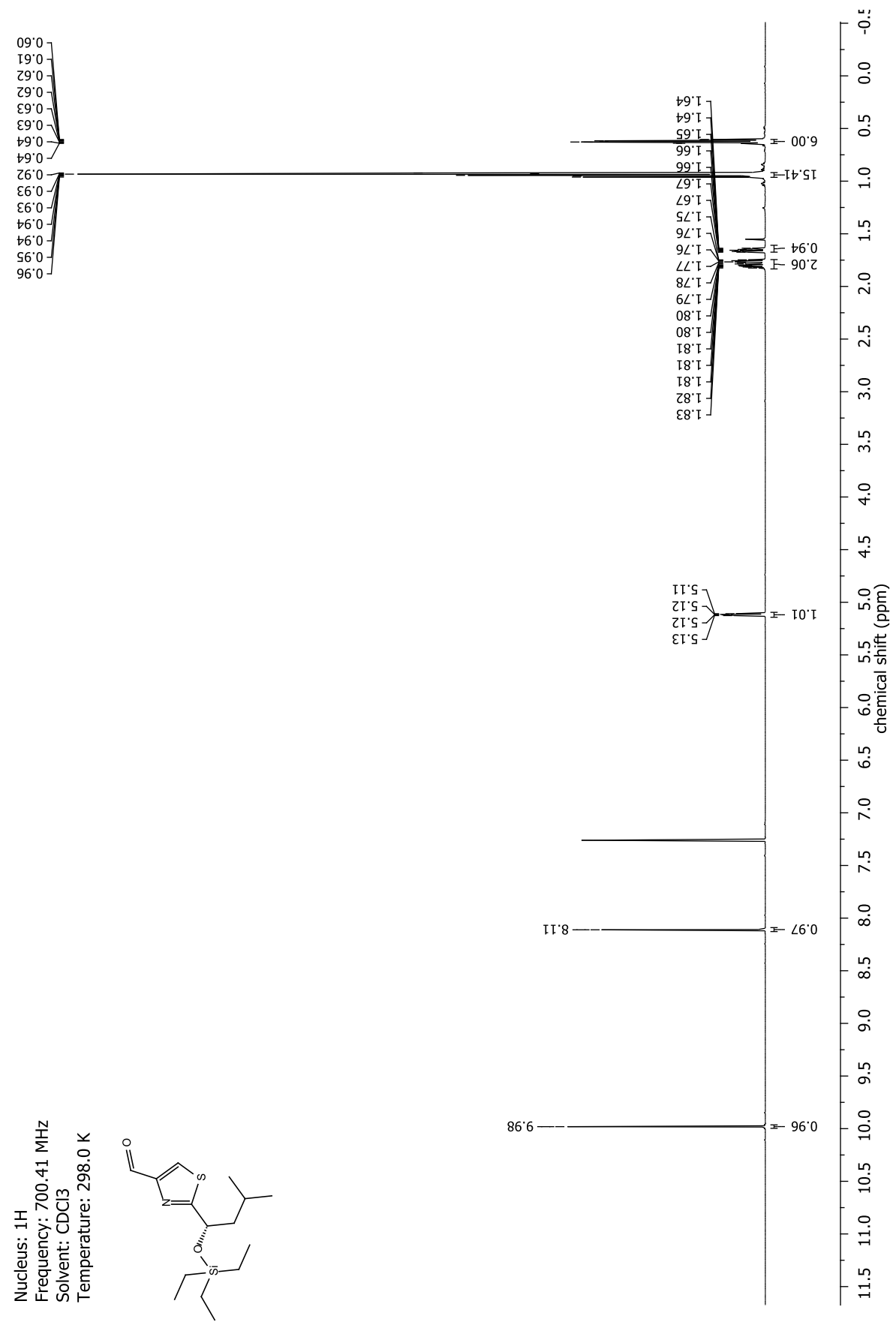




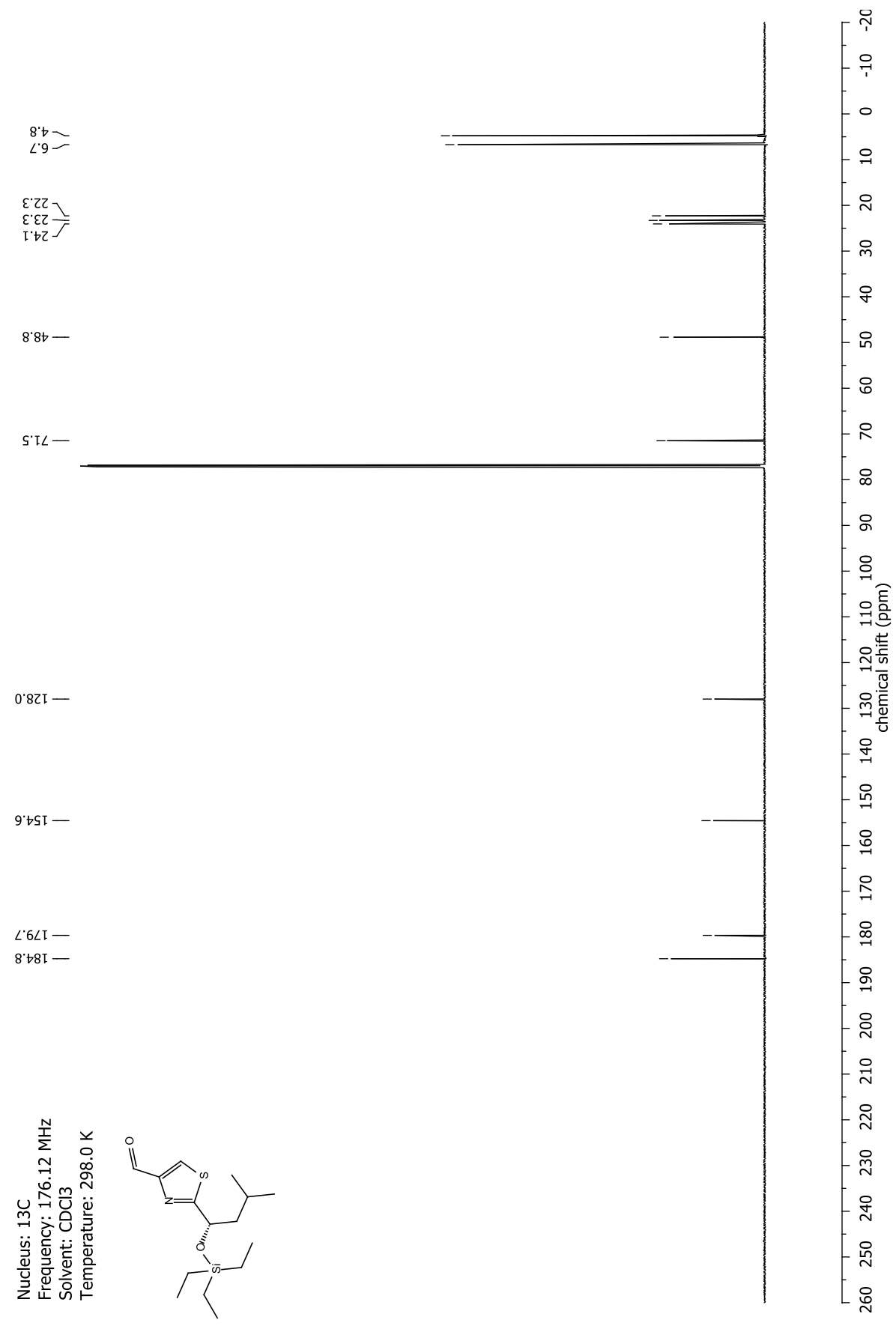


NMR-Spectra for Compound 53e

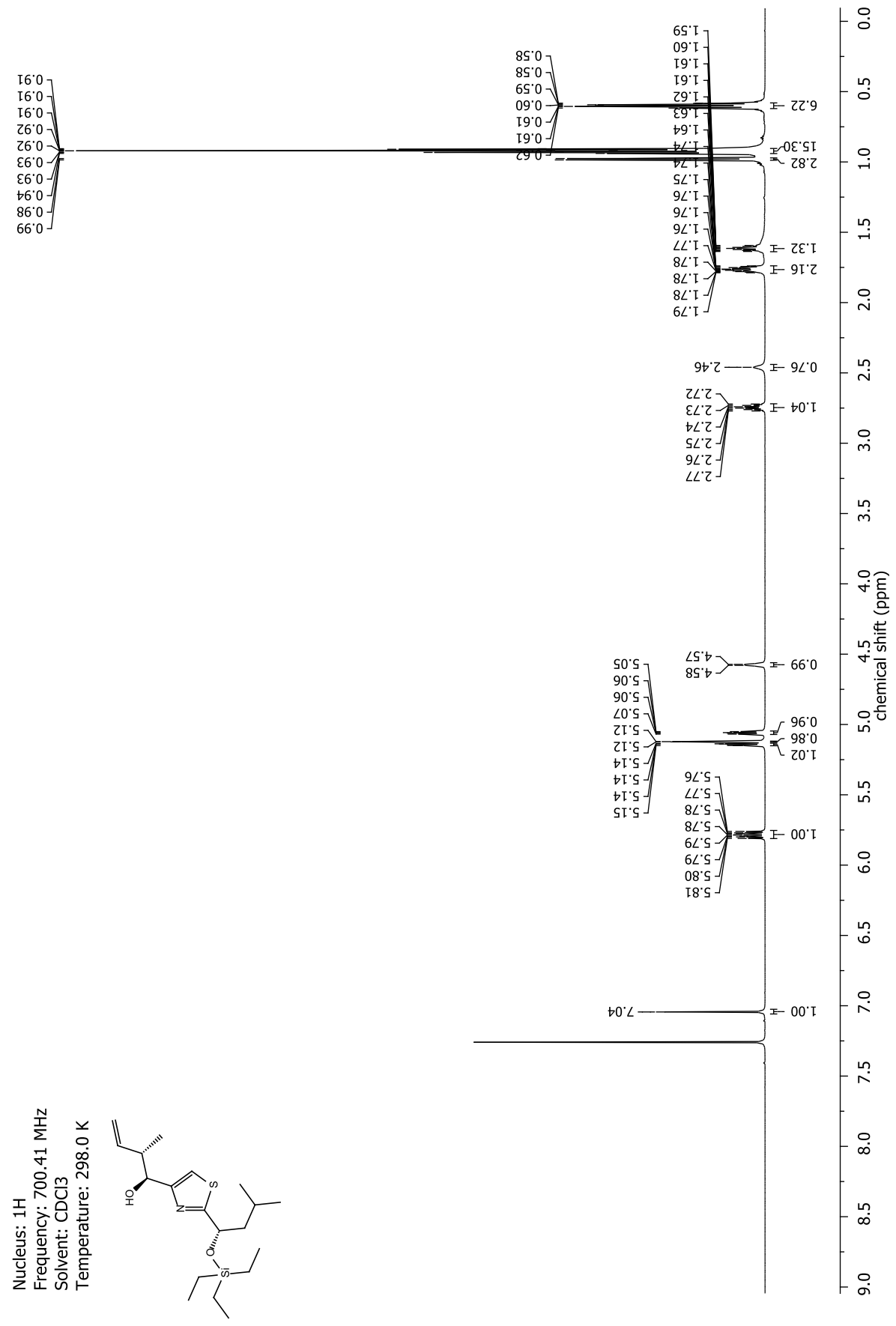



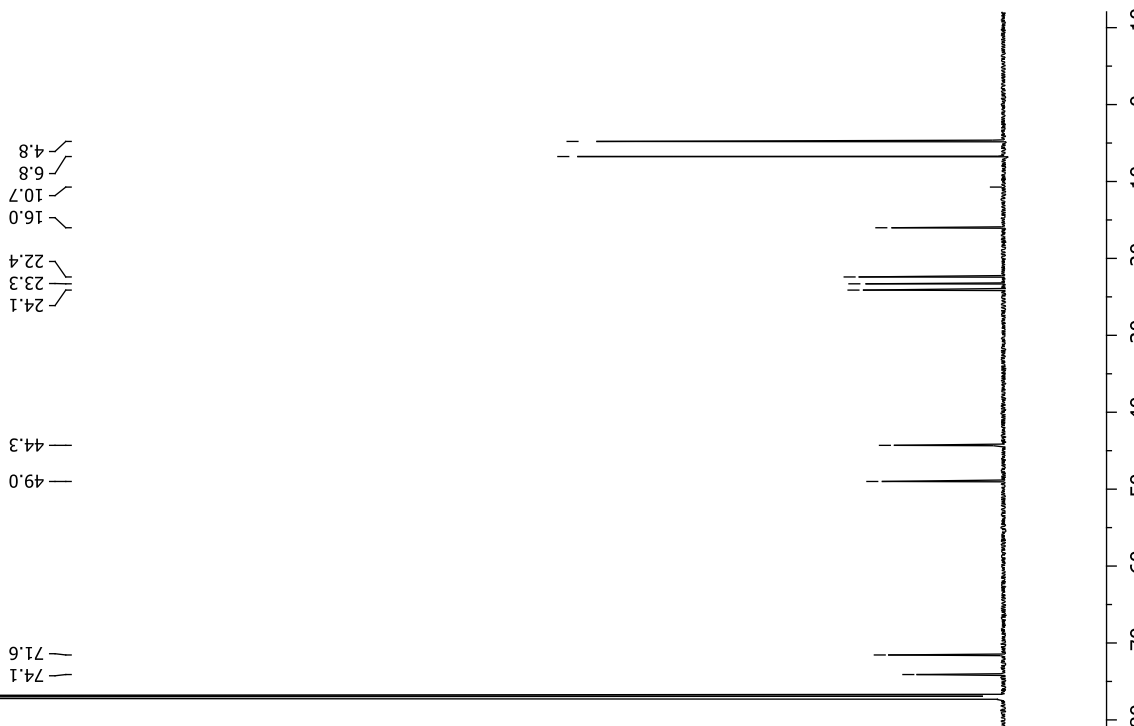

I'tII
$9 \cdot 9$ II

$\varepsilon \cdot t \downarrow-$
$0.6 เ-$

$9 \cdot T L-$

웅

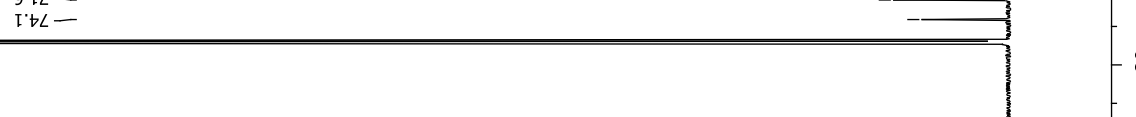

으를

$8 \frac{10}{20}$

을

L'6EI -

$0 . \angle S I-$

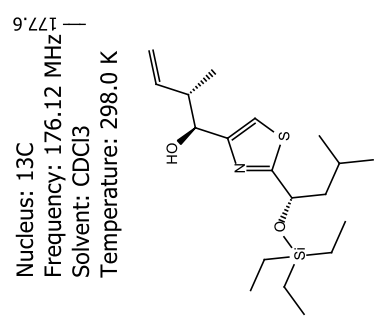


NMR-Spectrum for Compound 53e-M1

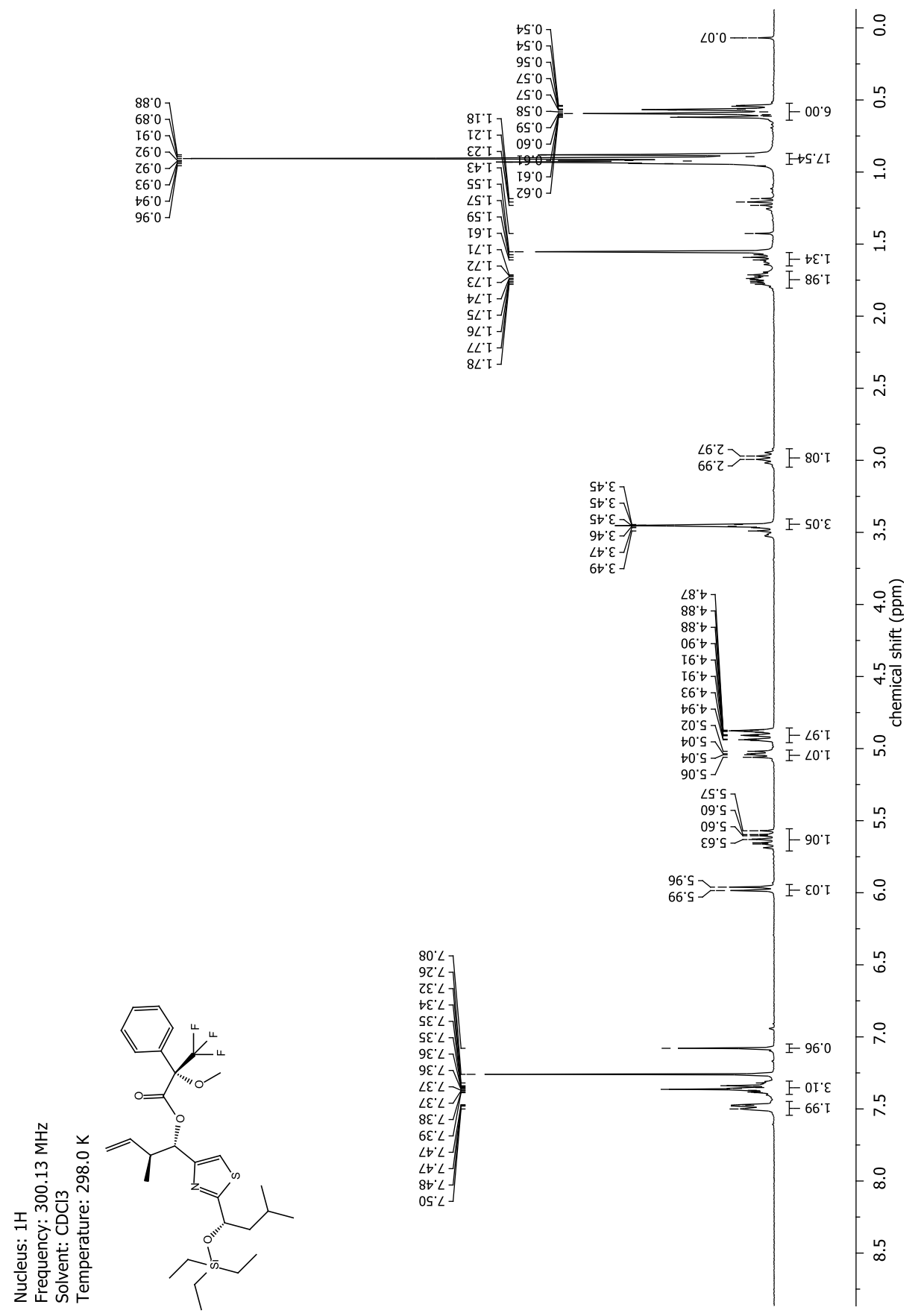


NMR-Spectrum for Compound 53e-M2

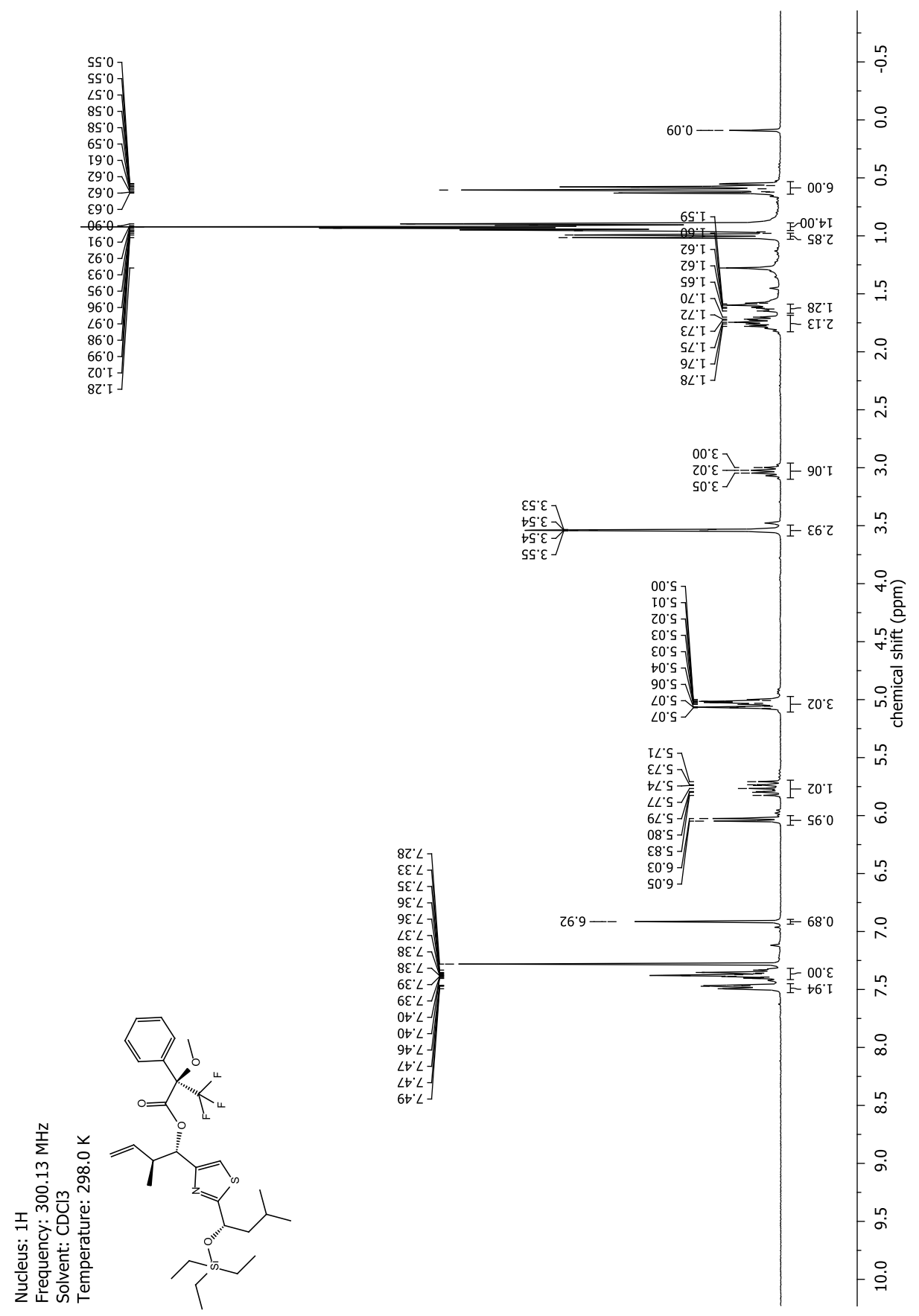


NMR-Spectra for Compound 54e

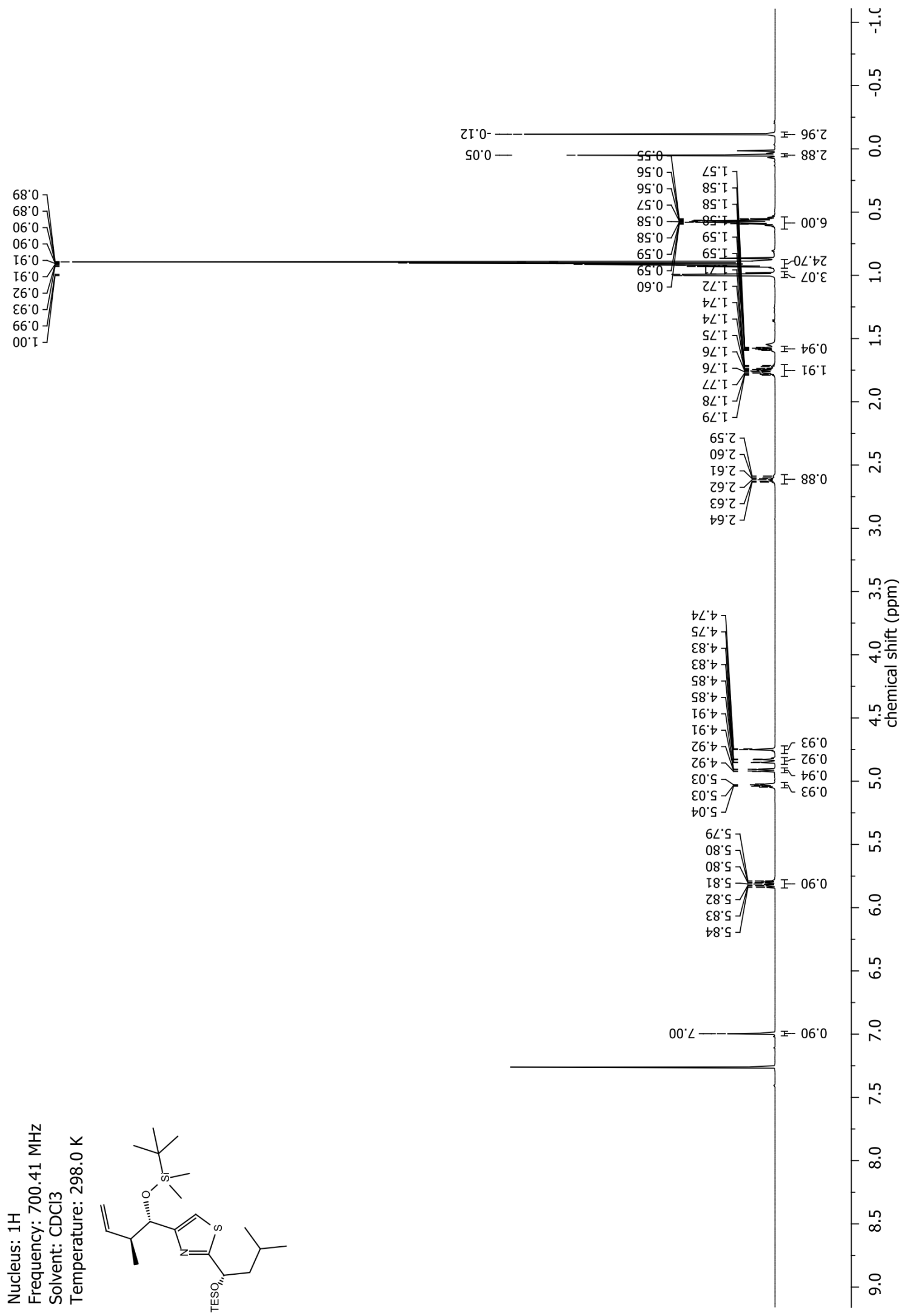


$8 \cdot t$
$4 \cdot 9$

8.917

2.812

$2 \cdot 22$

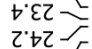

6.525

8'tb-

$\varepsilon \cdot 6 t-$
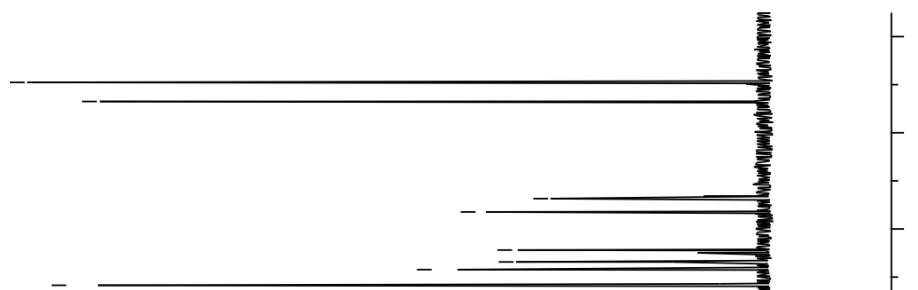

우

ำ

-

요

o

$8.5 L$

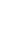

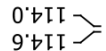

$0.0 t \mathrm{~L}$

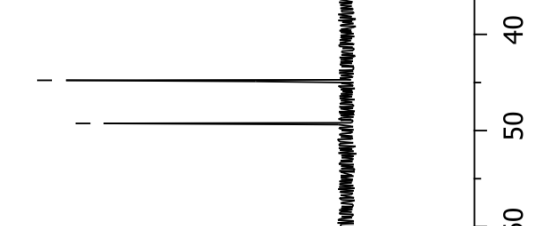

8

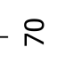

$\infty$

8

으믐

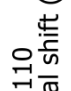

원든

음

d

$\frac{-1}{2}$

욱

8.8SI -

S'9LI -

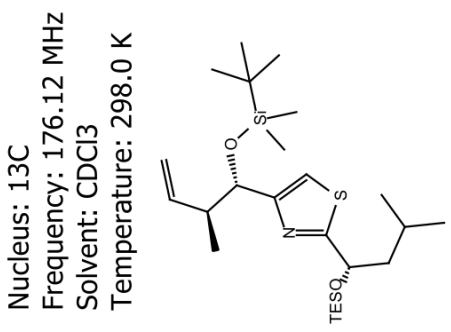

옥

ஓ्̀

$\stackrel{\text { ㄱ }}{2}$

고ำ 
NMR-Spectra for Compound 56

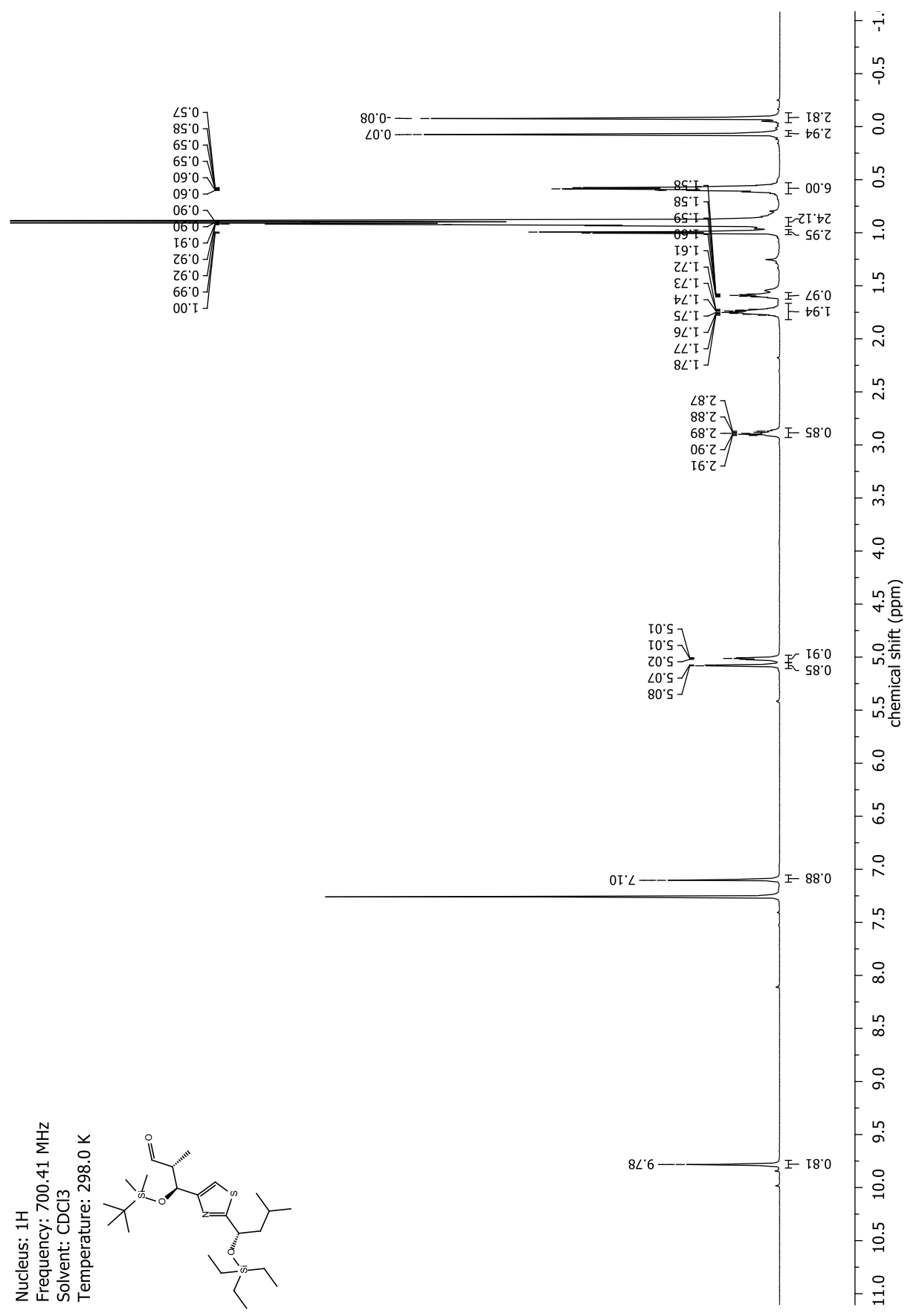




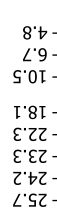

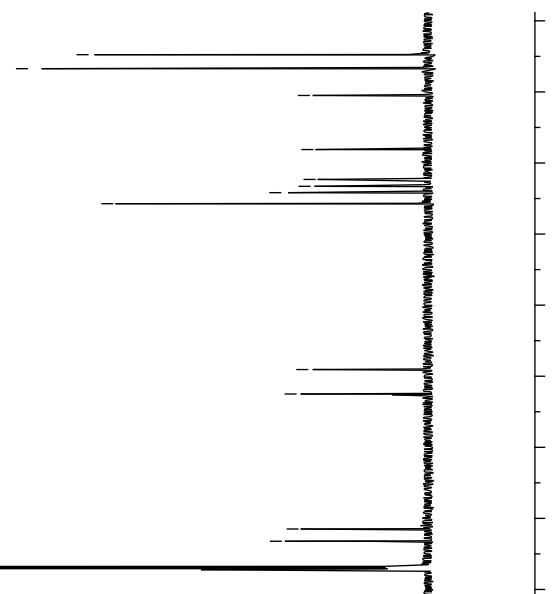

I. $6 b$

s'zs-

s't $\angle-$

$\tau^{\prime} \varepsilon L=$

1

8 tII -

$0 \cdot$ LSI -

LLLI -

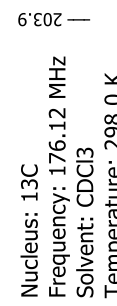

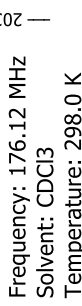

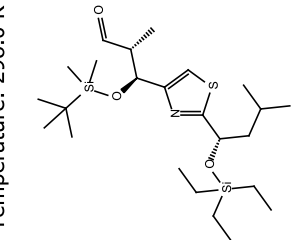

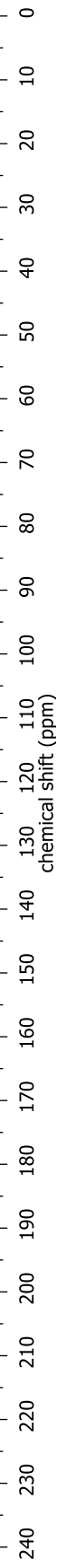


NMR-Spectra for Compound 57

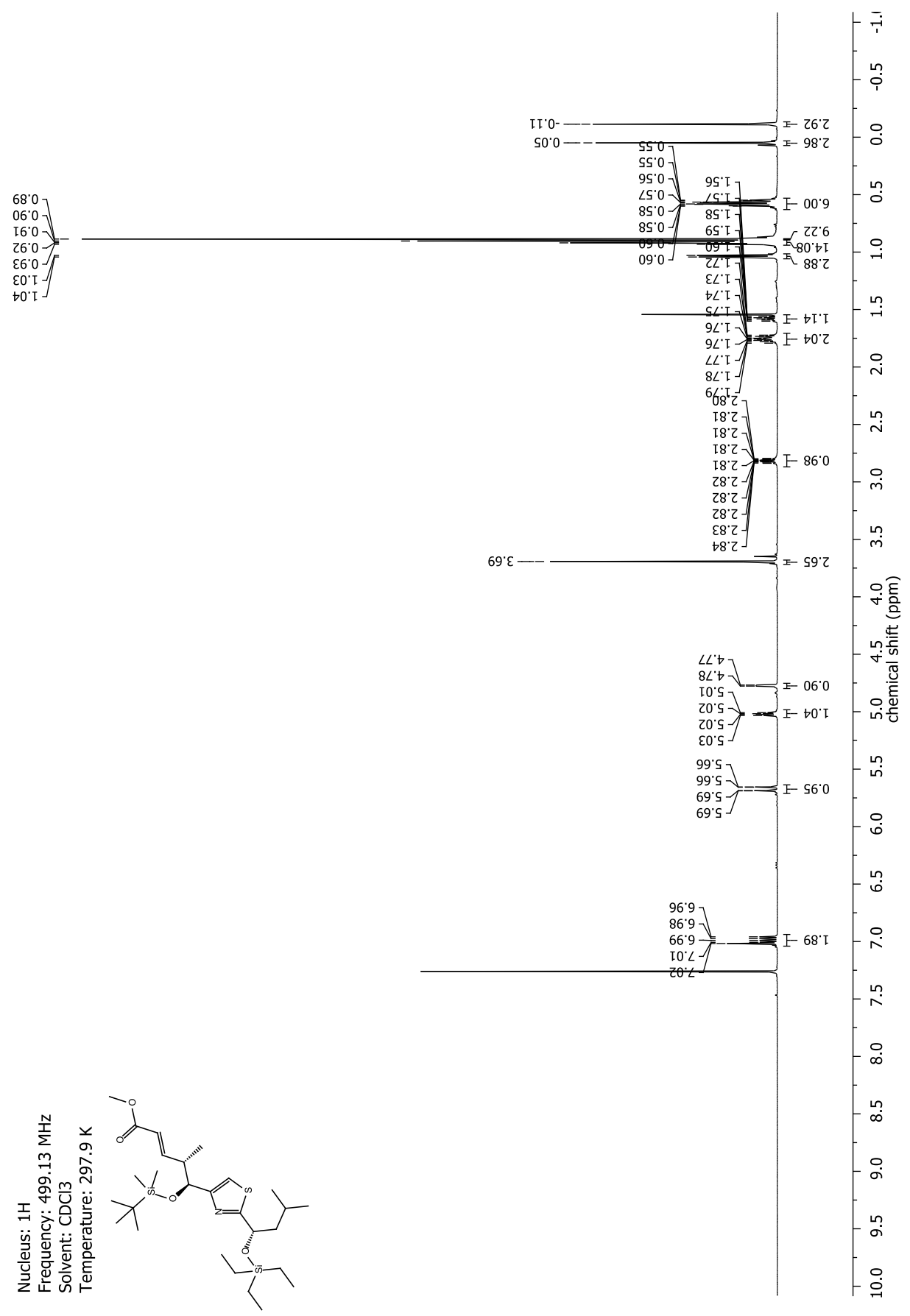




$$
\begin{aligned}
& \begin{array}{l}
i \cdot s- \\
\langle\cdot b-
\end{array} \\
& 8.6 \\
& \text { ¿.91 }
\end{aligned}
$$

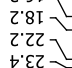

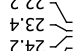

$$
\begin{aligned}
& \begin{array}{l}
\text { 2. } \\
\text { 8. } \mathrm{xz}
\end{array}
\end{aligned}
$$

L.Et-

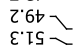

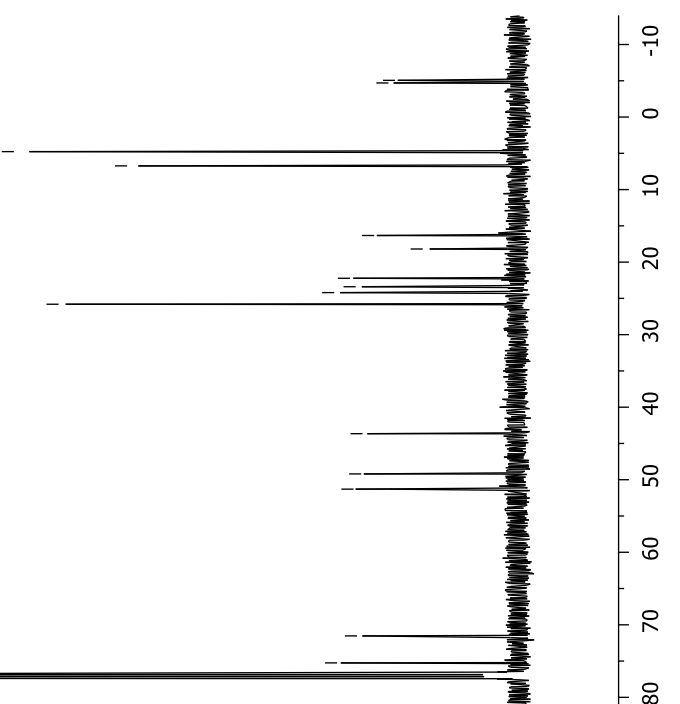

$S^{\prime} T \angle-$

เ๋เI -

เ'เนโ-

8.0ST -

0.85 I -

0. $\angle 9 I-$

I'LLI -
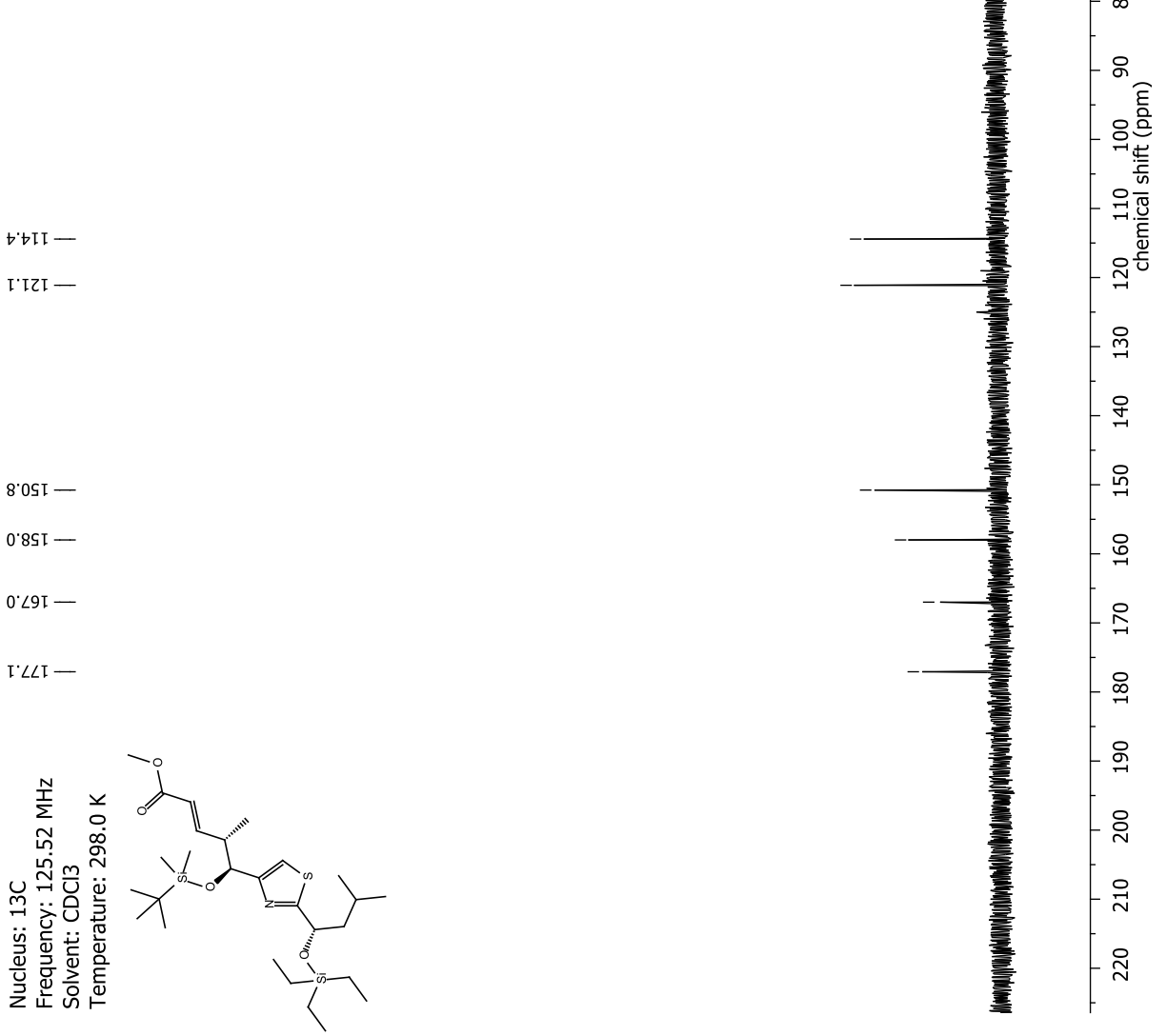
NMR-Spectra for Compound 46e

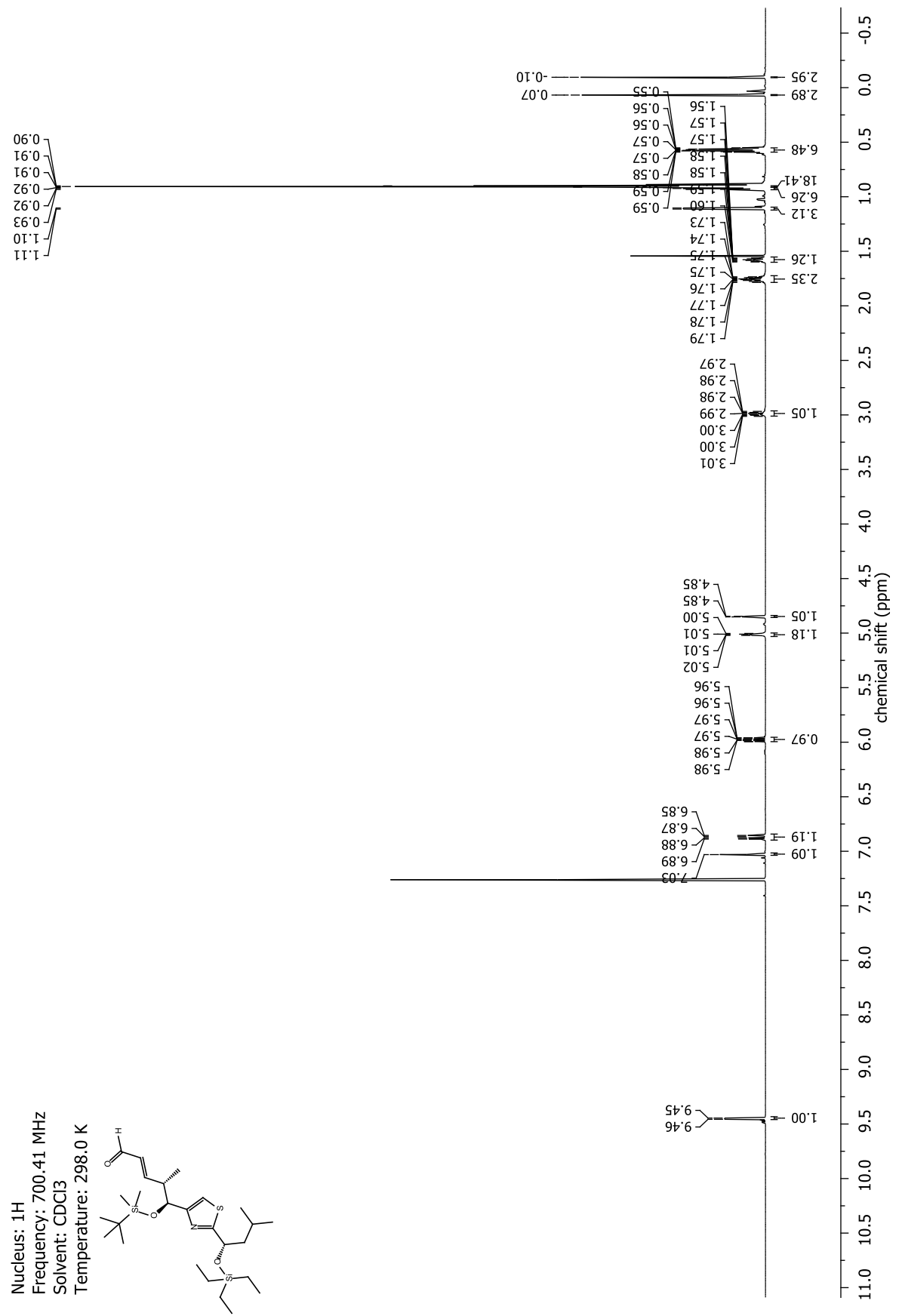




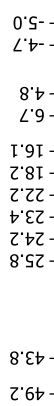

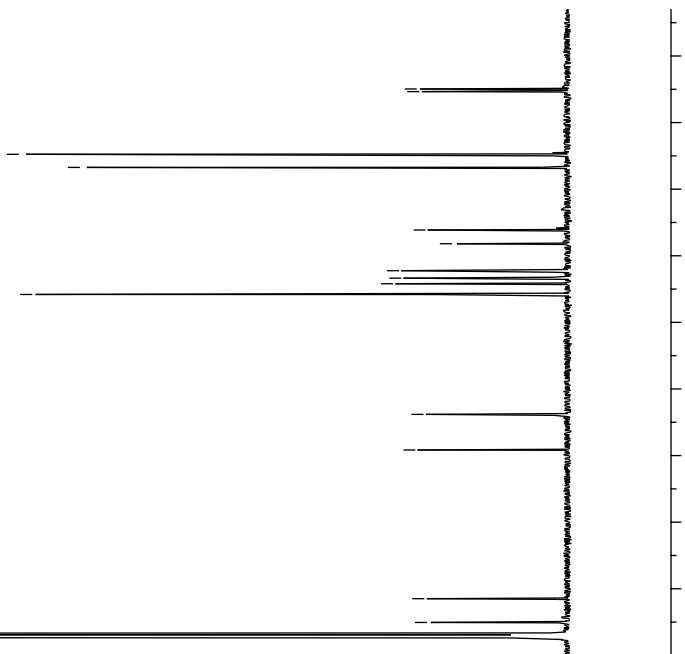

S.IL -

$\longrightarrow$ -

S'tDI -

०.๕๕I -

$\angle C S I=-$
$0.09 I=-$

S'LLI -

I'เ6I -

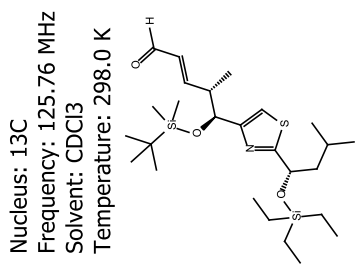


NMR-Spectra for Compound 58a

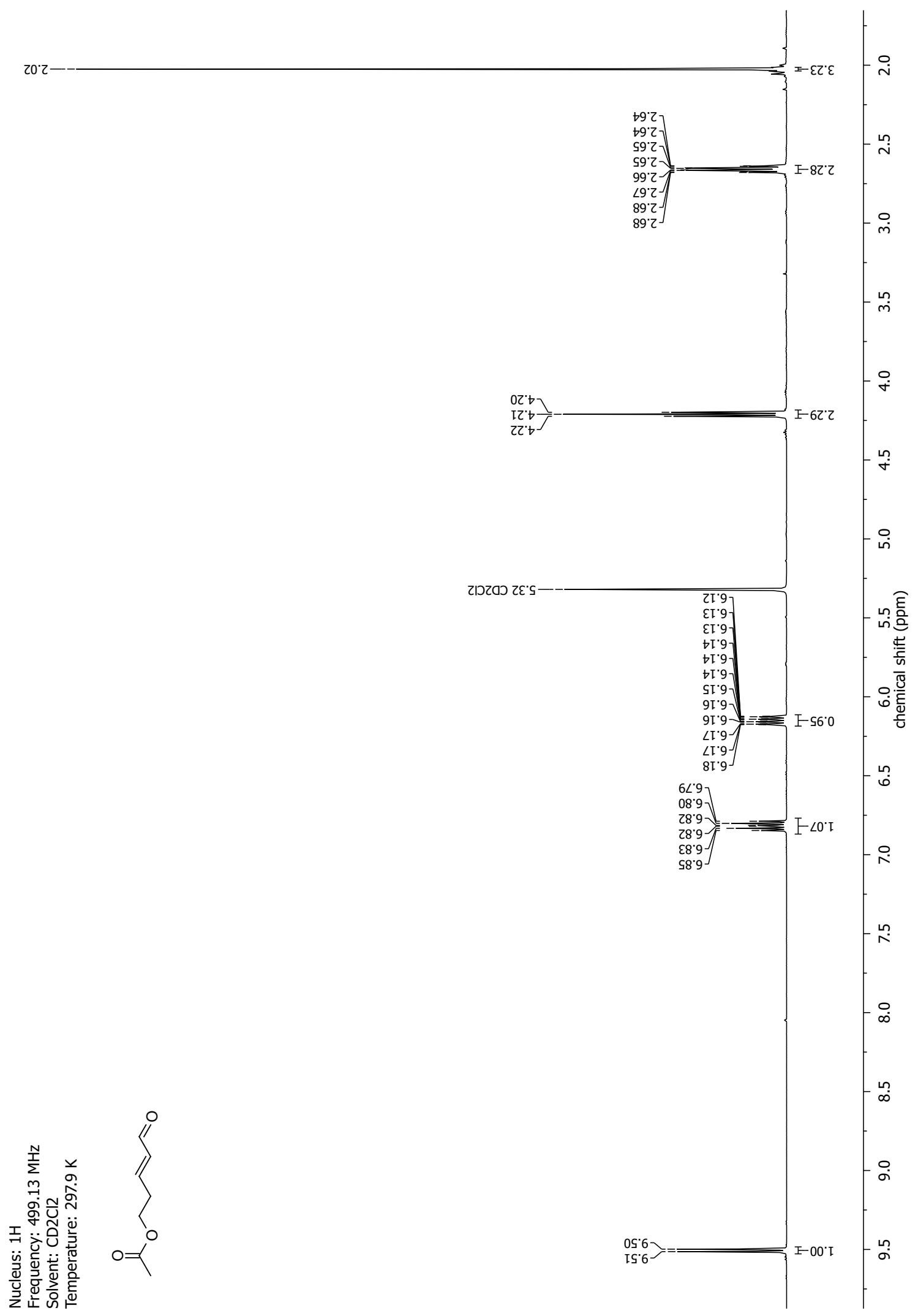




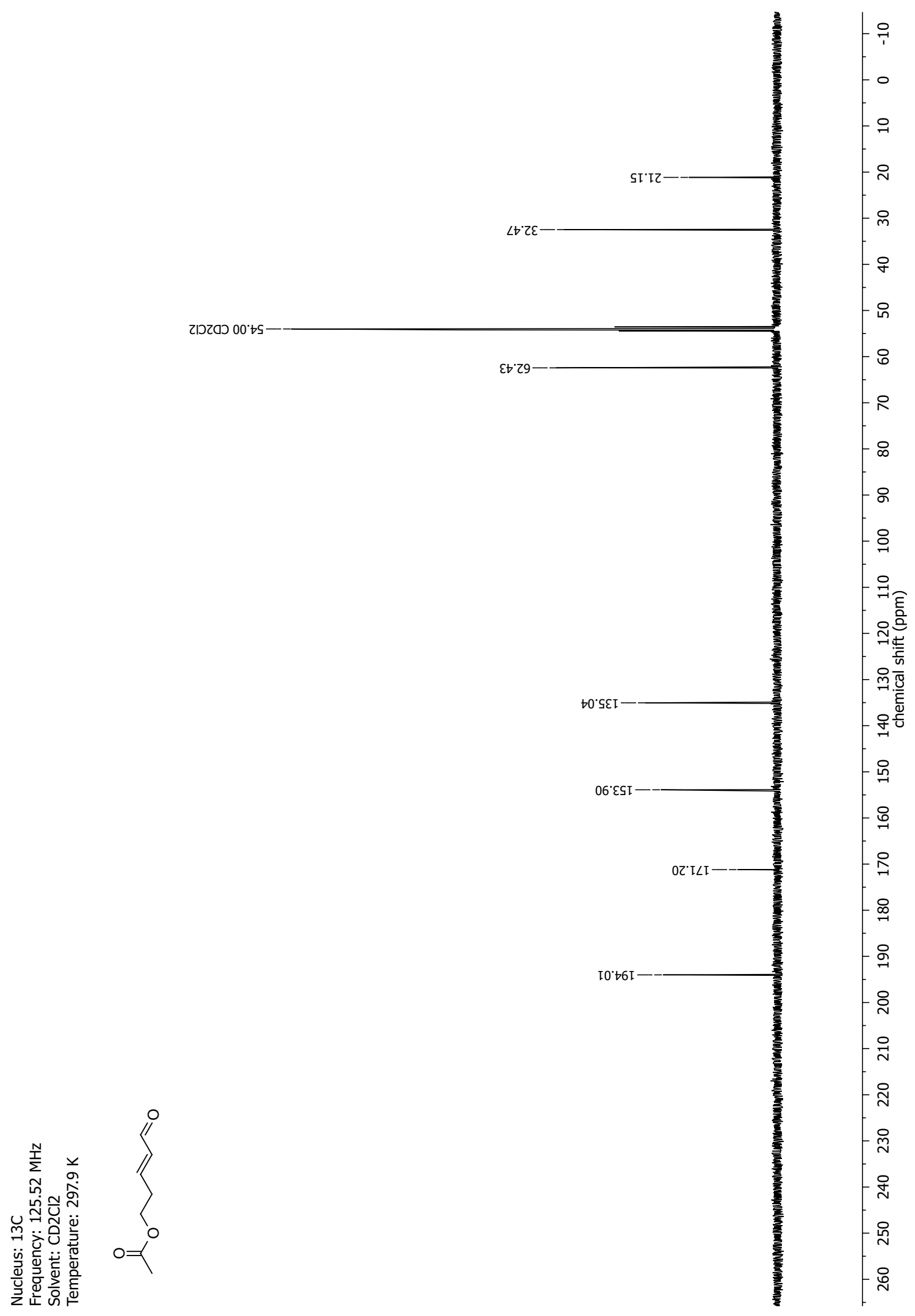


NMR-Spectra for Compound 58b

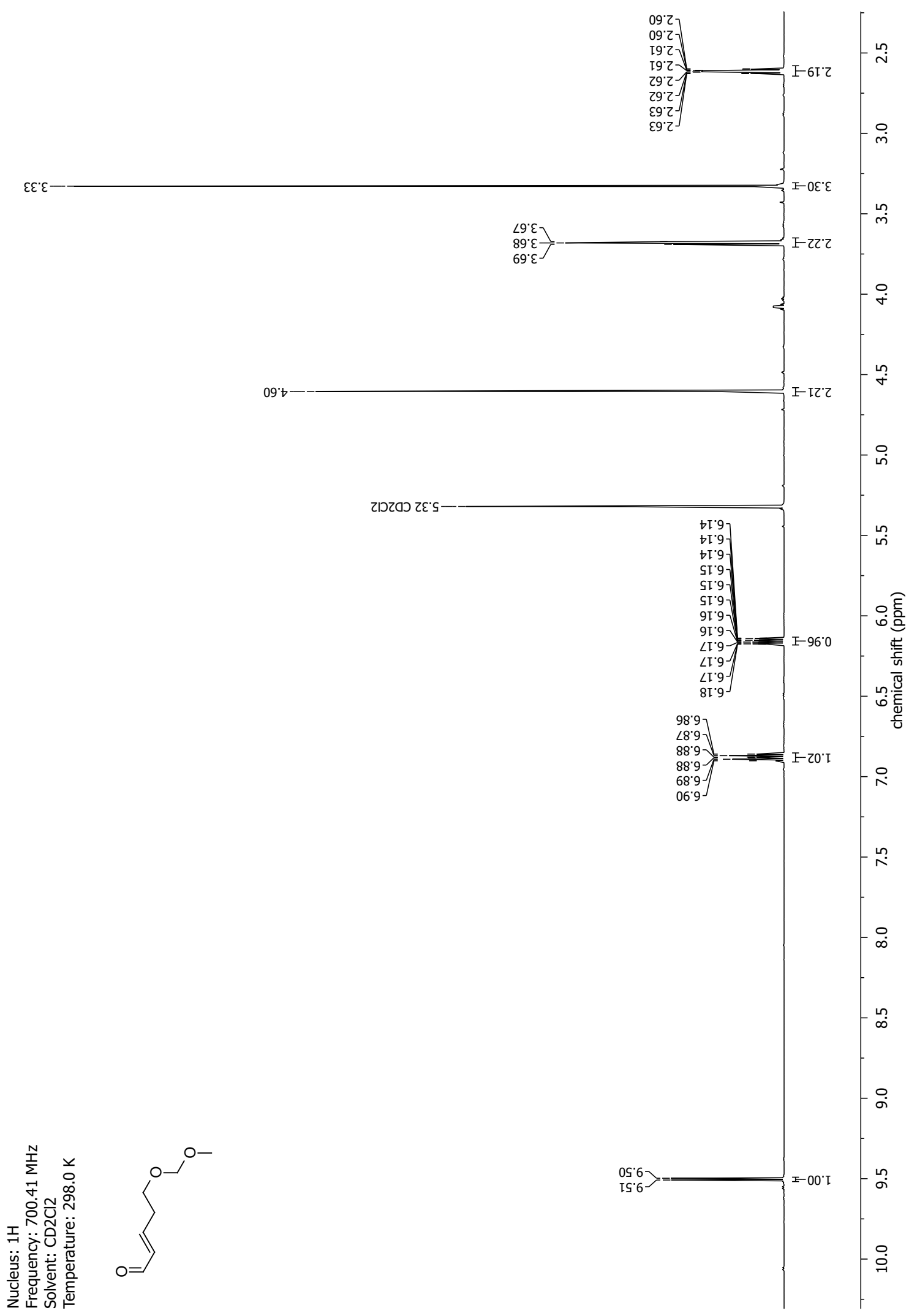




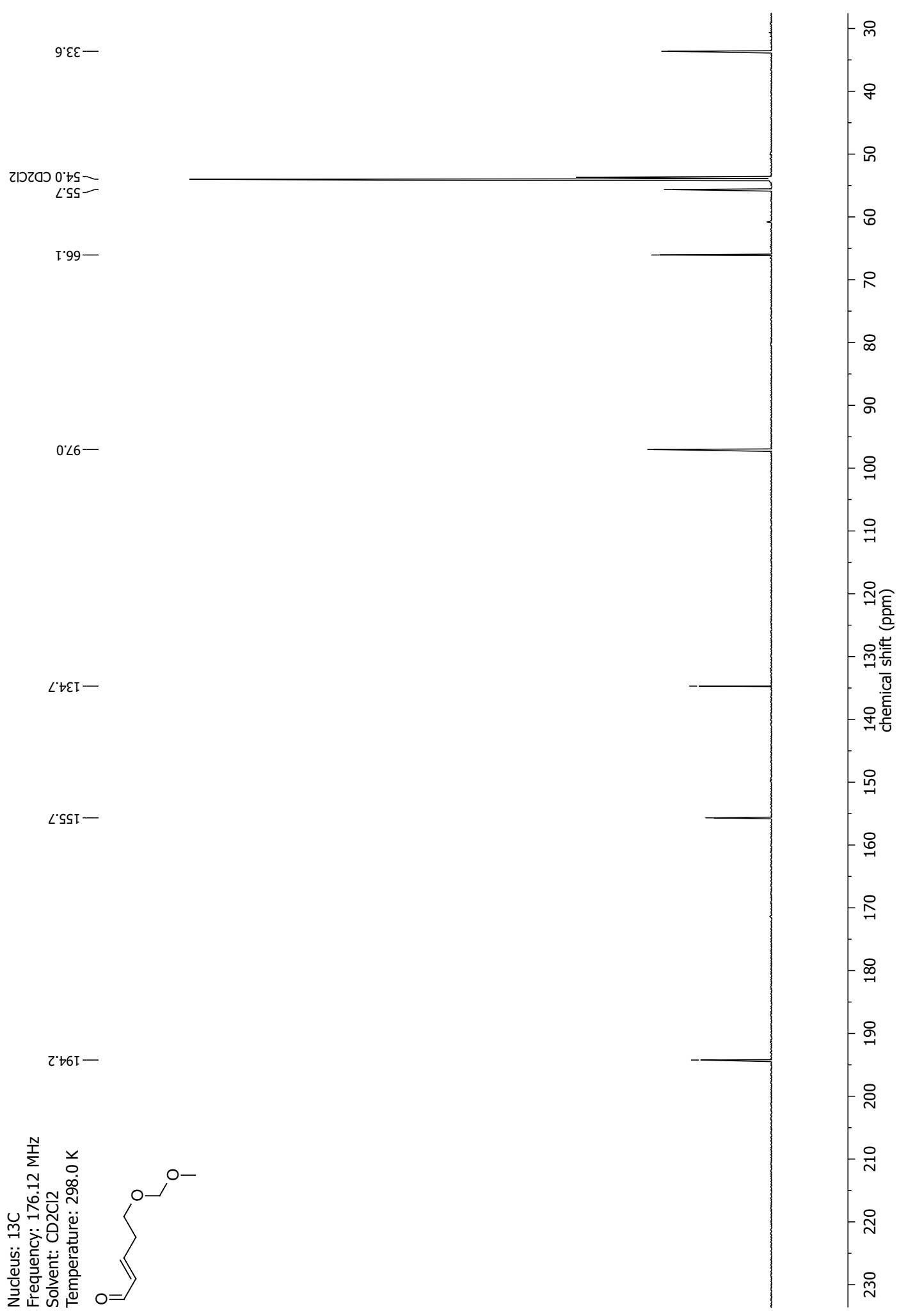


NMR-Spectra for Compound 58c

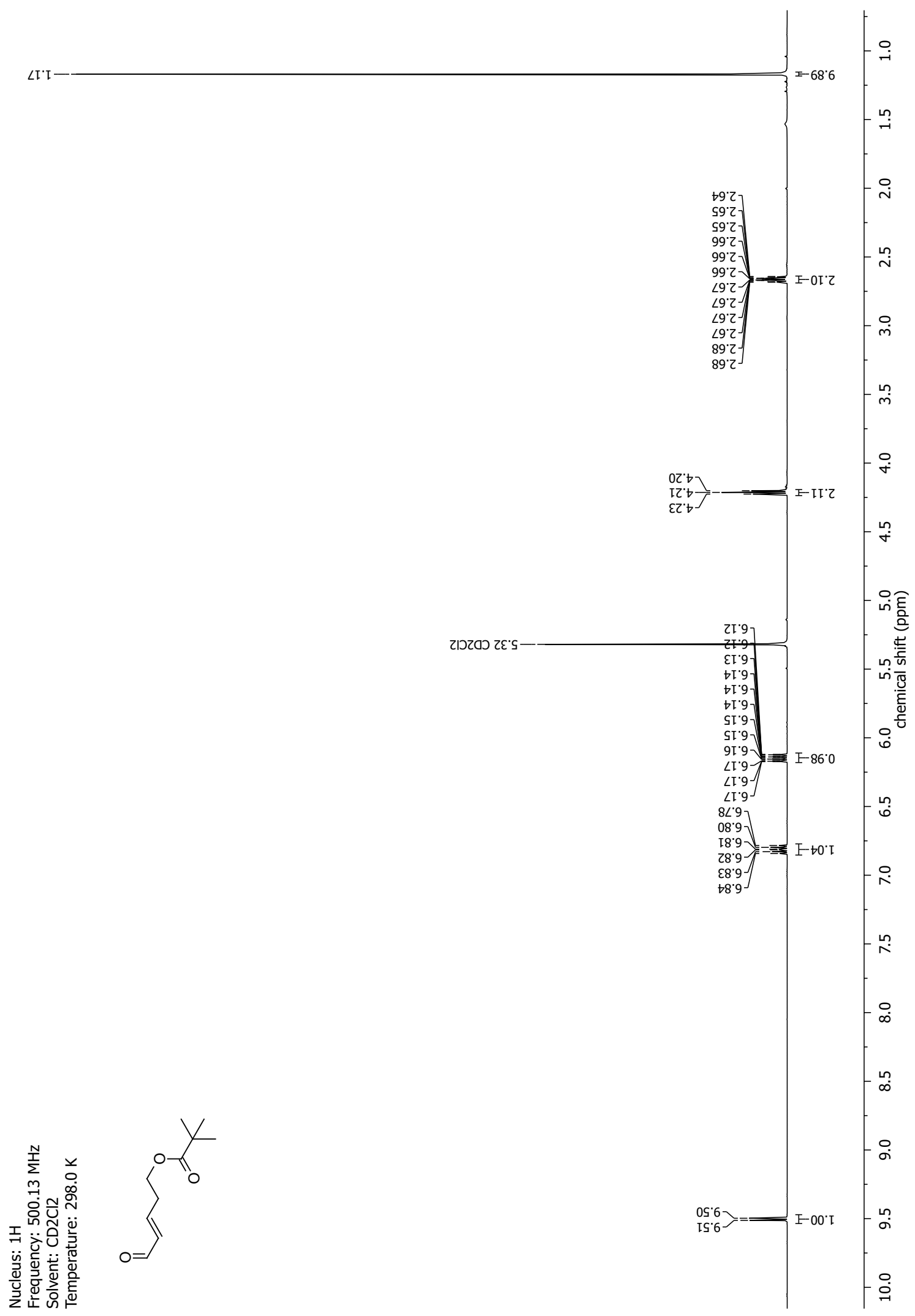




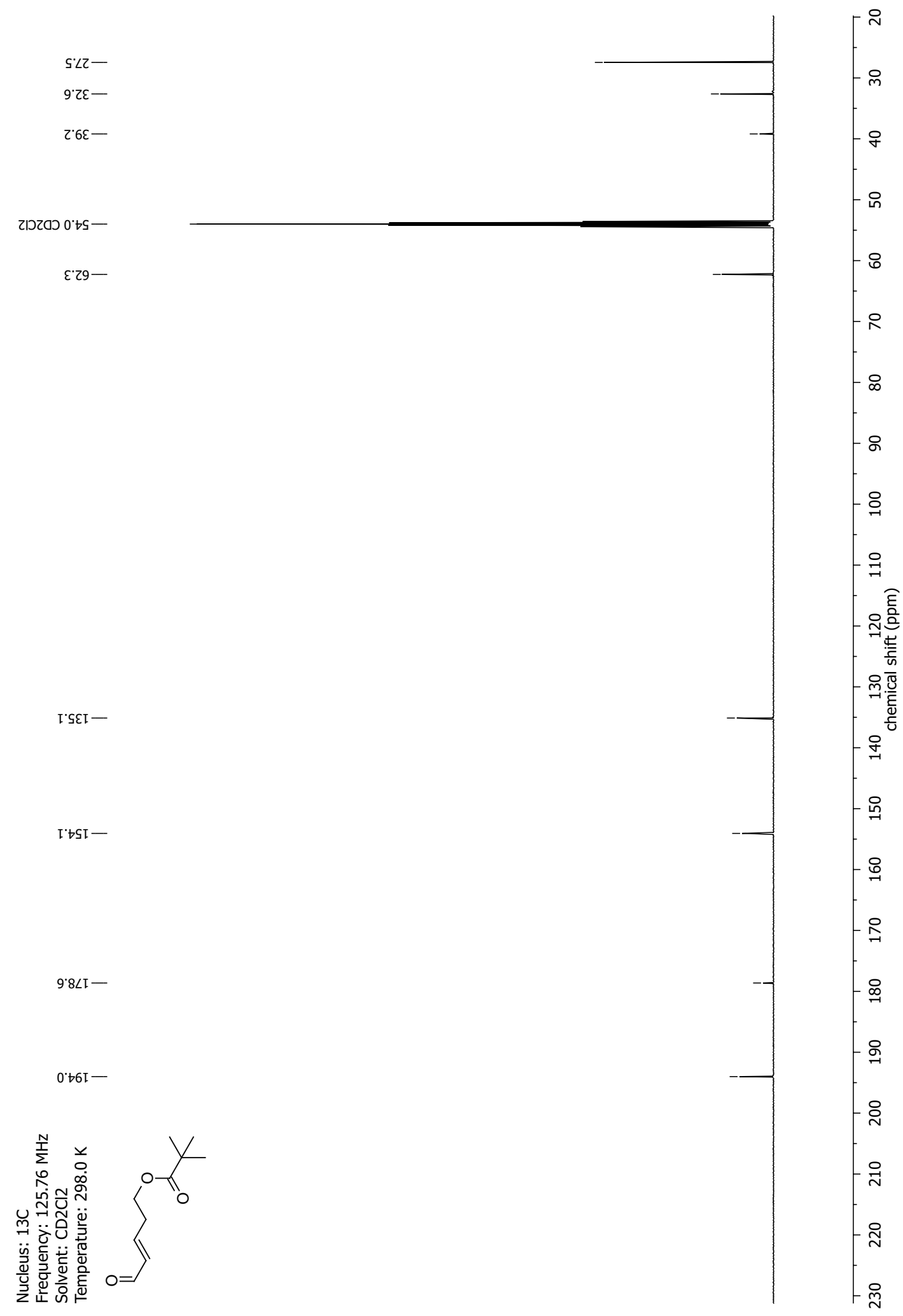


NMR-Spectra for Compound 58d

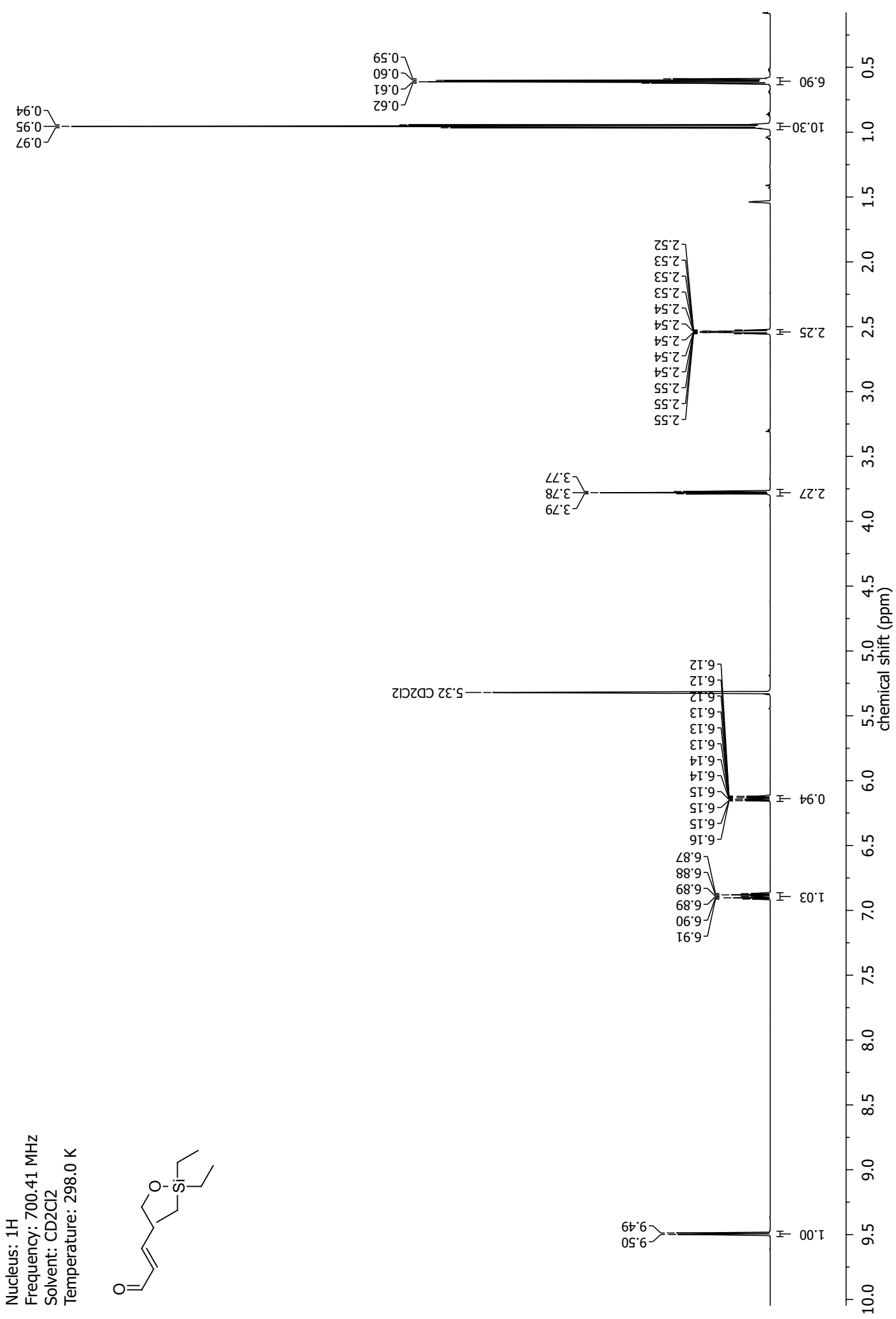




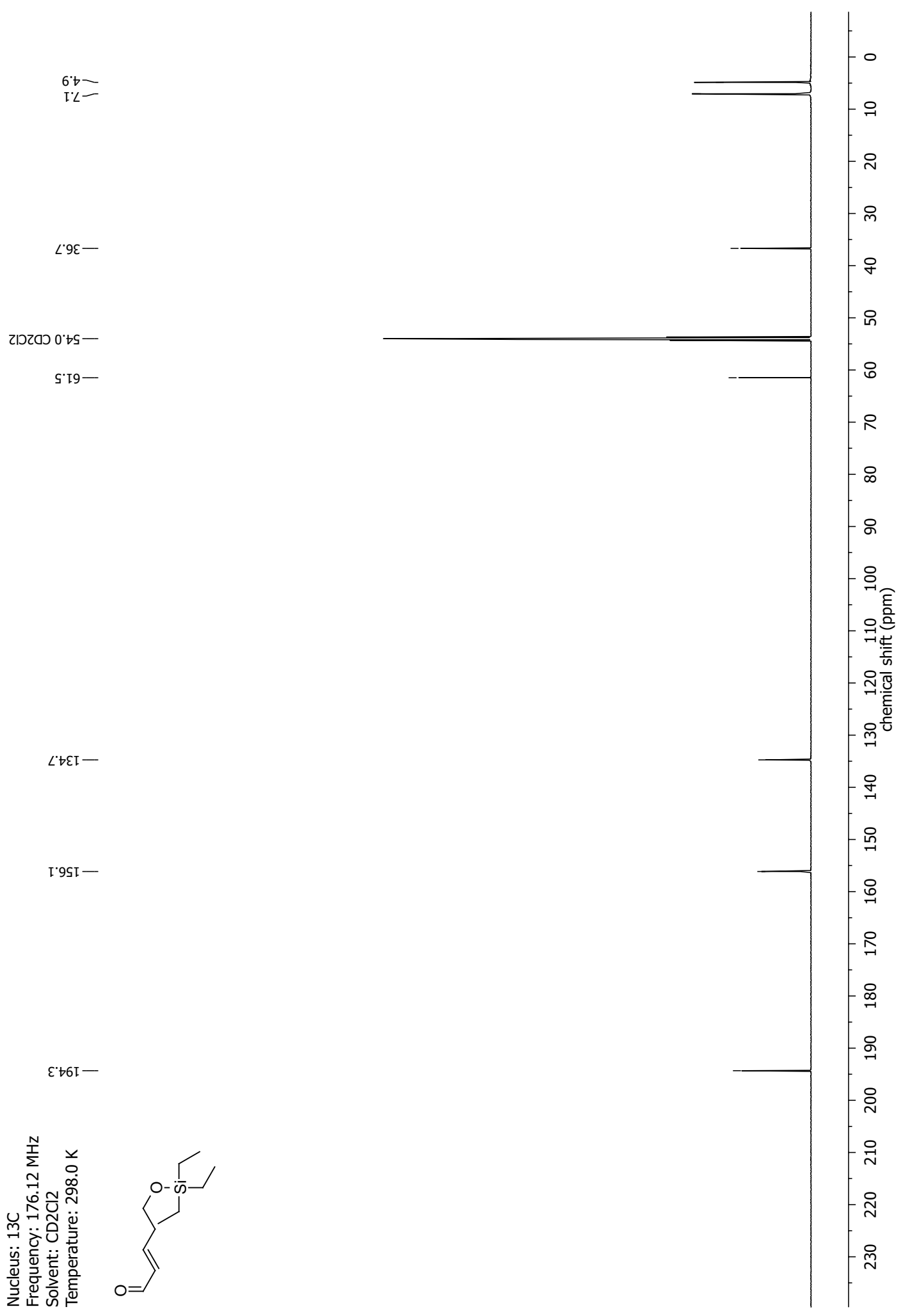


NMR-Spectra for Compound 58e

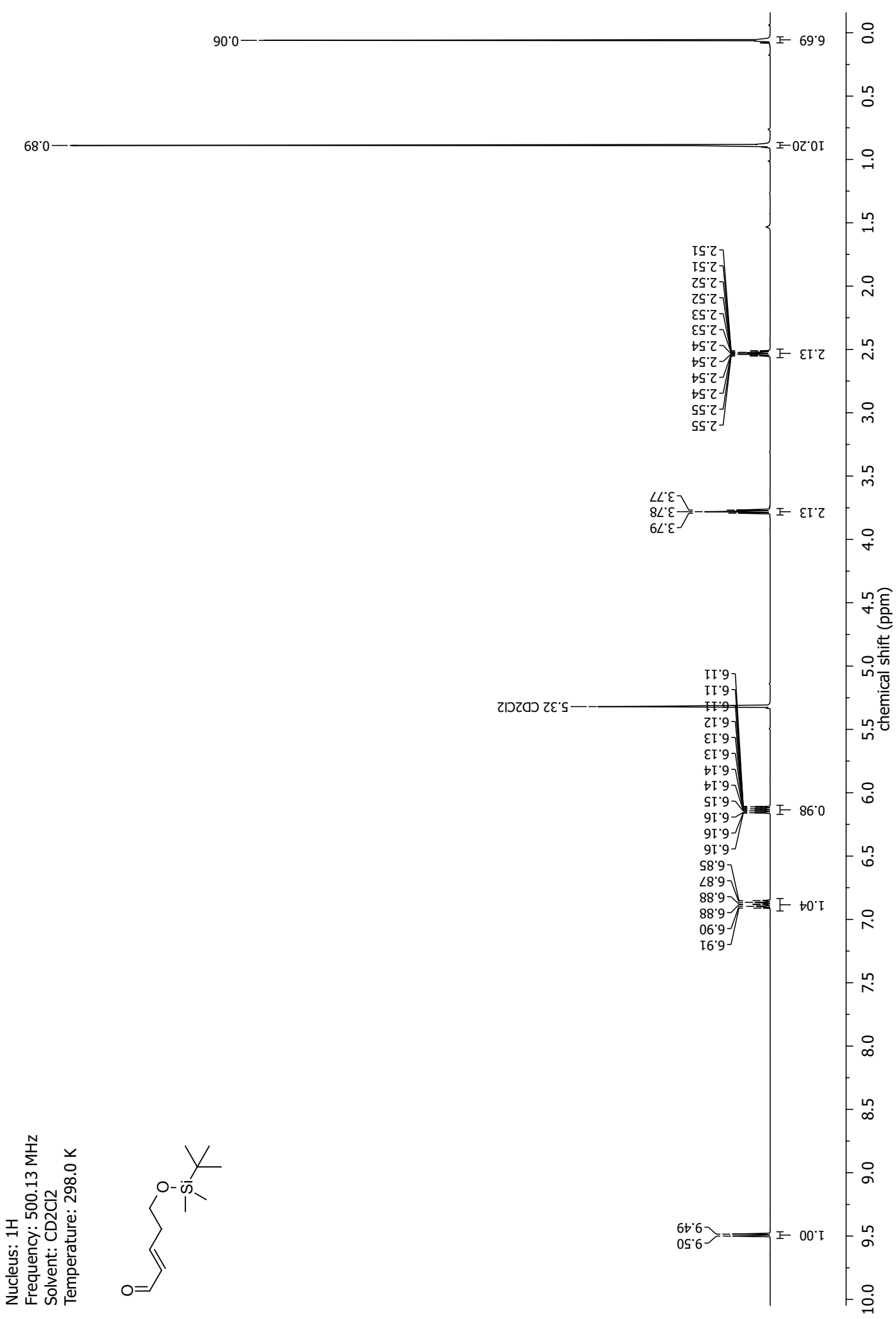




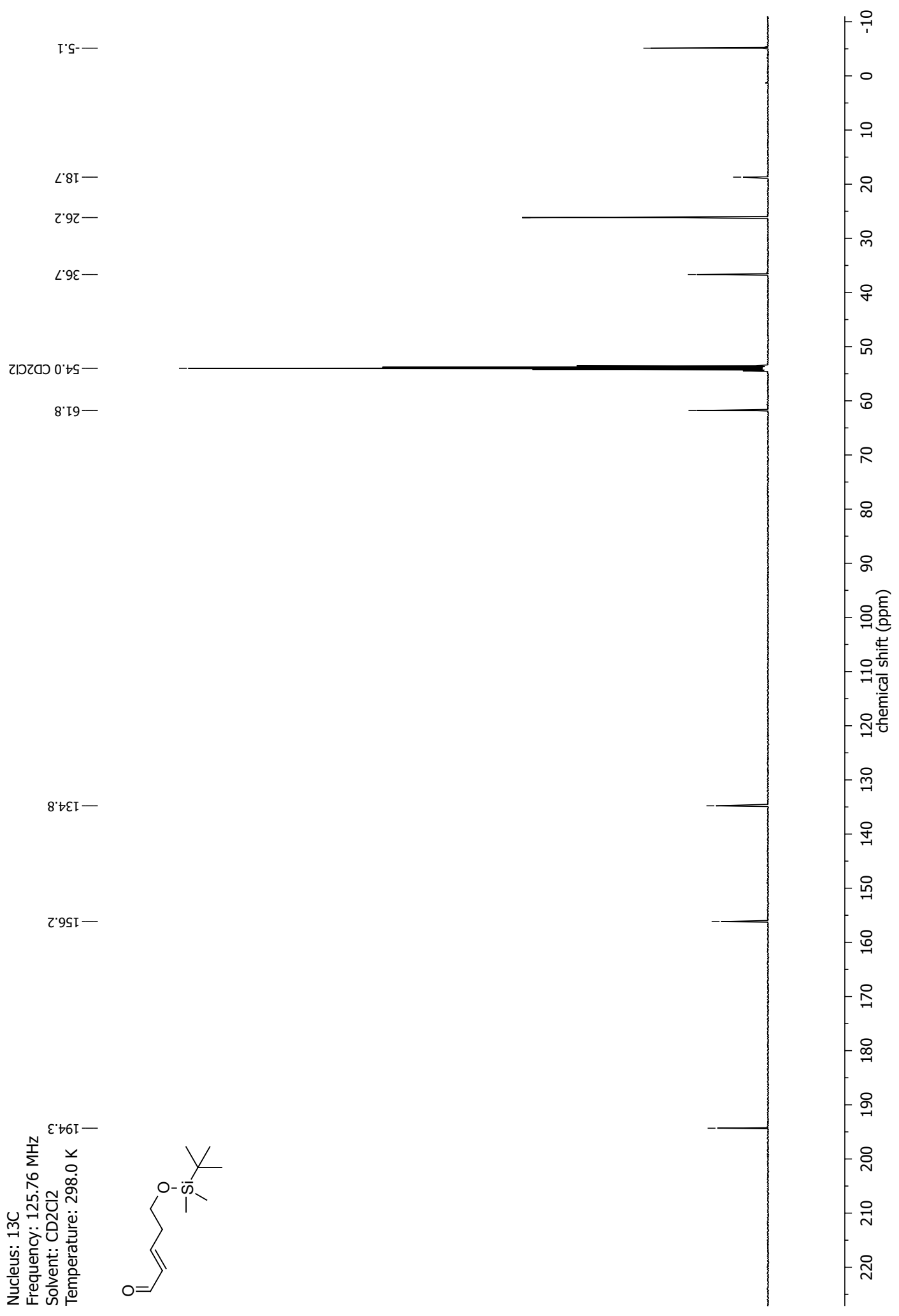


NMR-Spectra for Compound 63

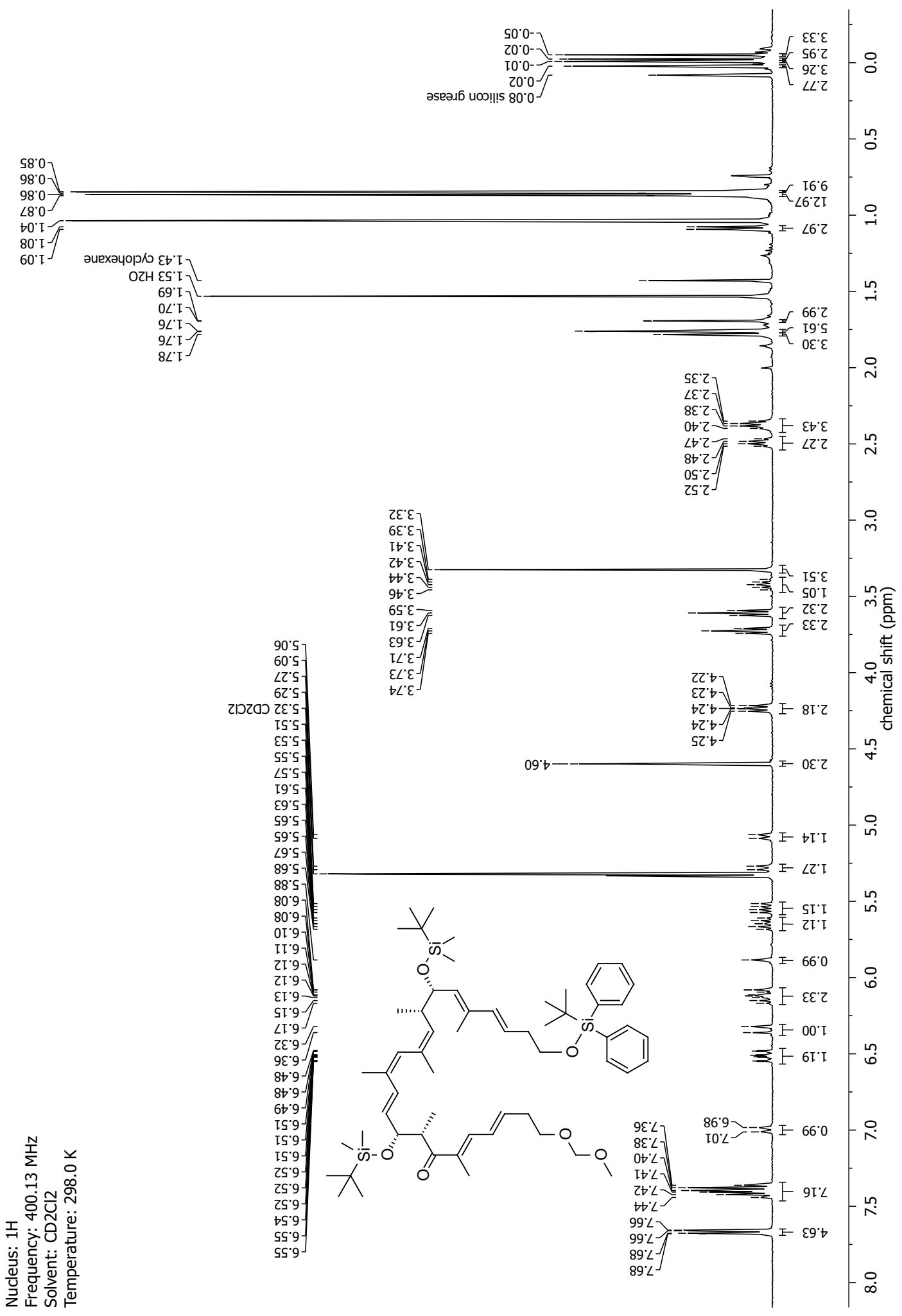




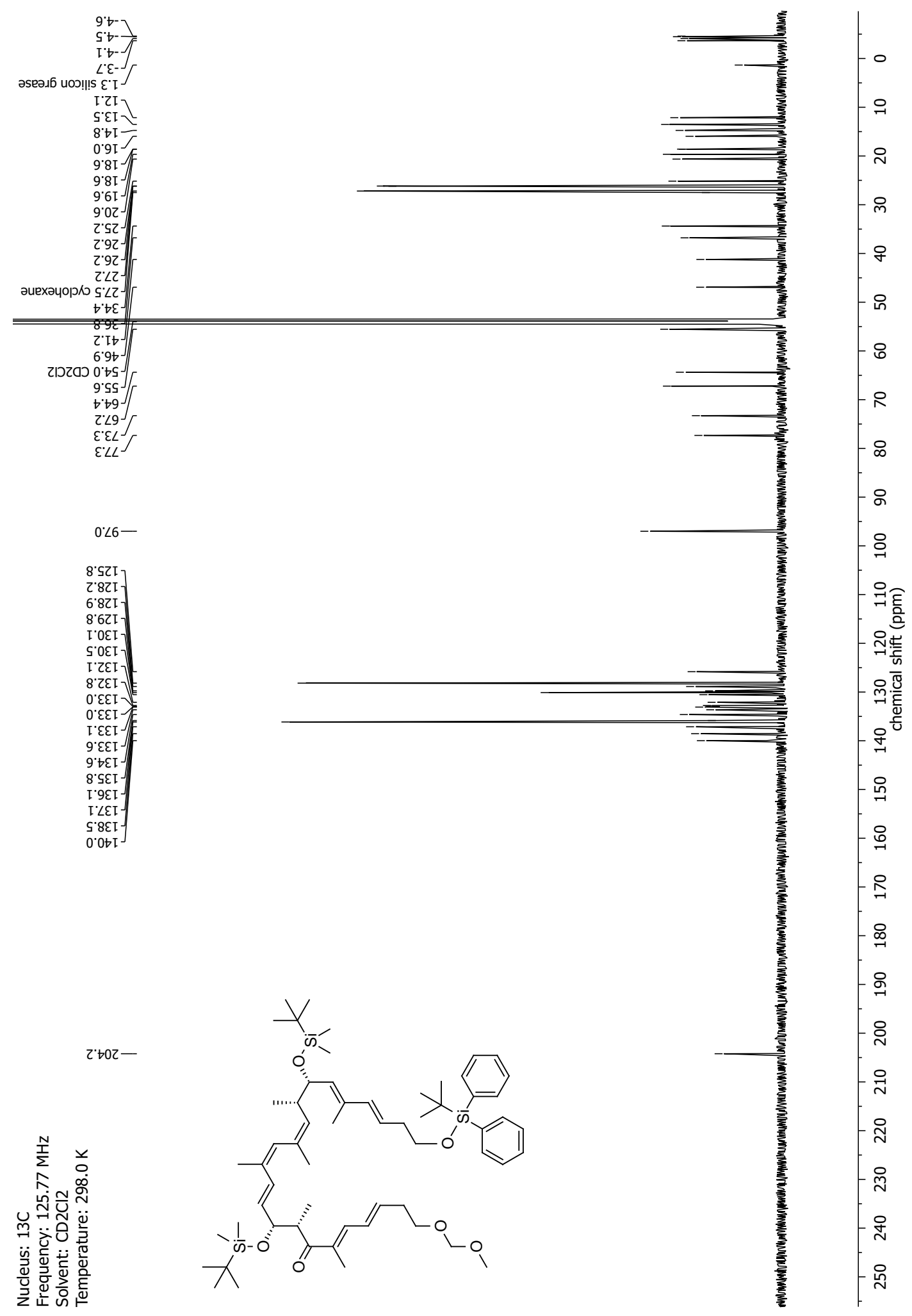




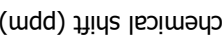

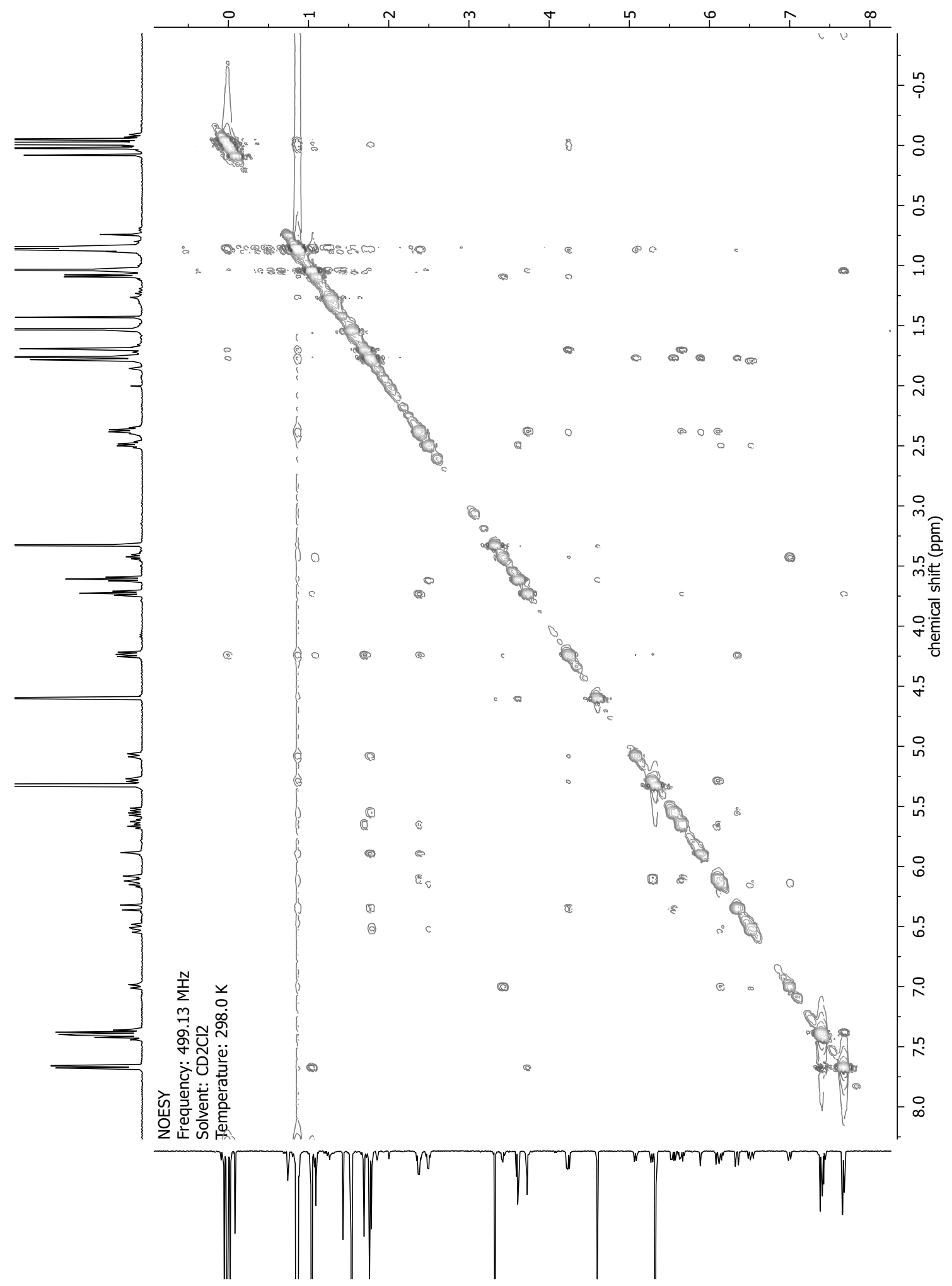


NMR-Spectra for Compound 65a

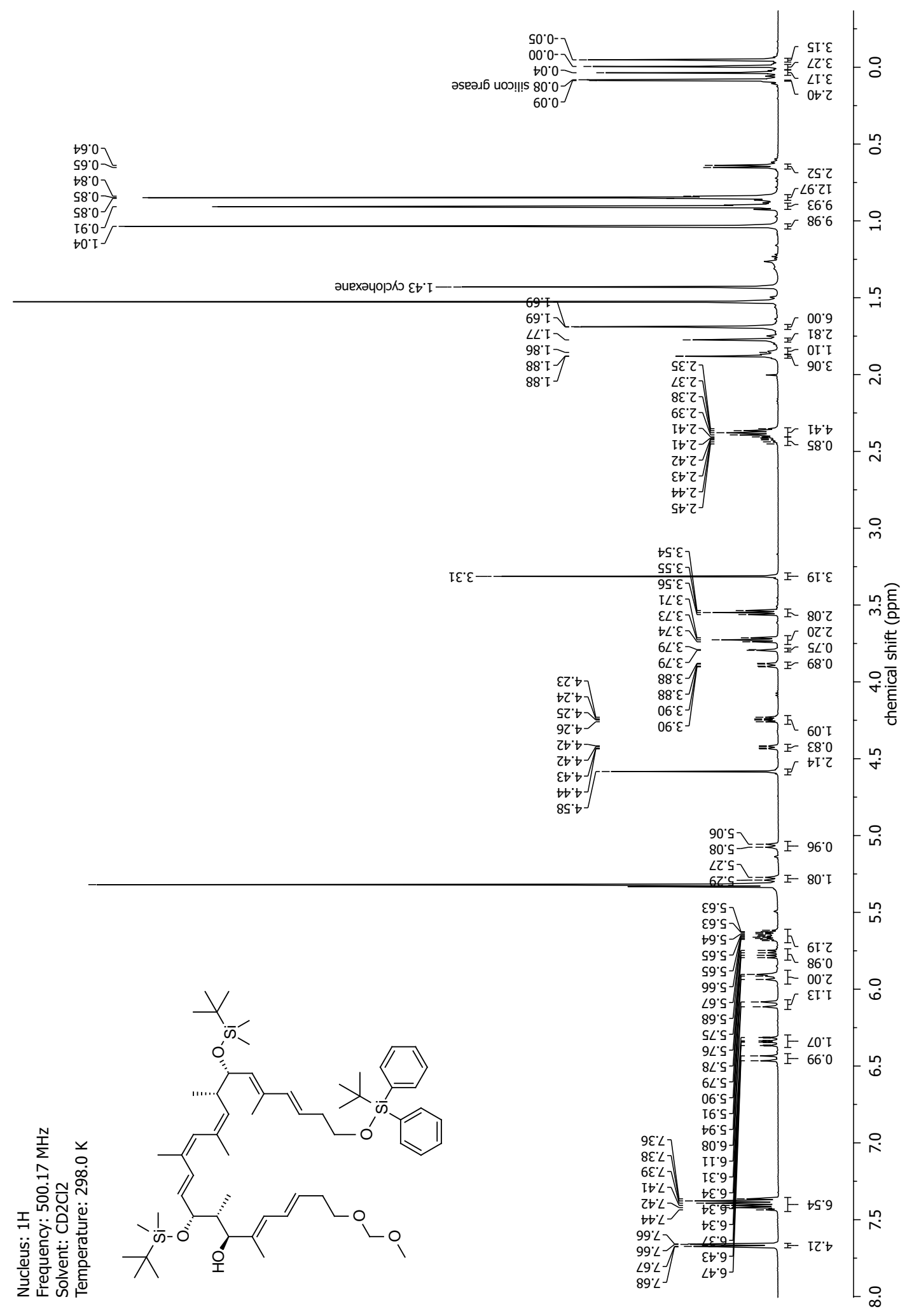




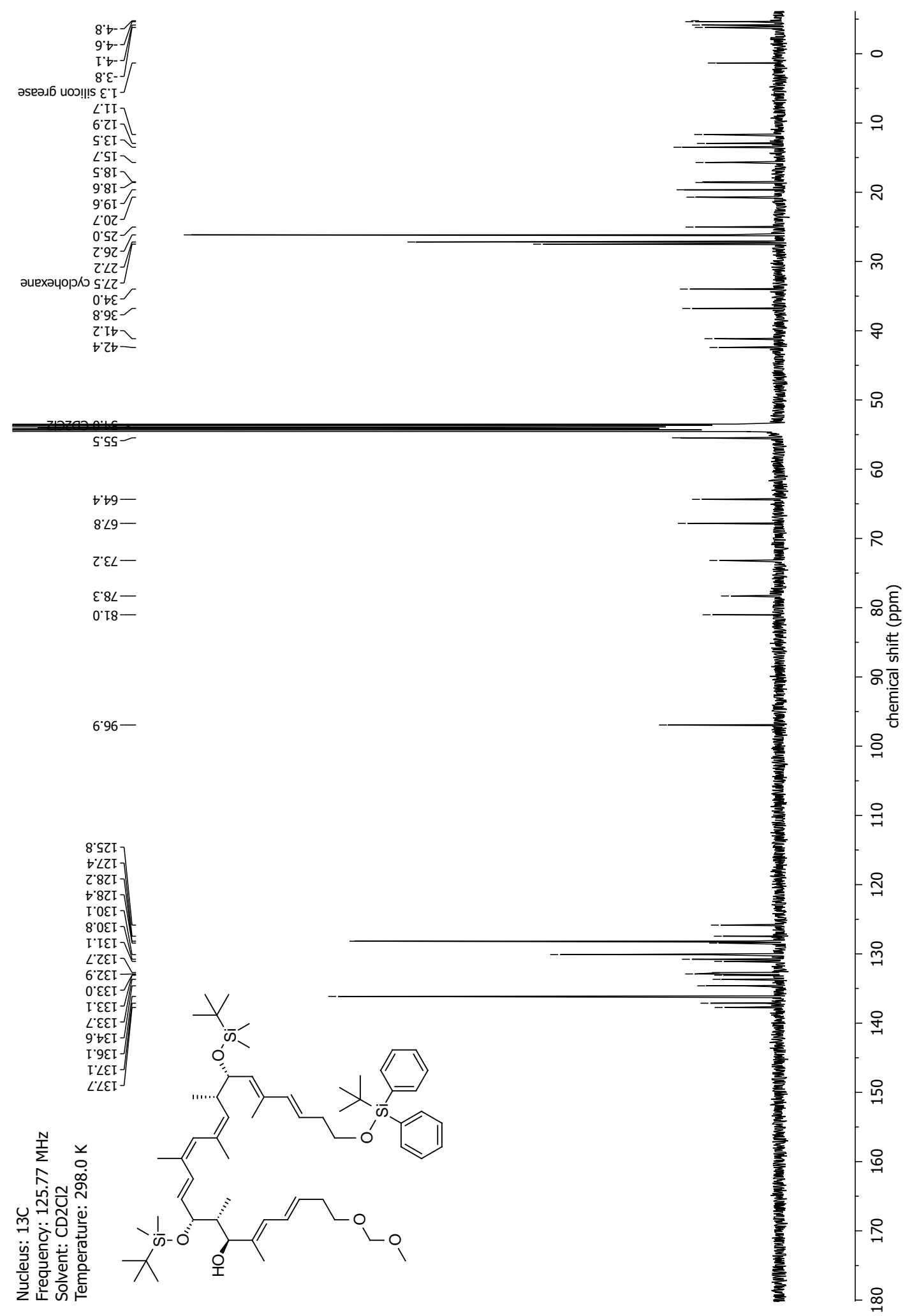




\section{NMR-Spectrum for Compound 65a-M1}

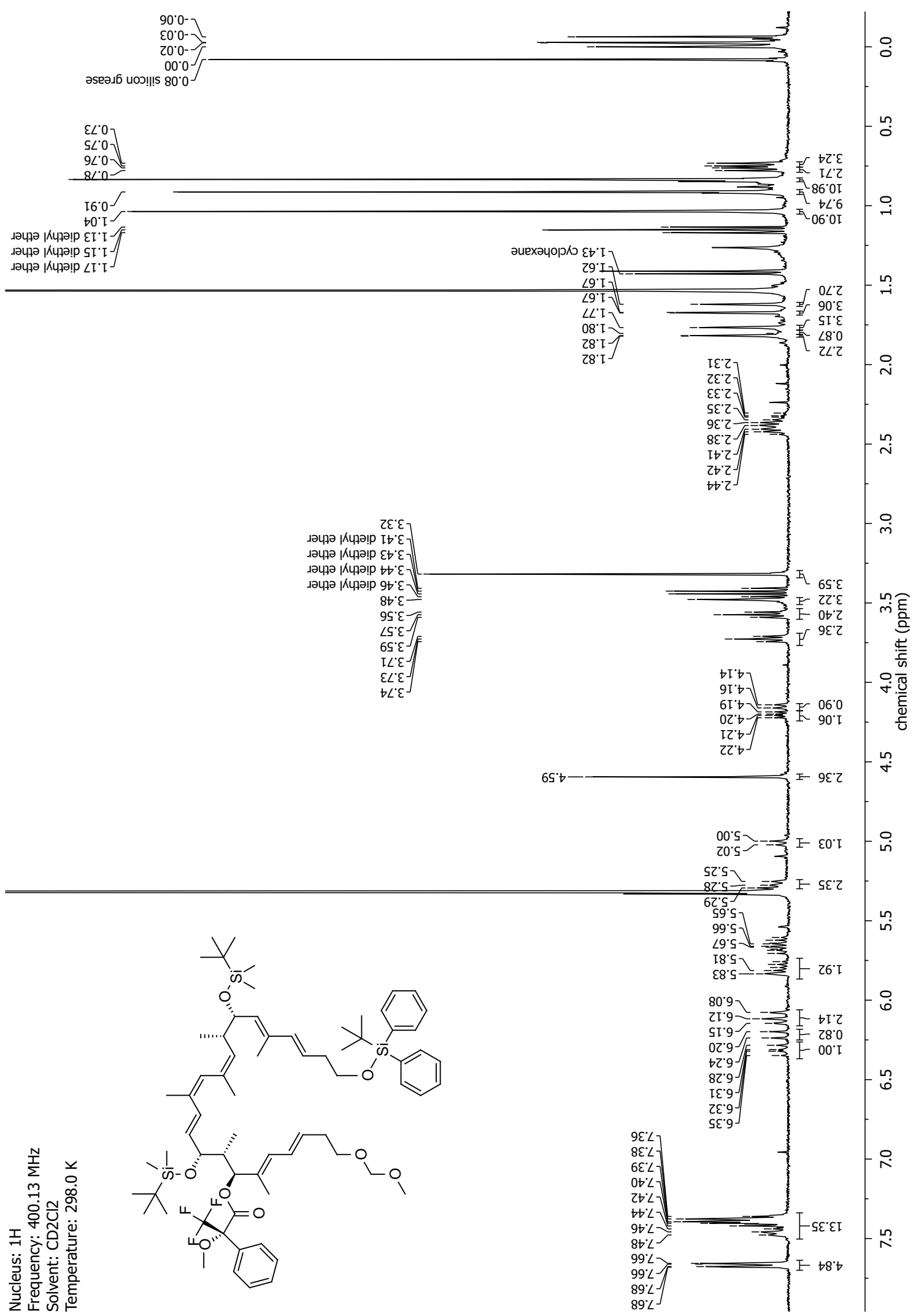


NMR-Spectrum for Compound 65a-M2

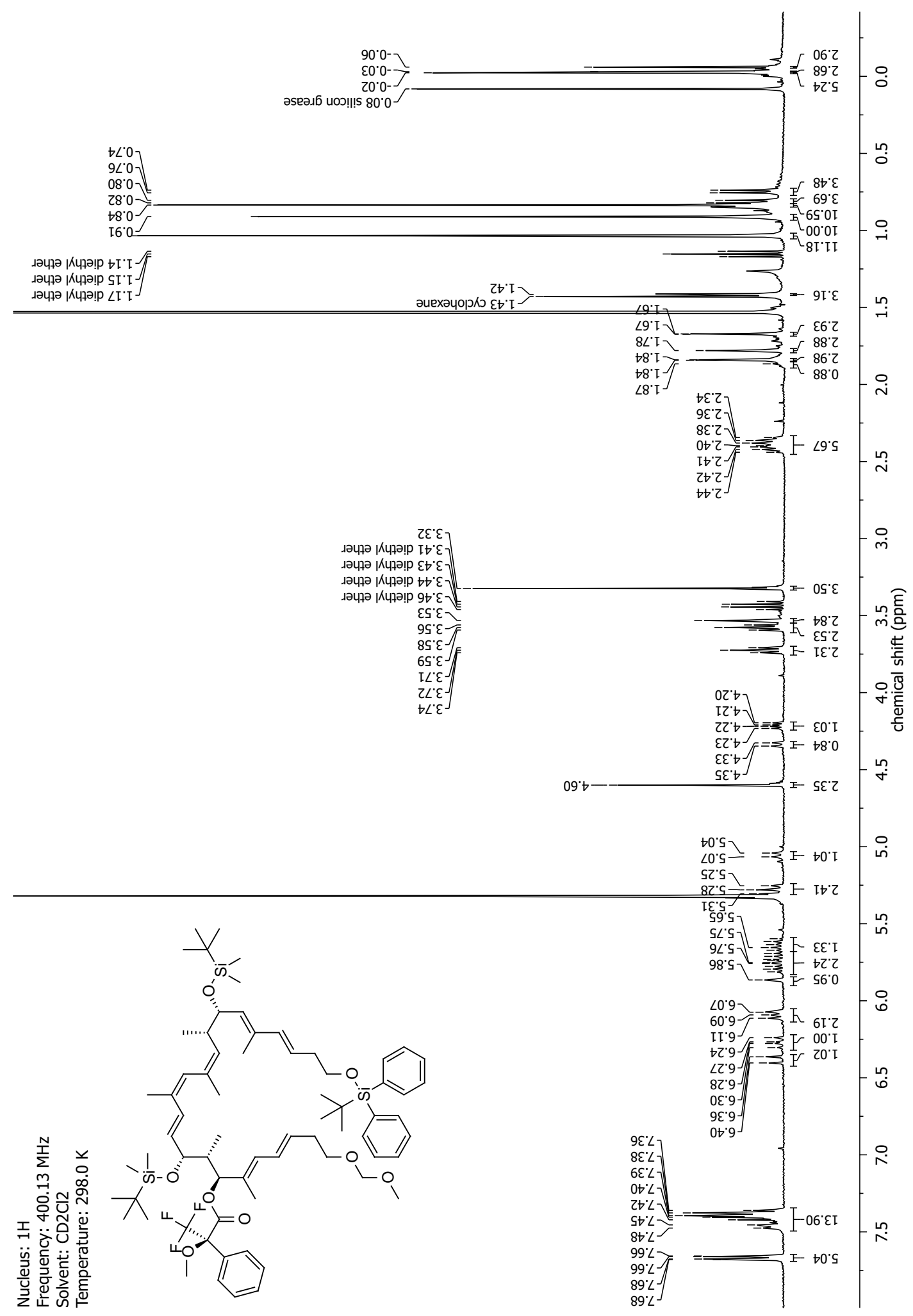


NMR-Spectra for Compound 66a

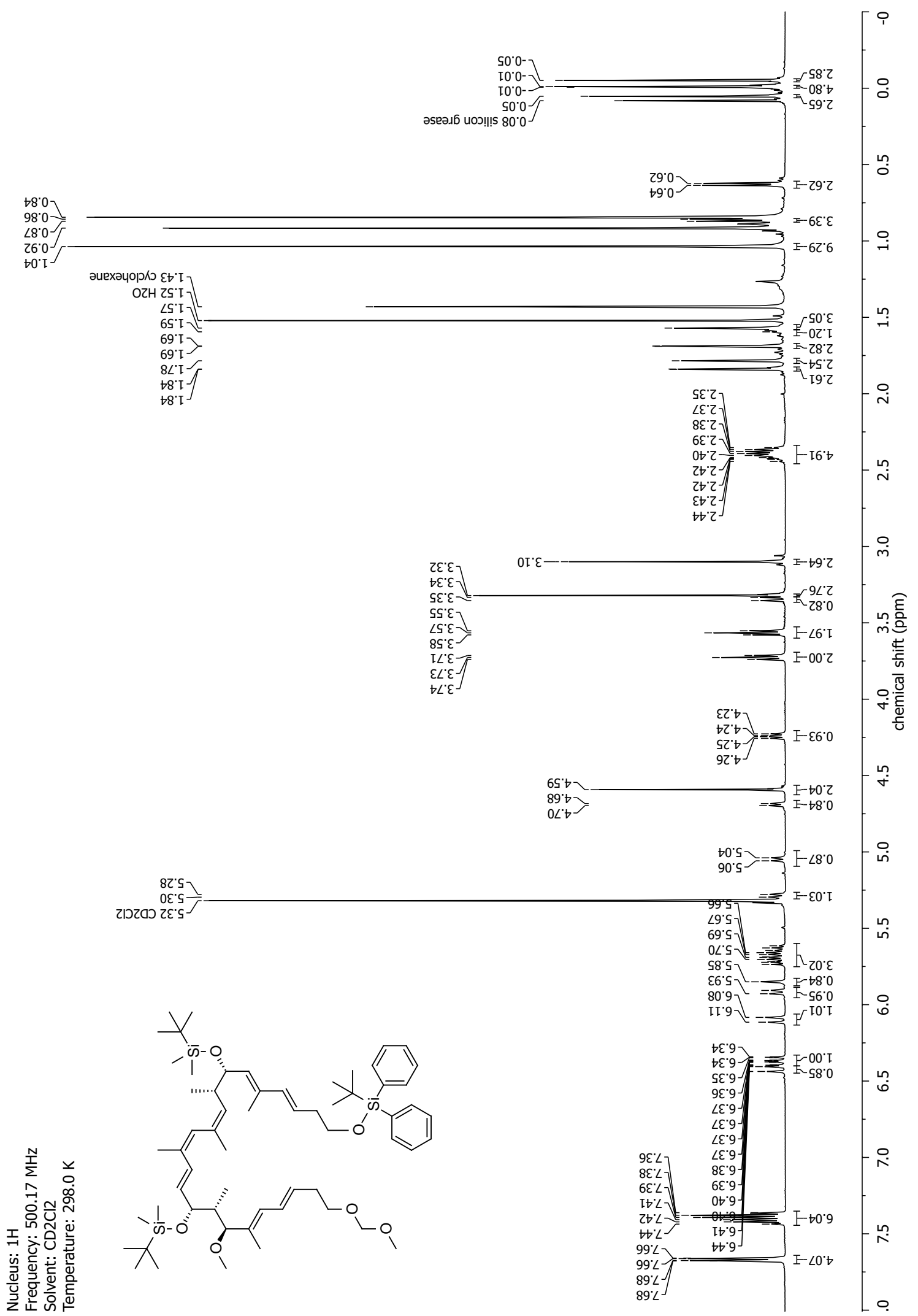




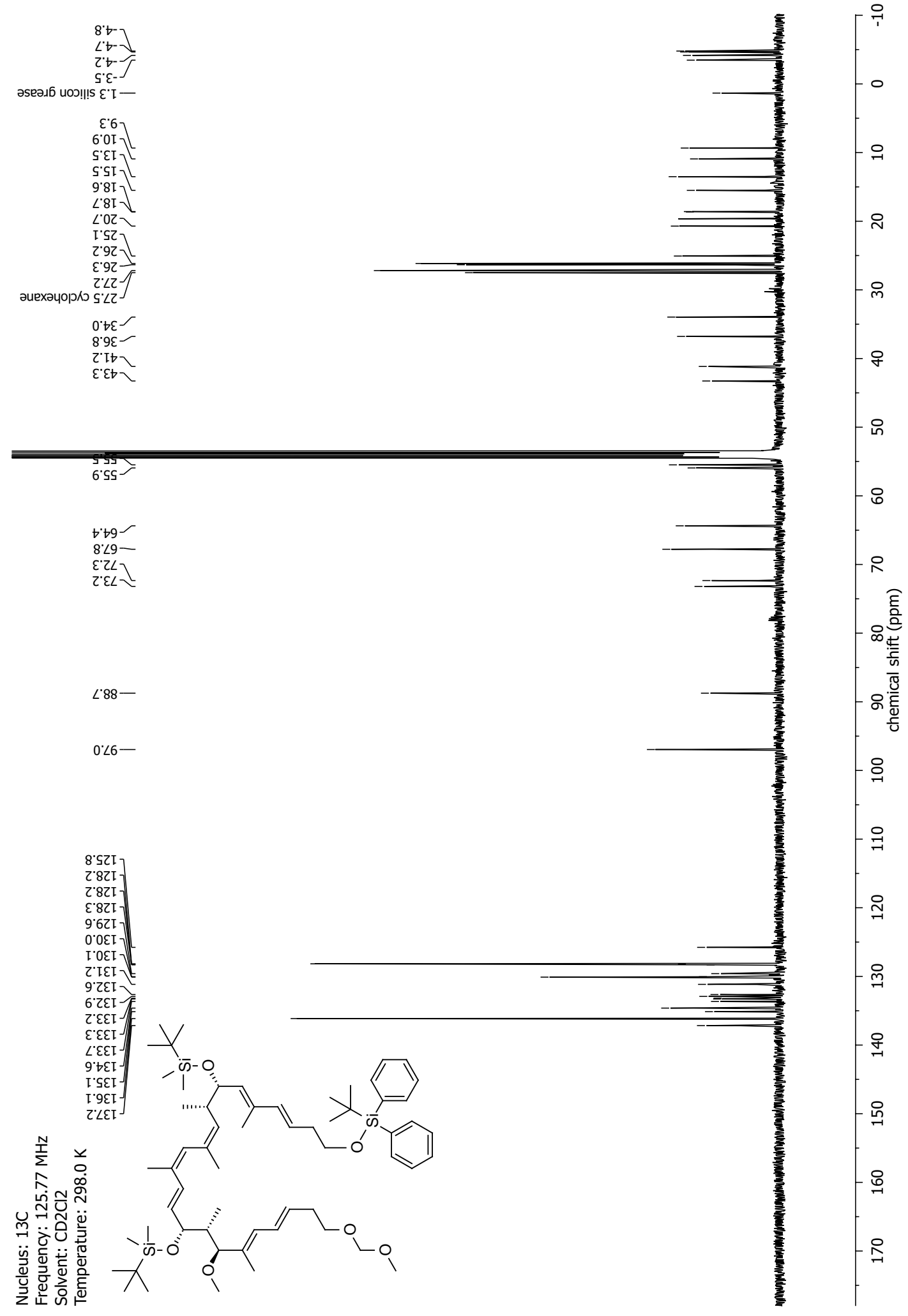




\section{NMR-Spectra for Compound 67}

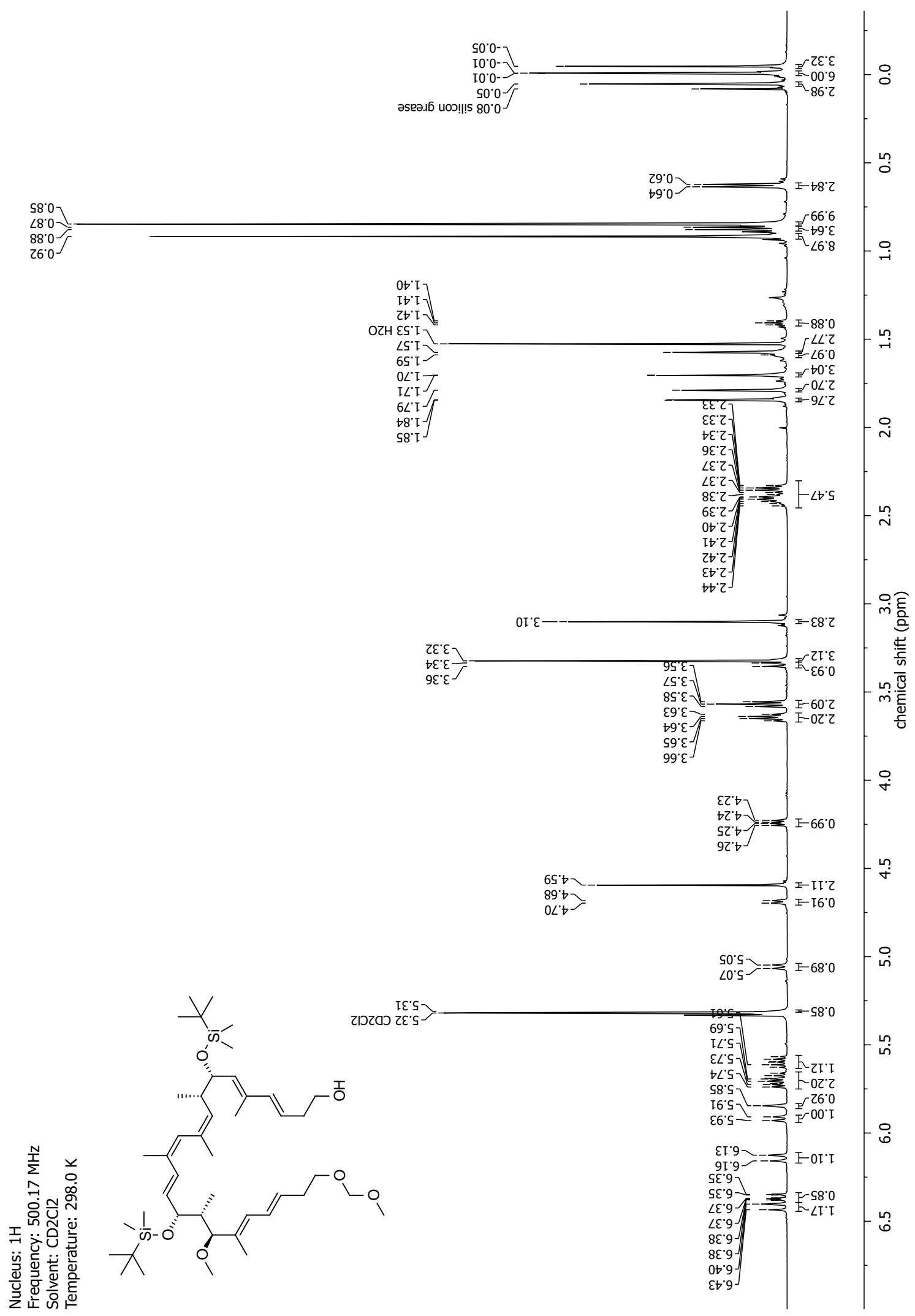




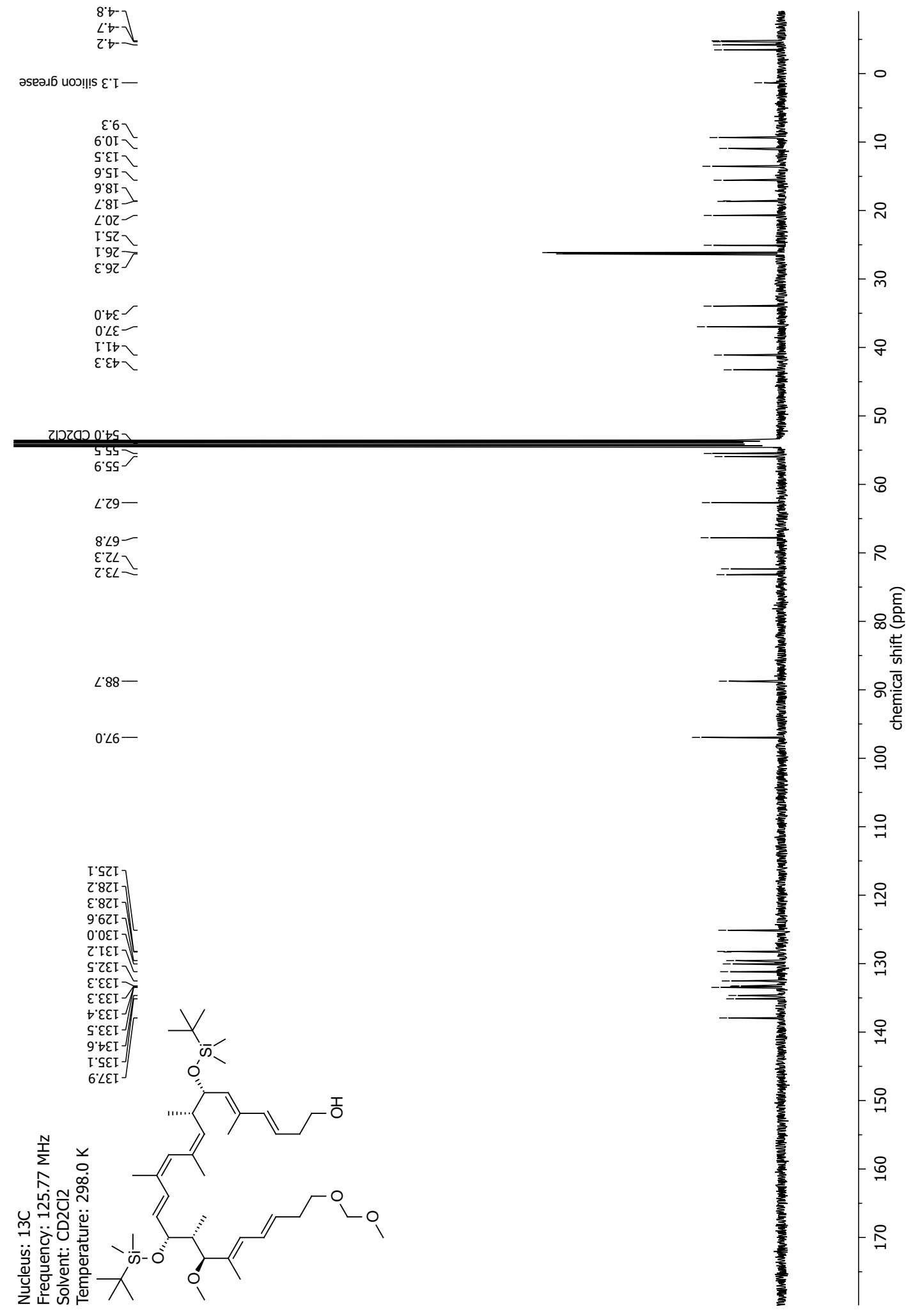


NMR-Spectra for Compound 64

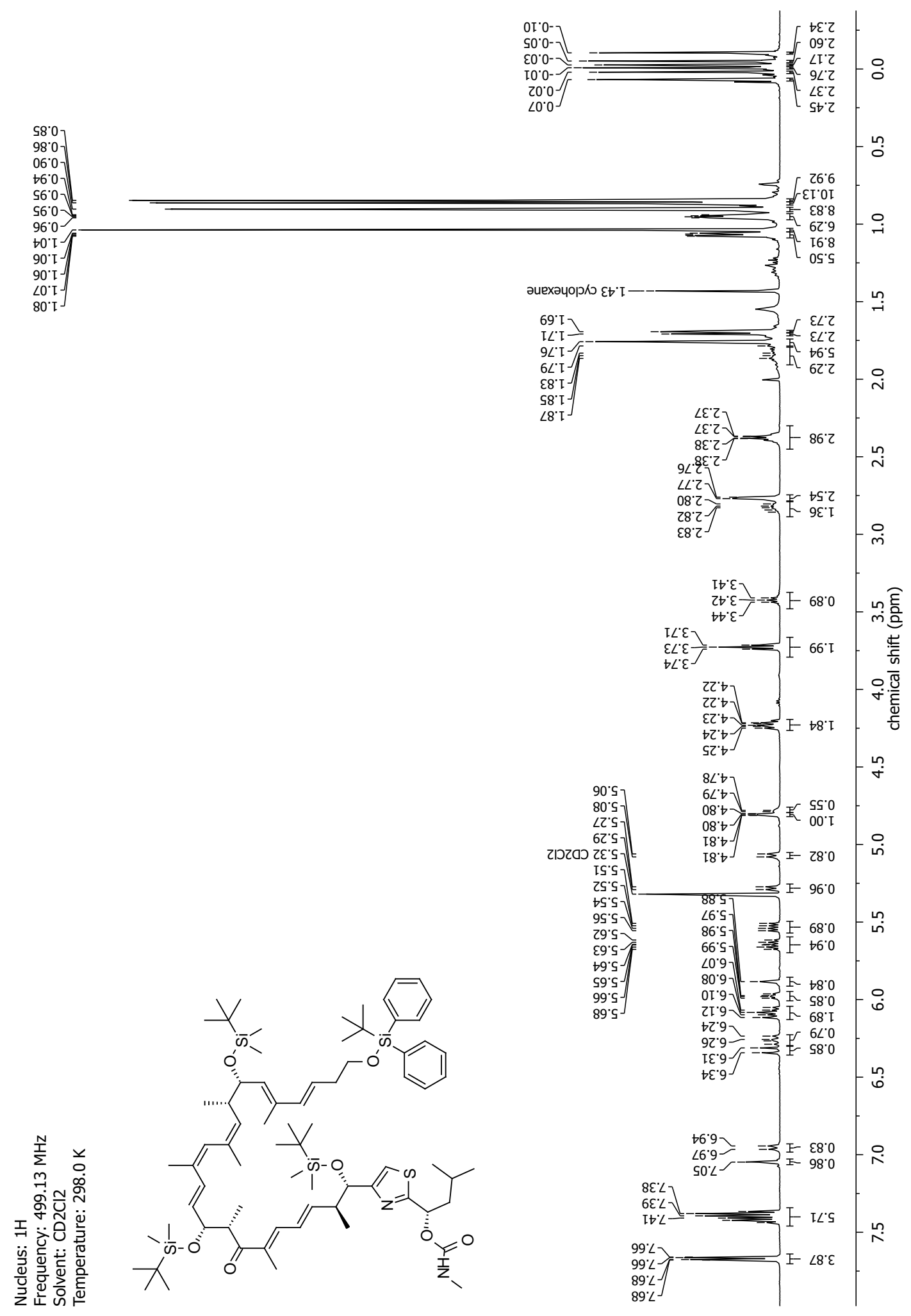




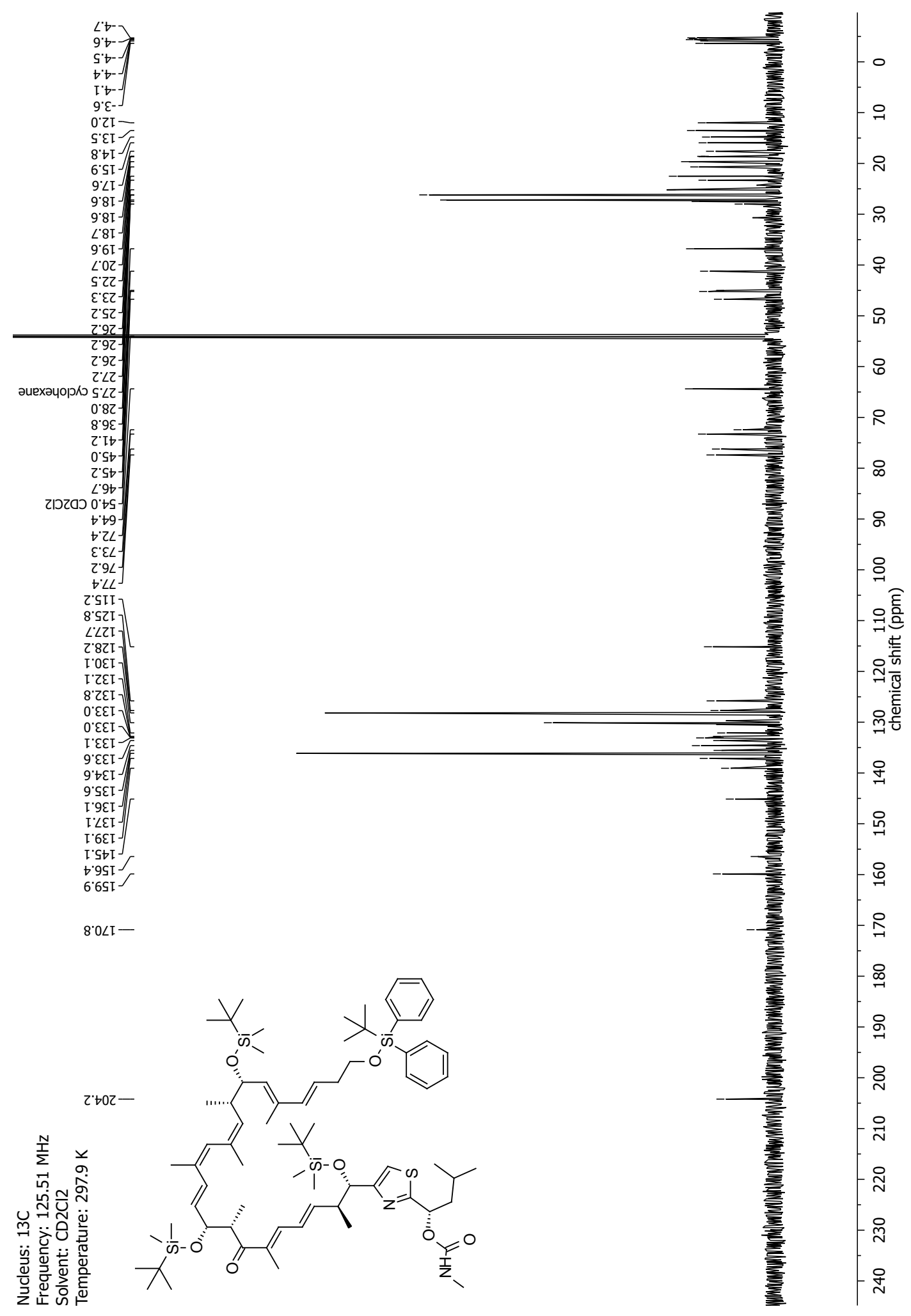




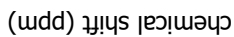

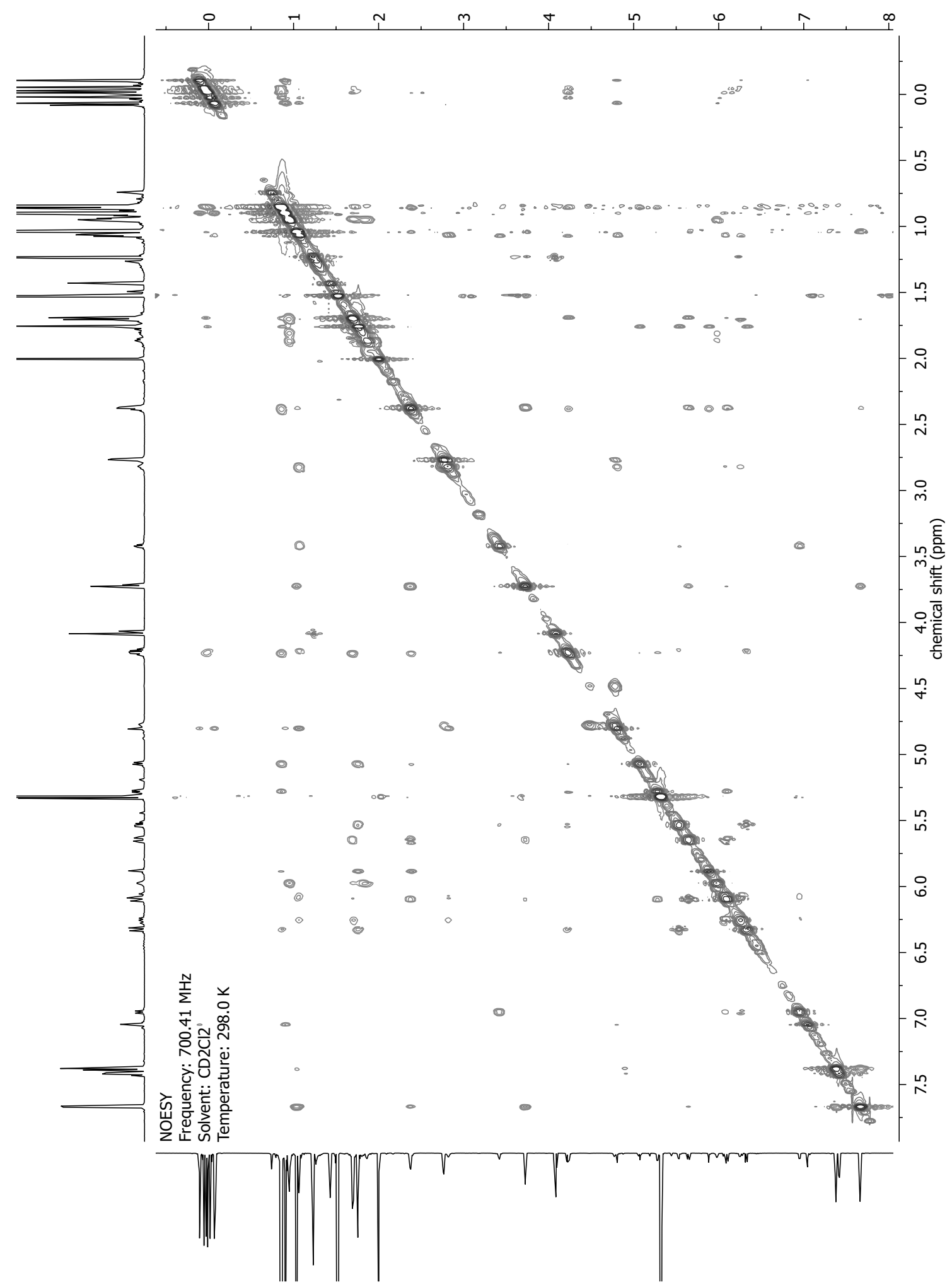


NMR-Spectra for Compound 65b

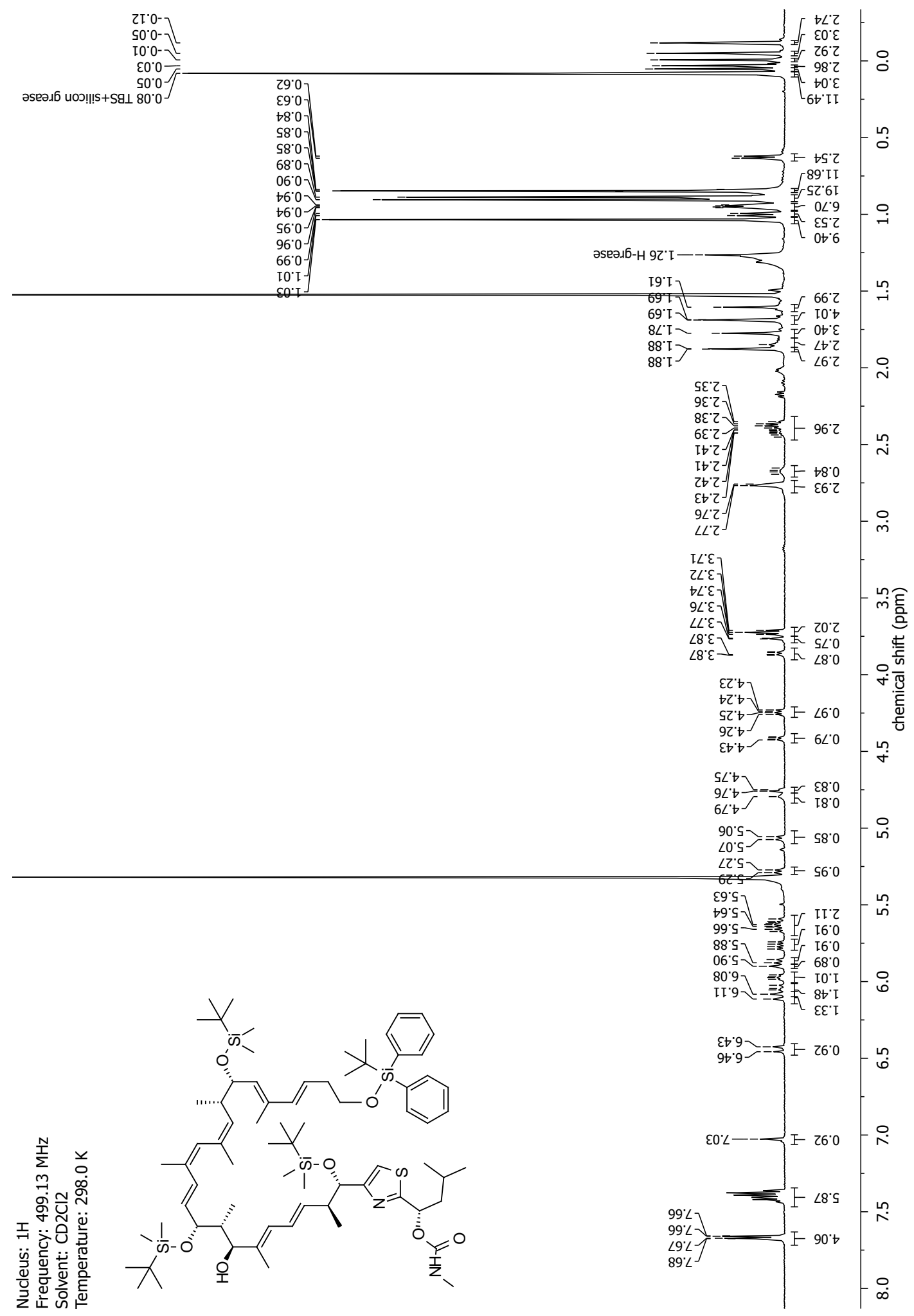




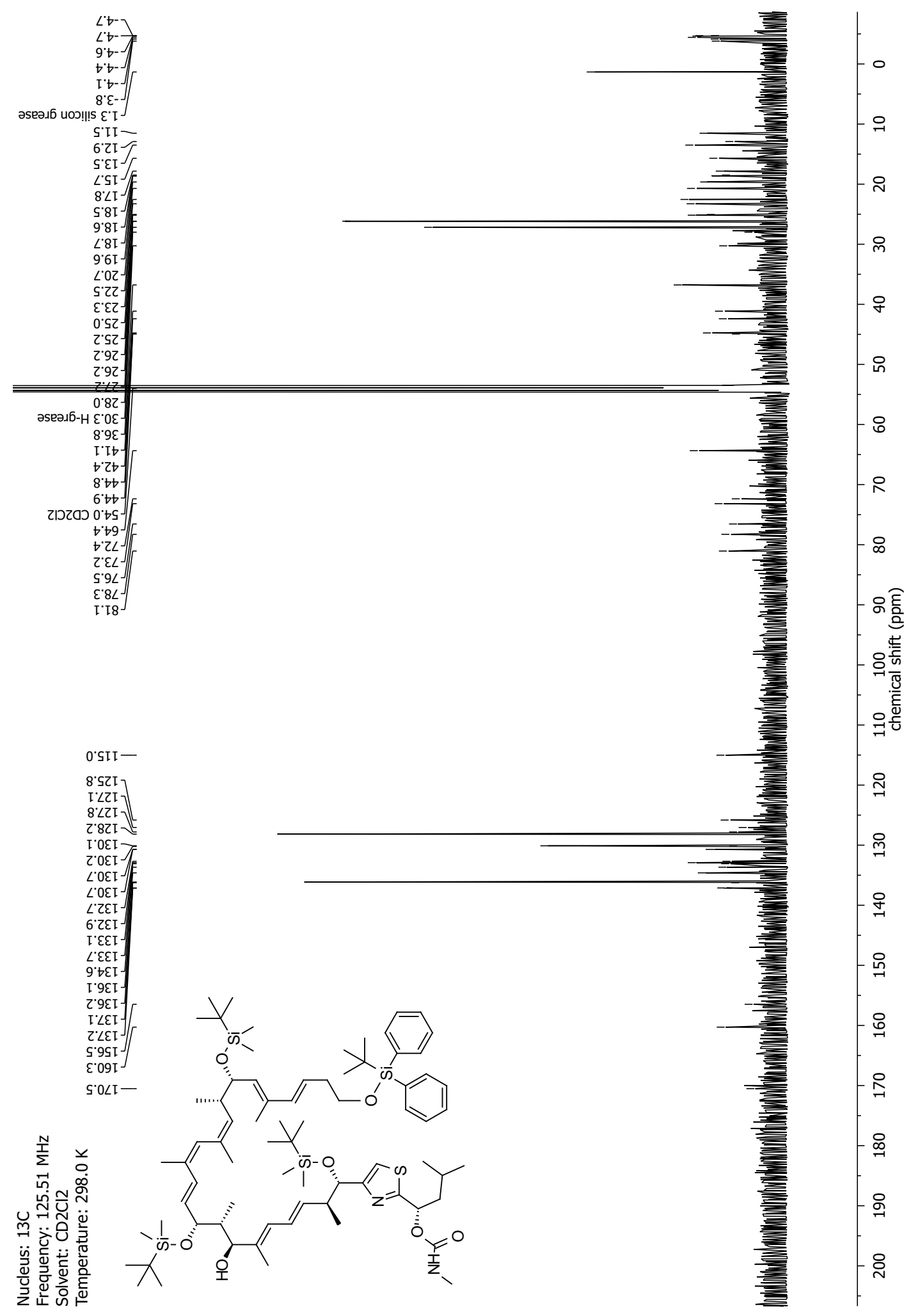


(mdd) भ!บऽ ןеग!шәцว

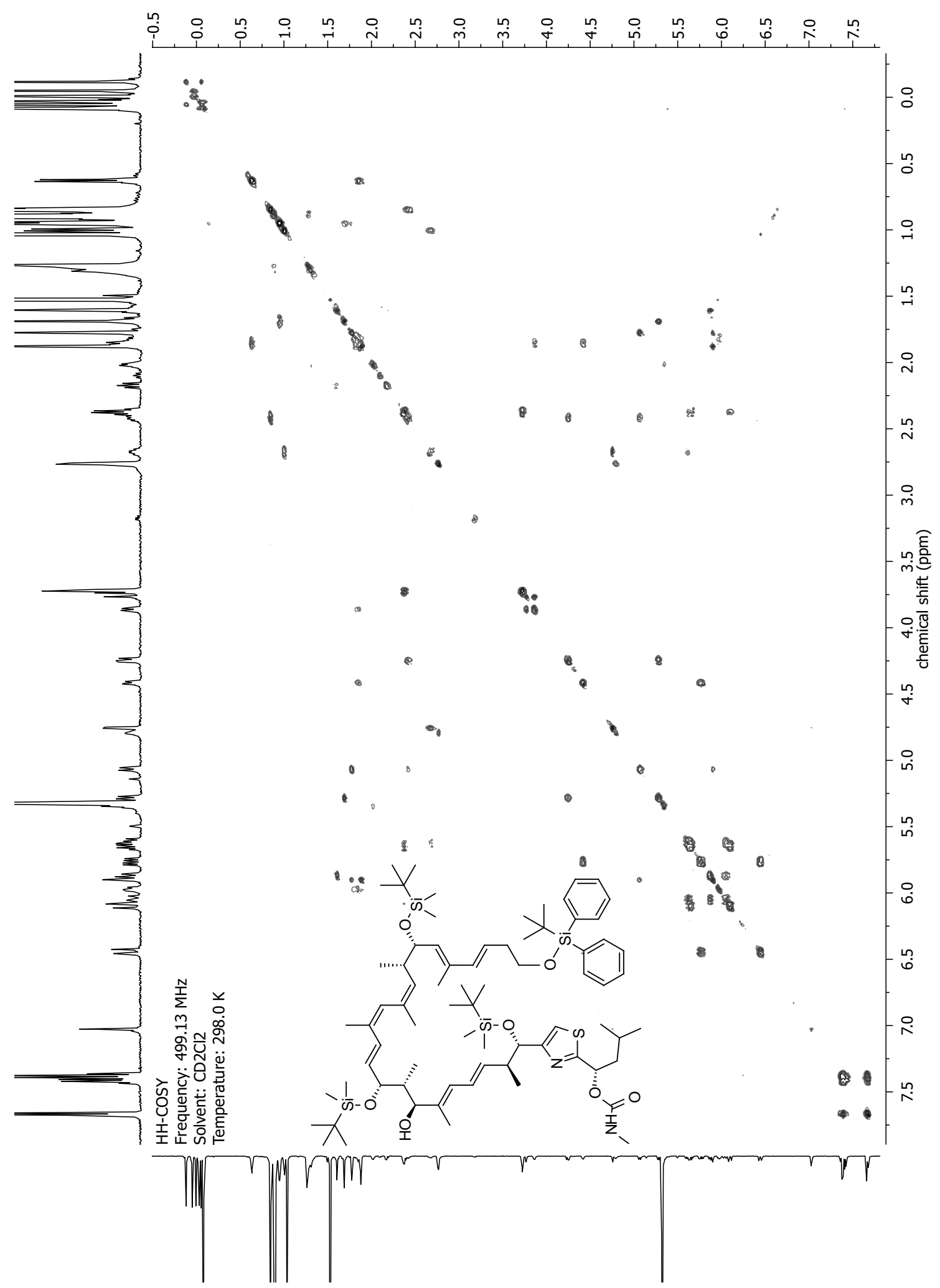


(mdd) भ!บऽ ןеग!шәцว

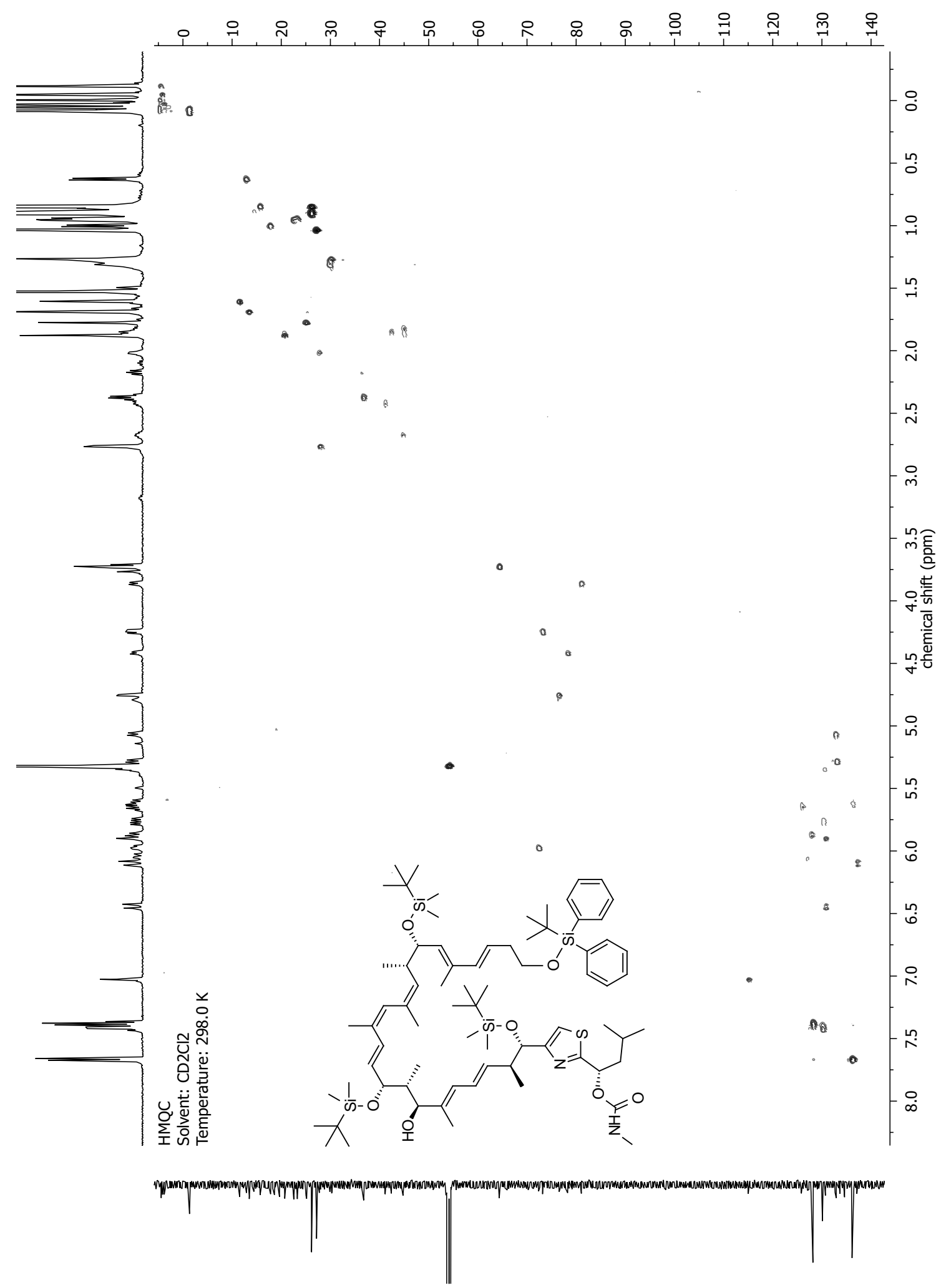


(mdd) भ!บऽ ןеग!шәцว

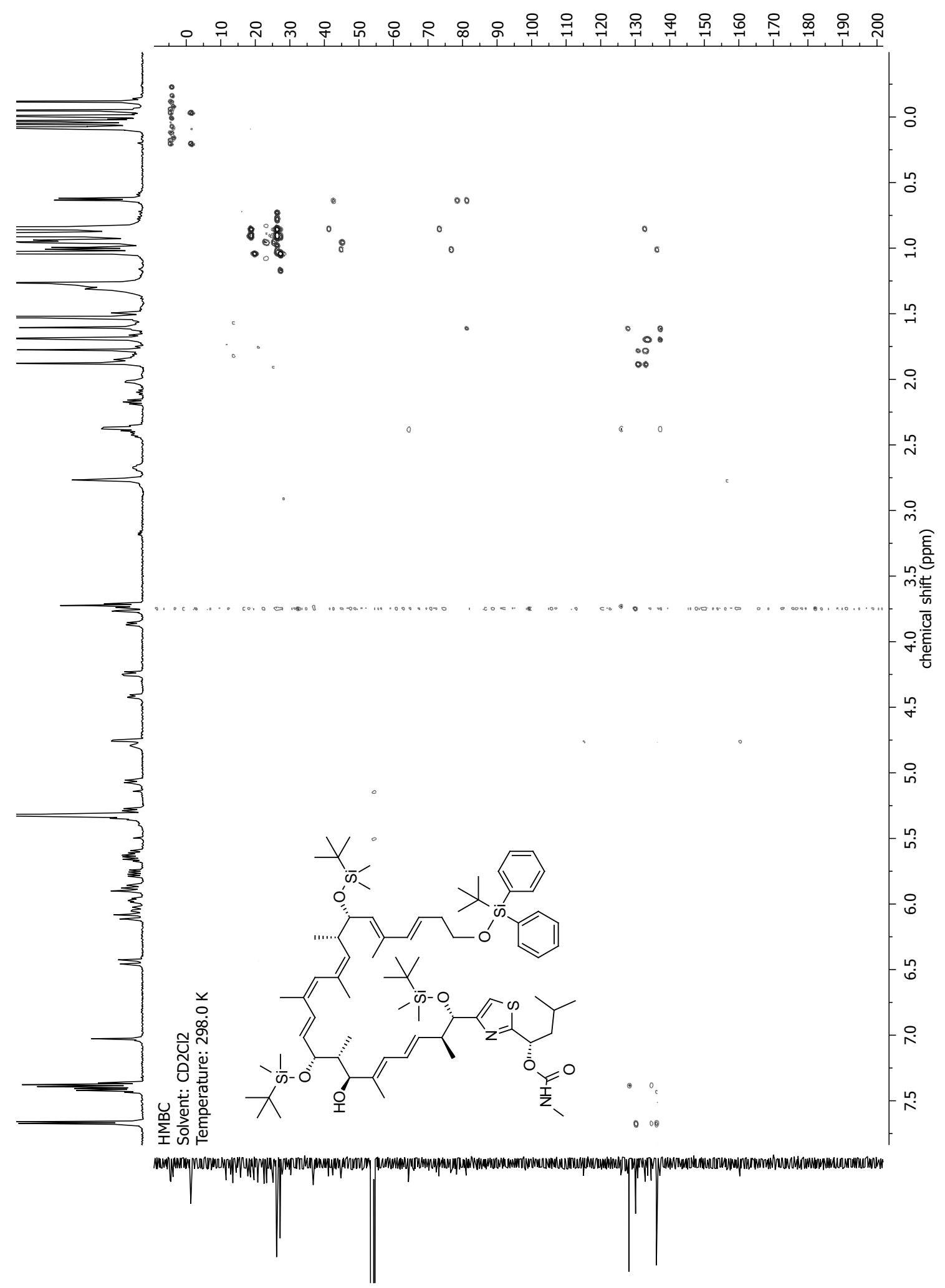


NMR-Spectra for Compound 73

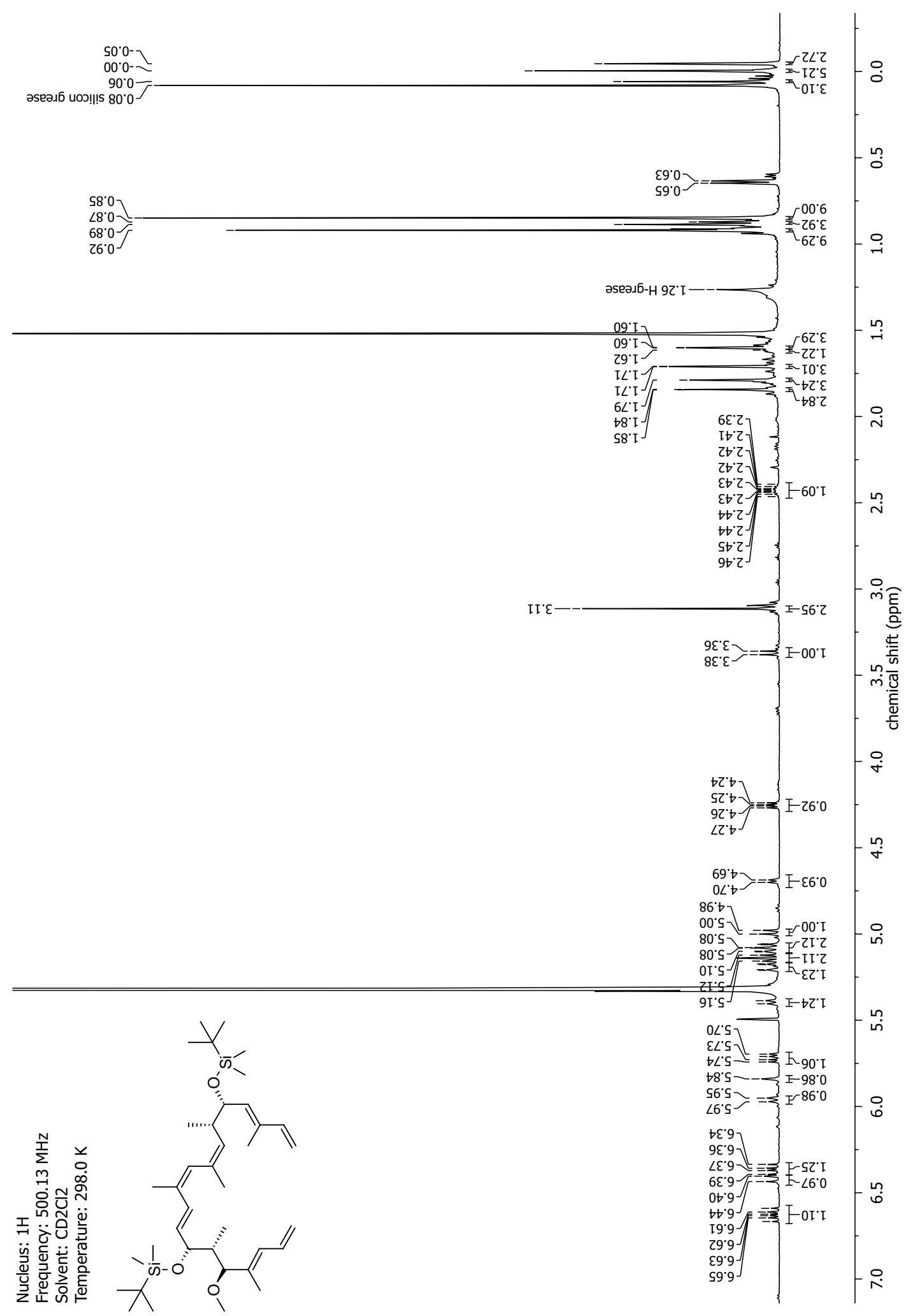



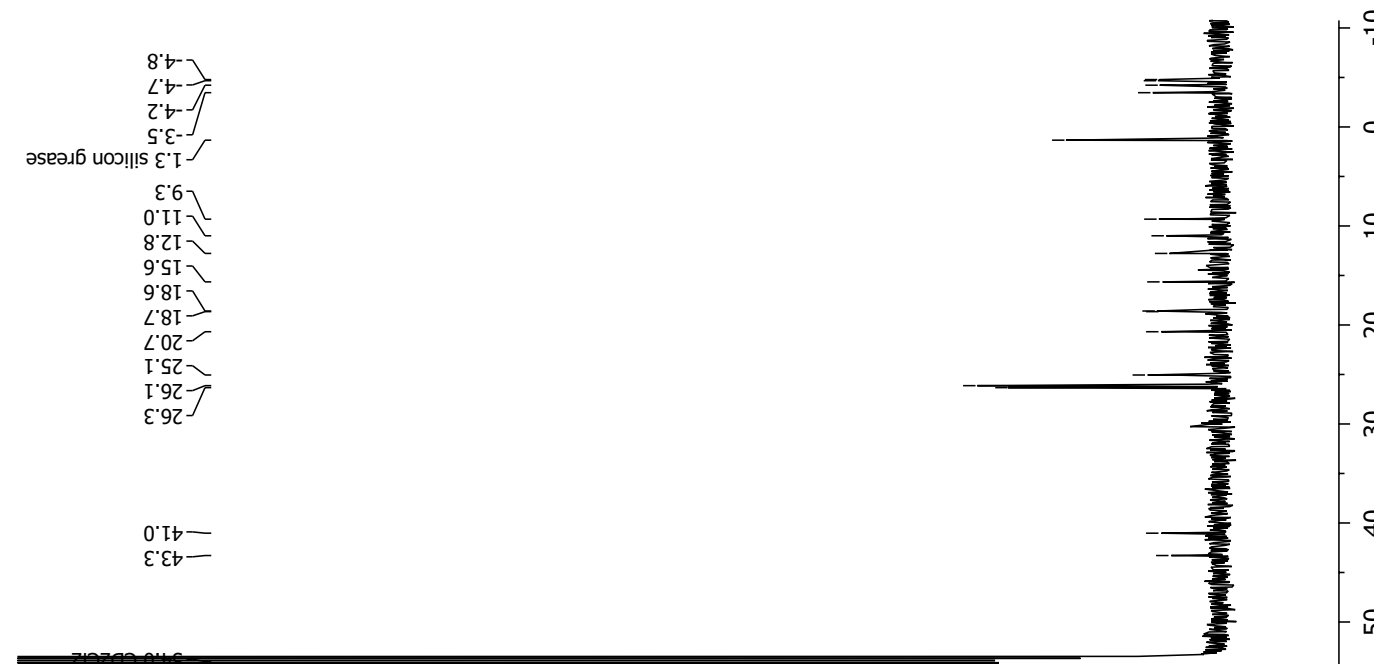

$0.95-$

$\varepsilon \cdot Z L$
$Z \cdot \varepsilon L$

$9 \cdot 88-$

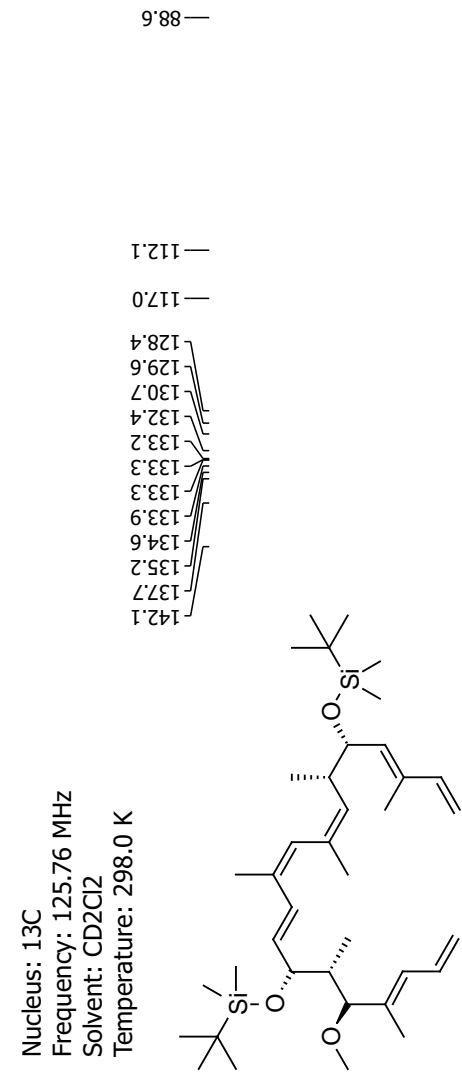




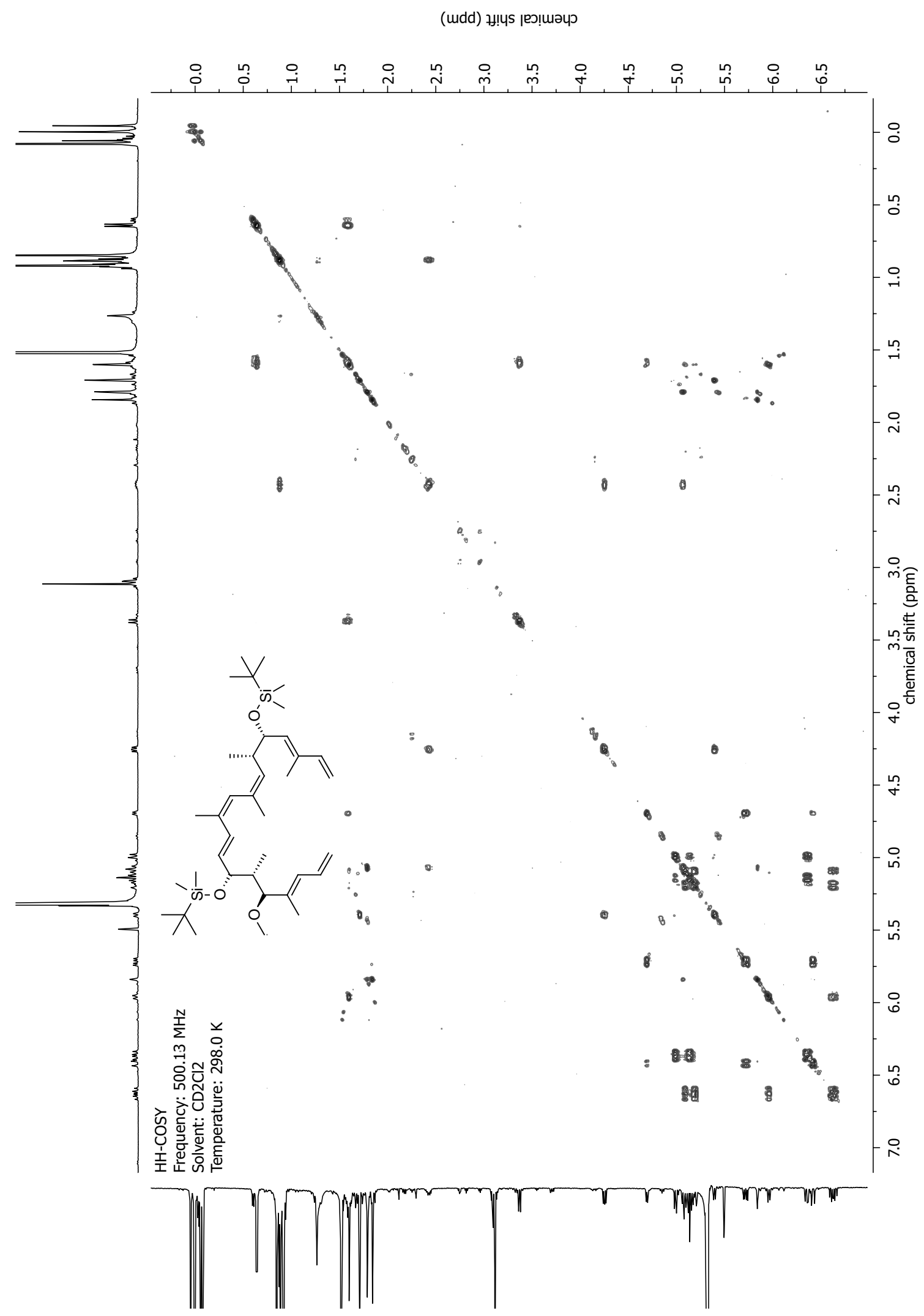


(udd) भ!บऽ ןеว!щәцว

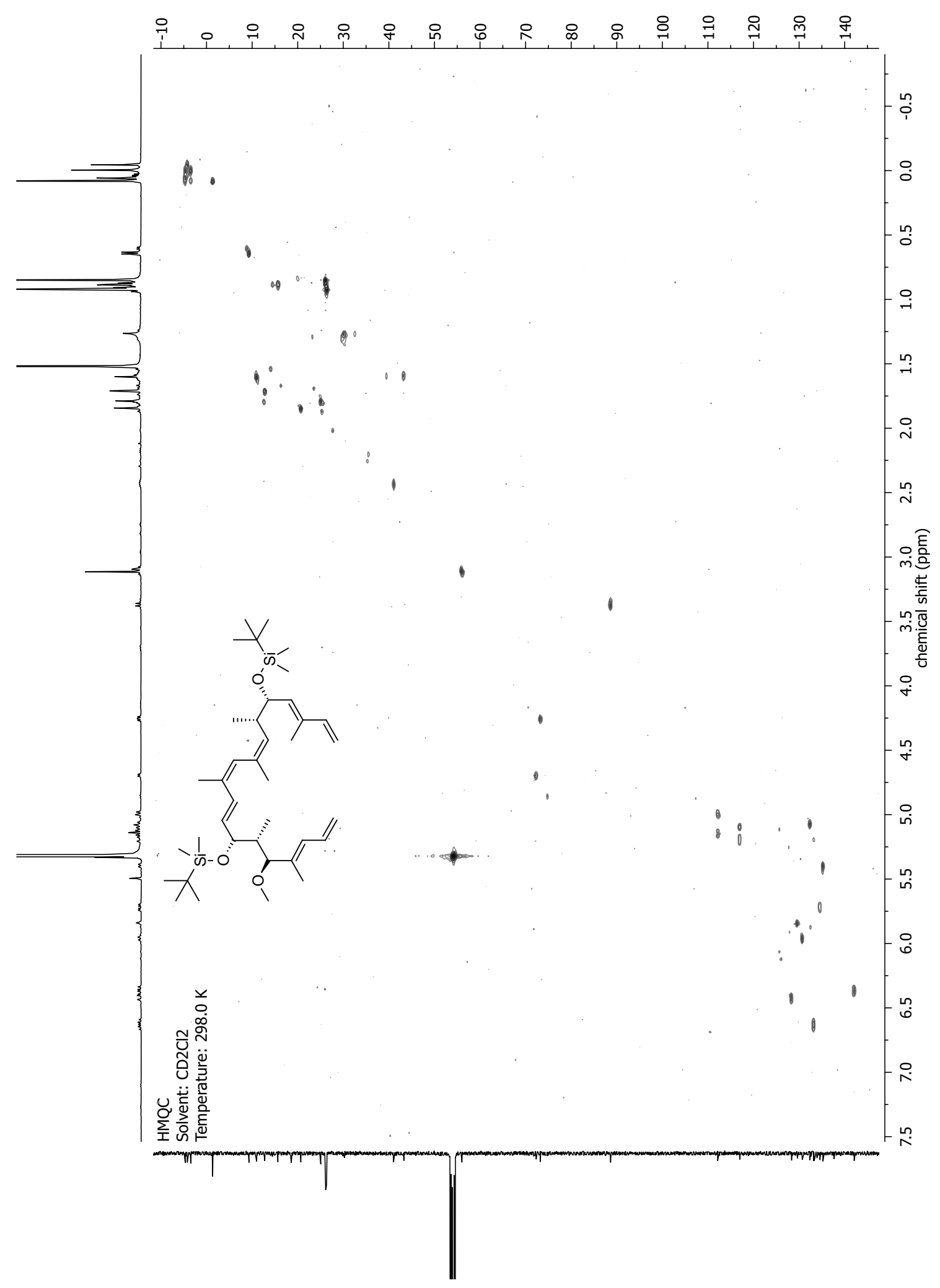




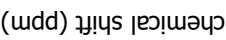

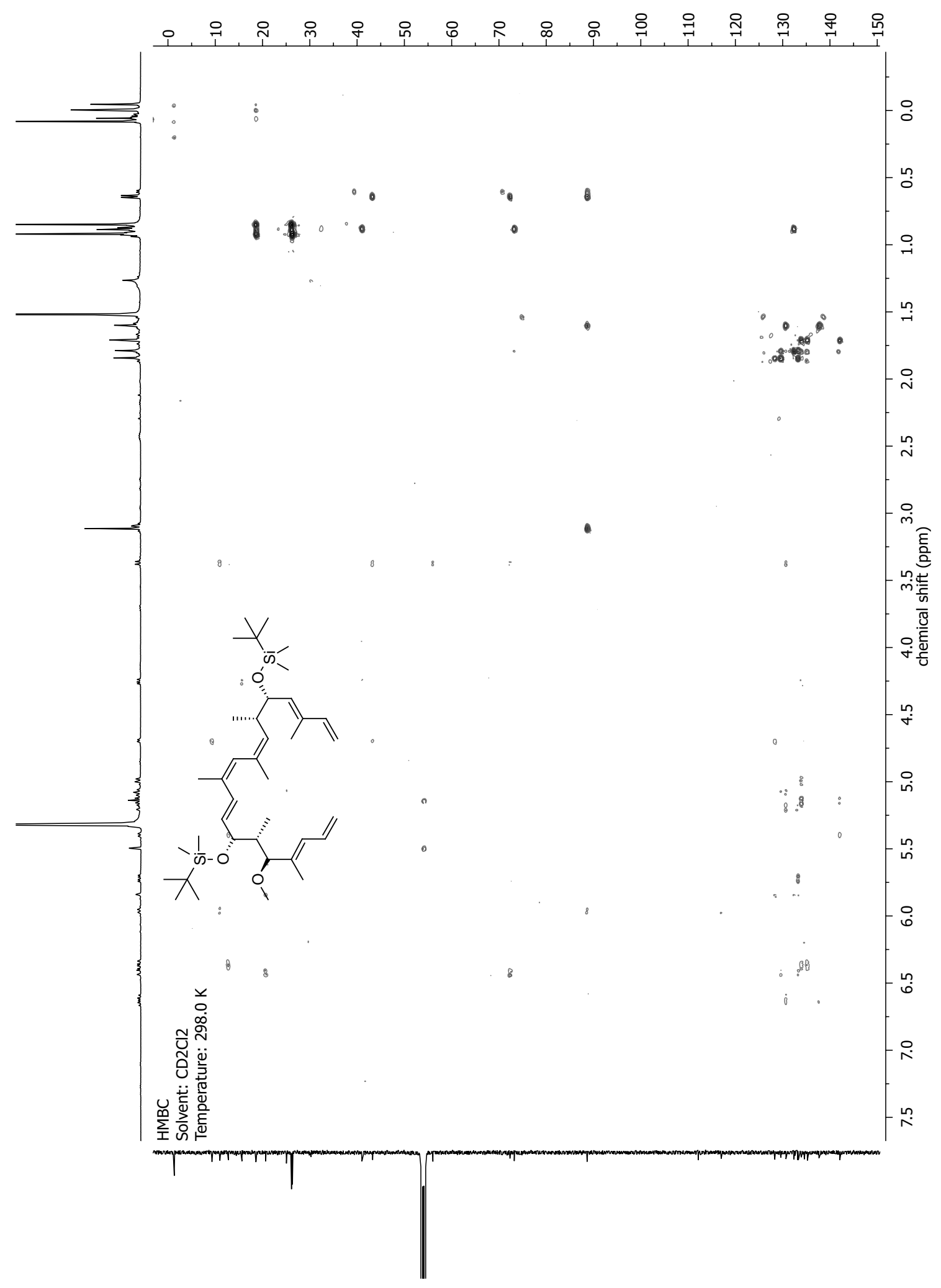


NMR-Spectra for Compound 103

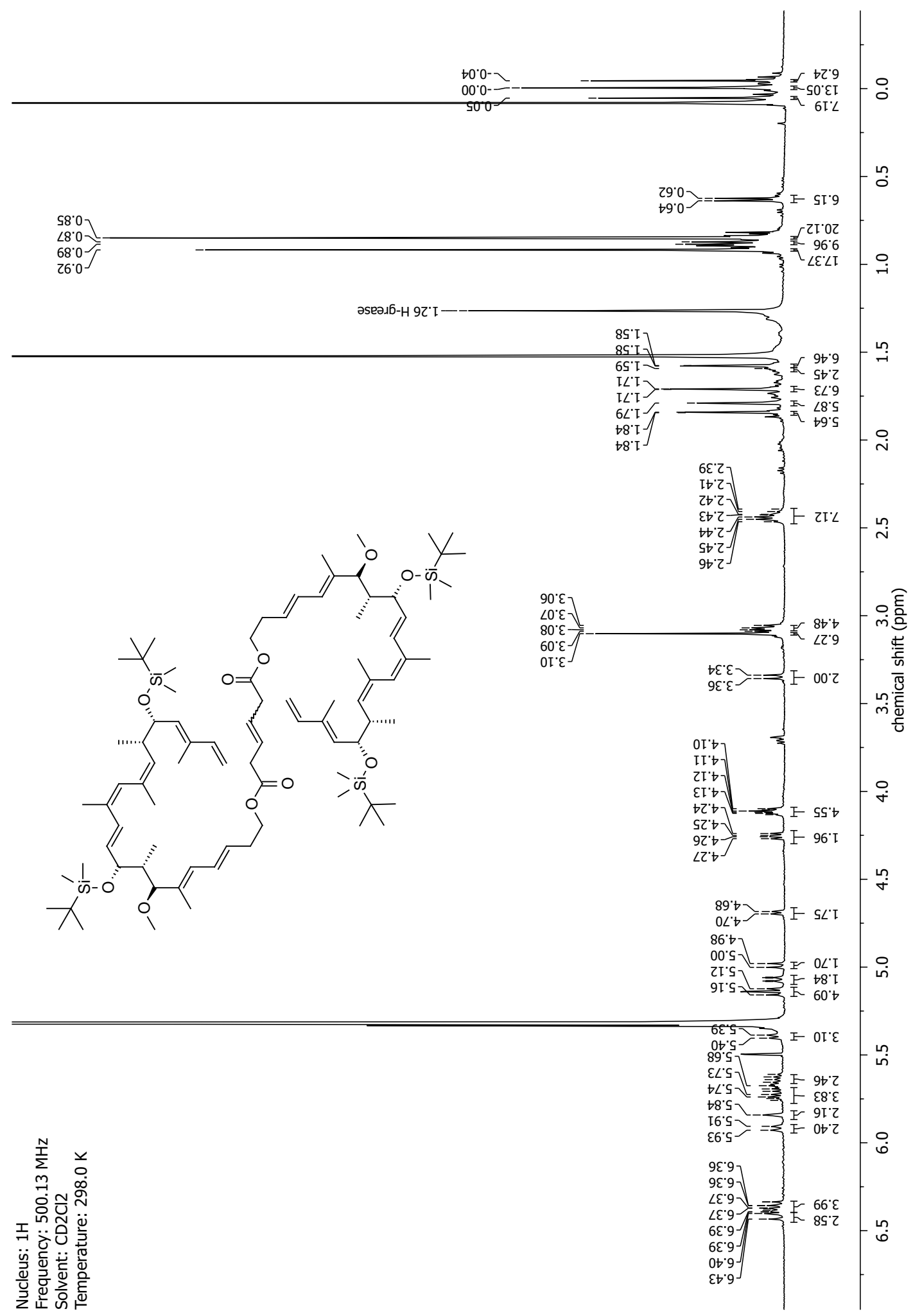




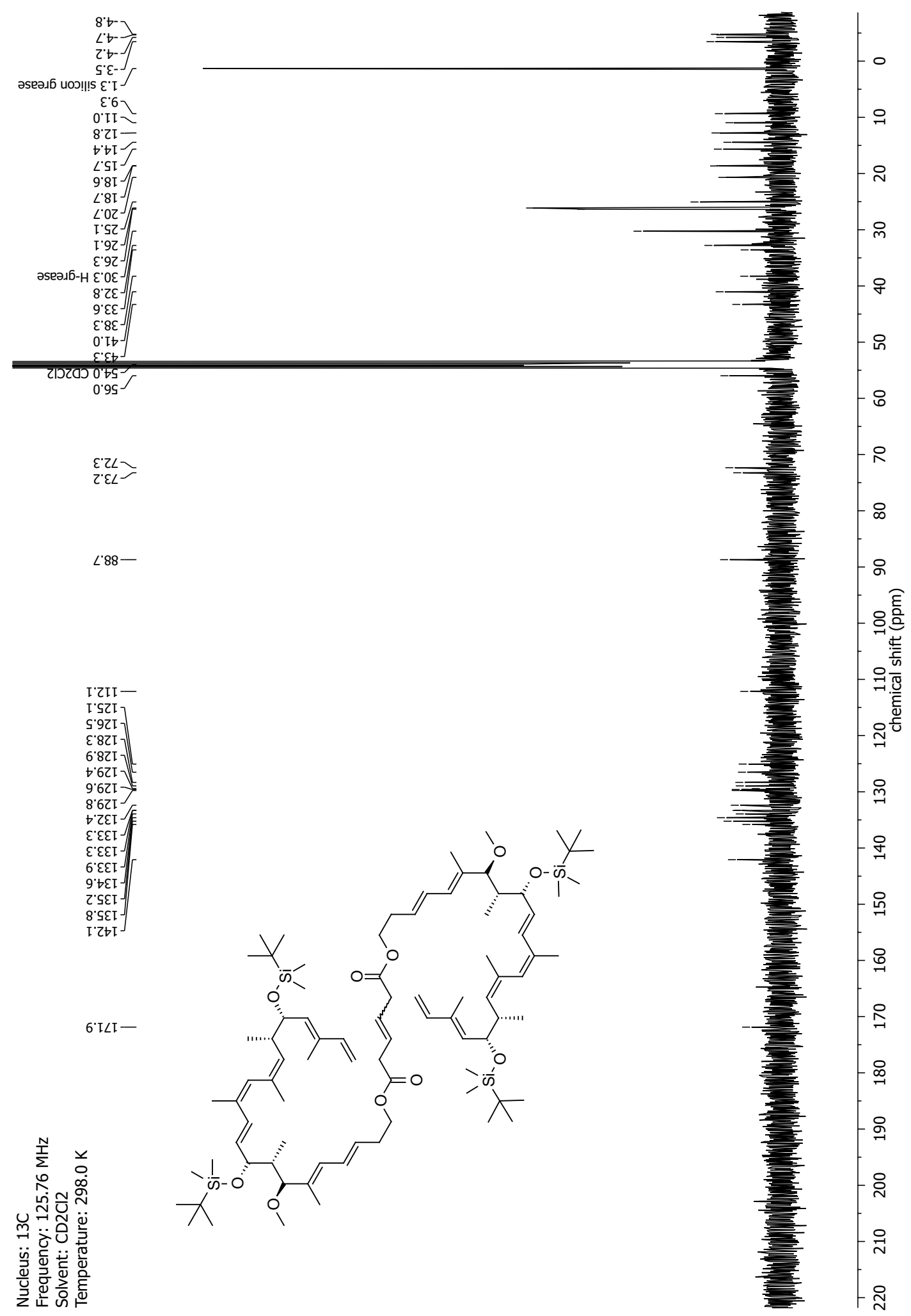




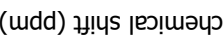

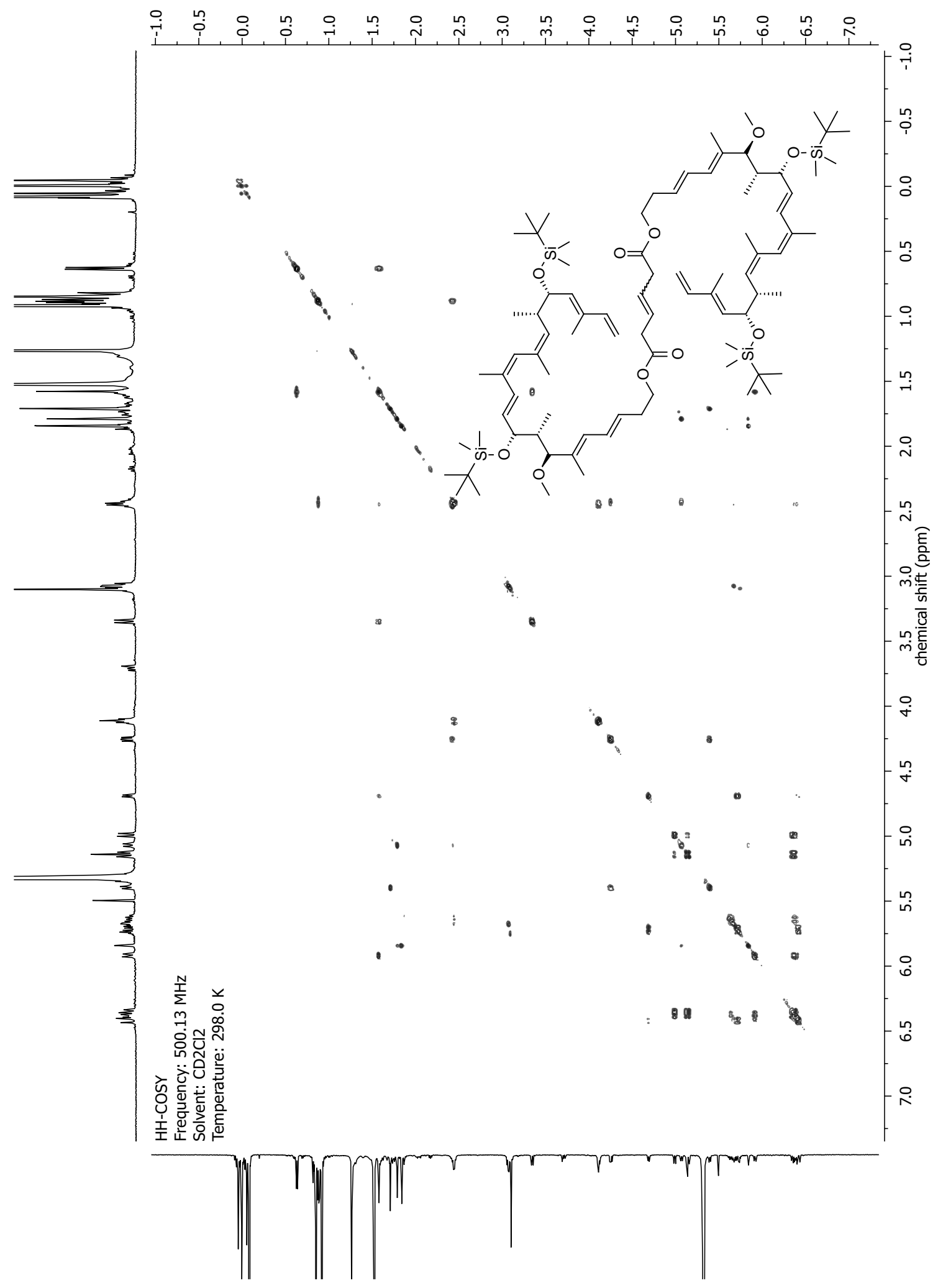


(udd) भ!บऽ ןеว!щәцว

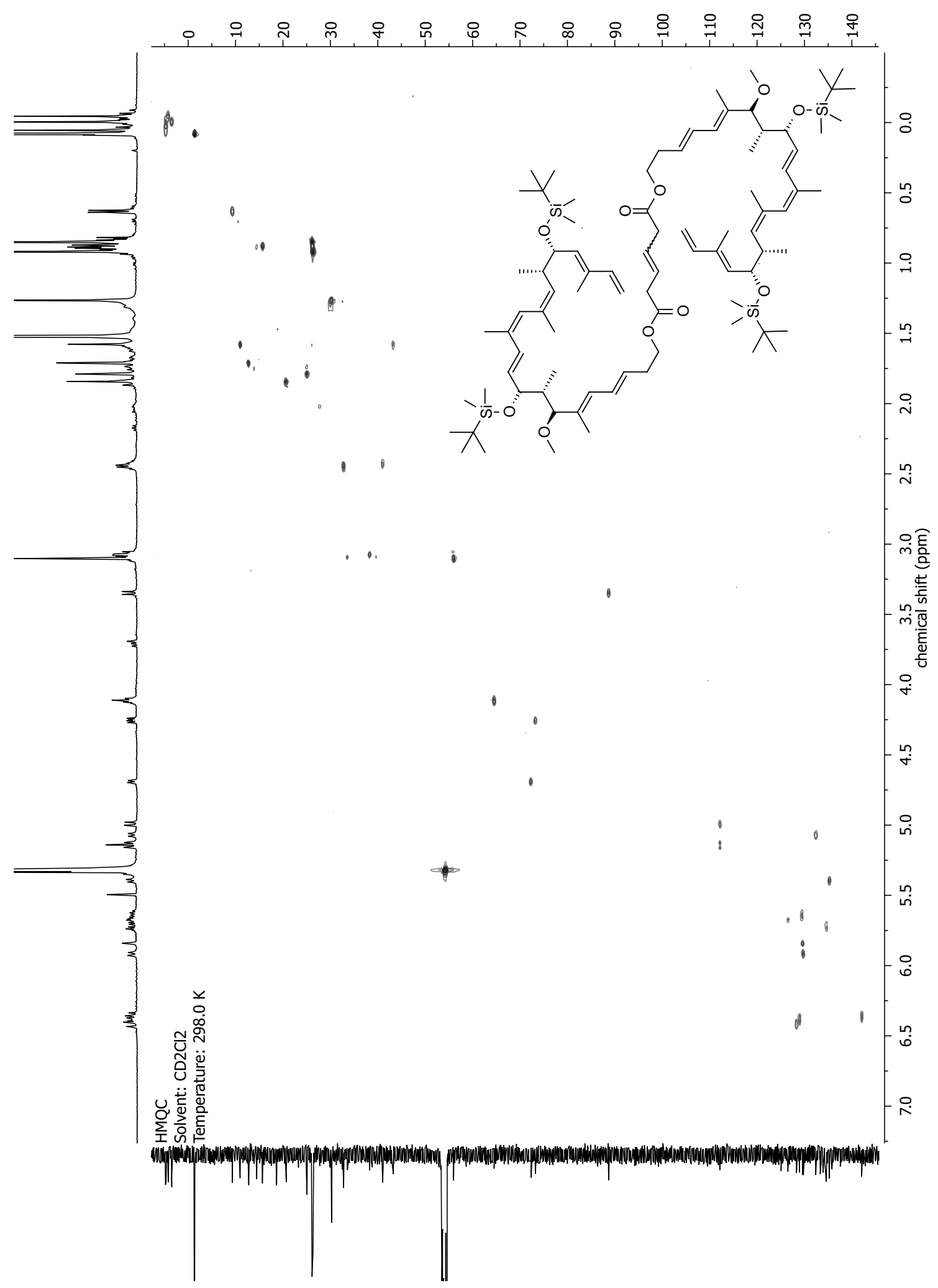


(udd) भ!บऽ ןеว!щәцว

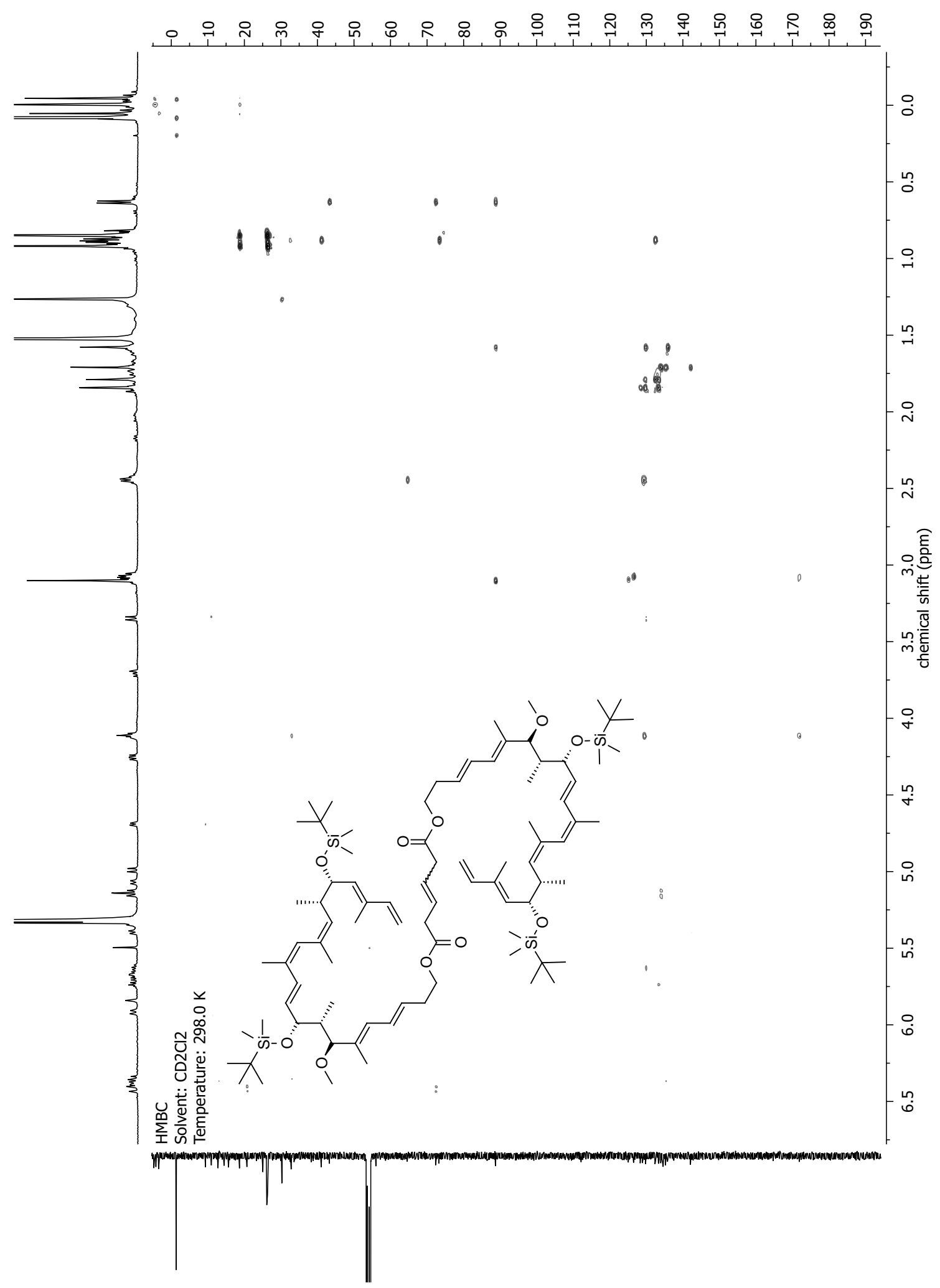


NMR-Spectra for Compound 75

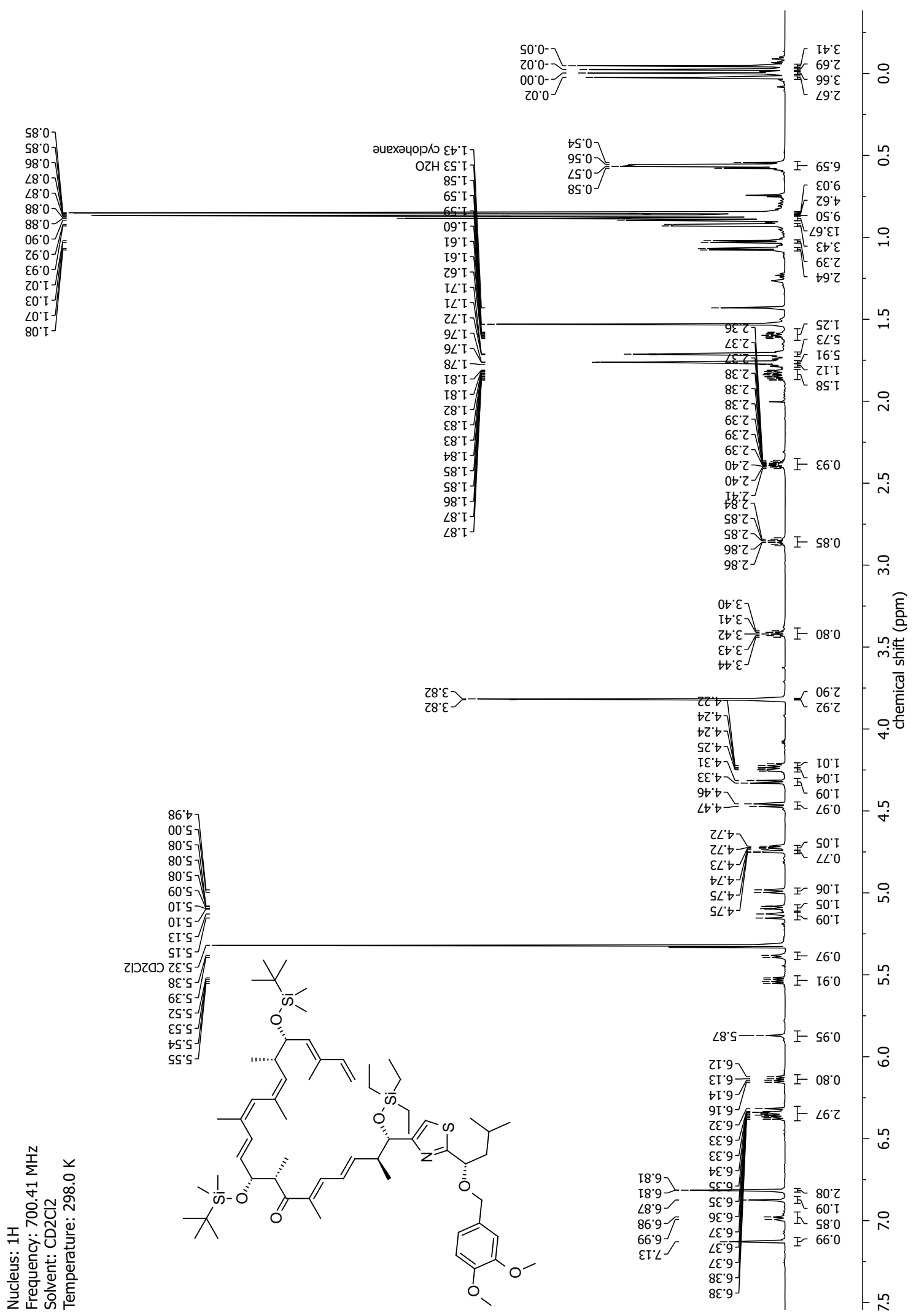




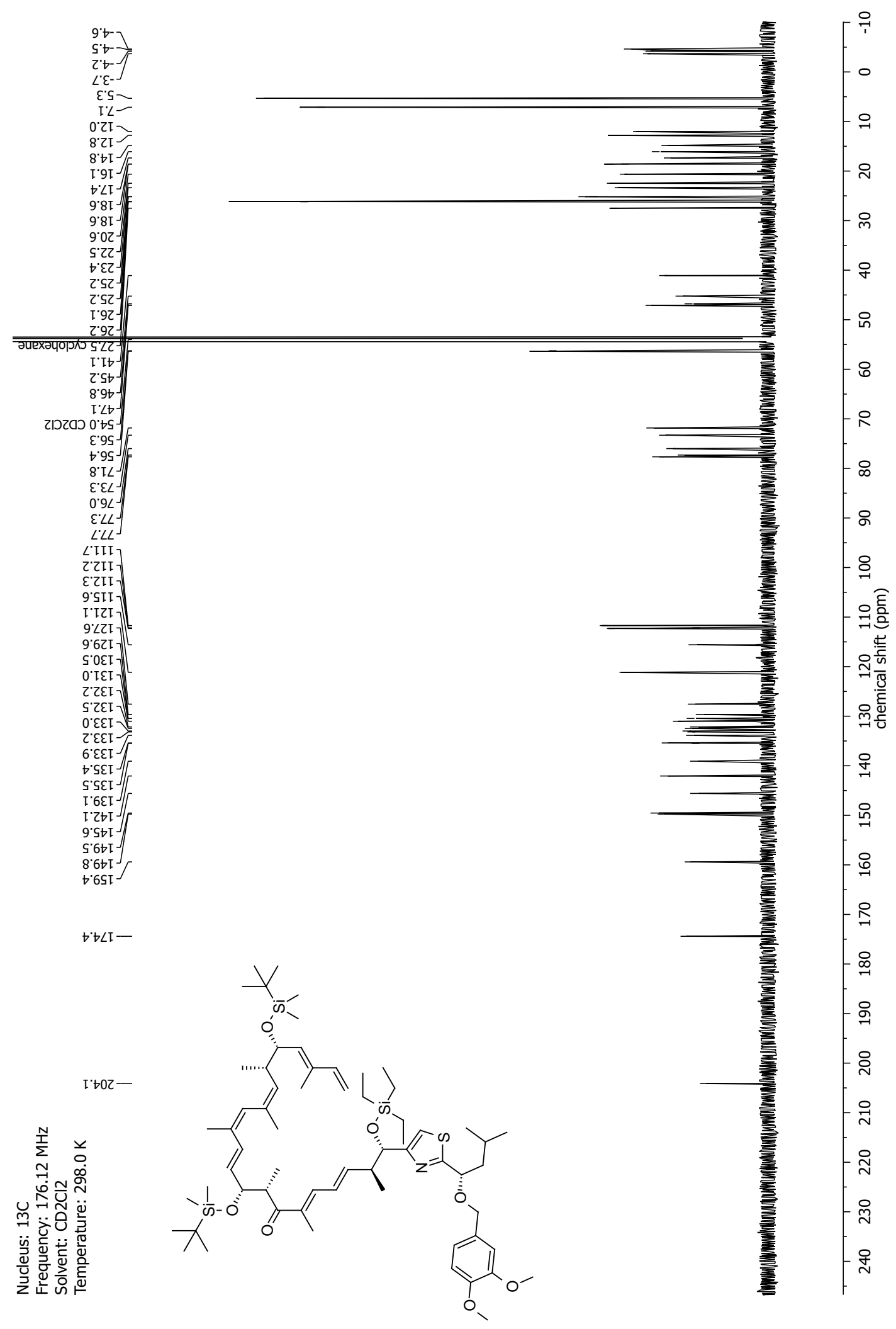




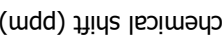

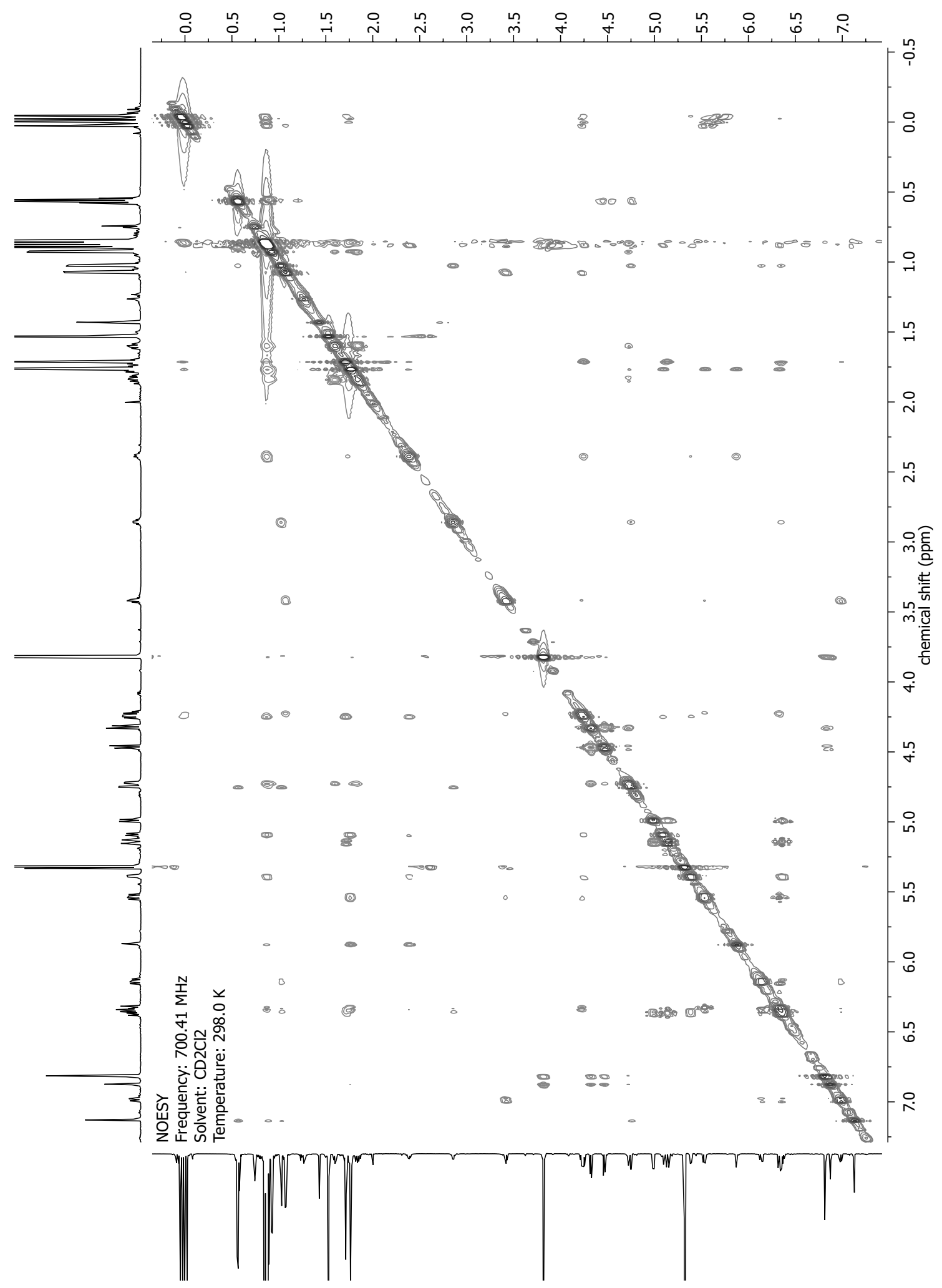


NMR-Spectra for Compound 104

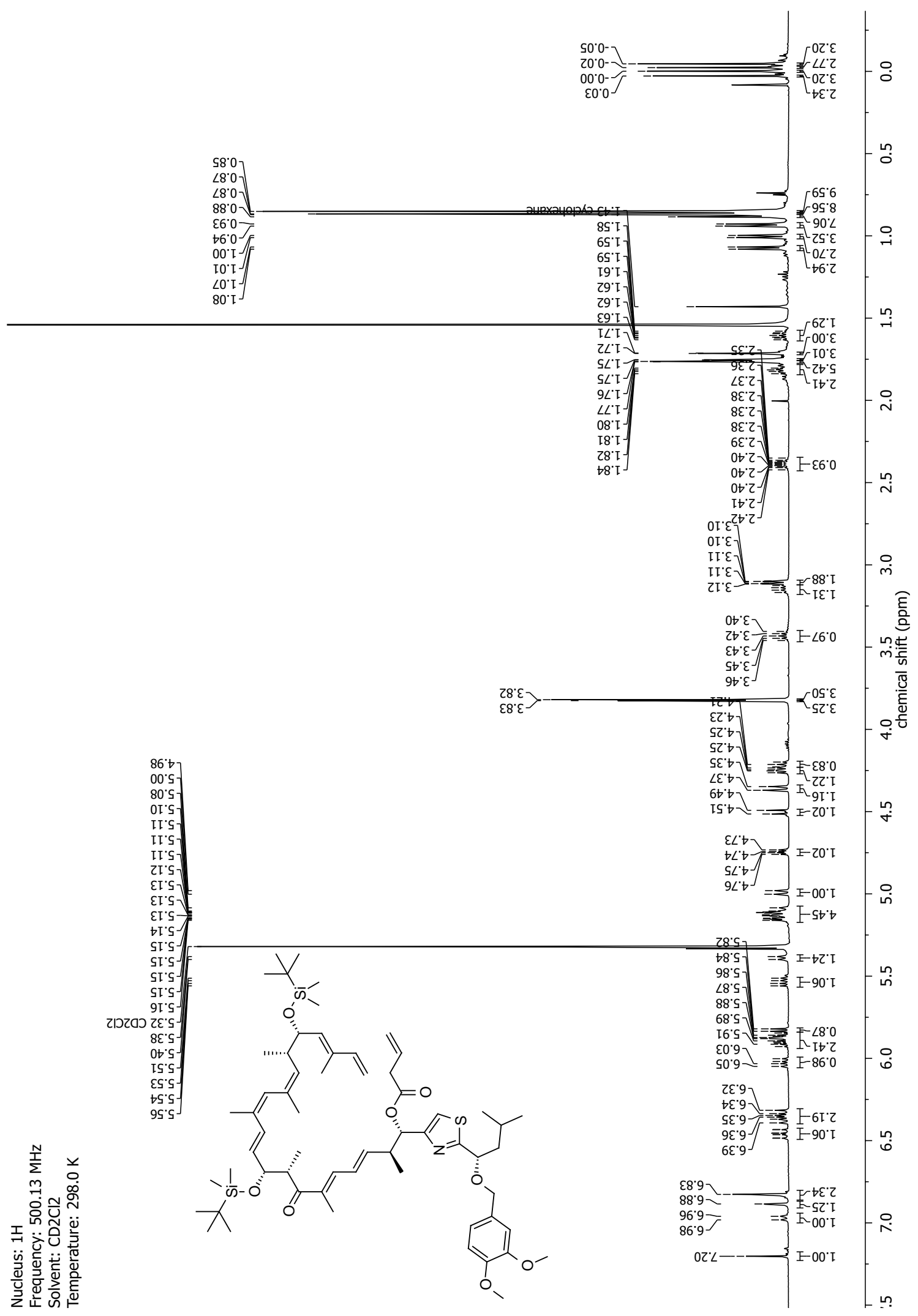




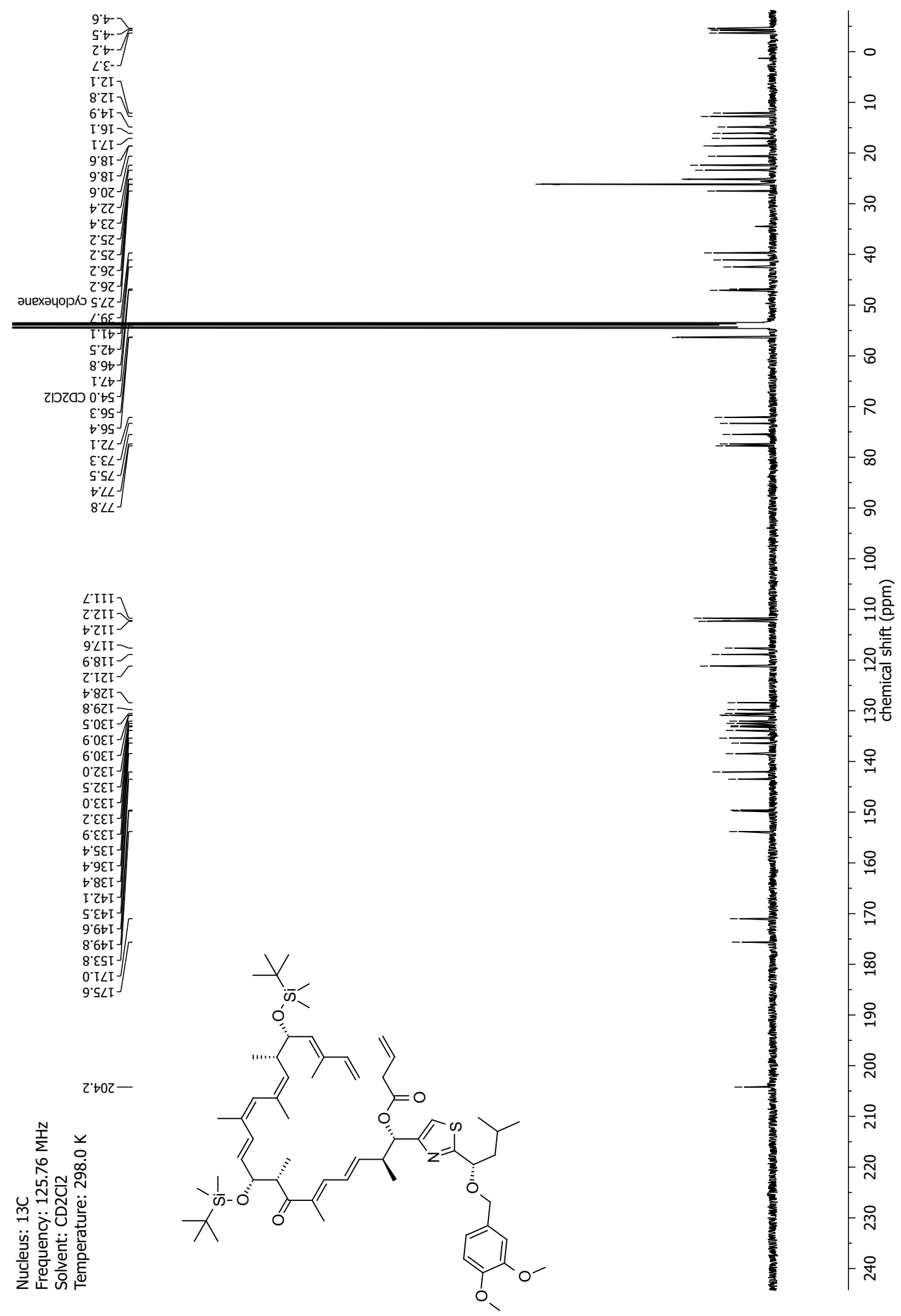


NMR-Spectra for Compound 105

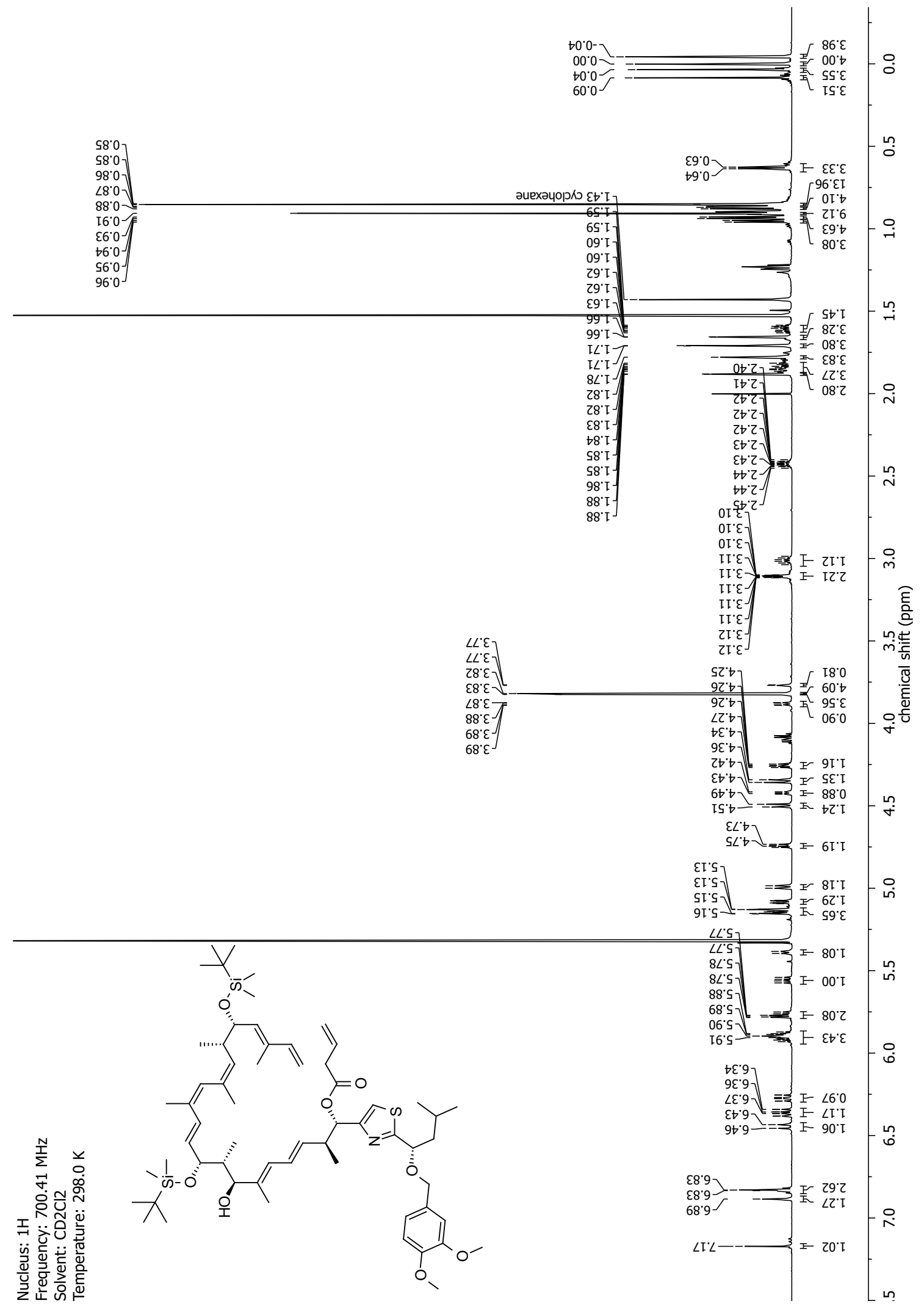




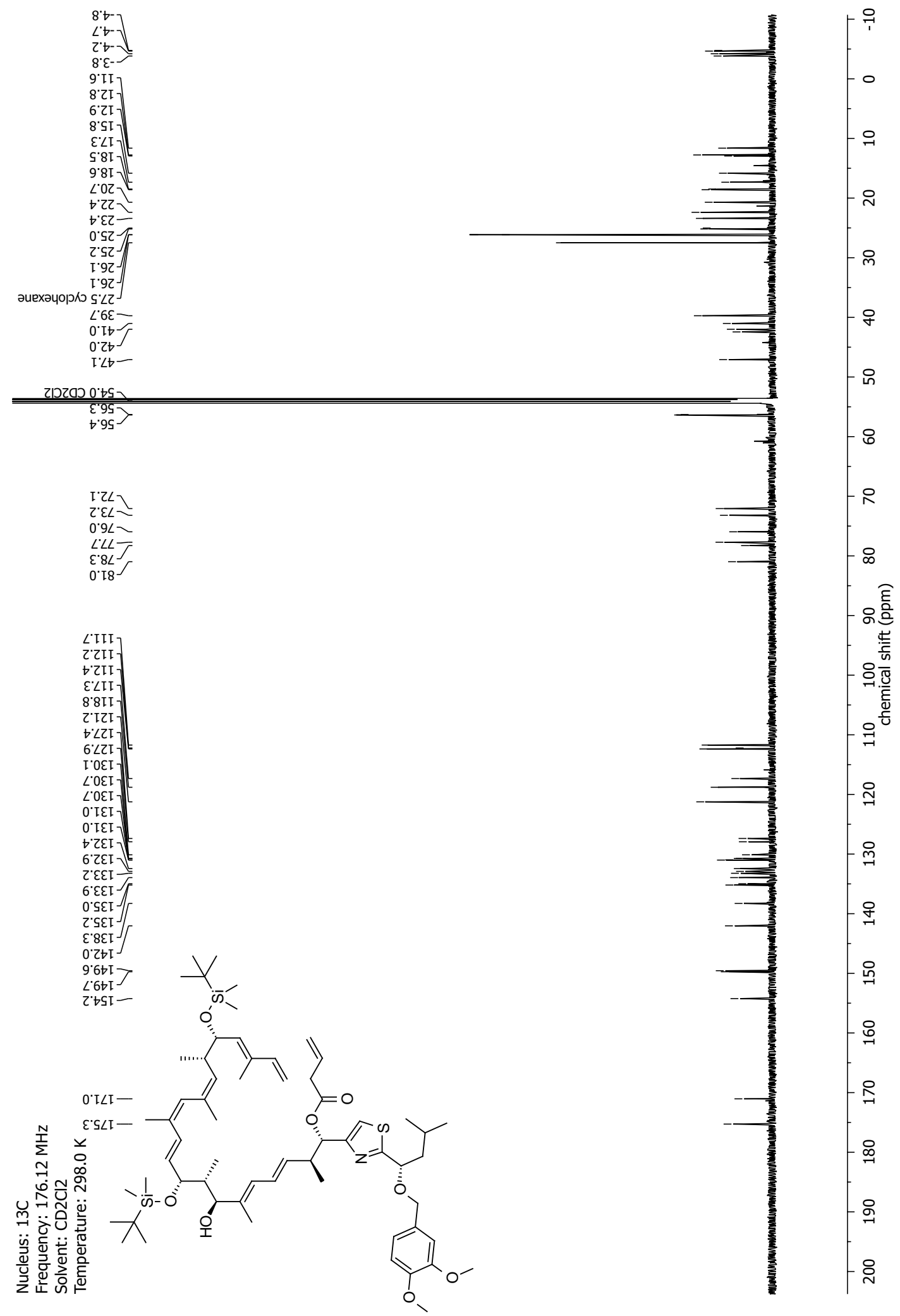




\section{NMR-Spectra for Compound 76}

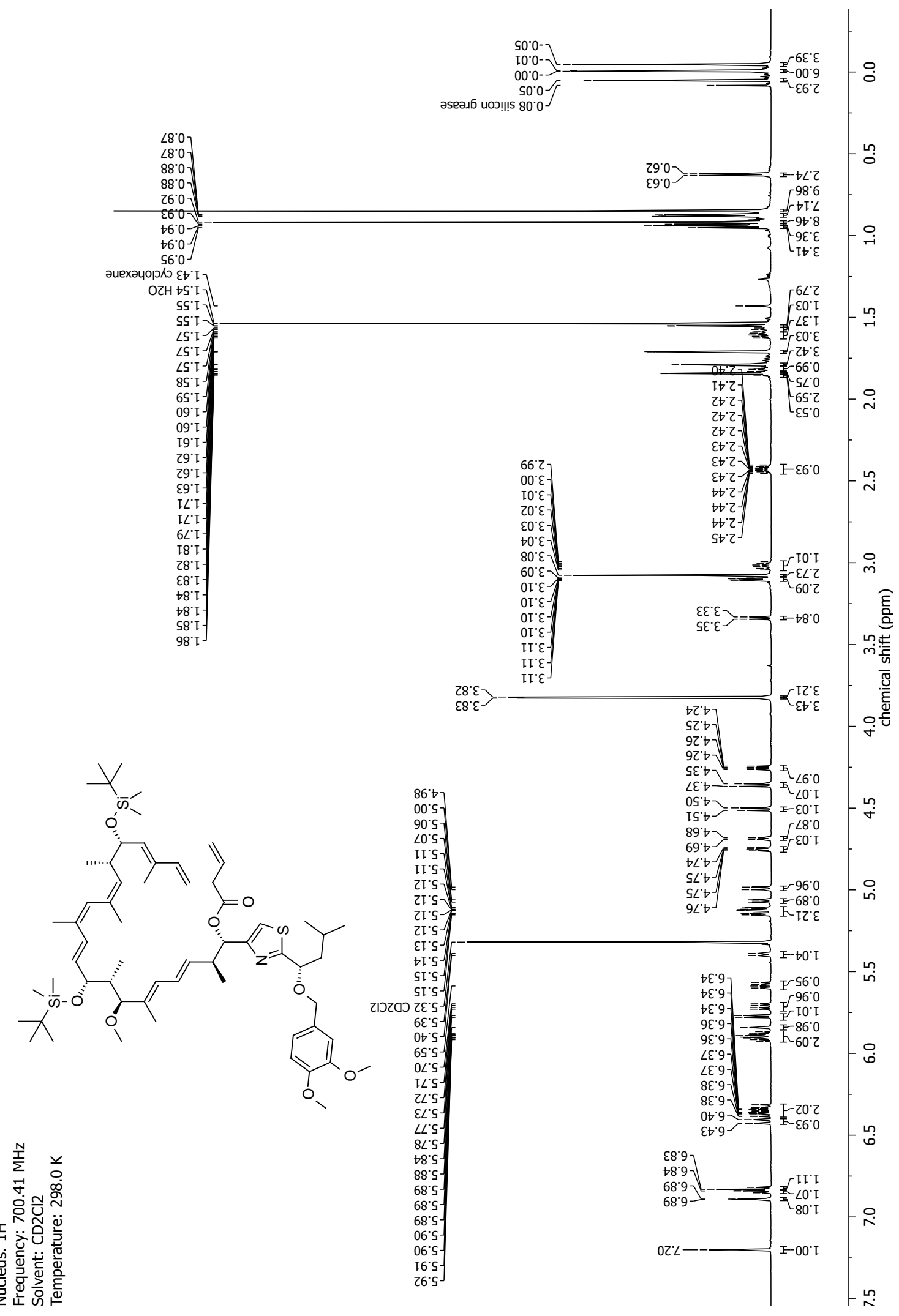




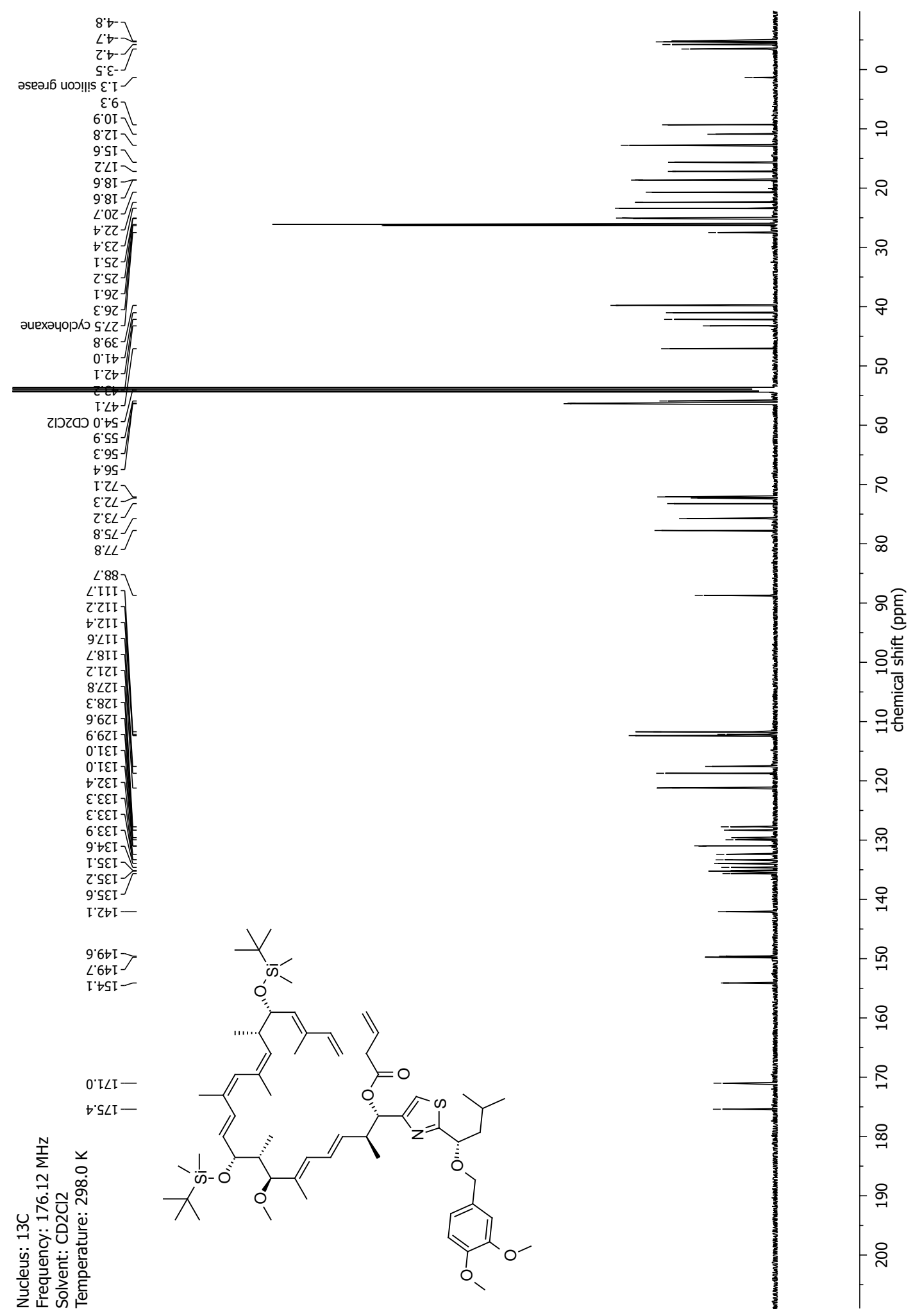


NMR-Spectra for Compound 77

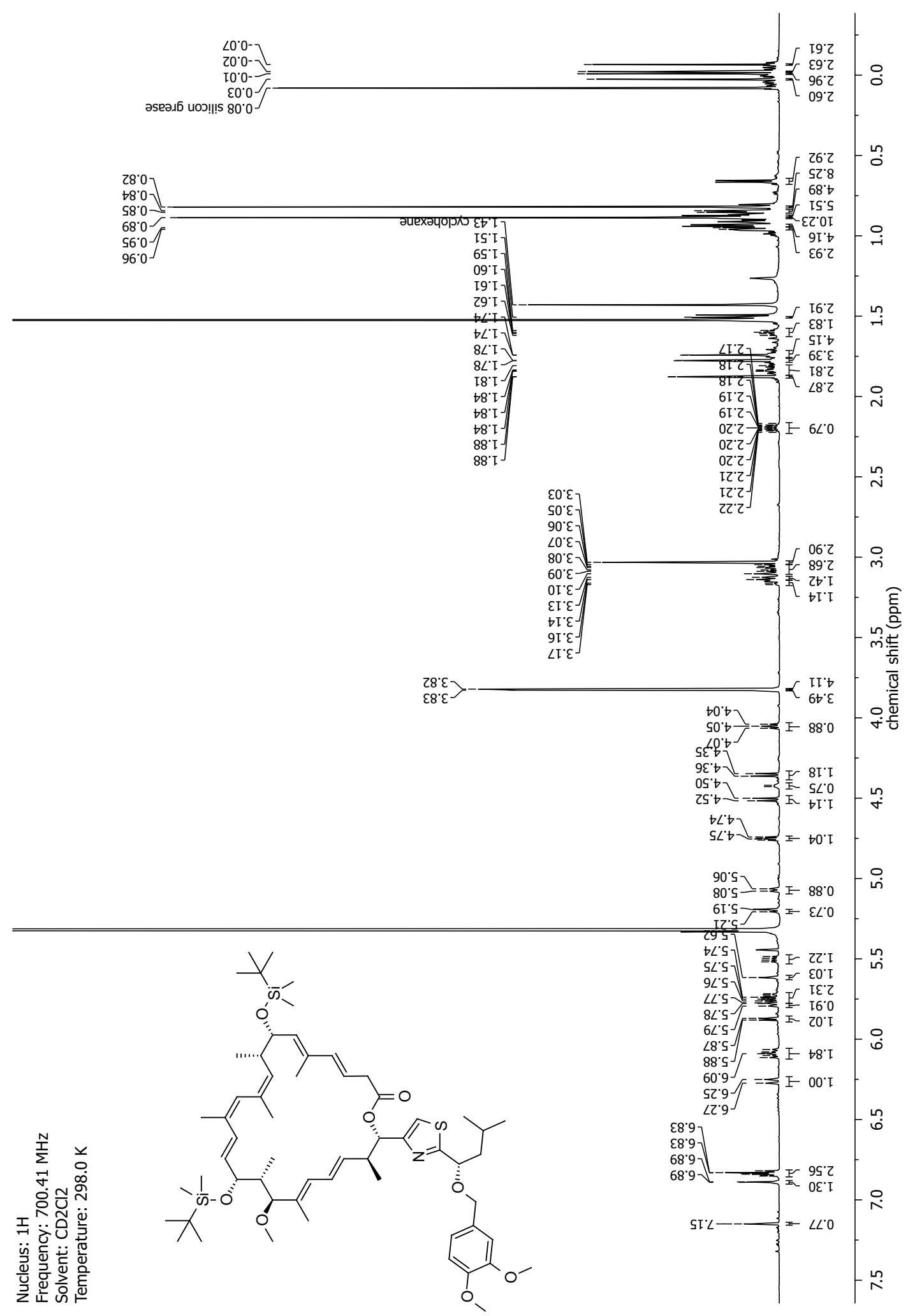




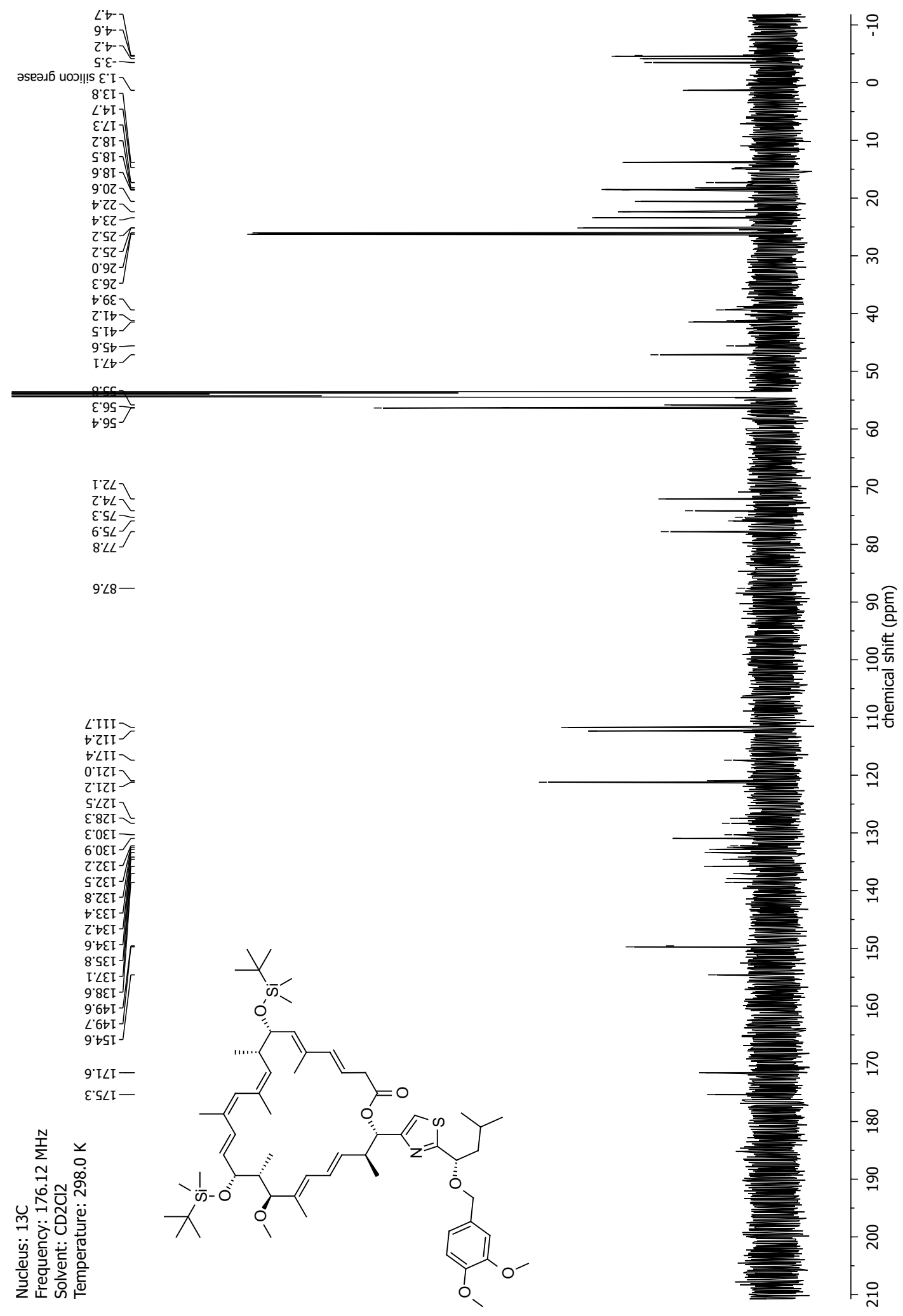


(udd) भ!บऽ ןอว!ฒәцว

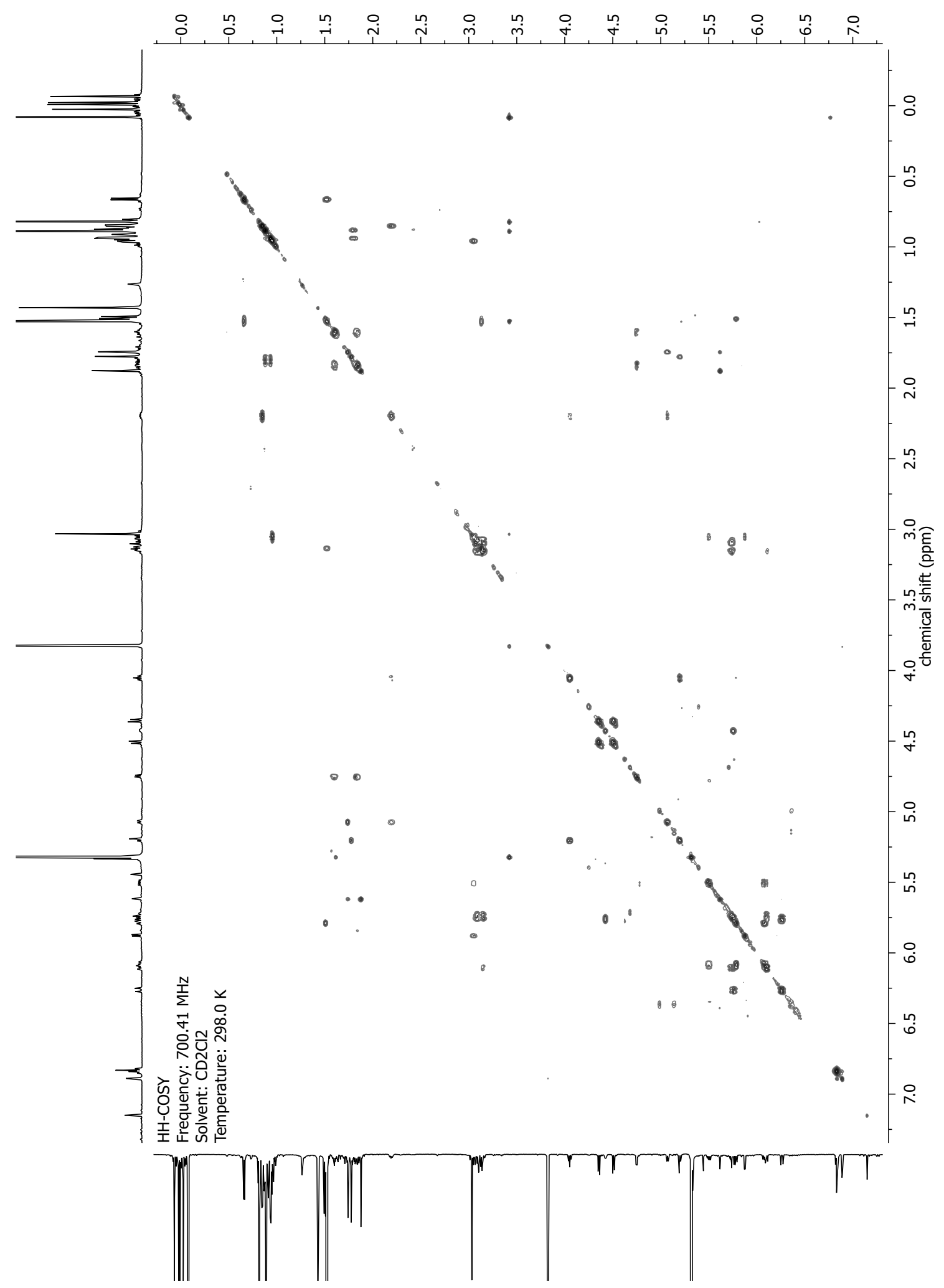


(udd) भ!บऽ ןеว!щәцว

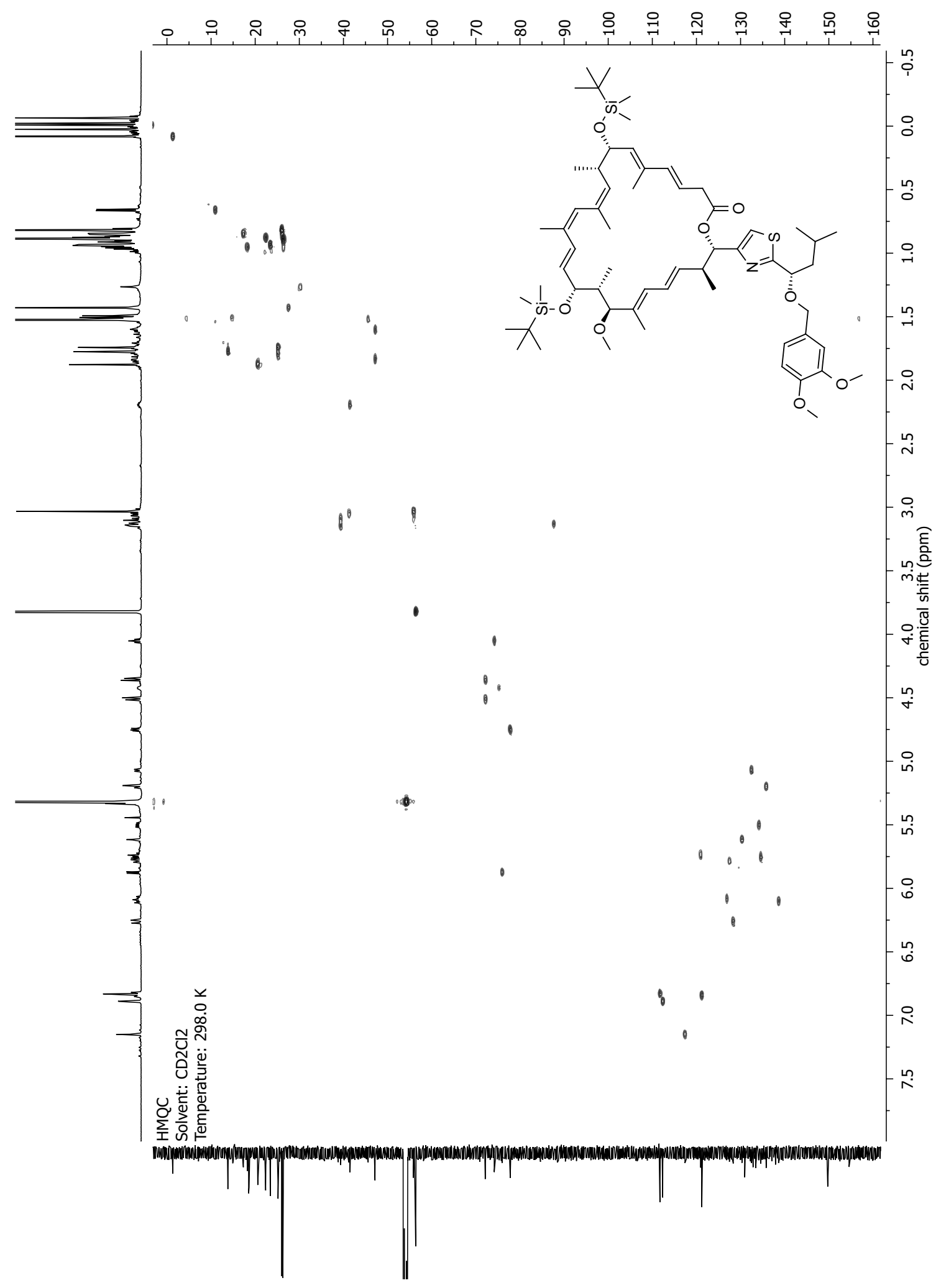




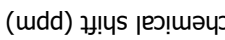
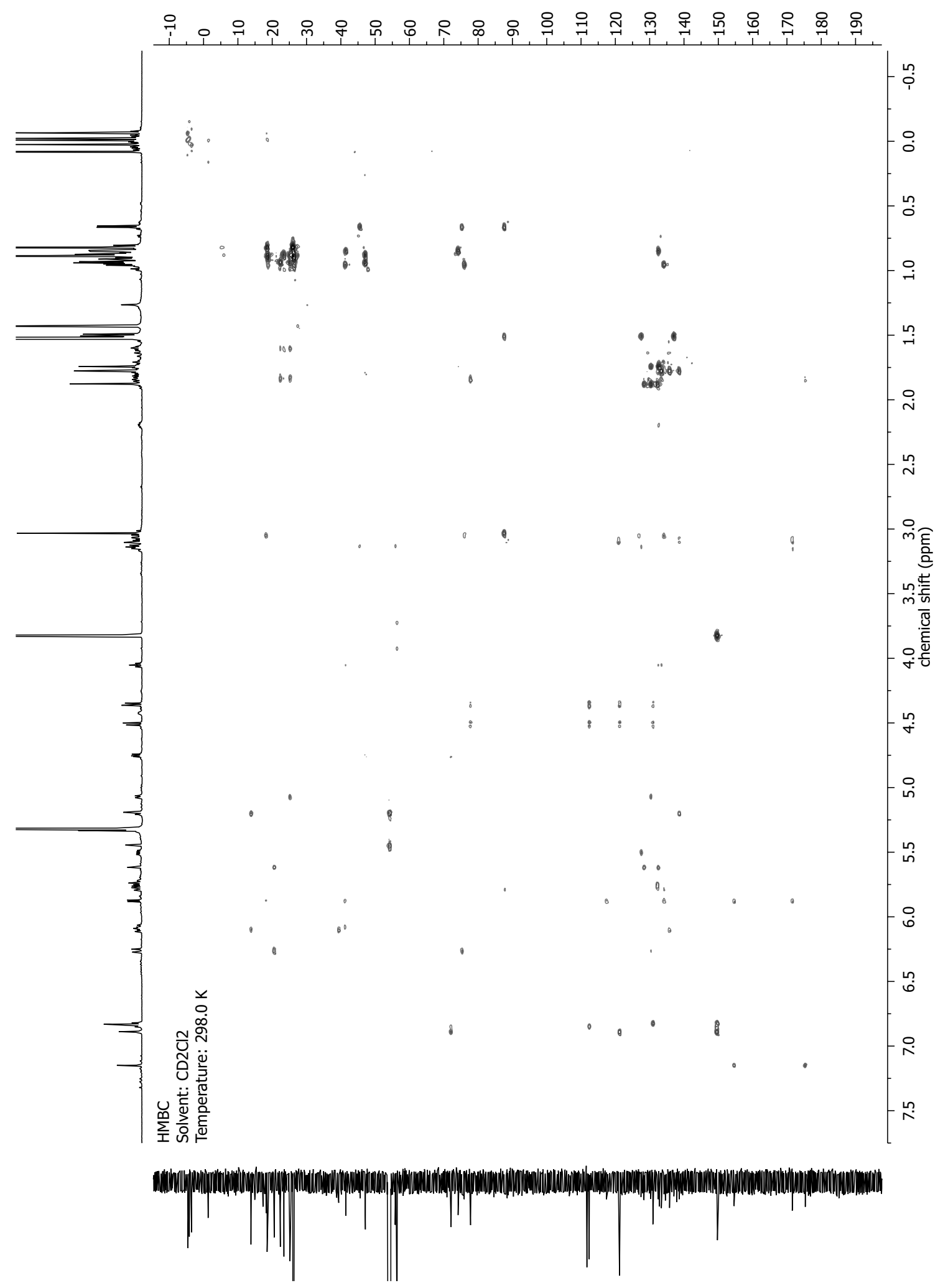
NMR-Spectra for Compound 106

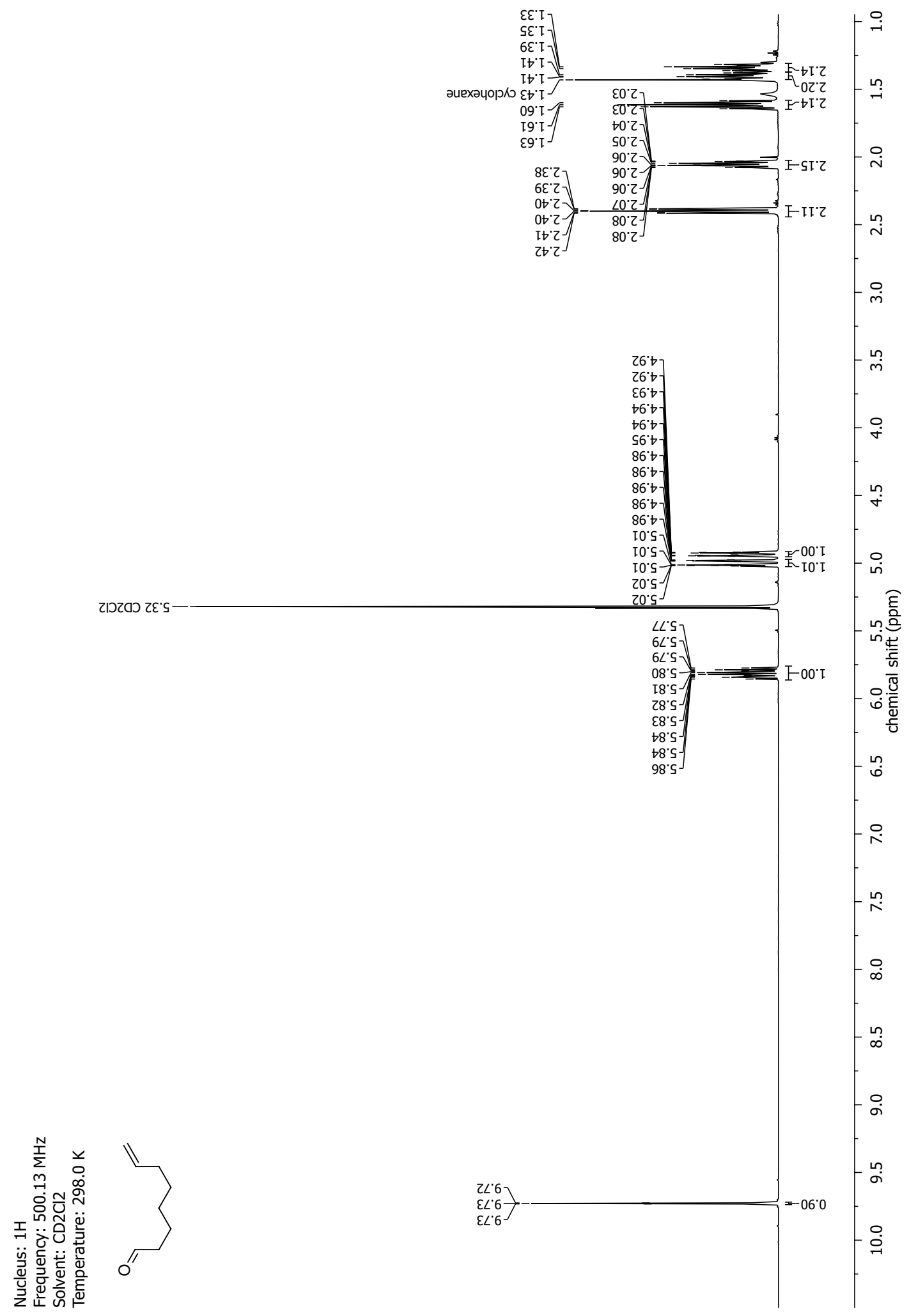




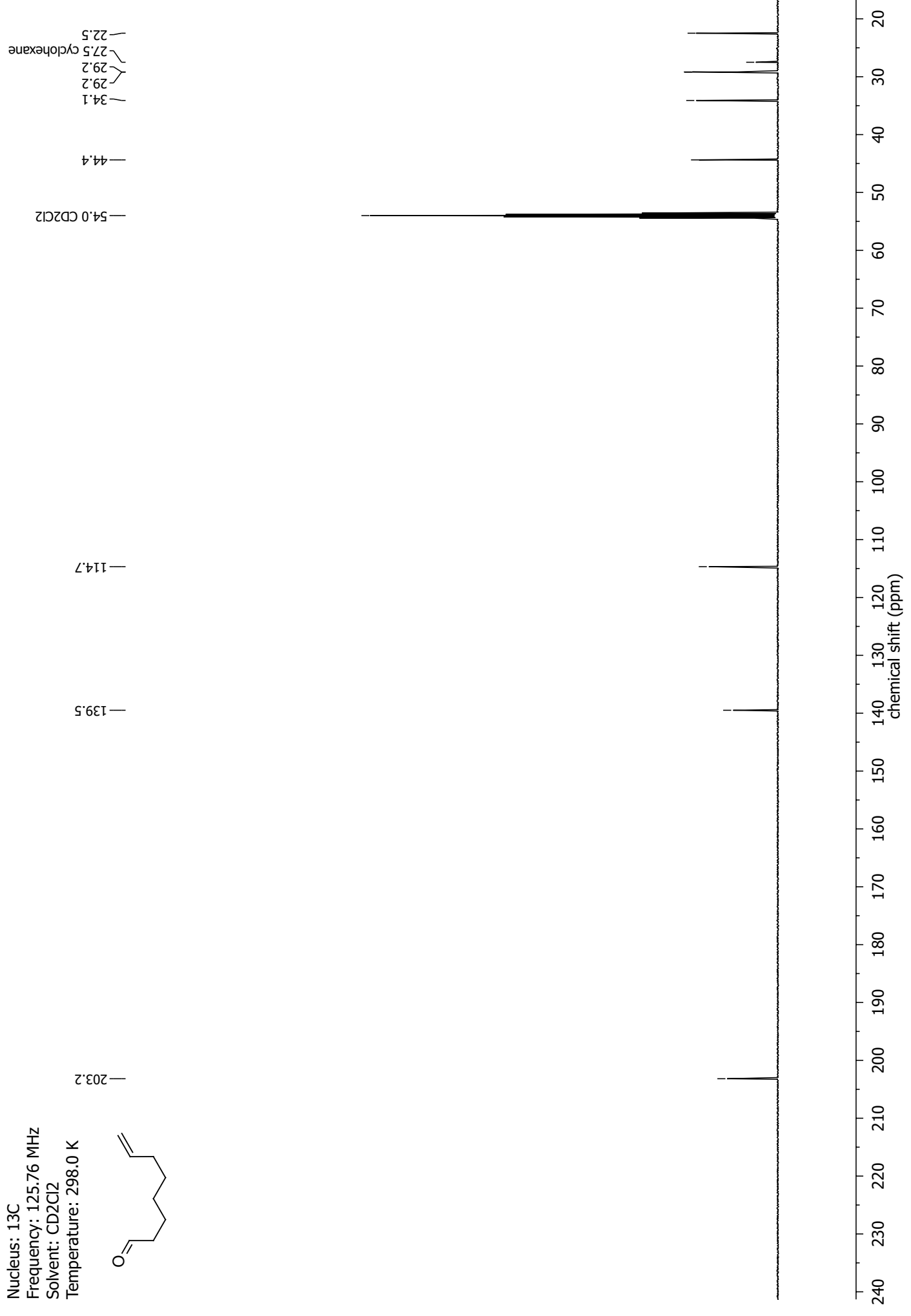


NMR-Spectra for Compound 107

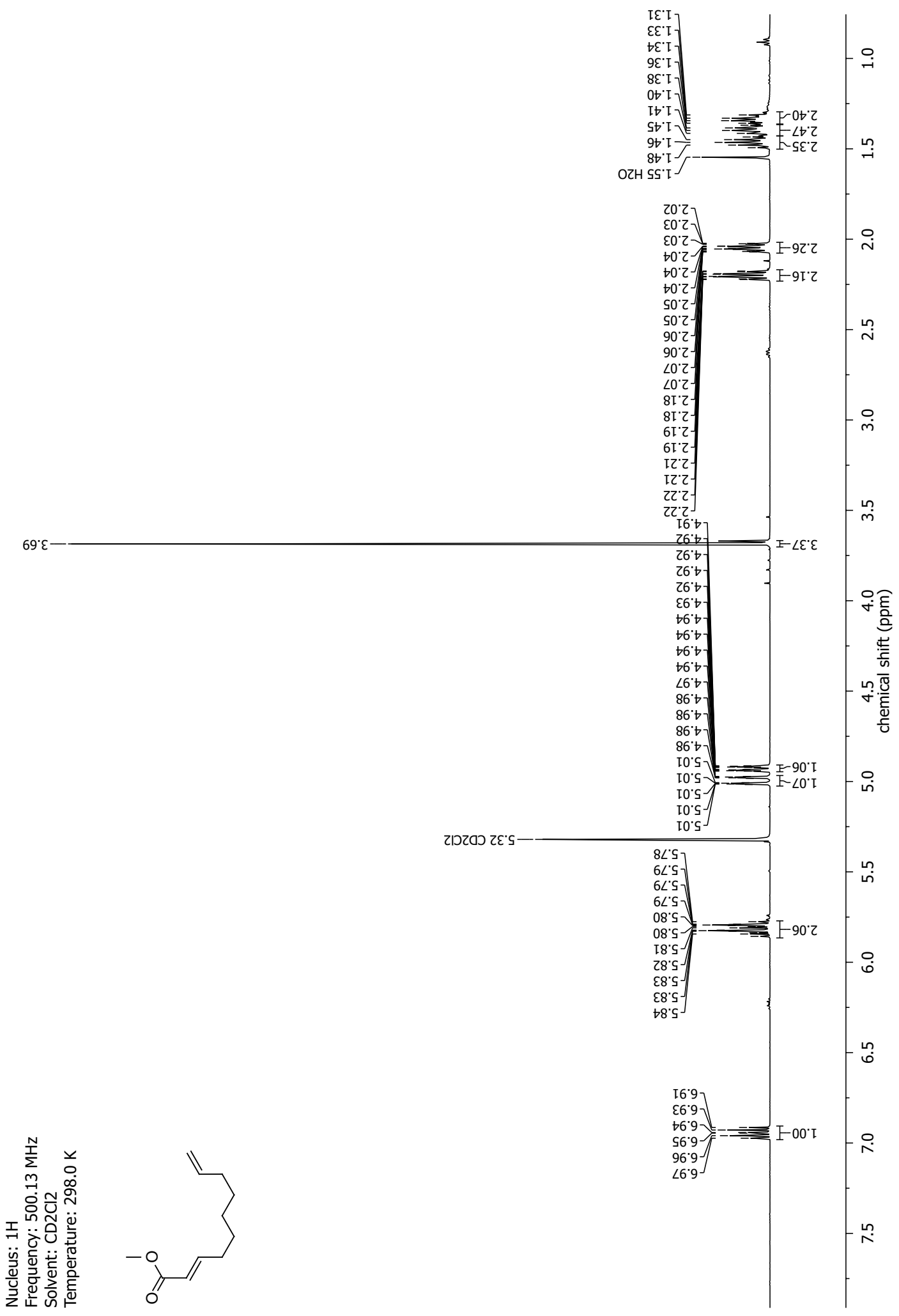




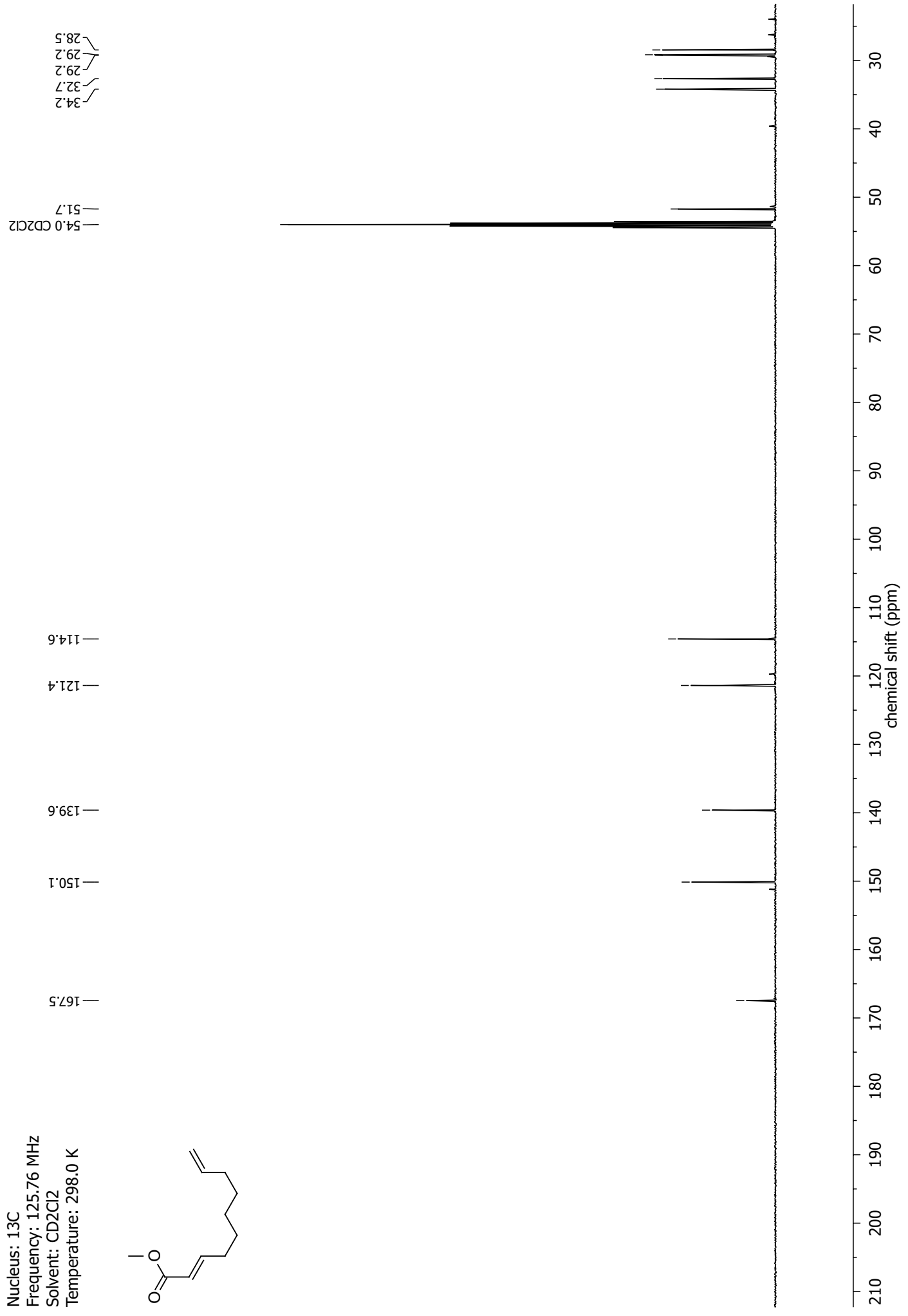


NMR-Spectra for Compound 82

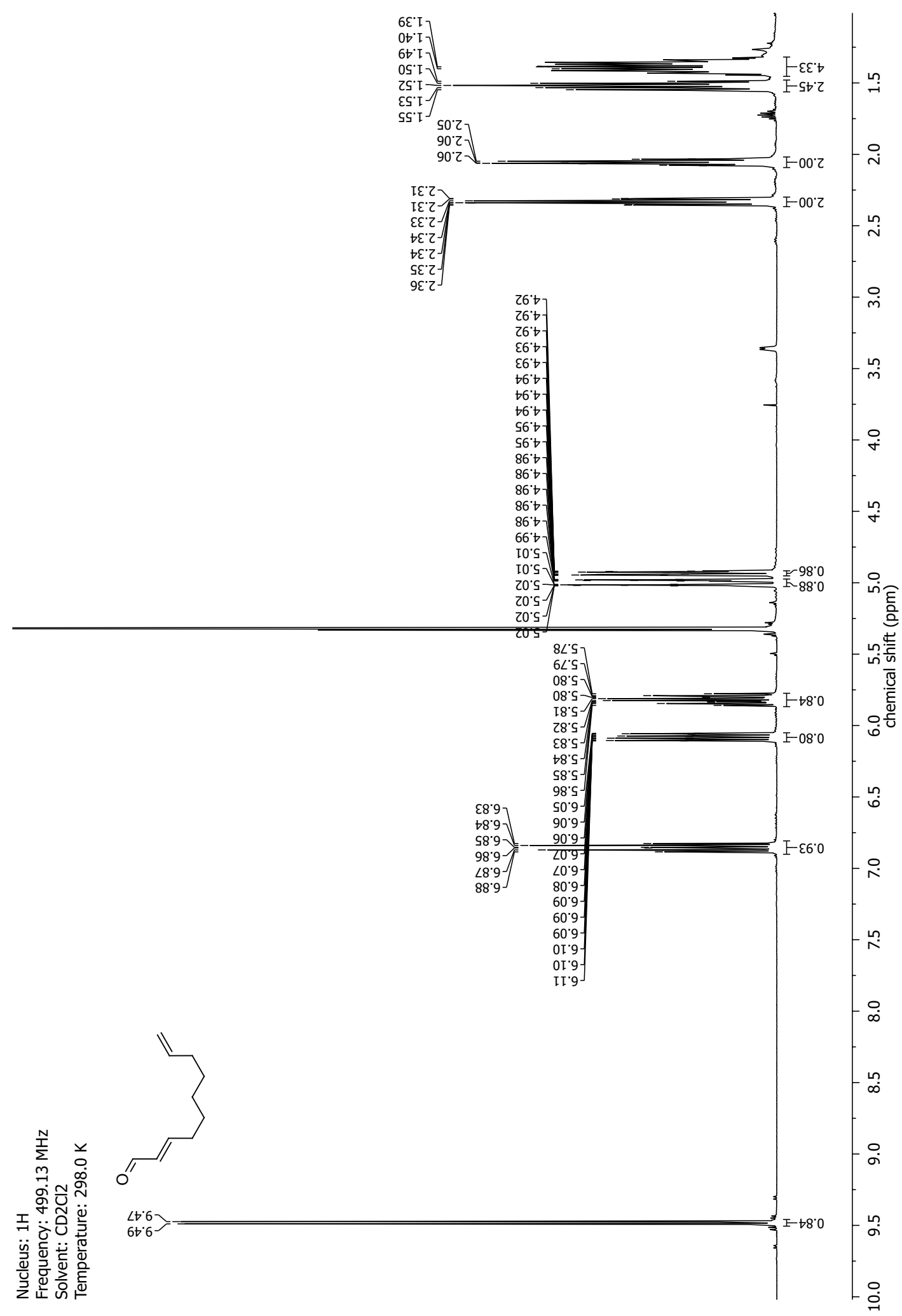




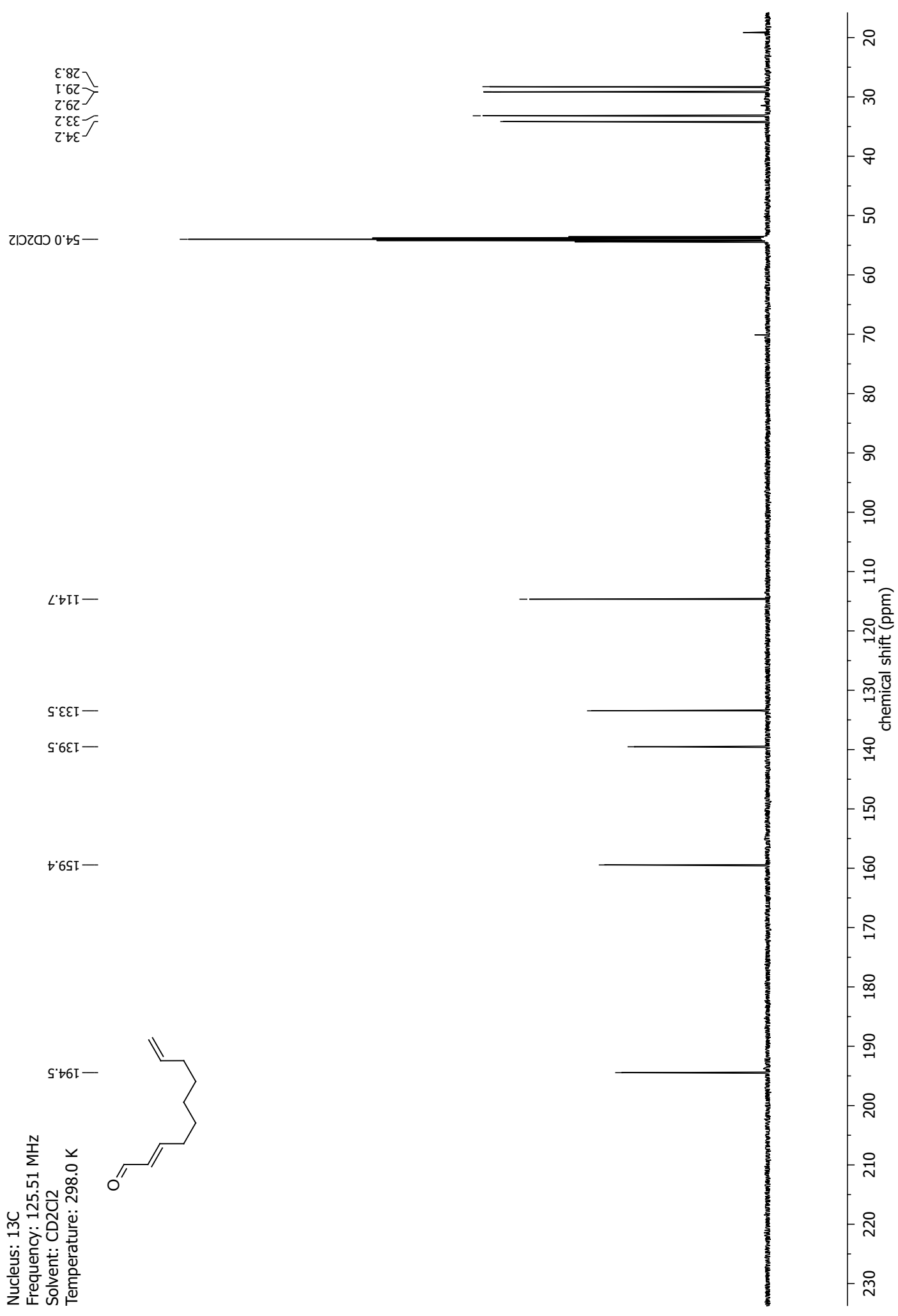


NMR-Spectra for Compound 108

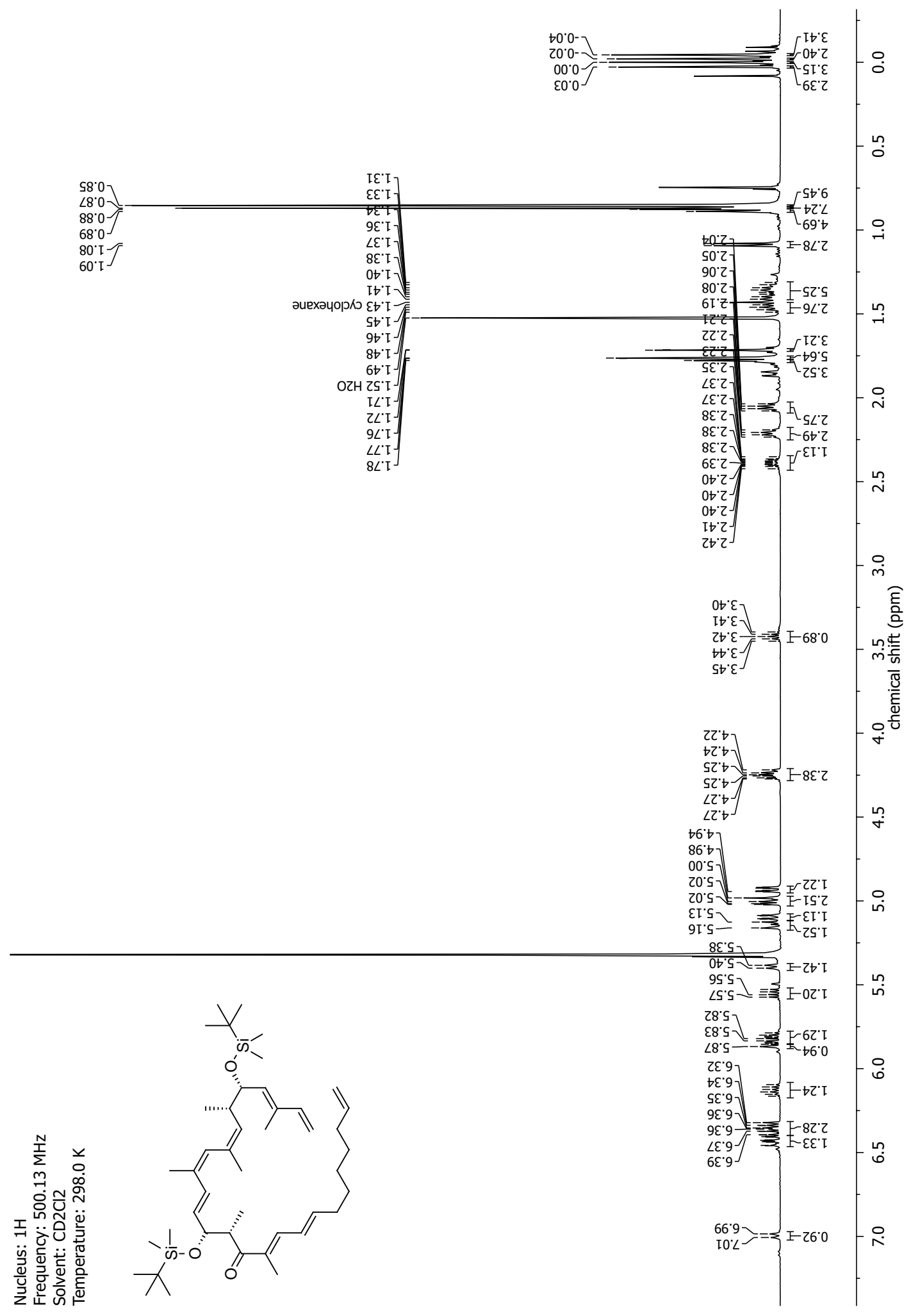




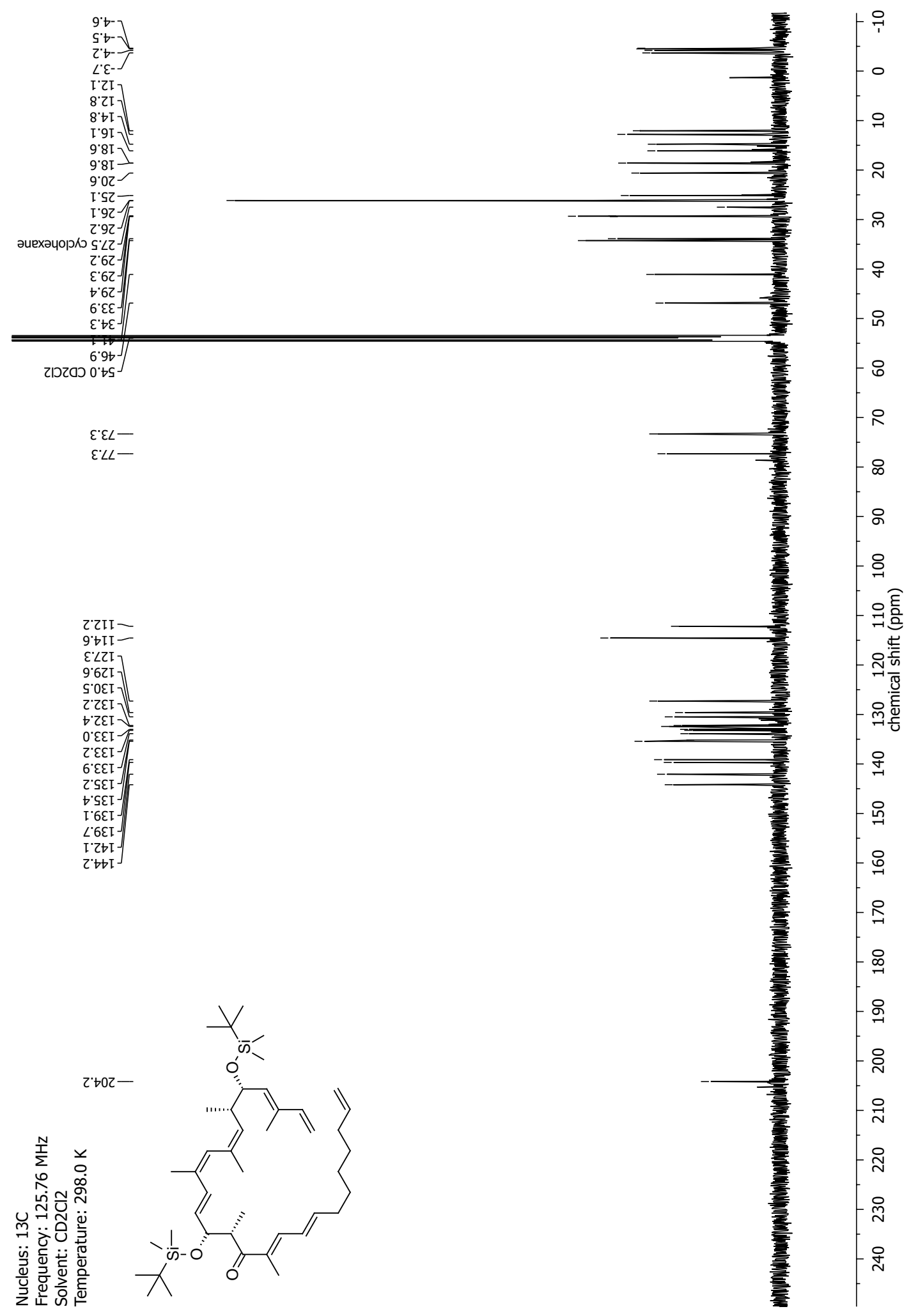


NMR-Spectra for Compound 109

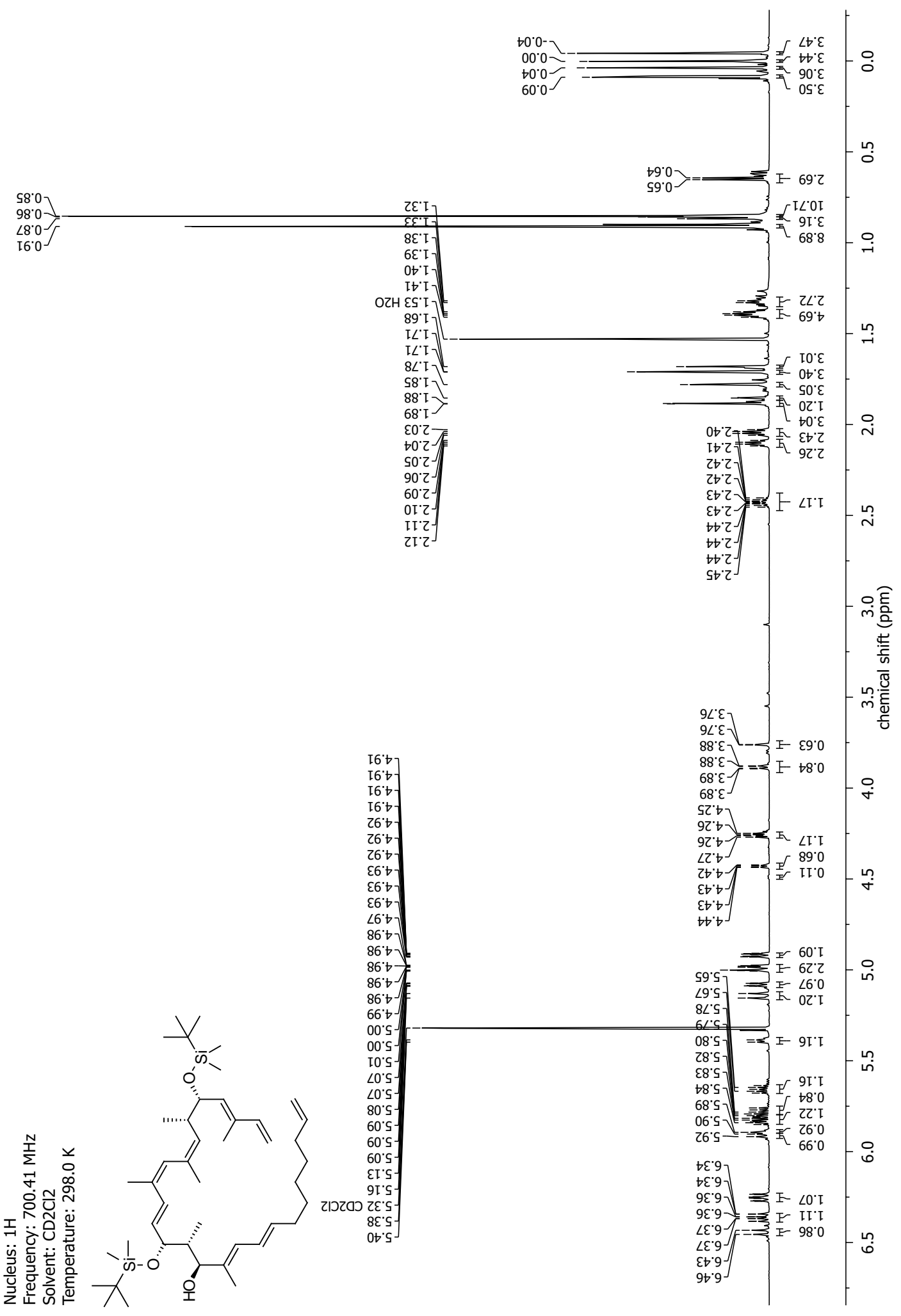




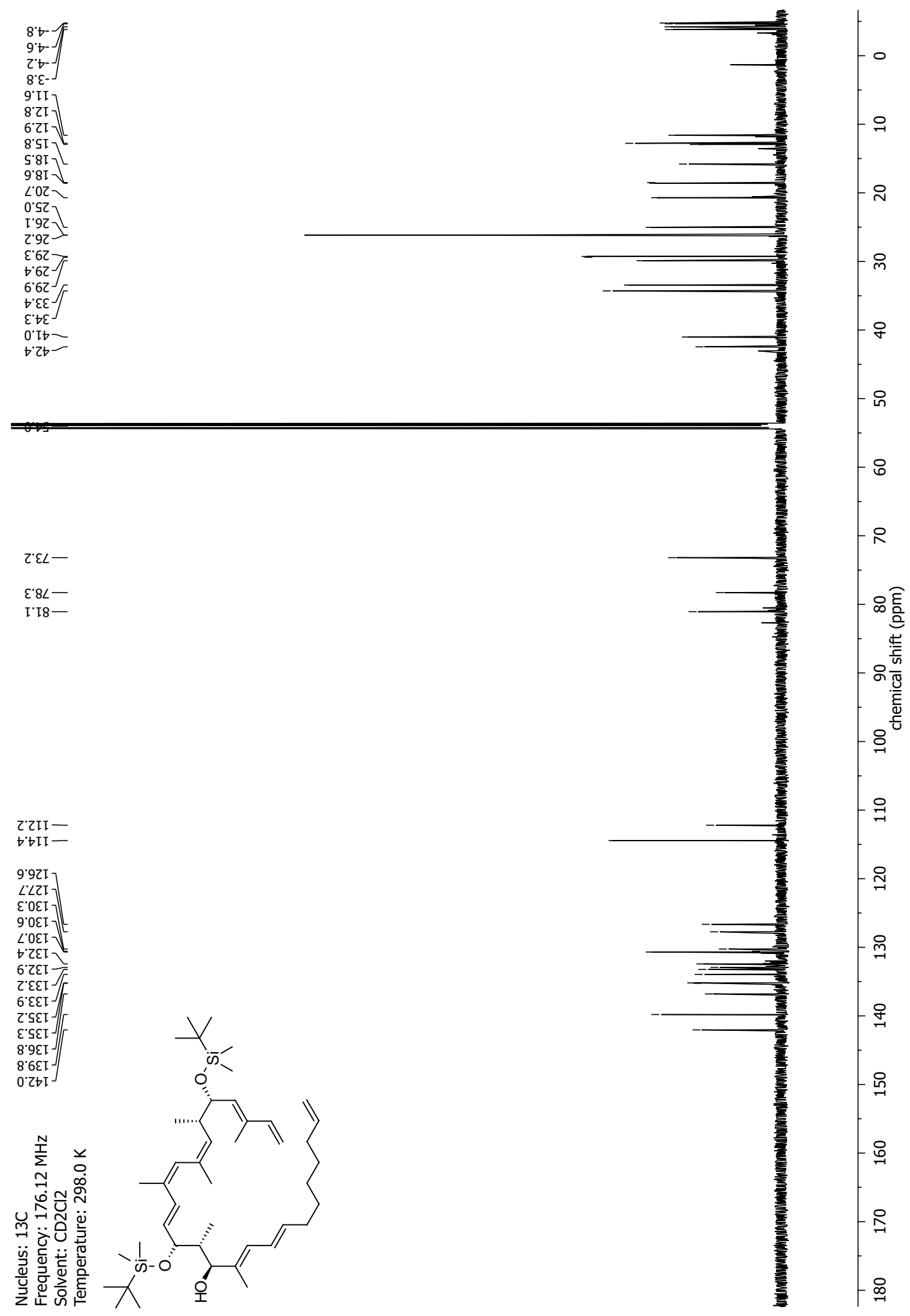


NMR-Spectra for Compound 83

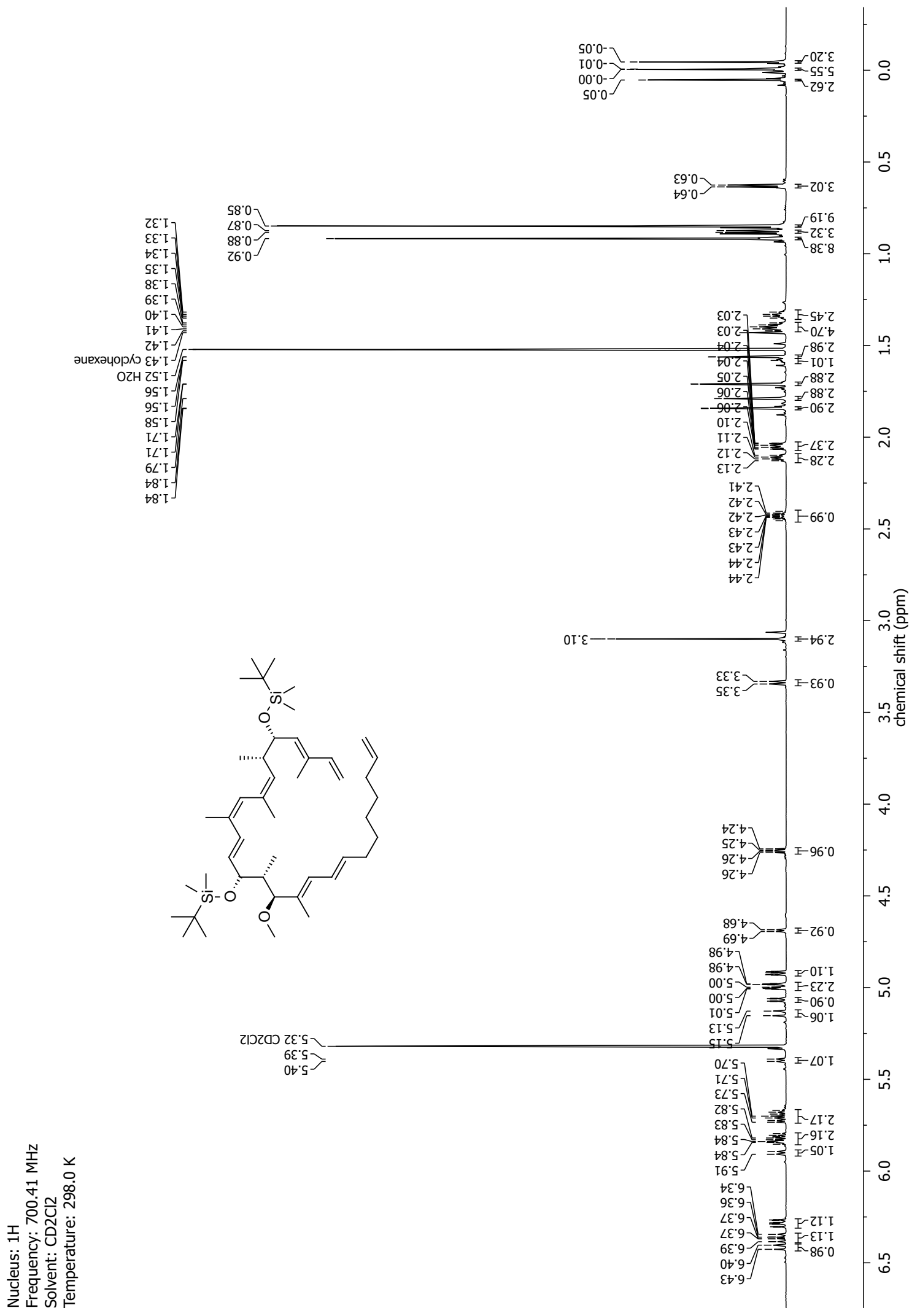




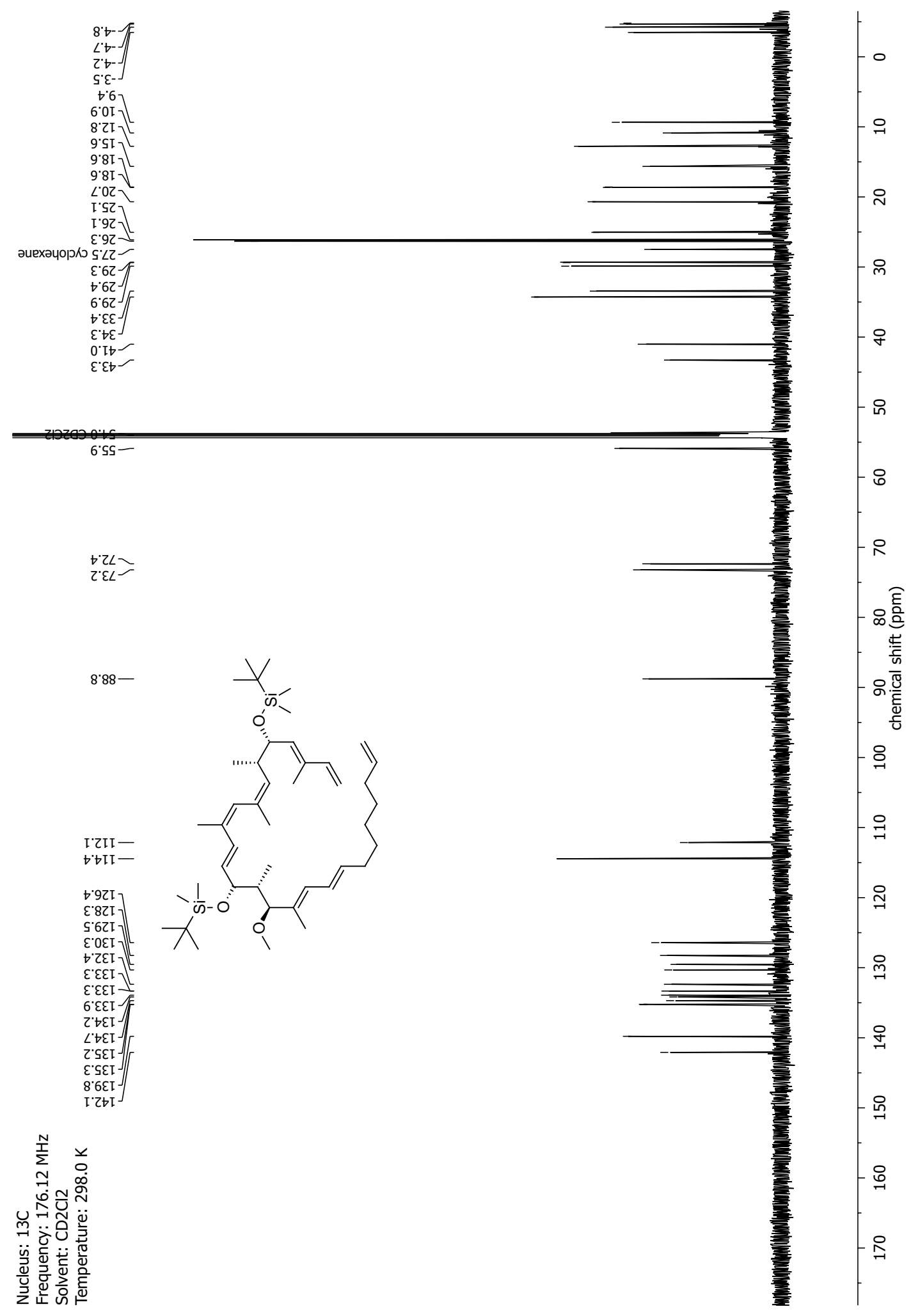

